\title{
Publications of Los Alamos Research 1988
}

\author{
Compiled by
}

Kathryn Varjabedian

Shirley A. Dussart

Wilma J. Mcclary

Jeannette A. Rich

\section{DISCLAIMER}

This report was prepared as an account of work sponsored by an agency of the United States Government. Neither the United States Government nor any agency thereof, nor any of their employees, makes any warranty, express or implied, or assumes any legal liability or responsi. bility for the accuracy, completeness, or usefulness of any information, apparatus, product, or process disclosed, or represents that its use would not infringe privately owned rights. Reference herein to any specific commercial product, process, or service by trade name. trademark, manufacturer. or otherwise does not necessarily constitute or imply its esdorsemen1, recommendation, or favoring by the United States Government or any agency thereof. The views and opinions of authors expressed herein do not necessarily state or reflect those of the United States Government or any agency thereof 


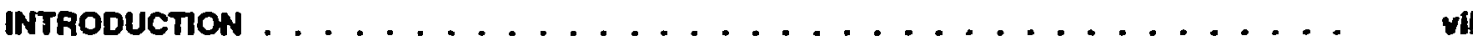

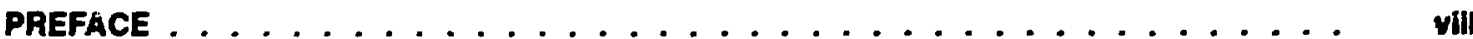

1. BIOLOGY AND MEDICINE $\ldots \ldots \ldots$

2. CHEMICAL HIGH EXPLOSIVES . . . . . . . . . . . . . . . . . . . . .

3. CHEMICAL SEPARATIONS PROCESSES FOR PLUTONIUM AND URANIUM . . . . 12

4. CHEMISTRY . . . . . . . . . . . . . . . . . . . . 13

6. COAL CONVERSION AND UTILIZATION-COAL SCIENCE AND ANALYSIS . . . . . 24

7. CRITICALITY STUDIES . . . . . . . . . . . . . . . . . . 25

8. ELECTRIC ENERGY SYSTEMS-SYSTEMS DEVELOPMENT AND CONTAOL . . . . 26

9. ENERGY CONSERVATION . . . . . . . . . . . . . . . . . 27

10. ENERGY-GENERAL . . . . . . . . . . . . . . . . . . . . . 28

12. ENERGY STORAGE . . . . . . . . . . . . . . . . . . . 29

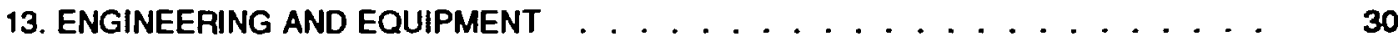

14. ENVIRONMENTAL CONTROL TECHNOLOGY AND EARTH SCIENCES . . . . . 38

16. GENERAL REACTOR TECHNOLOGY . . . . . . . . . . . . . . . 44

17. GENERAL, MISCELLANEOUS, AND PROGRESS REPORTS (NONNUCLEAR) . . . 47

18. GENERAL, MISCELLANEOUS, AND PROGRESS REPORTS (NUCLEAR) . . . . . . 50

19. GEOTHERMAL ENERGY . . . . . . . . . . . . . . . . . 54

20. GEOTHERMAL ENERGY-EXPLORATION TECHNOLOGY . . . . . . . . . . . . 55

21. GEOTHERMAL ENERGY-RESOURCE DEVELOPMENT _............ $\ldots 56$

22. HEALTH AND SAFETY . . . . . . . . . . . . . . . . . . . 57

23. INERTIAL CONFINEMENT FUSION .................. 58

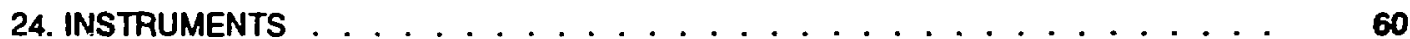

25. ISOTOPE SEPARATION ......................... 66

26. LIGHT WATER REACTOR TECHNOLOGY . . . . . . . . . . . . . . 67

27. LIQUID METAL FAST BREEDER REACTORS . . . . . . . . . . . . . . . . 68

29. MAGNETIC FUSION ENERGY . . . . . . . . . . . . . . . . . . 69

30. MAGNETIC FUSION ENERGY-FUS!ON SYSTEMS . . . . . . . . . . . . . . 74

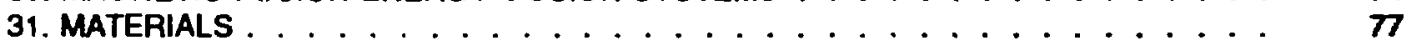

32. MATHEMATICS AND COMPUTERS . . . . . . . . . . . . . . . .

33. NUCLEAR MATERIALS . . . . . . . . . . . . . . . . . . . . . . . . . . . . 101

34. NUCLEAR PROPULSION SYSTEMS AND AEROSPACE S.AFETY . . . . . . . . 102

35. NUCLEAR WASTE MANAGEMENT . . . . . . . . . . . . . . 103

37. PARTICLE ACCELERATORS AND HIGH VOLTAGE MACHINES .......... 105

38. PETROLEUM AND NATURAL GAS ..................... 117

39. PHYSICS-ATOMIC AND MOLECULAR . . . . . . . . . . . . . . . . 118

40. PHYSICS-COSMIC AND TERRESTRIAL . . . . . . . . . . . . . . 126

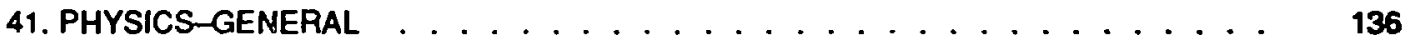

42. PHYSICS-NUCLEAR . . . . . . . . . . . . . . . . 157

43. PHYSICS-PARTICLES AND FIELDS . . . . . . . . . . . . . . 169

44. PLASMA PHYSICS, EXPERIME.JTAL . . . . . . . . . . . . . . . 178

45. PLASMA PHYSICS, THEORETICAL . . . . . . . . . . . . . . . 180 
46. RADIOISOTOPE AND RADIATION APPLICATIONS . . . . . . . . . . . . $18 i$

47. SAFEGUARDS-NUCLEAR MATERIALS SECURITY AND MANAGEMENT . . . . . . 182

49. SOLAR HEATING AND COOLING . . . . . . . . . . . . . . . . . . 196

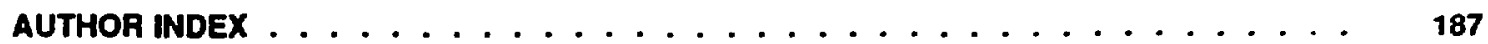

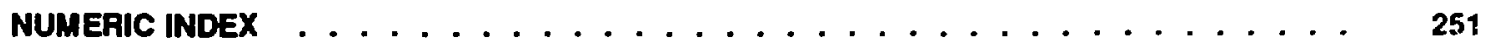

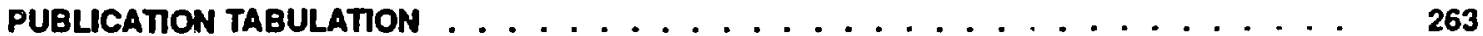




\title{
PIJBLICATIONS OF LOS ALAMOS RESEARCH 1988
}

\author{
Compiled by
}

Kathryn Varjabedian, Shirley A. Duseart, Wilma J. McClary, and Joannotte A. Rich

\begin{abstract}
ABSTAACT
This bibliography lists anclassified publleations of work done at the Los Alamos National Laboratory for 1963. The entries, which are subdivided by broad subject categorles, are cross-referenced with an author index and a numeric index.
\end{abstract}

\section{INTRODUCTION}

Los Alamos National Laboratory, which is operated for the US Department of Enargy (DOE) by the University of California, was founded in 1943 as part of the wartime Mantrattan Project at the site of the Los Alamos Ranch School, a boys' preparatory school in the mountains of ncrthern New Mexico. It had a single assignment-to create the world's first nuclear fission bombs. Success in that task, one of the greatest scientific and technological achievements of all time, was followed by equal success in developing the first thermonuclear weapons. Since 1952, the Laboratory has diversified its programs, and, while still serving as one of the nation's two development centers for nuclear weapons, Los Alamos now devotes much of its total effort to unclassitied research, exploring several peaceful applications of nuclear, conventional, and alternative forms of energy. Los Alamos research covers a broad spectrum, ranging from medium- and lowenergy nuclear physics research to programs involving superconductivity, medical and biological effects of radiation, and basic work in molecular and cellular biology.

Major nonweapons activities at Los Alamos are energy research in fields such as geothermal energy development, laser fusion research, and centrolled thermonuclear research using magnetic confinement.
Facilities used in such research at the Laboratory include specialized laboratories; a nuclear reactor designed for a variety of experiments; and particle accelerators such as the 24-MeV, three-stage Van de Graatt particle accelerator and the 27-MeV electron accelerator designed to produce a very intense, very stron burst of $x$ rays for high-speed flash radiography. At the Los Alamos Meson Physics Facility, where physics experiments are carried out and where production of a variety of radioisotopes for niedical use is under way, an 800-MeV, 1-mA-beam linear accelerator produces beams of protons, mesons, and other nuclear particles with intensities up to 10000 times greater than those available elsewhere. Another of the Laboratory's facilities, $Z T-40$, is a toroidal reversed-field device for controlled thermonuclear research involving magnetic confinement of hot plasmas for fusion research. A Central Computing Facility, with two Cray Y-MPs, five Cray X-MPs. and a large selection of other capabilities, including the CM-2 (Connection Machine) in the Advanced Computing Laboratory, assists in the Laboratory's research.

The Laboratory as of 1988 employed approximately 8000 persons, about one-third of whom are scientists and engineers. The total operating costs of the Laboratory, excluding construction, are about $\$ 892$ million per year. The investment in DOE-oivned buildings and capital equipment at Los Alamos is over $\$ 1$ billion. 


\section{PREFACE}

The Los Alamos Libraries' continuing program of locating, recording, and announcing publications of Los Alamos-sponsored research has resulted in these previous publications:

"Selected Bibliography of Publications of LASL Research, 1957-1962" (LAMS-2895 and supplement),

"Publications of LASL Research" for each of the years 1963 through 1966 ,

"Publications of LASL Research, 1967-1971" (ILA5400-MS).

"Publications of LASL Research, 1972-1976" (LA6755-MS, Vols. I and II),

"Publications of Los Alamos Research, 1977-1981" (LA-9435-MS, Vols. I and II),

"Publications of Los Alamos Research, 1982" (LA9800-MS),

"Publications of Los Alamos Research 1983" (LA10155-MS).

"Publications of Los Alamos Research 1984" (LA10450-MS),

"Publications of Los Alamos Research 1985" (LA10760-MS),

"Publications of Los Alamos Research 1986" (LA11050-MS), and

"Publications of Los Alamos Research 1987" (LA11400-MS).

This bibliography is a compilation of unclassilied publications of work done at the Los Alamos National Laboratory for 1988. Publications not included in earlier compilations have also been listed. Declassitication of previously classified reports is considered to constitute publication. All classified issuances are omitted-even those papers, themselves unclassified, which were published only as part of a classified document. If a paper was published more than once, all known places of publication are included.

The bibliography includes Los Alamos National Laboratory reports, papers released as non-Laboratory reports, journal articles, books, chapters of books, conterence papers either published separately or as part of conference proceedings issued as books or reports, papers published in congressional hearings, theses, and US patents. Publications by Los Alamos authors that are not records of Laboratory-sponsored work are included when the Libraries become aware of them.

The arrangement in each bibliographical citation is author(s) first, title (indented), and facts of publication (further indented). The CHEMICAL ABSTRACTS abbreviation is used for journal citations except for the capitalization. The group designations given after the authors' names indicate the groups with which the authors were associated when the papers were written. Parenthetical information includes report and reprint numbers, book call numbers, and patent numbers. The parentheses indicate the information by which Los Alamos borrowers may obtain the material from the Lus Alamos Libraries.

The entries are arranged in seclions by broad subject categories. Within each section they are alphabetical by author. All types of publications are interfiled.

The author and numeric indexes refer to the item numbers, which consist of a number designating the section followed by a dash and a number for the alphabetic arrangement of the item within the section. In the numeric index, the LA- and LA- $x \times x \times x-M S$, etc., numbers of reports are interfiled.

A Keyword-in-Context index included in earlier compilations is not provided.

Most of the reports listed in this publication are available for sale from

National Technical Information Service

US Department of Commerce

Springfield, VA 22161

or on request by government agencies and their contractors from

Office of Scientific and Technical Information

Technical Information Center

US Department of Energy

P.O. Box 62

Oak Ridge, TN 37831

Reprints of some journal articles are available as long as the supply lasts from the first author listed. Address requests to the first author at

Los Alamos National Laboratory

University of California

P.O. Box 1663

Los Alamos, NM 87545

This bibliography has been prepared by the Los Alamos Library Services Group, IS-4. from the records of the Classification Office, from abstracting and indexing journals, and from supplementary library files. Inquiries regarding any of the materials included should be directed to the Cataloging Section of the Los Alamos Libraries.

The Libraries gratefully acknowledge the assistance of members of the Laboratory's Administrative Data Processing Division for assistance with the computerized system for handling $\boldsymbol{p}$ jlication information. 
$1-1$

BELL, GEORGE I. (T-DO).

HUMAN GENOME-COMPUTATHONAL CHALLENGES.

TIC, 1988. 19P. MN (LA-UR-88-0450)

$1-2$

BRADBURY, JANES N. (MP-3).

PION PRODUCTION CROSS-SECTIONS AND ASSOCIATED PARAMETERS.

IN: ADVISORY GROUP MEETING ON NUCLEAR AND ATOMIC DATA FOA RADIOTHERAPY A!ID RELATED RADIOBIOLOGY, 1985, RIJSWIJK, NETHERLANDS. PROC., P.297-308. INTERNATIONAL ATO MC ENERGY AGENCY, 1987. (OC784.5 A38 1985X)

ALSO PUBUSHED: TIC, 1985. 18P. MN (LA-UR-85-3366)

1-3

BURKS, CHRISTIAN $(T-10)$.

GENBANK DATABASE AND THE FLOW OF SEOUENCE DATA FOR THE HUMAN GENOME.

IN: SCIENCE WRITERS WORKSHOP ON BIOTECHNOLOGY AND THE HUMAN GENONE, 1987. BROOKHAVEN NATIONAL LABOHATOPY. PROC., P.51-6. PLENUM, 1988. (CH301.837X V.46)

1-4

BUSH, DANIEL R. (INC-4); LANGSTON-UNKEFER, PAT J. (INC-4).

AMINO ACID TRANSPORT INTO MEMBRANE VESICLES ISOLATED FROM ZUCCHINI.

PLANT PHYSIOL., V.88, P.497-90. 1988.

1.5

CLARK, JOHN W. T-11/CNLS).

STATISTICAL MECHANICS OF NEURAL NETWORKS.

PHYS. REP., V.158, P.92-157. 1988.

1-6

COOPER, JANET L. (LS-4); WAKSHULL, ERIC (LS-4); WHARTON, W. R. (LS-4).

CHARACTERIZATION OF THE REBINDING OF 125 LEPIDERAML GROWTH FACTOR RELEASED FRCM

BALBC-3T3 CELLS FOLLOWING ACCUMIULATION IN PRESENCE OF CHLOAOCUINE.

J. CELL PHYSKOL., V.134, P.387-95. 1988.

$1-7$

CFAM, L. SCOTT (LS-4); BARTHOLDI, MARTY FRANK (LS-4); RAY, FRANK A. (LS-4); MEYNE, NULIANNE (LS-3); MOVZIS, ROBERT K. (LS-3); SCHWARZACHER-AOBINSON, TRUDE (LS-4); KRAEMER, PAUL M. (LS-4).

OVERVIEW OF FLOW CYTOGENETICS FOR CLINICAL APPLICATIONS.

CYTOMETRY SUPPL, V.3, P.94-100. 1988.

$1-8$

CRISSMAN, HARRY A. (LS-4); WILDER, MARK E. (LS-4); TOBEY, ROBERT A. (LS-1).

FLOW CYTOAETRIC LOCALIZATION WITHIN THE CELL CYCLE AND ISOLATION OF VABLE CELLS

FOLIOWNG EXPOSURE TO CYTOTOXIC AGENTS.

CANCER RES., V.48, P.5742-6. 1988.

1.9

CRISSMAN, HARAY A. (LS-4); HOFLAND, MARIANNE H.; STEVENSON, ANITA P. (LS-4);

WILDER, MARK E. (LS-4); TOBEY, ROBEAT A. (LS-1).

USE OF DIO-C5-3 TO IMPROVE HOECHST 33342 UPTAKE, RESOLUTION OF ONA CONTENT, AND SUPMVAL OF CHO CELLS.

EXP. CELL RES., V.174, P.388-96. 1988.

$1-10$

D'ANNA, JOSEPH A. (LS-3); GPADY, DEBORAH L (LS-1); TOBEY, ROBEAT A. (LS-3).

CHROMATIN STRUCTURAL CHANGES PAECEDE HEPUCATION IN INITUATED REPLCONS DURHG INHIBITION OF ONA ELONGATION.

IN: UCLA SYMPOSIUM ON MECHANISMS AND CONSECUENCES OF DNA DAMAGE, 1988, TAOS, N.M. PROC., P.550-43. A.R. LISS, 1988. (OH506 .1262X V.89)

ALSO PUBLSHED: TIC, 1988. 6P. MN (LA-UR-88-0266) 
$1-11$

DEAVEN, LARRY L. (LS-4); VAN DILLA, M. A.

NATIONAL LABORATORY GENE LIBRARY PROJECT.

TIC, 1988. 8P. MN (LA-UR-88-3544)

$i-12$

DURBIN, RICHARD D.; LANGSTON-UNKEFER, PAT J. (INC-4).

MECHANISMS FOR SELF-PROTECTION AGAINST BACTERIAL PHYTOTOXINS.

ANN. REV. PHYTOPATHOL, V.26, P.313-29. 1988.

$\hat{i} \cdot 13$

FARMER, J. DOYNE (CNLS); KAUFFMAN, STUART A.; PACKARD, NORMAN H. (CNLS).

AUTOCATALYTIC REPLICATION OF POLYMERS.

PHYSICA, V.22D, P.50-67, 1986.

ALSO PUBLISHED: EVOLUTION, GAMES, AND LEARNING MODELS FOR ADAPTATION IN MACHINES AND NQTURE. INTERNATIONAL CONFERENCE OF THE CENTER FOA NONLINEAR STUDIES. 5TH. 1985. LOS ALAMOS, N.M. PROC., P. 50-67. NORTH-HOLLAND, 1986. (CP408 .C46 1985)

1-14

FARMER, J. DOYNE (CNLS); PACKAFD, NORMAN H. (CNLS); PERELSON, ALAN S. (T-10).

IMMUNE SYSTEM, ADAPTATION, AND MACHINE LEARNING.

PHYSICA, V.22D, P.187-204. 1986.

ALSO PUBLISHED: EVOLUTION, GAMES, AND LEARNING-MODELS FOR ADAPTATION IN MACHINES AND NATURE. INTERNATIONAL CONFEFENCE OF THE CENTER FOR NONLINEAR STUDIES, $5 T H, 1985$. LOS ALAMOS, N.M. PROC., P. 187-204. NORTH-HOLLAND, 1986. (QP408 .C46 1985)

$1-15$

FEE, JAMES A. (INo-4); MATHER, MICHAEL W. (INC-4); SPRINGER, PENELOPE (INC-4); HENSEL, S.; 8USE, G. ISOLATION AND PARTIAL SEQUENCE OF THE A-PROTEIN GENE OF THERMUS THERMOPHILUS CYTOCHROME CIAAB.

ANN. N. Y. ACAD. SCI., V.550, P.32-8. 1988.

ALSO PUBLISHED: TIC, 1988. 13P. MN (LA-UR-88-2954)

$1-16$

FREYER, JAMES P. (LS-1).

ROLE OF NECROSIS IN REGULATING THE GROWTH SATURATION OF MULTICELLULAR SPHEAOIDS.

CANCER RES., V.48, P.2432-9. 1988.

$1-17$

FREYER, JAMES P. (LS-4); SCHOA, P. L. (LS-4); SAPONARA, ARTHUR G. (LS-2).

PARTIAL PURIFICATION OF A PROTEIN GROWTH INHIBITOR FROM MULTICELLULAR SPHEROIDS.

BIOCHEM. BIOPHYS. RES. COMMUN., V.152, P.463-8, 1888.

1.18

GARCIA, ANGEL E. (T-10); KRUMHANSL, JAMES ARTHUR.

ROLE OF THE COULOMB INTERACTION IN THE LOW-FREQUENCY DENSITY OF STATES OF DNA DOUBLE HELICES.

PHYS. REV., V.37A, P.4875-8. 1988.

1-19

GOLDSTEIN, BYRON (T-10); WIEGEL, FREDERIK W.

DISTRIBUTION OF CELL SURFACE PROTEINS ON SPREADING CELLS-COMPARISON OF THEOAY WITH

EXPERIMENT.

BIOPHYS. J., V.53, P.175-84. 1988.

1-20

GOLOSTEIN, BYAON (T-10); WOFSY, CARLA; HERAS, HECTOR E.

EFFECT OF MEMBRANE FLOW ON THE CAPTURE OF RECEPTORS BY COATED PITS- THECRETICAL

RESULTS.

BIOPHYS. J., V.53, P.405-14. 1888. 
$1-21$

GURLEY, LAWRENCE R. (LS-1); VALDEZ, JOSEPH G. (LS-2); LONDON, JERRY E. (LS-1);

SPALL, W. DALE (LS-1); DETHLOFF, L. A. (LS-1); LEHNERT, BRUCE E. (LS-1).

DEVELOPMENT AND CHARACTERIZATION OF AN HPLC SYSTEM FOR THE FRACTIONATION OF BRONCHOALVEOLAR LAVAGE FLUID.

LOS ALAMOS NATIONAL LABORATORY, 1988. 41P. (LA-11309-MS)

$1-22$

GURLEY, LAWRENCE R. (LS-2); LONDON, JERRY E. (LS-1); DETHLOFF, L. A. (LS-1);

STAVERT, DOUGLAS M. (LS- i); LEHNERT, BRUCE E. (LS-1).

ANALYSIS OF PROTEINS IN BRONCHOALVEOLAR LAVAGE FLUIDS DURING PULMONARY EDEMA RESLILTING FROM NITROGEN DIOXIDE AND CADMIUM EXPOSURE.

TIC, 1988. 1OP. MN (LA-UR-88-2790)

$1-23$

HALLIDAY, KAREN R.; PREISER, CECILIA F.; SILLERUD. LAUREL O. (LS-3).

DIFFERENTIATION OF HUMAN TUMORS FROM NONMALIGNANT TISSUE BY NATURAL-ABUNDANCE

CARBON-13 NMR SPECTROSCOPY.

MAGN. RESON. MED., V.7, P.384-411. 1988.

$1-24$

HEIDORN, DOUGLAS B. (LS-7); TREWHELLA, J. (LS-7).

COMPARISON OF THE CRYSTAL AND SOLUTION STRUCTURES OF CALMODULIN AND TROPONIN C.

BIOCHEMISTRY, V.27, P.909-15. 1988.

1-25

HJELM, REX P. (P-LANSCE); THIYAGARAJAN, PAPPANNAN; ALKAN, M. HF.YAT.

SMALL-ANGLE NEUTRON SCATTERING STUDY OF THE EFFECTS OF DILUTION ON PARTICLE

MORPHOLOGY IN MIXTURES OF GLYCOCHOLATE AND LECITHIN.

J. APPL. CAYSTALLOGR., V.21, P.858-63. 1988.

$1-26$

JACKSON, PAUL J. (LS-3); ROBINSON, N. J. (LS-3); DELHAIZE, EMMANUEL (LS-3).

CADMIUM TOLERANCE IN PLANT CELLS-A COMPARISON OF BIOCHEMICAL AND MOLECULAR

PROPERTIES OF TOLERANT AND SENSITIVE CELLS.

IN: UCLA COLLOQUIUM ON METAL ION HOMEOSTASIS-MOLECULAR BIOLOGY AND CHEMISTRY, 1988.

FRISCO, COLO. PROC., P.337-46. LISS, 1989. (QH506 .1262X V.98)

ALSO PUB: ISHED: TIC, 1988. 10P. MN (LA-UR-88-1584)

$1-27$

KNIGHT, THOMAS J. (INC-4); LANGSTON-UNKEFER, PAT J. (INC-4).

ADENINE NUCLEOTIDES AS ALLOSTERIC EFFECTORS OF PEA SEED GLUTAMINE SYNTHETASE.

J. BIOL. CHEM., V.263, P.11084-9. 1988.

1-28

KNIGHT, THOMAS J. (INC-4); LANGSTOH-!JNKEFER, PAT J. (INC-4).

ENHANCEMENT OF SYMBIOTIC DINITROGEN FIXATION BY A TOXIN-RELEASING PLANT PATHOGEN. SCIENCE, V.241, P.951-4. 1988.

1.29

KNIGHT, THOMAS J. (INC-4); BUSH, DANIEL R. (INC-4); LANGSTON-UNKEFER, PAT J. (INC-4).

OATS TOLERANT OF PSEUDOMON,AS SYRINGAE PV. TABACI CONTAIN TABTOXININE-BETA-

LACTAM-INSENSITIVE LEAF GLUTAMINE SYNTHETASES.

PLANT PHYSIOL., V.88, P.333-9. 1988.

$1-30$

LAPEDES, ALAN S. (T-DOT); FARBER, ROBERT M. (T-10).

HOW NEURAL NETS WORK.

IN: IEEE CONFERENCE ON NEURAL INFORMATION PRCCESSING SYSTEMS, 1ST, 1987, DENVER,

COLO. PROC., P.442-56. AMERICAN INSTITUTE OF PHYSICS, 1988. (OC76.5 N45 1988X)

$1-31$

LAYNE, SCOTT P. (CNLS); MARR, T. G. (T-10); STANLEY, E. ANN (T-7); HYMAN, JAMES M. (T-7);

COLGATE, STIRLING A. (T-6).

A NATIONAL HIV [HUMAN IMMUNODEFICIENCY VIRUS] DATABASE THAT FACILITATES DATA SHARING

TIC. 1988. 13P. MN (LA-UR-8B-0125) 
$1-32$

LEHNERT, BRUCE E. (LS-1); TOEVS, K. E. (LS-1); VALDEZ, Y. E. (LS-1); SEBAING, ROBERT J. (LS-1).

PARTICLE-MACROPHAGE RELATIONSHIPS DURING THE CLEARANCE OF PARTICLES FROM THE

ALVEOLAR MACROPHAGE COMPARTMENT.

LOS ALAMOS NATIONAL LABORATORY, 1988. 33P. (LA-11428-MS)

$1-33$

LONDON, JEARY E. (LS-1).

TOXICOLOGICAL STUDY OF 5,6,7,8 TETRAFLURO- 1,4-BENZODIOXIN.

LOS ALAMOS NATIONAL LABORATORY, 1988. 5P. (LA-11 168-MS)

$1-34$

LONDON, JERRY E. (LS-1).

TOXICOLOGICAL STUDY OF GADOLINIUM NITRATE.

LOS ALAMOS NATIONAL LAEORATORY, 1888. 5P. (LA-11261-MS)

1-35

MAPAONE, BABETTA L (LS-4).

LIFE SCIENCES DIVISION ANNUAL REPOAT 1987.

LOS ALAMOS NATIONAL LABORATORY, 1988. 107P. (LA-11235-PR)

$1-36$

MARRONE, BABETTA L (LS-7); CRISSMAN, HARRY A. (LS-4).

CHARACTERIZATION OF GRANULOSA CELL SUBPOPULATIONS FPOM AVIAN PREOVULATORY FOLUCLES

BY MULTIPARAMETER FLOW CYTOMETRY.

ENDOCRINOLOGY, V.122, P.651-8. 1988.

$1-37$

MARRONE, BABETTA L (LS-7); ASEM, ELIKPLIMI K.

CYCUC ADENOSINE MONOPHOSPHATE (CAMP) PRODUCTION IN AVIANJ THECA CELLS DURING FOLLICULAR MATURATION.

J. REPROD. FERTIL., V.83, P.67-71. 1988.

1-38

MATHEA, MICHAEL W. (INC-4).

BASE COMPOSITION-INDEPENDENT HYBRIDIZATION IN DRIED AGAROSE GELS-SCREENING AND RECOVERY FOR CLONING OF GENOMIC DNA FRAGMENTS.

BIOTECHNICUES, V.6, P.444-7. 1988.

$1-39$

MAYER-KRESS, GOTTFRIED (GNLS); LAYNE, SCOTT P. (CNLS).

DIMENSIONAUTY OF THE HUMAN ELECTROENCEPHALOGRAM.

ANN. N. Y. ACAD. SCI., V.504, P.62-87. 1988.

$1-40$

MC ELROY, JOHN F. (LS-7); O'DONNELL, JAMES M. (LS-7).

DISCRIMINATIVE STIMULUS PROPERTIES OF CLENBUTEROL-EVIDENCE FOR BETA ADRENERG!C INVOLVEMENT.

J. PHARMACOL. EXP. THER., V.245, P.155-63. 1988.

$1-41$

MERCER-SMITH, JANET A. (INC-11); ROBERTS, JEANETTE C. (INC-11); FIGARD, STEVE D. (INC-11);

LAYALLEE, DAVID K.

DEVELOPMENT OF COPPER-67-LABELED PORPHYRIN-ANTIBODY CONJUGATES.

IN: ANTIBODY-MEDIATED DELIVERY SYSTEMS, JOHN D. AODWELL, ED. P.317-52. S. DEKKER, 1988.

1-42

MYERS, GERALD (T-10).

HIV ENVELOPE DRIFT.

TIC, 1988. 9P. MN (LA-UR-89-1715) 
$1-43$

NIEDERHOFFER, ERIC C. (INC-4); NARANJO, CLEO M. (LS-3); FEE, JAMES A. (INC-4).

RELATIONSHIP OF THE SUPEROXIDE OISNUTASE GENES, SODA AND SODB, TO THE IRON UPTAKE (FUR) REGULON IN ESCHERICHIA COLJ K-12.

IN: UCLA COLLOQUIUM ON METAL ION HOMEOSTASIS-MOLECULAR BIOLOGY AND CHEMISTRY, 1988. FRISCO, COLO. PROC., P.149-158. USS, 1989. (OH506 .1262X V.98)

ALSO PUBUSHED: TIC, 1988. 10P. MN (LA-UR-B8-1812)

$1-44$

O'DONNELL, JAMES M. (LS-1).

BEHAVIORAL CONSECUENCES OF ACTIVATION OF BETA ADAENERGIC RECEPTORS BY

CLENBUTEROL-EVIDENCE FOR MEDIATION BY THE CENTRAL NERVOUS SYSTEM.

BRAIN RES. BULL., V.21, P.491-7. 1988.

$1-45$

PERRY, YMLLAM L; BASS, WILIAM M.; AIGGSBY, W. STUAFT; SIROTKIN, KARL (T-10).

AUTODEGRADATION OF DEOXYRIBONUCLEIC ACID (DNA) IN HUNAN RIB BONE AND ITS RELAT:ONSHIP TO THE TIME INTERVAL SINCE DEATH.

J. FOAENSIC SCI., JAN., P.144-53. 1988.

$1-46$

RCDRIGUEZ, MANUEL M. (LS-3); HEYSER, JAMES W. (LS-3).

GROWTH INHIBITON BY EXOGENOUS PPOUNE AND ITS METABOLISM IN SALTGRASS (DISTICHLIS SPICATA) SUSPENSION CULTURES.

PLANT CELL REP., V.7, P.305-8. 1988.

$1-47$

SALZMAN, GAPY C. (LS-2); KRALL, AANDY B. (LS-2); MARINUZZI, JOHN G. (DAAAMO).

KNOWLEDGE ENGINEERING SOFTWARE-A DEMONSTRATION OF A HIGH END TOOL.

TIC. 1887. 25P. MN (LA-UA-87-3924)

$1-48$

SCHRANDT, ROBERT G. (X-6): ULAM, STANISLAW M. (T-DO).

SOME ELEMENTARY ATTEMPTS AT NUMERICAL MODEUNG OF PROBLEMS CONCERNING RATES CF EVOLUTIONATY PROCESSES.

IN: EVOLUTION, GAMES, AND LEARNIVG-MODELS FOR ADAPTATION IN MACHINES AND NATURE INTERNATIONAL CONFERENCE OF THE CENTER FOR NONLINEAR STUDIES, 5TH, 1985, LOS ALAMOS. N.M. PACC., F. 4-12. NORTH-HOLLAND, 1986. (OP4O0 .C46 1985)

ALSO PUBLISHED: PHYSICA, V.22D, P. 4-12. 1986.

$1-49$

SCHWARZACHER-ROBINSON, TRUDE (LS-4); CRAM, L SCCTT (LS-4); MEYNE, JULIANNE (LS-3):

MOYZIS, ROBERT K. (LS-3).

CHARACTEAIZATION OF HUMAN HETEROCHROMATIN BY IN SITU HYBRIDIZATION WITH SATELLITE DNA CLONES.

CYTOGENET. CELL GENET., V.47, P.192-6, 1988.

$1-50$

SCHWARZACHER-ROBINSON, TRUDE (LS-4); KRAEMER, PAUL M. (LS-4); CRAM, L. SCOTT (LS-4). SPONTANEOUS IN VITRO NEOPLASTIC EVOLUTION OF CULTURED CHINESE HAMSTER CELLSNUCLEOLUS ORGANIZING AEGION ACTIVITY.

CANCER GENET. CYTOGENFT., V.35, P.119-28. 1988.

$1-51$

SCOTT, ALWMN c. (CNLS).

SOUTONS IN BIOLOGICAL MOLECULES.

IN: ENERGING SYNTHESES IN SCIENCE, 1984, SANTA FE, N.M. PROC., P.133-52. ADDISON-WESLEY. 1988. (O101 .E49 1988)

$1-52$

SEBFING, ROBEAT J. (LS-1); JOHNSON, N. F. (LS-1); SPALL, W. DALE (LS-1).

TRANSMISSION ELECTRYN MICFOSCOPY OF SMALL NUMBERS OF SOPTED CELS.

CVTOMETRY, V.9, P.88-82. 1988. 
$1-53$

SILLEAUD, LAUREL O. (LS-2); HALLIDAY, KAREN R.; GAIFFEY, AICHARD H.; FENOGLIO-PREISER, CECILIA; SHEPPARD, SCOTT.

IN VIVO SARBON-13 NMP SPECTROSCOPY OF THE HUMAN PROSTATE.

MAGN. RESON. MED., V.8, P.224-30. 1988.

$1-54$

SILLERUD, LALUEL O. (LS-7); KIRSCH, CLAUDIA F. (LS-7); PENNINO, RALPH P.; MILLEF, GARY;

CAPPON, JAMES P.; KORNFELD, MARIO; KIRSCH, WOLFF; OMER, GE,ORGE.

MONITORING OF EARLY WALLERIAN DEGENERATION IN RAT SCIATIC NEAVE USING HIGH-FIELD PROTON NMR SPECTROSCOPY

SURG. FORUM, V.38, P.555-8. 1987.

1.55

SILLERUD, LAUREL O. (LS-7); VAN HULSTEYN, DAVID B. (P-14); GRIF;FEY, AICHAFD H.

CARBON-13-POLARIZATION TRANSFER PROTON NMR IMAGING OF A SODIUM [CARBON-13]FORMATE

PHANTOM AT 4.7 TESLA.

J. MAGN. RESON., V.76, P.380-5. 1988.

$1-56$

SIROTKIN, KARL (T-10).

COMPUTER PROGRAM TO DISPLAY CODON CHANGES CAUSED BY MUTAGENESIS.

CABIOS, V.4, P.243-7. 1988.

1-57

SOUMPASIS, D. M: TUNG, CHANG-SHUNG (T-10).

RIGOROUS BASEPAIR ORIENTED DESCRIPTION OF DNA STRUCTURES

J. BIOMOL STRUCT. DYN., V.6, P.397-420. 1988.

1.58

STAVERT, DOUGLAS M. (LS-1); LEHNERT, BRUCE E. (LS-1).

CROSS-VENTILATING RAT MODEL-PULMONARY FUNCTIONAL STATUS OF PARTICIFATING ANIMALS.

J. AEROSOL MED., V.1, P.51-66. 1988.

$1-59$

STEWART, C. C. (LS-4); STEVENSON, ANITA P. (LS-4); HABBERSETT, RC (LS-4).

EFFECT OF LOW-DOSE IRRADIATION ON UNSTIMULATED AND PHA-STIMLULATED HUMAN LYMPHOCYTE SUBSETS

INT. J. RADIAT. BIOL., V.53, P.77-87. 1988.

1-60

STRIIISTE, GARY F. (LS-3); CHEN, DAVID JEN-CHI (LS-3); DE BRUIN, DERIK; LUKE, J. A. (LS-3);

MIC COY, LINDA S. (LS-3); MUDGETT, J. S. (LS-3); NICKOLS, JOYCE W. (LS-3); OKINAKA, RICHARD T. (LS-3);

TESMER, JUDITF: ज. (LS-3); MAC INNES, MARK A. (LS-3).

RESTOPATION OF CHINESE HAMSTER CELL RADIATION RESISTANCE BY THE HUMAN REPAIR GENE

ERCC-5 AND PROGRESS IN MOLECIJLAR CLONING OF THIS GENE.

IN: UCLA SYMPOSIUM ON MECHANISMS AND CONSEQUENCES OF DNA DAMAGE, 1988, TAOS, N.M PROC., P.301-6. A.A. LISS, 1988. (OH506.1262X V.83)

ALSO PUBLISHED: TIC, 1988. 7P. MN (LA-UR-88-0267)

1-61

TREWHELLA, J. (LS-2); HEIDORN, DOUGLAS B. (LS-2); SEEGER, PHILIP A. (LANSCE).

SOLUTION STRUCTURES OF CALCIUM REGULATING PROTEINS-A SMALL-ANGLE SCATTERING STUDY

TIC. 1987. 21P. MN (LA.UR-88-0413)

1-62

TREWHEL'A, J. (LS-7); CARLSON, VANICE A. (LS-7); CURTIS, ELIZABETH H. (LS-7);

HEIDORN, DOUGLAS B. (LS-7).

DIFFERENCES IN THE SOLUTION STRUCTURES OF OXIDIZED AND REDUCED CYTOCHROME C MEASURED BY SMALL-ANGLE X-RAY SCATTEAING.

BIOCHEMISTRY, V.27, P.1121-5. 1988. 
$1-63$

TUNG, CHANG-SHUNG (T-10); GARCIA, ANGEL E (T-10); MARA, T. G. (T-10). MISHRA, SANTOSH K (T-10). BURKS, CHRISTIAN (T-10).

MEASURES OF DNA CURVATURE.

IN: STRUCTURE \& EXPRESSION-PROCEEDINGS OF CONVERSATION IN BIONOLECULAR STEREODYNAMICS, 5TH, 1987, STATE UNIVERSITY OF NEW YORK AT ALBANY PROC.. V . . P 265.78 ADENINE PRESS, 1988.

1-64

VOELZ, GEORGE L. (HSE-2); STEVENSON, ANITA P. (LS-4); STEWART, C. C. (LS-4).

DOES PLUTONIUM INTAKE IN WORKERS AFFECT LYMPHOCYTE FUNCTION?

TIC, 1988. 16P. MN (LA-UR-88-1598)

$1-65$

WALKER, THOMAS E. (INC-4); EHLER. DEBORAH S. (INC-4); UNKEFER, CLIFFORO J. (INC-4)

SYNTHESIS OF 2-DEOXY-D-ARABINO- $(6-13 C)$ HEXOSE.

CARBOHYDR. RES., V.181, P.125-34. 1988.

$1-66$

YAMAZAKI, AKIO (LS-DO); TATSUMI, MASAHIRO (LS-7); BITENSKY, MARK. W. (LS-DO).

PURIFICATION OF ROD OUTER SEGMENT GTP-BINDING PROTEIN SUBUN:TS AND CGMP

PHOSPHODIESTERASE BY SINGLE-STEP COLUMN CHROMATOGRAPHY.

IN: INITIATION AND TERMINATION OF CYCLIC NUCLEOTIDE ACTION, J.D. CORBIN, R.A. JOHNSON,

EDS., (METHODS IN ENZYMOLOGY, V.159) P.702-3. ACADEMIC PRESS, 1988. (QP/601/M49/V.159)

$1-67$

ZBILUT, JHN P.; MAYER-KRESS, GOTTFRIED (CNLS): GEIST, KARL.HEINZ (CNLS).

DIMENSIONAL ANALYSIS OF HEART RATE VARIABILITY IN HEART TRANSPLANT RECIPIENTS.

MATH. BIOSCl., V.90, P.4970. 1988.

ALSO PUBLISHED: TIC, 1987, 36P. MN (LA-UR-87-2747) 
2-1

AKST, IRVING B. (M-3).

INTERMOLECULAR EXPLOSIVES.

IN: SYMPOSIUM (INTEANATIONAL) ON DETONATION, 8TH, 1985, ALBUQUEROUE. PREPRINTS, P.178-87. NAVAL SURFACE WEAPONS CENTER, 1986. (CD516 .S924 1985XA)

ALSO PUBLISHED: SYMPOSIUM (INTERNATIONAL) ON DETONATION, 8TH, 1985, ALBUQUERQUE.

PROC., P.1001-10. NAVAL SURFACE WEAPONS CENTER, 1986. (QD516 .S924 1985X)

2-2

BLAIS, NOAMAND C. (CHM-2); VALENTINI, JAMES J. (CHM-4).

REAL TIME ANALYSIS OF PETN DETONATION PRODUCTS.

IN: SYMPOSIUM (INTERNATIONAL) ON DETONATION, 8TH, 1985, ALBUQUERQUE. PREPRINTS, P.425-31. NAVAL SURFACE WEAPOHS CENTER, 1986. (CD516 .S924 1985XA)

ALSO PUBLISHED: TIC, 1985. 8P. MN (LA-UA-85-0860)

ALSO PUBLSHED: SYMPOSIUM (INTERNATIONAL) ON DETONATION, 8TH, 1985, ALBUQUERQUE.

PROC., P.701-9. NAVAL SURFȦCE WEAPONS CENTER, 1986. (OD516 .\$924 1985X)

$2-3$

CAGUJOSTRO, DOMNIC J. (M-4); WARNES, R. H. (M-4); JOHNSON, NORMAN LEE (T-3); FUJITA, R. K. (WX-4). SPALL MEASUREMENTS IN SHOCK-LOADED HEMISPHERICAL SHELLS FROM FREE-SURFACE VELOCITY HISTORIES.

IN: SHOCK WAVES IN CONDENSED MATTER, 1987, MONTEREY. PROC., F.367-70. ELSEVIER, 1988.

(CC173.4.C65 S57 1987X)

2-4

CAMPBELL, ARTHUR W. (M-3); TRAVIS, JOHN R. (T-3).

SHOCK DESENSITIZATION OF PBX-9404 AND COMPOSITION B-3.

IN: SYMPOSIUM (INTERNATIONAL) ON DETONATION, 8TH, 1985, ALBUQUEROUE. PREPRINTS, P.458-69. NAVAL SURFACE WEAPONS CENTER, 1986. (CD516 .5924 1985XA)

ALSO PUBLISHED: TIC, 1985. 13P. MN (LA-UR-85-1114)

ALSO PUBUSHED: SYMPOSIUM (INTERNATIONAL) ON DETONATION, 8TH, 1985. ALBUQUERQUE.

PROC., P.1057-68. NAVAL SURFACE WEAPONS CENTER, 1986. (OD516 .S924 1985X)

2-5

DALLMAN, JOHN C. (M-S).

MEASUREMENTS OF DETONATION-WAVE SPREADING AND LOCAL PARTICLE VELOCITY AT THE SURFACE

OF 17-MM LX-07 HEMISPHERICAL BOOSTERS.

LOS ALAMOS NATIONAL L BORATORY, 1988. 43P. (LA-11414-MS)

2-6

DAVIS, WILLIAM C. (M-3); CHHLES, WILLIAM C. (M-3).

DETONATION PROPERTIES OF LIOUID NITRIC OXIDE.

IN: SYMPOSIUM (INTERNATIONAL) ON DETONATION, 8TH, 1985. ALBUQUERQUE. PREPRINTS, P.12.

NAVAL SURFACE WEAPONS CENTER, 1986. (CD516 .S924 1985XA)

2-7

DAVIS, WILLIAM C. (M-3).

EQUATION OF STATE FOR DETONATION PAODUCTS

IN: SYMPOSIUM (INTERNATIONAL) ON DETONATION, 8TH, 1985, ALBUQUERQUE. PREPRINTS, P.119-30.

NAVAL SURFACE WEAPONS CENTER, 1986. (CC516 .\$924 1985XA)

ALSO PUBLISHED: TIC, 1985. 13P. MN (LA-UR-85-1129)

ALSO PUBLSHED: SYMPOSIUM (INTERNATIONAL) ON DETONATION, 8TH, 1985, ALBUQUERCUE.

PROC., P.785-95. NAVAL SURFACE WEAPONS CENTER, 1986. (CD516 .S924 1985X)

2-8

DICK, JERAY JOEL (M-9); FOREST, CHARLES A. (M-9); RAMSAY, JOHN B. (M-8); SEITZ, WENDELL LEE (M-9).

HLGONIOT AND SHOCK SENSITIVITY OF A PLASTIC-BONDED TATB EXPLOSIVE, PBX 9502.

J. APPL. PHYS., V.63, P.4881-8. 1988. 
$2-9$

GOLDSTEIN, SELMA (M-3); MADER, CHARLES L. (T-14).

DETONATION IN TUNGSTEN-LOADED HMX.

IN: SYMPOSIUM (INTERNATIONAL) ON DETONATION, 8TH, 1985, ALBUOUEROUE. PREPRINTS, P.157-62 NAVAL SURFACE WEAPONS CENTER, 1086. (CD516 .S924 1985XA)

ALSO PUBLSHED: TIC, 1085. 7P. MN (LA-UR-85-1122)

ALSO PUBUSHED: SYMPOSIUM (INTERNATIONAL) ON DETONATION, 8TH, 1985. ALBUOUEROUE

PROC., P.970-84. NAVAL SURFACE WEAPONS CENTER, 1986. (OD516 .S924 1985X)

2-10

GREINER, NORMAN R. (CLS-1); PHILIPS, DAVID S. (MST-6); JOHNSON, JAMES D. (T-1); VOLK, FRED.

DIANONDS IN DETONATION SOOT.

NATURE, V.333, P.440-2. 1988.

2-11

MADER, CHARLES L (T-14); KERSHNER, JAMES D. (T-14).

NUMERICAL MODEUNG OF THE EFFECT OF PARTICLE SIZE OF EXPLOSIVES ON SHOCK INITIATION PROPERTIES.

LOS ALAMOS, 1989. 21P. (LA-11239-MS)

2-12

MADER, CHARLES L. (T-14); KERSHNER, JAMES D. (T-14).

THREE-DIMENSIONAL HYORODYNAMIC HOT-SPOT MODEL.

IN: SYMPOSIUM (INTERNATIONAL) ON DETONATION, 8TH. 1995. ALBUOUERQUE. PREPRINTS. P.366-74 NAVAL SURFACE WEAPONS CENTER, 1986. (OD516 .S924 1985XA)

ALSO PUBUSHED: TIC, 1985, 10P. MN (LA-UR-P5-0742)

ALSO PUBUSHED: SYMPOSIUM (INTERNATIONAL) ON DETONATION, 8TH, 1985, ALBUQURQUE. PROC.. P.42-51. NAVAL SURFACE WEAPONS CENTER, 1986. (OD516 .\$924 1985X)

2-13

RAMSAY, JOHN B. (M-3).

EFFECT OF CONFINEMENT ON FAILURE IN 95 TATB/5 KEL-F

IN: SYMPOSIUM (INTERNATIONAL) ON DETONATION, 8TH, 1985, ALBUOUEROUE. PREPRINTS, P.6-11 NAVAL SURFACE WEAPONS CENTER, 1886. (CD516 .S924 1985XA)

ALSO PUBUSHED: SYMPOSIUM (INTERNATIONAL) ON DETONATION', BTH, 1985, ALBUQUERQUE.

PROC., P.372-9. NAVAL SURFACE WEAPONS CENTER, 1986. (OD516 .5924 1985X)

2-14

RITCHIE, JAMES P. (T-14); CROMER, DON T. (INC-4); RYAN, ROBERT R. (INC-4); STEWART, ROBERT F.: WASSERMAN, HARVEY J. (INC-4).

ELECTRON DENSITY DISTRIBUTION ANALYSIS FOR NITROGUANIDINE.

IN: SYMPOSIUM (INTERNATIONAL) ON DETONATION, 8TH, 1985, ALBUOUEROUE. PREPRINTS, P.213-20. NAYAL SURFACE WEAPONS CENTER, 1986. (CDE16 .S924 1985XA)

ALSO PUBUSHED: TIC, 1985. 10P. MN (LA-UR-85-1082)

ALSO PUBLSHEO: SYMPOSIUM (INTERNATIONAL) ON DETONATION, 8TH, 1985, ALBUQUERQUE. PROC., P.839-46. NAVAL SURFACE WEAPONS CENTER, 1986. (OD516 .S924 1995X)

2-15

SCHOTT, GARPY L. (M-1): CHICK, KENNETH M. (M-9).

PHOTOGRAPHICAUY OBSEPVED WAVES IN DETONATION OF LUUID NITAIC OXIDE.

IN: INTERNATIONAL COLLOOUIUM OFi DYNAMCS OF EXPLOSIONS AND REACTIVE SYSTEMS, 11TH, 1997, WARSAW, POLAND. PROC., P.372-385. AMERICAN INSTITUTE OF AERONAUTICS AND ASTRONAUTICS, 1888. (TLS07.P75 V.114)

2-16

SCHWALBE, LARRY A. (X-4); MUELLER, KARL H., JR. (M-4); CHRISTIAN, JOHN M. (M-4); DI BONA, P. J.

IN-FLIGHT RADIOGRAPHIC STUDY OF TWO NONAXISYMMETRIC. EXPLOSIVELY FORMED PENETRATORS LOS ALAMOS, 1988. 23P. (LA-11131) 
2.17

SEITZ, WENDELL LEE (M-1); STACY, HOWARD L. (M-1); WACKERLE, JERRY D. (M-1).

DETONATION REACTION ZONE STUDIES ON TATB EXPLOSIVES.

IN: SYMPOSIUM (INTERNATIONAL) ON DETONATION, 8TH, 1985, ALBUOUEROUE. PREPRINTS.

P.999-1008. NAVAL SURFACE WEAPONS CENTER, 1986. (QD516 .S924 1985XA)

ALSO PUBLISHED: TIC, 1985. 11P. MN (LA-UR-85-2460)

ALSO PUBLISHED: SYMPOSIUM (INTERNATIONAL) ON DETONATION, 8TH, 1985. ALBUQUERQUE.

PROC., P. 123-32. NAVAL SURFACE WEAPONS CENTER, 1986. (OD516 .S924 1985X)

2-18

SHAW, MILTON S. (T-14); JOHINSON, JAMES D. (T-1).

SLOW REACTION RATE IN DETONATIONS DUE TO CARBON CLUSTERING.

IN: SHOCK WAVES IN CONDENSED MATTER, 1987, MONTEREY. PROC., P.503-6. ELSEVIER, 1988.

(OC173.4C65 S57 1987X)

2-19

STEWART, DONALD S.; BDZIL, JOHN B. (M-9).

SHOCK DYNAMICS OF STABLE MULTIDIMENSIONAL DETONATION.

COMBUST. FLAME, V.72, P.311-23. 1988.

2-20

STINECIPHER, MARY M. (M-1); STRETZ, LARRY A. (M-1).

SENSITIVITY AND PERFORMANCE CHARACTERIZATION OF DINGU,

IN: SYMPOSIUM (INTERNATIONAL) ON DETONATION, 8TH, 1985. ALBUQUERQUE. PREPRINTS, P.603-13. NAVAL SURFACE WEAPONS CENTER, 1986. (QD516 .S924 1985XA)

ALSO PUBLISHED: TIC, 1985. 7P. MN (LA-UR-85-1390)

ALSO PUBLISHED: SYMPOSIUM (INTERNATIONAL) ON DETONATION, 8TH, 1985, ALBUQUEROUE.

PROC., P.351-60. NAVAL SURFACE WEAPONS CENTER, 1986. (CD516 .S924 1985X)

2-21

SWANSON, BASIL 1. (INC-4); AGNEW, STEPHEN F. (INC-4); GREINER, NORMAN R. (CHM-1).

STATIC HIGH PRESSURE STUDY OF NITRIC OXIDE CHEMISTRY-PROPOSED MECHANISM FOR NITRIC

OXIDE DETONATION.

IN: SYMPOSIUM (INTERNATIONAL) ON DETONATION, 8TH, 1985, ALBUQLIEROUE. PREPFINTS, P.432-40.

NAVAL SURFACE WEAPONS CENTER, 1986. (CD516 \$924 1985XA)

ALSO PUBLISHED: TIC, 1985. 10P. MN (LA-UA-85-1176)

ALSO PUBLISHED: SYMPOSIUM (INTERNATIONAL) ON DETONATION, BTH, 1985, ALBUQUEROUE.

PROC., P.715-24. NAVAL SURFACE WEAPONS CENTER, 1986. (OD516.S924 1985X)

2-22

TANG, P. K. $(X-4)$.

INITIATION AND DETONATION OF HETEROGENEOUS HIGH EXPLOSIVES-A UNIFIED MIODEL.

LOS ALAMOS NATIONAL LABORATORY, 1988 15P. (LA-11352-MS)

2.23

TANG. P. K. $(X-7)$; JOHNSON, JAMES N. (T-14); FOREST, CHARLES A. (M-1).

MODELING HETEROGENEOUS HIGH EXPLOSIVE BURN WITH AN EXPLICIT HOT-SPOT PROCESS

IN: SYMPOSIUM (INTERNATIONAL) ON DETONATION, 8TH, 1985, ALBUOUERQUE. PREPRINTS, P.375-84 NAVAL SURFACE WEAPONS CENTER, 1986. (CD515 S924 1985XA)

ALSO PUBLISHED: TIC, 1985. 11P. MN (LA-UR-85-0769)

ALSO PUBLISHED: SYMPOSIUM (INTERNATIONAL) ON DETONATION, 8TH, 1985, ALBUQUEROUE. PROC., P.52-61. NAVAL SURFACE WEAPONS CENTER, 1986. (QD516.S924 1985X)

2-24

TANG, P. K. (X-7)

STUDY OF DETONATION PROCESSES IN HETEROGENEOUS HIGH EXPLOSIVES.

J. APPL. PHYS., V.63, P.1041-5. 1988.

2-25

VORTHMAN, JOHN E. (M-1); ANDREWS, GEORGE H., JR. (M-1); WACKERLE, JERRY D. (M-1).

REACTION RATES FROM ELECTROMAGNETIC GAUGE DATA.

IN: SYMPOSIUM (INTERNATIONAL) ON DETONATION, 8TH, 1985, ALBUQUEROUE. PREPRINTS, P.976-86. NAVAL SURFACE WEAPONS CENTER, 1986. (CD516 .S924 1985XA)

ALSO PUBLISHED: TIC, 1985. 12P. MN (LA-UR-85-2459)

ALSO PUBLISHED: SYMPOSIUM (INTERNATIONAL) ON DETONATION, 8TH, 1985, ALBUOUEROUE.

PROC., P.99-110. NAVAL SURFACE WEAPONS CENTER, 1986. (OD516 .S924 1985X) 
WACKEPLE, JERPY D. (M-9); STACY, HOWARD L. (M-9).

REFRACTIVE INDEX OF SHOCKED ALKAL HALIDES.

IN: SHOCK WAVES IN CONDENSED MATTER, 1987, MONTEREY. PROC., P.599-702. ELSEVIER, 1988. (CC173.4.Ce6 557 1987X) 
3-1

ASPREY, LAPNED B. (INC-4); ELLER, P. GARY (INC-4).

METHOD FOR RECOVERY OF ACTINIDES FROM REFRACTORY OXIDES THEREOF USING OXYGEN-FLOURIDE.

PATENTED FEB. 9, 1988, FILED 1987. (U.S.PATENT-4.724,127)

3-2

ATTREP, MOSES, JR. (INC-11); EFURD, DEWARD W. (INC-11); RCENSCH, F. P. (INC-11).

DEVELOPNENT OF A RAPID RADIOCHEMICAL PROCEDURE FOR THE SEPARATION OF URANIUM- 235M FROM PLUTONIUM-239.

TIC, 1987. 12P. MN (LA-UR-88-0592)

3-3

CHRISTENSEN, DANA CARL (MST-13); BOWERSOX, DAVID F. (MST-13); MC KERLEY. BILLY J. (MST-12):

NANCE, ROBERT L. (MST-12).

WASTES FROM PLUTONIUM CONVERSION AND SCRAP RECOVERY OPERATIONS.

LOS ALAMOS, 1988. 99P. (LA-11069-MS)

3-4

MARSH, SINCLAIR F. (MST-12); YARBRO, STEPHEN L (MST-12).

CONPARAT!:E EVALUATION OF DHDECMP AND CMPO AS EXTRACTANTS FOR RECOVERING ACTINIDES FROM NITRIC ACID WASTE STREAMS.

LOS ALAMOS, 1988. 15P. (LA-11191-T)

3-5

MARSH, SINCLAIR F. (MST-12); YARBRO, STEPHEN L. (MST-12).

NEAR-QUANTITATIVE RECOVERY OF ACTINIDES FHOM NITRIC ACID WASTE STREAMS BY SOLVENT EXTRACTION LSING DHDECMP.

TIC, 1988. 4P. MN (LA-UR-88-1366)

3-6

MARTEU., CALVIN J. (CLS-1); HANSEL, JAMES M. (CLS-1).

DETERMINING CERIUM IN PLUTONIUM BY ANION EXCHANGE AND X-RAY FLUORESCENCE.

LOS ALAMOS NATIONAL LABORATORY, 1988. 5P. (LA-11298)

3-7

MULFORD, POBERT N. R. (MST-5); SHELDON, R. I. (MST-5).

DENSITY AND HEAT CAPACITY OF LIOUID URANIUM RT HIGH TEMFERATURES.

J. NUCL MATER., V.154, P.268-75. 1988.

3-8

PALMER, MICHAEL J. (MST-12).

USE OF OCTYLLPHENYL)-N,N,-DISOBUTYLCARBAMOYLMETHYLOPHOSPHENE OXIDE TO

DECONTAMNATE HYDROCHLORIC ACID WASTE STREAMS.

TIC. 1988. 17P. MN (LA-UR-88-3250) 


\section{CHEMISTRY}

4-1

AGNEW, STEPHEN F. (INC-4); SWANSON, BASIL I. (INC-4).

MODEL FOR THE DENSITY DEPENDENCE OF ELECTRONIC ABSORPTION BANDS-APPLICATION TO

CARBON DISULFIDE AND OTHER MOLECULES.

IN: SHOCK WAVES IN CONDENSED MATTER, 1997, MONTEREY. PROC., P.485-8. ELSEVIER, 1988.

(OC173.4.C65 S57 1987X)

4-2

AGNEW, STEPHEN F. (INC-4); MISCHKE, RACHEL (INC-4); SWANSON, BASIL I. (INC-4).

PRESSURE- AND TEMPERATURE-INDUCED CHEMISTRY OF CARBON DISULFIDE

J. PHYS. CHEM., V.92, P.4201-4. 1988.

$4 \cdot 3$

AGNEW, STEPHEN F. (INC-4): ALDISSI, MAHMOUD (MST-7).

RESONANCE RAMAN EXCITATION PROFILES OF FOLYACETYLENE/POLYISOPRENE BLOCK CO-POLYMERS

IN TOLUENE.

SYNTH. MET., V.28, P.D727-34. 1989.

ALSO PUBLISHED: TIC, 1989. 13P. MN (LA-UR-88-3043)

4-4

ALBERS, ROBERT C. (T-11)

LMTO [UNEAR MUFFIN-TIN ORBITAL] ELECTRONIC BAND-STRUCTURE CALCULATIONS FOR LINEAR

PLATINUM-BAOMINE CHAINS.

TIC. 1988. 7P. MN (LA-UR-88-2508)

4-5

APEL, CHARLES T. (CHM-1); BEUGELSDIJK, TONY J. (MST-9); GALLIMORE, DAVID L. (CHM-1);

MYERS, W. H. (CHM-1): FAIRES, LYNDA M. (CHM-1)

LOS ALAMOS FACILITY FOR INDUCTIVELY COUPLED PLASMA ATOMIC EMISSION SPECTROMETRIC

ANALYSIS OF RADIOACTIVE SOLUTIONS.

IN: PLASMA SPECTROSCOPY FOR THE ANALYSIS OF HAZARDOUS MATERIALS-DESIGN AND APPLICATION OF ENCLOSED PLASMA SOURCES, 1986, NEW ORLEANS. PROC., P.121-8. ASTM, 1987. (QD96.P62P58 1986X)

ALSO PUBLISHED: TIC, 1986. 19P. MHA (LA-UR-86-0889)

4-6

ARKO, AL J. (P-10); KOELUNG, D. D.; DUNLAP. B. D.; CAPASSO, C.: DEL GIUDICE, M.

SPECTROSCOPIC EVILENCE FON SF BANDS AT RDOM TEMPERATURE IN URANIUM BASED HEAVY FERMIONS.

TIC, 1988. 27P. MN (LA-UR-88-2440)

4-7

ARNDT, T.; BACKE, H.; STEINHOF, A.; DYER, PEGGY (P.3); FOWLER, MALCOLM M. (INC-11);

ELLER, P. GARY (INC-4); WILHELMY, JERRY B. (INC-11)

RADIATIVE LIFETIMES OF AMERICIUM I ATOMIC LEVELS.

PHYS. REV., V.38A, P.5084-91. 1988.

4-8

BASTIAN, NEIL R.; DIEKERT, GABRIELE; NIEDERHOFFER, ERIC C. (INC-4); TEO, BOON-KENG;

WALSH, CHAISTOPHER T.; ORME-JOHNSON, WILLIAM H.

NICKEL AND IRON EXAFS OF CARBON MONOXIDE DEHYDROGENASE FROM CLOSTRIDIUM

THERMOACETICUM STRAIN DSM.

J. AM. CHEM. SOC., V.110, P.5581-2. 1988.

4-9

BIGIO, IRVING J. (CLS-5); SZE, RO8ERT C. (CLS-5); TAYLOR, ANTOINETTE J. (CLS-5):

GIBSON, ROBERT B. (CLS-5).

OVERVIEW OF RECENT ADVANCES IN EXCIMER LASER TECHNOLOGY AT LOS ALAMOS

TIC. 1988. 6P. MN (LA-UR-88-2745) 
4-10

BISH, DAVID L. (ESS-1); HOWARD, SCOTT A.

QIIANTITLIIVE PHASE ANALYSIS USING THE RIETVELD METHOD.

J. APPL. CRYSTALLOGR., V.21, P.86-91. 1988.

4-11

BLAIS, NORMAND C. (CLS-2); TRUHLAR, DONALD G.; MEAD, C. ALDEN.

EFFECT OF A CONICAL INTERSECTION ON CFOSS SECTIONS FOR COLLISION-INDUCED DISSOCIATION J. CHEM. PHYS., V.89, P.E204-8. 1988.

4-12

BLAIS, NORMAFYD C. (CLS-2); TRUHLAF, DONALD G.

FINAL STATE AND VELOCITY DISTRIBUTION OF THE REACTION DEUTERIUM PLUS HYOROGEN-2 GOING TO HYDROGEN DEUTERIUM PLUS HYDROGEN AS A FUNCTION OF SCATTERING ANGLE.

J. CHEM. PHYS., V 88, P.5457-64. 1988.

4-13

BLAISE, JEAN; WYART, J. F.; ENGLEMAN, ROLF, JR. (CLS-2); PALMER, BYRON A. (CLS-4).

PRECISION ISOTOPE SHIFTS FOR THE HEAVY ELEMENTS-IV. THEORETICAL INTERPRETATION OF THE SHIFTS IN THE LOW EVEN CONFIGURATIONS OF THOAIUM I AND THORIUM II.

J. OPT. SOC. AM., V.5B, P.2087-92. 1988.

4-14

BORGES, HORTENCIO A. (P-10); THOMPSON, JOE D. (P-10); ARONSON, MEIGAN C. (P-10):

FISK, ZACHARY (P-10); SMITH, JAMES L (CMS).

ELECTRICAL RESISTIVITY OF URANIUM 1-X METALUIC ELEMENT X BERYLLIUM 13 UNDER PRESSURE

TIC, 1988. 9P. MN (LA-UR-88-1914)

4-15

BORING, A. MICHAEL (T-11); SCH.ADLER, GERHARD $H$. (T-11); ALBERS, ROBERT C. (T-11); WEINBERGER, P. ELECTRONIC STRUCTURE OF NEPTUNIUM.

J. LESS-COMMON MET., V.144, P..71-8. 1988.

4-i 6

BUANS, C. J. (INC-4); SATTELBERGEH, ALFRED P. (iNC-4).

URANIUM(VI)OXOALKOXIDE CLUSTER SYNTHESIS VIA LIGAND REDISTRIBUTION INORG. CHEM., V.27, P.3692-3. 1988.

4-17

CHEONG, SANG-WOOK (P-10); THOMPSON, JOE D. (P-10; FISK, ZACHARY (P-10);

KUBAT-MARTIN, KIMBEFLY A. (INC-4): GARCIA, EDUARDO (INC-4).

FIELD-INDUCED TRANSITIONG IN :TTRIUNA2 COPPEA-2 OXYGEN-5.

PHYS. REV., V.38B, P.7013-5. 1988.

4-18

CHRISTOPH, GARY G. (C-8); LARSON, ALLEN C. (P-8); ELLER, P. GARY (INC-4); PURSON, JOHN D. (INC-4);

ZAHRT, JOHN D. (X-5); PENNEMAN, ROBERT A. (INC-4); RINEHART, GARY H. (MST-11).

STRUCTURE OF BARIUM PLUTONATE BY NEUTRON POWDER DIFFRACTION.

ACTA CRYSTALLOGR., V.B44, P.575-80. 1988.

4-19

CHUNG, YUN C. (CLS-5); SHAY, T, M. (CLS-5).

EXPERIMENTAL DEMONSTRATION OF A DIODE LASEREXXCITED OPTICAL FILTER IN ATOMIC RUBIDIUM VAPOR.

IEEE J. QUANTUM ELECTRON., V.24, P.709-11. 1988.

4-20

CONRADSON, STEVEN D. (INC-4); STROUD, MARY ANN (INC-4); ZIETLOW, MIRIAM H;

SWANSON, BASIL I. (INC-4); BAERISWYL, DIONYS; BISHOP, ALAN R. (T-11).

CHARGE DENSITY WAVES AND LOCAL STATES IN OUASI-ONE-DIMENSIONAL MIXED VALENCE INORGANIC COMPLEXES

SOLID STATE COMMUN., V.65, P.723-9. 1988 
4-21

CCNRADSON, STEVEN D. (INC-4); SATTELBERGER, ALFRED P. (INC-4): WOODRUFF, WILLIAM H. (INC 4) $X$-RAY ABSORPTION STUDY OF OCTAFLUORODIRHENATE (III)-EXAFS STRUCTURES AND RESONANCE RAMAN SPECTROSCOPY OF OCTAHALODIRHENATES

J. AM. CHEM. SOC., Y.110, P.130911. 1988.

422

COPE, S. D.; RUSSELL, D. K.; FRY, HERBERT A. (CLS-3); JONES, LLEWELIYN H (INC-4);

BAREFIELD, J. E., II (CLS-4).

ANALYSIS OF THE NU-1 FUNDAMENTAL MODE OF HTO [MONOTITRATED WATER].

J. MOL. SPECTROSC., V.127, P.464-71. 1988.

4-23

CREMERS, DAVID A. (CLS-4).

OVERVIEW OF APPLICATIONS OF LASER-INDUCED BREAKDOWN SPECTROSCOPY (LIBS)

TIC, 1987. YP. MN (LA-UR-87-4033)

4-24

CROMER, DON T. (INC-4): HALL, JANE H. (P-DO); LEE, KIEN Y. (M-1): RYAN, ROBERT R. (INC.4). STRUCTURE OF THE ETHYLENEDIAMMONIUM SALT OF 3-NITRO- 1,2,4-TRIAZOL-5-ONE, CARBON 2 HYDROGEN 4(NITROGEN HYDROGEN 3)2.2 CARBON 2 NITROGEN 4 OXYGEN 3 HYDROGEN. ACTA CRYSTALLOGR., V.C44, P.1144-7. 1988.

$4-25$

CROMER, DON T. (INC-4); LEE, KIEN Y. (M-1); RYAN, ROBERT R. (INC-4).

STRUCTURES OF TWO POLYMORPHS OF 1,1'-DINITRO-3,3'-AZO-1,2,4-TRIAZOLE

ACTA CRYSTALLOGR., V.C44, P.1673-4. 1988.

4-26

CROSS, JON B. (CLS-2); BLAIS, NOAMAND C. (CLS-2).

HIGH ENERGY-INTENSITY ATOMIC OXYGEN BEAM SOURCE FOR LOW EARTH ORBIT MATERIALS

DEGRADATION STUDIES

TIC, 1988. 14P. MN (LA-UR-88-218B)

4-27

CROSS, JON B. (CLS-2); LAN, E. H.; SMITH, C. A.

TECHNIQUE TO EVALUATE COATINGS FOR ATOMIC OXYGEN RESISTANCE.

IN: INTEANATIONAL SAMPE SYMPOSIUM AND EXHIBITION, 33AD, 1988, ANAHEIM, CALIF. PROC., P.693-724. SOCIETY FOR THE ADVANCEMENT OF MATERIAL AND PROCESS ENGINEERING, 1988. (TA401.3.\$63/1988X)

4-28

DAHLBY, JOEL W. (CLS-1); GEORGE. WILLIAM E. (CLS-1); GONZALES, MANUEL (HSE-10);

STAFFORD, RONA:D G. (HSE-10).

VENTILATION SYSTEM HISTORY OF A PLUTONIUM ANALYTICAL CHEMISTRY LABORATORY.

LOS ALAMOS NATIONAL LABORATORY, 1988. 21P. (LA-11381-NS)

4-29

DAVID, SHELLE J. (MST-11); KIM, KYU C (MST-11).

VIBRONIC STATES OF PLUTONIUM HEXAFLUORIDE IN THE VISIBIE AND NEAA INFRAAED SPECTRAL REGIONS.

J. CHEM. PHYS., V.89, P.1780-6. 1988.

4-30

ECKERT, JUERGEN (P-8); KUBAS, GREGORY J. (INC-4); DIANOUX, ALBERT J.

AOTATIONAL TUNNELNG OF BOUND HYDROGEN IN A TUNGSTEN COMPLEX

J. CHEM. PHYS., V.88, P.466-8. 1989

4-31

ELLER, P. GARY (INC-4); MALM, JOHN G.; PENNEMAN, ROBERT A. (iNC-4)

METHOD FOR FLUORINATION OF ACTINIDE FLUOAIDES AND OXYFLUOAIDES THEREOF USING

DIOXYGEN DIFIUUORIDE.

PATENTED NOY. 8, 1988, FILED 1984. (U S.PATENT-4.783.322) 
4-32

ENGELKE, RAY P. (M-9); SCHIFERL, DAVID (M-6); STORM, CARLYLE B. (M-1); EARL, WILLIAM L. (INC DOT). PRODUCTION OF THE NITROMETHANE ACI ION BY STATIC HIGH PRESSURE.

J. PHYS. CHEM., V.92, P.6815-9. 1988.

4-33

ERICKSON, GEORGE F. (CLS-6).

SOLID HOSTS FOA DYE LASER RODS-PART 2, SOME EXPERIMENTAL RESULTS.

TIC, 1987. 8P. MN (LA-UR-88-0415)

4-34

ESTLER, R. C.; NOGAR, NICHOLAS S. (CLS-2).

CHEMICAL PRECUASOR TO OPTICAL DAMAGE DETECTED BY LASER IONIZATION MASS SPECTROMETAY. APPL. PHYS. LETT., V.52, P.2205-7. 1938.

4-35

FAIRBANK, WILLIAM M., JR.; LA BELLE, ROBERT D.; KELLER, RICHARD A. (CLS-2);

MILER, CHARLES M. (INC-7); POTHS, JANE (INC-7); FEAREY, BRYAN L. (INC-7).

PROSPECTS FOR LARGE DYNAMIC RANGE ISOTOPE ANALYSIS USING PHOTON BURST MASS

SPECTROMETRY.

TIC, 1988. 4P. MN (LA-UR-88-1407)

4-36

FEAREY, BRYAN L. (INC-7); PARENT, DENISE C. (CLS-2); KELLER, RICHARD A. (CLS-2);

MILLER, CHARLES M. (INC-7).

ISOTOPICALLY SELECTIVE, DOPPLER-FREE, SATUFATION SPECTROSCOPY OF LUTETIUM ISOTOPES VIA RESONANCE IONIZATION MASS SPECTROMETRY.

TIC, 1988. 5P. MN (LA-UR-88-1250)

4-37

FEAREY, BRYAN L. (INC-7); MILLER, CHARLES M. (INC-7); ROWE, M. W.; ANOERSON, JOHNNY E. (CLS-2); NOGAR, NICHOLAS S. (CLS-2).

PULSED LASER RESONANCE IONIZATION MASS SPECTROMETRY FOR ELEMENTALLY SELECTIVE DETECTION OF LEAD AND BISMUTH MIXTURES.

ANAL CHEM., V.50, P.1786-91. 1988.

4-38

FEAREY, BAYAN L. (INC-7); MILLE, CHARLES M. (INC-7); ANDERSON, JOHNNY E. (CLS-2);

NOGAR, NICHOLAS S. (CLS-2); ROWE, M. W.

RESONANCE IONIZATION MASS SPECTROMETRY OF :EAD AND BISMUTH MIXTURES.

TIC, 1988. 5P. MN (LA-UR-88-1248)

4-39

FEAREY, BRYAN L. (INC-7); MILLER, CHARLES M. (INC-7); KELLER, AICHARD A. (CLS-2);

PARENT, DENISE C. (CLS-2).

SECONDARY, NON-RESONANT CW I ASER IONIZATION EFFICIENCY ENHANCEMENT FOR RESONANCE IONIZATION MASS SPECTROMFTRY.

TIC, 1988. 5P. MN (LA-UR-88-1248)

4-40

FENG, XI-ZHARG (INC-11); XU, YING-TING (INC-11); SUN, PENG-NIAN (INC-11)

BALANCE BETWEEN VAN CER WAALS AND COORDINATION BOND ENERGIES-UNIFIED WIODEL OF COORDINATION BOND LENGTH IN LANTHANIDE COMPLEXES.

CAN. J. CHEM., V.66, P.109-10. 1988.

4-41

GARCIA, EDUARDO (INC-4); SAUER, NANCY N. (INC-4); RYAN, ROBERT R. (INC-4);

WILIANS, ARTHUR (LANSCE); ELLER, P. GARY (INC-4).

NEUTRON POWDER DIFFRACTION STUDY OF THE PRODUCTS FROM FLUORIISATING YTTRIUM-BARIUM-2

COPPEA-3 OXYGEN-7-MINUS-DELTA AT ROOM TEMPERATURE AND AT 400 DEGREES $C$.

J. MATER. RES., V.3, P.819-24. 1988. 
4-42

GARCIA, EDUARDO (INC-4); RYAN, ROBERT R. (INC-4); SAUER, NANCY N. (INC-4); FISK, ZACHARY (P-10): PIERCE, C. BALLARD (P-10); FLUSS, MICHAEL; BERARDEZ, LEWIS.

SYNTHESIS AND SUPERCONDUCTING CRITICAL TEMPEAATURE OF YTTRIUM BARIUM 2 COPPER 318 OXYGEN 7-DELTA.

PHYS. REV., V.38B, P.2900-2. 1988.

4-43

GEORGE, S.; GRAYS, A.; ENGLEMAN, ROLF, JR. (CLS-2).

SPECTRUM OF GOLD I IN THE INFRARED USING A FOURIER-TRANSFORM SPECTROMETER.

J. OPT. SOC. AM., V.5B, P.1500-2. 1988.

4-44

GILBERT, T.M. (INC-4); RYAN, ROBERT R. (INC-4); SATTELBERGER, ALFRED P. (INC-4).

MONO(CYCLOOCTATETRAENYL) ACTINIDE COMPLEXES-1. PREPARATIOR: OF THE DIAMIDES

(CBHB)AN[N(SIME3)2]2 (AN EQUALS TH, U), \& THE STRUCTURE OF (C8H8)THIN(SIME32]2.

ORGANOMETALLICS, V.7, P.2514-8. 1988.

4-45

HAIDER, QUAMRUL; LU, LON CHANG (INC.11).

ON THE ETA-NUCLEUS INTERACTION STRENGTH

PHYS. LETT., V.209B, P.11-3. 1988.

4-46

HARRADINE, DAVID M. (CLS-4); LYMAN, JOHN L (CLS-4); OLDENBORG, RICHARD C. (CLS-4);

SCHOTT, GARRY L. (M-1); WATANABE, HAFRY H. (CLS-3).

HYDROGENIAIR COMBUSTION CALCULATIONS-THE CHEMICAL BASIS OF EFFICIENCY IN HYPERSONIC FLOWS.

IN: AIAA THERMOPHYSICS, PLASMADYNAMICS AND LASERS CONFERENCE, 1988, SAN ANTONIO, TEX PROC., P.1-7. AIAA, 1988.

$4-47$

JACKSON, JOHN P.; ARTHURS, EUGENE; SCHWALBE, LARRY A. (X-4); SEGA, RONALD M.; WINDISH, DAVID; LONG, WILIAM H.; STAPPAERTS, EDDY A.

ACCELERATED AGING OF CELLULOSE BY LASER IRAADIATION.

TIC, 1988. 6P. MN (LA-UR-88-1185)

4-48

JACOBSON, LOREN A. (MST-6); MARTIN, PATRICK L (MST-5); MITCHELL, T. E. (CMS;.

RAPIDLY SOLILIFIED AND THEPIMALLY TREATED MICROSTRUCTURES OF BERYLLIUM-1WT\% YTTRIUM ALLOY.

IN: ELECTRON MICAOSCOPY SOCIETY. MEETING, 46TH, 1988, MILWAUKEE. PROC., P.782-3. SAN FRANCISCO PRESS, 1988.

4-49

JANECKY, DAVID R. (INC-T).

COUPLED SULFUR ISOTOPIC AND CHEMICAL MASS TRANSFER MODELING-APPROACH AND

APPLICATION TO DYNAMIC HYDROTHERMAL PROCESSES.

TIC, 1988. 11P. MN (LA-UR-88-3243)

4-50

JANECKY, DAVID R. (iNC-7).

MASSIVE SULFIDE DEPOSITS AND HYDROTHERMAL SOLUTIONS INCREMENTAL REACTION MODELING

OF MINERAL PAECIPITATION AND SULFUR ISOTOPIC EVOLUTION.

IN: INTERNATIONAL ASSOCIATION ON THE GENESIS OF ORE DEPOSITS. SYMPOSIUM, 7TH, 1986,

LULEA, SWEDEN, PROC., P.481-8. E. SCHWEIZERBART, 1988. (QE390 .1575 1986X)

ALSO PUBLISHED: TIC, 1986. 12P. MN (LA-UR-86-2791)

4-51

JARVINEN, GORDON D. (INC-4); LARSON, ELIZABETH M. (INC-4); WASSERMAN, HARVEY J. (C-3);

BUANS, C. J. (INC-4); RYAN, ROBERT R. (INC-4).

STRUCTURE OF TAIBENZYLAMMONIUM 1,1,1,1,2,2,2,3,3,3-DECACARBONYL-2,3-MU-

HYDRIDO-2,3-HU-SULFONYL-TRIANGULO-TRIOSMIUM.

ACTA CRYSTALLOGR., V.C44, P.1701-3. 1988. 
4-52

JONES, CLAUDE RANDOL (DRAFST).

OVERVIEW OF THE SDI [STRATEGIC DEFENSE INITIATIVE] PROGRAM IN SHORT WAVELENGTH CHEMICAL LASERS.

TIC, 1988. 19P. MN (LA-UR-88-0640)

4-53

JORGENSEN, BETTY S. (MST-6); ALDISSI, MAHMOUD (MST-7); UEPINS, RAIMOND (MST-7);

AGNEW, STEPHEN F. (INC-4).

HIGHLY ORIENTED UNSUBSTITUTED POLYDIACETYLENE.

IN: NONLINEAR OPTICAL PAOFERTIES OF POLYMERS, MATERIALS RESEARCH SOCIETY SYMPOSIUM, 1987, BOSTON. MRS, 1938. PROC., P.379-84.

4-54

KANG, J. S.; ALLEN, JAMES W.; SHEN, Z. X.; ELLIS, WALTON P. (CLS-2); YEH, J. J.; LEE, B. W.;

MAPLE, MERPILL B.; SPICER, W. E.; LINDAU, $I$.

ELECTRONIC STRUCTUAE OF THE QUENCHED SUPERCONDUCTIVITY MATEAIALS YTTAIUM-1-X PRASEODYMIUM-X BARIUM-2 COPPEA-3 OXYGEN-7-DELTA.

TIC, 1988. 21P. MN (LA-UR-88-2112)

$4-55$

KIM, KYU C. (MST-11); KROHN, BURTON J. (T-12); BRIESMEISTER, RICHAFD A. (MST-11):

CAVID, SHELLE J. (MST-11).

ANALYSIS OF THE FOURIEFI TRANSFORM AND DIODE LASER SPECTRA OF THE NU3 FUNDAMENTAL OF PLUTONIUM HEXAFLUORIDE.

J. MOL. SPECTFOSC., V.132, P.207-15. 1988.

4-56

KLEINSCHMIDT, PHILLIP D. (MST-5).

THERIOCHEMISTAY OF T:-AE PLUTONIUM FLUORIOES FROM ELECTRON !MPACT STIDIES.

J. CHEM. PHYS., V.89, P.6897-904. 1988.

4-57

KOBEA, EDWARO M. (INC-4): HAY, P. JEFFREY (T-12).

AB INITIO STUDIES OF THANSITION-METAL DIHYDROGEN CHEMISTRY.

TIC. 1988. 19P. MN (LA-UR-89-1791)

4-58

KOBER, EDWARD M. (T-14); RITCHIE, JAMES P. (T-14).

CALCULATED PROPERTIES AND SORPTION BEHAVIOR OF VARIOUS MOLECULES.

TIC, 1988. 8P. MN (LA-UR-88-3796)

4-59

KROHN, BURTON J. (T-12); MC DOWELL, AOBIN S. (CLS-4); PATTERSON, CHRIS W. (T-12);

NERESON, NORAIS G. (AP-2); REISFELD, MARTIN J. (LS-1); KIM, KYU C. (MST-11).

ANALYSIS OF THE O BRANCH OF THE 3 NU3 OVERTONE OF URANIUM HEXAFLUOR!DE-THE IMPLIED STRUCTURE OF THE N NU3 LADDER.

J. NOL. SPECTROSC., V.132, P.285-309. 1988.

4-60

LARSON, ELIZABETH M. (INC-4); ELLER, P. GARY (INC-4); PURSON, JOHN D. (INC-4);

PACE. CHARLES F. (INC-4); EASTMAN, MICHAEL P.; GREEGOR, R. B.; LYTLE. F. W.

SYNTHESIS AND STRUCTURAL CHARACTERIZATION OF CALCIUM TITANIUM OXYGEN-3 DOPED WITH 0.05-7.5 MOLE\% GADOLINIUM (III).

J. SOLID STATE CHEM., V.73, P.480-7. 1933.

4-61

LAWSON. A. C. (MST-5); OLSEN. CLAYTON E. (MST-13); RICHARDSON, J. W., JP.; MUELLER. M. H.;

LANOER, G. H.

STRUCTURE OF BETA-URANIUM.

ACTA CRYSTALLOGA., V.B44, P.89-96. 1988 
4-62

LIEFINS, PAIMOND (MST-7); ALDISSI, MAHMOUD (NST-7).

ELEGTAON-DONOR COPANT, METHOD OF IMPROVING CONDUCTIVITY OF POLYMERS BY DOPING

THEREWITH, AND A POLYMER SO TREATED.

PATENTED JULY 5, 1988, FILED 1984. (U.S.PATENT-4,755,326)

4-63

LOGE, GARY WAYNE (CLS-4); TIEE, JOE J. (CLS-4).

LIFETIME OF THE A DOUBLET SIGMA PLUS, $V^{\prime}=0$ LEVEL OF HS MEASUREU USING THE HANLE EFFEC?. J. CHEM. PHYS., V.89, P.7167-71. 1988.

4-64

LYMAN, JOHN L (CLS-4); HOLLAND, REDUS F. (CLS-4).

OXYGEN FLUORIDE CHEMICAL KINETICS.

J. PHYS. CHEM., V.92, P.7232-41. 1988.

4-65

MARLEY, NANCY A. (INC-7); GAFFNEY, JEFFREY S. (INC-7); MINAI, Y.; CHOPPIN, GREGORY R. (INC-4). CONFORMATIONAL CHANGES IN POLYELECTROLYTES AND THE EFFECT ON METAL BINDING.

TIC, 1988. 15P. MN (LA-UR-88-3687)

4-66

MARLEY, NANCY A. (INC-7); OTT, MARTIN ADOLF (INC-11); FEAREY, BRYAN L. (INC-7);

BENJAMIN, TIMOTHY M. (INC-7); ROGERS, PAMELA S. 2. (INC-7); GAFFNEY, JEFFREY S. (INC-7). HIGH-TEMPERATURE AND -PRESSURE SYSTEM FOR LASER RAMAN SPECTROSCOPY OF AOUEOUS SOLUTIONS.

REV. SCI. INSTRUM., V.59, P.2247-53. 1988.

4-67

MARTELL, CALVIN J. (CLS-1); HANSEL, JAMES M. (CLS-1).

DETERMINING GALLIUM FROM PLUTONIUM USING ANION EXCHANGE AND X-RAY FLUORESCENCE. LOS ALAMOS NATIONAL LABORATORY, 1988. 12P. (LA-11435)

4-68

MARTIN, JOE A. (CLS-2); NASTASI, MICHAEL (MST-7); TESMER, JOSEPH R. (P-10); MAGGIORE, CARL J. (CMS). HIGH-ENERGY ELASTIC BACKSCATTERING OF HELUUM IONS FOA COMPOSITIONAL ANALYSIS OF HIGH-TEMPERATURE SUPERCONDUCTOR THIN FILMS.

APPL. PHYS. LETT., V.52, P.2177-9. 1988.

4-69

MARTIN, JOE A. (CLS-2); CROSS, JON B. (CLS-2); POPE, LARRY E.

MOLYBDENUM SULFUR-2 INTERACTIONS WITH 1-3 EV ATOMIC OXYGEN.

TIC, 1988. 9P. MN (LA-UA-B8-3550)

4-70

MARTZ, JOSEPH C. (MST-13); HESS, DENNIS W.; ANDERSON. WALLACE E. (MST-13). MASS SPECTROMETRIC ANALYSIS OF CALIFORNIUM-4OXYGEN-2 PLASMAS-EFFECT OF OXYGEN CONCENTRATION AND PLASMA POWER.

TIC, 1988. 25P. MN (LA-UR-88-2751)

4-71

MAURO, BILLIE R. (CLS-6); FOLIYN, STEPHEN R. (CLS-6); SANDERS, VIRGIL E. (CLS-6). DAMAGE TO FUSED SILICA WINDOWS WHILE UNDER SIMULTANEOUS EXPOSURE TO FLOWING SOLVENTS AND LASER RADIATION AND 3OBNM.

TIC, 1987. 6P. MN (LA-1 :88-1094)

4-72

MC DOWELL, ROBIN S. (CLS-4).

ROTATIONAL PARTITION FUNCTIONS FOR LINEAR MOLECULES.

J. CHEM. PHYS., V.88, P.356-61. 1988.

4-73

MEEK, THOMAS T. (MST-6); SHEINBERG, HASKELL (MST-6); BLAKE, RODGER D. (MST-4).

SYNTHESIS OF ULTRAFINE POWDERS BY MICROWAVE HEATING.

PATENTED NOV. 15, 1988, FILED 1987. (U.S.PATENT- $4,784,686$ ) 
4-74

MILLER, CHARLES M. (INC-7); FEAREY, BAYAN L. :IIYC-7); PALMER, BYRON A. (CLS-4);

NOGAR, NICHOLAS S. (CLS-2).

HIGH-FIDELITY IN ISOTOPE RATIO MEASUREMENTS FOR RESONANCE IONIZATION MASS

SPECTROMETRY.

TIC, 1988. 5F. MN (LA-UR-88-1251)

4-75

MILLS, THOMAS R. (INC-4); MC IIJTEER, BERTHUS B. (INC-4); MONTOYA, JOE G. (INC-4).

SULFUR AND SELENIUM ISOTOPE SEPARATION BY DISTILLATION.

TIC. 1988. 7P. MN (LA-UA-88-2421)

4-76

MISKOWSKI, VINCENT M.; DALLINGER, RICHARD F.; CHRISTOPH, GARY G. (P-8); MORRIS, DAVID E. (INC-4):

SPIES, GEORGE H. (INC-4); WOODRUFF, WILLIAM H. (INC-4).

ASSIGNMENT OF THE RHODIUM-RHODIUM STRETCHING FREQUENCY IN BISLIGAND (TETRAACETATE) DIRHODIU!A COMPLEXES AND THE CRYSTAL AND MOLECULAR STRUCTURE OF GUANIDINIUM DICHLORU (TETRAACETATE) DIRHODIUM-RELATIONSHIP BETWEEN VIBRATIONAL SPECTPA AND STRUCTUAE. INORG. CHEM., V.26, P.2127-32. 1987.

4-77

MOORE, DAVID S. (CLS-4); SCHMIDT, STEPHEN C. (M-6).

EXPEPIMENTAL MOLECULAR SPECTROSCOPY IN SHOCK-COMPRESSED MATERIALS.

IN: SHOCK WAVES IN CONDENSED MATTER, 1987, MONTEREY. PROC., P.35-42. ELSEVIER, 1986. (QCi73.4.C65 $\$ 57$ 1987X)

4-78

MORALES, RAUL (CLS-1); BARTHOLDI, CATHERINE S. (CLS-1); CUNNIFGGAM, PAUL T. (CLS-1).

HPLC SEPARATION OF HETEROY.YCLIC BETA-DIKETONATES OF ACTINIDE,, LANTHANIDE AND TRANSITION METALS.

TALANTA, V.35, P.461-4. 1988.

4-79

MORALES, RAUL (CLS-1); NEKIMKEN, HOWARD L. (CLS-1); BARTIIOLDI, CATHERINUE S. (CLS-:);

CUNNINGHAM, PAUL T. (CLS-1).

SPECTRAL STUDIES OF HETEROCYCLIC BETA-DIKETONATES OF ACTINIDE, LANTHANIDE, AND

TRANSITION METALS.

SFECTROCHIM. ACTA, V.44A, P.165-9. 1988.

4.80

MUK'HERJEE, PPITISH (CLS-5); GOSNELL, TIMOTHY R. (CLS-5); BIGIO, IRVING J. (CLS-5).

BROADBAND MICROWAVE ABSORPTION SPECTROMETEF FOR LIQUID MEDIA.

REV. SCI. INSTRUM., V.59, P.2577-82. $1988^{\circ}$

4-81

NASTASI, MICHAEL (MST-7); ' ESMER, JOSEPH R. (P-16). HOLLANDER, M. G. (MEE-11);

SMITH, JAMES F. (CMS); MAG GIORE, CARL J. (CMS!

FORMATION OF YTTRIUM B.ARIUM-2 COPPER-3 OXYGEN-7 SUPERCONDUCTING FILMS BY ION IMPLANTATION.

APPL. PHYS. LETT., V.52, P.1729-31. 1938

4-82

NEKIMKEN, HOWARD L. (CLS-1); SMITH, BARBARA F. (CLS-1); JARVINEN, GOADON D. (INC-4);

PETERSON, EUGENE J. (CLS-1); JONES, MAPIANNE M. (CLS-1).

CCMPUTER-CONTROLLED FLOW INJECTION ANALYSIS SYSTEM FOR ON-LINE DETERMINATION OF

DISTRIBUTION RATIOS.

ANAL. CHEM., V.60, P.1390-3. 1988.

4-83

NESTOR, O. H.; FIGUEIRA, JOSEPH F. (CLS-DO).

PRODUCTION AND PROPERTIES OF PERRHENATE-DOPED ALKALI HALIDE CRYSTALS.

TIC, 1987. 8P. MN (IA-UR-8B-2006) 
4-84

NOGAR, NICHOLAS S. (CLS-2); ESTLER, R. C.

CHEMICAL PRECURSOR TO OPTICAL DAMAGE? STUDIES BY LASER IONIZATION MASS SPECTROMETRY

TIC, 1987. 10P. MN (LA-UR-E7-4002)

4-85

NOGAR, NICHOLAS S. (CLS-2); ESTLER, R. C.: ROWE, M. W.; FEAREY, BRYAN L. (INC-7);

MILLER, CHARLES M. (INC- 7 ).

LASER DESORPTHONABLATION STUDIES BY RESONANCE IONIZATION MASS SPECTROMETRY.

TIC, 1988. 5P. MN (LA-UA-88-1281)

4-86

OTT, KEVIN C. (CLS-1); SNITH, JAMES L. (CMS).

PREPARATION AND CHARACTERIZATION OF ISOTOPIC OXYGEN-ENAICHED YTTAIUM BARIUM COPPER OXIDE.

TIC, 1988. 15P. MN (LA-UR-88-1591)

4-87

PASTERNAK, MOSHE; TAYLOR, R. DEAN (WX-12).

VERY HIGH PRESSURE MOSSBAUER SPECTROSCOPY USING DIAMOND ANVIL CELLS

TIC, 1988. 23P. MN (LA-LRR-88-2559)

4-88

RAO. D.; TOVAR, M.; OSEROFF, S. B.; VIER, D. C.; SCHULTZ, S.; THOMPSON, JOE D. (P-10):

CHEONG, SANG-WOOK (P-10); FISK, ZACHARY (P-10).

CAYSTAL-FIELD AND EXCHANGE INTERACTIONS OF DILUTE GADOLINIUM-3+ IONS IN EUROPIUM-2 COPPER OXIDE-4.

PHYS. REV., V.38B, P.8920-2. 1988.

4-89

ROKOP, DONALD J. (INC-7); SCHROEDER, N. C.; WOLFSBERG, KURT (INC-7).

HIGH SENSITIVITY TECHNETIUM ANALYSIS USING NEGATIVE THERMAL IONIZATION MASS

SPECTROMETRY.

TIC, 1988. 3P. MN (LA-UR-88-2819)

4-90

RUNDBERG, ROBERT S. (INC-11); MITCHELL, ALAN J. (INC-11); TRIAY, INES R. (INC-11);

TORSTENFELT, N. BORJE.

SIZE AND DENSITY OF A PLUTONIUM-242 COLLOID.

IN: INTERNATIONAL SYMPOSIUM ON THE SCIENTIFIC BASIS FOR NUCLEAR WASTE MANAGEMENT. 11TH, 1987, BOSTON, MASS. PROC., P.243-8. MATEAIALS RESEARCH SOCIEIY, 1988. (TA401.M37X V.112)

ALSO PUBLISHED: TIC, 1987. 5P. MN (LA-UA-87-3951)

4-91

RYAN, ROBERT R. (INC-4); LARSON, ELIZABETH M. (INC-4); PAYNE, G. F.; PETERSON, JOSEPH R. STRUCTURE OF FAC-TAICHLORO (TRISTAIPHENYLARSINEOXIDE) CERIUM ACETONITAILE TRINITRO (TRISTRIPHENYLARSINEOXIDE) CERIUM-EVIDENCE FOR A STRUCTURAL TRANS INFLUENCE.

INORG. CHIM. ACTA, V.131, P.267-71. 1987.

4-92

SANDENAW, THOMAS A. (MST-13).

INCOMMENSURATE/COMMENSURATE CHARGE-DENSITY-WAVE STATES AS A SOURCE FOR PLUTONIUM METAL BEHAVIOR.

LOS ALAMOS, 1988. 28P. (LA-11083-MS)

4-93

SANDER, ROBERT K. (CLS-4); TIEE, JOE J. (CLS-4); QUICK, CHARLES ROBERT (CLS-4);

ROMERO, ROBERT J. (CLS-4); ESTLER, R. C.

OUENCHING OF CARBON-2 HYDROGEN PRODUCED BY VACUUM ULTRAVIOLET PHOTOLYSIS OF

ACETYLENE.

J. CHEM. PHYS., V.89, P.3495-501. 1988. 
4-94

SAUER, NANCY N. (INC-4); GARCIA, EDUARDO (INC-4); MARTIN, JOE A. (CLS-2); RYAN, ROBERT R (INC-4):

ELLER, P. GARY (INC-4); TESMER, JOSEPH R. (P-10); MAGGIORE, CARL J. (CMS).

FLUORINATION OF THE HIGH-TC SUPERCONDUCTORS YTTRIUM BARIUM-2 COPPER.3 OXIDE-7- DELTA AND GADOLINIUM QAARIUM-2 COPPER-3 OXIDE 7-DELTA

J. MATER. RES., V.3, P.813-8. 1988.

4-95

SEVERING, ANDREA (MST-5); REI:HARDT, W.; HOLLANDMORITZ, E.; WOHLLEGEN, D.; ASSMUS, W OBSERVATION OF PHONON ANOMALIES IN THE INTERMEDIATE-VALENCE COMPOUND CERIUMPALLADIUM-3.

PHYS. REV., V.38B, P.1773-8. 1988.

4-96

SHERA, E. BROOKS (P-DO).

SINGLE MOLECULE TRACKING.

PATENTED DEC. 27, 1988, FILED 1987. (U.S.PATENT-4,793,705)

4-97

SMITH, BARBARA F. (CLS-1); JARVINEN, GORDON D. (INC-4); MILLER, GEOFFREY G. (INC-11);

RYAN, ROBERT R. (INC-4); PETERSON, EUGENE J. (CLS-1).

SYNERGISTIC EXTRACTION STUDIES OF AMERICIUM (III) AND EUAOPIUM (III) FROM PERCHLORIC ACID WITH 4-BENZOYL-2, 4-DIHYDRO-5-METHYL-2-PHENYL-3-HYDROGEN-PYRAZOL-3-THIONE (BMPPT) AND TRI-N-OCTYLPHOSPHINE OXIDE (TOPO) IN BENZENE.

SOLVENT EXTR. ION EXCH., V.5, P.895-908. 1987.

4-98

SPANGLER, LEE H. (CLS-2); BOSMA, WAYNE B.; VAN ZEE, ROGER D.; ZWIER, TIMOTHY S. HINDERED INTERNAL ROTATION IN SOME SINGLY METHYLATED TRANS-STILBENES.

J. CHEM. PHYS., V.88, P.6768-77. 1988.

4-99

SZE, ROBERT C. (CLS-5); GREENE, DENNIS P. (CLS-9); BIGIO, IRVING J. (CLS-5), SHAY, T. M. (CLS-5):

MC COWN, ANDREW W. (CLS-5); FIGUEIRA, JOSEPH F. (CLS-5); SMITH, P.; VANNINI, MATTEO

DISCHARGE EXCITATION OF THE XENON FLUORIDE C TO A TRANSITION.

IN: INTERNATIONAL CONFERENCE ON LASERS, 1987, LAKE TAHOE, PROC., P.268-71. STS PRESS, 1988. ALSO PUBLISHED: TIC, 1987. 5P. MN (LA-UR-88-0656)

4-100

TAYLOR, ANTOINETTE J. (CLS-5); GOSNELL, TIMOTHY R. (CLS-5); ROBERTS, JEFFREY P. (CLS-5);

LESTER, C. S. (CLS-5); GIBSON, ROBERT B. (CLS-5); HARPER, S. E. (CLS-5); TALLMAN, CHARLES R. (CLS-5). SUBPICOSECOND, HIGH-BRIGHTNESS EXCIMER LASER SYSTEMS.

TIC, 1988. 4P. MN (LA-UR-88-2569)

4-101

TAYLOR, ANTOINETTE J. (CLS-5); GIBSON, ROBERT B. (CLS-5); ROBERTS, JEFFREY P. (CLS-5).

TWO-PHOTON ABSORPTION AT 248 MN IN ULTRAVIOLET WINDOW MATERIALS. OPT. LETT., V.13, P.814-6. 1988.

4-102

TESMER, JOSEPH R. (P-10); MAGGIORE, CARL J. (MEE-11); NASTASI, MICHAEL (MST-7);

CHEONG, SANG-WOOK (P-10); DICK, CHI-MM (CMS)

EFFECTS AND BEHAVIOH OF IMPLANTED FLUORINE IN HIGH TEMPERATURE SUPERCONDUCTORS IN: HIGH-TEMPERATURE SUPERCONDUCTORS, 1987, BOSTON, MASS. PROC., P.643-6. MATERIALS RESEARCH SOCIETY, 1988. (TK/7872/.SQ/H55/1987X)

4-103

TIEE, JOE J. (CLS-4); QUICK, CHARLES ROBERT (CLS-4); LOGE, GARY WAYNE (CLS-4);

WAMPLER, FRED B. (CLS-4).

TWO-PHOTON PUMPED CARBON DIOXIDE (B-A) LASER.

J. APPL. PHYS., V.63, P.288-90. 1988. 
4-104

VAN DER SLUYS, WILLIAM G. (INC-4); BURNS C. J. (INC-4); HUFFMAN, J. C.;

SATTELBERGER, ALFRED P. (INC-4).

URANIUM ALKOXIDE CHEMISTRY-1. SYNTHESIS AND THE NOVEL DIMERIC STRUCTURE OF THE FIRST HOMOLEPTIC URAMIUM(III) ARYLOXIDE COMPLEX.

J. AM. CHEM. SOC., V.110, P.5924-5. 1988.

4-105

VOTER, ARTHUR F. (T-12); COHEN, JENNIFER M. (T-12).

CLASSICALLY EXACT SURFACE DIFFUSION CONSTANTS AT ARBITRARY TEMPERATURE

TIC, 1988. 16P. MN (LA.UR-88-2784)

4-106

WALKER, THOMAS E. (INC-4); UNKEFER, CLIFFORD J. (INC-4); EHLER, DEBORAH S. (INC-4)

SYNTHESIS OF CARBON-13 ENAICHED MONOSACCHARIDES DERIVED FROM GLUCOSE AND MANNOSE J. CARBOHYDR. CHEM., V.7, P.115-32 1988

4-107

WANGEN, LAWRENCE E. (CLS-1); PHILLIPS, MARILYN VIRGINIA (CLS-1); WALKER, L. F. (CLS-1)

USE OF MULTIVARIATE CALIBRATION FOR PLUTONIUM OUANTITATION BY THE PLUTONIUM-3

SPECTROPHOTOMETRIC METHOD.

LOS ALAMOS NATIONAL LABORATORY, 1988. 8P. (LA-11297-MS)

4-108

WANTUCK, PAUL JOSEPH (CLS-8); OLDENBORG, RICHARD C. (CLS-4); BAUGCHUM, STEVEN L.;

WINN, KENNETH R. (CLS-4).

DIRECT MEASUREMENTS OF METHOXY REMOVAL RATE CONSTANTS FOR COLLISIONS WITH METHANE, ARGON, NITROGEN-2, XENON, AND CARBON FLUORIDE IN THE TEMPERATURE RANGE 673-973K IN: SYMPOSIUM (INTERNATIONAL) ON COMBUSTION, 22ND, 1988, UNIVERSITY OF WASHINGTON PROC., P.973-81. COMBUSTION INSTITUTE, 1989. (OD516.S92 1988)

ALSO PUBLISHED: TIC, 1988. 16P. MN (LA-UR-88-0186)

4-109

WEST, MIKE H. (MST-13); FERRAN, MICHELLE D. (MST-13); FIFE, KEITH W. (MST-13)

CHLORINATION OF PLUTONIUM DIOXIDE

LOS ALAMOS NATIONAL LABORATORY, 1988. 13P. (LA-11256)

4-110

WILSON, MICHAEL A.; POHORILLE, A.; PRATT, LAWRENCE R. (CLS-2).

SURFACE POTENTIAL OF THE WATER LIOUID- VAPOR INTERFACE. J. CHEM. PHYS., V.88, P.3281-5. 1988.

4-111

WOODRUFF, WILLIAM H. (INC-4); DYER, R. BRIAN (INC-4): SCHOONOVER, JON R. (INC-4).

RESONANCE RAMAN SPECTROSCOPY OF BLUE COPPER PROTEINS

IN: RESONANCE RAMAN SPECTRA OF HEME AND METALLOPROTEINS, T.G. SPIRO, ED. P $413-38$

WILEY, 1988. (QP519.9.R36 B56 1987 V.3)

4-112

ZIMMERMANN, BARBARA H. (INC-4); NITSCHE, CARMEN I. (INC-4); FEE, JAMES A. (INC-4);

RUSNAK, FRANK M: MUNCK, ECKARD.

PROPERTIES OF A COPPER-CONTAINING CYTOCHAOME BA3-A SECOND TERMINAL OXIDASE FROM THE

EXTREME THERMOPHILE THERMUS THERMOPHILUS

PROC. NATL. ACAD. SCI. USA, V.85, P.5779-83. 1988 
6. COAL CONVERSION AND UTILIZATION-COAL SCIENCE ANO ANALYSIS

6-1

SHALEK, PETER D. (MST-4); PHILLIPS, DAVID S. (MST-4); CHFISTIANSEN, DAVID E. (MST-7):

KATZ, J. D. (MST-4); PARKINSON. WILLIAM J. (WX-4); PETROVIC, JOHN J. (MST-4).

SYNTHESIS AND CHARACTERIZATION OF VLS-DERIVED SILICON CARBIDE WHISKERS.

TIC, 1988. 11P. MN (LA-UR-88-2536) 
7. CRITICALITY STUDIES

7-1

MALENFANT, PACHARD E. (DIR-EAD).

SELF UMMTING FEATURES OF ACCIDENTAL CRITICALITY IN A SOLUTION SYSTEM.

TIC, 1988. 9P. MN (LA-UR-88-3124)

7-2

PLASSMANN, EUGENE A. (N-2).

TECHNICAL SPECIFICATIONS FOR THE LOS ALAMOS CRITICAL EXPERIMENTS FACILITY LOS ALAMOS NATIONAL LABORATORY, 1988. 34P. (LA-06016.SOP,REV.2)

7-3

SPAIGGS, GPEGORY D. (N-2); MARTIN, E. RAY (AT-1).

COMPUTERIZED CONTROL SYSTEM FOR CRITICAL FACILITIES AT LOS ALAMOS NATIONAL LABORATORY ABSTRACT IN: TRANS. AFA. NUCL. SOC., V.56, P.331-2. 1988. 
8. ELECTRIC ENERGY SYSTEMS-SYSTEMS DEVELOPMENT AND CONTROL

Q-1

SWIFT, GREGORY W. (P-10).

LIOUID-METAL MAGNETOHYDRODYNAMIC ACOUSTIC TRANSDUCER.

J. ACOUST. SOC. AM., V.83, P.350-51. 1988. 
91

GAFFNEY, JEFFHEY S. (INC-7); TANNER, ROGER L.

ALCOHOL FUEL USE-IMPLICATIONS FOR ATMOSPHERIC LEVELS OF ALDEHYDES, ORGANIC NITRATES, PANS, AND PEROXIDES. SEPARATING SOURCES USING CARBON ISOTOPES.

TIC. 1988. OP. MN (LA-UR-88-1533)

92

HARRISON, RALPH J.; VOTER, ARTHUR F. (T-12); CHEN, SHAO P. (T-11).

EMBEDDED ATOM POTENTIAL FOR BCC IRON.

TIC, 1988. 6P. MN (LA-UR-88-3794)

9-3

HARRISON, RALPH J.; SPAEPEN, FRANS; VOTER, ARTHUR F. (T-12); CHEN, SHAO P. (T-11) STRUCTURE OF GRAIN BOUNDARIES IN IRON.

TIC, 1988. 26P. MN (LA-UR-88-2128)

$9-4$

HUFF, JAMES R. (MEE-11); VAMDERBORGH, NICHOLAS E. (MEE-11); ROACH, JAMES F. (S-2);

MURRAY, HUGH S. (MEE-11).

FUEL CELL POWERED PROPULSION SYSTEMS FOR HIGHWAY VEHICLES.

TIC, 1987. 18P. MN (LA-UR-87-0820)

$9-5$

MURRAY, HUGH S. (E-11).

PROGRESS IN FUEL CELLS FOR TRANSPORATION APPLICATIONS.

TIC, 1986. 2OP. MN (LA-UR-86-1476)

$9-6$

MURRAY, HUGH S. (MEE-11); HUFF, JAMES R. (MEE-11); ROACH, JAMES F. (S-2); THAYER, GARY R. (S-4). DOT FUEL-CELL-POWERED BUS FEASIBILITY STUDY-FINAL REPOAT-DECEMBER 1986.

LOS ALAMOS, 1987. 369P. (LA-10933-MS)

97

MURRAY. HUGH S. (MEE-11); HLIFF. JAMES R. (MEE-11).

FUEL CELLBATTERY HYBRID VEHICLE ASSESSMENT.

LOS ALAMOS, 1987. 128P. (LA-10948-MS)

$9-8$

SRINIVASAN, S. (MEE-11); TICIANELLI, EDSON A. (MEE-11); DEROUIN, CHARLES R. (MEE-11);

REDONDO, ANTONIO (MEE-11).

ADVANCES IN SOLID POLYMER ELECTROLYTE FUEL CELL TECHNOLOGY WITH LOW PLATINUM LOADING ELECTRODES

J. POWER SOURCES, V.22, P.359-75. 1988. 
10. ENERGY-GENERAL

10-1

ALTSEIMER, JOHN H. (S-4); ROACH, JAMES F. (S-2).

POTENTIALS FOA FUIEL CELLS IN REFINERIES AND CHLOR-ALKALI PLANTS.

TIC. 1986. 16P. MN (LA-UR-86-2044)

$10-2$

SPINIVASAN, S. (MEE-11).

FUEL CELLS FOR EXTRATERRESTRIAL AND TERRESTRIAL APPLICATIONS.

TIC, 1997. 32P. MN (LA-UR-87-2303)

$10-3$

SWIFT, GREGORY W. (P-10); MIGLORI, ALBERT (P-10): WHEATLEY, JOHN C. (CMS/P-10).

LIQUID METAL THERMOACOUSTIC ENGINE.

IN: INTERSOCIETY ENERGY CONVERSION ENGINEERING CONFERENCE, 21ST, 1986, SAN DIEGO. PROC., P.32-7. ACS, 1926.

ALSO PUBLISHED: TIC, 1986. 7P. MN (LA-UR-86-1548) 
12-1

GOTTESFELD, SHIMSHON (MEE-11); PAFFOAD, JUDITH (MEE-11).

NEW APPROACH TO THE PROBLEM OF CARBON MONOXIDE POISONING IN FLEL CELLS OPERATING AT LOW TEMPERATURES.

J. ELECTFOCHEM. SOC., V.135, P.2651-2. 1988.

$12-2$

PAFFETT, MARK THOMAS (MEE-11); BEERY, JER.OME G. (MEE-11); GOTTESFELD. SHIMSHON (MEE-11) OXYGEN REDUCTION AT PLATINUM 0.65 CHAOMIUM 0.35, PLATINUM 0.2 CHRCMIUM 0.8 AND ROUGHENED PLATINUM.

J. ELECTROCHEM. SOC., Y.135, P.1431-6. 1988.

$12-3$

TICIANELLI, EDSON A. (MEE-11); DEROUIN, CHARLES R. (MEE-11); SRINIVASAN, S. (MEE-11).

LOCALIZATION OF PLATINUM IN LOW CATALYST LOADING ELECTRODES TO ATTAIN HIGH POWER DENSITIES IN SPE FUEL CELLS.

J. ELECTROANAL CHEM., V.251, P.275-95. 1988.

$12-4$

TICIANELLI, EDSON A. (MEE-11!; DEROUIN, CHARLES R. (MEE-11); REDONDO, ANTONIO (MEE-11): SRINIVASAN, S. (MEE-11).

METHOOS TO ADVANCE TECHNOLOGY OF PROTON EXCHANGE MEMBRANE FUEL CELLS. IN: SYMPOSIUM ON ELECTRODE MATERIALS AND PROCESSES FOR ENERGY CONVEASION AND STORAGE, 2ND, 1997, PHILADELPHIA. PROC., P.166-88. ELECTROCHEMICAL SOCIETY, 1987 (TKV2901/.S9/1997X)

ALSO PUBLISHED: J. ELECTROCHEM. SOC., V.135, P.2209-14. 1988.

$12-5$

TICIANELL!, EDSON A. (MEE-11); SRINIVASAN, S. (MEE-11); GONZALEZ, E. R. RECENT ADVANCES IN SOUD POLYMER ELECTROLYTE FUEL CELL TECHNOLOGY.

TIC, 1988. 15P. MN (LA-UR-88-0100) 
13-1

ALLISON, S. W.; CATES, MICHAEL REID; SIMPSON, M. L.; NOEL, BRUCE W. (MEE-5); TURLEY, D.; GILLIES, G. T. SENSING APPLICATIONS OF RARE-EARTH DOPED LUMINESCENT MATERIALS.

TIC, 1988. 12P. MN (LA-UR-88-3866)

13-2

ALTSEIMER, JOHN H. (A-4); EDESKUTY, FREDERICK J. (A-4).

SURVEY OF GEOTHERMAL PROCESS HEAT APPLICATIONS IN GUATEMALA-AN ENGINEERING SURVEY. LOS ALAMOS NATIONAL LABORATORY, 1988. 86P. (LA-11326-MS)

13-3

APEL, E. C. (CLS-5); GOSNELL, TIMOTHY R. (CLS-5); SHAY, T. M. (CLS-5); SAMADANI, R. (CLS-5). FUNDAMENTAL NOISE PROCESSES IN A LASER EXCITED OPTICAL FILTER.

TIC, 1987. 7P. MN (LA-UR-88-0573)

13-4

BIGIO, IRVING J. (CLS-5); KURNIT, NORMAN A. (CLS-6); DONALDSON, W. R.; GEISSLER, K.;

SRINIVASAN-RAO, T.

LASER WORKING GROUP.

TIC, 1988. 7P. MN (LA-UR-88-4237)

13-5

BINDER, PHILIPPE M. (CNLS).

DIFFUSION IN LATTICE FLUIDS.

TIC. 1988. 11P. MN (LA-UR-88-2654)

13-6

BOROVSKY, JOSEPH E. (ESS-8); MC COMAS, DAVID J. (ESS-8); BARRACLOUGH, BRUCE L. (ESS-8). SECONDARY-ELECTRON YIELD MEASURED FOR 5-24 MEV PROTONS ON ALUMINUM-OXIDE AND GOLD TARGETS.

NUCL. INSTRUM. METHODS PHYS. RES., V.B30, P.191-5. 1988.

13.7

BUTLER, THOMAS A. (MEE-13); WEINACHT, DANIEL J. (MEE-13); TAFOYA, JOSE 1. (Q-11).

TRANSPORTATION LOADS WITH AIR VERSUS LEAF SPRING SUSPENSIONS.

LOS ALAMOS NATIONAL LABORATORY, 1988. 42P. (LA-11320-MS)

13-8

COOPER, RICHARD K. (AT-6); CHAN, K. C. (AT-6); WANG, TAI-SEN F. (AT-6); THODE, LESTER E. (ADDRA).

TRANSVERSE EFFECTS OF A WAVEGUIDE COUPLING SLOT.

TIC, 1988. 3P. MN (LA-UR-88-3251)

13-9

COSIMI, RONALD A. (WX-9).

GAS SHUTTER CLOSURE FOR SEALING LOS PIPES.

LOS ALAMOS, 1988. 7P. (LA-11207-MS)

13-10

CROSS, JON B. (CLS-2).

LABORATORY INVESTIGATIONS OF LOW EARTH ORBIT ENVIRONMENTAL EFFECTS ON SPACECRAFT

MATERIALS.

IN: ENGINEERING, CONSTRUCTION, AND OPERATIONS IN SPACE, 1988, ALBUQUERQUE, N.M. PROC. P.607-19. AMERICAN SOCIETY OF CIVIL ENGINEERS, 1988.

ALSO PUBLISHED: TIC, 1988. 16P. MN (LA-UR-88-1229)

13-11

CROSS, JON B. (CLS-2); CREMERS, DAVID A. (CLS-4).

LASER SUSTAINED DISCHARGE NOZZLE APPARATUS FOR THE PRODUCTION OF AN INTENSE BEAM OF HIGH KINETIC ENERGY ATOMIC SPECIES.

PATENTED OCT. 25, 1988, FILED 1988. (U.S.PATENT-4,780,608) 
13-12

CULLINGFORD, HATICE S.; KELLER, M. DEAN (ENG-DO).

LUNAR CONCFETE FOR CONSTRUCTION.

TIC. 1988. 16P. MN (LA-UR-88-2405)

13-13

DAVIOSON, RICH FRANK (WX-4); COOK, WILLIAM A. (WX-4); RABERN, DONALD A. (WX-4);

SCHNURR, NORMAN M. (WX-4).

PREDICTING BORE DEFORMATIONS AND LAUNCHER STRESSES IN RAILGUNS

IN: SYMPOSIUM ON ELECTROMAGNETIC LAUNCH TECHNOLOGY, 3RD, 1986, AUSTIN, TEX. PROC., P.31-6. IEEE, 1986 (TK7872.P8 S94 1986X)

ALSO PUELISHED: TIC, 1996. 7P. MN (LA-UR-86-0941)

13-14

DIXON, RAY D. (MST-6); LEWIS, GARY K. (MST-6); FLICK, FRED F. (MST-6).

LASER TACKING FOR MINIMIZING DISTORTION.

WELO. J., V.67, P.31-2. 1988.

13-15

EVANS, CHRIS; HANNAH, PHIL; RHORER, RICHARD L. (MEC-6).

PRELIMINARY EXPERIMENTS WITH THAEE IDENTICAL ULTRAPRECISION MACHINE TOOLS.

IN: ADVANCES IN FABRICATION AND METROLOGY FOR OPTICS AND LARGE OPTICS, 1988, SAN

DIEGO, CALIF. PROC., P.19-28. SPIE, 1988. (TA1505 .S64X V.966)

ALSO PUBLISHED: TIC, 1988. 24P. MN (LA-UR-88-2688)

$13-16$

FEBER, ROY C., JR. (MST-3); MERRIGAN, MICHAEL A. (MEE-13).

THERMOCHEMICAL MODELING OF MASS TRANSPORT IN HIGH-TEMPERATURE HEAT PIPES

IN: INTERNATIONAL HEAT PIPE CONFERENCE, 6TH, 1987, GRENOBLE, FRANCE. PROC., P.308-14. STT CENG, 1987. (TJ264.158 1987X)

ALSO PUBLISHED: TIC, 1987. 7P. MN (LA-UR-87-0911)

13-17

FORMAN, PETER R. (CTR-8); JAHODA, FRANZ C. (CTR-8).

LINEAR BIREFRINGENCE EFFECTS ON FIBER-OPTIC CURRENT SENSORS

APPL. OPT., V.27, P.3088-96. 1988.

13-18

FOWLER, CLARENCE M. (M-6).

LOSSES IN MAGNETIC FLUX COMPRESSION GENERATORS, PART 2-RADIATION LOSSES

LOS ALAMOS NATIONAL LABORATORY, 1988. 49P. (LA-09956-MS, V.2)

13-19

FOWLER, CLARENCE M. (M-6); ZIMMERMANN, EUGENE L. (WX-4); CUMMINGS, CHARLES E. (WX-4);

DAVIDSON, RICH FRANK (WX-4); FOLEY, EDWARD (WX-4); HAWKE, R. S.; KERRISK, JERRY F. (WX-4);

PARKER, JERALD V. (P-7); PARSONS, W. M. (CTR-9); PETERSON, DENNIS R. (WX-4);

SCHNURR, NORMAN M. (WX-4); STANLEY, PATRICK M. (WX-4).

RAILGUNS POWERED BY EXPLOSIVE DRIVEN FLUX COMPRESSION GENERATORS

IN: SYMPOSIUM ON ELECTROMAGNETIC LAUNCH TECHNOLOGY, 3RD, 1986, AUSTIN, TEX. PROC.,

P.69-74. IEEE, 1986. (TK7872.P8 $\$ 94$ 1986X)

ALSO PUBLISHED: TIC, 1986. 7P. MN (LA-UA-86-0926)

$13-20$

FUGELSO, LEIF ERIK (MEE-13); DEETER, MICHEL.

CALCULATION OF SUBSEOUENT STRUCTURAL EFFECTS AFTER AN ACCIDENTAL EXPLOSION IN A TEST FACILITY

TIC, 1988. 16P. MN (LA-UA-88-2439)

13-21

FUKUSHIMA, EIICHI (INC-4); RATH, ALAN R.; ROEDER, S. B. W.

APPARATUS FOR UNILATERAL GENERATION OF A HOMOGENEOUS MAGNETIC FIELD

PATENTED JAN. 26, 1988, FILED 1984. (U.S.PATENT-4.721,914) 
13-22

GAC. FRANK D. (MST-4); BLAKE, RODGER D. (MST-6); DAY, D. E.; HAGGERTY, JOHN S.

FABAICATION OF PRECISION GLASS SHELLS BY JOINING GLASS RODS

PATENT FEB. 23, 1:288, FILED 1986. (U.S.PATENT-4,726,829)

13-23

GOFORTH, JAMES H. (M-6); CAIRD, ROBERT S., JR. (M-6); GREENE, ARTHUR E. $(X-10)$ :

LINDEMUTH, I. R. $(X-10)$; MARSH, STANLEY P. (M-6); OONA, HENN (M-6); REINOVSKY, R. E. (M-6).

ADVANCES IN EXPLOSIVELY FORIMED FUSE OPENING SWITCHES.

IN: IEEE INTERNATIINAL PULSED POWER CONFERENCE, 6TH, 1987, ARLINGTON, VA. PROC., P.290.9.

IEEE, 1987. (TK2896.115 1987X)

13-24

GOFORTH, JANES H. (M-6); CAIRD, ROBERT S., JR. (M-6); FOWLER, CLARENCE M. (M-6);

GREENE, ARTHUR E. (X-10); KRUSE, HERALD W. (P-14); LINDEMUTH, I. R. (X-10); OONA, HENN (M-6):

REINOVSKY, R. E. (M-6).

PERFORMANCE OF THE LAGUNA PULSED POWEA SYSTEM.

IN: IEEE INTERNATIONAL PULSED POWER CONFERENCE, 6TH, 1987, ARLINGTON, VA. PROC., P.445-8. IEEE, 1987. (TK2896 .I15 1987X)

ALSO PUBLISHED: TIC, 1987. 9P. MN (LA-UR-B7-2124)

13-25

HANNIGAN, JAMES W. (CMS); SCHWARZ, RICARDO B. (CMS).

COMPACT FRICTION AND WEAR MACHINE.

REV. SCI. INSTRUM., V.59, P.1420-2. 1988.

13-26

HOFI.ER, THOMAS J. (P-10); WHEATLEY, JOHN C. (CMSIP-10); SWIFT, GREGORY W. (P-10):

MIGLIORI, ALBERT (P-10).

ACOUSTIC COOLING ENGINE.

PATENTED FEB. 2, 1988, FILED :986. (U.S.PATENT-4,722,201)

13-27

HONIG, EMANUEL M. (CLS-7); NUNNALLY, WILLIAM C. (E-11).

MULTIPLE RESONANT RAILGUN POWER SUPPLY.

PATENTED JAN. 12, 1988, FILED 1985. (U.S.PATENT-4,718,322)

13-28

HONIG. EMANUEL M. (CLS-7); NUNNALLY, WILLIAM C. (E-11).

REPETITIVE RESONANT RAILGUN POWER SUPPLY.

PATENTED JAN. 12, 1988, FILED 1985. (U.S.PATENT-4.718,321)

$13-29$

HOOTEN, DAVID T. (MEC-6); STANLEY, PATRICK M. (WX-4).

REAMING EXPERIMENTS FOR THE LETHALITY TEST SYSTEM.

T7C, 1988. 5P. MN (LA-UR-88-0782)

13-30

HULL, DONALD E. (MST- $\theta$ ); BIENIEWSKI, THOMAS M. (CHM-1). COMBINATION INDUCTION PLASMA TUBE AND CURRENT CONCENTAATOR FOR INTRODUCING A SAMPLE INTO A PLASMA.

PATENTED FEB. 23, 1988, FILED 1986. (U.S.PATENT-4,727,236)

13-31

JENSEN, REED J. (ADR); GIBSON, ROBERT B. (CLS-5); SCHAPPERT, GOTTFRIED T. (P-1);

TAYLOA, ANTOINETTE J. (CLS-5); ROBERTS, JEFFREY P. (CLS-5); CASPERSON, DONALD E. (P-1);

JONES, LARRY A. (P-1); KYRALA, GEORGE A. (P-1); LEE, PETER H. Y. (P-1); COBBLE. JAMES A. (P-4).

BRIGHT SOURCE LASERS AND EXPERIMENTS AT VERY HIGH IRRADIANCE.

TIC, 1997. 11P. MN (LA-UR-87-4015)

13-32

JOHNSON, JAMES O. (ESS-6): DINEGAR, ROBERT H. (M-7).

HIGH TEMPERATURE DETONATOR.

PATENTED APR. 5, 1988, FILED 1987. (U.S.PATENT-4,735,145) 
JOHNSTON, ROGER G. (LS-2).

INTERFEROMETRIC APPARATUS AND METHOD FOR DETECTION AND CHARACTERIZATION OF PARTICLES US!NG UGHT SCATTERED THEREFROM.

PATENT AUG. 16, 1988, FILED 1987. (U.S.PATENT-4,764,013)

13-34

KANEKO, K. (CNLS).

SPATIOTEMPORAL CHAOS IN ONE. AND TWODIMENSIONAL COUPLED MAP LATTICES.

TIC, 1988. 35P. MP! (LA-UPi-8S-3285)

13-35

KANIA, DON R. (P-1).

DESIGN OF X-RAY OPTICAL SYSTEMS TO PERFORM IN PULSED, HIGH FLUX X-RAY ENVIRONMENTS.

IN: MULTILAYER STRUCTURES AND LABORATOAY $X$-RAY LASER RESEARCH, 1986, SAN DIEGO, CALJF. PROC., P.157-62. SPIE, 1987. (TA1775I.MB5/1986X)

13-36

KEDDY, EDWARD S. (MEE-13); SENA, J. T. (MEE-13); MERAIGAN, MICHAEL A. (MEE-13); HEIDENAEICH, GARY; JOHNSON, STEVE.

DEVELOPMENT OF AN INTEGRATED HEAT PIPE - THERMAL STORAGE SYSTEM FOR A SOLAR RECEIVER. TIC, 1987. 10P. MN (LA-UR-87-3950)

13-37

KERRISK, JERRY F. (WX-4).

RAILGUN CONDUCTOR HEATING FROM MULTIPLE CURRENT PULSES.

IN: SYMPOSIUM ON ELECTROMAGNETIC LAUNCH TECHNOLOGY, 3FD, 1986, AUSTIN, TEX. PROC.,

P.127-32. IEEE, 1986. (TK7872.P8 S94 1986X)

ALSO PUBLISHED: TIC, 1986. 7P. MN (LA-UR-86-0815)

13-38

KRAUSSE, GEOAGE J., III (MP-4).

AXIAL FLOW PLASMA SHUTTER.

PATENTED JAN. 26, 1988, FILED 1986. (U.S.PATENT-4,721,891)

13-39

IWAN, THOMAS J. T. (X-10); SNELL, CHARLES M. $(X-10)$.

VIRTUAL CATHODE MICROWAVE GENERATOR HAVING ANNULAR ANODE SLIT.

PATENTED MAR. 8, 1988, FILED 1987. (U.S.PATENT-4,730,170)

13-40

LOONEY, LARRY D. (P-14); LYONS, PETER B. (ADDRA).

RADIATION-INDUSED TRANSIENT ABSORPTION IN SINGLE MODE CN TCAL FIBERS.

TIC, 1988. 9P. MN (LA-UR-88-2782)

13-41

LUCHT, ROY A. (M-8).

IN-BORE RADIOGRAPHY FOR LARGE-CALIBER GUN.

IN: INTERNATIONAL SYMPOSIUM ON BALUSTICS, 10TH, 1987, SAN DIEGO. PROC., VOL.1. P.1-11.

AMERICAN DEFENSE PREPAREDNESS ASSOCIATION, 1987. (UF/820.15/1987X)

ALSO PUBUSHED: TIC, 1987. 9P. MN (LA-UR-87-2788)

13-42

LUNDBERG, LYNN B. (MEE-13).

ALKAL METAL HEAT PIPE CORROSION PHENOMENA.

IN: INTERNATIONAL HEAT PIPE CONFERENCE, 6TH, 1987, GRENOBLE, FRANCE. PROC., P.280-4. STT CENG, 1987. (TJ264.158 1987X)

13-43

MARSH, STANLEY P. (N-6).

EXPLOSIVE PLANE-WAVE LENS.

PATENTED MAR. 8. 1988, FILED 1987. (U.S.PATENT-4,729,318) 
13-44

MARTIN, RICHARD A. (MEE-13); GREGOAY, WILLIAM S. (N-6); RICKETTS, C. I.; SMITH, PHILLIP R.;

LITTLETON, P. E. (WX-11); TALBOTT. D. V. (Q-6).

ANALYTICAL AND EXPERIMENTAL STUDIES OF VENTILATION SYSTEMS SUB.ECTED TO SIFAULATED

TOANADO CONDITIONS-VERIFICATION OF THE TVENT COMPUTER CODE.

LOS ALAMOS, 1988. 107P. (LA-11252-MS)

13-45

MENIKOFF, RALPH (T-14).

NUMERICAL IMPLICATION OF REIMANN PROBLEM THEORY FOR FLUID DYNAMICS

TIC. 1988. BP. MN (LA-UR-88-2672)

13-46

MERPIGAN, MICHAEL A. (MEE-13); REID, ROBERT S. (MEE-13).

HEAT PUMP AUGMENTED RADIATORS FOR SPACECRAFT THERIMAL MANAGEMENT.

TIC, 1988, 24P. MN (LA-UR-88-0847)

13-47

MIGLIORI, ALBERT (P-10); SWIFT, GREGORY W. (P-10).

LIOUID-SODIUM THEFRMACOUSTIC ENGINE.

APPL. PHYS. LETT., V.53, P.355-7. 1988.

13-48

MIKATARIAN, R. R.; JUMPER, E J.; WOOLHISER, C.

FLUID DYNAMIC ISSUES IN CONTINUOUS WAVE SHORT WAVELENGTH CHEMICAL LASERS

TIC. 1988. 35P. MN (LA-UR-88-2019)

13-49

MILENSKI, JOHN O. (MST-6); DIXON, RAY D. (MST-6).

DATA ACQUISITION SYSTEM FOR AN ELECTRON BEAM WELDER

J. VAC. SCI. TECHNOL., V.A6, P.2586-8. 1988.

13-50

NGUYEN, DINH C. (CLS-6); WATKINS, DAVID E. (CLS-6); WEBER, MICHAEL E. (CLS-6).

APPLICATION OF PULSE COMPRESSION AND SHAPING TO THE FEL PHOTOELECTRIC INJECTOR

TIC, 1988. 16P. MN (LA-UR-88-0696)

13-51

O'ROURKE, PETER J. (T-3); COLLINS, LANCE R. (T-3).

TURBULENT HEAT FLUX IN LOW MACH NUMBER FLOWS WITH LARGE DENSITY VARIATIONS.

TIC, 1988. 19P. MN (LA-UR-88-0633)

$13-52$

PAEZ, THOMAS L; HUNTER, NORMAN F. (WX-11).

EXPERIMENTAL IDENTIFICATION OF NONLINEAR STRUCTURAL MODELS

TIC, 1988. 24P. MN (LA-UR-87-3582)

$13-53$

PARKER, JERALD $\checkmark .(P-1)$.

WHY PLASMA ARMATURE RAILGUNS DON'T WORK (AND WHAT CAN BE DONE ABOUT IT).

TIC. 1988. 9F. MN (LA-UR-88-0789)

ALSO PUBLISHED: SYMPOSIUM ON ELECTROMAGNETIC LAUNCH TECHNOLOGY, 4TH, 1988, AUSTIN. TEX. PROC.. [NO PAG.] IEEE, 1988. (TK7872.P8 S94 1988X)

13-54

PARKER, JERALD V. (P-7); PARSONS, W. M. (CTR-9).

EXPERIMENTAL MEASUREMENT OF ABLATION EFFECTS IN PLASMA ARMATURE RAILGUNS IN: SYMPOSIUM ON ELECTROMAGNETIC LAUNCH TECHNOLOGY, 3RD, 1986, AUSTIN, TEX. PROC. P.181-8. IEEE, 1986. (TK7872.P8 S94 1986X)

ALSO PUBLISHED: TIC, 1986. 9P. MN (LA-UR-86-0943)

13-55

PARKER, JERALD V. (P-7)

SRS [SEGMENTED RAIL SURFACE] RAILGUN-A NEW APPROACH TO RESTRIKE CONTROL. TIC, 1988. 7P. MN (LA-UR-88-0709) 
$13-56$

PARSONS, W. M. (CTR-9); SIMS, JAMES R. (WX-4); PARKER, JERALD V. (P-7)

LETHALITY TEST SYSTEM.

IN: SYMPOSIUM ON ELECTROMAGNETIC LAUNCH TECHNOLOGY, 3RD, 1986, AUSTIN, TEX. PROC.,

P.189-92. IEEE, 1986. (TK7872.PB S94 1986X)

ALSO PUBLISHED: TIC, 1986. 5P. MN (LA-UR-86-0922)

13-57

PARSONS, W. M. (CTR-9); FORD, RICHARD D.; WILDI, PAUL; CHOWDHURI, PRITINDRA.

SWITCHING DEVICES USED IN THE LETHALITY TEST SYSTEM.

IN: IEEE INTERNATIONAL PULSED POWER CONFERENCE, 6TH, 1987, ARLINGTON, VA. PROC, P 33-7. IEEE, 1987. (TK2896 .115 1987X)

ALSO PUBLISHED: TIC, 1987. 6P. MN (LA-UR-87-2192)

13-58

RANKEN, WILUAM A. (MEE-13).

IRRADIATION OF HIGH TEMPERATURE HEAT PIPES.

IN: INTERNATIONAL HEAT PIPE CONFERENCE, 6TH, 1987, GRENOBLE, FRANCE. PROC., P.755-60. STT CENG, 1987. (TJ264.158 1987X)

13-59

REID, DONALD W. (AT-5).

HIGH-POWERED, SOLID-STATE RF SYSTEMS.

IN: IEEE PARTICLE ACCELERATOR CONFERENCE, 1987, WASHINGTON, D.C PROC., P.1701-4. IEEE. 1987. (OC787.P3 137 1987)

ALSO PUBLISHED: TIC, 1987. 5P. MN (LA-UR-87-0502)

13-60

SCHNURR, NORMAN M. (WX-4).

THERMAL ANALYSIS OF ELECTROMAGNETIC LAUNCHER RAILS

LOS ALAMOS, 1987. 13P. (LA-11087-MS)

13-61

SCHRAGE, DALE L. (AT-1); YOUNG, LLOYD M. (AT-1); CAMPBELL, BILLY M. (AT-4); BILLEN, JAMES H. (AT-1): WANGLER, THOMAS P. (AT-1); STOVALL, JAMES E. (AT-1); MARTINEZ, FELIX A. (AT-1):

CLARK, WILLIAM L. (AT-1); GIBBS, W. SCOTT (MST-6); BOLME, GERALD O. (AT-1); OSHEA, PATRICK G. (BPO): LYNCH, MICHAEL T. (BPO); DEVENPORT, JAMES C. (BPO); RATHKE, J.; MICICH. R.; ROSE, J.; RICHTER, R.; ROSATO, G.

FLIGHT-OUALIFIED RFO FOR THE BEAR PROJECT. TIC, 1988. 5P. MN (LA-UR-88-3008)

13-62

SCOTT, MARION L. (MST-7).

REFLECTANCE OF ALUMINUM REFLECTORS IN THE EXTREME ULTRAVICLET.

TIC. 1988. 7P. MN (LA-UR-88-3436)

13-63

SEYFAIED, W. E., JR.; JANECKY, DAVID R. (INC-7); BERNDT, M. E.

ROCKING AUTOCLAVES FOR HYDROTHERMAL EXPERIMENTS II. THE FLEXIBLE REACTION-CELL SYSTEM IN: HYDROTHERMAL EXPERIMENTAL TECHNIQUES, G.C. ULMER AND H.L. BARNES. EDS., P.216-39. WILEY, 1987. (OC/281/.H93/1987)

13-64

SIMS, JAMES R. (WX-4); CHRISTENSEN, KIRK ELDON (WX-4); CUMMINGS, CHARLES E. (WX-4);

CALKINS, NOEL C. (WX-4).

LAUNCHER BARREL FOR THE LETHALITY TEST SYSTEM RAILGUN

IN: SYMPOSIUM ON ELECTROMAGNETIC LAUNCH TECHNOLOGY, 3RD, 1986, AUSTIN, TEX. PROC., P.281-4. IEEE, 1986. (TK7872.PB S94 1986X)

ALSO PUBLISHED: TIC, 1986. 5P. MN (LA-LJA-B6-0942)

13-65

STEELE, RAYMOND DOUGLAS (M-1); TAN, TAI HO (M-6).

FAST SHCCK TUBE ASSEMBLIES. IN: SHOCK WAVES IN CONDENSED MATTER, 1987, MONTEREY. PROC., P.661-7. ELSEVIER, 1988. (CC173.4.C65 557 1987X)

ALSO PUBLISHED: TIC, 1987. 5P. MN (LA-UR-87-2199) 
$13-66$

SWIFT, GREGORY W. (P-10).

WATER AS A THERMOACOUSTIC WORKING FLUID.

TIC. 1988. 10P. MN (LA-UR-88-3671)

13.67

TAKEDA, HARUNORI (AT-6); SOLLID, JON E. (CLS-6).

ONE-DIMENSIONAL SIMULATION OF A FREE-ELECTRON LASER OSCILLATOR USING A DIFFRACTION GRATING AS A CAVITY MIRAOR.

TIC, 1988. 22P. MN (LA-UR-88-3613)

$13-68$

TESANOVIC, ZLATKO B. (T-11); BISHOP, ALAN R (T-11); MARTIN, RICHARD L. (T-12).

NOVEL EXCITONIC MECHANISM FOR HIGH TEMPERATURE SUPERCONDUCTIVITY

SYNTH. MET., V.29, P.F627-34. 1989.

ALSO PUBLISHED: TIC, 1988. 9P. MN (LA-UR-88-2535)

$13-69$

VAN GULICK, LEONARD A. (WX-11).

FINITE ELEMENT WELDING COMPUTATIONS USING GENERAL PURPOSE NONLINEAR ANALYSIS CODES. IN: ASME PRESSURE VESSELS AND PIPING CONFERENCE, 1988, PITTSBURGH. PA. PROC., P.13-22.

AMERICAN SOCIETY OF MECHANICAL ENGINEERS, 1988. (TA347.F5 A86 1988)

ALSO PUBLSHED; TIC, 1988. 29P. MN (LA-UR-88-0313)

13-70

VEESER, LYNN R. (P-14); GEORGE, M. J. (M-4); MENIKOFF, RALPH (T-14); PAPATHEOFANIS, B. (P-3);

MC GURN, JOHN S. (P-4); GOBBY. PETER L. (MST-7).

SENSING OF HIGH PRESSURE BY SHOCK HEATING QUARTZ OPTICAL FIBERS.

IN: FIBER OPTIC AND LASER SENSORS, 5TH, 1987, SAN DIEGO. PROC., P.60-8. SPIE, 1988

13-71

VORTHMAN, JOHN E. (M-6).

OPTIMUM SIZE OF STAGED FLUX COMPRESSION GENERATORS.

IN: IEEE INTERNATIONAL PULSED POWER CONFERENCE, 6TH, 1987, ARLINGTON, VA. PROC.. P.438-40. IEEE, 1987. (TK2896 .I15 1987X)

ALSO PUBLISHED: TIC, 1987. 4P. MN (LA-UP-87-2138)

13-72

WALLEA, CLYDE R. (MP.8).

STANDARD OPERATING PROCEDURE-GAS ATIMOSPHERE MELCO BRAZING FURNACE. LOS ALAMOS NATIONAL LABORATORY, 19813. 41P. (LA-11353-SOP)

13-73

WANTUCK, PAUL JOSEPH (CLS-8); APPERT, QUENTIN D. (CLS-8); TONG, KWON-ON; PISTORESI, DENIS J. EFFECTIVENESS OF METAL COATED DIFFRAC'TION GRATINGS AT GRAZING AND NEAR NORMAL INCIDENCE AS HARMONIC SCRUBBERS.

TIC, 1988. 25P. MN (LA-UR-88-3148)

13-74

WARREN, ROGER W. (AT-7); SOLLID, JON E. (CLS-5): FELDMAN, DONALD W. (AT-7); STEIN, WILL:AM E. (AT-7): JOHNSON, W. JOEL D. (AT-5); GOLDSTEIN, JOHN C. (X-1); LUMPKIN, ALEX H. (P-15).

NEAR-IDEAL LASING WITH A UNIFORM WIGGLER.

TIC, 1988. 28P. MN (LA-UR-88-3586)

13-75

WARREN, POGER W. (AT-7); ELLIOTT, C. JAMES (X-1).

NEW SYSTEM FOR WIGGLER FABRICATION AND TESTING.

IN: ADRIATICO RESEARCH CONFERENCE -UNDULATOR MAGNETS FOR SYNCHROTRON RADIATION AND FREE ELECTRON LASERS", 1987, TRIESTE, ITALY. PROC., P.28-38. WORLD SCIENTIFIC, 1988 (OC756.7.A37 1987X)

ALSO PUBLISHED: TIC, 1987. 12P. MN (LA-UR-87-2981) 
13-76

WENDER, STEVE A. (P-3): SEESTROM-MORRIS, SUSAN J. (P-3): NELSON, RONALD O. (P-3).

WHITE NEUTAON SOURCE FPOM 1 TO 400 MEV.

IN: INTERNATIONAL SYMPOSIUM ON CAPTURE GAMMA-RAY SPECTROSOOPY AND RELATED TOPICS. 6TH, 1987, LEUYEN, BELGIUM PAOC., P.S417-24. INSTITUTE OF PHYSICS. 1988. (QC1.157X NO.88)

13-77

WHITCOMB, BRUCE M.; FLURER, RANDY L.; WOO, L; TOMS, K. W.; OGLE. J. W. (P. 14).

CALIBRATION SYSTEM FOA SUB-10O-PS RELATIVE TIMING.

IN: HIGH BANDWIDTH ANALOG APPLICATIONS OF PHOTONICS, 1986, CAMBRIDGE, MASS. PROC. P.59-64. SPIE, 1987. (TA1505'.H528/1986X)

13-78

WILDE, DALE K. (MEE-11); LYNN, DAVID K. (MEE-11); HAMILTON, DOUGLAS J.

FINAL REPORT-THEFIMIONIC INTEGRATED CIRCUIT PROGRAM. LOS ALAMOS NATIDNAL LABORATORY, 1988. 165P. (LA-11284-MS)

$13-79$

WOLCSHUN, KEITH A. (MEE-13).

MEMBRANE HEAT PIPE SPACE RADIATOR DESIGN.

INTERNATIONAL HEAT PIPE CONFERENCE, 6TH, 1987, GRENOBLE, FRANCE. PPOC., P. 427-32. STT CENG, 1987. (TJ264.158 1987X)

$13-80$

YAN, YITON T. $(X-10)$.

OUTPUT POWER ENHANCEMENT OF AC RAMAN FREE ELECTRON LASERS.

NUCL INSTRUM. METHODS PHYS. RES., V.A272, P.120-4. 1988.

ALSO PUBLSSHED: TIC, 1987. 19P. MN (LA-UA-87-3013)

13-81

ZIMMERMANN, EUGENE L. (P-1); FOWLER, CLARENCE M. (M-6); FOLEY, EDWARD (W/X-4);

PARKER, JERALD V. (P-7).

HIMASS ELECTROMAGNETIC LAUNCHER AT LOS ALAMOS.

IN: SYMPOSIUM ON ELECTPOMAGNETIC LAUNCH TECHNOLOGY, 3RD, 1986, AUSTIN. TEX. PROC. P.347-9. IEEE, 1986. (TK7872.Pa S94 1986X)

ALSO PUBLISHED: TIC, 1986. 4P. MN (LA-UF-86-0925) 


\section{ENVIRONMENTAL CONTROL TECHNOLOGY AND EARTH SCIENCES}

14-1

ANDER, MARK E. (ESS-3); GOLDMAN, TERRENCE J. (T-5); HUGHES, RICHARD J. (?-8);

NIETO, MICHAEL MARTIN (T-8).

POSSIBLE RESOLUTION OF THE BROOKHAVEN AND WASHINGTON EOTVOS EXPERIMENTS.

PHYS. REV. LETT., V.50, P.1225-8. 1988.

14-2

ARMBRUUSTMACHER, THEODORE J.; SHANNON, SPENCER S., JR. (ESS-1).

CARBONATITE COMPLEX AT IRON HILL, POWDERHORN DISTRICT, GUNNISON COUNTY, COLORADO

IN: CENTENNIAL FIELD GIJIDE. P.325-7. GEOLOGICAL SOCIETY OF AMERICA, 1987. (GE/77/.C46)

$14-3$

BROWN, FRED (HSE-8); PURTYIAUN, WILLIAM D. (HSE-8); STOKER, ALAN K. (HSE-8); BARR, ALICE R. (HSE-8).

SITE GEOLOGY AND HYDROLOGY OF TECHNICAL AREA 16, AREA P. LOS ALAMOS, 1988. 8P. (LA-11209MS)

$14-4$

BROWN, JOSEPH A. (ESS-3); GAFFNEY, E. S.; BLAISDELL, G. L.; JOHNSON, J. B.

TECHNICUES FOR GAS GUN STUDIES OF SHOCK WAVE ATTENUATION IN SNOW. IN: SHOCK WAVES IN CONDENSED MATTER, 1987, MONTEREY. PROC., P.657-60. ELSEVIER, 1988. (QC173.4.C65 557 1987X)

ALSO PUBLISHED: TIC, 1987. 5P. MN (LA-UR-87-2221)

14-5

BROWN, WILBUR K. (P-15).

THEORY OF SEOUENTIAL FRAGMENTATION AND ITS ASTRONOMICAL APPLICATIONS.

LOS ALAMOS NATIONAL LABORATORY, 1988. 24P. (LA-11043)

14-6

BUMSTED, M. PAMELA (CHM-1); ARMELAGOS, GEORGE J.: BATESON, M. CATHERINE; DIRKS, ROBERT:

JONES, ERIC M. (ESS-5); NADER, LAUPA.

NUCLEAR WINTER-THE ANTHROPOLOGY OF HUMAN SURVIVAL.

IN: AMERICAN ANTHROPHOLOGICAL ASSOCIATION, 84TH, 1985, WASHINGTON, D.C. PROC, M.P. BUMSTEAD, SESSION ORGANIZER. AMERIGAN ANTHROPOLOGICAL ASSOCIATION, 1985. (U263 A4 $1985 X)$

14-7

CARR, MICHAEL D.; WADDEIL, SANDPA J.; VICK, GREG S.; STOCK, JOANNE M.; MONSEN, SUSAN A:

HARAIS, ANITA G.; CORK, BYRON W.; BYERS, FRANK M., JA. (ESS-1).

GEOLOGY OF DRILL HOLE UE25P\#1-A TEST HOLE INTO PRE-TERTIAAY ROCKS NEAR YUCCA MOUNTAIN. SOUTHERN NEVADA.

U.S. GEOLOGICAL SURYEY, 1986. 89P. (USGS-OFR-86-175)

14-8

CONAWAY, JOHN G. (ESS-3).

TEMPERATURE LOGGING AS AN AID TO UNDERSTANDING GROUNDWATER FLOW IN BOREHOLES IN: INTERNATIONAL SYMPOSIUM ON BOREHOLE GEOPHYSICS FOR MINERALS, GEOTECHNICAL, AND GROUNDWATER APPLICATIONS, 2ND, 1987, GOLDEN, COLO. PROC., P.51-9. SOCIETY OF

PROFESSIONAL WELL LOG ANALYSIS, 1988. (TN269 157 1987X)

ALSO PUBLISHED: TIC, 1987. 10P. MN (LA-UR-87-3355)

$14-9$

DENNIS, BERT R. (ESS-11); JERMANCE, RAYMOND (ESS-11); LAWTON, ROBERT G.;

OUINTINILLA, ALEJANDRO; BERGANZA, JUAN.

RESULTS OF WELL LOGGING OPERATIONS AT THE AHUACHAPAN GEOTHERMAL FIELD, EL SALVADOR. TIC, 1988. 7P. MN (LA-UR-88-4257)

$14 \cdot 10$

DEUPREE, ROBERT G. (ESS-5); NOEL, SUSAN D. (ESS-5).

MONTE CARLO SIMULATIONS OF AN EPITHERMAL NEUTRON LOGGING TOOL.

LOG ANAL., P.95-102. MAR. APR. 1988. 
14-11

DEVAURS, M. A. (HSE-12); SPRINGER, EVERETT P. (HSE-12); LANE, LEONARD J.;

LANGHORST, GARY J. (HSE-12).

PREDICTION METHODOLOGY FOR CONTAMINANT TRANSPORT FROM RANGELAND WATERSHEDS.

TIC. 1988, 27P. MN (LA-UR-88-2071)

14. 12

ELSTON, WOLFGANG E.; WOHLETZ, KENNETH H. (ESS-1).

OUATERNARY BASALT FIELDS OF WES : CENTRAL NEW MEXICO-MC CARTYS PAHOEHOE FLOW, ZUNI

CANYON AA FLOW, ZUNI ICE CAVE. BANDERA CRATER, ZUNI SALT LAKE MAAR.

IN: CENTENNIAL FIELD GUIDE, VOL.2, ROCKY MOUNTAIN SECTION, P.431-6. GEOLOGICAL SOCIETY OF AMERICA, 1987. (QET7I.C46)

14-13

FEHLER, MICHAEL C. (ESS-4); ROBERTS, PETER; FAIRBANKS, TOM.

TEMPORAL CHANGE IN CODA WAVE ATTENUATION OBSERVED DURING AN ERUPTION OF MOUNT SAINT HELENS.

J. GEOPHYS. RES., V.93E, P.4367-73. 1988.

14-14

GAFFNEY, JEFFREY S. (INC-7); TANNER, ROGER L.

ALCOHOL FUEL USE-IMPL!CATIONS FOR ATMOSPHEAIC LEVELS OF ALDEHYDES, ORGANIC NITRATES.

PANS, AND PE:ROXIDES. SEPARATING SOURCES USING CARBON ISOTOPES.

TIC, 1988. 6P. MN (LA-UR-88-1533)

14-15

GAUTIER, MARGARET A. (HSE-9); GLADNEY, E. S. (HSE-9); PHILLIPS, MARYBETH (HSE-9);

O'MALLEY, BRYAN T. (HSE-9).

OUALITY ASSURANCE FOR HEALTH AND ENVIRONMENTAL CHEMISTRY-1987.

LOS ALAMOS NATIONAL LABORATORY, 1988. 36:2P. (LA-11454-MS)

14-16

GEISSMAN, JOHN W.

PALEOMAGNETISM AND ROCK MAGNETISM OF QUARTERNARY VOLCANIC ROCKS AND LATE PALEOZOIC STRATA, VC-1 CORE HOLE, VALLES CALDERA, NEW MEXICO, WITH EMPHASIS ON REMAGNETIZATION OF LATE PALEOZOIC STRATA.

J. GEOPHYS. RES., V.93B, P.6001-25. 1988.

14-17

GLATZMAIER, GARY ANDREW (ESS-5).

NUMERICAL SIMULATIONS OF MANTLE CONVECTION-TIME-DEPENDENT, THREE-DIMENSIONAL,

COMPRESSIBLE, SPHERICAL SHELL

GEOPHYS. ASTROPHYS. FLUID DYN., V.43, P.223-64. 1988.

14-18

GOFF, FRASER E. (ESS-1); GARDNER, JAMIE N. (ESS-1).

VALIES CALDERA REGION, NEW MEXICO, AND THE EMERGING CONTINENTAL SCIENTIFIC DRILLING PROGRAM.

J. GEOPHYS. RES., V.93B, P.5997-9. 1988.

14-19

GOFF, SUE JACOBSEN (ESS- 1 ).

FIELD PROCEDURES MANUAL-SHADY REST, CALIFORNIA, AND SULPHUR SPRINGS, NEW MEXICO.

LOS ALAMOS NATIONAL LABDRATORY, 1988. 13P. (LA-11453-OBES)

$14-20$

HARRINGTON, CHARLES D. (ESS-1); DETHIER, DAVID P. (ESS-1); WHITNEY, J. W.

REPLY ON 'SCANNING ELECTRON MICROSCOPE METHOD FOR ROCK-VARNISH DATING' GEOLCGY, V.16, P.1051-2. 1988. 
14-21

HAWKINS, WARD L. (ESS-1); OLIVER, RONALD D. (ESS-3); LAVELLE, MORGAN J.

BOREHOLE INSPECTION SYSTEM FOR LARGE DIAMETER HOLES.

IN: INTERNATIONAL SYMPOSIUM ON BOREHOLE GEOPHYSICS FOR MINERALS, GEOTECHNICAL, AND GROUNDWATER APPLICATIONS, 2ND, 1987, GOLDEN, COLO. PROC., P.341-8. SOCIETY OF

PROFESSIONAL WELL LOG ANALYSIS, 1989. (TN269.157 1987X)

ALSO PUBLISHED: TIC, 1987. 10P. MN (LA-UR-87-3354)

14-22

HEIKEN, GRANT (ESS-1); WOHLETZ, KENNETH H. (ESS-1); EICHELBERGER, J.

FRACTURE FILLINGS AND INTRUSIVE PYROCLASTS, INYO DOMES, CALIFORNIA.

J. GEOPHYS. RES., V.938, P.4335-50. 1988.

14-23

HOLDEN, DANIEL N. (ESS-7); ULBRICH, CARITON.

RADAR STUDY OF THE INTERACTION BETWEEN LIGHTNING AND PRECIPITATION.

TIC, 1988. 10P. MN (LA-UR-88-1328)

14-24

HOUSE, LEIGH S. (ESS-3); CASH, DANIEL J. (ESS-3).

REVIEW OF HISTORIC AND INSTRUMENTAL EARTHQUAKE ACTIVITY AND STUDIES OF SEISMIC HAZARDS NEAP LOS ALAMOS, NEW MEXICO.

LOS ALAMOS NATIONAL LABORATORY, 1988. 41P. (LA-11323-MS)

14-25

JALBERT, ROLAND A. (HSE-1); MURPHY, CHARLES E.

CANADIAN EXPERIMENTAL HT RELEASE OF JUNE 10, 1987, U.S. MEASUREMENTS.

LOS ALAMOS NATIONAL LABORATORY, 1988. 27P. (LA-1 1368-MS)

14-26

JALBERT, ROLAND A. (HSE-1); MURPHY, CHARLES E.

US TSAM MEASUREMENTS DURING THE JUNE 1987 EXPERIMENTAL HT RELEASE AT THE CHALK RIVER NUCLEAR LABORATORIES, ONTARIO, CANADA.

TIC, 1988. 6P. MN (LA-UR-88-0820)

14-27

JOHNSTON, ROGER G. (LS-2); SALZMAN, GARY C. (LS-2).

PHASE DIFFERENTIAL SCATTERING AND POLYDISPERSITY.

TIC, 1988. 10P. MN (LA-UR-88-3418)

14-28

KAC, CHIH-YUE JIM (ESS-5); YAMADA, TETSUJI (ESS-5).

NUMEAICAL SIMULATIONS OF A NIGHTTTIME STRATOCUMULUS-CAPPED BOUNDARY LAYER OBSERVED

OVER LAND USING A TURBULENCE CLOSURE MODEL.

IN: SYMPOSIUM ON TURBULENCE AND DIFFUSION, 8TH, 1988, SAN DIEGO, CALIF. PROC., P.285-8.

AMERICAN METEOROLOGICAL SOCIETY, 1988. (OC880 .S9 1988X)

14-29

KAO, CHIH-YUE JIM (ESS-5); YAMADA, TETSUJł (ESS-5).

USE OF THE CAPTEX DATA FOR EVALUATIONS OF A LONG-RANGE TRANSPORT NUMERICAL MODEL WITH A FOUR-DIMENSIONAL DATA ASSIMILATION TECHNIQUE.

MO. WEATHER REV., V.116, P.293-306. 1988.

$14-30$

KARL, ROBERT RAYMOND, JR. (ESS-7).

FIELD TEST RESULTS OF ATMOSPHERICALLY DISPERSED VEGETATIVE CELLS FOR A TRACER USING A SOLAR BLIND FLUORESCENCE LIDAR.

IN: JOINT CONFERENCE ON APPLICATIONS OF AIR POLLUTION METEOROLOGY, 6TH, 1989, ANAHEIM.

CALIF. PROC., P.125-7. AMERICAN METEOROLOGICAL SOCIETY, 1988. (TD881 .J64 1989X)

ALSO PUBLISHED: TIC, 1988. 6P. MN (LA-UR-88-3316)

$14-31$

MAESTAS, L. M.; RAYMOND, ROBERT, JA. (ESS-1); COHEN, ARTHUR D. (ESS-1); MANN, DAVID A. (ESS-1).

ADVANCES IN PREPARATION TECHNIQUES FOR PAOCESSING MICFOTOME SECTIONS OF PEAT.

LOS ALAMOS, 1987. 20P. (LA-11171-OBES) 
$14-32$

MONDT, JOHANNES P. (CTR-6).

THEORETICAL BASIS FOR AN EXPERIMENTAL TEST WITH REGARO TO THE ORIGIN OF VHF RADIATION

FROM NATURAL LIGHTNING.

LOS ALAMOS, 1988. 27P. (LA-11276-NS)

$14-33$

MURPHY, TINOTHY C. (ESS-7).

ELECTROMAGNETIC PULSE (EMP) FROM SURFACE BURSTS.

LOS ALAMOS, 1987. 13F. (LA-1 1060-MS)

$14-34$

ORGILL, MONTE M. (ESS-7); BARR, SUMNER (ESS-7).

INFLUENCE OF EXTERNAL WINDS AND CLOUDINESS ON THE TRANSITION LAYER ABOVE NOCTURNAL.

VALLEY DRAINAGE.

IN: CONFERENCE ON MOUNTAIN METEOROLOGY, 4TH, SEATTLE, WASH. PREPRINT VOLUME. PROC., P.76-80. AMERICAN METEOROLOGICAL SOCIETY, 1987. (QC993.6 .C65 1987X)

ALSO PUBLISHED: TIC, 1987. 6P. MN (LA-UR-87-1704)

14-35

ORTH, CHARLES J. (INC-11); ATTREP, MOSES, JA. (INC-11); MAO, XUEYING (INC-11); KAUFFMAN, ERLE G:

DINER, RICHARD; ELDER, WILL. P.

IRIDIUM ABUNDANCE MAXIMA IN THE UPPER CENOMANIAN EXTINCTION INTERVAL.

GEOPHYS. RES. LETT., V.15, P.346-9. 1988.

14-36

ORTH, CHARLES J. (INC-11); ATTREP, MOSES, JR. (INC-11); OUINTANA, LEONARD R. (INC-11);

DINER, FIICHARD; ELDER, WILL P.

SIDEAOPHILE ABUNDANCE MAXIMA AT UPPER CENOMANIAN MARINE INVERTEBRATE EXTINCTION HORIZON-WESTERN INTERIOR OF NORTH AMERICA.

IN: LUNAR AND PLANETARY SCIENCE CONFERENCE, 19TH, 1988, HOUSTON, TEX. PROC., P.893-4.

LUNAR AND PLANETARY INSTITUTE, 1988. (OB592 .L84 1988X)

14-37

PERAY, FRANK V. (INC-7); BALDRIDGE, W. S. (ESS-1); DE PAOLO, D. J.

CHEMICAL AND ISOTOPIC EVIDENCE FOR LITHOSPHERIC THINNING BENEATH THE RIO GRANDE RIFT. NATURE V.332, P.432-4. 1988.

14-38

PORCH, WILLIAM M. (ESS-7); NEFF, WILLIAM; KING, CLARK.

CONPARISONS OF METEOROLOGICAL STRUCTURE PARAMETERS IN COMPLEX TERRAIN USING OPTICAL AND ACOUSTICAL TECHNIUES.

APPL. OPT., Y.27, P.2222-8. 1988.

$14-39$

PORCH, WILLIAM M. (ESS-7); KAO, CHIH-YUE JIM (ESS-5); KYLE, THOMAS GAIL (IT-1); KELLEY, RICHARD G.

SHIP TRAIUCLOUD DYNAMIC EFFECTS FROM APOLLOSOYUZ PHOTOGRAPH JULY 16, 1975. TIC. 1988. 15P. MN (LA-UR-88-3341)

$14-40$

PORCH, WILLIAM M. (ESS-7); GIFFORD, FPANKLIN A. (ESS-7); HOARD, DONALD E. (ESS 7 )

STUDY OF STABILITY ZONE INFLUENCES AND TRACER PATTERNS FROM THE 1987 ANATEX [ACROSS NORTH AN:ERICA TRACER EXPERIMENT] EXPERIMENT

IN: JOINT CONFERENCE ON APPUCATIONS OF AIR POLLUTION METEOROLOGY, 6TH, 1989, ANAHEIM, CALIF. PROC., P.647. AMERICAN METEOROLOGICAL SOCIETY, 1988. (TD881 .J64 1989X)

ALSO PUBLSHED: TIC, 1988. 15P. MN (LA-UR-88-3317)

14-41

PURTYMUN, WLUAM D. (HSE-B); FERENBAUGH, ROGFR WILLIS (HSE-9); MAES, MAX N. (HSE-8) OUALTY OF SURFACE AND GROUND WATER AT AND ACJACENT TO THE LOS ALAMOS NATIONAL LABORATORY-REFERENCE ORGANIC COMPOUNDS.

LOS ALAMOS NATIONAL LABORATOFY, 1988. 25P. (LA-11333-MS) 
14.42

PUATYMUN, WILLIAM D. (HSE-8); FERENBAUGH, ROGER WILLIS (HSE-8); WILLIAMS, MARY C. (HSE-9);

MAES, MAX N. (HSE-8).

WATER QUALITY IN THE VICINITY OF FENTON HILL, 1985 AND 1986.

LOS ALAMOS, 1988. 36P. (LA-11210-PR)

$14-43$

PURTYMUN, WILLIAM D. (HSE-8); STOKER, ALAN K. (HSE-8).

WATER SUPPLY AT LOS ALAMOS-CURRENT STATUS OF WELLS AND FUTURE WATER SUPPLY LOS ALAMOS NATIONAL LABORATORY, 1988. 39P. (LA-11332-MS)

14-44

RAYMOND, ROBERT, JR. (ESS-1); BISH, DAVID L. (ESS-1); COHEN, ARTHUR D. (ESS-1).

INORGANIC CONTENTS OF PEATS.

TIC, 1988. 16P. MN (LA-UR-88-0535)

14-45

ROBINSON, RICHARD D. (HSE-9); BELL, MICHAEL G. (HSE-9); BUANS, COLLEEN E. (HSE-8):

KNAB, DARYL (HSE-9).

COMPARISON OF TRACE LEVEL CONCENTRATIONS FOR SILVER, ARSENIC, BARIUM, CADMIUM, AND LEAD IN DRINKING WATERS OBTAINED BY INDUCTIVELY COUPLED PLASMA-MASS SPECTROMETRY AND

ELECTROTHERMAL-VAPORIZATION ATOMIC ABSORPTION SPECTROSCOPY.

LOS ALAMOS, 1987. 47P. (LA-11095-MS)

$14-46$

AOBINSON, RICHARD D. (HSE-9); KNAB, DARYL (HSE-9).

ESTABLISHING A LEVEL OF CONFIDENCE FOR THE ANALYTICAL VALUES FOR THE TRACE METALS IN

THE LOS ALAMOS DRINKING WATER SUPPLY AND DISTRIBUTION SYSTEM.

LOS ALAMOS, 1987. 28P. (LA-11091-MS)

$14-47$

SMYTH, JOSEPH R. (ESS-1); BISH, DAVID L. (ESS-1).

CRYSTAL STRUCTURES AND CATION SITES OF THE ROCK-FORMING MINERALS.

ALLEN AND UNWIN, 1988. 332 P. (QE397 .S64 1987X)

$14-48$

SPRINGER, EVERETT P. (HSE-12): CUNDY, TERRANCE W.

EFFECTS OF SPATIALLY-VAFYING SOIL PROPERTIES ON SOIL EROSION.

TIC, 1988. 3OP. MN (LA-UR-88-2376)

$14-49$

STALLINGS, E. A. (HSE-9); CANDELARIA, LINDA M. (HSE-9); GLADNEY, E. S. (HSE-9).

INVESTIGATION OF A FUSION TECHNIQUE FOR THE DETERMINATION OF TOTAL SULFUR IN GEOLOGICAL SAMPLES BY ION CHROMATOGRAPHY.

ANAL. CHEM., V.60, P.1246-8. 1988.

$14-50$

STIX, JOHN; GOFF, FRASER E. (ESS-1); GORTON, MICHAEL P.; HEIKEN, GRANT (ESS-1);

GARCIA, SAMMY R. (INC-5).

RESTORATION OF COMPOSITIONAL ZONATION IN THE BANDELIER SILICIC MAGMA CHAMBEH BETNEEN TWO CALDERA-FORMING ERUPTIONS-GEOCHEMISTRY AND OPIGIN OF THE. CERRO TOLEDO RHYOLITE, JEMEZ MOUNTAINS, NEW MEXICO.

J. GEOPHYS. RES., V.93B, P.6129-47. 1988.

14.51

STONE, GREGORY L. (ESS-7); HOARD, DONALD E. (ESS-7).

WIND FLUCTUATIONS IN A NOCTURNAL DRAINAGE FLOW

IN: CONFERENCE ON MOUNTAIN METEOROLOGY, 4TH, 1987, SEATTLE, WASH. PREPRINT VOLUME

PROC., P.52-7. AMERICAN METEOROLOGICAL SOCIETY, 1987. (QC993.6 .C65 1987X)

ALSO PUBLISHED: TIC, 1987. 7P. MN (LA-UR-87-1745)

$14-52$

TRENT, BRUCE C. (ESS-5).

EFFECT OF MICRO-STRUCTURE ON THE MACROSCOPIC BEHAVIOA OF CEMENTED GRANULAR

MATERIAL.

LOS ALAMOS, 1988. 263P. (LA-11169-T) 
$14-53$

TRENT, BRUCE C. (ESS-5).

MICROSTRUCTURAL EFFECTS IN STATIC AND DYNAMIC NUMERICAL EXPERIMENTS.

IN: U.S. SYMPOSIUM ON ROCK MECHANICS, 19TH, 1988, MINNEAPOLIS. RROC, P.395-402. BALKEMA, 1988.

ALSO PUBLISHED: TIC, 1988. 9P. MN (LA-UR-87-4218)

14-54

VALENTINE, GREG A. (ESS-5).

FIELD AND THEORETICAL ASPECTS OF EXPLOSIVE VOLCANIC TRANSPORT PROCESSES

LOS ALAMOS NATIONIAL LABORATOAY, 1988. 232P. (LA-11441-T)

14-55

WACHS, DANIEL; HARRINGTON, CHARLES D. (ESS-1); GARDNER, JAMIE N. (ESS-1);

MAASSEN, LARRY W. (ESS-1).

EVIDENCE OF YOUNG FAULT MOVEMENTS ON THE PAJARITO FAULT SYSTEM IN THE AREA OF LOS

ALAMOS, NEW MEXICO.

LOS ALAMOS, 1988. 23P. (LA-11156-MS)

14-56

WILLIAMS, MICHAEL D. (A-4).

UPDATE ON THE MESO-MICROSCALE METEOROLOGICAL HIERARCHY.

TIC, 1987. 19P. MN (LA-UR-88-1238)

14-57

WILLIAMS, RAY E. (ESS-4); DEY, TOM N. (ESS-5); RAUENZAHN, RICK (T-3); KRANZ, ROBERT (ESS-3);

TESTER, JEFFERSON W.; POTTER, ROBERT M. (ESS-4): MURPHY, HUGH D. (ESS-DOT).

ADVANCEMENTS IN THERMAL SPALLATION DRILLING TECHNOLOGY.

LOS ALAMOS NATIONAL LABORATORY, 1988. 45P. (LA-11391-MS)

14-58

WILSON, ROBERT D.; COOK, TAVONI K.; DEAN, SUMNER H. (X-7).

EFFICIENT SIMULATION MODEL FOR NUCLEAR GEOPHYSICAL MEASUREMENTS.

IN: INTERNATIONAL SYMPOSIUM ON BOREHOLE GEOPHYSICS FOR MINERALS, GEOTECHNICAL, AND GROUNDWATER APPLICATIONS, 2ND, 1987, GOLDEN, COLO. FROC., P.203-8. SOCIETY OF

PROFESSIONAL WELL LOG ANALYSIS, 1988. (TN269 157 1987X)

ALSO PUBLISHED: TIC, 1987. 7P. MN (LA-UR-87-4129)

14-59

YAMADA, TETSUJI (ESS-5); BUNKER, SUSAN S. (ESS-5).

DEVELOPMENT OF A NESTED GRID, SECOND MOMENT TURBULENCE CLOSURE MODEL AND APPLICATION TO THE 1982 ASCOT BRUSH CREEK DATA SIMULATION.

J. APPL. METEOROL., V.27, P.562-78. 1988.

14-60

YAMADA, TETSUJI (ESS-5); BUNKER, SUSAN S. (ESS-5); NICCUM, E. (ESS-5).

NUMERICAL SIMULATIONS OF LONG-RANGE POLLUTANT TRANSPORT FROM COASTAL TO INLAND MOUNTAINOUS REGIONS.

IN: SYMPOSIUH ON TURBULENCE AND DIFFUSION, 8TH, 1988, SAN DIEGO, CALIF. PROC., P.20-2.

AMERICAN METEC.ROLOGICAL SOCIETY, 1988. (OC880 .59 1988X)

14-61

YAMADA, TETSUJI (ESS-5); BUNKER, SUSAN S. (ESS-5); NICCUM, E. (ESS-5). NUMERICAL SIMULATIONS OF LONG-RANGE POLLUTANT TRANSPORT FROM COAST TO INLAND MOUNTAINOUS REGIONS.

TIC, 1988. 11P. MN (LA-UR-88-0113)

14-62

YAMADA, TETSUJI (ESS-5); BUNKER, SUSAN S. (ESS-5); NICCUM, E. (ESS-5).

SIMULATIONS OF THE ASCOT BRUSH CREEK DATA BY A NESTED-GRID, SECOND-MOMENT

TURBULENCE'-CLOSURE MODEL AND A KERNEL CONCENTRATION ESTIMATCR.

IN: CONFERENCE ON MOUNTAIN METEOROLOGY, 4TH, 1987, SEATTLE, WASH. PREPRINT VOLUME

PROC., P.175-9. AMERICAN METEOROLOGICAL SOCIETY, 1987. (OC993.6 .C65 1987X)

ALSO PUBLISHED: TIC, 1987. 6P. MN (LA-UR-87-1657) 
16-1

ANDERSON, CHARLES A. (Q 13); FLY, GERALD W. (Q-13); LUNDBERG, LYNN B. (Q-13);

ROMERO, JOE A. (O-13).

INVESTIGATION OF THE STRENGTH OF H440 GRAPHITE WHEN SUBJECTED TO COMBINED PRIMARY AND

SECONDARY STRESS.

LOS ALAMOS, 1986. B1P. (LA-10652-MS)

$16-2$

BENNETT, JOEL G. (MEE-13); DOVE, RICHARD C. (MEE-13); DUNWOODY, WADE E. (MEE-13);

FARRAR, CHARLES R. (MEE-13); GOLDMAN, PEGGY (MEE-13).

SEISMC CATEGORY I STRUCTURES PROGRAM-RESULTS FOR FY 1925.

LOS ALAMOS, 1987. 46P. (LA-11117-MS)

16-3

BENNETT, JOEL G. (MEE-13); DOVE, RICHARD C. (MEE-13); DUNWOODY, WADE E. (MEE-13);

FARRAR, CHARLES R. (MEE-13); GOLDMAN, PEGGY (MEE-13).

SEISMC CATEGOAY I STRUCTURES PROGRAM-RESULTS FOR FY 1986.

LOS ALAMOS NATIONAL LABORATORY, 1988. 6OP. (LA-11377-MS)

$16-4$

BOYACK, B. E. (N-12).

USE OF 2D/3D DATA FOR PEAK CLADDING TEMPERATURE UNCERTAINTY STUDIES.

TIC, 1988. 10P. MN (LA-UR-B8-3405)

16-5

BUTLER, THOMAS A. (Q-13); BENNETT, JOEL G. (Q-13); BABCOCK, C. D., JP. (Q-13); NATSIAVAS, S.

RESPONSE OF SEISMIC CATEGORY I TANKS TO EARTHOUAKE EXCITATION.

LOS ALAMOS, 1987. 66P. (LA-10871-MS)

16-6

BYERS, HELEN M. (X-6); O'DELL, R. DOUGLAS (X-6).

RADIATION TRANSPORT OCTOBER 1, 1986 - DECEMBER 31, 1986.

LOS ALAMOS, 1:88. 47P. (LA-11154-PR)

16-7

DOVE, RICHARD C. (MEE-13); BENNETT, JOEL G. (MEE-13); FARRAR, ChARLES R. (MEE-13);

ANDERSON, CHARLES A. (MEE-13).

SEISMIC CATEGORY I STRUCTURES PROGRAM FINAL REPORT, FY $1983-84$.

LOS ALAMOS, 1987. 91P. (LA-11013-MS)

$16-8$

GEORGE, DENISE C. (T-2); LA BAUVE, RAPHAEL J. (T-2).

PERTV-A STANDARD FILE VERSION OF THE PERT-V CODE.

LOS ALAMOS, 1988. 2âP. (LA-11206-MS)

16-9

GREGOPY, WILLIAM S. (N-6); NICHOLS, BILL 0. (N-6); WHITE, BRUCE W. (N-6); SMITH, PHILLIP R.;

LESUE, IAN H.; COAKRAN, J. R.

FIRE AEPOSOL EXPERIMENT AND COMPARISONS WITH COMPUTER CODE PREDICTIONS

TIC, 1988. 14P. MN (LA-UR-88-2666)

16-10

HAGAN, PATRICK S. (T-7); HERSKOWITZ, M.; PIRKLE, C.

RUNAWAY IN HIGHLY SENSITIVE TUBULAR REACTORS.

SIAM J. APPL. MATH., V.48, P.1437-50. 1988.

16-11

HAGAN, PATRICK S. (T-7); HERSKOWITZ, Ni.; PIRKLE, C.

SIMPLE APPROACH TO HIGHLY SENSITIVE TUBULAR REACTORS

SIAM J. APPL. MATH., V.48, P.1083-101. 1989. 
$16-12$

HANSEN, GORDON E. (N-2); AUDAS, JAMES H. (N-2); MARTIN, E. RAY (N-2); PEDERSON, RAYMOND A. (N-2); SPRIGGS, GAEGORY D. (N-2); WHITE, ROGER H.

CRITICAL EXPERIMENTS IN SUPPORT OF THE CNPS [COMPACT NUCLEAR POWER SOURCE] PROGRAM. TIC, 1988. 9P. MN (LA-UR-88-2272)

$16-13$

JAFFE, L.; BEATTY, R.; BHANDARI, P.; CHOW, E.; DEININGER, W.; EWELL, R.; FUJITA. T.; GROSSMAN, M.; KIA, T.; NESMITH, B.; NEWELL, T.; SHIH, C. F.; SPANOS, J.; STALLKAMP, J.; STEVENS. J.; SHULTZ, J.; WANCHOO, A. K.; WHITE, L.; BELL, CHARLES R. (N-6); CARLSON, DONALD E. (N-12);

METZGER, JOHN D. (A-4); VOSS, SUSAN; BLOOMFIELD, H.; HELLER, J. NUCLEAR REACTOR POWER AS APPLIED TO A SPACE-BASED RADAR MISSION. TIC, 1988. 17P. MN (LA-UR-88-3253)

16-14

JAFFE, L; FUJITA, T.; BEATTY, R.; BHANDARI, P.; CHOW, E.; DEININGER, W.; EWELL, R.; GROSSMAN, M.; KIA, T.; NESMITH, B.; NEWELL, T.; SHIH, C. F.; SPANOS, J.; STALLKAMP, J.; STEVENS, J.; SHULTZ, J.; WANCHOO, A. K; WHITE, L; BELL, CHARLES R. (N-6); CARLSON, DUNALD E. (N-12);

METZGER, JOHN D. (A-4); VOSS, SUSAN; SLOOMFIELD, H.; HELLER, J.

SYSTEMS ASPECTS OF A SPACE NUCLEAR REACTOR POWER SYSTEM.

TIC, 1988. 18P. MN (LA-UR-88-3252)

16-15

JENKS, RICHARD P. (N-9)

PRELIMINARY MODELING OF THE TMI-2 ACCIDENT WITH MELPROG-TRAC.

TIC, 1988. 9P. MN (LA-UA-88-1364)

$16-16$

KELLY, ELIZABETH J. (A-1); HEMPHILL, GERALYN M. (A-1).

ESTIMATION TECHNIQUES FOR COMMON CAUSE FAILURE EVENTS.

LOS ALAMOS, 1988. 48P. (LA-11179-MS)

16-17

LLES, DENNIS R. (N-9); SPORE, JAY W. (N-9); KNIGHT, THAD D. (Q-9); NELSON, R. A. (N-9);

CAPPIELLO, MICHAEL W. (N-9); PASAMEHMETOGLU, KEMAL O. (N-9); MAHAFFY, JOHN H. (Q-9);

GUFFEE. LAURA A.; STUMPF, HENRY (N-9); DOTSON, PAUL J. (Q-9); STEINKE, ROBERT G. (N-9);

SHIRE, PHILLIP R. (N-9); GREINER, SONYA E.; SHERWOOD, KENNETH B.

TPAC-PF1/MOD1 CORRELATIONS AND MODELS.

LOS ALAMOS NATIONAL LABORATORY, 1988. 558P. (LA-11208-MS)

$16-18$

MASON, R. E. (MST-11); MATTHEWS, ROBERT BRUCE (MST-11).

COMPATIBILITY IN SPACE REACTOR FUEL SYSTEMS.

LOS ALAMOS, 1988. 4OP. (LA-11071-NS)

16-19

MERAIGAN, MICHAEL A. (MEE-13); REID, ROBERT S. (MEE-13).

MULTIMEGAWATT SPACE POWER SYSTEMS-THERMAL MANAGEMENT ANNUAL REPORT 1987

LOS ALAMOS NATIONAL LABORATORY, 1988. 254P. (LA-11254-MS)

$16-20$

MOORE, LESLIE M. (A-1); BECKMAN, RICHARD J. (A-1).

APPROXIMATE ONE-SIDED TOLERANCE BOUNDS ON THE NUMBER OF FAILURES USING POISSON REGRESSION

TECHNOMETRICS, V.30, P.283-90. 1588.

16-21

MOTLEY, FRANK E. (N-9); JENKS, RICHARD P. (N-9).

MODELING OF THE TMI-2 [THREE MILE ISLAND UNIT-2] ACCIDENT WITH MELPROGITAC AND

CALCULATICN RESULTS FOR PHASES 1 AND 2.

TIC, 1988. 23P. MN (LA-UR-88-3348)

16-22

O'DELL, R. DOUGLAS (X-6); BYERS, HELEN M. (X-6)

RADIATION TRANSPORT OCTOBER 1, 1987-DECEMBER 31, 1987.

LOS ALAMOS NATIONAL LABORATORY, 1988. 38P. (LA-11358-PR) 
$16-23$

O'DELL, R. DOUGLAS (X-6); NAGY, ANN (X-6).

RADIATION TRANSPORT PROGRESS REPORT JANUARY 1, 1988-MARCH 31, 1888.

LOS ALAMOS NATIONAL LABORATORY, 1988. 42P. (LA-11390-PR)

16-24

O'DELL, R. DOUGLAS (X-6); BYERS, HELEN M. (X-6).

RADIATION TRANSPORT, APRIL 1, 1987- JUNE 30, 1987.

LOS ALAMOS, 1988. 79P. (LA-11188-PR)

16-25

O'DELL, R. DOUG'AS (X-6); NAGY, M. ANN (X-6).

RADIATION TRANSPORT, JANUARY 1, 1987- MARCH 31, 1987.

LOS ALAMOS, 1988. 47P. (LA-11180-PR)

$16-26$

O'DELL, R. DOUGLAS (X-6); NAGY, M. ANN (X-6).

RADIATION TRANSPORT, JULY 1, 1997- SEPTEMBER 30, 1987.

LOS ALAMOS, 1988. 44P. (LA-11249-PR)

16-27

PALMER, RONALD G. (N-12).

POSTANALYSIS OF THE CNPS [COMPACT NUCLEAR POWER SOURCE] CRITICAL EXPERIMENT

TIC, 1988. 13P. MN (LA-UR-88-2258)

$16-28$

SIEBE, D. A. (N-12); BOYACK, B. E. (N-12); STEINER, J. L. (N-12).

TRAC PF1/MOD1 CALCULATIONS AND DATA COMPARISONS FOR MIST FEED AND BLEED AND STEAM GENERATOR TUBE RUPTURE EXPERIMENTS.

TIC, 1988. 31P. MN (LA-UR-88-3557)

16-29

SPORE, JAY W. (N-9); NELSON, RALPH A. (N-9); STEINKE, ROBERT G. (N-6); CAPPIELLO, MICHAEL W. (N-6): JENKS, R. (N-6).

TRAC-PF1/MOU2 STATUS AND PLANS

TIC, 1988. 22P. MN (LA-UR-88-3846)

16-30

STEINER, J. L. (N-12): SIEBE, D. A. (N-12).

ONCE-THROUGH STEAMGENERATOR SENSITIVITY CALCULATIONS.

TIC, 1988. 29P. MN (LA-UR-88-3845)

$16-31$

SULLVAN, L. HAROLD (N-6).

UNCERTAINTY IN LOCA (LOSS-OF-COOLANT ACCIDENT! ANALYSIS HISTORICAL DISCUSSION. TIC, 1988. 15P. MN (LA-UR-88-2631)

$16-32$

WENDROFF, BURTON (T-7).

OUENCH FRONT REVISITED.

TIC, 1988. BP. MN (LA-UR-88-1919) 
17. GENERAL, MISCELLANEOUS, AND PROGRESS REPORTS (NONNUCLEAR)

17-1

ALESSI, VICTOR E.; BINGAMAN, JEFF; HECKER, SIEGFRIED S. (DIR): TWINING, BRUCE G.;

WHETTEN, JOHN T. (ADERA).

LOS ALAMOS NATIONAL LABORATORY VERIFICATION TECHNOLOGY-DEDICATION OF THE SPACE SCIENCE LABORATORY APRIL 5, 1888.

LOS ALAMOS NATIONAL LABORATORY, 1988. 15P. (LALP-88-019)

17-2

APT, KENNETH E. (CLS-DO).

CHEMICAL WARFARE ARMS CONTROL-ISSUES AND CHALLENGES

LOS ALAMOS NATIONAL LABORATr RY, 1988. 39P. CNSS REPORT NO.6 (LA-11451)

$17-3$

BEMENT, THOMAS R. (A-1); PICARD, RICHARD R. (A-1); PECK, CLAUDIA (A-1); MOORE, LESLIE M. (A-1): HEMPHILL, GERALYN M. (A-1).

STATISTICAL EFFORTS FOR LOS ALAMOS CREW MEMBER LETHALITY PROGRAM.

TIC, 1988. 16P. MN (LA-UR-88-3471)

$17-4$

BROWNE, JOHN C. (ADR).

IMPACT OF THE STRATEGIC OEFENSE INITIATIVE ON RESEARCH AT LOS ALAMOS NATIONAL

LABOAATORY.

TIC, 1986. 17P. MN (LA-UR-86-3767)

$17-5$

BUMSTED, M. PAMELA (CLS-1).

RECOGNIZING WOMEN IN THE ARCHAEOLOGICAL RECORO.

TIC, 1887. 10P. MN (LA-UR-87-4288)

$17-6$

BUSSOLINI, P. L. (MST-9); OAVIS, ALVIN H. (NST-9); GEOFFRION, R. RONALD (MST-9). NEW APPROACH TO QUALITY FOR NATIONAL RESEARCH LABS.

QUAL PROG., JAN., P.24-7. 1998.

17-7

COOPER, NECIA G. (PAO).

LOS ALAMOS SCIENCE-NUMBER 16.

TIC, 1988. 84P. MN (LA-UR-88-1000)

17-8

COVER, LESTER E. (MEC-DO).

PLANNING AN MIS [MANUFACTURING INFORMATION SYSTEM] FOR A JOB SHOP ENVIRONMENT TIC, 1987. 17P. MN (LA-UR-87-3909)

17-9

CRUZ, JAMES M. (IS-12); MACHEN, JUDY (IS-11); RHOADES, JOHN (IS-8).

DELUVERING A MESSAGE VISUALLY-ADAPTING MUSEUM PRINCIPLES TO THE PRODUCTION OF TECHNICAL EXHIBITS.

IN: INTERNATIONAL TECHNICAL COMMUNICATION CONFERENCE, 35TH, 1988, PHILADELPHIA. PA.

PAOC., P.VC3. SOCIETY FOR TECHNICAL COMMUNICATION, 1988. (T10.5 157 1988X)

17-10

EDESKUTY, FREDERICK J. (A-4); STEWART, WALTER F. (A-4).

SAFETY ASPECTS OF LARGE-SCALE HANDLING OF HYDROGEN.

TIC, 1888. 14P. MN (LA-UR-88-2045)

17-11

FRANK, JOSEPH A.

EMPLOYMENT-GENERATING PROJECTS FOR THE ENERGY AND MINERALS SECTORS OF HONDURAS LOS ALAMOS NATIONAL LABORATORY, 1968. 79P. (LA-108B6-MS) 
$17-12$

GARRITY, PATRICK J. (CNSS).

UNITED STATES SPACE POLICY-REVIEW AND ASSESSMENT.

LOS ALAMOS NATIONAL LABORATORY, 1988. 41P. CNSS REPORT NO.3 (LA-11i81)

$17-13$

GERE, SCOT B. (ACTO).

INTERPRETATIONS UNDERSTANDING SOVIET ARMS CONTROL MOTIVATIONS AND VERIFICATION ATTITUDES.

LOS ALAMOS NATIONAL LABORATORY, 1988. 29P. (LA-11475-MS)

$17-14$

HECKER, SIEGFRIED S. (DIR).

ROLE FOR NATIONAL LABORATORIES IN ENHANCING ECONOMIC COMPETITIVENESS-THE EXAMPLE OF HIGH-TEMPERATURE SUPERCONDUCTORS.

LOS ALAMOS, 1987.2OP.CNSS PAPER NO.1(LA-11092-MS)

17-15

JONES, CLAUDE RANDOL (DRAFST).

OVERVIEW OF THE SDI [STRATEGIC DEFENSE INITIATIVE] PROGRAM IN SHORT WAVELENGTH CHEMICAL LASERS.

TIC, 1988. 19P. MN (LA-UR-B8-0640)

17-15

JONES, ERIC M. (ESS-5).

BASIS OF SETTLEMENT-ECONOMIC FOUNDATIONS OF PERMANENT PIONEER COMMUNITIES.

TIC, 1988. 17P. MN (LA-UR-88-1110-REV)

17-17

JONES, EAIC M. (ESS-5).

ECONOMIC FOUNDATIONS OF PERMANENT PIONEER COMMUNITIES.

TIC, 1988. 18P. MN (LA-UAR-88-1110)

17.18

JONES, ERIC M. (ESS-5).

PUTTING SPACE RESOURCES TO WORK.

TIC, 1988. 11P. MN (LA-UA-88-2801)

17-19

JONES, ERIC M. (ESS-5).

STEPS TOWARD LUNAR SETTLEMENT.

TIC, 1988. 12P. MN (LA-UR-8B-1192)

$17-20$

LOS ALAMOS NATIONAL LABORATORY.

RESEARCH HIGHLIGHTS 1987.

LOS ALAMOS NATIONAL LABORATORY, 1987. 80P. (LALP-88-016)

$17-21$

MARTZ, HARRY F., JR. (A-1): WALLER, RAY' A. (S-DO).

ON THE MEANING OF PROBABILITY.

RELAB. ENG. SYST. SAF., V.23, P.299-304. 1988.

$17-22$

MORTENSEN, JEANNETTE J. (IS-11); RUMINER, MAPY K. (IS-11); SINORADZKI, HELEN M. (IS-11): SNYDER, CONNIE M. (IS-11).

STRATEGIC PLANNING-ADVENTURES IN WONDERLAND.

IN: INTERNATIONAL TECHNICAL COMMUNICATION CONFERENCE, 35TH, 1988, PHILADELPHIA, PA.

PROC., P.MPD-93-4. SOCIETY FOR TECHNICAL COMMUNICATION, 1988. (T10.5 157 1988X)

17-23

POWELL, DENNIS R. (A-5); STORM, GREGORY (A-5)

AVENUE OF APPROACH GENERATION.

TIC, 1988. 10P. MN (LA-UR-88-3753) 
$17-24$

POWELL, DENNIS R. (A-5); WRIGHT, JAMES C. (A-5); SLENTZ, G. E. (MEE-5); KNUDSEN, PAUL D. (A-5). REPRESENTATIONS TO SUPPOAT TF.RPAIN REASONING.

TIC, 1988. 9P. MN (LA-UR-88-3754)

$17-25$

SANDOVAL, DONALD L. (T-3); HARLOW, FRANCIS H. (T-3); GENIN, KENT E. (T-3).

HUMAN COLLECTIVE DYNAMICS-TWO GROUPS IN ADVERSARIAL ENCOUNTER.

LOS ALAMOS, 1988. 72P. (LA-11247-MS)

17-26

STUTZ, ROGER ALAN (ENG-4); ZOCHER, MARC (ENG-8).

NEW DEVELOPMENTS IN CAPITAL COST ESTIMATING.

TIC, 1988. 7P. MN (LA-UR-88-0194)

17-27

TRAINOR, MARY STODDARD (A-6); ANDREWS, ANDREW E. (A-6).

HIGH-TECHNOLOGY CLASSROOM-A PREVIEW OF COMING ATTRACTIONS.

ilC, 1988. BP. MN (LA-UR-88-2550)

$17-28$

WELTMAN, JOHN J. (CNSS).

WHITHER THE GENERAL PURPOSE FORCES?

CENTER FOA NATIONAL SECURITY STUDIES, LOS ALAMOS NATIONAL LABORATORY, 1988. 4 P. (UA23 W45 1988X)

17-29

WHEELER, KYLE T. (C-2).

ESTABLISHING STANDARDS FOR COMPUTER DOCUMENTATION.

IN: INTERNATIONAL TECHNICAL COMMUNICATION CONFERENCE, 35TH, 1988, PHILADELPHIA, PA.

PROC., P.WE-166-7. SOCIETY FOR TECHNICAL COMMUNICATION, 1968. (T10.5 .157 1988X)

$17-30$

WILLAMS, JOEL M. (MST-7).

INNOVATIVE ARRANGEMENTS FOR INDUSTRYIDOE LABORATORY COLLABORATION IN HIGH TEMPERATURE SUPERCONDUCTIVITY.

TIC. 1938. 8P. MN (LA-UR-87-4010)

$17-31$

YEAPWOOD. DEIDRA D. (NST-12).

TRAINING AND EXERCISES OF THE EMERGENCY RESPONSE TEAM AT THE LOS ALAMOS PLUTONIUM FACILITY.

TIC, 1988. 10P. MN (LA-UR-88-0177) 
18-1

CANAVAN, GREGORY H. (P-DU).

ADAPTIVE PREFERENTIAL DEFENSE AND DISCRIMINATION.

LOS ALAMOS NATIONAL LABORATORY. 1983. 45P. (LA-11376-MS)

18-2

CANAVAN, GREGORY H. (P-DO).

ADVANCED DEFENSE TECHNOLOGIES IN TRANSITION.

LOS ALAMOS NATIONAL LABORATORY, 1988. 32P. (LA-11361-MS)

18-3

CANAVAN, GREGORY H. (P.DO); HAMMOND, JOHN H.

CONGTELLATION SIZING FOR MODEST DIRECTED ENERGY PLATFORMS.

LOS ALAMOS, 1988. 27P. (LA-11238-MS)

18-4

CANAVAN, GREGORY H. (P-DO).

DIRECTED ENERGY ARCHITECTURES

LOS ALAMOS NATIONAL LABORATORY, 1988. 64P. (LA-11285-MS)

$18-5$

CANAVAN, GREGORY H. (P-DO); BROWNE, JOHN C. (ADDRA).

FREE ELECTRON LASERS IN STRATEGIC DEFENSE.

LOS ALAMOS, 1988. 32P. (LA-11225-MS)

18-6

CANAVAN, GREGORY H. (P-DO).

IMPACT OF CARRIE 9 VEHICLE MASS AND COST ON BOOST INTERCEPTORS.

LOS ALAMOS NATIONAL LABORATORY, 1988. 21P. (LA-11406-MS)

18-7

CANAVAN, GREGORY H. (P-DO).

LASER COUNTERMEASURE IMPACTS AND PENALTIES.

LOS ALAMOS, 1988. 26P. (LA-11264-MS)

18-8

CANAVAN, GREGORY H. (P-DO).

MILITAFY USES OF SPACE.

LOS ALAMOS NATIONAL LABORATORY, 1988. 2OP. (LA-1 1344-MS)

18-9

CANAVAN, GREGORY H. (P-DO).

ROLE OF ADVANCED TECHNOLOGIES IN STAATEGIC DEFENSE

LOS ALAMOS NATIONAL LABORATORY, 1988. 37P. (LA-11374-MS)

18-10

CANAVAN, GREGORY H. (P-DO): BROWNE, JOHN C. (ADDRA)

ROLES FOR NEUTRAL PARTICLE BEAMS IN STRATEGIC DEFENSE

LO'S ALAMOS, 1988. 125P. (LA-11226-MS)

18-11

CANAVAN, GREGORY H. (P.DO).

SDI-MYTH OR REALITY?

I.OS ALAMOS NATIONAL LABORATORY, 1988. 17P. (LA-11322-MS)

18-12

CANAVAN, GREGORY H. (P.DO).

SDI-MYTH OR REALTY?

TIC, 1988. 15P. MN (LA-UR-88-1326) 
18-13

CANAVAN, GREGORY H. (P-DO).

STRATEGIC DEFENSE IN TRANSITION

LOS ALAMOS NATIONAL LABORATORY, 1988. 14P. (LA-11375-MS;

18-14

CANAVAN, GAEGOAY H. (P-DO); BROWNE, JOHN C. (ADDRA)

WHERE DIRECTED ENERGY STANDS IN STRATEGIC DEFENSE

LOS ALAMOS, 1988. 30P. (LA-11172-MS)

$18-15$

DAVIS, ALVIN H. (MST-9).

AUDITING NUCLEAR WEAPONS OUALITY PROGRAMS AT LOS ALAMOS

TIC, 1988. 7P. MN (LA-UR-88-1452)

18-16

DREICER, JARED S. (A-5); MICHELSEN, CHRISTIE D. (A-5); MORGESON, J. DARRELL (A-5)

SEAT-A STRATEGIC ENGAGEMENT ANALYSIS TOOL.

TIC. 1988. 6P. MN (LA-UR-87-3131)

18-17

GARRITY, PATRICK J. (CNSS); PENDLEY, ROBERT E. (CNSS); SELDEN, ROBERT W. (CNSS).

FUTURE OF NUCLEAR WEAPONS-THE NEXT THREE DECADES. CONFERENCE SUMMARY.

LOS ALAMOS NATIONAL LABORATORY, 1988. 19P. CNSS PAPER NO.16 (LA-11399-MS)

$18-18$

GARRITY PATRICK J. (CNSS).

INF TREATY-PAST, PRESENT, AND FUTURE.

LOS ALAMOS, 1988. 19P. CNSS PAPER NO.4 (LA-11234-MS)

18-19

GARRITY, PATRICK J. (CNSS); GORE, RAYMOND A. (E-DG); PENDLEY, ROBERT E. (CNSS);

PILAT, JOSEPH F. (CNSS).

MONITORING SPACE WEAPONRY-DETECTION AND VERIFICATION ISSUES

LOS ALAMOS NATIONAL LABORATOFY, 1988. 39P. CNSS REPORT NO.2 (LA-11278)

$18-20$

GARRITY, PATRICK J. (CNSS).

U.S.-SOVIET START NEGOTIATIONS-STATUS AND IMPLICATIONS.

LOS ALAMOS NATIONAL LABORATORY, 1988. 16P. CNSS PAPER NO.7 (LA-11280-MS)

18-21

HECKER, SIEGFRIED S. (D.R).

DOE WEAPONS LABORATORIES' CONTRIBUTIONS TO THE NATION'S DEFENCE TECHNOLOGY BASE

LOS ALAMKS NATIONAL LABORATORY, 1988. 36P. CNSS PAPER NO.9 (LA-11319-MS)

18-22

HECKER, SIEGFRIED S. (DIR).

THE ROLE OF THE DOE WEAPONS LABORATORIES IN A CHANGING NATIONAL SECURITY ENVIRONMENT

LOS ALAMOS NATIONAL LABORATORY, 1988. 27P. CNSS PAPER NO.8 (LA-11318-MS)

18-23

KUPPERMAN, R. H.; SELDEN, ROBERT W. (CNSS); SMITH, H. A.

ACHIEVING MUTUAL DETERRENCE WITH ACTIVE DEFENSES.

LOS ALAMOS NATIONAL LABORATORY, 1988. 52P. CNSS REPORT NO.4 (LA-11405)

18-24

KUPPERMAN, R. H.; SELDEN, ROBERT W. (X-DO); SMITH, H. A.

MODEL OF THE DEFENSE OF LAND-BASED MISSILE FORCES BY A MULTILAYER ANTIMISSILE SYSTEM LOS ALAMOS NATIONAL LABORATORY, 1987. 138P. CNSS REPORT NO.1 (LA.UR-B7-14B9)

18-25

LE CLAIRE, RENE J., JR. (A-4)

BEAM PROFILE EFFECTS ON NPB PERFORMANCE

LOS ALAMOS, 1988. 15P. (LA-11228-MS) 
$18-26$

LUNDY, ARVID S. (IT-3); PANKFIATZ, MICHAEL C. (IT-3): HCGSETT, VICTOR H. (IT-3): MUNROE. JAMES L. (IT-3). NUCLEAR TECHNOLOGY AND THE EXPORT CONTROL LAWS.

TIC, 1828. 7P. MN (LA-UIR-88-1996)

18-27

MAC FARLANE, ROBERT E. (T-2).

IMPACT OF ENDF-6 ON CROSS SECTION PROCESSING CODES

TIC, 1988. 10P. MN (LA.UAR-88-2129)

18-28

MARK, J. CARSON (DIRICN).

PURPOSE OF NUCLEAR TEST EXPLOSIONS. PAPER 1.

IN: NUCLEAR WEAPON TESTS-PROHIBITION OR LIMITATION? J. GOLDBLAT AND D. COX, EDS.

P.[31]-42. OXFORD UNIVERSITY PRESS, 1988. (UA12.5I.N83/1987)

$18-29$

MORALES, RE: YNALDO (WPC.1); PENDLEY, ROBERT E. (DRA-ACV).

LOS ALAMOS STRATEGIC DEFENSE RESEARCH AND THE ABM TREATY.

LOS ALAMOS NATIONAL LABORATORY, 1988. 34P. CNSS PAPER NO.10 (LA-11262-MS)

18-30

PENG, JEN CHEIH (P-2); SUNIER, JULES W. (P-2); BROWN, RONALD E. (P-3); DRAKE, DARRELL M. (ESS-8).

NELSON STEIN [OBITUARY]

PHYS. TODAY, V.41, P.88. 1988.

18-31

PILAT, JOSEPH F. (CNSS).

ARMS CONTROL AND SOVIET GRAND STRATEGY

LOS ALAMOS, 1988. 9P. CNSS PAPER NO.5 (LA-11218-MS)

$18-32$

POTTER, WILLIAM C.

EMERGING NUCLEAR SUPPLIEAS.

LOS ALAMOS NATIONAL LABORATORY, 1988. 15P. CNSS PAPER NO.13. (LA-11314-MS)

18-33

RAMBERG, BENNETT

LESSONS OF CHEANOBYL.

LOS ALAMOS NATIONAL LABORATORY, 1988. 17P. CNSS PAPER NO.14 (LA-11315-MS)

$18-34$

SAPIR, JOSEPH L. (N-12); BRANDON, DENISE I. (N-12); COLLINS, P. J.; COWAN, C. L; POATER, C. A.; ANDAE, S. V.

ANALYSIS OF SP-100 CAITICAL EXPERIMENTS

TIC. 1988. 15P. MN (LA-UR-88-2008)

$18-35$

TUCKER, ROBERT W. (CNSS).

NUCLEAR FUTURE-POUTICAL AND SOCIAL CONSIDERATIONS.

LOS ALAMOS NATIONAL LABORATORY, 1988. 12P. CNSS PAPER NO.11 (LA-11270 MS)

18-36

WESTERVELT, DONALD R. (CNSS).

ROLE OF LABORATORY TESTS.

IN: NUCLEAR WEAPON TESTS-PAOHIBITION OR LIMITATION? J. GOLDBLAT AND D. COX. EDS.

P.[47]-58. OXFOAD UNIVEASITY PRESS, 1988. (UA/12.5/.N83/1987)

$18-37$

WILSON, WILLIAM B. (T-2); ARTHUR, EDWARD D. (T-DO); LA BAUVE, RAPHAEL J. (T-2); PERRY, ROBERT T. CALCULATED PROTON-INDUCED THICK-TARGET RADIONUCLIDE ACTIVATION YIELDS AND NEUTRON YIELD SPECTRA FOA EP LESS THAN OR EQUAL TO 50 MEV. WITH COMPARISONS TO OTHER CALCULATIONS AND MEASURED DATA.

TIC, 1988. 5P. MN (LA-UR-88-2370) 
18-38

YOUNG, PHILUP G., JR. (T-2).

STATUS OF NUCLEAR DATA EVALUATIONS FOR VERSION VI OF ENDF/B.

TIC, 1938. 14P. MN (LA-UR-88-2130) 
19-1

BERGER, MICHAEL E. (MEE-DO); MURPHY, HUGH D. (ESS-DOT).

PROSPECTS FOR HOT DRY ROCK IN THE FUTURE.

TIC, 1988. 10P. MN (LA-UR-88-1261)

19-2

DEUPREE, ROBERT G. (ESS-5).

COMPARISON BETWEEN TWO EPITHEPMAL NEUTRON LOGGING TOOLS

LOS ALAMOS NATIONAL LABORATORY, 1988. 12F. (LA-11251-MS)

19-3

GOFF, SUE JACOBSEN (ESS-1); LAUGHIIN, A. WILLIAM (ESS-1); RUFENACHT, HEINZ D.;

GOFF, FRASER E. (ESS-1); HEIKEN, GRANT (ESS-1); ADAMS, ANDREW I. (ESS-1); MUSGRAVE, JOHN (ESS-1): PLANNER, H. N. (ESS-1); RAMOS, N.

EXPLORATION GEOTHERMAL GRADIENT DRILLING, PLATANARES, HONDURAS, CENTRAL AMERICA. LOS ALAMOS NATIONAL LABORATORY, 1988. 33P. (LA-11349-MS)

$19-4$

LAUGHLIN, A. WILLIAM (ESS-1).

RECOMMENDATIONS REPORT OF THE PLATANARES GEOTHERMAL SITE, DEPARTMENT OF COPAN. HONDURAS.

LOS ALAMOS NATIONAL LABORATORY, 1988. 73P. (LA-11410-MS)

19-5

LIU, JOANNE; BADALAMENTE-ALEXANDER, TIA M.

GEOTHERMAL POWER GENERATION OPTIONS FOR FENTON HILL RESERVOIR TESTING

LOS ALAMOS NATIONAL LABORATORY, 1988. 134P. (LA-1147C-MS)

19-6

WHETTEN, JOHN T. (ADERA); MURPHY, HUGH D. (ESS-DOT); HANOLD, ROBERT J. (ESS-DOT):

MYERS, C. WESLEY (ESS-1): DUNN, J. C.

ADVANCED GEOTHERMAL TECHNOLOGIES.

TIC, 1988. 16P. MN (LA-UR-87-4125) 
20-1

ALBRIGHT, JAMES N. (ESS-4); JOHNSON, P. A. (ESS-4); PHILLIPS, WILLIAM S. (ESS-4);

BRADLEY, CHRISTOPHER R. (ESS-4); RUTLEDGE, JAMES T. (ESS-4).

CROSSWELL ACOUSTIC SURVEYING PROJECT.

LOS ALAMCS, 1988. 131P. (LA-11157-MS)

$20-2$

BENNETT, GLORIA A. (MEE-11)

ACTIVE COOLING FOR DOWNHOLE INSTRUMENTATION-PRELIMINARY ANALYSIS AND SYSTEM

SELECTION

LOS ALANOS, 1988. 148P. (LA-11102-MS)

$20-3$

CONAWAY, JOHN G. (ESS-3).

TEMPERATURE LOGGING AS AN AID TO UNDERSTANDING GROUNDWATER FLOW IN BOREHOLES. IN: INTERNATIONAL SYMPOSIUM ON BOREHOLE GEOPHYSICS FOR MINERALS, GEOTECHNICAL, AND GPOUNDWATER APPLICATIONS, 2ND, 1987, GOLOEN, COLO. PROC., P.51-9. SOCIETY OF PROFESSIONAL WELL LOG ANALYSIS, 1988. (TN269.157 1987X)

ALSO PUBLSHED: TIC, 1987. 1OP. MN (LA-UR-87-3355)

20-4

GOFF, SUE JACOBSEN (ESS-1); LAUGHLIN, A. WILLIAM (ESS-1); RUFENACHT, HEINZ D.;

GOFF, FRASER E. (ESS-1); HEIKEN, GRANT (ESS-1); RAMOS, N.

EXPLORATION GEOTHERMAL GRADIENT DRILLING, PLATANARES, HONDURAS, CENTRAL AMERICA. IN: GEOTHERMAL ENERGY SYMPOSIUM, 1988, NEW ORLEANS, LA. PROC., P.55-64. ASME, 1988. (TJ280.7.G446 1988X)

ALSO PUBLISHED: TIC, 1988. 11P. MN (LA-UR-87-3603)

20-5

LAUGHLIN, A. WILLIAM (ESS-1); FRANK, JOSEPH A. (ADIII); FLORES, $\mathbf{W}$.

GEOTHERMAL RESOURCE ASSESSMENT IN HONDURAS-HOW WE GOT TO PLATANARES.

IN: GEOTHERMAL ENERGY SYMPOSIUM, 1988, NEW ORLEANS, LA. PROC., P.65-9. ASME, 1988.

(TJ280.7.G446 1988X)

ALSO PUBLISHED: TIC, 1988. 6P. MN (LA-UR-87-3609)

$20-6$

MOORE, TROY K. (ESS-4).

INITIAL BOREHIOLE ACOUSTIC TELEVIEWER DATA PROCESSING ALGORITHMS. LOS ALAMIS NATIONAL LABORATORY. 1989. 185P. (LA-11327-MS)

$20-7$

SHEVENELL, LISA (ESS-1); GOFF, FRASER E. (ESS-1); MILES, D. J. (ESS-1); WAIBEL, AL;

SWANBERG, CHANDLER.

UTHOLOGIC DESCRIPTIONS AND TEMPERATURE PROFILES OF FIVE WELLS IN THE SOUTHWESTERN

VALLES CALDERA REGION, NEW MEXICO.

LOS ALAMOS, 1988. 43P. (LA-11165-OBES)

20-8

STAROUIST, VIRGINIA L

CORE LOG-VALLES CALDERA *2A, NEW MEXICO.

LOS ALAMOS, 1988. 87P. (LA-11176-OBES)

$20-9$

VUATAZ, FRANCOIS D.; GOFF, FRASER E. (ESS-1).

WATER GEOCHEMISTRY AND HYDROGEOLOGY OF THE SHALLOW AQUIFER AT ROOSEVELT HOT SPRINGS, SOUTHERN UTAH-A HOT DRY ROCK PRUSPECT.

LOS ALANOS, 1987. 7OP. (LA-11160-HDR) 
21-1

ALTSEIMER, JOHN H. (A-4); EDESKUTY, FREDERICK J. (A-4).

DIRECT APPLICATION OF GEOTHERMAL ENERGY TO PROVIDE PROCESS HEAT IN GUATEMALA.

IN: GEOTHERMAL ENERGY SYMPOSIUM, 1988, NEW ORLEANS, LA. PROC., P.49-53. ASME, 1988.

(TJ280.7.G446 1988X)

ALSO PUBLSHED: TIC, 1987. 18P. MN (LA-UR-87-2734)

21.2

CHAALES, ROBERT W. (INC-7); JANECKY, DAVID R. (INC-7); GOFF, FRASER E. (ESS-1): MC KIBBEN, M. A. CHEMOGRAPHIC AND THERMODYNAMIC ANALYSIS OF THE PARAGENESIS OF THE MAJOR PHASES IN THE VICINITY OF THE 6120-FOOT (1866 M) FLOW ZONE, CALIFORNIA STATE WELL 2-14.

J. GEOPHYS. AES., V.93B, P.13145-7. 1988.

21-3

FRANKE, PAUL R., JR. (ESS-DOT).

U.S. HOT DRY ROCK GEOTHERMAL ENERGY DEVELOPMENT PROGRAM

IN: GEOTHERMAL ENERGY SYMPOSIUM, 1988, NEW ORLEANS, LA. PROC., P.11-15. ASME, 1988.

(TJ280.7.G446 1988X)

ALSO PUBLISHED: TIC, 1987. 7P. MN (LA-UA-87-3085)

21.4

GOFF, FRASER E. (ESS-1); SHEVENEL, LISA (ESS-1); GARDNER, JAMIE N. (ESS-1); VUATAZ, FRANCOIS-D.; GRIGSBY. CHARLES O. (ESS-1).

HYDROTHERMAL OUTLFOW PLUME OF VALLES CALDERA, NEW MEXICO AND A COMPARISON WITH OTHEA OUTFLOW PLUMES.

J. GEOPHYS. RES., V.938, P.6041-58. 1988.

21.5

MURPHY, HUGH D. (ESS-DOT); FEHLER, MICHAEL C. (ESS-4); ROBINSON, BRUCE A. (ESS-4);

TESTER, JEFFERSON W.; POTTER, ROBERT M. (ESS-4); BIADSELL, STEVE (ESS-4). HOT DRY AOCK FRACTURE PROPAGATION AND RESERVOIR CHARACTERIZATION.

TIC, 1988. 10P. MN (LA-L'R-88-1358)

21-6

ZYVOLOSKI, GEORGE A. (T-3); DASH, ZORA V. (ESS-4); KELKAR, SHARAD (ESS-4).

FEHM-FINITE ELEMENT HEAT AND MASS TRANSFER CODE.

LOS ALAMOS, 1988. 55P. (LA-11224-MS) 


\section{HEALTH AND SAFETY}

22-1

ENVIRONMENTAL SURVEILLANCE GROUP (HSE-8)

ENVIRONMENTAL SURVEILLANCE AT LOS ALANOS DURING 1987.

LOS ALAMOS NATIONAL LABORATORY, 1986. 287P. (LA-11306-ENV)

22-2

GAUTIER, MARGARET A. (HSE-9); GLADNEY, E. S. (HSE-9); MOSS, WILLIAM D. (HSE-9);

PHILLPS, MARYBETH (HSE-9); O'MALLEY, BRYAN T. (HSE-9).

QUAUTY ASSUAANCE FOR HEALTH AND ENVIRONMENTAL CHEMISTRY-1986.

LOS ALAMOS, 1987. 357P. (LA-11114-MS)

$22-3$

LONDON, JERAY E. (LS-1).

PRELIMINARY TOXICOLOGICAL STUDY OF YTTRIUM BARIUM COPPER OXIDE.

LOS ALAMOS NATIONAL LABORATORY, 1988. 13P. (LA-11437-MS)

22-4

LONDON, JERRY E. (LS-1).

TOXICOLOGICAL STUDY OF 1,2,4-TRIAZOLE-5-ONE.

LOS ALAMOS NATIONAL LABORATORY, 1988. 11P. (LA-11461-MS)

$22-5$

ORTIZ, LAWRENCE W. (HSE-5); SODERHOLM, SIDNEY C. (HSE-5); VALDEZ, FRANK O. (HSE-5).

PENETRATION OF RESPIRATOR FILTERS BY AN ASBESTOS AEROSOL.

AM. IND. HYG. ASSOC. J., V.49, P.451-60. 1988.

22-6

ROSENTHAL, MARY A. (HSE-DO).

HEALTH, SAFETY, AND ENVIRONMENT DIVISION ANNUAL REPORT 1987.

LOS ALAMOS NATIONAL LABORATORY, 1988. 109P. (LA-11257-PR)

22-7

SCOGGINS, WAYNE A. (HSE-8); VAN ETTEN, DON M. (HSE-8).

REAL-TIME RADIATION MAPPING EYSTEM.

TIC. 1988. 7P. MN (LA-UR-88-3062)

$22-8$

SKAGGS, BARBARA JAN (HSE-5); LOIBL, JOHN (HSE-5); CARTER, KEITH D. (HSE-5); HYATT, EDWIN C. (HSE-5). EFFECTS OF TEMPERATURE AND HUMIDITY ON RESPIRATOR FIT UNDER SIMULATED W!ORK CONDITIONS.

LOS ALAMOS NATIONAL LABORATORY, 1988. 40P. (LA-11236)

$22-8$

VOELZ, GEORGE L. (HSE-2).

IONIZING RADIATION.

IN: OCCUPATIONAL MEDICINE-PRINCIPLES AND PRACTICAL APPLICATIONS, C. ZENZ, ED., P.426-62.

YEAR BOOK MEDICAL, 1988. (RC/963/.O23/1988)

22-10

WILKINSON, GAEGG S. (HSE-14).

MOATALTY AMONG ROCKY FLATS WORKERS.

IN: INTERNATIONAL CONFERENCE ON HEALTH EFFECTS OF LOW DOSE IONISING RADIATION, 1987.

LONDON. PROC., P.91-4. BAITISH NUCLEAR ENERGY SOCIETY, 1988. (OP82.2.I53 H4

22-11

WOODPOW, LISA M. (HSE-5).

EVALUATION OF FOUA OUANTITATIVE LABORATORY FUME HOOD PERFORMANCE TEST METHODS. LOS ALAMOS, 1987. 219P. (LA-11143-T) 
23-1

DUDZIAK, DONALD J. (A-4); HARRIS, DAVID B. (A-4); PENDERGRASS, JOHN H. (A-4).

GOALS, RECUIREMENTS, AND DESIRABLE CHARACTERISTICS OF THE LMC INERTIAL FUSION DRIVER. IN: SYMPOSIUM ON FUSION ENGINEERING, 12TH, 1987, MONTEREY, CALIF. PROC.. P.997-1000. IEEE, 1987. (TK9204.S93 1987X)

23.2

FOREMAN, LARRY R. (MST-7); HOFFER, JAMES K. (P-10).

FABRICATION OF ICF REACTOR TARGETS BASED ON SYMMETRIZATION OF SOLID FUEL

TIC, 1988. 6P. MN (LA-UR-B8-3273)

23-3

HARRIS, DAYID B. (A-4); DUDZIAK, DONALD J. (A-4); CARTWRIGHT, DAVID C. (DRAIFLT).

INERTIAL FUSION IN THE NINETIES.

TIC, 1987. 21P. MN (LA-UR-88-0568)

23-4

HARRIS, DAVID B. (A-4).

LOS ALAMOS NATIONAL LABORATORY PLANS FOR A LABORATORY MICAOFUSION FACILITY TIC, 1988. 5P. MN (LA-UR-88-2663)

23-5

HAUER, ALLAN (P-4); FORSLUND, DAVID W. (X-DO); MC KINSTRIE, C. J. (CNLS); WARK, J. S.;

HARGIS, PHILIP J., JR.; HAMIL, ROY A.; KINDEL, JOSEPH M. (X-1).

CURRENT NEW APPLICATIONS OF LASER PLASMAS.

LOS ALAMOS NATICNAL LABORATORY, 1988. 41P. (LA-11295-MS)

23-6

HAUER, ALLAN (P-4); BALDIS, HECTER A.

INTRODUCTION TO LASER PLASMA DIAGNOSTICS.

LOS ALAMOS NATIONAL LABORATORY, 1988. 49P. (LA-11178-MS)

23-7

HOFFER, JAMES K. (P-10); FOREMAN, LARRY R. (MST-7)

RADIOACTIVELY INDUCED SUBLIMATION IN SOLID TRITIUM.

PHYS. REV. LETT., V.60, P.1310-3. 1988.

23-8

JENSEN, REED J. (ADR).

TRAVELING WAVE APPROACH TO PLASMA PUMPING FOR X-RAY SOURCES.

TIC, 1988. 13P. MN (LA-UR-88-3347)

23-9

JENSEN, REED J. (P-DO).

LARGE EXCIMER LASERS FOR FUSION.

IN: EXCIMER LASERS AND OPTICS, 1986, CAMBRIDGE, MASS. PROC., P.15-21. SPIE, 1987.

(TA/1695/.E934/1986X)

ALSO PUBLISHED: TIC, 1986. 14P. MN (LA-UR-86-3319)

23-10

KRISTAL, RICHARD (P-4); BLAIR, LARRY S. (CLS-DO); BUAROWS, MICHAEL D. (CLS-9);

CARTWRIGHT, DAVID C. (DRAMIFLT); GOLDSTONE, PHILIP D. (P-4); GREENE. DENNIS P. (CLS-9);

HANLON, JOHN ARTHUR (CLS-8); HAUER, ALLAN (P-4); KANG, MICHAEL (CLS-7); KORTEGAARD, B. L. (CLS-8);

MC LEOD, JOHN (CLS-B); MEAD, W. C. (X-1); ROSE, EVAN A. (CLS-7); ROSOCHA, LOUIS A. (CLS-7). AURORA STATUS AND PLANS.

TIC, 1987. 27P. MN (LA-UR-88-0409)

23-19

MASON, RODNEY J. (X-1); JONES, MICHAEL E. (X-1).

SIMULATION OF PLASMA EROSION OPENING SWITCHES.

TIC, 1988. 7P. MN (IA-UR-86-2029) 
23-12

MEAD, W. C. $(X-1)$; STOVER, ELMER K. (X-1); KAUFFMAN, R. L.; KORNBLUM, H. N.; LASINSKI, B. $F$ MODELING, MEASUREMENTS, AND ANALYSIS OF $X$-RAY EMISSION FROM 0.26-MICRON.

LASER-IRRADIATED GOLD DISKS.

PHYS. REV., V.38A, P.5275-88. 1988.

23-13

ROSOCHA, LOUIS A. (CLS-7); BLAIR, LARRY S. (CLS-DO)

RECENT PROGRESS ON THE LOS ALAMOS AURORA ICF [INERTIAL CONFINEMENT FUSION] LASER SYSTEM.

TIC, 1987. 13P. MN (LA-UR-88-0154)

23-14

STOVALL, JAMES E. (AT-1); GUY, FRANK W. (AT-1); STOKES, RICHARD H. (AT-1);

WANGLER, THOMAS P. (AT-1).

BEAN FUNNELING STUDIES AT LOS ALAMOS

TIC, 1988. 15P. MN (LA-UR-88-3277)

23-15

YOUNG, AINSUE T., JA. (MST-7)

POLYMER-SOLVENT PHASE SEPARATION AS A ROUTE TO LOW DENSITY. MICROCELLULAR PLASTIC FOAMS.

J. CELL. PLAST., V.23, P.55-72. 1987. 


\section{INSTRUMENTS}

24-1

ACKERHALT, JAY R. (T-12); HANSON, DAVID E. (T-12), A.DAMS, RICHARD C.; RAYMOND, T. D.; REISER, CHAISTOPHER; RICE, JAMES K.; MICHIE, ROBERT B.

AMPLIFIED SPONTANEOUS EMISSION AND PULSE TRAIN AMPLIFICATIION IN A KRYPTON FLUORIDE AMPLFIER.

TIC, 1988. 8P. MN (LA-UA-88-3777)

24-2

APEL, CHARLES T. (CLS-1); LAYMAN, LARRY R. (CLS-1); GALLIMOHE, DAVID L (CLS-1). EXTERNALLY PRESSUAIZED POROIJS CYLINDER FOR MULTIPLE SURFACE AEROSOL GENERATION AND METHOD OF GENERATION

PATENTED MAY 10, 1988, FILED 1986. (U.S.PATENT-4,743,407)

24-3

BANAR, JOSEPH C. (INC-7); PERRIN, RICHARD E. (INC-7); OSTRENGA, RAYMOND A. (MEC-1).

ROTARY TURRET AND REUSABLE SPECIMEN HOLDER FOR MASS SPECTROMETER.

PATENTED MAY 17, 1988, FILED 1986. (U.S.PATENT-4,745,277)

24-4

BARRON, MICHAEL H. (MEE-5); GALLAGHER, T.; GOULDING, CHARLES A. (N-2);

HEDGES, ROBERT ORRIN (MEE-5); HENNEKE, K. M. (MEE-5); MOSS, CALVIN E. (N-2); SHROUF. K. C. (MEE-5); SLENTZ, G. E. (MEE-5).

SEGMENTED CALORIMETER FOR LOW-ENERGY PARTICLE BEAMS IN SPACE.

TIC, 1987. 4P. MN (LA-UA-87-3575)

24-5

BENJAMIN, ROBERT F. (M-6)

MICPOBALLOON TIMING SENSOR.

IN: PHOTONICS-HIGH BANDWIDTH ANALOG APPLICATIONS, 1986, HOWEY-IN-THE-HILLS, FLA. PROC., P.228-36. SPIE, 1987. (TA1505 .SS4X V.648)

24-6

BENNETT, GLORIA A. (MEE-11); HEDSTROM, JAMES C. (MEE-13).

CHIP COOUNG CONCEPTS-COMBINED GROUNDPLANE AND COLDPLATE. LOS ALAMOS, 1988. 38P. (LA-11243-MS)

24-7

BENNETT, GLOAIA A. (MEE-11); MOORE, TROY K. (ESS-4).

THERMAL PROTECTION APPARATUS.

PATENTED JAN. 26, 1988, FILED 1986. (U.S.PATENT-4,722,026)

24-8

BOLE, VICTOR $W$.

PAECISION ELECTRONIC SPEED CONTROLLER FOR AN ALTERNATING-CURRENT.

PATENTED MAR. 15, 1988, FILED 1982. (U.S.PATENT-4,731,572)

24-9

BOWMAN, J. DAVID (MP-4).

CONCEPTUAL DESIGN OF A HIGH-RESOLUTION PI ZERO SPECTROMETER.

IN: PION-NUCLEUS PHYSICS-FUTURE DIRECTIONS AND NEW FACIUTIES AT LAMPF, 1987, LOS ALAMOS NATIONAL LABORATORY. PROC., P.119-41. AMERICAN INSTITUTE OF PHYSICS, 1988. (OC/793.5/.M42/P56/1987X)

24-10

BPIXNER, BERLYN (M-DO); KLEIN, MORPIS M. (X-5).

OPTIMIZATION EXPERIMENTS WITH A DOUBLE GAUSS LENS. OPT. ENG., V.27, P.420-3. 1988.

24-11

BRIXNER, BERLYN (M-DO); KLEIN, MORAIS M. (X-5).

OPTIMIZATION TO ELIMINATE TWO LENS ELEMENTS THAT HAVE UNDESIRABLE SHAPES.

OPT. ENG., V.27, P.1027-30. 1988. 
24-12

CHUNG, YUN C. (CLS-5); SHAY, T. M. (CLS-5).

FREQUENCY STABILIZATION OF A DIODE LASER TO A FABRY-PEROT INTERFEROMETER.

OFT. ENG., V.27, P.424-7. 1988.

24-13

DOSS, JAMES D. (MP-3); COOKE, D. W. (MP-14); MC CABE, CHARLES W. (MP-3). MAEZ, M. (MP-14). NONCONTACT METHODS USED FOR CHARACTERIZATION OF HIGH-TC SUPERCONDUCTORS. REV. SCI. INSTRUM., V.59, P.659-61. 1988.

24-14

FARAAR, CHARLES R. (MEE-13); DOVE, RICHARD C. (MEE-13); HEDSTROM, JAMES C. (MEE-13).

INSTRUMENTATION OF THE SANTA CLARA AND NAMBE BRIDGES DURING THE CONFINED PHYSICS RESEARCH FACILITY GENERATOR TRANSPORATION.

LOS ALAMOS NATIONAL LABORATORY, 1988. 33P. (LA-11373-MS)

24-15

FRITZ, JOSEPH N. (M-6); OLINGER, BARTON W. (M-6); VORTHMAN, JOHN E. (M-6); WILDER, L. (M-6)

SMALL ASM PROBES.

LOS ALAMOS NAIIONAL LABORATORY, 1988. 21P. (LA-11384-MS)

24.16

FUKA, MAPY A. (E-B); CLOUT, PETER N. (AT-3); CONLEY, ANDREW P. (AT-3); HILL, J. O. (AT-3);

ROTHROCK, RICHARD (AT-3); TREASE, L. L. (AT-3); ZANDER, M. (AT-3).

ARGONNE BEAMLINE-B TELESCOPE CONTROL SYSTEM-A STUDY OF ADAPTABILITY.

IN: IEEE PARTICLE ACCELERATOR CONFERENCE, 1987, WASHINGTON, D.C. PROC., P.652-4. IEEE, 1987. (CC787.P3 I37 1987)

ALSO PUBLISHED: TIC, 1987. 4P. MN (LA-UA-87-0501)

24-17

GAVRON, AVIGDOR I. (P-2).

FLOW ANALYSIS USING CALORIMETERS.

NUCL INSTRUM. METHODS PHYS. RES., V.A273, P.371-6. 1988

24-18

GEISIK, CARL (AT-2).

BEGINNER'S GL ,DE TO SPICE.

LOS ALAMOS NATIONAL LABORATORY, 1988. 43P. (LA-11321-MS)

24-19

GEORGE, M. J. (M-4); MENIKOFF, RALPH (T-14); VEESER, LYNN R. (P-14).

USE OF OPTICAL FIBERS FOR SHOCK PRESSURE AND TIMING MEASUREMENTS AT HIGH PRESSURES. LOS ALAMOS, 1988. 33P. (LA-11148-MS)

24-20

GOELLER, ROY M. (MEE-11); THOMPSON, M. CLARK (MEE-11); HAMMOND, ROBERT B. (MEE-11):

LEMONS, ROSS A. (MEE-11); NUNNALLY, WILLAM C.

INVESTIGATION OF CRYOGENIC PHOTOCONDUCTIVE POWER SWITCHES.

IN: IEEE INTERNATIONAL PULSED POWER CONFERENCE, 6TH, 1987. ARLINGTON, YA. PROC., P.157-60. IEEE, 1987. (TK2896 .I15 1987X)

24-21

HEMSING, WILLARD F. (M-7).

VISAR-INTERFEROMETER QUADRATURE SIGNAL RECORDING BY ELECTRONIC STREAK CAMERA.

IN: SYMPOSIUM (INTERNATIONAL) ON DETONATION, 8TH, 1985, ALBUQUERQUE. PREPRINTS, P.801-7. NAVAL SURFACE WEAPONS CENTER, 1986. (CD516 .S924 1985XA)

ALSO PUBLISHED: TIC, 1985. 8P. MN (LA-UR-85-2510)

ALSO PUBLISHED: SYMPOSIUM (INTERNATIONAL) ON DETONATION, 8TH, 1985, ALBUOUEROUE. PROC., P.468-72. NAVAL SURFACE WEAPONS CENTER, 1986. (OD516 .S924 1985X)

24-22

HJELM, REX P. (P-LANSCE).

RESOLUTION OF TOF LOW-O DIFFRACTOMETERS-INSTRUMENTAL, DATA ACQUISITION AND REDUCTION FACTORS.

II. APPL. CRYSTALLOGR., V.21, P.618-28. 1988 
24-23

HOCKADAY, MARY P. (P-14); WILKE, MARK D. (P-15); BLAKE, RICHARD L (P-4); VANINETTI, JOE (P-14):

GRAY, NORMAN T. (P-15); NEDROW, PAUL.

TIME-RESOLVED DOUBLY BENT CRYSTAL X-RAY SPEC'TROMETER.

TIC, 1888. 16P. MN (LA-UR-88-0797)

ALSO PUBLISHED: REV. SCI. INSTRUM., V.59, P.1822-4. 1988.

24-24

HOFLER, THOMAS J. (P-10).

ACCURATE ACOUSTIC POWER MEASUREMENTS WITH A HIGH-INTENSITY DRIVER. J. ACOUST. SOC. AM., V.83, P.777-86. 1988.

24-25

HOLT, DAVID M.; WINCHENBACH, G. L.

ELECTRONIC SHADOWGRAPH STATION AND CONTROL SYSTEM.

TIC, 1888. 1OP. MN (LA-UR-88-1303)

24-26

HYER, DIANNE K. (P-DQ).

NEUTRON REFLECTOMETEP FOR LANSCE? REPOAT OF A WORKSHOP HELD AT THE LOS ALAMOS

NATIONAL LABORATORY NOVEMBER 2-3, 1987.

LOS ALAMOS NATIONAL LABORATORY, 1988. 38P. (LA-11301-C)

24-27

IVERSON, ARTHUR E. (MEE-11); WYSIN, GARY M. (MEE-11); SMITH, DOUGLAS L. (MEE-11):

REDONDO, ANTONIO (MEE-11).

OVERSHOOT IN THE RESPONSE OF A PHOTOCONDUCTOR EXCITED BY SUBPICOSECOND PULSES. APPL. PHYS. LETT., V.52, P.2148-50. 1988.

24-28

KAPUSTINSKY, JON S. (P-2); LILLBERG, JOHN W. (P-2); BOISSEVAIN, JAN G. (P-2)

PRECISION HALF-WAVE RECTIFIER.

LOS ALANOS NATIONAL LABORATORY, 1988. 7P. (LA-11407-MS)

24-29

LOONEY, LARRY D. (P-14); LYONS, PETER B. (ADDRA); KELLY, R. E.

STRESS-RELATED PHENOMENA IN TRANSIENT RADIATION-INDUCED ABSORPTION IN OPTICAL FIBERS TIC, 1988. 12P. MN (LA-UR-88-1458)

24-30

LUTZ, S. S.; TURLEY, W. D.; BORELLA, H. M.; NOEL, BRUCE W. (MEE-5); CATES, MICHAEL REID;

PROBERT, M. R.

REMOTE TEMPERATURE-MEASUREMENT INSTRUMENTATION FOR A HEATED ROTATING TURBINE DISK. TIC, 1988. 20P. MN (LA-UR-88-0599)

24-31

MAC ARTHUR, DUNCAN W. (N-2); ADRIAN, STEVEN; MATTHEWS, $S$.

WIRE CHAMBER ASSEMBLY TECHNIQUES.

LOS ALAMOS NATIONAL LABORATORY, 1988. 15P. (LA-11237-MS)

24-32

MARTIN. ALBERT D. (M-8).

HIGH RESOLUTION DIGITAL DELAY TIMER

PATENTED JAN. 12, 1988, FILED 1986. (U.S.PATENT-4,719,375)

24-33

MAULDIN, G. H.; SCHREINER, E. T.; HARRIS, J. O.; THOMPSON, M. CLARK (MEE-11); SOMOANO. R. B.:

YEN, S. P.

DIELECTTRIC MODIFICATION OF REPETITION RATE TESTED POLYCARBONATE PERFLUOROCARBON

CAPACITORS-THE G. 11. MAULDIN MEMORIAL REPORT.

IN: IEEE INTERNATIONAL PULSED POWER CONFERENCE, 6TH, 1997, ARLINGTON, VA. PROC., P.417-21.

IEEE, 1987. (TK2896 .I15 1987X) 
24-34

MC COWN, ANDRES W. (CLS-9); GODARD, JODY A. (CLS-9).

ENHANCEMENTS IN THE OUTPUT ENERGY OF A KRYPTON FLUORIDE OSCILLATOR. J. APPL. PHYS., V.64, P.2879-84. 1988.

24-35

MOAPIS, CHIPISTOPHER L. (MP-10); ATENCIO, L. G. (MP-10); IDZOREK, GEORGE C. (P-14); MORRIS. SHIELA L. TWO-DIMENSIONAL BEAM IMAGE MONITOR. NUCL. INSTRUM. METHODS PHYS. RES., V.A269, P.333-4. 1988.

24-36

OBST, ANDREW W. (P.15); KING, NICHOLAS S. P. (P.15); HANCOCK, A. D.; HILKO, R. A.;

KLINGLEA, QUENTIN G.; BOYER, K. G.; ENGE, H. A.; KOWALSKI, S.; HOLSINGER, AONALD F.; LOWN, $R$. $R$. COMPACT PEAMANENT MAGNET PROTON SPECTROMETER.

NUCL. INSTRUM. METHODS PHYS. RES., V.A263, P.407-13. 1988.

24-37

OGLE, J. W. (P-14); THAYER, D. R. (P-14); LOONEY, LARFY D. (P-14); CVERNA, F. (P-15);

YATES, GEORGE J. (P.15); IVERSON, C. E.; LUTZ, S. S.; NELSON, M. A.; WHITCOMB, BRUCE M.

RADIATION-INDUCED IMAGING SYSTEM OVER LONG FIBER-OPTIC BUNDLES

IN: HIGH BANDWIDTH ANALOG APPLICATIONS OF PHOTONICS, 1986, CAMBRIDGE, MASS. PROC., P.24-30. SPIE, 1987. (TA1505/.H528/1986X)

ALSO PUBLISHED: TIC, 1986. 8P. MN (LA-UR-86-2914)

24-38

OLSHER, RICHARD H. (HSE-1); HAYNIE, JOHN S. (HSE-1).

SR-9ON-90 FIELD CALIBRATOR FOR PEAFORMANCE TESTING OF BETA-GAMMA SURVEY INSTRUMENTS. TIC, 1998. 13P. MN (LA-UR-88-2669)

24-39

PAISLEY, D. L. (MT).

RECENT ADVANCES IN HIGH SPEED PHOTOGRAPHY AND ASSOCIATED TECHNOLOGIES IN THE. USA. TIC. 1988. 10P. MN (LA. UR-88-4131)

24-40

PAULTER, NICHOLAS G. (MEE-11); GIBBS, ALAN J. (MEE-11); SINHA, DIPEN N. (MEE-11).

FABRICATION OF HIGH-SPEED GALLIUM ARSENIDE PHOTOCONDUCTIVE PULSE GENERATORS AND

SAMPUNG GATES BY ION IMPLANTATION.

IEEE TRANS. ELECTRON DEVICES, V.35, P.2343-8. 1988.

24-41

PAULTER, NICHOLAS G. (MEE-11).

HIGH-SPEED OPTOELECTRONIC PULSE GENEAATION AND SAMPLING SYSTEM.

IEEE TRANS. INSTRUM. MEAS., V.37, P.449-53. 1988.

24-42

PETOUHOFF, MICHAEL L. (AT-2).

TAACKING A MONOCHROMATIC LIGHT SOURCE.

LOS ALAMOS NATIONAL LABORATORY, 1988. 9P. (LA-11312-MS)

24-43

PORCH, WILLIAM M. (ESS-7); NEFF, WILLIAM; KING, CLARK.

COMPARISONS OF METEOROLOGICAL STRUCTURE PARAMETERS IN COMPLEX TERFIIN USING OPTICAL AND ACOUSTICAL TECHNIQUES.

APPL. OPT., V.27, P.2222-8. 1988

$24-4$

ROBERTS, JEFFREY P. (CLS-5); TAYLOR, ANTOINETTE J. (CLS-5); LEE, PETER H. Y. (P-1);

GIBSON, ROBERT B. (CLS-5)

HIGH-IRRADIANCE 248-NMI LASER SYSTEM.

OPT. LETT., V.13, P.734-6. 1988.

24-45

ROBINSON, ROBERT A. (LANSCE); PYNN, R. (LANSCE)

ON THE KINEMMTICS OF CRYSTAL ANALYZER SPECTROMETERS AT PULSED NEUTRON SOURCES. NUCL. INSTRUM. METHOOS PHYS. RES., V.A272, P.758-62. 1988. 
24-46

ROBINSON, ROBERT A. (P-8); NUTTER, MURLIN J. (P-8); SILVER, RICHARD N. (T-11);

HOOTEN, DAVID T. (MEC-6); RICKETTS, RALPH L. (WX-4).

NEW CHOPPER SPECTROMETER AT LANSCE, PHAROS.

TIC, 1988. 11P. MN (LA-UR-88-3664)

24-47

SAUNDERS, JIMMY D.; RINGLER, THOMAS J.; BUILTA, LEE A. (M-4); KAUPPILA, T. J. (M-4);

MOIR, DAVID C. (M-4); DOWNEY, STEPHEN W.

SIMPLE LASER-DRIVEN, METAL PHOTOCATHODES AS COLD, HIGH-CURRENT ELECTRON SOURCES. IN: IEEE PARTICLE ACCELERATOR CONFERENCE, 1987, WASHINGTON. PROC. P.337-9. IEEE, ALSO PUBLISHED: TIC, 1987. 4P. MN (LA-UR-87-0750)

24-48

SCHALM, FOBERT W. (OS-8).

USE OF FIBER OPTIC EQUIPMENT FOR SECURITY.

TIC, 1988. 6P. MN (LA-UR-88-0798)

24-49

SHAY, T. M. (CLS-5); CHUNG, YUN C. (CLS-5)

ULTRAHIGH-RESOLUTION, WIDE-FIELD-OF-VIEW OPTICAL FILTER FOA THE DETECTION OF

FR:QUENCY-DOUBLED ND:YAG [NEODYMIUM:YTTRIUM ALUMINUM GARNET] RADIATION.

OPT. LETT., V.13, P.443-5. 1988.

24-50

GMITH, DOUGLAS L. (MEE-11); MAILHIOT, CHRISTIAN.

PIEZOELECTRIC EFFECTS IN STRAINED-LAYER SUPERLATTICES

J. APPL. PHYS., V.63, P.2717-9. 1988.

24-51

SMITH, RUSS C. (P-14).

OPTIMAL DESIGN OF HIGH SPEED ANALOG APD RECEIVERS

TIC, 1988. 9P. MN (LA-UR-88-3731)

24-52

STUPIN, DAVID M. (MST-7).

NEAR-REAL-TIME RADIOGRAPHY DETECTS 0.1\% CHANGES IN AREAL DENSITY WITH 1-MILLIMETER

SPATIAL RESOLUTION.

IN: REVIEW OF PROGRESS IN QUANTITATIVE NONDESTRUCTIVE EVALUATION, 1987, WILLIAMSBURG PROC., P.1723-30. PLENUM, 1988.

ALSO PUBLISHED: TIC, 1987. 14P. MN (LA-UR-87-3026)

24-53

SZE, ROBERT C. (CHM-5).

INDUCTIVELY STABILIZED EXCIMER LASERS.

IN: EXCIMER LASERS AND OPTICS, 1986, CAMBRIDGE, MASS. PROC., P.5-6. SPIE, 1987. (TA1695/.E934/1986X)

24-54

SZE, ROBERT C. (CLS-5); GREENE, DENNIS P. (CLS-9); BIGIO, IRVING J. (CLS-5); SHAY, T. M. (CLS-5);

MC COWN, ANDREW W. (CL.S-5); FIGUEIRA, JOSEPH F. (CLS-5); SMITH, P.; VANNINI, MATTEO.

DISCHARGE EXCITATION OF THE XENON FLUORIDE C TO A TRANSITION.

IN: INTERNATIONAL CONFERENCE ON LASERS, 1987, LAKE TAHOE, PROC., P.268-71. STS PRESS, 1988. ALSO PUBLISHED: TIC, 1987. 5P. MN (LA-UR-88-0656)

24-55

TAYLOR, ANTOINETTE J (CLS-9); GIBSON, ROBERT B. (CLS-9); ROBERTS, JEFFREY P. (CLS-9).

PICOSECOND GAIN STUDIES IN KRYPTON FLUORIDE AMPLIFIERS.

APPL. PHYS. LETT. V.52, P.773-5. 1988.

24-56

THAYER, D. R. (P.14).

INTEGRATED OPTICS INTERFEROMETRIC MODULATOAS

IN: PHOTONICS-HIGH BANDWIDTH ANALOG APPLICATIONS, 1986, HOWEY-IN-THE-HILLS, FLA. PROC. P.254-8. SPIE, 1987. (TA1505 .S64X V.648) 
VEESER, LYNN R. (P-14); CHANDLER, GEOAGE I. (CTR-8); DAY, GORDON W.

FIBER OPTIC SENSING OF PULSED CURAENTS.

IN: PHOTONICS-HIGH BANDWIDTH ANALOG APPLICATIONS, 1986, HOWEY-IN-THE-HILLS, FLA. PROC. P.197-212. SPIE, 1987. (TA1505 .564X V.648)

24-58

WAGNER, RONALD S. (MEE-11); BORDES, NICOLE (MEE-11); BRADELY, JEFFREY M. (MEE-11);

MAGGIORE, CARL J.; KNUDSON, ALVIN; CAMPBELL, ARTHUR.

ALPHA, SILICON, AND IRON ION INDUCED CURRENT TRANSIENTS IN LOW CAPACITANCE SILICON AND GALLIUM ARSENIDE DIODES.

TIC, 1989. 28P. MN (LA-UR-88-2073)

24-59

WILKE, MARK D. (P-15); KING, NICHOLAS S. P. (P-15).

TWO-DIMENSIONAL TIME-DEPENDENT IMAGING UTILIZING TOMOGRAPHIC CONCEPTS

IN: PHOTONICS HIGH BANDWIDTH ANALOG APPLICATIONS, 1986, HOWEY-IN-THE-HILLS, FLA. PROC. P.24-43. SPIE, 1987. (TA1505 .S64X V.648)

$24-60$

WINSLOW, ORVILLE G. (P-9); DAVIS, WILLIAM C. (M-9).

ROTATING MIRROR STREAK CAMERA WITH AN OPTIONAL IMAGE INTENSIFIER CAMERA BACK. IN: HIGH SPEED PHOTOGRAPHY, VIDEOGFAPHY, AND PHOTONICS, 5TH, 1987, SAN DIEGO. PROC.. P.96-100. SPIE, 1988.

24-61

YATES, GEORGE J. (P-15); JARAMILLO, STEVEN A. (P-15); HOLMES, VANNER H., JR.; BLACK, J. P. CHARACTERIZATION OF NEW FPS VIDICONS FOR SCIENTIFIC IMAGING APPLICATIONS. LOS ALAMOS NATIONAL LABORATORY, 1988. 35P. (LA-11035-MS)

24-62

YATES, GEORGE J. (P-15); WILKE, MARK D. (P-15); KING, NICHOLAS S. P. (P-15); LUMPKIN, ALEX H. (P-15). ULTRAVIOLET IMAGING OF HYDAOGEN FLAMES.

IN: ULTRAVIOLET TECHNOLOGY II, 1988, ORLANDO, FLA. PROC., P.271-8. 1988. SPIE, 1988. ALSO PUBLISHED: TIC, 1988. 9P. MN (LA-UR-88-1121) 
25. ISOTOPE SEPARATION

25-1

ROBINSON, C. PAUL (NSP); JENSEN, REED J. (ADR); COTTER, THEODORE P. (S-4); BOYER, KEITH (OIR-O). GREINER, NORMAN R. (CLS-1).

LASER ISOTOPE SEPARATION.

PATENTED MAR. 19, 1988, FILED ;975. (U.S.PATENT-4,734,177) 
26-1

BOYACK. B. E. (N-12); DUFFEY, R. B.; GRIFFITH. P.; KATSMA, K. R.; LELLOUCHE, G. S.; LEVY. S.; ROHATGI, U. S.; WILSON, G. E.; WULFF, W.; ZUBER, N.

QUANTIFYING REACTOR SAFETY MARGINS-PAAT 1. AN OVERVIEW OF THE CODE SCALING. APPLICABILITY, ANO UNCERTAINTY EVALUATION METHODOLOGY.

TIC, 1988. 19P. MN (LA-UR-89-3571)

26-2

BOYACK, B. E. (N-12); SHIRE, PHILUIP R. (N-9); HARMONY, S. C. (N-9); RHEE, G.

TRAC CODE ASSESSMENT USING DATA FROM SCTF CORE-III, A LARGE-SCALE 2D/3D FACILITY TIC, 1988. 12P. MN (LA-UR-88-3158)

26-3

EICH. WALTER J.; EISENHART, LAURANCE D.; LITTLE, ROBERT C. (X-6): MOSTELLER, R. D.; CHAO, JASON. ENDF/B-V CELL COMPARISONS AND MCNP-3A. TIC. 1988. 8P. MN (LA-UR-88-0336)

26-4

PASAMEHMETOGLU, KEMAL O. (N-9); NELSON, R. A. (N-9).

FURTHER CONSIDERATIONS OF CRITICAL HEAT FLUX IN SATURATED POOL BOILING DURING POWER TRANSIENTS.

IN: NATIONAL HEAT TRANSFER CONFERENCE, 1988, HOUSTON, TEX. PROC., P.395-404. AMERICAN SOCIETY OF MECHANICAL ENGINEERS, 1988. (TJ260 .N36 1988)

ALSO PUBLSHED: TIC, 1988. 23P. MN (LA-UR-87-4016)

26-5

PASAMEHMETOGLU, KEMAL O. (N-9); NELSON, R. A. (N-9).

INVESTIGATIONS OF THE OUASI-STEADY APPROACH USED IN TRANSIENT TWOPHASE FLOW ANALYSIS TIC, 1988. 24P. MN (LA-UR-87-4161)

26-6

PASAMEHMETOGLU, KEMAL O. (N-9); NELSON, R. A. (N-9); GUNNERSON, F. S.

THEORETICAL PREDICTION OF CRITICAL HEAT FLUX IN FORCED CONVECTION BOILING DURING POWER TAANSIENTS.

TIC, 1988. 19P. MN (LA-UR-87-4171)

26-7

PASAMEHMETOGLU, KEMAL O. (N-9); NELSON, R. A. (N-9); GUNNERSON, F. S.

THEORETICAL PREDICTION OF CRITICAL HEAT FLUX IN SUBCOOLED POOL BOILING DURING POWER TRANSIENTS.

TIC, 1988. 21P. MN (LA-UR-87-4162) 
27-1

BAUMUNG, K.; LUMPKIN, ALEX H. (P-15).

FUEL MOTION MEASUREMENT WITH THE CABRI HODOSCOPE.

IN: SCIENCE AND TECHNOLOGY OF FAST REACTOR SAFETY, 1986, GUERNSEY. PROC., V.1., P.141-6 BRITISH NUCLEAR ENERGY SOCIETY, 1987. (TK9203.B7 S38 1886X)

27-2

BOHL, WILLIAM R. (N-6); WILHELM, DIRK (N-8); BERTHIER, JEAN; PARKER, F. (N-6); ICHIKAWA, SCHINICHI; GOUTAGNY, LUC; NINOKATA, H. (N-6); MAUDLIN, PAUL J. (N-6).

MULTIPHASE FLOW IN THE ADVANCED FLUID DYNAMICS MODEL.

TIC, 1988. 28P. MN (LA-UR-87-4231)

27-3

LA BAUVE, RAPHAEL J. (T-2); ENGLAND, TALMADGE R. (T-2); GEORGE, DENISE C. (T-2);

MAC FARLANE, ROBERT E. (T-2): WILSON, WIUAM B. (T-2).

DANDE-A LINKEO GENERAL CODE SYSTEM FOR CORE NEUTRONICSIDEPLETION ANALYSIS.

TIC, 1986. 13P. MN (LA-UR-66-1675)

ALSO PUBLSHED: NUCL. SCI. ENG., V.95, P.152-63. 1987.

27-4

LOUIE, DAVID L. Y. (MST-11).

CHARACTERIZATION OF FUEL SWELLING IN HELIUM-BONDED CARBIDE FUEL PINS. LOS ALAMOS, 1987. 292P. (LA-11051-T)

27-5

LUMPKJN, ALEX H. (P-DO).

CONMENTS ON THE POSSIBLE ROLES OF VOLATILE FISSION PAODUCTS (CESIUM) IN CABRI TESTS. IN: SCIENCE AND TECHNOLOGY OF FAST REACTOP SAFETY, 1986, GUERNSEY. PROC., V.2., P.419-25. BRITISH NUCLEAR ENERGY SOCIETY, 1987. (TK9203.B7 S38 1986X)

27-6

ROCHE, L.; SCHMITZ, F.; FREUND. D.; PFRANG, W.; CAMERON, R. F.; SATO, I.; BAARS, RALPH E. (N-12).

DESCRIPTION OF THE STATE OF CABRI FUEL PINS AFTER PREIRRADIATION AND JUST BEFORE THE TRANSIENT (TISUB O]) STATE.

IN: SCIENCE AND TECHNOLOGY OF FAST REACTOR SAFETY, 1986, GUERNSEY. PROC., V.1, P.121-7. BRITISH NUCLEAR ENERGY SOCIETY, 1987. (TK9203.87 S38 1986X) 
29-1

ALMAGUER, JUAN ANTONIO; HAMEIRI, ELIEZER; HERRERA, JULIO; HOLM, DARRYL D. (T-7). LYAPUNOV STABILITY ANALYSIS OF MAGNETOHYDRODYNAMIC PLASMA EOUILIBAIA WITH AXISYMMETRIC TOROIDAL FLOW.

PHYS. FLUIDS, V.31, P.1930-9. 1988.

29-2

ANDERSON, JAMES L. (MST-3); BARTLIT, JOHN R. (MST-3); CARLSON, RICHARD V. (MST-3);

COFFIN, DON OWEN (MST-3); DAMIANO, FORTUNATO A. (MST-3); SHERMAN, ROBERT H. (MST-3);

WILMS, RICHARD S. (MST-3); YOSHIDA, H.; YAMANISHI, TOSHIHIKO; NAITO. TAISEI; HIRATA, SHINGO; NARUSE, $Y$.

EXPERIENCE OF TSTA [TRITIUM SYSTEMS TEST ASSEMBLY] MILESTONE RUNS WITH 100 GRAMS-LEVEL OF TAITIUM.

TIC, 1988. 12P. MN (LA-UR-B8-0873)

29-3

ANDERSON, JAMES L. (MST-3); BARTLIT, JOHN R. (MST-3).

RECENT RESULTS AT THE TRITIUM SYSTEMS TEST ASSEMBLY

TIC, 1988. 9P. MN (LA-UR-88-2915)

29-4

ANDERSON, JAMES L. (MST-3)

TAITIUM SYSTEMS TEST ASSEMBLY APPLICABILITY TO ITER.

TIC, 1988. 6P. MN (LA-UR-88-2388)

29-5

BARTUT, JOHN R. (MST-3); ANDERSON. JAMES L. (MST-3).

TRITIUM SYSTEMS TEST ASSEMBLY-OVERVIEW AND RECENT RESULTS

TIC, 1988. 9P. MN (LA-UR-88-2796)

29-6

BATHKE, CHARLES G. (CTR-12); KRAKOWSKI, ROBERT A. (CTR-12); MANZANARES, ROBERT G. (CTR-4);

MILER, RONALD L. (CTR-12); WERLEY, KENNETH A. (CTR-12).

CONCEPTUAL DESIGN OF A REVERSED-FIELD PINCH FUSION NEUTRON SOURCE.

TIC, 1988. 2OP. MN (LA-UR-B8-0814)

29-7

BATHKE, CHARLES G. (CTR-12); KRAKOWSKI, ROBERT A. (CTR-12); SCHOENBERG, KURT F. (CTR-2).

TECHNOLOGY AND PHYSICS IMPLICATIONS OF OSCILLATING-FIELD CURRENT DRIVE IN

REVERSED-FIELD PINCHES.

TIC, 1988. 7P. MN (LA-UR-88-2888)

29-8

CARAMANA, EDWARD J. (CTR-6); NEBEL, R. A. (CTR-6).

REVERSED-FIELD PINCH OHMIC EOUILIBRIA DURING CURRENT DECAY AND TERMINATION.

PHYS. FLUIDS, V.31, P.3322-9. 1989.

29-9

CASEY, MARJORIE A. (MEE-9); GRUETZMACHER, KATHLEEN M. (MST-3); BARTLIT, JOHN R. (MST-3);

CADWALLADER, LEE C.

DATA COLLECTION SYSTEM FOR FAILUREMAINTENANCE AT THE TRITIUM SYSTEMS TEST ASSEMBLY.

TIC. 1988. 9P. MN (LA-UR-88-0897)

29-10

CHFIEN, ROBERT E. (CTR-3); CRAWFORD, EDWARD A.; HUGRASS, WAHEED N.; OKADA, SHIGEFUMI (CTR-3);

REJ, DONALD J. (CTR-3); SIEMON, RICHARD E. (CTR-3); TAGGART, DANIEL P. (CTR-3);

TUSZEWSKI, IICHEL G. (CTR-3); WEBSTER, R. B. (CTR-6); WRIGHT, BRADFORD L. (CTR-3)

FORMATION AND CONFINEMENT OF FRCS IN FRX-CILSM.

TIC. 1988. 5P. MN (LA-UR-88-3737) 
29-11

CLINARD, FRANK W., JR. (MST-5).

EFFECT OF IRRADIATION-INDUCED DEFECTS ON FUSION REACTOR CERAMICS

TIC, 1886. 28P. MN (LA-UA-86-3123)

29-12

COFFIN, DON OWEN (MST-3).

SAFETY RECORD AT THE TRITIUM SYSTEMS TEST ASSEMBLY.

TIC. 1888. 18P. MN (LA-UR-88-0444)

29-13

COFFIN, DON OWEN (MST-3); COLE, STEVEN P. (MST-3); WILHELM, RICHARD C. (MST-9).

TAITIUM-COMPATIBLE PIEZOELECTAIC VALVE FOR THE TOKAMAK FUSION TEST REACTOR.

TIC, 1988. 12P. MN (LA-UR-88-0443)

29-14

COGGESHALL, S. V. (X-1); MEAD, W. C. $(X-1)$; JONES, ROGER D. $(X-1)$.

FLICKER IN LASER-PLASMA SELF-FOCUSING.

TIC, 1988. 5P. MN (LA-UR-88-3318)

29-15

DAVIDSON, J. WILEY (A-4); DUDZIAK, DONALD J. (A-4); PELLONI, S.; STEPANEK, JIRI.

TWO-DIMENSIONAL CROSS-SECTION SENSITIVITY AND UNCERTAINTY ANALYSIS OF THE LBM [LITHIUM BLANKET MODULE] EXPERIMENTS AT LOTUS.

TIC, 1988. 9P. MN (LA-UR-88-32i2)

29-16

DI MARCO, JOSEPH N. (CTR-DO).

UPDATE OF RFP SCALING DATA

TIC. 1988. 11P. MN (LA-UA-88-3375)

29-17

DREICER, HARAY (CTR-DO)

RATIONALE FOR THE DESIGN OF CPRF/ZTH, THE NEXT STEP IN THE US RFP [REVERSED FIELD PINCH] PROGRAM.

IN: COURSE AND WORKSHOP ON PHYSICS OF MIRRORS, REVERSED FIELD PINCHES AND COMPACT TORI, 1987, VARENNA, ITALY. PROC., P.359-95. SOCIETA ITALIANA DI FISICA, 1988. (QC718.5.P45 P48 1987X)

ALSO PUBUSHED: TIC, 1987. 6P. MN (LA-UR-87-3210)

$29-18$

FORMAN, PETER R. (CTR-8); JAHODA, FRANZ C. (CTR-8); MILLER, GUTHRIE (CTR-2).

DETECTION OF PLASMA EQUIUBRIUM SHIFTS WITH FIBER OPTIC SENSING OF IMAGE CURRENTS.

TIC, 1988. 16P. MN (LA-UR-88-0811)

29-19

GIRRENS, STEVE P. (MEE-13); BENNETT, JOEL G. (MEE-13); MURPHY, DENNIS M.

POLOIDAL FIELD COIL STRESS ANALYSIS FOR THE ZTH MACHINE.

LOS ALAMOS, 1988. 52P. (LA-11203-MS)

29-20

GLASSER, ALAN H. (CTR-6); HAMMEL, JAY E. (CTR-8); LEWIS, H. RALPH, JR. (CTR-6); LINDEMUTH, I. R. (X-1): MC CALL, GENE H. (X-DO); NEBEL, R. A. (CTR-6); SCUDDER, DAVID W. (CTR-8);

SHLACHTER, JACK S. (CTR-8); LOVBERG, RALPH H.; ROSENAU, PHILIP; SHEEHEY, P.

PHYSICS OF THE HIGH DENSITY Z-PINCH

TIC. 1988. 11P. MN (LA-UR-88-2840)

29-21

GRUETZMACHER, KATHLEEN M. (MST-3); CARLSON, RICHARD V. (MST-3); STENCEL. J. R.; SISSINGH, R. A. TRANSPORTATION OF TRITIATED WASTE FROM FUSION FACILITIES

TIC, 1988. 18P. MN (LA-UR-88-0870) 
HSIAO, MING Y. (CTR-6); WERLEY, KENNETH A. (CTR-12): LING, KUOK M. (CTR-6).

ONE-AND-A-QUARTER-DIMENSIONAL TRANSPORT CODE FOR FIELD-REVERSED CONFIGURATION STUDIES -A USER'S GUIDE FOR CFRX.

LOS ALAMOS NATIONAL LABORATORY, 1988. 47P. (LA-11212-MS)

29-23

JARBOE, THOMAS R. (CTR-5); BARNES, CHRISTOPHER W. (CTR-5); FERNANDEZ, JUAN C. (CTR-5); HENINS, IVARS (CTR-5); WYSOCKI, F. J. (CTR-5); MARKLIN, GEORGE J. (CTR-5).

MODIFIED MAGNETIC CONFINEMENT CONFIGURATIONS BASED ON UNDERSTANDING HELICITY DISSIPATION AND PRESSURE DRIVEN MODES IN THE SPHEROMAK.

TIC. 1988. 2P. MN (LA-UR-B8-1104)

\section{9-24}

KHERANI, NAZIR P.; SHMAYDA, WALTER T.; JALBERT, ROLAND A. (HSE-1).

TRITIUM REMOVAL FROM INERT GASES USING ZR2FE.

IN: SYMPOSIUM ON FUSION ENGINEERING, 12TH, 1987, MONTEREY, CALIF. PROC., P.1239-42. IEEE. 1987. (TK9204 .S93 1987X)

29-25

KONKEL, H. E. (CTR-4).

LIGHTNING PROTECTION SCHEME FOR THE CPAF/ZTH SYSTEM COMPLEX. IN: SYMPOSIUM ON FUSION ENGINEERING, 12TH, 1987, MONTEREY, CALIF. PROC., P.816-18. IEEE. 1987. (TK9204.S93 1987X)

\section{9-26}

KRAKOWSKI, AOBERT A. (CTR-12).

REVERSED-FIELD PINCH AS A POLOIDAL-FIELD-DOMINATED, COMPACT, HIGH-POWER-DENSITY FUSION SYSTEM.

TIC, 1988. 8P. MN (LA-UR-88-2923)

29-27

LEWIS, H. RALPH, JR. (CTR-6); BARNES, DANIEL C.; BISHOP, R. C.; KRALL, NICHOLAS A.; MIKIC, Z;

MILPOY, RICHARD D.; MIRIN, ART A.; SGRO, ANTHONY G. (CTR-6); SHUMAKER, DANA E.;

STAUDENMEIER, J. L. (CTR-6); WEBSTER, R. B. (CTR-3).

TILT MODE, TURBULENCE AND TRANSPORT IN FIELD-REVERSED CONFIGURATIONS.

TIC, 1988. 10P. MN (LA-UR-88-2708)

29-28

MC CALL, GENE H. (X-DO).

HIGH DENSITY Z-PINCH.

TIC, 1988. 15P. MN (LA-UR-88-2912)

29-29

MILLER, GUTHRIE (CTR-2); INGRAHAM, JOHN C. (CTR-2).

EQUIUBRIUM AND FLUCTUATION ANALYSIS FOR ZTH ELECTRICAL DIAGNOSTICS.

LOS ALAMOS NATIONAL LABORATORY, 1988. 26P. (LA-11434-MS)

29-30

MILLE, GUTHAIE (CTR-2); FABER, VANCE (C-3); WHITE, ANDREW B., JR. (C-3).

FINDING PLASMA EQUILIBRIA WITH MAGNETIC ISLANDS.

J. COMPUT. PHYS., V.79, P.417-35. 1988.

29-31

NASISE, JOSEPH E. (MST-3).

SONE NEW TECHNIQUES IN TRITIUM GAS HANDLING AS APPLIED TO METAL HYDRIDE SYNTHESIS. TIC, 1988. 16P. MN (LA-UR-88-0871)

29-32

PARSONS, W. M. (CTR-9).

DESIGN AND TESTING OF A PROTOTYPE WATER-COOLED VACUUM INTERRUPTER FOR USE IN SUPERCONDUCTING MAGNET PROTECTION CIRCUITS.

TIC, 1988. 5P. MN (LA.UR-88-0865) 
29-39

SCHOENBERG, KURT F. (CTR-2); INGRAHAM, JOHN C. (CTR-2); MOSES, RONALD W., JR, (CTR-6);

WEBER, PAUL G. (CTR-2); BURKHARDT, LOUIS C. (CTR-2); CAYTON, THOMAS E. (CTR-6);

DI MARCO, JOSEPH N. (CTR-DO); DOWNING, JAMES N., JR. (CTR-2); ELUS, RICHARD F.;

GERWIN, RICHARD A. (CTR-6); MILLER, GUTHRIE (CTR-2); MUNSON, CARTER P. (CTR-2);

NEBEL, R. A. (CTR-6); SCHOFIELD, ALDRED E. (CTR-2); VEERASINGAM, R.; WERLEY, KENNETH A. (CTR-12); WURDEN, GLEN A. (CTR-12).

CONFINEMENT DYNAMICS AND BOUNDARY CONDITION STUDIES IN THE REVERSED FIELD PINCH.

TIC. 1988. 14P. MN (LA-UR-88-3323)

29-34

SCHOENBERG, KURT F. (CTR-2).

CONFINEMENT DYNAMICS IN THE REVERSED FIELD PINCH.

TIC, 1988. 2P. MN (LA-UR-88-1103)

29-35

SHERMAN, ROBERT H. (MST-3); BARTLIT, JOHN R. (MST-3); YOSHIDA, H.; YAMANISHI, TOSHIHIKO; NAITO, TAISEI; HIRATA, SHINGO; NARUSE, Y.

OPERATION OF THE TSTA [TRITIUM SYSTEMS TEST ASSEMBLY] WITH 100 GRAM TRITIUM.

TIC, 1988. 12P. MN (LA-UR-88-1230)

$29-36$

SIEMON, RICHARD E. (CTR-3); CHRIEN, ROBERT E. (CTR-3); HUGRASS, WAHEED N.;

OKADA, SHIGEFUMI (CTR-3); REJ, DONALD J. (CTR-3); TAGGART, DANIEL P. (CTR-3);

TUSZEWSKI, MICHEL G. (CTR-3); WEBSTER, R. B. (CTR-6); WRIGHT, BRADFORD L. (CTR-3)

FIELD-REVERSED CONFIGURATION (FRC) EXPERIMENTS.

TIC, 1988. 15P. MN (LA-UR-88-2878)

29-37

TALLEY, THURMAN L. (T-2); HALE, GERALD M. (T-2).

FUSION PRODUCT SPECTRA.

TIC, 1988. 5P. MN (LA-UR-88-1643)

29-38

TUSZEWSKI, MICHEL G. (CTR-3).

SEMIEMPIRICAL FORMATION MODEL FOR FIELD-REVERSED CONFIGURATIONS.

PHYS. FLUIDS, V.31, P.3754-9. 1988.

29-39

TUSZEWSKI, MICHEL G (:TR-3).

STATUS OF THE FIELD-REVERSED CONFIGURATION AS AN ALTERNATE CONFINEMENT CONCEPT. TIC, 1988. 18P. MN (LA-UR-88-2821)

29-40

WEBER, PAUL G. (CTR-2).

LOS ALAMOS RFP [REVERSED FIELD PINCH] PROGRAM PLANS AND PROJECTED NEEDS.

TIC, 1988. 9P. MN (LA-UR-88-2531)

29-41

WEBSTER, R. B. (CTR-6); LEWIS, H. RALPH, JR. (CTR-6); STAUDENMEIER, J. L. (CTR-6); MILROY, RICHARD D.; BARNES, DANIEL C.; BISHOP, R. C.; SPENCER, ROSS L. (CTR-6); XU, YING-TING (INC-11); MATHESON, P. L RECENT THEORETICAL STUDIES OF INTERNAL STRUCTIJRE AND STABILITY OF FIELD- REVERSED CONFIGURATIONS.

TIC, 1988. 5P. MN (LA-UR-88-3761)

29-42

WERLEY, KENNETH A. (CTR-12); BATHKE, CHARLES G. (CTR-12); KRAKOWSKI, ROBERT A. (CTR-12).

EDGE-PLASMAS AND WALL PROTECTION IN RFPS [REVERSED-FIELD PINCH].

TIC, 1988. 6P. MN (LA-UR-88-2890)

29-43

WERLEY, KENNETH A. (CTR-12); BATHKE, CHARLES G. (CTR-12).

HIGH-RECYCLE DIVERTOR FOR ITER [INTERNATIONAL THERMONUCLEAR EXPERIMENTAL REACTCR].

TIC, 1988. 7P. MN (LA-UR-88-2889) 
20-44

WURDEN, GLEN A. (CTR-2); WEBER, PAUL G. (CTR-2); SCHOENBERG, KUAT F. (CTR-2);

SCHOFIELD, ALDRED E. (CTR-2); PHILLIPS, JAMES A. (CTR-DO); MUNSON, CARTER P. (CTR-2);

MILLER, GUTHRIE (CTR-2); INGRAHAM, JOHN C. (CTR-2); HOWELL, ROBFRT B. (CTR-2):

DOWNING, JAMES N., JR. (CTR-2); CHRIEN, ROBERT E. (CTR-3); CAYTON, THOMAS E. (CTR-6);

BURKHARDT, LOUIS C. (CTR-2); BASTASZ, ROBERT J.; WALKER, S. E.; PRESZLER, ALAN M.; CAROLAN, P. G.; BUNTING, C. A.

ION HEATING STUDIES IN THE ZT-40M REVERSED FIELD PINCH.

TTC. 1988. 5P. MN (LA-UR-88-0895)

29-45

WURDEN, GLEN A. (CTR-2); WEBER, PAUL G. (CTR-2); MUNSON, CARTER P. (CTR-2);

CAYTON, THOMAS E. (CTR-6); BUNTING, C. A.; CAROLAN, P. G.

PELLET INJECTION IN THE RFP [REVERSED FIELD PINCH].

TIC, 1988. 7P. MN (LA-UR-88-3831)

29-46

WYSOCKI, F. J. ¡CTR-5); FERNANDEZ, JUAN C. (CTR-5); HENINS, IVARS (CTR-5):

JARBOE, THOMAS R. (CTR-5); MARKUN, GEOPGE J. (CTR-6).

EVIDENCE FOA A PRESSURE-DRIVEN INSTABIUTY IN THE CTX SPHEROMAK.

PHYS. REV. LETT., V.61, P.2457-60. 1988. 
30-1

ANDERSON, JAMES L. (MST-3); BARTLIT, JOHN R. (MST-3).

FUSION TRITIUM PROGRAM IN THE UNITED STATES.

TIC, 1988. 9P. MN (LA-UR-88-0876)

30-2

BARTLT, JOHN R. (MST-3); GRUETZMACHER, KATHLEEN M. (MST-3); FLEMING, ROBERT B.

OVERVIEW OF TRITIUM SYSTEMS FOR THE COMPACT IGNITION TOKAMAK.

IN: SYMPOSIUM ON FUSION ENGINEERING, 12TH, 1987,MONTEREY, CALIF. PROC., P.1230-4. IEEE, 1987. (TK9204 .S93 1987X)

ALSO PUBLISHED: TIC, 1987. 13P. MN (LA-UR-87-3346)

30-3

BATHKE, CHARLES G. (CTR-12); KRAKOWSKI, ROBERT A. (CTR-12); MILLER, RONALD L. (CTR-12);

WERLEY, KENNETH A. (CTR-12).

DT FUS!ON NEUTRON SOURCE BASED ON THE REVERSED-FIELD PINCH.

IN: SYMPOSIUM ON FUSION ENGINEERING, 12TH, 1987, MONTEREY, CALIF. PROC., P.829-34. IEEE, 1987. (TK9204.S93 1987X)

ALSC PUBLISHED: TIC, 1987. 5P. MN (LA-UR-87-3468)

30-4

BATHKE, CHARLES G. (CTR-12).

TITAN MAGNET CONFIGURATION.

IN: TITAN REVERSED-FIELD PINCH FUSION REACTOR STUDY-PRESENTED AT SYMPOSIUM ON FUSION ENGINEERING, 12TH, 1987, MONTEREY, CALIF. PROC., UNIVERSITY OF CALIFORNIA, LOS ANGELES, 1987. (UCLA-PPG-1110)

ALSO PUBUSHED: TIC, 1987. 11P. MN (LA-UR-87-3462)

30-5

BATHKE, CHARLES G. (CTR-12).

TITAN OSCILLATING-FIELD CURRENT-DRIVE S ISTEM.

IN: TITAN REVERSED-FIELD PINCH FUSION REACTOR STUDY-PRESENTED AT SYMPOSIUM ON FUSION ENGINEERING, 12TH, 1987, MONTEREY, CALIF. PROC., UNIVERSITY OF CALIFORNIA, LOS ANGELES, 1987. (UCLA-PPG-1110)

ALSO PUBLISHED: TIC, 1987. 5P. MN (LA-UR-87-3466)

ALSO PUBLSHED: SYMPOSIUM ON FUSION ENGINEERING, 12TH, 1987, MONTEREY, CALIF. PROC., P.900-3. IEEE, 1987. (TK9204.S93 1987X)

$30-6$

BINNING, KIRK E. (MST-3); JENKINS, EVERETT M. (MST-3).

TIPS FOR THE FABRICATION OF TEMPORARY TRITIUM EXPERIMENTS.

TIC, 1988. BP. MN (LA-UR-88-0845)

30-7

CARLSON, RICHARD V. (MST-3); BINNING, KIRK E. (MST-3); COLE, STEVEN P. (MST-3);

JENKINS, EVERETT M. (MST-3); WILHELM, RICHARD C. (MST-3).

OPERATING EXPERIENCE AND PROCEDURES AT THE TRITIUM SYSTEMS TEST ASSEMBLY.

TIC. 1988. OP. MN (LA-UR-88-0877)

30-8

COFFIN, DON OWEN (MST-3)

SAFE TRITIUM HANDLING AT THE TRITIUM SYSTEMS TEST ASSEMBLY.

ABSTRACT IN: J. VAC. SCI. TECHNOL., V.A6, P.1264-5. 1988.

30.9

CAEICER, HARRY (CTR-DO).

SHORT INTRODUCTION TO THE STATUS AND MOTIVATION FOR REVERSED FIELD PINCH AND COMPACT TOROID RESEARCH.

IN: COURSE AND WOAKSHOP ON PHYSICS OF MIRRORS, REVERSED FIELD PINCHES AND COMPACT TORI, 1987, VARENNA, ITALY. PROC., P.31-49. SOCIETA ITALIANA DI FISICA, 1988. (OC718.5.P45 P48 1987X)

ALSO PUBLISHED: TIC, 1997. 22P. MN (LA-UR-87-3209) 
30-10

FERNANDEZ, JUAN C. (CTR-5); BARNES, CHRISTOPHER W. (CTR-5); JARBOE, THOMAS R. (CTR-5); HENINS, IVARS (CTR-5); HOIDA, HIROSHI W. (CTR-5); KLINGNER, PHILLIP L. (CTR-5);

KNOX, STEPHEN O. (CTR-5); MARKLIN. GEORGE J. (CTR-5); WRIGHT, BRADFORD L. (CTR-3).

ENERGY CONFINEMENT STUDIES IN SPHEROMAKS WITH MESH FLUX CONSERVERS.

NUCL. FUSION, V.28, P.1555-94. 1988.

$30-11$

FORMAN, PETER R. (CTR-8); JAHODA, FRANZ C. (CTR-8).

LINEAR BIREFRINGENCE EFFECTS ON FIBER-OPTIC CURRENT SENSORS. APPL. OPT., V.27, P.3088-96. 1988.

30-12

GOHAR, Y.; BAKER, C.: BROOKS, J.; FINN, P.; HASSANEIN, A.; WILLMS, RICHARD S. (MST-3): BARR, W.; BUSHIGIN, A.; KALYANAM, K. M.; HAINES, J.

NUCLEAR MODULES OF ITER TOKAMAK SYSTEMS CODE.

IN: SYMPOSIUM ON FUSION ENGINEERING, 12TH, 1987, MONTEREY, CALIF. PROC., P.1271-8. IEEE, 1987. (TK9204.S93 1987X)

30-13

KRAKOWSKI, ROBERT A. (CTR-12); DELENE, JERRY G.

CONNECTIONS BETWEEN PHYSICS AND ECONOMICS FOR TOKAMAK FUSION POWER PLANTS. J. FUSION ENERGY, V.7, P.49-89. 1988.

30-14

KRAKOWSKI, ROBERT A. (CTR-12); DELENE, JERRY G.

CONNECTIONS BETWEEN PHYSICS AND ECONOMICS FOR TOKAMAK FUSION POWER PLANTS. IN: SYMPOSIUM ON FUSION ENGINEERING, 12TH, 1987. MONTEREY, CALIF. PROC., P.938-42. IEEE. 1987. (TK9204.S93 1987X)

ALSO PUBLISHED: TIC, 1987. 6P. MN (LA-UR-87-3467)

$30-15$

MARTIN, R.; SZE, D. K.; BARTLIT, JOHN R. (MST-3); GIERSZEWSKI, P. J.

TRITIUM SYSTEMS FOA THE TITAN REVERSED-FIELD PINCH FUSION REACTOR DESIGN.

IN: TITAN REVERSED-FIEI_D PINCH FUSION REACTOR STUDY-PRESENTED AT SYMPOSIUM ON FUSION ENGINEERING, 12TH, 1987, MONTEREY, CALIF. PROC., UNIVERSITY OF CALIFORNIA, LOS ANGELES, 1987. (UCLA-PPG-1110)

$30-16$

MILLER, RONALD L. (CTR-12).

TITAN REVERSED-FIELD PINCH REACTOR-DESIGN-POINT DETERMINATION AND PARAMETRIC STUDIES. IN: SYMPOSIUM ON FUSION ENGINEERING, 12TH, 1987. MONTEREY, CALIF. PROC., P.951-4. IELE, 1987. (TK9204.S93 1987X)

ALSO PUBLISHED: TIC, 1987. 10P. MN (LA-UR-87-3465)

30-17

MUIR, DOUGLAS W. (T-2); DAVIDSON, J. WILEY (A-4): DUDZIAK, DONALD J. (A-4); DAVIERWALLA, D. M.; HIGGS, COLLIN E.; STEPANEK, JIRI.

BENCHMARK-PROBLEM SPECIFICATION AND CALCULATION USING SENSIBL, A ONE-AND TWO-

DIMENSIONAL SENSITIVITY AND UNCERTAINTY ANALYSIS CODE OF THE AARE SYSTEM.

TIC, 1988. 15P. MN (LA-UR-88-0672)

$30-18$

SHERMAN, ROBERT H. (MST-3).

DESIGN OF AN ISOTOPE SEPARATION SYSTEM FOR JET.

IN: SYMPOSIUM ON FUSION ENGINEERING, 12TH, 1987, MONTEREY, CALIF PROC., P.1243-6. IEEE, 1987. (TK9204.S93 1987X)

ALSO PUBLISHED: TIC, 1987. 5P. MN (LA-UA-87-3591)

30-19

WERLEY, KENNETH A. (CTR-12); BATHKE, CHARLES G. (CTR-12); KRAKOWSKI, ROBERT A. (CTR-12).

EDGE PLASMAS AND PLASMAMIALL INTERACTIONS IN AN IGNITION-CLASS REVERSED FIELD PINCH. IN: SYMPOSIUM ON FUSION ENIGNEERING, 12TH, 1987, MONTEREY, CAILF. PROC., P, $414-8$. IEEE, 1987. (TK9204 .S93 1987X)

ALSO PUBLISHED: TIC, 1987. 6P. MN (LA-UR-87-3464) 
$30-20$

WRIGHT, BRADFCRD L. (CTR-5); BARNES, CHRISTOPHER W. (CTR-5); FERNANDEZ, JUAN C. (CTR-5); HENINS, IVARS (CTR-5); HOIDA, HIPOSHI W. (CTR-5); JAREOE, THOMAS R. (CTR-5):

KNOX, STEPHEN O. (CTR-5); MARKLIN, GEORGE J. (CTR-6); MAYO, ROBERT M.; PLATTS. D. A. (CTR-5) HELICITY CONSERVATION AND ENERGY CONFINEMENT IN CTX SPHEROMAKS.

TIC, 1986. 14P. MN (LA-UR-86-3384)

$30-21$

YAMANISHI, TOSHIHIKO; YOSHIDA, H.; HIRATA, SHINGO; NAITO, TAISEI; NARUSE, Y.;

SHERMAN, ROEERT H. (MST-3); BARTLIT, JOHN R. (MST-3); ANDERSON, JAMES L. (MST-3).

SINGLE COLUMN AND TWO-COLUMN H-D-T DISTILLATION EXPERIMENTS AT TSTA.

TIC. 1088. 22P. MN (LA-UR-88-0872)

30-22

YAVORNIK, EDWARD J. (MEE-12); MOSES, RONALD W., JR. (CTR-6); DRANSFIELD, G. D. (MEE-12);

CUSTER, DANIEL. M. (MEC-7).

CP.JFRTH SHELL POLOIDAL GAP DESIGN AND FABAICATION.

IN: SYMPOSIUM ON FUSION ENGINEERING, 12TH, 1987, MONTEREY, CALIF. PROC., P.881-2. IEEE. 1887. (TK9204.S93 1987X) 


\section{MATERIALS}

31-1

ABE, KATSUNORI; LOGAN, CLINTON M.; SANEYOSHI, KEIJi; CLIAARD, FRANK W., JR. (MST-4) IRRADIATION EFFECTS IN KAPTON POLYIMIDE FILM FROM 14-MEV NEUTRONS AND COBALT-60 GAMMA RAYS.

IN: INFLUENCE OF FADIATION ON MATERIAL PROPERTIES, 13TH, 1986, SEATTLE. PROC., P.669-81. ASTM, 1987. (TA/418.6I,I54/1986XA)

$31-2$

ANDERSON, M. P.; GREST, G. S.; SROLOVITZ, DAVID J. (T-11).

MCPOSTRUCTURAL DYNAMIC STUDY OF GRAIN GROWTH.

IN: COMPUTER-BASED MICROSCOPIC DESCRIPTION OF THE STRUCTURE AND PROPERTIES OF MATERIALS, 1985, BOSTON, MASS. PROC., P.225-31. MATERIALS RESEARCH SOCIETY, 1986. (TA418.5 .C66 1985X)

ALSO PUBLISHED: TIC, 1986. 8P. MN (LA-UR-86-0916)

31-3

ANLAGE, S. M.; NASH, P.; RAMACHANDRAN, RAM; SCHWARZ, RICARDO B. (CMS).

PHASE EOUILIERIA FOR THE ALUMINUM-RICH REGION OF THE ALUMINUM-RUTHENIUM SYSTEM. J. LESS-COMMON MET., V.136, P.237-47. 1988.

$31-4$

ARMES, S. P. (MST-7); ALDISSI, MAF:MOLD (MST-7); AGNEW, STEPHEN F. (INC-4).

POLY(VINYL PYRIDINE)-BASED STABILIZERS FOR AQUEOUS POLYPYRPOLE LATICES

TIC, 1988. 12P. MN (LA-UA-88-2705)

$31-5$

AXLER, KEITH M. (MST-5); FOLTYN, ELIZABETH MAY (MST-5); PETERSON, DEAN E (MST-5):

HUTCHINSON, WILLIAM B. (CLS-1).

PRODUCTION ANO EXAMINATION OF TERNARY PLUTONIUM-ALUMINUM-IRIDIUM INTERMETALLIC PHASES J. NUCL MATER., V.158, P.279-80. 1988.

$31-6$

BAER, DONALD R.; FRYDRYCH, DANIEL J.; JERVIS, THOMAS R. (MST-7).

NEAR SURFACE CHEMISTRY AND CORROSION BEHAVIOR OF EXCIMER LASER SURFACE-MELTED AISI TYPE 304 STAINLESS STEEL.

TIC, 1988. 13P. MN (LA-UR-88-1960)

$31-7$

CARTER, DAVID H. (MST-6); GIBBS, W. SCOTT (MST-6); PETROVIC, JOHN J. (MST-4).

MECHANICAL CHARACTERIZATION OF SIC WHISKER-REINFORCED MOLYGDENUM SILICON-2

TIC, 1988. 11P. MN (LA-UR-88-3776)

$31-8$

CARTER, DAVID H. (MST-6).

SILICON CARBIDE WHISKER-REINFORCED MOLYBDENUM SILICON-2.

LOS ALAMOS NATIONAL LABORATCAY, 1988. 138P. (LA-11411-T)

$31-9$

CASTRO, AICHARD G. (MST-6); STANEK, PAUL W. (MST-6).

MCROLAMINATE COMPOSITE STRUCTURES BY LOW PRESSURE PLASMA SPRAY DEPOSITION

IN: INTERNATIONAL POWDER METALLURGY CONFEFENCE 1988, ORLANDO, FLA. PROC., P.743.67.

METAL POWDER INDUSTRIES FEDERATION, 1968. (TN695 . M62X)

ALSO PUBLISHED: TIC, 1988. 12P. MN (LA-UR-B8-0589)

$31-10$

CHEN, SHAO P. (T-11); VOTER, ARTHUR F. (T-12); ALBERS, ROBERT C. (T-11); BORING, A. MICHAEL (T-11): HAY, P. JEFFREY $(T-12)$

THEORETICAL STUDIES OF GRAIN BOUNDAFIES IN NICKEL, ALUMINUM, AND NICKEL-3 ALUMINIDE WITH AND WITHOUT BORON.

IN: INTERFACIAL STRUCTURE, PROPERTIES, AND DESIGN, 1988, RENO, NEV. PROC., P.355.60.

MATERIALS RESEARCH SOCIETY, 1988. (TA401.M37X V.122)

ALSO PUBLISHED: TIC, 1988. 8P. MN (LA-UR-88-2260) 
31-11

COGHLAN, WILLIAM A.; CLINARD, FRANK W., JR. (MST-5).

MODELING OF IRRADIATION-INDUCED AMIORPHIZATION IN A TITANATE CERAMIC.

J. LESS-COMMON MET., V.140, P.255-65. 1988.

ALSO PUBLISHED: TIC, 1987. 23P. MN (LA-UR-87-2873)

31-12

COST, JAMES RICHARD (MST-5); BROWN, ROBERT D. (MP-7); GIORGI, ANGELO L. (MST-5);

STANLEY, JAMES T. (MST-5).

EFFECTS OF NEUTRON IRRADIATION ON NEODYMIUM-IRON-BORON MAGNETIC PROPERTIES. IEEE TRANS. MAGN., V.24, P.2016-9. 1988.

$31-13$

COST, JAMES RICHARD (MST-5); WILLIS, JEFFREY O. (P-10); THOMPSON, JOE D. (P-10);

PETERSON, DEAN E. (MST-5).

FAST-NEUTRON IRRADIATION OF YTTRIUM BARIUM-2 COPPER-3 OXYGEN-X.

PHYS. REV., V.37B, P.1563-8. 1988.

$31-14$

COX, LAWRENCE E. (MST-13).

X-RAY PHOTOEMISSION STUDY OF DELTA-PLUTONIUM STABILIZED BY 1 WT.\% GALLIUM-ELECTRONIC

STRUCTURE AND SPUTTER-INDUCED SURFACE PHASE TRANSFORMATION.

PHYS. REV., V.37B, P.8480-3. 1988.

$31-15$

CROSS, JON B. (CLS-2); LAN, E. H.; SMITH, C. A.

TECHNIQUE TO EVALUATE COATINGS FOR ATOMIC OXYGEN RESISTANCE.

IN: INTERNATIONAL SAMPE SYMPOSIUM AND EXHIBITION, 33RD, 1988. ANAHEIM, CALIF. PROC. P.693-724. SOCIETY FOR THE ADVANCEMENT OF MATERIAL AND PROCESS ENIGINEERING. 1988 (TA401.3/.S63/1988X)

$31-16$

EBERHARDT, JAMES J.; HAY, P JEFFREY (T-12); CAPPENTER, JUSEPH A., JR.

MATERIALS BY DESIGN-A HIERARCHICAL APPROACH TO THE DESIGN OF NEW MATERIALS. IN: COMPUTEA-BASED MICROSCOPIC DESCRIPTION OF THE STRUCTURE AND PROPERTIES OF MATERIALS, 1985, BOSTON, MASS. PROC., P 19!-206. MATERIALS RESEARCH SOCIETY, 1986 (TA418.5 Ce6 1985X)

ALSO PUBLISHED: TIC. 1985. 23P. MN (LA-UR-85-4322)

$31-17$

EBERHART, M. E. (MST-5); VVEDENSKY. D. D

BOND STRAIN AT GRAIN BOUNDARIES

PHYS. REV. LETT., V.60, P.1381-2. 1988.

31-18

EBEFHART, M. E. (MST-5); VVEDENSKY, D. D.

ENVIRONMENTALLY SPECIFIC MECHANICAL PROPERTIES-BEYOND ATOMIC PARAMETEFS

PHYS. REV., V.378, P.8483-90. 1988.

31.19

EBERHART, M. E. (MST-5); VVEDENSK'Y. D. D

MODEL FOR QUCTILITY-ENHANCEMENT IN TTHE LITHIUM-2 INTERMETALLIC COSIPOUNDS

SCR. METALL., V.22, P.1183-8. 1988.

$3 !-20$

EK'LUND, P. C.; HOFFMAN, D. M.: DE LONG, LANCE E.; ARAKAWA. E. T.; SMITH, JAMES L (CMS): FISK, ZACHARY (CMS).

OPTICAL DIELECTRIC FUNCTION OF URANIUM-BERYLLIUM-13, CERIUM-BERYLLIUM-13. ANU

THORIUM-BERYLLIUM-13.

IN: INTERNATIONAL CONFERENCE ON VALENCE FLUCTUATIONS, 5TH, 1987, BANGALORE. INCIA PROC., P.671-4. PLENUM, 1987. (OC/176.8/.V34/157/1987)

31-21

ERICKSON, GEORGE F. (CLS-6).

SOLID HOSTS FOR DYE LASER RODS-PART 1, CRITERIA FOR CHOOSING A HOST MATERIAL.

TIC, 1987. 5P. MN (LA-UR-88-0414) 
31-22

FISHER, R. A.; LACY, S. E : MARCENAT, C.; OLSEN, J. A.; PHILLIPS. N. E.; FISK, ZACHARY (CMS);

GIORGI, ANGELO L (MST-5), SMITH, JAMES L. (CMS); STEWART, GREGORY R. (MST-5).

LOW-TEMPERATURE SPECIFIC HEAT OF URANIUM BERYLLIUM 13.

IN: INTERNATIONAL CONFERENCE ON VALENCE FLUCTUATIONS, 5TH, 1987, BANGALORE, INDIA. PROC., P.345-8. PLENUM, 1987. (OC/176.8/.V34/157/1987)

ALSO PUBLISHED: TIC, 1997. 5P. MN (LA-UR-87.0309)

$31-23$

FOLLANSBEE, PAUL S. (MST-5); KOCKS, U. FRED (CMS).

CONSTITUTIVE DESCRIPTION OF THE DEFORMATION OF COPPER BASED ON THE USE OF THE MECHANICAL THRESHOLD STRESS AS AN INTERNAL STATE VARIABLE. ACTA METALL., V.36, P.81-93. 1988.

31-24

FOLLANSBEE, PAUL S. (MST-5).

HIGH-STRAIN-RATE DEFORMATION MECHANISMS IN COPPER AND IMPLICATIONS FOR BEHAVIOR DURING SHOCK-WAVE DEFORMATION. IN: SHOCK WAVES IN CONDENSED MATTER, 1987, MONTEREY. PROC., P.249-54. ELSEVIER, 1988 (OC173.4.C65 557 1987X)

ALSO PUBLISHED: TIC, 1987. 19P. MN! (LA-UR-87-2198)

31-25

FOLLANSBEE, PAUL S. (MST-5).

RECENT RESULTS AND CONTINUED DEVELOPMENT OF THE MTS [MECHANICAL THRESHOLD STRESS] MODEL.

TIC, 1988. 28P. MN (LA-UR-88-2521)

31-26

FOLTYN, ELIZABETH MAY (MST-5); PETERSON, DEAN E. (MST-5).

PB-PU (LEAD-PLUTONIUM) SYSTEM.

BULL. ALLOY PHASE DIAGRAMS, V.9, P.267-71. 1988.

31-27

FOLTYN, ELIZABETH MAY (MST-5); PETERSON, DEAN E. (MST-5).

PU-SN (PLUTONIUM-TIN) SYSTEM.

BULL. ALLOY PHASE DIAGRAMS, V.9, P.152-5. 1988.

31-28

GEORGE, TNOTHY G. (MST-5).

HIGH-STRAIN-RATE, HIGH-TEMPERATURE BIAXIAL TESTING OF DOP-26 IRIDIUM

LOS ALAMOS NATIONAL LABORATORY, 1988. 47P. (LA.11065)

31.29

GEORGE, TIMOTHY G. (MST-5); STEVENS, MICHAEI. F. (MS:-5).

HIGH-TEMPERATURE IMPACT PROPERTIES OF DOP-26 IIIDIUM.

J. MET., V.40, P.32-5. 1988

31.30

GIORGI, ANGELO L. (MST-5): LAWSON, A. C. (MST-5); GOLDSTONE, JOYCE A. (P.8), VOLIN, K G JORGENSEN, J. D.

MAGNETIC STRUCTURE OF URANIUM COPPER-2 SILICON-2 AND URANIUM (COPPER 0 75 MANGANESE $0.25) 2$ SILICON-2.

J. APPL. PHYS., V.63, P.3604-5. 1988.

31-31

GIORGI, ANGELO L. (MST-5).

TERNARY SUPERCONDUCTORS.

IN: INTERNATIONAL CONFERENCE ON TERNARY AND MULTINARY COMPOUNDS, 7TH. 1986. SNOWMASS, COLO. PROC., P.29-35. MATERIALS RESEARCH SOCIETY, 1987. (OC610.9 I565 ALSO PUBLISHED: TIC, 1986. 9P. MN (LA-UR-86-3124)

31-32

GRAY, GEORGE T., III (MST-5).

DEFOFMATION TWINNING IN ALUMINUM - 4.8 WT\% MAGNESIUM ACTA METALL., V.36, P.1745-54. 1988. 
$31-33$

GRAY, GEORGE T., III (MST-5); MORRIS, CHARLES E. (M-6).

INFLUENCE OF PEAK PRESSURE ON THE SUBSTRUCTURE EVOLUTION AND SHOCK WAVE PROFILES OF TITANIUM-ALUMINUM-6-VANADIUM-4.

TIC. 1986. BP. MN (LA-UR-88-0625)

$31-34$

GRAY, GEORGE T., III (MST-5); FOLLANSBEE, PAUL S. (MST-5).

INFLUENCE OF PEAK PRESSURE ON THE SUBSTRUCTURE EVOLUTION AND MECHANICAL PROPERTIES OF SHOCK-LOADED 6061-T6 ALUMINUM.

IN: SHOCK WAVES IN CONDENSED MATTER, 1987, MONTEREY. PROC., P.339-42. ELSEVIER, 1988 (CC173.4.C65 S57 1987X)

ALSO PUBLISHED: TIC, 1987. I1P. MN (LA-UR-87-2134)

31-35

GRAY, GEORGE T., III (MST-5); FOLLANSBEE, PAUL S. (MST-5)

INFLUENCE OF STRAIN RATE ON THE SUBSTRUCTURE EVOLUTION AND YIELD BEHAVIOR OF

THALLUM-6ALUMINUM-4VANADIUM.

TIC, 1988. 9P. MN (LA-UR-88-0604)

31-36

HIRVONEN, J. P.; NASTASI, MICHAEL (MST-7); JERVIS, THOMAS R. (MST-7); TESMER, JOSEPH R. (P-10)

UNLUBRICATED SLIDING PROPERTIES OF ION BEA!A AND EXCIMER LASER MIXED

IRON-TITANIUM-CARBON MULTILAYERED FILMS

TIG, 1988. 7P. MN (LA-UR-88-3667)

31-37

HOCKETT, JOHN E., JR. (WX-5); ZUKAS, EUGENE G. (MST-5).

RESPONSE OF IRON TO DYNAMIC COMPRESSION.

IN: CONFERENCE ON MIECHANICAL PROPERTIES OF MATERIALS AT HIGH RATES OF STRAIN. IST, 1974, OXFORD. ENGLAND. PROC., P.53-61. INSTITUTE OF PHYSICS, 1974. (TA417.6 C65

$31-38$

HOFFER, JAMES K. (P-10): FOREMAN. LARRY R. (MST-7).

RADIOACTIVELY INDUCED SUBLIMATION IN SOLID TRITIUM

PHYS. REV. LETT., V.60, P.1310-3. 1988.

31-39

HUANG, J. C. (MST-5); GRAY, GEORGE T., III (MST-5)

MCROSTRUCTURAL CHAR CCTERIZATION OF SILVER SHOCK-LOADED AT HIGH PRESSURE SCR. METALL., V.22, P.545-50. 1988

$31-40$

HUANG, J. C. (MST-5); GRAY, GEORGE T., III (MST T-5).

SUBSTRUCTURE EVOLUTION AND DEFORMATION MODES IN SHOCK-LOADED NIOBIUM. MATER. SCI. ENG., V.A103, P.241-55. 1988

31-41

JACOBSON, LOREN A. (MST-6); RICHARDSON, SHERRI A. (MST-6).

MICROSTRUCTURE AND PROPERTIES OF RAPIDLY SOLIDIFIED BERYLLIUM-TRANSITION METAL ALLOYS TIC, 1988. 11P. MN (LA-UR-88-1586)

$31-42$

JACOBSON, LOREN A. (MST-S): MARTIN, PATRICK L. (MST-5): MITCHEUL, T. E. (CMS).

RAPIOLY SOLIDIFIED AND THERMAL.LY TREATED MICROSTRUCTURES OF BERYLLIUM-1WT\% YTTRIUM

ALLOY.

IN: ELECTRON MICROSCOPY SOCIETY. MEETING, 46TH, 1988, MILWAUKEE. PROC., D.782-3. SAN FRANCISCO PRESS, 1988.

$31-43$

JERVIS, TiHOMAS R. (MST-3); NASTASI, MICHAEL (MST-7); ZOCCO, THOMAS G. (MST-5)

EXCIMER LASER MIXING OF TITANIUM LAYERS ON AISI 304 STAINLESS STEEL.

IN: FUNDAMENTALS OF BEAM-SOLID INTERACTIONS AND TRANSIENT THERMAL PROCESSING. MATERIALS RESEARCH SOCIETY SYMPOSIUM, 1987, BOSTON. PROC., P.621-5. MATERIALS RESEARCH SOC., 1988. (TA418.6 .F86 1987X) 
$31-44$

JERVIS, THOMAS R. (MST-7); HIRVONEN, J. P.; NASTASI, MICHAEL (MST-7).

EFFECT OF EXCIMER LASER ALLOYING OF TITANIUM ON THE SLIDING FRICTION OF AISI 304 STAINLESS STEEL

J. MATER. RES., V.3. P.1104-7. 1988.

$31-45$

JERVIS, THOMAS R. (MST-7); NASTASi, MICHAEL (MST-7); ZOCCO, THOMAS G. (MST-5).

EXCIMER LASER MIXING OF TITANIUM LAYERS ON AISI 304 STAINLESS STEEL.

IN: MATERIALS RESEARCH SOCIETY SYMPOSIUM ON FUNDAMENTALS OF BEAM-SOLID

INTERACTIONS AND TRANSIENT THERMAL PROCESSING, 1987, BOSTON, MASS. PROC., P.621.5.

MATERIALS RESEARCH SCCIETY, 1988. (TA401.M37X V.100)

$31-46$

JERVIS, THOMAS R. (MST-7); NASTASI, MICHAEL (MST-7); ZOCCO, THOMAS G. (MST-5);

MARTIN, JOE A. (CLS-2).

FORMATION OF AMORPHOUS TITANIUM ALLOY LAYERS BY EXCIMER LASER MIXING OF TITANIUM ON

AISI 304 STAINLESS STEEL SURFACES.

APPL. PHYS. LETT., V.53, P.75-7. 1888.

31-47

JERVIS, THOMAS R. (MST-7); HIRVONEN, J. P. (MST-7); NASTASI, MICHAEL (MST-7);

ZOCCO, THOMAS G. (MST-5); MARTIN, JOE A. (CLS-2); PHARR, GEORGE M.; OLIVER, W. C.

SURFACE MECHANICAL PROPERTIE'S OF TITANIUM ALLOYS PRODUCED BY EXCIMER LASER MIXING OF TITANIUM ON AISI 304 STAINLESS STEEL.

TIC, 1988. 7P. MN (LA-UR-88-3036)

$31-48$

JOHNSON, KAYE ALLAN (MST-13); STAUDHAMMER, KARL P. (MST-7); MEDINA, WALTER J. (MST-13):

PIERCE, C. BALLARD (P-10); ELLIOTT. NORMAN E. (NST-7).

OBSERVATION OF CRYSTALLOGRAPHIC AND JC ANISOTROPY IN BULK SHOCK-COMPACTED YTTRIUM BARIUM 2 COPPER 3 OXYGEN 7-X POWDERS

IN: WORKSHOP ON INDUSTAIAL APPLICATIONS OF SHOCK PROCESSING OF POWDERS. IST, 1988. SOCORAO, N.M. PAOC., P.223-8. CENTER FOR EXPLOSIVES TECHNOLOGY RESEAPCH. NEW MEXICO INSTITUTE OF MNING AND TECHNOLOGY, 1988. (TP156.C50 W67 1988X)

$31-49$

JOHNSON, KAYE ALLAN (MST-13); STAUDHAMMER, KARL P. (MST-7); ELLOTT. NORMAN E. (MST-7): MEDINA, WALTER J. (MST-13).

STRAIN AND AMBIENT TEMPERATURE MICROSTRUCTURAL EFFECTS IN SHOCK LOADED 304-STAINLESS STEEL

IN: SHOCK WAVES IN CONDENSED MATTER, 1987, MONTEREY, PROC., P.347-50. ELSEVIER, 198B (OC173.4.C65 557 1987X)

$31-50$

JOYCE, EDWARD L., JR. (MST-7); JERVIS, THOMAS R. (MST-7).

ANALYSIS OF THE EFFECT OF SYSTEM VARIABLES ON THE OUAUTY OF THIN FILMS AND POWDERS

PRODUCED BY LASER-BREAKDOWN CHEMICAL VAPOR DEPOSITION.

IN: LASER AND PARTICLE BEAM CHEMCAL PROCESSING FOR MCROELEC TRONICS. 1987. BCSTON. MASS. MATERIALS RESEARCH SOCIETY, 1988. (TA401.M37X V.101)

ALSO PUBUSHED: TIC, 1987. 5P. MN (LA-UR-87-3574)

31-51

KATZ, J. D. (MST-4); BLAKE, RODGER D. (MST-6); PETROVIC, JOHN J. (MST-4).

MCROWAVE SINTERING OF ALUMINA - SHLICON CARBIDE COMPOSITES AT 2.45 AND 60 GHZZ

TIC, 1988. 24P. MN (LA-UR-88-0597)

$31-52$

KOCKS, U. FAED (CSSI. STOUT. MCHAEL G. (MST-5); ROLLETT. ANTHONY DAVID (MST-5)

INFLUENCE OF TEXTURE ON STRAIN HARDENING.

IN: INTERNATIONAL CONFERENCE ON THE STRENGTH OF METALS AND ALLOYS, 8TH, 1988, TAMPERE, FINLAND. PROC., P.25-34. PERGAMON, 1988.

ALSO PUBLISHED: TIC, 1988. 11P. MN (LA-UR-88-1168) 
$31-53$

KOCKS, U. FRED (CMS).

SYMMETRIC SET OF EULER ANGLES AND OBLIOUE ORIENTATION SPACE SECTIONS.

IN: INTERNATIONAL CONFERENCE ON TEXTURES OF MATERIALS, 8TH, 1987, SANTA FE, N.M. PRGC., P.31-6. METALLURGICAL SOCIETY, 1988. (TA418.5 .157 1987X)

ALSO PUBLISHED: TIC, 1887. 7P. MN (LA-UA-88-0005)

31-54

LAWSON, A. C. (MST-5).

APPLICATIONS OF PULSED NEUTRON POWDER DIFFRACTION TO ACTINIDE ELEMENTS

IN: CONFERENCE ON APPLICATIONS OF X-RAY ANALYSIS, 1987, DENVER, COLO. PROC., P.385-93.

PLENUM, 1988. (543.085 C748A V.31)

31-55

LE SAR, RICHARD A. (T-11).

ELECTRONIC STRUCTURE OF CONDENSED MOLECULAR SYSTEMS.

TIC, 1888. 21P. MN (LA-UR-88-1015)

31-56

LEWIS, GARY K. (MST-6); CREMERS, DAVID A. (CLS-4); DIXON, PAY D. (MST-6).

LASER PLUME TEMPERATURE MEASUREMENTS IN VARIOUS GASES

TIC, 1888. 6P. MN (LA-UR-88-0540)

31.57

UST, R. S. (P-10): ARKO, AL J. (P-10); FISK, ZACHARY (P-10); CHEONG, SANG-WOOK (P- 10):

CONRADSON, STEVEN D. (INC-4); THOMPSON, JOE D. (P-10); PIERCE, C. BALLARD (P-10):

PETERSON, DEAN E. (MST-5); BAFTLETT, ROGER J. (P-14); O'ROURKE, JOHN A. (MST-5); SHINN. N D

SCHIRBER, J. E.; OLSON, C. G.; YANG, A-B.; PI. T-W.: VEAL. B. W.: PAULIKAS, A P., CAMPUZANO. J C

PHOTOEMISSION FPOM SINGLE-CRYSTAL EUROPIUM BARIUM-2 COPPER-3 OXYGEN-G-PLUS $X$ CLEAVED BELOW 2OK-METALLIC-TO-INSULATING SURFACE TRANSFORMATION

TIC. 1938. 7P. MN (LA-UR-88-3413)

31-58

LUNDBERG, LYNN B. (MEE-13;

HIGH TEMPERATURE INTERDIFFUSION AND PHASE EOUIUBRIA IN URANIUM-MOLYBDENUM TIC, 1988. 19P. MN (LA-UR-88-2660)

$31-50$

LYONS. PETER B. (DRANDR)

RADIATION EFFECTS

IN: PHOTONICS-HIGH BAHDWIDTH ANALOG APPLICATIONS. 1986. HOWEY.IN. THE.HILLS. FLA PROC P.128-33. SPIE. 1997. (TA1505 .S64X V.648)

$31-60$

MAGGOAE. CARL J. (CMS); BLACIC, JAMES D (ESS-3); BLONDIAUX. G . DEBRUN. J L. ALI. M haGE

MATHEZ, E.; MISDAQ, M. A : VALLADON, M

CHANNELED AND MEAOACTIVATION OF MATERIALS

TIC. 1988. 12F. TN (LA-UR-88-3492)

$31-61$

MARIANER, SHLOMO (T-11); FLORIA, L. M

APPLICATION OF THE METHOD OF EFFECTIVE POTENTIALS TO A MODEL FOR TWINNING IN ELASTIC

MATERIALS.

PHYS. REV., V.38B, P.12n54-7 1388

$31-62$

MARTIN, JOE A. (CLS-2); NASTASI, MICHAEL (MST-7); TESMER, JOSEPH R. (P-10); MAGGIORE, CAFL J (CMS) HIGH-ENERGY ELASTIC BACKSCATTERING OF HEIIUM IONS FOR COMPOSITIONAL ANALYSIS OF HIGH-TEMPERATURE SUPERCONDUCTOA THIN FILMS.

APPL. PHYS. LETT., V.52, P.2177-9. 1988

$31-63$

MAYER, ANTON (MST-7).

ELECTRODEPOSITION OF MAGNESIUM AND MAGNESIUMNALUMINUM ALLOYS.

PATENTED OCT. 18, 1988, FILED 1988. (U.S.PATENT-4,778,575) 
$31-64$

MC ELFRESH, M. W. (P-10).

EFFECT OF PRESSURE ON THE HEAYY-ELECTRON SUPERCONDUCTORS URANIUM-BERYLLIUM-13 AND UPANIUM-RUTHENIUM-2-SILICON-2.

LOS ALAMOS NATIONAL LABORATORY, : \$88. 164P. (LA-11366-T)

31-65

MC HENRY, M. E.; VVEDENSKY, D. D.; EBERHART, M. E. (MST-5); O'HANDLEY, R. C.

SYMMETAY-INDUEED LOCAL MAGNETIC MOMENTS IN ICOSAHEDRAL ALUMINUM MANGANESE ALLOYS. PHYS. REV., V.37B, P.10887-90. 1988.

$31-66$

MITCHELL, T. E. (CMS).

ELECTRON MUROSCOPY OF CERAMIC SUPERCONDUCTORS-A BIBLIOGRAPHY J. ELECTRON MICAOSC. TECH., V.8, P.339-41. 1988.

31-67

MITCHELL, T. E. (CMS); ROY, TAPPAN (CMS).

ELECTRON MCROSCOPY OF CEPAMIC SUPERCONDUCTORS.

TIC, 1988. 7P. MN (LA-UR-88-0642)

$31-68$

MITCHELL, T. E. (CMS); SCHWARZ, RICARDO B. (CMS).

TEM STUDIES OF AMORPHIZATION PROCESSES AND AMORPHOUS STRUCTURES

IN: ELECTRON MICROSCOPY SOCIETY. MEETING, 46TH, 1988, MILWAUKEE. PROC. P.448-9. EMS, 1988

31-69

MITCHELL, T.E.; ROY. TAPPAN (CMS); SCHWAPZ, AICARDO B. (CMS); SMITH, JAMES F. (CMS);

WOHLLEBEN, D. (CMS).

ELECTRON NMCAOSCOPY STUDY OF TWIN BEHAVIOR IN GADOLINIUM BARIUM-2 COPPLE.3 OXYGEN.X

AND YTTRIUM BARIJIA-2 COPPER-3 OXYGEN-X

J. ELECTPON MICPOSC. TECH., V.8, P.317-323. 1988.

$31-70$

MOARIS, CHARLES E. (M-6); WINKLER, MNX A. (M-5); MITCHELL, ARTHUR C (M-6)

TITANIUM-6\%ALUMNUM-4\%VANADIUM ALLOY WAVE PAOFILE MEASUREMENTS IN THE SHADOW REGION IN: SHOCK WIAVES IN CONDENSED MATTER, 1987. MONTEREY PROC. P 265-8 ELSEVIER, 1988 (OC173.4.C65 S57 1987X)

ALSO PUBLISHED TIC. 1997. 5P. MN (LA-UR-87-2284)

$31-71$

NASH, P.: SCHWARZ, RICARDO B. (CMS)

CALCULATION OF THE GLASS FORMING RANGE IN BINARY METALUC SYSTEMS USING

THERMODYNAMIC NODELS.

ACTA METALL., V 36, P.3047-53. 1988

31-72

NASTASI, MICHAEL (MST- $n$ ) TESMER, JOSEPH R. (12.10); HOLLANDER, M. G (MEE-11).

SMITH, JAMES F. (CMS); MAGGIORE, CARL J. (CMS)

FORMATION OF YTTRIUM BARIUM-2 COPPER-3 OXYGEN-7 SUPERCONDUCTING FILMS BY ION IMPLANTATION

APPL. PHYS. LETT., V.52, P.1729-31, 1988

31.73

NASTASI, MICHAEL (MST-7); OKAMOTO, P. R.; AVERBACK, R. S; HUNG, L S., BARBOUR, J C., MAYER, J W IRRADIATION ST.ABILTTY OF PALLADIUM-2-SILICON.

J. LESS-COMMON MET., V.140, P.277-86. 1988.

31-74

NASTASI, MICHAEL (MST- 7 ; HIRVONEN, J. P.; JERVIS, THOMAS R. (MST-7); PHARR, GEORGE M .

OLIVER, W. C.

SURFACE MECHANICAL PROPERTIES OF CARBON IMPLANTED NICKEL.

J. MATER. RES., V.3, P.226-32. 1988. 
31-75

NYITRAY, A. M. (MST-7); WILLIAMS, JOEL M. (MST-7).

COMPOSITE FOAMS.

TIC, 1988. 14P. MN (LA-UR-88-3019)

31.76

OTT, HANS R.; FISK, ZACHARY (P-10).

HEAVY ELECTRONS IN METALS.

IN: INTERNA TIONAL CONFERENCE ON RECENT PROGRESS IN MANY-BODY THEORIES, 5TH, 1987. OULU, FINLAND. PROC., P.1-16. PLENUM PRESS, 1988. (OC612.S8 1496 1987)

31-77

PETERSON, DEAN E. (MST-5).

PREPARATION OF OXIDE SUPERCONDUCTORS.

IN: IDEAS AND SCIENCE ELECTRONICS EXPOSITION AND SYMPOSIUM, 10TH, 1988, ALBUOUEROUE. N.M. PROC., P.94-7. IDEAS IN SCIENCE AND ELECTRONICS, INC., 1988. (T6 .183

ALSO PUBLISHED: TIC, 1988. 5P. MN (LA-UR-88-1226)

$31-78$

PHILLIPS, N. E.; FISHER, R. A.; LACY, S. E.; MARCENAT, C.; OLSEN, J. A.; FLOUQUET, JACQUES; AMATO, A. JACCARD, D.; FISK, ZACHARY (CMS;; GIORGI, ANGELOL. (MST-5); SMITH, JAMES L. (CMS):

STEWAFT, GREGORY R. (MST-5).

PRESSURE DEPENDENCE OF THE SPECIFIC HEAT OF HEAVY-FERMION COMPOUNDS. IN: INTERNATIONAI. CONFERENCE ON VALENCE FLUCTUATIONS, 5TH, 1987, BANGALORE, INDIA. PROC., P.141-50. PLENUM, 1987. (OC/176.8/.V34/157/1987)

31-79

ROLLETT, ANTHONY DAVID (MST-6).

STRAIN HARDENING AT LARGE STRAINS IN ALUMINUM ALLOYS.

LOS ALAMOS, 1986. 367P. (LA-11202-T)

$31.8 \mathrm{C}$

ROLLETT, ANTHONY DAVID (MST-6); KOCKS, U. FAED (CMS); EMBUAY, J. D. (MST-DO);

STOUT, MICHAEL G. (MST-5); DOHERTY, ROGER D.

STRAIN HARDENING AT LARGE STRAINS.

TIC. 1988. 7P. MN (LA-UR-88-0674)

$31-81$

ROLLETT, ANTHONY DAYID (MST-6): KOCKS, U. FRED (C'MS); SIOUT, MICHAEL G. (MST-5):

EMBURY, J. D. (MST-DO); DOHERTY, ROGER D.

WORK HARDENING AT LARGE STRAINS. THEOAY AND EXPERIMENT.

IN: INTERNATIONAL CONFERENCE ON COMPUTATIONAL ENGINEERING SCIENCE, 1988, ATLANTA, GA PROC., P.16.V.1-2. SPRINGER-VERLAG, 1988. (QA801/.1375/1988)

31-82

ROY, TAPPAN (CMS); MITCHELL, T. E. (CMS)

LOW TEMPERATUFE TPANSFOAMATIONS AND TWINNING IN LANTHANUM $2-X$ STRONTIUM $X$ COPPER

OXYGEN 4-DELTA AND LANTHANUM 2 COPPER OXYGEN 4.

IN: ELECTRON MICROSCOPY SOCIETY, NEETING, 46TH, 1988, MILWAUKEE. PROC., P.854-5. SAN

FRANCISCO PRESS, 1988

$31-83$

SANDENAW, THOMAS A. (MST-13).

INCOMMENSURATE/COMKENSURATE CHARGE-DENSITY-WAVE STATES AS A SOURCE FOR PLUTONIUM METAL BEHAVIOR.

LOS ALAMOS, 1988. 28P. (LA-11083-NS)

31-84

SAW, C. K.; SCHWIARZ, RICARDO B. (CMS).

CHEMICAL SHORT-RANGE ORDER IN DENSE RANDOM-PACKED MODELS

J. LESS-COMMAN MET., V.140, P.385-93. 1988. 
31.85

SCHWARZ, RICARDO B. (CMS); HANNIGAN, JAMES W. (CMS); SHEINBERG, HASKELL (MST-6);

TIAINEN, TUOMO J. (CMS).

AMORPHOUS POWDERS OF ALUMINUMHAFNIUM PREPARED BY MECHANICAL ALLOYING.

IN: INTERNATIONAL POWDER METALLURGY CONFERENCE, 1988, ORLANDO. FLA. PROC., P.415-27. METAL POWDER INDUSTRIES FEDERATION, 1988.

ALSO PUBLISHED: TIC, 1988. 13P. MIJ (LA-UR-88-1820)

$31-86$

SCHWARZ, RICARDO B. (CMS); PETRICH, RICHARD R. (CMS).

CALORIMETRY STUDY OF THE SYNTHESIS OF AMORPHOUS NICKEL TITANIUM ALLOYS BY MECHANICAL

ALLOYING.

J. LESS-COMMON MET., V.140, P.171-84. 1988.

ALSO PUBLISHED: TIC, 1988. 32P. MN (LA-UR-88-0427)

31-87

SCHWARZ, RICARDO B. (CMS).

FORMATION OF AMORPHOUS ALLOYS BY SOLID STATE REACTIONS.

MATER. SCI. ENG., V.97, P.71-8. 1988.

31-88

SCHWARZ, RICARDO B. (CMS)

GLASS FORMING RANGES OF AMORPHOUS ALLOYS

TIC. 1988. 1OP. MN (LA-UR-88-0809)

$31-89$

SCHWARZ, RICARDO B. (CMS).

METASTABLE ALLOY PHASES PREPARED BY SOLID STATE REACTIONS AND BALL MILLING

TIC, 1988. 20P. MN (LA-UR-88-1167)

$31-90$

SCHWARZ, RICARDO B. (CMS)

RECENT ADVANCES IN THE SYNTHESIS OF AMORPHOUS METALLIC MATERIALS.

IN: SYMPOSIUM ON ADVANCES IN PHASE TRANSITIONS, 1987, MC MASTER IINIVERSITY, ONTARIO.

CANADA. PROC., P.166-77. PEFGAMON PRESS, 1988. (OD503 A28 1987X)

31-91

SCHWARZ, RICARDO B. (CMS); JOHNSON, W. L

REMARKS ON SOLID STATE AMOAPHIZING TRANSFOAMATIONS

J. LESS-COMAON MET, Y.140, P.1-6. 1988.

ALSO PUBLISHED: TIC. 1987. 14P. MN (LA-UP-88-0588)

31-92

SCOTT, MARION L (MST-7); ARENDT, PAUL N. (MST-7); CAMERON, BERNARD J. (AIST-7);

NEWNAM, BRIAN E. (CLS-5).

CONTAMNATION LAYERS ON EUV REFLECTORS

IN: SOFT X-RAY OPTICS AND TECHNOLOGY, 1986, BERLN, FRG. PROC. P. 156-62. SPIE, 1937 (TA1505 $.564 \times$ V.7331

31-93

SCOTT, MARЮN L (MST-7).

OPTICAL IAEASUREMENTS OF SURFACE OXIDE LAYER FC̄ñVIATION ON METAL FILMS

TIC, 1987. BP. MN (LA-UR-87-3888)

31-94

SHALEK, PETER D. (MST-4); KATZ, J. D. (MST-4); HURLEY, GEORG: F. (MST-4).

PREALLOYED CATALYST FOR GROWING SILICON CARBIDE WHISKERS:

PATENTED DEC. 6, 1998, FILED 1987. (U.S.PATENT-4,789,537)

$31-95$

SHELDON, R. I. (MST-5); FOLTYN, ELIZABETH MAY (MST-5); PETERSON, DEAN E. (MST-5)

PE-U (LEAD-UAANIUM) SYSTEM.

BULL. ALLOY PHASE DIAGRAMS, V.8, P.536-41. 1987. 
31-96

SMITH, JAMES L. (CMS); FISK, ZACHARY (CMS); OTT, WANS R.

ACTINIDES AND HEAVY FERMIONS.

IN: INTERNATIONAL CONFERENCE ON VALENCE FLUCTUATIONS, 5TH, 1987, BANGALORE. PROC. P.11-5, 1987. PLENUM, 1987. (CC176.8.V34 157 1987)

ALSO PUBLISHED: TIC, 1987. 6P. MN (LA-UR-87-0012)

31-97

SMITH, JAMES L. (CMS); FISK, ZACHARY (P-10); THOMPSON, JOE D. (P-10); WILLIS, JEFFREY O. (P-10); BORGES, HORTENCIO A. (P-10).

SUPERCONDUCTING PROPERTIES OF ACTINIDE COMPOUNDS AND OTHER RECENT RESULTS

PHYSICA, V.148B, P.14-6. 1987.

$31-88$

SOMMER, WALTER F., JR. (MP-13); LOHMANN, W. (MP-13); GRAF, KARL; TAYLOR, IVAN K. (MP-13);

CHAVEZ, RAYMOND M. (MP-19).

OPERATING EXPERIENCE AT THE LOS ALAMOS SPALLATION RADIATION EFFECTS FACILITY AT LAMAPF. IN: INFLUENCE OF RADIATION ON MATERIAL PROPERTIES, 1987, SEATTLE. PROC., P.718-29. ASTM, 1988. (TA4 18.6.E333 1986XA)

31-99

STAUDHAMMER, KARL P. (MST-7); JOHNSON, KAYE ALLAN (MST-4): MEDINA, WALTER J. (MST-4)

SHOCK CONSOLIDATION OF YTTRIUM BARIUM-2 COPPER-3 OXYGEN-7-X POWDERS

TIC, 1988. 13P. MN (LA-UR-88-3864)

$31-100$

STAUDHAMMER, KARL P. (MST-7).

SUPERCONDUCTOR CONSOLIDATION

TIC, 1988. TP. MN (LA-UR-B8-1588)

31-101

TAKESHITA, TORU (CMS); KOCKS, U. FRED (CNS); WENK, H. R

PATH DEPENDENCE OF DEFORMATION TEXTURE DEVELOPMENT

IN: INTERNATIONAL CONFERENCE ON TEXTURES OF MATERIALS, 8TH, 1987, SANTA FE, N.M. PROC. P.445-8. METALLURGICAL SOCIETY, 1988. (TA418.5 .157 1987X)

ALSO PUBUSHED: TIC, 1887. 5P. MN (LA-UR-88-0007)

31-102

TAYLOR, THOMAS N. (CLS-2); PHILLIPS, DAVID S. (MST-6)

SURFACE COMPOSITION AND BONDING OF SILICON CARBIDE POWDERS AND WHISKERS

ABSTRACT IN: J. VAC. SCI. TECHNOL., V.A6, P.982-3. 1988.

31-103

TESMER, JOSEPH R. (P-10); MAGGIORE, C.ARL J. (CMS); PARKIN, DON M. (CMS)

NEW FACILITY FOR ION BEAM MATERIALS CHARACTERIZATION AND MODIFICATION AT LOS ALAMOS NUCL INSTRUM. METHODS PHYS. RES., V.B4O/41, P.718-21. 1989.

ALSO PUBLISHED: TIC, 1988. 13P. MN (LA-UR-88-3481)

31-104

TIAINEN, TUOMO J. (CMS); SCHWARZ, RICARDO B. (CMS)

SYNTHESIS AND CHARACTERIZATION OF MECHANICALIY ALLOYED NICKEL-TIN POWDERS J. LESS-COMMON MET., V.140, P.99-112. 1988.

31-105

VANNINI, MATTEO; SZE, ROBERT C. (CLS-5); HOMMEAU, FRANOIS.

USE OF MAGNETIC MATERIALS IN EXCIMER LASER CIRCUITS

IN: INTERNATIONAL CONFERENCE ON LASERS '87, 1987, LAKE TAHOE. PROC., P. 103-10. 1988 ALSO PUBLISHED: TIC, 1987. 9P. MN (LA-UR-88-0657)

31-105

VOGL, P.; LEISING, G.

IN TRINSIC ENERGY GAP OF CRYSTALLINE TRANS-POLYACETYLENE

TIC, 1988. 7P. MN (LA-UR-88-2265) 
31-107

VOGL, P.; CAMPBELL, DAVID K. (CNLS); SANKEY, O. F.

THEORY OF 3-D STRUCTURE AND INTRINSIC DEFECTS OF TRANS-POLYACETYLENE.

TIC, 1988. 9P. MN (LA-UR-88-2264)

$31-108$

WEERTMAN, JOHANNES (MST-5); FOLLANSBEE, PAUL S. (MST-5).

DISLCCATION DYNAMICS AND PLASTIC SHOCK WAVES.

NECH. MATER., V.7, P.177-89. 1988.

31-100

WILLIAMS, ARTHUR (LANSCE); KWEI, GEORGE H. (LANSCE); VON DREELE, R. B. (LANSCE);

LARSON, ALLEN C. (P-B); RAISTRICK, IAN D. (MEE-11); BISH, DAVID L. (ESS-1).

JOINT X-RAY AND NEUTRON REFINEMENT OF THE STRUCTURE OF SUPERCONDUCTING Y ITRIUM

BARIUM-2 COPPER-3 OXYGEN-7-X-THERMAL PARAMETERS, STRAIN, AND CATION DISORDEF.

PHYS. REV., V.378, P.7960-2. 1988.

31-110

WILLLAMS, JOEL M. (MST-7); FOREMAN, LARRY R. (MST-7).

PARYLENE COATED MICROSPHERES-OPERATIONAL PARAMETERS AND ROUND ROBIN RESULTS. TIC. 1987. 22P. MN (LA-UR-88-0849)

$31-111$

WILLIAMS, JOEL M. (MST-7); WROBLESKI, DEBRA A. (MST-7).

SPATIAL DISTRIBUTION OF THE PHASES IN WATER-IN-OIL EMULSIONS. OPEN AND CLOSED

MCPOCELUULAR FOAMS FROM CROSS-LINKED POLYSTYRENE

LANGMUIR, V.4, P.656-62. 1988

$31-112$

WILLJAMS, JOEL M. (MST-7).

TOROIDAL MICROSTRUCTURES FROM WATER-IN-OIL EMULSIONS.

LANGMUIR, V.4, P.44-9. 1988.

31-113

WILLIS, JEFFAEY O. (P-10); COST, JAMES RICHARD (MST-5); BROWN, ROBERT D. (MP-7)

THOMPSON, JOE D. (P-10); PETERSON, DEAN E. (MST-5).

RADIATION DAMAGE IN YTTRIUM BARIUM(2) COPPER(3) OXYGEN(7) BY FAST NEUTRONS

IN: HIGH-TEMPERATURE SUPERCONDUCTORS, 1987, BOSTON, MASS PROC . P $391-4$ MATERIALS RESEARCH SOCIETY, 1988. (TK/7872/S8/H55/1987X)

ALSO PUBLISHED; TIC, 1987. 5P. MN (LA-UR-87-3803)

$31-114$

WOHLLEBEN, D. (CMS).

LATICE INSTABILITIES IN HEAVY FERMION SUPERCONDUCTORS IN: INTERNATIONAL CONFERENCE ON VALENCE FLUCTUATIONS, 5TH, 1987. BANGALORE. INDIA PROC., P.707-19. PLENUM, 1987. (OC/176.8/.V34/57/1987)

ALSO PUBUSHED: TIC, 19a7. 14P. MN (LA-UR-87-1715)

31-115

WROBLESKI, DEBRA A. (MST-7); CASH, D. L. (MST-7); HERMES, ROBERT E

COMPARISON OF SURFACE MODIFICATION OF POLY(ETHER URETHANES) ON PHYSICAL PAOPERTIES

AND BLOOD COMPATIBILITY

TIC. 1988. 6P. MN (LA-UR-88-2650)

31-116

WROBLESK!, DEBRA A. (MST-7); ABㄷㄴ. KEITH H. (INC-5); GRAY, ALTON JAMES (MST-7).

WILLIAMS, JOEL M. (MST-7)

POTENTIAL FOAMS AND METAL FOILS FOR COSMIC DUST CAPTURE

LOS ALAMOS NATIONAL LABORATORY, 1988. 46P. (LA-11271-MS)

$31-117$

YOUNG, KATHLEEN M. (MST-13).

EFFECTS OF VIBRATION AND ELECTROMAGNETIC FORCES ON CASTING-A LITERATURE REVIEW LOS ALAMOS, 1987. 3P. (LA-11170-MS) 
31-118

ZIRNGIEBL, ERBERHARD (MST-5); THOMPSON, JOE D. (P-10); SMITH, JAMES L. (CMS); FISK, ZACHARY (CMS). NORMAL STATE MAGNETIC BEHAVIOR OF (URANIUM 1-X RARE EARTH X)BERYLLIUM 13 PSEUDOEINARIES.

IN: INTERNATHNAL CONFERENCE ON VALENCE FLUCTUATIONS, 5TH, 1987, BANGALORE, INDIA. PROC., P.349-52. PLENUM, 1987. (QC/176.8/.V34/157/1987)

ALSO PUBLISHED: TIC, 1987. 5P. MN (LA-UR-87-0015)

31-119

ZOCCO, THOMAS G. (MST-5); ROHR, DANA L. (MST-5).

DEVELOPMENTS IN THE TEM EXAMINATION OF PLUTONIUM AND PLUTONIUM ALLOYS.

IN: SPECIMEN PREPARATION FOR TRANSMISSION ELECTRON MICROSCOPY OF MATERIALS, MATERIALS RESEARCH SOCIETY SYMPOSIUM, 1987, BOSTON, PROC., P.259-64. MATERIALS RESEARCH SOCIETY, 1988. (TA417.23/S64/1987X)

ALSO PUBLISHED: TIC. 1987. 8P. MN (LA-UA-67-3717)

$31-120$

ZUKAS, EUGENE G. (MST-5); TAYLOR, JOHN W. (ADDRA).

EFFECT OF STRAIN AGING ON THE DYNAMIC ELASTIC-PLASTIC PROPERTIES OF IRON.

IN: CONFERENCE ON MECHANICAL PROPERTIES OF MATERIALS AT HIGH RATES OF STRAIN. IST. 1974, OXFORD, ENGLAND. PROC., P.177-86. INSTITUTE OF PHYSICS, 1974. (TA417.6 .C65 1974)

$31-121$

ZUREK, ANNA K. (MST-5).

BRITTLE CRACKS IN INTRINSICALLY DUCTILE COPPER.

SCR. METALL., V.21, P.1619-22. 1987

$31-122$

ZUREK, ANNA K. (MST-5): JOHNSON, JAMES N. (T-1): FRANTZ. CHARLES E. (MST-5).

CHARACTERIZATION OF DYNAMIC FRACTURE IN COPPER UNDER UNIAXIAL STRESS AND UNIAXIAL STRAN.

TIC, 1988. OP. MN (LA.UR-88-1001) 
32-1

ABDALLAH, JOSEPH, JR. (T-4); CLARK, ROBERT EDWARD HOLMES (X-6). SUPERCOMPUTERS AND ATOMIC PHYSICS DATA.

TIC, 1988. 8P. MN (LA-UR-88-0771)

$32-2$

ABDALLAH, JOSEPH, JR. (T-4); CLARK, ROBERT EOWARD HOLMES (X-6); COWAN, ROBERT D. (T-4).

THEORETICAL ATOMIC PHYSICS CODE DEVELOPMENT I. CATS-CONAN ATOMIC STRUCTURE CODE. LOS ALAMOS NATIONAL LABORATORY, 1988. 26P. (LA-11436-M.V.1)

$32-3$

ABDALLAH, JOSEPH, JR. (T-4); CLARK, ROBERT EDWARD HOLMES (X-6).

THEORETICAL ATOMIC PHYSICS CODE DEVELOPMENT IV. LINES A CODE FOR COMPUTING. ATOMIC UINE SPECTRA.

LOS ALAMOS NATIONAL LABORATOAY, 1988. 37P. (LA-11436-M.V.4)

$32-4$

ADDESSIO, F. L. (T-3); CLINE, MICHAEL C. (T-3); CUUKOWICZ, JOHN K. (T-3).

GENERAL TOPOLOGY, GODUNOV METHOD.

COMPUT. PHYS. COMMUN., V.48, P.65-73. 1998.

32-5

AN, ZHI-GANG (CNLS); NNISZEWSKI, SUSAN MARIE (C-10); LEE, Y. C.;

PAPCUN, GEORGE JACK VICTOR (C-10); DOOLEN, GARY D. (ADDR).

HIERTALKER-A DEFAULT HIERARCHY OF HIGH ORDER NEURAL NETWORKS THAT LEARNS TO READ ENGUSH ALOUD.

TIC, 1988. 9P. MN (LA-UR-88-1849)

32-6

ANDERSON, W. J.: FEIN, RICHARD S.; MICHELSEN, CHRISTIE D. (A-5); SIBLEY, LEWIS B.; TUCKER, ROBERT C

PRODUCTS AND SERVICES DATABASE.

IN: PLANNING WOAKSHOP PROGRAM ON COMPUTERIZED TRIBOLOGY INFORMATION SYSTEM

DEVELOPMENT, 1985, GAITHEASBURG, MO. PROC., P.45-52. U.S. G.P.O., 1987. (TJ1075 .T68 1985X)

32-7

BAILEY, DAVID J. (OS-DO).

MONITORING THE USAGE OF A COMPUTER SYSTEM.

TIC. 1987. 14P. MiN (LA-UR-87-4103)

$32-8$

BENSON, MALURICE W.; FREDERICKSON, PAUL O. (C-3).

FAST PARALLEL ALGORITHMS FOA THE MOORE- PENROSE PSEUDQINVERSE

IN: CONFEAENCE ON HYPERCUBE MULTIPROCESSOAS, 2ND, 1986, KNOXVILLE, TENN PROC.,

P.597-604. SIAM, 1987. (QA76.5/.C6192/1986)

ALSO PUBLISHED: TIC, 1986. 10P. MN (LA-UR-86-3887)

$32-9$

BENSCN, MAURICE W.; FREDERICKSON, PAUL O. (C-3)

FAST PSEUDO-INVERSE ALGOAITHNS ON HYPERCUBES.

IN: MULTIGRID METHODS-COPPER MOUNTAIN CONFERENCE ON MULTIGRID METHODS, 3RD, 1987.

COPPER MOUNTAIN, COLO. PROC., P.23-33. M. DEKKER, 1988. (QA377.M943 1987X)

32-10

BERKBIGLER, KATHAYN P. (C-10); MAX, PATRICIA A. (C- 90).

GRAPHICS ADVISOR-A PROTOTYPICAL ADVISORY EXPERT SYSTEM

TIC, 1998. 8P. MN (LA-UR-88-3917)

32-11

BIEDENHARN, LAWRENCE C.; LOUCK, JAMES D. (T-7).

SOME PROPERTIES OF GENERALIZED HYPERGEOMETAIC COEFFICIENTS.

ADV. APPL. MATH., V.9, P.447-64. 1989. 
$32-12$

BOETTGER, JONATHAN C. (T-1).

SESAME EQUATION OF STATE NUMBER 2293, LITHIUM.

LOS ALAMOS NATIONAL LABORATOAY, 1988. 11P. (LA-11338-MS)

32-13

BOOTH, THOMAS E. $(X-6)$.

INTELLIGENT RANDOM NUMBER TECHNIOUE IN MCNP.

NUCL. SCI. ENG., V.100, P.248-54. 1988.

32-14

BRICKNER, RALPH G. (C-3); WASSERMAN, HARVEY J. (C-3); HAYES, ANN H. (C-3): MOORE. JAMES W. (C-3) BENCHMARKING THE IBM 3090 WITH VECTOF FACILITY.

TIC, 1986. 15P. MN (LA-UA-B6-3300)

$32-15$

BRUCKNER, LAWRENCE A. (S-1); HUME, M. W.; DELVIN, W. L

ON PRECISION AND ACCURACY (BIAS) STATEMENTS FOR MEASUREMENT PROCEDURES.

LOS ALAMOS, 1988. 27P. (LA-11190-MS)

32-16

BUChaNAN, BRUCE G.; SUlLIVAN, JOHN (CLS-1); CHENG. T. P.; CLEARWATER, SCOTT H. (AT-8). SIMULATION-ASSISTED INDUCTIVE LEARNING.

TIC, 1988. 8P. MN (LA-UR-88-2462)

32-17

BUCHER, INGRID Y. (C-3).

VECTOR PERFORMANCE OF REGISTER-TO- REGISTER VECTOR COMPUTERS. LOS ALAMOS, 1988. 32P. (LA-11198-MS)

32-18

BUTTERFIELD, KENNETH B. (N-2).

MENU-A FOATH MENU COMPILER.

TIC, 1988. 8P. MN (LA-UR-88-3372)

32-19

BUZBEE, BILLY L. (C-DO).

SUPERCOMPUTERS-VALUES AND TRENDS.

IN: SUPCONDUCTING-STATE OF THE ART, PROC., P.1-9. NORTH-HOLLAND. 1987. (OC76.5 5898 1987)

$32-20$

CAMPBELL, JOHN R. (S-6/C-3).

DISTRIBUTED MATHEMATICS IN A HETEROGENEOUS ENVIRONMENT

TIC, 1987. 14P. MN (LA-UR-88-1259)

32-21

CAFAPBELL, LAURENCE J. (T-11).

VORTEX LATTICES IN THEORY AND PRACTICE

TIC, 1988. 11P. MN (LA-UR-88-2429)

$32-22$

CARLSON, JOSEPH A. (T-5).

GREEN'S FUNCTION MONTE CARLO CALCULATIONS OF HELIUM-4 TIC, 1988. 16P. MN (LA-UF-88-2920)

32-23

CHAMBERLAN, R. M.; FREDERICKSON. PAUL O. (C-3): LINDHEIM. J.: PETERSEN. J.

HIGH-LEVEL LIBRARY FOR HYPERCUBES.

IN: CONFERENCE ON HYPERCUBE MULTIPROCESSORS, 2ND, 1986, KNOXVILLE, TENN. PROC. P.651-5. SIAM, 1987 (OA76.5/.C6192/1986)

32-24

CHAN, K. C. (AT-6).

ELECTROMAGNETIC TIME-DOMAIN CALCULATIONS IN TWO AND THREE DIMENSIONS.

TIC, 1988. 16P. MN (LA-UR-89-3206) 
CIOSLOWSKI, JEFZY (T-12).

DENSITY DRIVEN SELF-CONSISTENT FIELD METHOD-I. DERIVATION AND BASIC PROPERTIES.

J. CHEM. PHYS., V.89, P.4871-4. 1988.

32-26

CLARK, GARY L. (C-3); ANKENY, LEE A. (C-3).

GRID GENERATION SOFTWARE ENGINEERING AT LOS ALAMOS.

TIC, 1988. 10P. MN (LA-UR-88-2732)

32-27

CLARK, ROBERT EDWARD HOLMES (X-6); ABDALLAH, JOSEPH, JR. (T-4); KRAMER, S. P. (X-6).

THEORETICAL ATOMIC PHYSICS CODE DEVELOPMENT III. TAPS-A DISPLAY CODE FOR ATOMIC PHYSICS

DATA.

LOS ALAMOS N.ATIONAL LABORATORY, 1988. 31P. (LA-11436-M.V.3)

$32-28$

CLARK, AOBERT EDWARD HOLMES (X-6): ABDALLAH, JOSEPH, JR. (T-4): CSANAK, G. Y. (T-4);

MANN, JOSEPH B., JR. (T-4); COWAN, ROBERT D. (T-4).

THEORETICAL ATOMIC PHYSIS CODE DEVELOPMENT II. ACE-ANOTHER COLLISIONAL EXCITATION CODE. LOS ALAMOS NATIONAL LABORATORY, 1988. 25P. (LA-11436-M,V.2)

$32-29$

COLLINS, MAURICE W. (C-8); MC LARTY, TYCE T. (C-B).

MASS STORAGE SYSTEM REFERENCE MODEL SYSTEM MANAGEMENT.

TIC, 1988. 9P. MN (LA-UR-B8-2678)

$32-30$

COLLINS, MAURICE W. (C-B); DEVANEY, MARNORIE A. (C-8); KITTS, DAVID (C-DO).

PROFILES IN MASS STORAGE-A TALE OF TWO SYSTEMS.

TIC, 1988. 8P. MN (LA-UR-88-2752)

32-31

COOK, WILLIAM A. (WX-4); GENIN, JOSEPH.

GENERALIZED FINITE STRAINS, GENERALIZED STRESSES, AND A PRINCIPLE OF VIRTUAL WORK FOR

FINITE-ELEMENT COMPUTER PROGRAMS.

LOS ALAMOS NATIONAL LABORATORY, 1988. 29P. (LA-11371-MS)

$32-32$

COOK, WILLIAM A. (WX-4).

GENERALIZED FINITE STRAINS, GENERALIZED STRESSES, AND A HYBRID VARIATIONAL PRINCIPLE FOR

FINITE-ELEMENT COMPUTER PROGRAMS.

LOS ALAMOS NATIONAL LABORATORY, 1988. 26P. (LA-11372-MS)

$32-33$

COOK, WILLIAM A. (WX-4); GENIN, JOSEPH.

LARGE NONLINEAR DEFORMATION OF SHELL STRUCTURES.

IN: INTERNATIONAL CONFERENCE ON COMPUTATIONAL ENGINEERING SCIENCE, 1988, ATLANTA. GA.

PROC., P.28.V.1-2. SPRINGER-VERLAG, 1988. (QASO1/.1375/1988)

32-34

COOPER, FREDERICK M. (T-B); GINOCCHIO, JOSEPH N. (T-5); WIPF. ANDREAS (T-B).

DERIVATION OF THE S-MATRIX USING SUPERSYMMETRY.

PHYS. LETT., V.129A, P.145-7. 1988.

32-35

COOPER, RICHARD K. (AT-6); BROWMAN, M. JEAN (AT-6); WEILAND. T.

MAFIA APPROACH TO SOLVING MAXWELL'S EQUATIONS IN THREE DINENSIONS.

TIC, 1988. 11P. MN (LA-UR-88-0978)

32-36

COPT, GAPY P. (LANSCE).

ECOLOGY OF SOFTWARE CONFIGURATION MANAGEMENT.

TIC, 1988. 13P. MN (LA-UR-88-3176) 
DAVIDSON, J. WILEY (A-4); DUDZIAK, DONALD J. (A-4); HIGGS, COLIN E.; STEPANEK, JIPI. ONE- AND TWO-DIMENISIONAL CROSS-SECTION SENSITIVITY AND UNCERTAINTY PATH OF THE AARE [ADVANCED ANALYSIS FOR REACTOR ENGINEEFING] MODULAR CODE SYSTEMS.

TIC, 1988. 9P. MN (LA-UR-88-3371)

32-38

DAVIS, CECIL G. (P-15).

IMPROVEMENTS TO SOIL (AN EULERIAN HYDRODYNAMICS CODE).

LOS ALAMOS, 1988. 23P. (LA-:1199-MS)

$32-30$

DUERING, CHARLES R. (CNLS); GIBBON, JOHN D.; HOLM, DARRYL D. (T-7); NICOLAENKO, BASIL (T-7/CNLS).

LOW-DIMENSIONAL BEHAVIOUR IN THE COMPLEX GINZBURG-LANDAU EOUATION.

NONLINEARITY, V.1, P.279-309. 1988.

$32-40$

DOERING, CHARLES R. (T-7); GIBBON, JOHN 0.; HOLM, DARRYL D. (T-7); NICOLAENKO, BASIL (T-7).

FINITE DIMENSIONALITY IN THE COMPLEX GINZBURG-LANDAU EQUATION.

TIC. 1987. 31P. MN (LA-UR-88-0358)

32-41

DOU, JIMMIE D. (CLS-2); BECK. THOMAS L (CLS-2); FREEMAN, DAVID L.

OUANTUM MONTE CARLO DYNAMICS-THE STATIONAAY PHASE MONTE CARLO PATH INTEGRAL

CALCULATION OF FINITE TEMPERATURE TIME CORRELATION FUNCTIONS.

J. CHEM. PHYS., V.89, P.5753-63. 1988.

$32-42$

DOU, JINMIE D. (CLS-2); FREEMAN, DAVID L; GILAN, M. M.

STATIONARY PHASE MONTE CARLO METHODS-AN EXACT FORMULATION.

CHEM. PHYS. LETT , V.143, P.277-83. 1988.

$32-43$

DONAHUE, STEVE (LANSCE); CORT, GARY P. (LANSCE).

LANSCE [LOS ALANOS NEUTAON SCATTERING CENTER] SOFTWARE MANAGEMENT ENVIRONMENT.

TIC, 1888. 14P. MN (LA-UR-88-1988)

$32-44$

DRE广CER, JARED S. (A-5).

POPA-A PERSONAUTY AND OEJECT PROFILING ASSISTANT.

LOS ALAMOS NATIONAL LABORATOFY, 1988. 30P. (LA-11413-MS)

32-45

DUKOWICZ, JOHN K. (T-3).

EFFICIENT VOLUME COMPUTATION FOR THREE-DIMENSIONAL HEXAHEDRAL CELLS.

J. COMPUT. PHYS., V.74, P.493-6. 1988.

$32-46$

EGDOAF, HARAY W. (A-5); ROBERTS, DOUGLAS J. (A-5).

DISCRETE EVENT SIMULATION IN THE ARTIFICIAL INTELLIGENCE ENVIRONMENT.

TIC, 1987. BP. MN (LA-UR-B7-4256)

$32-47$

FABER, VANCE (C-3); WING. G. NILTON (C-3).

EFFECTIVE BOUADS FOR THE SINGULAR VALUES OF INTEGRAL OPERATORS.

J. INTEGRAL EOUAT. APPL., V.1, P.55-64. 1988.

$32-48$

FABER, VANCE (C-3); SETH, DANIEL L (C-3); WING, G. MILTON (C-3). INVARIANT IMBEDDING IN TWO DIMENSIONS.

TIC, 1988. 23P. MN (LA-UR-88-2512) 
$32-49$

FARMER, J. DOYNE (CNLS/T-7): LAPEDES, ALAN S. (T-DOT): PACKARD, NORMAN H. (CNLS):

WENDROFF, BURTON (T-7).

EVOLUTION, GAMES, AND LEARNING-MODELS FOR ADAPTATION IN MACHINES AND NATURE.

NOATH-HOLLAND, 1986. 402 P. (QP 408 .C46 1985)

32-50

FICKETT, WILDON (M-3).

MATHEMATICAL PROBLEM FROM DETONATION THEORY.

O. APPL. MATH., V.66, P.459-71. 1988.

32-51

FILIPPONE, W. L.; ALCOUFFE, RAYMOND E. (X-6).

SN/MONTE CARLO RESPONSE MATRIX HYBRID METHOD.

NUCL. SCI. ENG., V.100, P.209-17. 1988.

ALSO PUBLISHED: TIC, 1987. 16P. MN (LA-UR-87-0087)

$32-52$

FISHER, HARLO M. (X-6).

XDATAP.

LOS ALAMOS, 1988. 82P. (LA-11155-MS)

32-53

FLORYAN, J. M.; ZEMACH, CHARLES (T-DO).

QUADRATURE RULES FOR SINGULAR INTEGRALS WITH APPLICATION TO SCHWARZ-CHRISTOFFEL MAPPINGS.

J. COMPUT. PHYS., V.75, P.15-30. 1988.

$32-54$

FOWLER, JOHN D. (X-7); MC GOWEN, MICHAEL (C-5).

DESIGN AND IMPLEMENTATION OF A SUPERCOMPUTER FRAME BUFFER SYSTEM.

IN: SUPERCOMPUTING '88, 1988, ORLANDO, FLA. PROC., P.140-7. IEEE COMPUTER SOCIETY, 1988. (OA76.5 S897 1988X)

ALSO PUBLISHED: TIC, 1988. 25P. MN (LA-UR-88-0890)

32-55

FREDERICKSON, PAUL O. (C-3); MC BRYAN, OLIVER A. (C-3).

PARALLEL SUPERCONVERGENT MULTIGRID.

IN: COPPER MOUNTAIN CONFERENCE ON MULTIGAID METHODS, 3RD, 1987, COPPER MOUNTAIN, COLO. PROC., P.195-210. M. DEKKER, 1988. (OA377I.M943/1987X)

ALSO PUBLISHED: TIC, 1987. 21P. MN (LA-UR-87-2329)

$32-56$

FUNITA, R. K. (WX-4); CORT, GEORGE E. (WX-4).

LONG ROD IMPACTS ON OBLIQUE PLATES-THREE-DIMENSIONAL CALCULATIONS WITH HULL. LOS ALAMOS NATIONAL LABORATORY, 1988. 16P. (LA-11455-MS)

32-57

GEORGE, DENISE C. (T-2); LA BAUVE, RAPHAEL J. (T-2).

PERTV-A STANDARD FILE VERSION OF THE PERT-V CODE.

LOS ALAMOS, 1988. 22P. (LA-11206-MS)

$32-58$

GIRRENS, STEVE P. (MEE-13); BENNE7T, JOEL G. (MEE-13).

INFINITE ELEMENT FOR THE ABAOUS FINITE ELEMENT CODE.

LOS ALAMOS NATIONAL LABORATORY, 1988. 27P. (LA-11363-MS)

$32-59$

GUPTA, RAJAN (T-DO).

HYBRID MONTE CARLO ALGORITHM AND THE CHIRAL TRANSITION

TIC, 1987. 1OP. MN (LA-UR-87-4146) 
$32-60$

HALL, JANE H. (P-DO); HIROMOTO, ROBERT E. (C-3).

EXPERIENCES WITH GLOBAL OPTIMIZATION TECHNIQUES IN MASSIVELY PARALLEL PROCESSING ENVIRONMENTS.

TIC, 1988. 1OP. MN (LA-UR-88-1423)

32-61

HANSON, FLOYD; MOHER, THOMAS; SABELLI, NORA; SOLEM, ANN (C-2).

TRAINING PROGRAM FOR SCIENTIFIC SUPERCOMPUTING USERS.

IN: SUPERCOMPUTING'88, 1988, ORLANDO, FLA. PROC., P.342-9. IEEE COMPUTER SOCIETY, 1988. (QA76.5 .S897 1988X)

ALSO PUBLISHED: TIC, 1988. 18P. MN (LA-UR-88-2083)

$32-62$

HANSON, KENNETH M. (M-4).

METHOD TO EVALUATE IMAGE-RECOVERY ALGORITHMS BASED ON TASK PERFORMANCE IN: MEDICAL IMAGING II, 1988, NEWPORT BEACH. PROC., P.336-43. SPIE, 1988.

ALSO PUBLISHED: TIC, 1988. 9P. SNN (LA-UR-88-0111)

$32-63$

HANSON, KENNETH M. (M-4).

POPART-PERFOFMANCE OPTIMIZED ALGEBRAIC RECONSTRUCTION TECHNIOUE.

TIC, 1988. 9P. MN (LA-UR-88-3126)

$32-64$

HODGDON, MARION L. (M-6).

MATHEMATICAL THEORY AND CALCULATIONS OF MAGNETIC HYSTERESIS CURVES.

TIC, 1988. 16P. MN (LA-UR-88-1470)

32-65

HONG, JUNG PYO (E-10); TOMLINSON, ROBERT D. (E-10); PATEL, NISHEETH R.; POLLARD. L. HOWARD. HYPERCUBE PROJECT AND A SIMULATOR FOR A HYPERCUBE OF COMPUTERS.

IN: CONFERENCE ON HYPERCUBE MULTIPROCESSORS, 1ST, 1985, KNOXVILLE. PROC., P.11-18. SIAM. 1986. (OA76.5/.C6192/1985)

ALSO PUBLISHED: TIC, 1985. 9P. MN (LA-UR-86-1151)

32.66

HOSHINO, TSUTOMU; HIROMOTO, ROBERT E. (C-3); SEKIGUCHI, SATOSHI; MAJIMA, SUMIKO.

MAPPING SCHEMES OF THE PARTICLE-IN-CELL METHOD IMPLEMENTED ON THE PAX COMPUTER

PARALLEL COMPUT., V.9, P.53-75. 1988.

32-67

JAEGER, DWIGHT L. (WX-11).

POINT-DEFINED GEOMETRY FOR SOLID MODELING.

LOS ALAMOS NATIONAL LABORATORY, 1988. 17P. (LA-11334-MS)

$32-68$

KENNEOY, F.; BLAU, P.; FRIES, J.; GRATTIDGE, WALT; PETERSON, MARSHALL; REISZ, GERALD E. (ADP-2);

SCHADE, $M$.

BIBLIOGRAPHIC DATABASE

IN: PLANNING WORKSHOP PROGRAM ON COMPUTERIZED TRIBOLOGY INFORMATION SYSTEM

DEVELOPMENT, 1985, GAITHERSBURG, MD. PROC., P.39-44. U.S. G.P.O., 1987. (TJ1075 .T68 1985X)

32-69

KEVREKIDIS, I. G. (CNLS); ECKE, ROBERT E. (P.10).

GLOBAL BIFURCATIONS IN MAPS OF THE PLANE AND IN RAYLEIGH-BENARD CONVECT!ON.

TIC, 1987. 25P. MN (LA-UR-88-3790)

32-70

KRESS, JOEL D. (T-12); WOODFUFF, SUSAN B. (X-7); PARKER, GREGORY A.; PACK, RUSSELL T. (T-12) SOME STRATEGIES FOR ENHANCING THE PERFORMANCE OF THE BLOCK LANCZOS METHOD.

TIC, 1988. 8P. MN (LA-UF-88-2482) 
LANGTON, CHRIS (CNLS).

ARTIFICIAL LIFE.

IN: INTERDISCIPLINARY WORKSHOP ON ARTIFICIAL LIFE, 1987. LOS ALAMOS. N.M. PROC., P.1-47.

ADDISON.WESLEY, 1989. (QH324.8 .A77 1987X)

ALSO PUBLISHED: TIC, 1987. 48P. MN (LA-UR-88-3397)

$32-72$

LAPEDES, ALAN S. (T-DOT); FARBER, ROBERT M. (T-10).

HOW NEURAL NETS WORK.

IN: IEEE CONFERENCE ON NEURAL INFORMATION PROCESSING SYSTEMS, 1ST, 1987, DENVEA,

COLO. PROC., P.442-56. AMERICAN INSTITUTE OF PHYSICS, 1988. (OC76.5 .N45 1988X)

$32-73$

LAPEDES, ALAN S. (T-DOT); FARBER, ROBERT M. (T-10).

NONLINEAR SIGNAL PROCESSING USING NEURAL NETWORKS-PREDICTION AND SYSTEM MODELLING.

TIC, 1987. 52P. MN (LA-UR-87-2662)

32-74

LAYNE, SCOTT P. (CNLS); MARR, T. G. (T-10); STANLEY, E. ANN (T-7); HYMAN, JAMES M. (T-7):

COLGATE, STIRLING A. (T-6).

A NATIONAL HIV [HUMAN IMMUNODEFICIENCY VIRUS] DATABASE THAT FACILITATES DATA SHARING.

TIC, 1388. 13P. MN (LA-UR-88-0125)

32.75

LEE, KENNETH (X-1); LEE, Y. C.; BARNES, CHRISTOPHER W. (CTR-5); ALDRICH, CHARLES H.;

KINDEL, JOSEPH M. $(X-1)$.

TIME SERIES PREDICTION USING A RATIONAL FRACTION NEURAL NETWORK.

TIC, 1980. 7P. MN (LA-UR-88-1203)

$32-76$

LEE, WEN HO (X-7); KWAK, D.

ELASTIC-PLASTIC FLOW USING OPERATOR SPLITTING AND PARTICLE-IN-CELL METHODS IN A

TWO-DIMENSIONAL EULERIAN HYDRODYNAMIC CODE.

LOS ALAMOS, 1988. 63P. (LA-10857)

32- 77

LEE, WEN HO (X-7); PAINTER, JAMES W. ( $X-7)$.

MATERIAL VOID OPENING COMFUTATION USING PARTICLE METHOD.

TIC, 1988. 15P. MN (LA-UR-88-1691)

32-78

LEWIS, PAUL S. (MEE-3).

ALGORITHMS AND ARCHITECTURES FOR ADAPTIVE LEAST SOUARES SIGNAL PROCESSING, WITH

APPLICATIONS IN MAGNETOENCEPHALOGRAPHY.

LOS ALAMOS NATIOPAL LABORATORY, 1988. 298P. (LA-11409-T)

$32-79$

LOUCK, JAMES D. (T-7); BIEDENHARN, LAWRENCE C.: LOHE, MAX A.

ON THE DENOMINATOR FOR CANONICAL SU(3) TENSOR OPERATORS-II. EXPLICIT POLYNOMIAL FORM. J. MATH. PHYS., V.29, P.1106-17. 1988.

32-80

LUBECK, OLAF M. (C-3); MOORE, JAMES W. (C-3); MENDEZ, RAUL.

PERFORMANCE OF THE NEC SX-2 AND CRAY X-MP SUPERCOMPUTERS.

TIC, 1987. 14P. MN (LA-UR-87-0227)

32-81

LUBECK, OLAF M. (C-3).

SUPERCOMPUTER PERFORMANCE-THE THEORY, PRACTICE, AND RESULTS. LOS ALAMOS, 1988. 63P. (LA-11204-MS)

32-82

MANDELL, DAVID A. $(X-7)$; TREASE, HAROLD E. $(X-7)$.

PARALLEL PROCESSING A REAL CODE-A CASE HISTORY.

TIC, 1988. 21P. MN (LA-UR-88-1836) 
MANTEUFFEL, THOMAS A. (C-3); WHITE. ANDREW B., JR. (C-DO).

SECOND-OADER ACCURATE DIFFERENCE SCHEMES ON HIGHLY IRREGULAR MESHES.

TIC, 1988. 7P. MN (LA-UR-88-2832)

32-84

MARGOLIN, LEONARD G.; BURTON, D. E.; CROWLEY, W. P.; TRENT, BRUCE C. (ESS-5).

COMPUTER SIMULATION OF NUCLEAR WEAPONS EFFECTS.

IN: MILITARY COMPUTING CONFERENCE, 2ND, 1988, ANAHEIM, CALIF. PROC., P.17-26. MILITARY

COMPUTING INSTITUTE, 1988. (UG478 .M54 1988X)

$32-85$

MARINUZZI, JOHN G. (DRAAMO).

BUILDING AN ARTIFICIAL INTELLIGENCE CAPABILITY AT LOS ALAMOS.

TIC, 1988. 5P. MN (LA-UR-88-1166)

32-86

MARTIN, E. RAY (N-2); JONES, DAVID F. (JCMAR).

JNC-16-A 16-CHANNEL DATA ACQUISITION AND ANALYSIS MODULE.

LOS ALAMOS NATIONAL LABORATORY, 1988. 20P. (LA-11424-MS)

32-87

MARTINEZ, RONALD (A-5).

RAPID PROTOTYPING OF SIMULATIONS IN ARTIFICIAL INTELLIGENCE ENVIRONMENTS.

TIC. 1988. 8P. MN (LA-UR-87-4255)

$32-88$

MATALON, M.; ROSENAU, PHILIP.

REGULARIZED KS EQUATION DESCRIBING THE FORMATION OF CELLULAR FLAMES.

PHYS. LETT., V.133A, P.23-8. 1988.

32-89

MC BRYAN, OLIVER A. (C-3).

NUWERICAL COMPUTATION ON MASSIVELY PARALLEL HYPERCUBES.

IN: CONFERENCE ON HYPERCUBE MULTIPROCESSORS, 2ND, 1986, KNOXVILLE, TENN. PAOC., P.706-19. SIAM, 1987. (QA76.5/.C5192/1986)

ALSO PUBLISHED: TIC. 1986. 20P. MN (LA-UR-86-4218)

$32-90$

MC KAY, MICHAEL D. (S-1).

SENSITIVITY AND UNCERTAINTY ANALYSIS USING A STATISTICAL SAMPLE OF INPUT VALUES.

IN: UNCERTAINTY ANALYSIS, Y. RONEN, ED., P.145-86. CRC PRESS, 1988. (C/375/.U53/1988)

$32-91$

MC KINSTRIE, C. J. (CNLS).

RELATIVISTIC SOLITARY-WAVE SOLUTIONS OF THE BEAT-WAVE EQUATIONS.

PHYS. FLUIDE, V.31, P.288-97. 1988.

32-92

MC KINSTRIE, C. J. (CNLS); LUTHER, GREG G. (CNLS).

SOLITARY-WAVE SOLUTIONS OF THE GENERALISED THREE-WAVE AND FOUR-WAVE EOUATIONS.

PHYS. LETT., V.127A, P.14-8. 1988.

32-93

METPOPOLIS. NICHOLAS (T-7); ROTA, GIAN-CARLO (DIR OFF).

SYMMETRIC FUNCTIONS-A BIJECTIVE IDENTITY.

PAOC. AM. MATH. SOC., V.102, P.218-20. 1988.

32-94

MICHELSEN, CHRISTIE D. (A-5); DREICER, JARED S. (A-5); MOAGESON, J. DARRELL (A-5).

GRAPHICAL, INTELLIGENT INTERFACE FOR DISCRETE-EVENT SIMULATIONS.

TIC. 1988. 8P. MN (LA-UR-87-3090) 
MILEWSKI, JOHN O. (MST-6); FETZKO, STEPHEN (MST-6); OXXON, RAY D. (MNST-6).

DATA ACOUISTION AND ANALYSIS FOR EB WELOING H' FERSPECTIVE.

WELD. J., V.67, P.255S-63S. 1988.

$32-96$

MILLER, WARREN F., JR. (DDERT).

SUPERCOMPUTING-AN INDISPENSABLE COMPONENT OF APPLIED RESEARCH.

LOS ALAMOS NATIONAL LABORATORY, 1988. 12P. CNSS PAPER NO. 15 (LA-11392-MS)

$32-97$

MNISZEWSKI, SUSAN MARIE (C-10); SPRINGER, FDWARD A. (E-8); RRENNER, DAVID P. (E-5).

METHOD FOR ENCRYPTION AND TRANSMISSION OF DIGITAL KEYING DATA.

PATENTED MAR. 15, 1988, FILED 1985. (U.S.PATENT-4.731,840)

$32-98$

MORSE, NORMAN R. (C-DO).

C-DIVISION ANNUAL REVIEW AND OPERATING PLAN, JANUARY 1988.

LOS ALAMOS, 1938. 159P. (LA-11216-MS)

$32-99$

MURPHY, TIMOTHY C. (ESS-7).

DEVELOPMENT AND TESTING OF KITES

LOS ALAMOS NATIONAL LABORATORY, 1988. 44F. (LA-11329-NS)

32-100

PARTER, SEYMOUR V.; STEUERWALT, MICHAEL $(X-7)$.

ITERATIVE METHODS FOR DISCRETE ELLIPTIC EQUATIONS.

IN: INTERNATIONAL SYMPOSIUM ON COMPUTING METHODS IN APPLIED SCIENCES AND

ENGINEERING, 6TH, 1983, VERSAILLES, FRANCE. PROC., P.47-5E. ELSEVIER, 1984. (QA/297/.157/1983)

32-101

PAXSON, VERN; JACOBSON, VICTORIA; THEIL, ED; LEE, MARTIN J.; CLEAFWATER, SCOTT H. (AT-8).

SCIENTIFIC WORKSTATION OPERATOR-INTERFACE FOR ACCELERATOR CONTROL

IN: IEEE PARTICLE ACCELERATOR CONFERENCE, 1987, WASHINGTON, D.C. PROC., P.556-8. IEEE. 1987. (QC787.P3 137 1987)

32-102

PEDERSON, SHANE (A-1); BECKMAN, RICHARD J. (A-1).

MEAN ESTIMATION IN HIGHLY SKEWED SAMPLES.

TIC, 1989. 14P. MN (LA-UR-88-1590)

$32-103$

PEDERSON, SHANE (A-1).

REVIEW OF "ANALYSIS OF LINEAR MODELS" BY R.R. HOCKING.

J. AM. STAT. ASSOC., V.83, P.271. 1988.

32-104

PETERSON, JANET S. (C-3).

ON THE FINITE ELEMENT APPROXIMATION OF INCOMPRESSIBLE FLOWS OF AN ELECTRICALLY

CONDUCTING FLUID.

NUMER. METHODS PARTIAL DIFFER.EQUAT., V.4, P.57-68. 1988.

32-105

PETERSON, MARSHALL; FORNWALL, MARK; GRATTIDGE, WALT; JAHANMIR, SAID; LOCKWOOD, FRAN;

MONEYHUN, DORA; SHAFER, DONALO F. (A-6).

RESERACH-IN-PROGRESS DATABASE.

IN: PLANNING WOAKSHOP PROGRAM ON COMPUTERIZED TRIBOLOGY INFOAMATION SYSTEM

DEVELOPMENT, 1985, GAITHEASBURG, MD. PROC., P.53-5. U.S. G.P.O., 1987. (TJ1075 .T68 1965X)

32-106

PHILLIPS, RICHARD LANG (C-6)

SCIENTIFIC VISUALIZATION WORKBENCH.

TIC, 1988. 9P. MN (LA-UA-88-2454) 
32-107

PIMBLEY, GEORGE H. (T-14).

SEMIGROUP ASSOCIATED WITH A OUASI-LINEAA SYSTEM IN WHICH THE COUPLING TERMS ARE LINEAR-II.

NONLINEAR ANAL. THEOAY HETHODS APPL., V.12, P.321-40. 1988.

$32-108$

POLLAATD. L. HOWARD; CYRUS, TAIT; THERMAN, RUSSEL: HONG, JUNG PYO (MEE-10);

KELLNER, FICHARD G. (E-10); TOMLINSON, ROBERT D. \{E-10).

UNMHC-THE LOS ALAMOS AND UNIVERSITY OF NNEW MEXICO HYPERCUBE.

IN: CONFERENCE ON HYPERCUBE MULTIPROCESSORS, 2ND, 1986, KNOXVILLE, TENN. PROC., P.271-5. SIAM, 1987. (OA76.5/.C6192/1986)

32-109

POORE, RAY V. (P-9); BYANES, LORIE L. (MP-6); HARAINGTON, MARGYE P. (MP-6);

STUEWE, ROBERT B. (AF-6); WESTERVELT, R. T. (E-11)

IMPLEMENTATION OF A DISTRIBUTED COMPU'TER CONTROL SYSTEM AT THE LOS ALAMOS PAOTON STORÁGE RING.

TIC, 1988. 6P. MN (LA-UR-88-3414)

32-110

OUEST. KEVIN B. (X-1).

HYBRID SIMULATIONS.

TIC. 1987. 7P. MN (LA-UR-88-1276)

32-111

SALZMAN, GARY C. (LS-2); KRALL, RANDY B. (LS-2); MARINUZZI, JOHN G. (DRAAMO).

KNOWLEDGE ENGINEERING SOFTWARE-A. DENONSTRATION OF A HIGH END TOOL. TIC, 1987. 25P. MN (LA-UR-87-3924)

32-112

SANDERS, CLAUDIA (C-2).

MDOC MACRO PACKAGE-A SOFTWARE TOOL TO SUPPORT COMPUTER DOCUMENTATION STANDARDS. IN: INTERNATIONAL TECHNICAL COMMUNICATION CONFERENCE, 35TH, 1988, PHILADELPHIA, PA. PROC., P.WE-162-5. SOCIETY FOR TECHNICAL COMMUNICATION, 1988. (T10.5 .I57 1988X)

ALSO PUBLISHED: TIC, 1987. 13P. MN (LA-UR-87-3129)

$32-113$

SCHALLER, STUART C. (MP-1); BNORKLUND, ERIC A. (MP-1).

DISTAIBUTED DATA ACCESS IN THE LAMPF CONTROL SYSTEM.

IN: IEEE PARTICLE ACCELERATOR CONFERENCE, 1987, WASHINGTON, D.C. PROC., P.745 7. IEEE, 1987. (OC787.P3 137 1987)

$32-114$

SCHNEIDER, BARRY I. (T-12); COLLINS, LEE A. (T-11).

DIRECT ITERATIVE-VARIATIONAL METHOD FOR SOLVING LARGE SETS OF LINEAR ALGEBRAIC

EOUATIONS.

TIC, 1988. 20P. MN (LA-UR-88-2823)

32-115

SILVA, ALEX A. (AT-2); RANGEL, MANUAL (AT-5); HOEBERLING, ROBERT FREDERICK (AT-5).

PHOTODIGTIZE-A SOFTWARE/HARDWARE SYSTEM.

LOS ALAMOS NATIONAL LABORATORY, 1988. 9P. (LA-11272-MS)

32-116

SIMMONS, MARGARET L. (C-3); LUBECK, OLAF M. (C-3).

BENCHMARK OF THE CONVEX C-1 MINI SUPERCOMPUTER.

TIC, 1986. 7P. MN (LA-UR-86-2890)

$32 \cdot 117$

SIMMONS, MARGARET L. (C-3); WASSERMAN, HARVEY J. (C-3).

PERFORMANCE COMPARISON OF THE CRAY-2 AND CRAY X-MP/416 SUPERCOMPUTERS

IN: SUPERCOMPUTING '88, 1980, ORLANDO, FLA. PROC., P.288-95. i.EEE COMPUTER SOCIETY 1988. (OA76.5 .S897 1988X)

ALSO PUBLISHED: TIC, 1988. 9P. NN (LA-UR-88-2391) 
SIROTKIN, KARL (T-10).

COMPUTER PROGRAM TO DISPLAY CODON CHANGES CAUSED BY MUTAGENESIS

CABIOS, V.4. P.243-7. 1988.

32-119

SMITH, WAYNE (MP-1); BJORKLUND, ERIC A. (MP-1).

CAMAC-BASED KNOB CONTROLLER FOR THE LAMPF CONTROL SYSTEM

IN: IEEE PARTICLE ACCELERATOR CONFERENCE, 1987, WASHINGTON, D.C. PROC., P.544-6. IEEE, 1987. (QC787.P3 I37 1987)

$32-120$

SORSBY, CHARLES R. (MEE-10).

ASSIGNMENT OF POINTS TO THREE-DIMENSIONAL TRAJECTORIES.

LOS ALAMOS NATIONAL LABORATORY, 1988. 133P. (LA-11425-T)

32-121

STUTZ, ROGER ALAN (ENG-4); ZOCHER, MARC (ENG-8).

NEW DEVELOPMENTS IN CAPITAL, COST ESTIMATING.

TIC, 1988. 7P. MN (LA-UA-88-0194)

32-122

SWARTZ, BLAIR K. (T-7).

CONDITIONING COLLOCATION.

SIAM J. NUMER. ANAL., V.25, P.124-47. 1988.

32-123

TABOR, JAMES E. (C-10).

DYNAMIC RUN-TIME GRAPHICS.

TIC, 1987. 16P. MN (LA-UR-87-1270)

32-124

THORN, CHARLES T. (A-6)

APPLICATION OF COMPUTERS TO LEARNING IN THE COMMAND AND GENERAL STAFF COLLEGE (CGSC)-A FRONT END ANALYSIS STUDY-CGSC ANALYSIS, TASK A.

TIC, 1988. 68P. MN (LA-UR-88-1491)

32-125

TOLMIE, DONALD E. (C-5).

HIGH-SPEED CHANNEL (HSC) STANDARD.

TIC, 1988. 5P. MN (LA-UR-88-3903)

32-126

TORNEY, DAVID C. (T-10)

EIGENMODES OF A RANDOM WALK ON A 2-D LATTICE TORUS WITH ONE TRAP.

TIC, 1988. 15P. MN (LA-UR-88-0915)

$32-127$

TRAINOR, MARY STODDARD (A-6).

SELECTING AN AUTHORING SYSTEM 1988-HOW THE VENDORS MAKE IT MORE DIFFICULT.

TIC, 1988. 8P. MN (LA-UR-88-2572)

32-128

VIGIL, BENNY MANUEL (C-6); BOUCHIEA, STEPHANY C. (C-6); SANDERS, CLAUDIA (C-2);

SYDORIAK, STEPHEN J. (P-4); WHEELER, KYLE T. (C-2).

DOCUNENT PREPARATION SYSTEM IN A LARGE NETWORK ENVIRONMENT.

TIC. 1988. 12P. MN (LA-UA-B8-1792)

$32-129$

WALTERS, WALLACE F. (X-6).

USE OF THE CHEBYSHEV-LEGENDRE OUADRATURE SET IN DISCRETE-ORDINATE CODES.

TIC, 1987. 11P. MN (LA-UR-87-3621) 
32-130

WASSERMAN, HARVEY J. (C-3); SIMMONS, MARGARET L. (C-3); HAYES, ANN H. (C-3).

BENCHMARK OF THE SCS-40 COMPUTER-A MINI SUPERCOMPUTER COMPATIBLE WITH THE CAAY $X-M P / 24$.

TIC, 1987. 1OP. MN (LA-UR-87-0659)

32-13†

WASSERMAN, HARVEY J. (C-3).

LOS ALAMOS NATIONAL LABORATORY COMPUTER BENCHMARKING 1988.

LOS ALAMOS NATIONAL LABORATORY, 1988. 19P. (LA-11465-MS)

$32-132$

WEIR, DIANE R. (ADP-2).

HOW EXCELERATOR CHANGED MY JOB.

TIC. 1988. 10P. MN (LA-UR-88-2781)

32-133

WENDROFF, BURTON (T-7); WHITE, ANDREW B., JR. (C-3)

SOME SUPRACONVERGENT SCHEMES FOR HYPERBOLIC EQUATIONS ON IRREGULAR GRIDS.

TIC, 1988. 7P. MN (LA-UR-88-1394)

32-134

WENES, GEERT ( $\gamma-5)$; GINOCCHIO, JOSEPH N. (T-5).

ALGEBRAIC APPROACH TO MEDIUM ENERGY PROTON SCATTERING.

IN: INTERNATIONAL SYMPOSIUM ON CAPTURE GAMMA-RAY SPECTROSCOPY AND RELATED TOPICS.

6TH, 1987, LEUVEN, BELGIUM. PFOC., P.S65-70. INSTITUTE OF PHYSICS, 1988. (OC1.157X NO.88)

32-135

WEST, JAMES T. (X-6); MURPHY, JACK.

SABRINA - AN INTERACTIVE GEOMETRY MODELER FOR MCNP [MONTE CARLO NEUTRON PHOTON].

TIC, 1988. 7P. MN (LA-UR-88-3612)

32- 136

WII_SON, TIMOTHY L (Q-6); NICHOLS, BILL D. (N-6); HIRT, CYRIL W., JR.: STEIN. LELAND R. (T-3).

SOLA-DM-A NUMERICAL SOLUTION ALGORITHM FOR TRANSIENT THREE-DIMENSIONAL FLOWS.

LOS ALAMOS, 1988. 91P. (LA-11161-NS)

32-137

WOLOSHUN, KEITH A. (MEE-13); MERRIGAN, MICHAEL A. (MEE-13); BEST, ELAINE D. (MEE-13).

HTPIPE-A STEADY-STATE HEAT PIPE ANALYSIS PROGRAM-A USER'S MANUAL

LOS ALAMOS NATIONAL LABORATORY, 1988. 97P. (LA-11324-M)

32-138

YAMADA, TETSUJI (ESS-5); WILLIAMS, MICHAEL D. (A-4).

DEVELOPMENT OF FORECASTING CAPABILITY BY USING MICROCOMPUTERS.

TIC, 1988. 23P. MN (LA-UR-88-1394)

32-139

YANG, MING-JEN (P-DO).

MONTE CÚRLO CODE FOR A TIME PROJECTION CHAMBER.

NUCL INSTRUM. METHODS PHYS. RES., V.A270. P.126-39. 1988. 
33. NUCLEAR MATERIALS

33-1

GLADNEY, E. S. (HSE-9).

DETERMINATION OF URANIUM IN GSJ, CRPG, AND CCRMP REFERENCE SAMPLES BY DELAYED NEUTRON ASSAY.

GEOSTAND. NEWSL., V.9, P.275-6. 1985

33-2

GOFF, FRASER E. (ESS-1); GARDNER, JAMIE N. (ESS-1).

ISOTOPE GEOCHEMISTRY OF SULPHUR SPRINGS, AN ACID-SULFATE SYSTEM IN VALLES CALDEFA, NEW MEXICO.

IN: NEW ZEALAND GEOTHERMAL WORKSHOP, 5TH, 1983, UNIVERSITY OF AUCKLAND. PROC.,

P.145-49. UNIVERSITY OF AUCKLAND GEOTHERMAL INSTITUTE, 1983. 
34-1

BOHL, RICHARD J. (Q-6).

SP-100 SURETY AND NUCLEAR TECHNOLOGY-ANNUAL REPORT FOR FISCAL YEAR 1984. LOS ALAMOS, 1985. 384P. (LA-10318-PA)

34-2

GEORGE, TIMOTHY G. (MST-5).

SPACE NUCLEAR SAFETY PROGRAM APAIL - JUNE 1987.

LOS ALAMOS NATIONAL LABORATORY, 1988. 13P. (LA-11308-PR)

34-3

GEORGE, TIMOTHY G. (MST-5).

SPACE NUCLEAR SAFETY PROGRAM JANUARY - MARCH 1987.

LOS ALAMOS NATIONAL LABORATORY, 1988. 15P. (LA-11274-PR)

34-4

VOSS, SUSAN S. (N-12); MC DANIEL, PATRICK J.; WOODALL, DAVID M.

BURNUP VERSUS CPITICALITY LIMITATIONS FOR A POTENTIAL SPACE REACTOR DESIGN IN THE 1 TO 100 MEGAWATT RANGE.

IN: SYMPOSIUM ON SPACE NUCLEAR POWER SYSTEMS, 3RD, 1986, ALBUOUEROUE, N.M. PROC.,

P.427-34. ORBIT BOOK CO., 1987. (TL1102.N8 S96 1986)

34-5

ZOCKER, ROY W. (MST-5).

STATEMENT OF DR. ROY W. ZOCKER.

REVIEW OF RTG UTILIZATION IN SPACE MISSIONS. HEARING BEFORE THE SUBCOMMITTEE ON

ENERGY RESEAHCH AND PRODUCTION AND THE SUBCOMMITTEE ON SPACE SCIENCE AND APPLICATIONS OF THE COMMITTEE ON SCIENCE AND TECHNOLOGY, U.S. HOUSE OF REPRESENTATIVES, WASHINGTON, D.C., MAR. 4, 1986. P.116-9. U.S. G.P.O., 1986. (TL1102.N8 R47 1986XA) 
35.1

ABEELE, WILLY V. (HSE-12); NYHAN, JOHN W. (HSE-12); DRENNON, BARRY J. (HSE-12).

TECHNOLOGY DEVELOPMENT FOR THE DESIGN OF SHALLOW LAND BURIAL FACILITIES AT S-MIARID SITES.

TIC, 1986. 14P. MN (LA-UR-86-3243)

35-2

BECKMAN, RICHARD J. (A-1), THOMAS, KIMBERLY W. (INC-11); CROWE, BRUCE M. (INC-7).

PAELIMINARY REPORT ON THE STATISTICAL EVALUATION OF SORPTION DATA-SOAPTION AS A

FUNCTION OF MINEAALOGY, TEMPERATURE, TIME, AND PARTICLE SIZE.

LOS ALAMOS NATIONAL LABORATORY, 1988. 26P. (LA-11246-MS)

35-3

BISH, DAVID L. (ESS-1).

SMECTITE DEHYDAATION AND STABILITY-APPLICATIONS TO RADIOACTIVE WASTE ISOLATION AT YUCCA MOUNTAIN, NEVADA.

LOS ALAMOS, 1988. 31P. (LA-11023-MS)

35-4

BOOKER, JANE M. (A-1).

COMBINING OUALITATIVE AND OUANTITATIVE DATA-THE USE OF SUBJECTIVE JUDGMENT MODELING.

TIC, 1988. 23P. MN (LA-UR-88-1675)

$35-5$

CAMPBELL, KATHERINE (A-1).

STATISTICAL GUIDELINES FOR PLANNING A LIMITED DRILLING PROGRAM.

LOS ALAMOS NATIONAL LABORATORY, 1988. 54P. (LA-11293-MS)

35-6

CEDERBERG, GAIL A. (ESS-5); GREENWADE, L. ERIC (ESS-5).

PRELIMINARY GEOCHEMICAL/GEOPHYSICAL MODEL OF YUCCA MOUNTAIN.

IN: INTERNATIONAL SYMPOSIUM ON THE SCIENTIFIC BASIS FOR NUCLEAR WASTE MANAGEMENT, 11TH, 1987, BOSTON, MASS. PROC., P.331-40. MATERIALS RESEARCH SOCIETY, 1988. (TA401 .MBTX V.112)

ALSO PUBLISHED: TIC, 1987. 14P. MN (LA-UR-87-3854)

35-7

CHIPERA, STEVE J. (ESS-1); BISH, DAVID L. (ESS-1).

MINERALOGY OF DRILL HOLE UE-25P\#1 AT YUCCA MOUNTAIN, NEVADA.

LOS ALAMCS, 1988. 24P. (LA-11292-MS)

35-8

DEVAURS, M. A. (HSE-12); SPRINGER, EVERETT P. (HSE-12).

REPRESENTING SOIL MOISTURE IN EXPERIMENTAL TRENCH COVER DESIGNS FOR WASTE BURIAL WITH THE CREAMS MODEL.

HAZARD. WASTE HAZARD. MATER., V.5, P.295-312. 1988.

$35-9$

DEVAURS, M. A. (HSE-12).

USE OF THE CREAMS MODEL TO REPRESENT SOIL MOISTURE UNDER VARIOUS TRENCH COVER

DESIGNS FOR WASTE BURIAL.

TIC, 1988. 4P. MN (LA-UR-88-1560)

35-10

FOWLER, ERIC B. (LS-6); POLZER, WILFRED L. (HSE-12).

REVIEW OF RESEARCH CONDUCTED BY LOS ALAMOS NATIONAL LABORATORY FOR THE NRC WITH

EMPHASIS ON THE MAXEY FLATS, KY, SHALLOW LAND BURIAL SITE.

LOS ALAMOS NATIONAL LABORATORY, 1988. 68P. (LA-11354-MS) 
35-11

FUENTES, H. R. (HSE-12); ESSINGTON, EDWARD H. (HSE-12): POLZER, WILFAED L. (HSE-12). SOLUTE LEACHING FROM RESINITUFF MEOIA IN UNSATURATED FLOW-EXPERIMENTS AND CHARACTERIZATION.

RADIOACT.WASTE MANAGE.NUCL.FUEL CYCLE V.10, P.285-320. 1988.

35-12

LOPEZ, EDWARD A. (HSE-12); BA.RNES, FAIRLEY J. (HSE-12); ANTONIO, ERNEST J. (HSE-12).

EFFECTS OF VEGETATION AND SOIL-SURFACE COVER TREATMENTS ON THE HYDROLOGIC BEHAVIOR OF LOW-LEVEL WASTE TRENCH CAPS.

TIC, 1988. 14P. MN (LA-UR-88-0391)

35-13

NORRIS, ANDREW E. (INC-7); WOLFSBERG, KURT (INC-7); GIFFORD, S. K.; BENTLEY, HAROLD W.;

ELMORE, DAVID.

INFILTRATION AT YUCCA MOUNTAIN, NEVADA, TRACED BY CHLORINE-36.

NUCL. INSTRUM. METHODS PHYS. RES., V.B29, P.376-9. 1987.

ALSO PUBLISHED: TIC, 1987. 7P. MN (LA-UR-87-2252)

35-14

NYHAN, JOHN W. (HSE-12); ABEELE, WILLY V. (HSE-12).

DEVELOPMENT OF CORRECTIVE MEASURES AND SITE STABILIZATION TECHNOLOGIES FOR SHALLON LAND BURIAL FACILITIES AT SEMIARID SITES.

TIC, 1986. 16P. MN (LA-UR-86-3244)

35-15

ROFER, C. K. (ESS-1): STREIT, GERALD E. (A-4).

KINETICS AND MECHANISM OF METHANE OXIDATION IN SUPEACRITICAL WATER.

LOS ALAMOS NATIONAL LABORATORY, 1988. 32P. (LA-11439-MS)

$35-16$

THOMAS, KIMBERLY W. (INC-11)

RESEARCH AND DEVELOPMENT RELATED TO THE NEVADA NUCLEAR WASTE STORAGE INVESTIATKONS OCTOBER 1-DECEMBER 31, 1984.

LOS ALAMOS NATIONAL LABORATORY, 1988. 96P. (LA-11443-PR)

35-17

THOMPSON, JOE L. (INC-11).

LABORATORY ANO FIELD STUDIES RELATED TO THE RADIONUCLIDE MIGRATION PROJECT, OCTOBER 1. 1986-SEPTEMBER 30, 1987.

LOS ALAMOS, 1988. 31P. (LA-11223-PR)

$35-18$

VANIMAN, DAVID T. (ESS-1); BISH, DAVID L. (ESS-1); CHIPERA, STEVE J. (ESS-1).

PRELIMINARY COMPARISON OF MINERAL DEPOSITS IN FAULTS NEAR YUCCA MOUNTAIN, NEVADA, WITH POSSIBLE ANALOGS.

LOS ALAMOS NATIONAL LABORATORY, 1988. 54P. (LA-11289-MS)

35-19

WEST, KAREN A. (WX-4).

NEVADA NUCLEAR WASTE STORAGE INVESTIGATIONS EXPLORATORY SHAFT FACILITY FLUIDS AND

MATERIALS EVALUATION.

LOS ALAMOS NATIONAL LABORATORY, 1988. 152P. (LA-11398-MS)

35-20

ZYVOLOSKI, GEORGE A. (T-3): DASH, ZORA V. (ESS-4); KELKAR, SHARAD (ESS-4).

FEHM-FINITE ELEMENT HEAT AND MASS TRANSFER CODE.

LOS ALAMOS, 1988. 55P. (LA-11224-MS) 
37-1

BHATIA, TAROLCHAN S.; GUY, FRANK W. (AT-1); NEUSCHAEFER, G. H. (AT-1); PABST, $M$;

SCHRIBER, S. O. (AT-DO); STOVALL, JAMES E. (AT-1); WANGLER, THOMAS P. (AT-1);

WILSON, MAHLON T. (AT-DO); WORTH, G. T. (AT-2).

SSC LINAC INJECTOR.

TIC, 1988. 11P. MN (LA-UR-88-3909)

37-2

BIGIO, IRVING J. (CLS-5).

LASERS FOR SWITCHED-POWER LINACS.

TIC, 1988. 9P. MN (LA-UR-88-2374)

37-3

BILLEN, JAMES H. (AT-1).

ANALYSIS OF MEASURED POST-COUPLER FIELDS IN A RAMPED-GRADIENT DRIFT-TUBE LINAC

TIC, 1988. 3P. MN (LA-UA-88-3248)

$37-4$

BILLEN, JAMES H. (AT-1); SPALEK, GEORGE (AT-1): SHAPIAO, ALAN H. (AT-1).

FIELD STABILITY IN TWO-STCM DRIFT-TUBE LINACS.

TIC, 1988. 3P. MN (LA-UF-88-3262)

37-5

BILLEN, JAMES H. (AT-1); SHAPIRO, ALAN H. (AT-1)

POST-COUPLER STABILIZATION AND TUNING OF A RAMPED-GRADIENT DRIFT-TUBE LINAC.

TIC, 1988. 4P. MN (LA-UR-88-3264)

37-6

BOICOURT, GRENFELL P. (AT-6); MERSON, JEAN L. (AT-6).

CALCULATIONS ON PERMANENT-MAGNET OUADRUPOL $5 S$ WITH NONRECTANGULAR CPOSS SECTION. TIC, 1988. 4P. MN (LA-UR-88-3149)

37-7

BOICOURT, GRENFELL P. (AT-6).

PARMILA [PHASE AND RADIAL MOTION IN LINEAR ACCELERATOAS]-AN INTRODUCTION.

IN: WORKSHOP ON LINEAR ACCELERATOR AND BEAM OPTICS CODES, 1988, SAN DIEGO, CALIF. PROC., P.1-21. AMERICAN INSTITUTE OF PHYSICS, 1988. (CC1 .A38X NO.177)

ALSO PUBLISHED: TIC, 1988. 23P. MN (LA-UR-88-1544)

37-8

BOWLING, PLEAS S. (AT-8); BROWN, STANLEY K. (AT-8).

SEPARATION OF CONTROL VARIABLES IN AN HYDROGEN MINUS ION SOURCE.

TIC, 1988. 6P. MN (LA-UR-88-1826)

$37-9$

BRADLEY, JILL P. (WX-11).

CW RFO CORE-TANK STRUCTURE ANALYSES.

LOS ALAMOS, 1988. 41P. (LA-11164-MS)

$37-10$

BRAU, CHARLES A. (AT-7),

RF-LINAC FREE-ELECTRON LASERS.

IN: FREE-ELECTFON LASERS, 1987, LOS ANGELES. PROC., P.84-102. SPIE, 1988. (TA1677.F73 1987X)

37-11

BROWMAN, M. JEAN (AT-6): SPALEK, GEORGE (AT-1); FAIEDRICHS, P. B. (AT-1); BARTS. THERESE C. (AT-6).

STUDIES OF THE FOUR-ROD RFO IRADIO-FREQUENCY QUADRUPOLE] USING THE MAFIA CODES.

TIC, 1998. 4P. MN (LA-UR-88-3263) 
37-12

BROWMAN, M. JEAN (AT-6); SPALEK, GEORGE (AT-1); BARTS. THERESE C. (AT-6).

STUDYING THE END REGIONS OF RFOS USING THE MAFIA CODES.

TIC, 1988. 3P. MN (LA-UR-88-3276)

37-13

BROWMAN, M. JEAN (AT-6); COOPER, RICHARD K. (AT-6); FRIEDRICHS, CARL C. (AT-5); WEILAND, T. TESTING URMEL-3D BY MODELING A FERRITE-TUNED RF CAVITY.

IN: IEEE FARTICLE ACCELERATOR CONFERENCE, 1987, WASHINGTON, D.C. PROC., P.1892-1900. IEEE, 1987. (QC787.P3 137 1987)

ALSO PUBLISHED: TIC, 1987. 4P. MN (LA-UR-87-0763)

37-14

BURCZYK, LEONARD; DALESIO, LEO R. (AT-8); DINGLER, ROBERT D. (AT-B); HILL, J. O. (AT-8);

HOWELL, JO ANN (AT-8); KERSTIENS, DEBORA M. (AT-8); KING, REBECCA L. (AT-8);

KOZUBAL, ANDREW J. (AT-8); LITTLE, CYNTHIA K. (AT-8); MARTZ, VIRGINIA E. (AT-8);

ROTHROCK, RICHARD B. (AT-8); SUTTON, JOHN G. (AT-8).

GROUND TEST ACCELERATOR CONTROL SYSTEM SOFTWARE.

TIC, 1988. 8P. MN (LA-UR-88-1537)

37-15

CARLSTEN, BRUCE E. (AT-7).

EMITTANCE GROWTH CAUSED BY BENDS IN THE LOS ALANOS FREE-ELECTRON LASER ENERGY

RECOVERY EXPERIMENT.

IN!: IEEE PARTICLE ACCELERATOR CONFERENCE, 1987, WASHINGTON, D.C. PROC., P.1755-7. IEEE, 1987. (QC787.P3 137 1987)

ALSO PUBLISHED: TIC. 1987. AP. MN (LA-UR-87-0862)

$37-16$

CARLSTEN, BRUCE E. (AT-7).

NEW PHOTOELECTRIC INJECTOR DESIGN FOR THE LOS ALAMOS NATIONAL LABORATORY XUY FEL ACCELERATOR.

TIC, 1988. 16P. MN (LA-UR-88-3328)

$37-17$

CARLSTEN, BAUCE E. (AT-7); SHEFFIELD, RICHARD L. (AT-7).

PHOTOELECTRIC INJECTOR DESIGN CONSIDERATIONS.

TIC, 1988. 5P. MN (LA-UR-88-3327)

37.18

CARLSTEN, BRUCE E. (AT-7); CHAÑ, K. C. (AT-б).

PRELIMINAFY INJECTOR, ACCELERATOR, AND BEAMLINE DESIGN FOA RF-LINAC-DPIVEN XUV

FREE-ELECTRON LASERS.

TIC, 1987. 3OP. MN (LA-UR-87-3092)

37-19

CHAN, K. C. (AT-6); COUPER, RICHARD K. (AT-6).

LTRACK-BEAM-TRANSPORT CALCULATION INCLUDING WAKEFIELD EFFECTS.

IN: WORKSHOP ON LINEAR ACCELERATOR AND BEAM OPTICS CODES, 1988, SAN DIEGO, CALIF.

PROC., P.37-44. AMERICAN INSTITUTE OF PHYSICS, 1988. (QC1 A38X NO.177)

ALSO PUBLISHED: TIC, 1988. 10P. MN (LA-UR-88-1543-REV)

37-20

CHAN, K. C. (AT-6); FRASER, JOHN S. (AT-7).

MINIMUM BEAM-ENERGY SPREAD OF A HIGH CURRENT RF LINAC.

IN: IEEE PARTICLE ACCELERATOR CONFERENCE, 1987, WASHINGTON, D.C. PROC., P. 1075-80. IEEE. 1987. (QC787.P3 137 1987)

$37-21$

CHOW, WENG; SAXMAN, A. C. (CLS-8); MC VEY, BRIAN D. (X-1): APPERT, QUENTIN D. (CLS-8);

BENDER, STEVEN C. (CLS-8).

PHASED ARRAY CONCEPTS FOR FREE-ELECTRON LASERS.

APPL. OPT., V.27, P.1370-1. 1988. 
37-22

CLEARWATER, SCOTT H. (AT-8); LEE, MAATTIN J.

PROTOTYPE DEYELOPMENT OF A BEAM LINE EXPERT SYSTEM.

IN: IEEE PARTICLE ACCELERATOF CONFERENCE, 1987, WASHINGTON, D.C. PROC., P.532-4. IEEE. 1987. (CC787.P3 137 1987)

37.23

COLTON, EUGENE P. (MP- 1 A ): THIESSEN, HENRY A. (MP-10).

H MINUS INJECTION INTO THE LOW-ENERGY BOOSTER OF THE SSC.

TIC, 1988. 5P. MN (LA-UA-88-3954)

$37-24$

COLTON, EUGENE P. (MP-14); NEUFFER, DAVID (AT-6); THIESSEN, HENRY A. (MP-14);

BUTLER, HAROLD S. (MP-DO); SWAIN, GEORGE R. (MP-14; LOMBARDI. A. (MP-14);

FITZGERALD, D. H. (MP-5); MARIAM, FRANK (MP-13); PLUM, MICHAEL A. (MP-5); FYOER, F. (MP-5):

MACEK, ROBERT J. (MP-DO); CLARK, DAVIC A. (MP-5); HURD, JAMES W. (MP-5); HUTSON, RICHARD L. (MP-5):

MC GILL. JOHN A. (MP-13); HARDEK, THOMAS W. (AT-3); SHAFER, R. (AT-3); LAWRENCE, GEORGE P. (AT-3):

JASON, ANDREW JOHN (AT-3); BLIND, BARBARA (ATi-3); HARDEKOPF, ROBERT A. (AT-3).

STATUS REPORT ON THE LOS ALAMOS PROTON STORAGE RING.

TIC. 1988. 7P. MN (LA-UA-88-1800)

37-25

COLTON, EUGENE P. (MP-14)

STRAWMAN i.6-GEV COMPRESSOR DESIGN.

IN: AOVANCED HADRON FACILITY ACCELERATOR DESIGN WORKSHOP, 1988, LOS ALAMOS NATIONAL LABORATORY. PROC., P.444-60. LOS ALANOS NATIONAL LABORATORY, 1989. (LA-11432-C)

ALSO PUBLISHED: TIC, 1988. 18P. MN (LA-UR-88-1797)

37.26

COOPER, RICHARD K. (AT-6); MENZEL, MARY TSINGOU (AT-6), STOKES, HELEN K. (AT-6);

WARREN, JOHN L. (AT-6).

NEW DOCUMENTATION FROM THE LOS ALAMOS ACCELERATOR CODE GROUP

IN: IEEE PAHTICLE ACCELERATOR CONFERENCE, 1987, WASHINGTON, D.C. PROC., P.1780-4. IEEE, 1987. (OC787.P3 137 1987)

37-27

COOPER, RICHARD K. (AT-6); BROWMAN, M. JEAN (AT-6); WEILAND. T. (AT-6).

THREE-DIMENSIONAL RF STRUCTURE CALCULATIONS

TIC, 1988. 29P, MN (LA-UR-88-3205)

37-28

CRANDALL, KENNETM R. (AT-1); WANGLER, THOMAS P. (AT-1).

PARMTEQ [PHASE AND RADIAL MOTION IN TRANSVERSE ELECTRIC QUADRUPOLE\}-A BEAM DYNAMHICS CODE FOR THE RFO LINEAR ACCELERATOR.

IN: WORKSHOP ON LINEAR AC',ELERATOR AND BEAM OPTICS CODES, 1988, SAN DIEGO, CALIF.

PROC., P.22-8. AMERICAN INSTITITE OF PHYSICS, 1988. (OC1 .A3BX NO.177)

ALSO PUBLISHED: TIC, 1987. 8P. MN (LA-UR-88-1546)

$37-29$

DOMBECK, THOMAS W. (P-3).

PERFORMANCE OF THE LOS ALAMOS EXPANDING TELESCOPE.

TIC, 1988. 16P. MN (LA-UR-88-1476)

37-30

EATON, LAWRIE (AT-5); JACHIM, STEPHEN (AT-5); NATTER, ECKARD (AT-5).

ACAPTIVE CONTROL TECHNIOUE FOR ACCELERATORS USING DIGITAL SIGNAL PAOCESSING (DSP)

TECHNOLOGY.

TIC. 1987. 4P. MN (LA-UR-87-3258)

37-31

EDESKUTY, FREDERICK J. (A-4): STEWART, WALTER F. (A-4)

PRELIMINARY DESCRIPTIDN OF THE GROUND TEST ACCELERATOR CFIYOGENIC COOLING SYSTEM. TIC, 1988. 15P. MN (LA-UR-88-2498) 
37.32

FAZIO, MICHAEL V. (AT-5); HOEBERLING, ROBEAT FREDERICK (AT-5).

EXPERIMENTAL PROGRESS ON VIRTUAL CATHODE, VERY HIGH POWER, MICROWAVE SOURCE

DEVELOPMENT.

IN: IEEE PARTICLE ACCELERATOR CONFERENCE, 1987, WASHINGTON, D.C. PROC., P.1921-3. IEEE. 1987. (CC787.P3 137 1987)

ALSO PUBLISHED: TIC, 1987. 4P. MN (LA-UR-87-0872)

$37-33$

FAZIO, MICHAEL V. (AT-5); KINROSS-WRIGHT, JOHN (AT-5); HOEBERLING, ROBERT FREDERICK (AT-5):

VAN HAAFTEN, F. W. (AT-5).

HIGH-POWER MICROWAVE SOURCE DEVELOPMENT AT LOS ALAMOS.

TIC, 1988. 4P. MN (LA-UA-88-3278)

37-34

FELDMAN, DONALD W. (AT-7); WARREN, ROGER W. (AT-7); WATSON, J. M. (AT-7); STEIN, WILLIAM E. (AT-7); FRASER, JOHN S. (AT-7); SPALEK, GEORGE (AT-3): LUMPKIN, ALEX H. (P-DO); CARLSTEN, BRUCE E. (AT-7); TAKEDA, HARUNORI (AT-6); WANG, TAI-SEN F. (AT-6); BRAU, CHARLES A. (DAANEW).

LOS ALAMOS FREE-ELECTAON LASER ENERGY-RECOVERY EXPERIMENT.

IN: IEEE PARTICLE ACCELERATOR CONFERENCE, 1987, WASHINGTON, D.C. PROC., P.221-23. IEEE. 1987. (OC787.P3 137 1987)

ALSO PUBLISHED: TIC, 1987. 4P. MN (LA-UR-87-0633)

37-35

FELDMAN, DONALD W. (AT-7); WARAEN, ROGER W. (AT-7); CARLSTEN, BRUCE E. (AT-7):

STEIN, WILLIAM E. (AT-7); LUMPKIN, ALEX H. (P-15); BENDER, STEVEN C. (CLS-8); SPALEK, GECRIGE (AT-1); WATSON, J. M. (AT-7); YOUNG, LLOYD M. (AT-1); FRASER, JOHN S. (AT-7); GOLDSTEIN, JOHN C. (X-1);

TAKEDA, HARUNORI (AT-6); 'NANG, TAI-SEN F. (AT-6); CHAN, KWOK-CHI D. (AT-6); MC VEY, BRIAN D. (X-1): NEWNAM, BRIAN E. (CLS-6); LOHSEN, RICHARD A. (f.T-5); FELDMAN, R. B. (AT-E):

COOPER, RICHARD K. (AT-6); JOHNSON, WILLIAM J. (AT-5); BRAU, CHARLES A. (AT-7).

RECENT RESULTS FROM THE LOS ALAMOS FREE-ELECTRON LASER.

IN: BEIJING FEL SEMINAR, 1938, BEIJING UNIVERSITY. PROC., P.66-96. WORLD SCIENTIFIC, 1989. (OC1 B45X V.2)

ÁLSO PUBLISHED: TIC, 1988. 39P. MN (LA-UR-88-2136)

37-36

FOLTYN, STEPHEN R. (CLS-6).

VARIABLE LASER ATTENUATOR.

PATENTED OCT. 18, 1988, FILED 1987. (U.S.PATENT-4,778,263)

37-37

FORTGANG, CLIFFORD M. (AT-2); BOLME, GERALD O. (AT-1); COTTINGAME, W. B. (AT-2);

JOHNSON, K. F. (MP.10); MAROUARDT, J. H. (AT-2); RUSTHOI, DANIEL F. (AT-2); SANDER, OSCAR R. (AT-2); WORTH, G. T. (AT-2).

LONGITUDINAL BEAM DYNAMICS OF A 5-MEV DTL [DRIFT-TUBE LINAC]-A COMPARISON OF THEORY AND EXPERIMENT

TiC, 1988. 3P. MN (LA-UR-88-3247)

$37-38$

FRASER, JOHN S. (AT-7); SHEFFIELD, RICHARD L. (AT-7); GRAY, E. R. (AT-7); GILES, PAUL M. (AT-4): SPRINGER, ROBERT W. (MST-7); LOEBS, V. A. (MST).

PHOTOCATHODES IN ACCELERATOR APPLICATIONS

IN: IEEE PARTICLE ACCELERATOR CONFERENCE, 1987, WASHINGTON, D.C. PROC., P.1705-9. IEEE, 1987. (OC787.P3 137 1987)

ALSO PUBLSSHED: TIC, 1987. 6P. MN (LA-UR-87-0863)

37-39

FRIEDAICHS, CARL C. (AT-6); CARLINI, FIOGER D. (MP. 14); SPALEK, GEORGE (AT-i); SMNTHE, W. $R$.

TEST RESULTS OF THE LOS ALAMOS FERRITE-TUNED CAVITY.

IN: IEEE PARTICLE ACCELERATOR CONFERENCE, 1987, WASHINGTON. D.C. PROC., P. 1896-7. IEEE, 1987. (CC787.P3 137 1987)

ALSO PUBLISHED: TIC, 1987. 3P. MN (LA-UR-87-0909) 
37-40

FUKA, MARY A. (E-8); CLOUT, PETER N. (AT-3); CONLEY, ANDREW P. (AT-3); HILL, J. O. (AT-3);

ROTHROCK, RICHARD (AT-3); TREASE, L. L. (AT-3); ZANDER, M. (AT-3).

ARGONNE BEAMLINE-B TELESCCPE CONTROL SYSTEM-A STUDY OF ADAPTABILITY.

IN: IEEE PARTICLE ACCELERATOR CONFERENCE, 1987, WASHINGTON, D.C. PROC., P.652-4. IEEE. 1987. (OC787.P3 137 1987)

ALSO PUBLISHED: TIC, 1987. 4P. MN (LA-UR-87-0501)

37-41

GERSTL, S. A. W. (T-DOT); CHITANVIS, SHIRISH M. (T-DOT); ZARDECKI, ANDREW (T-DO); WALLACE, JIA;

GEBHARDT, FRED; DE KINDER, R. E., JR.

FOG-OIL ANOMALY CONFIRMED IN HELSMK-I TESTS.

TIC, 1988. 7P. MN (LA-UR-88-1738)

37-42

GOLDSTEIN, JOHN C. (X-1); MC VEY, BRIAN D. (X-1); ELLIOTT, C. JAMES (X-1).

CONCEPTUAL DESIGNS OF A 50 NM FEL [FREE. ELECTRON LASER] OSCILLATOR AND A 20-40 NM SASE

[SELF-AMPLIFIED SPONTANEOUS EMISSION] AMPLIFIEA.

NUCL. INSTRUM. METHODS PHYS. RES., V.A272, P.177.82. 1988.

ALSO PUBLISHED: TIC, 1987. 20P. MN (LA-UR-87-3964)

37-43

GOLOSTEIN, JOHN C. (X-1;: MC VEY, BRIAN D. (X-1); CARLSTEN, BRUCE E. (AT-7);

THODE, LESTER E. (ADDRA).

INTEGRATED NUMERICAL MODELING OF FREE-ELECTRON LASER OSCILLATORS

TIC, 1988. 17P. MN (LA-UR-88-3546)

$37-44$

GOLDSTEIN, JOHN C. $(X-\{)$; MC VEY, BRIAN D. $(X-1)$; TOKAR, ROBERT L. $(X-1)$; ELLIOTT, C. JAMES $(X-1)$;

SCHMITT, M.. .; CARLSTEN, BRUCE E. (AT-7): THODE. LESTER E. (ADDRA).

SIMULATION CODES FOR MODELING FREE-ELECTRON LASER OSCILLATORS.

TIC, 1988. 11P. MN (LA-UR-88-3881)

37-45

GRAY, E. R. (AT-1); FRASER, JOHN S. (AT-6).

DESIGN AND CONSTRUICTION OF THE PHOTOCATHODE ELECTRON GUN CAVITY.

TIC, 1988. 4P. MN (LA-UR-88-3258)

37-46

GRAY, E. R. (AT-1); SPALEK, GEORGE (AT-1); SHAPIRO, ALAN H. (AT-1).

RADIO-FREOUENCY OUADRUPOLE ACCELERATOR LONGITUDINAL FIELD STABILIZER.

TIC, 1988. 25P. MN (LA-UR-88-2465)

37-47

HUGHES, THOMAS P.; BUILTA, LEE A. (M-4); MACK, JOSEPH M., JR. (M-4); MOIR, DAVID C. (M-4).

TRAPPING OF THE PHERMEX BEAM IN A MIRROR FIELD.

IN: IEEE PARTICLE ACCELERATOR CONFERENCE, 1987, WASHINGTON, D.C. PROC., P.966-8. IEEE, 1987. (CC787.P3 137 1987)

ALSO PUBLISHED: TIC, 1987. 4P. MN (LA-UR-87-0809)

37.48

HURD, JAMES W. (MP-5); MC GILL, JOHN A. (MP-13).

MODIFICAT IN OF ACCELERATION ELEMENT IN "TRANSPORT".

IN: IEEE PARTICLE ACCELERATOR CONFERENCE, 1987, WASHINGTON, D.C. PROC., P.1198-1200. IEEE. 1987. (OC787.P3 137 1987)

ALSO PUBLISHED: TIC, 1987, 4P. MN (LA-UR-87-0660)

37-49

JAMESON, ROBERT A. (AT-DO); SCHRIEER, S. O. (AT-DO).

ACCELERATOR TECHNOLOGY PROGRAM, OCTOBER 1985-MARCH 1986, VOLUME I.

LOS ALAMOS NATIIONAL LABORATORY, 1988. 103P. (LA-11295-SA) 
37-50

JAMESON, ROBERT A. (AT-DO).

HIGH-BRIGHTNESS H MINUS ACCELERATORS.

IN: IEEE PARTICLE ACCELERATOR CONFERENCE, 1987, WASHINGTON, O.C. PROC., P.303-7. IEEE. 1987. (OC797.P3 137 1987)

ALSO PUBLISHED: TIC, 1987. 6P. MN (LA-UR-87-0666)

$37-51$

JAMESON, ROBERT A. (AT-DO).

HIGH-BRIGHTNESS RF LINEAR ACCELERATORS.

IN: NATO ADVANCED STUDY INSTITUTE ON HIGH-BRIGHTNESS ACCELERATORS, 1986, PITLOCHRY, SCOTLAND. PROC., P.169-99. PLENUM PRESS, 1988. (OC1 N37.K V.178)

ALSO PUBLISHED: TIC. 1986. 33P. MN (LA-UR-86-2243)

$37-52$

JAMESON, ROBERT A. (AT-DO).

RF BREAKDOWN LIMITS.

IN: NATO AOVANCED STUDY INSTITUTE ON HIGH-BRIGHTNESS ACCELERATORS, 1986, PITLOCHR!. SCOTLAND. PROC., P.497.506. PLENUM PRESS, 1988. (OC1 .N37X V.178)

37-53

JAMESON, ROBERT A (AT-DO): REID, DONALD W. (AT-5).

RF POWER SOURCES FOR HIGH-BRIGHTNESS RF LINACS

IN: NATO AOVANCED STUDY INSTITUTE ON HIGH-BRIGHTNESS ACCELERATORS, 1986. PITLOCHRY, SCOTLAND. PFOC., P.507-19. PLENUM PFiESS, 1988. (OC1 .N37X V.178)

37-54

JASON, ANDREW JOHN (AT-3); BLIND, BARBARA (AT-3); SVATON, ERNIE M. (AT-6).

UNIFORM RIBBON-BEAM GENERATION FOR ACCELERATOR PRODUCTION OF TRITIUM. TIC, 1988. 5P. MN (LA-UR-88-3326)

37-55

JONES, MICHAEL E. (AT-6); CARLSTEN, BRUCE E. (AT-7).

SPACE-CHARGE-INDUCED EMITTANCE GROWTH IN THE TRANSPORT OF HIGH-BRIGHTNESS ELECTFON BEAMS.

IN: IEEE PARTICLE ACCELERATOR CONFERENCE, 1987, WASHINGTON, D.C. PAOC., P. 1319-21. IEEE, 1987. (OC787.P3 137 1987)

ALSO PUBLISHED: TIC, 1987. 4P. MN (LA-UR-87-04 15)

37-56

KAUPPILA, T. J. (M-4); BUILTA, LEE A. (M-4); CRUTOHER, J. K. (M-4); ELLIOTT. JIMMY C. (M-4);

MOIR, OAVID C. (M-4).

PULSED ELECTRON INJECTOR USING A METAL PHOTOCATHODE IRRADIATED BY AN EXCIMER LASER. IN: IEEE PARTICLE ACCELERATOR CONFERENCE, 1987, WASHINGTON, D.C. PROC., P.273-5. :EE, 1987. (OC787.P3 137 1987)

37-57

KEINIGS, RHONALD K. (X-10); JONES, MICHAEL E. (X-1).

ANPULAR BEAM-DRIVEN HIGH-GRADIENT ACCELERATORS.

TIC, 1988. 7P. WNN (LA-UR-88-2109)

$37-58$

LAWRENCE, GEOAGE P. (AT-DO).

PERFORMANCE OF THE LOS ALAMOS PROTON STORAGE RING.

IN: IEEE PARTICLE ACCELERATOR CONFERNCE, 1987, WASHINGTON, D.C. PROC., F.825-0. IEEE, 1997. (OC787.P3 137 1987)

ALSO PUBLISHED: TIC, 1987. 6P. MN (LA-UR-87-0873)

37-59

LISKA, DONALD J. (AT-4); DAUELSBERG, LAWRENCE B. (AT-4).

DESIGN OF HIGH-POWER RADIO-FREOUENCY DRIVE LOOPS FOR OPERATION INTO 425-MHZZ LNEAR A.CELERATOAS.

LOS ALAMTOS NATIONAL LABORATORY, 1988. 20P. (LA-11265-MS) 
37-60

LISKA, LONALD J. (AT-4); KRAUS, ROBERT H., JA.; BROWN, ROBERT D. (MP-7);

COST, JAMES RICHARD (MST-5).

DEVELOPMENT OF SMALL-SCALE QUADRUPOLES TO OPEFATE AT 20 TO $50 \mathrm{~K}$. TIC, 1988. 3P. MN (LA-UR-88-3260)

37-61

LISKA, DONALD J. (AT-4); DAUELSBERG, LAWRENCE B. (AT-4)

DRIFT-TUBE LINAC INCORPORATING A RAMPED ACCELERATING FIELD.

IN: IEEE PARTICLE ACCELERATOR CONFERENCE, 1987, WASHINGTON, D.C. PROC., P.1797-9. IEEE, 1987. (QC787.P3 137 1987)

ALSO PUBLISHED: TIC, 1987. 4P. MN (LA-UR-87-0724)

37-62

LOONEY. LARFY D. (P-14): LYONS, PETER B. (DRANDR).

RADIATION-INDUCED TRANSIENT ATTENUATION OF OPTICAL FIEERS AT 800 AND $1300 \mathrm{NM}$. IN: OPTICAL TECHNIQLIES FOR SENSING AND MEASIJREMENIT IN HOSTILE ENVIRONMENTS, 1987. ORLANDO, FLA. PROC., P.44-52. SPIE. 1987. (TA1505 .S64X V.787)

ALSO PUBLISHED: TIC, 1987. 1OP. MN (LA-UR-87-2465)

$37-63$

LUMPKIN, ALEX H. (P-15); FELDMAN, R. B. (AT-6); FELDMAN, DONALD W. (AT-7):

APGAR, STEWART A., III (AT-7); CARLSTEN, BRUCE E. (AT-7); FIORITO, R. B:; RULE, D. W.

OPTICAL TRANSITION RADIATION MEASUREMENTS FOR THE LOS ALAMOS AND BCE!NG FREE

ELECTRON LASER EXPERIMENTS

TIC, 1988. 18P. MN (LA-UR-88-3793)

37-64

LUMPKTN, ALEX H. (P-15).

POTENTIAL APPLICATION OF THE LOS ALAMOS FREE-ELECTON LASER-HIGH-TEMPERATURE

SUPEACONDUCTORS.

TIC, 1988. 1GP. MN (LA-UR-FB-3792)

37-65

LUMPKIN, ALEX H. (P-15); KING, N!CHOLAS S. P. (P-15); WILKE, MARK D. (P-15); WEI, S. P.; DAVIS, K. J.

TIME-RESOLVED SPECTRAL MEASUREMENTS FOR THE BOEING FREE-ELECTRON LASER EXPERIMENTS

TIC. 1988. 17P. MN (LA-UR-88-3858)

$37-66$

LUMPKIN, ALEX H. (P-DO); FELDMAN, P. B. (AT-6); FELDMAN, DONALO W. (AT-7); STEIN, WILLIAM E. (AT-7).

ON-UNE ELECTRON BEAM MEASUREMENTS FOR THE LOS ALAMOS FREE-ELECTHON LASER.

IN: IEEE PARTICLE ACCELERATOR CONFERENCE, 1987, WASHINGTON, D.C. PROC., P.212-14. IEEE, 1987. (CC787.P3 I37 1387)

ALSO PUBLISHED: TIC, 1987. 15P. MN (LA-UR-87-0925)

37-67

LYNCH, MICHAEL T. (AT-5); SORUM, LOREN N.; KEFFELER, DAVID ROBERT (AT-5).

RF CONTROL SYSTEM FOR A ROCKET-BORNE ACCELERATOR

IN: IEEE PARTICLE ACCELERATOR CONFERENCE, 1987, WASHINGTON, D.C. PRCC., P.775-7. IEEE,

198\%. (OC787.P3 137 1987)

$37-68$

MACEK, ROBERT J. (MP-DO); FITZGERALD, D. H. (MP-13); HUTSON, RICHARD L. (MP-5):

PLUM, MICHAEL A. (MP-10); THIESSEN, HENRY A. (MP-14).

ANALYSIS OF BEAM LOSSES AT PSR IPROTON STORAGE RINII].

IN: DVVANCED HADRON FACILITY ACCEI ERATOR DESIGN WORKSHOP, 1988, LOS ALAMOS NATIONAL LABORATORY. PROC., P.44-6. LCS ALAMOS NATIONAL LABOAATORY, 1989. (LA-i1432-C)

ALSO PUBLISHED: TIC, 1988. 4P. MN (LA-UR-88-1682)

$37-69$

MOTTERSHEAD, C. T. (AT-6); LYSENKO, WALTER P. (AT-6).

OPTICS CODE DEVELOPMENT AT LOS ALAMOS.

IN: WORKSHOP ON LINEAR ACCELERATOR AND BEAM OPTICS CODES. 19R8, SANV DIEGO, CALIF PROC., F.220-7. AMERICAN INSTITUTE OF PUYYSICS, 1988. (OC1 A38X NO.177)

ALSC PUBLISHED: TIC, 1988. 9P MNi (LA.UR-88.1827) 
MOTTERSHEAD, C. T. (AT-6); LAWRENCE, GEORGE P. (AT-DO); CHANNELL, PAUL J. (AT-6); SCHWEITZER, L. B. SIMULATION OF BEAM LOSS IN THE LOS ALAMOS PROTON STORAGE AING.

IN: IEEE PARTICLE ACCELERATOR CONFERENCE, 1987, WASHINGTON, D.C. PROC.. F.1111-13. IEEE. 1987. (CC787.P3 137 1987)

ALSO PUBLISHED: TIC, 1987. 4P. MN (LA-UR-87-0871)

37-71

NEUFFER, DAVID (AT-6).

MULTIPOLE CORRECTION IN LARGE SYNCHROTRONS.

TIC, 1988. 14P. MN (LA-UR-88-1690)

37-72

NEWNAM, BRIAN E. (CLS-6).

DEVELOPMENT OF A FREE-ELECTRON LASER USER FACILITY FOR THE EXTREME ULTRAVIOLET.

TIC, 1987. 22P. MN (LA-UR-88-0837)

37-73

NEWNAM, BAIAN E. (CLS-Sิ).

PROJECTED PERFOAMANCE OF RF-LINAC-DRIVEN FREE-ELECTRON LASERS IN THE YUW AND SOFT $X$-RAY FEGIONS.

TIC, 1987. 8P. MN (LA-UR-87-4245)

37-74

NEWNAM, BRIAN E. (CLS-6).

XUV FREE-ELECTRON LASER DEVELOPMENT AT LOS ALAMOS.

TIC, 1988. 6P. MN (LA-UA-88-4163)

37-75

O'SHEA, PATAICK G. (BPO); SCHRAGE, DALE L. (AT-1); YOUNG, LLOYD M. (AT-1);

ZAUGG, THOMAS J. (DRA-BPO); LYNCH, MICHAEL T. (BFO); MC KENNA, KENNETH F. (BPO);

HANSBOROUGH, LASH D. (BPO).

LABORATORY PERFORMANCE OF THE BEAR [BEAM EXPERIMENT ABOARD ROCKET] RFO [RADIO-

FREOUENCY OUADRUPOLE].

TIC, 1988. 12P. MN (LA-UR-88-3236)

37.76

RANGARAJAN, G.; CHAN, K. C. (AT-6).

TRANSVERSE RESISTIVE-WALL INSTABILITY OF A BUNCHED ELECTRON BEAM IN A WIGGLER. THC. 1988. 4P. MN (LA-UR-88-3006)

37-77

REID, DONALD W. (AT-DO).

RECENT ADVANCES IN HIGH-POWER MICROWAVE AMPLIFIERS.

TIC, 1988. 4P. MN (LA-UR-38-1070)

37-78

RUSSELL, GARY J. (P-9); ROBINSON, HAROLD (P-9); LEGATE, G. L. (P-9); WOODS, RICHARD (P-9);

WH:TAKER, ELLIS RAY (P-9); BRIDGE, A. (P-9); HUGHES, K. J. (MP-5).

LANSCE (LOS ALAMOS NEUTRON SCATTERING CENTER) TARGET SYSTEM.

IN: MEETING OF INTEANATIONAL COLLABORATION ON ADVANCED NEUTRON SOURCES, 9TH, 1986, VILLUGEN, SWITZERLAND. PROC., P. 777-244. SWISS INSTITUTE FOR NUCLEAR RESEARCH, 1987. (OC/793.5/.N4622/M/4/1986X)

ALSO PUBLISHED: TIC, 1986. 69P. MN (LA-UR-87-1156)

$37-79$

SCHALLER, STUART C. (MP-1); SCHULTZ, DAVID E. (MP-1).

ON DESIGNING A CONTROL SYSTEM FOR A NEW GENERATION OF ACCELERATOAS.

TIC, 1987. 6P. MN (LA-UR-87-3177)

37-80

SCHERMER, ROBERT I. (AT-3).

TUNING PEPMANENT MAGNETS WITH ADJUSTABLE FIELD CLAMPS.

IN: IEEE PARTICLE ACCELERATOR CCNFERENCE, 1987, WASHINGTON, D.C. PROC., P.1514-16. IEEE, 1987. (QC787.P3 137 1987)

ALSO PUBLISHED: TIC, 1S27. 4P. MN (LA-UR-87-0646) 
37.81

SCHNEIDER, E. J. (MP-14).

TEST RESULTS ON DUAL RESONANT POWER SUPPLY WITH FLAT TOP AND FLAT BOTTOM CURRENT.

IN: IEEE PARTICLE ACCELERATOR CONFERENCE, 1987, WASHINGTON, D.C. PROC., P.1505-7. IEEE. 1987. (QC787.P3 137 1987)

ALSO PUBLISHED: TIC, 1997. 4P. MN (LA-UR-87-1068)

37-82

SCHRAGE, DALE L. (AT-1); YOUNG, LI.OYD M. (AT-1); CAMPBELL, BILLY M. (AT-4); BILLEN, JAMES H. (AT-1); STOVALL, JAMES E. (AT-1); MARTINEZ, FELIX A. (AT-1); CLARK, WILLIAM L. (AT-1); BOLME, GERALD O. (AT-1): GIBBS, W. SCOTT (MST-6); KING, JOE DAVID (P-3); O'SHEA, PATRICK G. (BPO); BUTLER, THOMAS A. (BPO):

RATHKE, J.; MICICH, R.; ROSE, J.; RICHTER, R.; ROSATO, G.

BEAR RFQ-BEAM EXPERIMENT ABOARD ROCKET.

TIC, 1988. 14P. MN (LA-UR-88-3042)

37-83

SCHRIBER, S. O. (AT-DO).

UNAC WORKING GROUP TECHNICAL SUMMARY.

IN: ADVANCED HADRON FACILITY ACCELERATOR DESIGN WORKSHOP, 1988, LOS ALAMOS NATIONAL LABORATORY. PROC., P.96-103. LOS ALAMOS NiTIONAL LABORATORY, 1989. (LA-11432-C)

ALSO PUBLISHED: TIC, 1988. 9P. MN (LA-UR-88-1687)

37-84

SCHULTZ, DAVID E. (MP-1); SILBAR, RICHARD R. (T-5).

TOWARD AUTOMATIC CONTROL OF PARTICLE ACCELERATOR BEAMS.

TIC, 1988. 16P. MN (LA-UR-88-1200)

37-85

SHERMAN, J. D.: PITCHER, ERIC J.: ALLISON, PAUL W. (AT-2).

H- BEAM NEUTRALIZATION MEASUREMENTS WITH A GRIDDED-ENERGY ANALYZER, A NONINTERCEPTIVE BEAM DIAGNOSTIC.

TIC, 1988. 3P. MN (LA-UR-88-3259)

37-86

SILBAP, RICHARD R. (T-5); SCHULTZ, DAVID E. (MP-1).

AUTOMATION OF PARTICLE ACCELERATOR CONTROL

IN: ASME INTERNATIONAL COMPUTERS IN ENGINEERING CONFERENCE AND EXHIBITION, 1988. SAN FRATICISCO, CALF. PROC., P.79-33. ASME, 1988.

ALSO PUBLSHED: TIC, 1988. 6P. MN (LA-UR-88-1058)

37-87

SMITH, HORACE V., JA. (AT-2); SCHNURR, NORMAN M. (WX-4); WHITAKER, DEAN H. (AT-2);

KALASH, K. E. (WX-4).

it MINUS ION SOURCE WITH HIGH DUTY FACTOR.

IN: IEEE PARTICLE ACCELERATOR CONFERENCE, 1987, WASHINGTON, D.C. P:7OC., P.301-3. IEEE, 1997. (QC787.P3 137 1987)

ALSO PUBLISHED: TIC, 1987. 4P. MN (LA-UR-87-0706)

37-B8

SMITH, HORACE V., JR. (AT-2); ALLISON, PAUL W. (AT-2); KELLER, RODERICH.

SPECTROSCOPIC INVESTIGATION OF HPLUS AND DPLUS ION SOUACE PLASMAS.

IN: INTERNATIONAL SYMPOSIUM ON THE PRODUCTION AND NEUTRALIZATION OF NEGATIVE IONS AND BEAMS, 4TH, 1986, BROOKHAVEN NATIONAL LABORATORY. PROC., P.181-92. AMERICAN INSTITUTE OF PHYSICS, 1987. (OC/702.7I.NA/P76/1986X)

ALSC) PUBLISHED: TIC, 1986. 13P. MN (LA-UR-86-3863)

37-89

STOKES, AICHARD H. (RT-1); WANGLER, THOMAS P. (AT-1); CRANDALL, KENNETH R. (AT-1).

RFO ACCELERATORS FOR HEATING THERMONUCLEAR PLASMAS. IN: IEEE PARTICLE ACCELERATOR CONFERENCE, 1987, WASHINGTON, D.C. PROC., P.334-6. IEEE. 1987. (CC787.P3 137 1987) 
37-90

SWAIN, GEORGE R. (MP.14).

DESIGN OF A MAIN RING CAVITY.

IN: ADVANCED HADRON FACILITY ACCELERATOR DESIGN WORKSHOP, 1988, LOS ALAMOS NATIONAL LABORATOFYY. PROC.. P.365-80. LOS ALAMOS NATIONAL LABORATORY, 1989. (LA-11432-C)

ALSO PUBLISHED: TIC, 1988. 17P. MN (LA-UR-88-2076)

37-91

TAKEDA, HARUNORI (AT-6).

TRANSIENT ANALYSIS OF A COUPLED ACCELERATOR ANO DECELERATOR SYSTEM.

IN: IEEE PARTICLE ACCELERATOR CONFERENCE, 1987, WASHINGTON, D.C. PROC., P.1161-63. IEEE, 1987. (OC787.P3 I37 1987)

ALSO PUBLISHED: TIC, 1987. 4P. MN (LA-UR-87-0779)

37-92

TALLERICO, PAUL J. (AT-5): COULON, JEAN-PIERRE.

COMPUTER SIMULATION OF THE LASERTRON WITH A RING MODEL.

IN: IEEE PARTICLE ACCELERATOR CONFERENCE, 1987, WASHINGTON. D.C. PROC., P.1806-8. IEEE,

1987. (CC787.P3 137 1987)

ALSO PUBLISHEO: TIC, 1987. 4P. MN (LA-UR-87-0517)

37-93

TALLERICO, PAUL J. (AT-5); COULON, J-P.

RING MODEL OF THE LASERTRON.

LOS ALAMOS, 1988. 28P. (LA-11189-MS)

37-94

THIESSEN. HENRY A. (MP-14).

ADVANCED HADRON FACILITY-A COMBINED KAON FACTORY AND COLD-NEUTRON SOURCE.

IN: INTERNATIONAL WORKSHOP ON HADRON FACILITY TECHNOLOGY, 1987, SANTA FE, N.M. PROC., P.138-81. LOS ALAMOS NATIONAL LABORATORY, 1987. (LA-11130-C)

ALSO PUBLISHED: TIC, 1987. 9P. MN (LA-UR-87-0915)

ALSO PUBLISHED: IEEE PARTICLE ACCELERATOR CONFERENCE, 1987, WASHINGTON, D.C. PROC..

P.86-8. IEEE, 1987. (OC787.P3 137 1987)

37-95

THIESSEN, HENAY A. (MP-14).

POSSIBLE APFLIGATIONS OF SUPERCONDUCTING RF AT LAMPF.

IN: PION-NUICLEUS PHYSICS-FUTURE DIRECTIONS AND NEW FACILITIES AT LAMPF, 1987, LOS

ALAMOS NATIONAL LABORATORY,. PROC., P.537-50. AMERICAN INSTITUTE OF PHYSICS, 1988.

(OC/793.5/.M42/P56/1987X)

$37-96$

THIESSEN, HENRY A. (MP.14)

PROCEEDINGS OF THE INTERNATIONAL WORKSHOP ON HADRCN FACILITY TECHNOLOGY, FEBRUARY

2-5, 1987.

LOS ALAMOS, 1987. 597P. (LA-11130-C)

37-97

THIESSEN, HENRY A. (MP-14).

TECHNICAL SUMMARY OF THE ACCELERATOR DESIGN WORKSHOP, 22-27 FEBRUARY 1988.

IN: ADVANCED HADRON FACILITY ACCELERATOF DESIGN WORKSHOP. 1988, LOS ALAMOS NATIONAL LABORATORY. PROC., P.470-4. LOS ALAMOS NATIONALL LABORATORY, 1989. (LA-11432-C)

ALSO PUBLISHED: TIC, 1988. 6P. MN (LA-UR-88-1349)

37-98

THIESSEN, HENRY A. (MP-14).

TOWARDS AN ADVANCED HADRON FACILITY AT LOS ALAMOS.

IN: ADVANCED HADRON FACILITY ACCELERATOR DESIGN WORKSHOP, 1988, LOS ALAMOS NATIONAL LABORATORY. PROC., P.31-43. LOS ALAMOS NATIONAL LABORATORY, 1989 (LA-11432-C)

ALSO PUBLISHED: TIC, 1988. 13P. MN (LA-UR-88-1349) 
THIESSEN, HENRY' A. (MP-DO); MISCHKE, RICHARD E. (MP-4); MOSS, JOEL MARSHALL (P-2);

SANDERS, GARY H. (MP-13); MACEK, ROBERT J. (MP-13); ROSEN, SIMON PETER (T-DO).

PROPOSAL TO EXTEND THE INTENSITY FRONTIER OF NUCLEAF AND PARTICLE PHYSICS TO 45 GEV (LAMPF II).

TIC, 1984. 281P. MN (LA-UR-84-3982)

37-100

WADLINGER, E. ALAN (AT-3).

FRINGE FIELDS OF CURRENT DOMINATED MULTIPOLE MAGNETS.

TIC, 1988. 4P. MN (LA-UF-88-3146)

37-101

WADLINGER, E. ALAN (AT-3).

MAPPING THE FRINGE FIELDS OF MULTIPOLE MAGNETS

TIC, 1988. 4P. MN (LA-UR-88-3020)

37-102

WADLINGER, E. ALAN (AT-3).

PARAMETER SCALING TO PRODUCE DIFFERENT CHARGED-PARTICLE BEAM-TRANSPORT SYSTEMS

HAVING IDENTICAL EQUATIONS OF MOTION.

LOS ALAMOS, 1988. 21P. (LA-11263-MS)

37. 103

WALIING, LINDA (AT-1); NEUFFER, DAVID (AT-6); THIESSEN, HENRY A. (MP-14); SPALEK, GECRGE (AT-1).

TRANGMISSION-LINE IMPEDANCE MEASUREMENTS OF BEAM PIPES FOR AN ADYANCED HADRON

FACILITY.

IN: INTERNATIONAL WORKSHOP ON HADRON FACILITY TECHNOLOGY, 1987, SANTA FE, N.M. PROC., P.279-81. LOS ALAMOS NATIONAL LABORATORY, 1987. (LA-11130-C)

ALSO PUBLISHED: TIC, 1987. 4P. MN (LA-UR-87.0888)

ALSO PUBLISHED: IEEE PARTICLE ACCELERATOR CONFERENCE, 1987, WASHINGTON, D.C. PROC., P.1164-6. IEEE, 1987. (OC787.P3 137 1987)

$37-104$

WANG, TAI-SEN F. (AT-6); THODE, LESTER E. (ADDRA); COOPER, RICHARD K. (AT-6).

CAVITY-WAVEGUIDE COUPLING THROUGH A LARGE APERTURE.

TIC, 1988. 4P. MN (LA-UR-88-3261)

37-105

WANG, TAI-SEN F. (AT-6); GOLDSTEIN, JOHN C. (X-1); NEWNAM, BRIAN E. (CLS-6); MC VEY, BRIAN D. (X-1).

GENERATION OF COHERENT SOFT X-RAYS USING A SINGLE-PASS FREE-ELECTRON LASER AMPLFIER. TIC, 1987. 9P. MN (LA-UR-88-1215)

ALSO PUBLISHED: TIC, 1988. 11P. MN (LA-UR-88-1215-REV)

37.106

WANG, TAI-SEN F. (AT-6); TAKEDA, HARUNOAI (AT-6).

RF STABILITY IN THE LOS ALAMOS FREE-ELECTRON LASER ENERGY-RECOVERY EXPERIMENTT IN: IEEE PARTICLE ACCELERATOR CONFERENCE, 1987, WASHINGTON, D.C. PROC., P.1102-4. IEEE, 1987. (OC787.P3 137 1987)

37-107

WANG. TAI-SEN F. (AT-6); GOLDSTEIN, JOHN C. (X-1); NEWNAM, BRIAN E. (CLS-5); MC VEY. BRIAN D. (X-1). SINGLE-PASS FREE-ELECTRON LASER FOR SOFT $X$-RAYS WITH WAVELENGTH LESS THAN OR EOUAL TO TEN NANOMETERS.

IN: IEEE PARTICLE ACCELERATOR CONFERENCE, 198?, WASHINGTON. D.C. PROC., P.202-4. IEEE, 1987. (OC787.P3 137 1987)

37-108

WANGLER, THOMAS P. (AT-1).

BRIGHTNESS LMITS IN LINEAR ION ACCELERATORS

IN: NATO ADVANCED STUDY INSTITUTE ON HIGH-BRIGHTNESS ACCELERATORS, 1986. PITLOCHRY, SCOTLAND. PROC., P.411-25. PLENUM PRESS, 1988. (OC1 .N37X V.178) 
37-109

WANGLER, THOMAS P. (AT-1).

DEVELOPMENTS IN THE PHYSICS OF HIGH CURRENT LINEAR ION ACCELERATORS.

IN: SEMINAR ON NEW TECHNIQUES FOR FUTURE ACCELERATOPIS, 1986, F:RICE, SICILY. P.OOC.,

P.181-200. PLENUM PRESS, 1987. (OC/787/.P3/S46/1986)

37-110

WANGLER, THUMAS P. (AT-1); MILLS, RENE SCOTT (AT-1;; CRANDAI.L, KENNETH R. (AT-i).

EMITTANCE GROWTH IN INTENSE BEAMS.

IN: IEEE PARTICLE ACCELERATOR CONFERENCE, 1987, WASHINGTON, D.C. PROC., P.1006-10. IEEE, 1987. (QC787.P3 137 1987)

ALSO PUBLISHED: T'C, 1987. 6P. MN (LA-UR-87-0861)

$37-11 !$

WANGLER, THOMAS P. (AT-1); STAPLES, JOHN.

SUMMARY FOR THE WORKING GROUP ON LINAC CODES.

IN: WORKSHOP ON LINEAR ACCELERATOR AND BEAM OPTICS CODES, 1988, SAN DIEGO CALF PROC., P.347-50. AMERICAN INSTITUTE OF PHYSICS, 1988. (OC1.A38X NO.177)

ALSO PUBLISHED: TIC, 1988. 5P. MN (LA-UR-88-1562)

37-112

WARREN, JOHN L. (HSE-7).

COMPUTER CODES USED IN PARTICLE ACCELERATOR DESIGN-FIRST EDITION.

TIC, 1987. 199P. MN (LA-UR-B6-3320)

37-113

WARREN, RCGER W. (AT-7).

ELECTRON BEAM DIAGNOSTICS FOR FREE-ELECTRON LASERS.

IN: FREE-ELECTRON LASERS, 1987, LOS ANGELES. PROC., P.146-54. SPIE, 1988. (TA1677 .F73 1987X)

37-114

WARREN, ROGER W. (AT-7).

FREE-ELECTRON :ASER OSCILLATOR EXPERIMENTS AT THE LOS ALAMOS NATIONAL LABOAATORY. IN: INTERNATIONAL CONFERENCE ON LASEAS, 1986, ORLANDO. PROC., P.6C2-7. STS PRESS.

17.115

WILSON, NORMAN G. (AT-2); PRECECHTEL, D.

MECHANICAL DESIGN AND FABRICATION OF A 425-MHZ H MINUIS BUNCHER.

IN: IEEE PARTICLE ACCELERATOR CONFERENCE, 1987, WASHINGTON, D.C. PROC., P.1851-3. IEEE. 1987. (QC787.P3 137 1987)

ALSO PUBLISHED: TIC, 1987. 4P. MN (LA-UR-87-0764)

37-116

WINJE, R.A. (MP-14).

PRELIMINARY DESIGN OF THE LOS ALAMOS FAST KICKER MAGNET PULSER AND POWER SUPPLY. TIC, 1988. 6P. MN (LA-UR-88-3363)

37-117

YAN, YITON T. $(X-10)$.

COLLECTIVE ACCELERATION OF PROTONS BY THE PI_ASMA WAVES IN A COUNTERSTREAMING

ELECTRON BEAM.

IN: IEEE PARTICLE ACCELERATOR CONFERENCE, 1987, WASHINGTON D.C. PROC., P.175-7. IEEE, 1987. (QC787.P3 I37 1987)

ALSO PUBLISHEU: TIC, 1987. 8P. MN (LA-UR-87-0870)

37-118

YOUNG, LLOYD M. (AT-1); BIDDLE, RODNEY S. (AT-2).

RESONANCE CONTROL FOR A CW ICONTINUOUS WAVE] ACCELERATOR.

IN: IEEE PAFTICLE ACCELERATOR CONFERENCE, 1987, WASHINGTON, D.C. PROC., P.634-6. IEEE, 1987. (QC787.P3 I37 1987)

ALSO PUBLISHED: TIC, 1987. 4P. MN (LA-UR-87-6647) 
38-1

ALBRIGHT, JAMES N. (ESS-4); JOHNSON, P. A. (ESS-4).

CROSSWELL ACOUSTIC SURVEYING IN GAS SANDS-TRAVEL-TIME PATTERN RECOGNITION SEISMIC O AND CHANNEL WAVES.

TIC, 1985. 20P. MN (1.A-UA-85-1140)

38-2

ALBRIGHT, JAMES N. (ESS-4); TERAY, DARRELL A. (ESS-4); BRADLEY, CHRISTOPHER R. (ESS-4).

PATTERN RECOGNITION AND TONOGRAPHY USING CROSSWELL ACOUSTIC DATA.

TIC, 1965. 10P. MN (LA-UR-85-1138)

38-3

ALTSEIMER, JOHN H. (S-4); ROACH, FRED (S-2); ANDERSON, JOSEPH M.; KRUPKA, MILTON C. (S-4). POTENTIAL APPLICATION OF FUEL CELL COGENERATION SYSTEMS IN PETROLEUM REFINERIES.

LOS ALAMOS. 1987. 123P. (LA-10814-MS)

38-4

JOHNSON, P. A. (ESS-4); ALBRIGHT, JAMES N. (ESS-4).

CONVERSION OF BOREHOLE STONELEY WAVES TO CHANNEL WAVES IN COAL.

TIC, 1987. 12P. MN (LA-UR-87-0877)

38-5

JOHNSON, P. A. (ESS-4); ALBRIGHT, JAMES N. (ESS-4); PHILLIPS, WILLIAM S. (ESS-4).

CROSSWELL SEISMIC MEASUREMENT OF TRANSVERSE ISOTROPY IN VISUB PI AND Q/SUB P/.

TIC, 1986. 13P. MN (LA-UR-86-1160)

$38-6$

JOHNSON, P. A. (ESS-4); ALBRIGHT, JAMES N. (ESS-4).

IN-SITU PHYSICAL PROPERTIES MEASUREMENTS USING CROSSWELL ACOUSTIC DATA.

TIC, 1985. 10P. MN (LA-UR-B5-1139)

39-7

PARKINSON, WILLIAM J. (WX-4).

FOSSIL FUEL FUANACE REACTOA.

PATENTED NOV. 17, 1987, FILED 1986. (U.S.PATENT-4,706,581)

$38-8$

SMITH, R. L. (A-DO).

DAMPENING TURMOIL IN THE OIL MARKET.

LOS ALAMOS NATIONAL LABORATORY, 1988. 15P. CNSS PAPER NO. 2 (LA-1 1313-MS)

$38-9$

TEPERMEISTER, IGOR; SMITH, JENNIFER.

METHANE TO METHANOL-REACTOR DESIGN ANO PROCESS EVALUATION.

LOS ALAMOS NATIONAL LABORATORY, 1988. 112P. (LA-11396-MS) 
39.1

ABDALLAH, JOSEPH, JR. (T-4); CLARK, ROBERT EDWARD HOLMES (X-6).

SUPERCOMPUTERS AND ATOMIC PHYSICS DATA.

TIC, 1988. 8P. IAN (LA-UR-88-0771)

39-2

ABDALLAH, JOSEPH, JP. (T-4); CLARK, ROBERT EDWARD HOLMES (X-6); COWAN, ROBERT D. (T-4).

THEORETICAL ATOMIC PHYSICS CODE DEVELOPMENT I. CATS-COWAN ATOMIC STRUCTURE CODE. LOS ALAMOS NATIONAL LABORATORY, 1988. 26P. (LA-11436-M.V.1)

39-3

ACKERHALT, JAY R. (T-12); MILONNI, PETER W. (T-12).

CHAOS AND INCOHERENECE IN A CLASSICAL ROTATION-VIBRATION MODEL OF INFRARED

MULTIPLE-PHOTON EXCITATIC:.

PHYS. REV., V.37A, P.1552-62, 1988.

39-4

ACKERHALT, JAY R. (T-12); MILONNI, PETER W. (T-12).

IR LASER EXCITATION IN MOLECULES-CHAOS AND DIFFUSIVE ENERGY GROWTH.

IN: NATO ADVANCED RESEARCH WORKSHOP ON ATOMIC AND MOLECULAR PROCESSES WITH SHORT INTENSE LASER PULSES, 1987, LENNOXVILLE, QUEBEC. PROC.. P.407-16. PLENUM PRESS. 1988. (QC793.5.P427 N38 1987)

ALSO PUBLISHED: TIC, 1987. 12P. MN (LA-UR-87-2311)

39-5

ARKO, AL J. (P-10); YATES, B. W.; DUNLAP, B. D.; KOELLING, D. D.; MITCHELL, A. W.; LAM, D. J.; OLSON, C. G.;

DEL GIUDICE, M.; FISK, ZACHARY (CMS); SMITH, JAMES L. (CMS).

UNDERSTANDING PHOTOEMISSION SPECTRA IN URANIUM BASED HEAVY FERMION SYSTEMS

IN: INTERNATIONAL CONFERENCE ON VALENCE FLUCTUATIONS, 5TH, 1987, BANGALOAE, INDIA.

PROC. P.39-46. PLENUM, 1987. (OC/176.8/.V34/157/1987)

39-6

ARONSON. MEIGAN C. (P-10); SALAMON, M. B.; CHRISTENSON, K. K.; GHIRON, K.

EFFECTS OF DOPING ON THE ELECTRONIC PROPERTIES OF NIOBIUM SELENIUM-3.

PHYS. REV., V.38B, P.10468-75. 1988.

39-7

ARONSON, MEIGAN C. (P-10); SALAMON, M. B.

MAGNETIZATION AND ELECTRON-SPIN-RESONANCE STUDIES OF DOPEO NIOBIUM SELENIUM-3.

PHYS. REV., V.38B, P.10476-82. 1988.

39-8

ASHERY, D.; LICHTENSTADT, J.; PIASETZKY, ELI; MORRIS, CHRISTOPHER L. (MP-10); ZUMBRO, J. D.;

GILMAN, R.; RAWOOL, M. W.; MORDECHAI, S.; BOYER, B.; FUENTES, A.; JOHNSON, K.; MC DONALD, J. W.;

SMITHSON, M. J.; WILLIAMS, A. L.; YOO, S. H.; MOORE, C. FRED.

SEARCH FOR PI NUCLEON NUCLEON BOUND SYSTEMS.

PHYS. LETT., V.215B, P.41-4. 1988.

39-9

BAERISWYL, DIONYS; RISHOP, ALAN R. (T-11).

LOCALISED POLARONIC STATES IN MIXED-VALENCE LINEAR CHAIN COMIPLEXES.

J. PHYS. C, V.21, P.339-56. 1988.

39-10

RARTH, S.; GYGAX, F. N.; HITTI, B.; LIPPELT, E.; OTT, HANS R.; SCHENCK, A.; FISK, ZACHARY (CMS).

MICROSCOPIC STUDY OF MAGNETISM IN HEAVY-ELECTRON UAANIUM-2 ZINC-17 BY POSITIVE

MAGNETIC MOMENT SR.

IN: INTERNATIONAL CONFERENCE ON VALENCE FLUCTUATIONS, 5TH, 1987, BANGALORE, INDIA.

PROC., P.361-4. PLENUM, 1987. (OC/176.8/.V34/157/1987) 
39-11

BOWLES, THOMAS J. (P-3); WILKERSON, J. F. (P-3); BROWNE, JOHN C. (ADR); MALEY, MARTIN P. (P-10): ROBERTSON, ROBERT G. H. (P-3); KNAPP, DAVID A.; HELFFRICH, J. A.

LIMIT ON THE ANTI-ELECTRON-NEUTRINO MASS IN FREE MOLECULAR TRITIUM BETA DECAY.

IN: INTERNATIONAL SYMPOSIUM ON WEAK AND ELECTROMAGNETIC INTERACTIONS IN NUCLEI, 1986. HEIDELBERG, GERMANY. PROC., P.782-5. \& PRINGER-VERLAG, 1986. (OC/794.8/W4/W36/1986)

ALSO PUBLISHED: TIC, 1986. 5P. MN (LA-UR-86-2766)

39-12

BRENTEN, H.; ZIRNGIEBL, ERBERHARD (MST-5); WIRE, MIKE S. (MST-5); BLUMENRODER, S.; POFAHL, G.; GUNTHERODT, GUNAT; FISK, ZACHARY (CMS).

RAMAN SCATTERING FRON SPIN FLUCTUATIONS AND PHONONS IN URANIUM PLATINUM-3. IN: INTERNATIONAL CONFERENCE ON VALENCE FLUCTUATIONS. 5TH, 1987, BANGALORE, INDIA. PROC., P.353-6. PLENUM, 1987. (OC/176.8/.V34/157/1987)

39-13

CAMPBELL, DAVID K. (CNLS); BAERISWYL, DIONYS; MAZUMDAR, SUMIT (CNLS).

COULOMB CORRELATION EFFECTS IN QUASI-ONE-DIMENSIONAL CONDUCTORS

TIC, 1986. 6P. MN (LA-UR-86-2804)

39-14

CAMPBELL, LAURENCE J. (T-11)

NORMAL MATTER STORAGE OF ANTIPROTONS.

IN: RAND WORKSHOP ON ANTIPROTON SCIENCE AND TECHNOLOGY, 1987, RAND CORPORATION PROC., P.342-58. WORLD SCIENTIFIC, 1988. (OC793.5.P72 R36 1987)

ALSO PUBLISHED: TIC, 1987. 18P. MN (LA-UR-B7-3823)

39-15

CARLSON, RANDOLPH L. (M-4).

LASER-GUIDING OF RELATIVISTIC ELECTRON BEAMS WITH APPLICATIONS TO FLASH X-RADIOGRAPHY. IN: IEEE PARTICLE ACCELERATOR CONFERENCE, 1987, WASHINGTON, D.C. PROC., P.939-41. IEEE, 1987. (OC787.P3 137 1987)

ALSO PUBLISHED: TIC, 1987. 4P. MN (LA-UR-87-0798)

39-16

CARTWRIGHT, DAVID C. (DRAIFLT); CSANAK, G. Y. (T-4).

CALCULATION OF THE ELECTRON IMPACT COHERENCE PARAMETERS FOR EXCITATION OF THE 3 ID STATE OF HELIUM. J. PHYS. B, V.20, P.L583-90. 1987.

39-17

CAUSA, M. T.; FAINSTEIN, C.; NIEVA, G.; SANCHEZ, R.; STEREN, L. B.; TOVAR, M.; ZYSLER, R.; VIER, D. C.; SCHULTZ, S.; OSEROFF, S. B.; FISK, ZACHARY (P-10); SMITH, JAMES L. (CMS).

CRYSTAL FIELD INTERACTION AND MAGNETIC ORDER IN GADOLINIUM BARIUM 2 COPPER 3 OXYGEN 7. TIC, 1988. 6P. MN (LA-UR-88-0366)

$39-18$

CIOSLOWSKI, JERZY (T-12).

ON EXTRACTING THE BULK PROPERTIES FROM RESULTS OF SMALL CLUSTER CALCULATIONS. CHEM. PHYS. LETT., V.153, P.446-50. 1988.

39-19

CLARK, POBERT EDWARD HOLMES (X-6); ABDALLAH, JOSEPH, JR. (T-4); CSANAK, G. Y. (T-4):

MANN, JOSEPH B., JR. (T-4); COWAN, ROBERT D. (T-4).

THEORETICAL ATOMIC PHYSIS CODE DEVELOPMENT II. ACE-ANOTHER COLLISIONAL EXCITATION CODE. LOS ALAMOS NATIONAL LABORATORY, 1988. 25P. (LA-11436-M.V.2)

39-20

COALSON, ROB D. (T-12).

TIME-DEPENDENT APPROACHES TO SPECTROSCOPY INVOLVING NON-ADIABATICALLY COUPLED EXCITEO ELECTRONIC STATES

ABSTRACT IN: INTERNATIONAL CONFERENCE ON RAMAN SPECTROSCOPY, 10TH, 1986, EUGENE, ORE. PROC., P.17-5. UNIVERSITY OF OREGON, 1986. (RC454.R36 157 1986) 
39-21

COHEN, JAMES S. (T-12); LEON, MELVIN (MP-DO); PADIAL, N. T. (T-12).

PRESSURE BROADENING OF THE [(DEUTERON-TRITON-MUON) DEUTERON-ELECTRON-ELECTRON] FORMATION RESONANCES.

IN: MUON-CATALYZED FUSION MEETING, 1988, SANIBEL ISLAND, FLA. PAOC., P.111-123. AMEFICAN INSTIIUTE OF PHYSICS, 1589. (OC1.A38X NO.181)

ALSO PUBLISHED: TIC, 1988. 14P. MN (LA-UR-88-1748)

39-22

COLLINS, LEE A. (T-11); SCHNEIDER, BARRY I. (T-12).

AB INITIO METHOOS FOR ELECTRON-MOLECULE COLLISIONS.

IN: INTERNATIONAL SYMPOSIUM ON ELECTRON- MOLECULE SCATTERING AND PHOTONONIZATION. 1987, DARESBURY LABORATORY. PROC., P.147-68. PLENUM PRESS, 1988. (OC793.5.E628 1583 1997) ALSO PUBLISHED: TIC. 1987. 26P. MN (LA.UR-87-2249)

39-23

COLLINS, LEE A. (T-4); MERTS, ATHEL L. (T-4).

MODEL CALCULATIONS FOR AN ATOM INTERACTING WITH AN INTENSE, TIME-DEPENDENT ELECTRIC FIELD.

PHYS. REV., V.37A, P.2415-31. 1988.

39-24

COLTON, EUGENE P. (MP-14); GIANFELICE, E.; BISOGNANO, J.

COUPLED-BUNCH INSTABILITIES IN RHIC.

TIC. 1988. 15P. MN (LA-UR-88-1734)

39-25

COMTET, G; HARVEY, CAROL J.; STEWART, JARES E. (N-1); BRYANT, HOWARD C.;

BUTTERFIELD, KENNETH B. (N-2); CLARK, DAVID A. (P-9); DONAHUE, JOEY B. (MP-7):

GRAM, PETER A. M. (MP-4); MAC ARTHUR, DUNCAN W. (MP-4); YUAN, VINCENT W. (AT-2); SMITH, W. W.; COHEN, STANLEY.

STABILITY OF THE ONE-P-ZERO [SINGLET P ODD] SHAPE RESONANCE IN HYDROGEN MINUS IN MODERATE ELECTRIC FIELDS.

PHYS. REV., V.35A, P.1547-54. 1987.

39-26

CSANAK, G. Y. (T-4): CARTWRIGHT, DAVID C. (DRPIFRA).

ELECTRON-IMPACT COHERENCE PARAMETERS FOR EXCITATION OF THE 1P STATES IN HEUUM.

PHYS. REV., V.38A, P.2740-61. 1988.

39-27

CSANAK, G. Y. (T-4); CARTWRIGHT, DAVID C. (DRPIFRA); BLUM, K.

FINE-STRUCTURE EFFECT FOR N3L STATES IN LS-COUPLED TARGETS.

J. PHYS. B, V.21, P.L107-12. 1988.

39-28

CSANAK, GEORGE (T-4); CARTWRIGHT, DAVID C. (DRPIFRA)

ROLE OF SYMMETRY OF THE FINAL TARGET STATE IN THE ANGULAF MOMENTUM TRANSFER BY

ELECTRON IMPACT EXCITATION OF ATOMS.

J. PHYS. B, V.20, P.L603-10. 1987.

39-29

DOWELL, FLONNIE (T-1).

INTRACHAIN FLEXIBILITY CONSTRAINTS ON PHASE STABILITIES AND ODD-EVEN EFFECTS IN MULTIPLE SMECTIC-A AND NEMATIC LIQUID CRYSTALS.

PHYS. REV., V.38A, P.382-94. 1988.

39-30

DOWELL, FLONNIE (T-1).

NEW THEORY FOR COMPETING INTERACTIONS AND MICROSTRUCTURES IN PARTIALLY ORDERED

(LIOUID-CRYSTALLINE) PHASES.

IN: CMS WORKSHOP ON COMPETING INTERACTIONS AND MICPOELUSTERS-STATIGS AND DYNAMICS, 1987, LOS ALAMOS, N.M. PROC., P.177.82. SPRINGER-VERLAG, 1988. (OC173.4.C65 C58 ALSO PUBLISHED: TIC. 1987. 8P. MN (LA-UR-87-2618) 
DOWELL, FLONNIE (T-1).

PREDICTION AND DESIGN OF FIRST SUPER-STRONG MOSTLY-RIGID POLYMERS FROM VERY MOLECULAR THEORIES FOR SMECTIC AND NEMATIC POLYMERS.

TIC, 1988. 15P. MN (LA-UR-88-3885)

39-32

DOWELL, FLCNNIE (T-1).

THEORETICAL PREDICTIONS FOR SIDE-CHAIN LIQUID-CRYSTAL POLYMERS AND COMPARISON TO EXPERIMENT.

TIC. 1988. 7P. MN (LA-UR-88-3884)

$39-33$

ERPENBECK, JEROME J. (T-11); COHEN, E. G. D.

EQUIPARTITION OF ENERGY IN A ONE-DIMENSIONAL MODEL OF DIATOMIC MOLECULES.

PHYS. REV. V.38A, P.3054-64. 1988.

39-34

GAMMEL, JAN T. (T-11); CAMPBELL, DAVID K. (CNLS).

COMMENT ON THE MISSING BOND-CHARGE REPULSION IN THE EXTENDED HUBBARD MODEL. PHYS. REV. LETT., V.60, P.71-3. 1988.

39-35

GODART, C.; KAPPLER, J. P.; THOMPSON, JOE D. (P-10); KRILL, G.; GUPTA, LAXMI C.

CORRELATION BETWEEN VALENCE AND ELECTRONIC STRUCTURE AS STUDIED BY EXAFS IN RARE

EARTH, TRANSITION METAL-2, (SILICON, GERMANIUM)-2 COMPOUNDS.

IN: INTERNATIONAL CONFERENCE ON VALENCE FLUCTUATIONS, 5TH, 1987, BANGALORE, INDIA. PROC., P.225-34. PLENUM, 1987. (OC/176.8/.V34/157/1987)

39-36

GOGGIN, MICHAEL E. (T-12); MILONNI, PETER W. (T-12).

DRIVEN MORSE OSCILLATOR-CLASSICAL CHAOS, OUANTUM THEORY, AND PHOTODISSOCIATION. PHYS. REV., V.37A, P.796-806. 1988.

39.37

GUNTHERODT, GUNAT; ZIRNGIEBL, ERBERHARD (MST-5); MOCK, R.; BRENTEN, H.; BLUMENRODER, S.; POFAHL, G.; FISK, ZACHAAY (CMS).

RAMAN AND BRILLOUIN SCATTERING SPECTROSCOPY OF INTERMEOIATE VALENCE AND HEAVY FERMION COMPOUNDS.

IN: INTERNATIONAL CONFERENCE ON VALENCE FLUCTUATIONS, 5TH, 1987, BANGALORE, !NDIA.

PROC., P.261-7. PLENUM, 1987. (OC/176.8/.V34/157/1987)

39-38

HALDING, JESPER (T-11); LOMDAHL, PETER S. (T-11).

TWO-COMPONENT SOLITON MODEL FOR PROTON TRANSPORT IN HYDROGEN-BONDED MOLECULAR

CHAINS.

PHYS. FEV., V.37A, P.2608-13. 1988.

39-39

HALE, GERALD M. (T-2); STRUENSEE, MICHAEL C. (T-12); PACK, RUSSELL T. (T-12); COHEN, JAMES S. (T-12).

BOUNDARY-VALUE APPROACH TO NUCLEAR EFFECTS IN MUON-CATALYZED DEUTERON-TRITON FUSION.

IN: MUON-CATALYZED FUSION MEETING, 1988, SANIBEL ISLAND, FLA. PROC., P.344-54. AMERICAN INSTITUE OF PHYSICS, 1989. (OC1.A38X NO.181)

ALSO PUBLISHED: TIC, 1988. 11P. MN (LA-UR-88-1788)

39-40

HAYES, EDWARD F.; WALKER, ROBERT B. (T-12).

ANGULAR MOMENTIJM DEPENDENT BARRIERS-POSSIBLE KEY TO UNDEASTANOING ANGULAR

DISTRIBUTIONS FOFI THE REACTIONS OF FLUORINE WITH HYOROGEN-2, DEUTERIUM-2, AND

HYOROGEN DEUTERIUM.

J. CHEM. PHYS., V.89, P.5598-606. 1988. 
39-41

HAYES, EDWARD F.; WALKER, ROBERT B. (T-12).

PREDICTION OF ANGULAR DISTRIBUTIONS FOR THE FLUORINE PLUS HYDROGEN-2 AND FLUORINE PLUS DEUTERIUM REACTIONS.

J. CHEM. PHYS., V.88, P.5978-9. 1988.

$39-42$

HOFFMAN, GARY G. (CLS-2); PRATT, LAWRENCE R. (CLS-2); HARRIS, ROBERT A.

MONTE CARLO INTEGRATION OF DENSITY FUNCTIONAL THEORY-FERMIONS IN A HARMONIC WELL CHEM. PHYS. LETT., V.148, P.313-6. 1968.

39-43

HOLIAN, BRAD LEE (T-1); GRADY, DENNIS E.

FRAGMENTATION BY MOLECULAR DYNAMICS-THE MICROSCOPIC "BIG BANG". PHYS. REV. LETT., V.60, P.1355-8. 1988.

$39-44$

HOLIAN, BRAD LEE (T-1).

MODELING SHOCK-WAVE DE.FORMATION VIA MOLECULAR DYNAMICS.

PHYS. REV., V.37A, P.2562-8. 1988.

39-45

HOWE, STEVEN D. (ERAET): HYNES, M. V. (P-15); PICKLESIMER, A. (P-15).

PORTABLE PBARS, TRAPS THAT TRAVEL.

IN: AAND WORKSHOP ON ANTIPAOTON SCIENCE AND TECHNOLOGY, 1987, RAND CORPORATION. PROC., P.481-501. WORLD SCIENTIFIC, 1988. (QC793.5.P72 R36 1987)

ALSO PUBLISHED: TIC, 1987. 2OP. MN (LA-UR-88-0737)

39-46

HYNES, M. V. (P-15).

ANTIPROTONS ARE ANOTHER MATTER

PHYS. SCR., V.T22, P.195-203. 1988.

ALSO PUBLISHED: TIC, 1987. 20P. MN (LA-UR-87-2386)

39-47

JACAK, B. V. (P-2).

RECENT HEAVY ION RESULTS FROM CERN AND BROOKHAVEN.

TIC, 1988. 28P. MN (LA-UR-88-3948;

39-48

KNOWLES, PETER J.; WERNER, HANS-JOACHIM; HAY, P. JEFFREY (T-12); CARTWRIGHT, DAVID C. (DRAMFLT).

A-DOUBLET-PI TO X-DOUBLET-SIGMA-PLUS RED AND B-DOUBLET-SIGMA-PLUS TO X-DOUBLET-

SIGMA-PLUS VIOLET SYSTEMS OF THE CN RADICAL-ACCURATE MULTIREFERENCE CONFIGURATION INTERACTION CALCULATIONS OF THE RADIATIVE TRANSITION PROBABILITIES.

J. CHEM. PHYS., V.89, P.7334-43. 1988.

39-49

LA GATTUTA, K. J. (X-7).

INTERFERENCE EFFECTS IN ELECTRON-ION RECOMBINATION-II. RESORIANCE AND DIRECT CHANNELS PHYS. REV., V.38A, P.1820-30. 1988.

39-50

LAWRENCE. J. M.; CHEN. Y. Y.; THOMPSON, JOE D. (P-10); BORGES. HORTENCIO A. (P-10).

PRESSURE DEPENDENCE OF THE RESISTIVITY OF CERIUM-3 ALUMINUM.

IN: INTERNATIONAL CONFERENCE ON VALENCE FLUCTUATIONS, 5TH, 1987, BANGALORE, INDIA.

PROC. P.433-6. PLENUM, 1987. (QC/176.8/.V34/157/1987)

ALSO PUBLISHED: TIC, 1987. 5P. MN (LA-LUR-87-0455)

$39-51$

LEON, MELVIN (MP.DO)

THEORETICAL SURVEY OF MUON CATALYZED FUSION.

IN: MUON-CATALYZED FUSION MEETING 1988, SANIBEL ISLAND, FLA. PROC., P.94-104. AMERICAN INSTITUTE OF PHYSICS, 1989. (CC1 .A38X NO.181)

ALSO PUBLISHED: TIC, 1988. 12P. MN (LA-UR-88-1802) 
39-52

MAILHIOT, CHRISTIAN; SMITH, OOUGLAS L. (E-11).

K.P THEORY OF SEMICONDUCTOR SUPERLATTICE ELECTRONIC STRUCTURE-II. APPLICATION TO

GALLIUM-INOIUM-ARSENIC-ALUMINUM-INDIUM ARSENIC [100) SUPERLATTICES.

PHYS. REV., V.33B, P.8360-72. 1988.

39-53

MAPLE, MERAILL B.; CHEN, J. W.; DALICHAOUCH, Y.; KOHARA, T.; LAMBERT, STEVEN E.; LEE, B. W.; ROSSEL, C.: TORIKACHVILI, MILTON S.; FISK, ZACHARY (CMS); MC ELFAESH, M. W. (P-10);

SMITH, JAMES L. (CMS): THOMPSON, JOE D. (P-10); WILLIS, JEFFREY O. (P-10); ALLEN, JAMES W.

EXPERIMENTS ON THE HEAVY ELECTRON SUPEACONDUCTORS URANIUM-BERYLLIUM-13 AND

URANIUM-RUTHENIUM-2-SILICON-2.

IN: INTERNATIONAL CONFERENCE ON VALENCE FLUCTUATIONS, 5TH,1987, BANGALORE, INDIA.

PROC., P.47-55. PLENUM, 1987. (OC/176.8/.V34/157/1987)

$39-54$

MARTIN, RICHARO L. (T-12); SAXE, PAUL W. (T-12)

AB INITIO ELECTRONIC STRUCTURE THEORY FOR A CLUSTER MODEL OF LANTHANUM 2-X STRONTIUM $X$ COPPER OXYGEN 4

INT. J. QUANTUM CHEM., SYMP., V.22, P.237-44. 1988.

39-55

MIGLIORI, ALBERT (P-10); MAXTON, PATRICK M. (P-10); CLOGSTON, ALBEHT M. (CMS):

ZIRNGIEBL, ERBERHARD (P-10); LOWE, MARY (P-10)

ANOMALOUS TEMPERATURE DEPENDENCE IN THE RAMAN SPECTRA OF L-ALANINE- EVIDENCE FOR

DYNAMIC LOCALIZATION.

PHYS. REV., V.38B, P.13464-7. 1988

39-56

MILONNI, PETER W. (T-12).

DIFFERENT WAYS OF LOOKING AT THE ELECTROMAGNETIC VACUUM.

PHYS. SCR. V.T21, P. 102-9. 1988.

ALSO PUBLISHED: TIC, 1987. 33P. MN (LA-UR-87-2315)

39-57

MILONNI, PETER W. (T-12).

SHORTCOMINGS OF THE KELDYSH APPROXIMATION.

PHYS. REV., Y.38A, P.2682-5. 1988

39-58

MILONNI, PETER W. (T-12); GIBSON, ROBERT B. (CLS-5); TAYLOR, ANTOINETTE J. (CLS-5).

ULTRASHORT PULSE PROPAGATION IN KRYPTON FLUORIDE LASER AMPLIFIERS.

J. CPT. SOC. AM., V.5B, P.1360-8. 1988.

39-59

MISCHKE, RICHARD E. (MP-4).

PARITY NONCONSERVATION IN THE PROTON-DEUTERON TOTAL CROSS SECTION AT 800 MEV.

TIC, 1988. 7P. MN (LA-UR-88-3031)

$39-60$

PACIOTTI, MICHAEL A. (MP-DO); BAKER, O. K. (MP-DO): BRADBURY, JAMES N. (MP-DO):

COHEN, JAMES S. (T-12); LEON, MELVIN (MP-DO); MALTRUD, HERMAN R. (WX-5); STURGESS. L. L (WX-5);

JONES, S. E.; LI, P.; REES, L. M.; SHEELY, E. V.; SHURTLEFF, J. K.: TAYLOR, S.; ANDERSON, ALAN N. (MP.7):

CAFFREY, A. J.: ZABRISKIE. JOHN; BROOKS. F. D.: CILLIERS, W. A.: DAVIES, G. D.: ENGLAND, J. B. A.:

PYLE, G. J.; SOUIER, G. T. A., BERTIN, A.; BRUSCHI, M.; PICCININI, M.; VITALE, A.; ZOCCOLI, A.; BOM, V. A.;

VAN EIJK, C. W. E.; DE LAAN, H.; EATON, G. H.

FIRST DIRECT MEASUREMENT OF ALPHA - MUON STICKING IN DEUTERON TAITON - MUON-CATALYZED

FUSION.

IN: MUON-CATALYZED FUSION MEETING, 1988, SANIBEL ISLAND, FLA PROC., P.38-51. AMERICAN

INSTITUTE OF PHYSICS, 1989. (OC1 A38X NO.181)

ALSO PUBLISHED: TIC, 1988. 15P. MN (LA-UR-88-2785)

$39-61$

PAD:AL, N. T.; COHEN, JAMES S. (T-12); LEON, MELVIN (MP.3).

IMPACT BROADENING OF THE DEUTERIUM-TAITIUM-MUON FORMATION RESONANCES

PHYS. REV., V.38A, P.1172-6. 1988. 
39-62

PADIAL, N. T.; COHEN, JAMES S. (T-12); WALKEFi, ROBERT B. (T-12).

ROTATIONAL TRANSITIONS IN COMPOUND MUONIC MOLECULES.

PHYS. REV., V.37A, P.329-39. 1988.

39-63

PASTERNAK, MOSHE; TAYLOR, R. DEA:J (P-10).

STRUCTURAL AND VALENCE PROPERTIES OF THE AMORPHOUS-METALLIC HIGH-PRESSURE PHASE OF TIN IODINE-4.

PHYS. REV., V.37B, P.8130-7. 1988.

$39-64$

PENTTILA, S. I. (MP-7): AMANN, JAMES F. (MP-10); JARMER, J. J. (MP-7); JONES, K. W. (MP-10);

TANAKA, NOBUYUKI (MP-10); BARLETT. M. L.; HOFFMANN, G. W. (T-10); KIELHORN, W. F.; PAULETTA, G.:

PUACELL, $M$.

FIRST POLARIZATION RESULTS FROM THE LAMPF CARBON-13 TARGET.

TIC, 1988. 6P. MN (LA-UR-88-3604)

39-65

PETERSON, OTIS G. (CHM-6).

LASER NEUTPALIZATION.

TIC, 1986. 33P. MN (LA-UR-86-3115)

39-66

PHILLPOT, SIMON R.; BISHOP, ALAN R. (T-11); HOROVITZ, BARUCH.

AMPLTUDE BREATHERS IN DIATOMIC PEIERLS SYSTEMS.

J. PHYS. C, V.20, P.L485-91. 1987.

39-67

OUEST, KEVIN B. $(X-1)$.

HYBRID SIMULATIONS.

TIC, 1987. 7P. MN (LA-UA-88-1275)

39-68

REAGOR, DAVID W. (P-10); CHEONG, SANG-WOOK (P-10); BROWN, STUART E. (P-10);

MIGLIORI, ALBERT (P-10); FISK, ZACHARY (P-10); THOMPSON, JOE D. (P-10).

ELECTRODYNAMICS OF SINGLE-CRYSTAL EUROPIUM-2 COPPER OXYGEN-4-A BOSON EXCITATION SPECTRUM.

TIC, 1988. 12P. MN (LA-UR-88-2277)

$39-69$

RITCHIE, JAMES P. (T-14).

ELECTRON DENSITY DISTRIBUTION ANALYSIS FOR NITROMETHANE, NITROMETHIDE, AND NITRAMIDE. J. AM. CHEM. SOC., V.107, P.1829-37. 1985.

39-70

SCHADLER, GERHARD H. (T-11); BORING, A. MICHAEL (T-11); WEINBERGER, P.; GONIS, A.

ELECTRONIC STRUCTURE OF STOICHIOMETRIC AND OFF-STOICHIOMETRIC TANTALUM CARBON-X. PHYS. REV., V.38B, P.9538-44. 1988.

39-71

SCHIFERL, DAVID (M-6); LE SAR, RICHARD A. (T-11); MOORE, DAVID S. (CLS-4).

RAMAN SPECTROSCOPY ON SIMPLE MOLECULAR SYSTEMS AT VERY HIGH DENSITY. IN: NATO ADVANCED RESEARCH WORKSHOPI EUROPEAN PHYSICAL SOCIETY WOAKSHOP ON SIMPLE MOLECULAR SYSTEMS AT VERY HIGH DENSITY, 1988, LES HOUCHES, HAUTE SAVOIE, FRANCE. PROC., P.303- 28. PLENUM, 1989. (CC1 .N37X V.186)

ALSO PUBLISHED: TIC, 1988. 27P. MN (LA-UR-B8-1671)

39-72

SCHNEIDER, BARRY I. (T-12); LYNCH, DIANE L. (T-12); GIBSON, T. L.

COMPARISON OF MODEL AND AB INITIO POLARIZATION POTENTIALS IN E-H SCATTERIING.

PHYS. REV., V.37A, P.3590-3. 1988. 
$39-73$

SMITH, DOUGLAS L (E-11); MAILHIOT, CHRISTIAN.

K.P THEORY OF SEMICONDUCTOR SUPERLATTICE ELECTRONIC STRUCTURE- - FORMAL RESULTS.

PHYS. REV., V.338, P.8345-59. 1988.

30-74

STRAUB, GALEN K. (T-1).

COULOMB ELECTROSTATIC CONTRIBUTIONS TO THE ENERGY, PRESSURE, BULK MODULUS. AHE

STRESS-STRAIN COEFFICIENTS IN THE ROCK SALT STRUC $\because U R E$.

LOS ALAKOS, 1988. 25P. (LA-11232-MS)

39-75

STRUENSEE, MICHAEL C. (T-12); HALE, GERALD * (T-2); PACK, RUSSELL T. (T-12); COMEN, JAMES S. (T-12). NUCLEAR EFFECTS ON THE FIGENVALUES OF THE DT MU MOLECULE.

PHYS. REV., V.37A, P.340-8. 1988.

39-76

STRUENSEE, MICHAEL C. (T-12); COHEN, JAMES S. (T-12).

STARK MIXING OF $2 S$ MUONIC HELIUM-1. COLLISIONS WITH HYDROGEN ATOMS

PHYS. REV., V.38A, P.44-52. 1988.

39- $\pi$

STRUENSEE, MCHAEL C. (T-12); COHEN, JAMES S. (T-12).

TRANSITIONS BETWEEN THE N EOUALS 2 FINE-STRUCTURE LEVELS IN HYDROGEN PLUS PROTON COLLISIONS.

PHYS. AEV., V.38A, P.3377-87. 1988.

3978

VOTER, ARTHUR F. (T-12).

SIMULATION OF THE LAYER-GROWTH DYNAMICS IN SILVER FILMS-DYNAMICS OF ADATOM CLUSTERS AND VACANCY CLUSTERS ON SILVER(100).

IN: MODELNG OF OPTICAL THIN FILMS, 1987, SAN DIEGO. PROC., P.214-26. SPIE, 1988.

ALSO PUBUSHED: TIC, 1987. 14P. MN (LA-UR-87-2807)

39-79

WEEKS, DAVID E. (T-12); HARTER, WILLIAM G. $(T-12)$.

VIBRATIONAL FREQUENCIES AND NORMAL MODES OF BUCKMINSTERFULLERENE.

CHEM. PHYS. LETT., V.144, P.366-72. 1988.

$39-80$

WEINBERGER, P.; BORING, A. MICHAEL (T-11); ALBERS, ROBERT C. (T-11); TEMMERMAN, W. M. THEORTICAL STUDY OF THE ELECTRONIC STRUCTURE OF ORDERED AND DISORDERED COPPER 3 GOLD.

PHYS. REV., V.388, P.5357-62. 1988 
40-1

ABAZOV, A. I.; ABDURASHITOV, D. N.; ANOSOV, O. P.; AVDEYEV, A. V.; BELOUSKO, YU. I.; BYCHUK, O. V.; DANSHIN, S. N.; EROSHKINA, L. A.; FAIZOV. E. L.; GAYEVSKY, V. I.; GAVRIN, V. N.; GRIGORJEV. A. M.;

KALIKHOV, A. V.; KIREYEV, S. M.; KNODEL, T. V.; KNYSHENKO, I. I.; KORNOUKHOV, V. N.;

MEZENTSEVAH, S. A.; MIRNOV, I. N.; EJIKBOSVERPDZE, SH. M.; OSTRINSKY, A. V.; PETUKHOV, V. V.;

PIKHULYA, O. E.; PSHUKOV, A. M.; REVZIN, N. YE.; SHIKHIN, A. A.; SHILO, YU. I.; SLYUSAREVA, YE. D.;

STEPANYUK, M. V.; TIKHONOV, A. A.; TIMOFEYEV, P. V.; VERETENKIN, E. P.; VERMUL, V.M.; YANTS, V. E.;

ZAKHOROV, YU. I. ' ZATSEPIN, G. T.; CLEVELAND, B. C. (P-3); BOWLES, THOMAS J. (P-3);

ELLIOT:, STEVEN R. (P-3); O'BRIEN, HAROLD A. (P-3); WARK, DAVID L. (P-3); WILKERSON, J. F. (P-3);

CHERRI, M. L.; KOUZES. R. T.

SOVIET AMERICAN GALLIUM EXPERIMENT (SAGE).

TIC, 1988. 6P. MN (LA-UR-88-1461)

$40-2$

AKIMOTO, KAZUHIRO (ESS-8).

ELECTROMAGNETIC EFFECTS ON PARAMETRIC INSTABILITIES OF LANGMUIR WAYES.

PHYS. FLUIDS, V.31, P.538-46. 1988.

40-3

AKIMOTO, KAZUHIRO (ESS-8); KARIMABADI, $H$.

RELATIVISTIC STRUCTURE OF STOCHASTIC WAVE-PARTICLE INTERACTION.

PHYS. FLUIDS, V.31, P.1505-14. 1988.

40-4

ALEXANDER, C.; NEUGEBAUER, M.: SMITH, E. J.; BAME, SAMUEL J., JR. (ESS-8).

RELATION OF SOLAR WIND STRUICTURE TO HYDROMAGNETIC DISCONTINUITIES.

TIC, 1987. 6P. MN (LA-UR-87-4159)

40-5

ARGO, PAUL E. (ESS-7); HUNSUCKER, R. D.

FOREWORD-THE WORLDWIDE ATMOSPHERIC GRAVITY WAVE STUDY (WAGS).

RADIO SC!., V.23, P.865. 1988.

40-6

BAME, SAMUEL J., JR. (ESS-8); MARTIN, RONALD H. (ESS-8); MC COMAS, DAVID J. (ESS-8); BURCH, J. L; MARSHALL, J. A.; YOUNG, DAVID T. (ESS-8).

THREE-DIMENSINAL PLASMA MEASUREMENTS FROM THREE-AXIS STABILIZED SPACECRAFT.

TIC, 1988. 36P. MN (LA-UR-88-3145)

40.7

BECKER, STEPHEN A. $(X-2)$

AGB [ASYMPTOTIC GIANT BRANCH]-STAR EVOLUTION

IN: SYMPOSIUM ON THE ORIGIN AND DISTRIBUTION OF THE ELEMENTS, 1987, NEW ORLEANS, LA.

PROC., P.387-97. WORLD SCIENTIFIC, 1988. (OB/450/.S97/1987)

ALSO PUBLISHED: TIC, 1987. 12P. MN (LA-UR-87-3448)

40-8

BECKER, STEPHEN A. (X-2).

EVOLUTION OF VARIABLE STARS

IN: STELLAR PULSATION-CONFERENCE HELD AS MEMORIAL TO JOHN P. COX, 1986, LOS ALAMOS. PROC., P.16-26. SPRINGER-VERLAG, 1987. (OB/B38/.574/1986X)

ALSO PUBLISHED: TIC, 1986. 12P. MN (LA-UR-86-3387)

40-9

BILOSTEN, M. LARS; ZUREK, WOJCIECH H. (T-6).

POSITRON ANNIHILATION RADIATION FROM THE GALACTIC CENTER- "CHESHIRE CAT" COMPTON SCATTERING AND THE ORIGIN OF EXCESS CONTINUUM.

ASTROPHYS. J., V.329, P.212-24. 1988 
40-10

BOROVSKY. JOSEPH E. (ESS-8).

DYNAMIC SHEATH-OBJECTS COUPLING TO PLASMAS ON ELECTRON-PLASMA-FREQUENCY TIME

SCALES.

PHYS. FLUIDS, V.31, P.1074-100, 1988.

40-19

BOROVSKY, JOSEPH E. (ESS-8).

PRODUCTION OF AURORAL KILOMETRIC RADIATION BY GYROPHASE-BUNCHED DOUBLE-LAYER-

EMITTED ELECTRONS-ANTENNAE IN THE MAGNETOSPHERIC CURRENT REGIONS.

J. GEOPHYS. RES., V.93A, P.5727-40. 1988.

40.12

BOROVSKY, JOSEPH E. (ESS-8).

PROPERTIES AND DYNAMICS OF THE ELECTRON BEAMS EMANATING FROM MAGNETIZED PLASMA DOUBLE LAYERS.

J. GEOPHYS. RES., V.93A, P.5713-25. 1988.

40-13

BROSIUS, J. W.: HOLMAN, G. D.; NIEONER, M. B.; BRANDT, J. C.; SLAVIN, J. A.; SMITH. E. J.;

ZWICKL, P.ONALD D. (ESS-8); BAME, SAMUEL J., JR. (ESS-8).

CAUSE OF TWO PLASMA-TAIL DISCONNECTION EVENTS IN COMET P/HALLEY DURING THE ICE-HALLEY

RADIAL PERIOD.

IN: ESLAB SYMPOSIUM, 20TH, 1986, HEIDELBERG, GERMANY. PROC., P.267-75. SPRINGER-VERLAG, 1988. (QB723.H2 E75 1986)

40-14

BRUNISH, WENDEE M. (ESS-5); WILLSON, L. A.

EVOLUTION OF CEPHEIDS WITH PULSATIONALLY-DRIVEN MASS LOSS.

IN: STELLAR PULSATION-CONFERENCE HELD AS MEMORIAL TO JOHN P. COX, 1986, LOS ALAMOS. PROC., P.27-30. SPRINGER.VERIAG, 1987. (QB/838/.S74/1986X)

ALSO PUBLISHED: TIC, 1986. 5P. MN (LA-UR-86-3407)

40-15

BRUNISH, WENDEE M. (ESS-5); GUZIK, JOYCE A.; WILLSON, L. A.; BOWEN. GEORGE.

MAIN SEQUENCE MASS LOSS

IN: SYMPOSIUM ON THE ORIGIN AND DISTRIBUTION OF THE ELEMAENTS, 1987, NEW ORLEANS, LA. PROC., P.373-6. WORLD SCIENTIFIC, 1988. (OB/450/S97/1987)

ALSO PUBLISHED: TIC, 1987. 5P. MN (LA-UR-87-3357)

40-16

COATES, A.; JOHNSTONE, A.; THOMSEN, MICHELLE F. (ESS-8); FORMISANO, V.; AMATA, E.; WILKEN, B.; JOCKERS, K.; WINNINGHAM, J. DAVID; BORG, H.; BRYANT, D. A.

SOLAR WIND FLOW THROUGH THE COMET HALLEY BOW SHOCK

IN: ESLAB SYMPOSIUM, 20TH, 1986, HEIDELBERG, GERMANY. PROC., P.55-60. SPAINGEP.VERLAG, 1988. (QB723.H2 E75 1986)

$40 \cdot 17$

COLGATE, STIRLING A. (T-6)

PULSAR FORMATION AND THE FALL BACK MASS FRACTION

IN: GEORGE MASON ASTROPHYSICS WORKSHOP, 4TH, 1987, GEORGE MASON UNIVERSITY, FAIRFAX,

VA. PROC., P.341-8. CAMBRIDGE UNIVERSITY PRESS, 1988. (OB843.S95 G46 1987)

40-18

COLGATE, STIRLING A. (T-6).

RELATIONSHIP BETWEEN HIGH-ENERGY PHYSICAL PHENOMENA ON THE SUN AND IN ASTROPHYSICS SOL. PHYS., V.118, P.1-15. 1988.

40-19

COX, ARTHUR N. (T-6): HILLS, JACK G. (T-6)

LOS AL:MOS NATIDNAL LABORATORY [OBSERVATORY REPORT, JULY 1, 1986-JUNE 30, 1987]

BULL. AM. ASTRON. SOC., V 20, P.286-307. 1988. 
40-20

COX, ARTHUR N. (T-6); GUZIK, JOYCE A.; KIDMAN, RL'SSELL B. (A-4).

OSCILLATION FREQUENCIES OF SOLAR MODELS.

TIC, 1988. 6P. MN (LA-UR-88-3271)

40-21

COX, ARTHUR N. (T-6); CAHN, JULIUS $H$.

PULSATIONAL STABILITY OF WOLF-RAYET STARS

ASTROPHYS. J., V.326, P.804-1: 1988.

40-22

COX, ARTHUA N. (T-6); CAHN, JULIUS H.

SOLAR-LIKE OSCILLATIONS IN LATE SPECTRAL CLASS STARS.

7IC. 1988. 6P. MN (LA-UR-88-3219)

40-23

COX, ARTHUR N. (T-6); PROFFITT, CHARLES R.

THEORETICAL INTERPRETATIONS OF ANOMALOUS CEPHEID PULSATIONS. ASTROPHYS. J., V.324, P.1042-7. 1988.

40-24

DEUPREE, ROBERT G. (ESS-5).

MIXING CORE MATERIAL INTO THE ENVELOPES OF RED GIANTS.

IN: STELAR PULSATION-CONFERENCE HELD AS MEMORIAL TO JOHN P. COX, 1986, LOS ALAMOS. PROC., P. 31-3. SPRINGER-VERLAG, 1987. (QB/838/S74/1986X)

ALSO PUBUSHED: TIC, 1986. 4P. MN (LA-UR-86-3385)

40-25

DINGUS, BRENDA L.

STUDY OF ULTRA-HIGH ENERGY EMISSION FROM CYGNUS X-3 AND HERCULES X-1.

LOS ALAMOS NATIONAL LABORATORY, 1988. 164P. (LA-11431-T)

40-26

DINGUS, BRENDA L; ALEXANDREAS, D. E.; ALLEN, R. C.; BURMAN, ROBERT L. (MP-4);

BUTTERFIELD, KENNETH B. (N-2); CAOY, ROBERT (MP-14); CHANG, C. Y.; ELLSWORTH, R. W.;

GOODMAN, J. A.; GUPTA, S. K.; HAINES, T. J.; KRAKAUER, D. A.; LLOYD-EVANS, JEREMY (MP-4);

NAGLE, DARRAGH E. (MP-4); POTTER, M. E.; SANDBERG, V. D. (MP-4); TALAGA, R. L.;

WILKINSON, C. A. (MP-4); YODH, G. B.

IJLTRAHIGH-ENERGY PULSED EMISSION FROM HERCULES $X-1$ WITH ANOMALOUS AIR-SHOWER MUON

PRODUCTION.

PHYS. REV. LETT., V.61, P.1906-9. 1988.

40-27

DRAKE, DARRELL M. (ESS-8); FELDMAN, WILLIAM C. (ESS-8); JAKOSKY. B. $M$.

MARTIAN NEUTRON LEAKAGE SPECTRA.

J. GEOPHYS. RES., V.93B, P.6353-68. 1988.

40-28

ENGLERT, P.; JULL, A. J.; DONAHUE, DOUGLAS J.; REEDY, ROBERT C. (ESS-8).

COSMOGENIC NUCLIDE PRODUCTION RATES-DEPTH DEPENDENCE OF CARBON-14 PRODUCTION.

IN: LUNAR AND PLANETARY SCIENCE CONFERENCE, 19TH, 1988, HOUSTON, TEX. PROC., P.303-4.

LUNAR AND PLANETARY INSTITUTE, 1988. (QB592 .L84 1988X)

40-29

EPSTEIN, RICHARD I. (ESS-9!)

ACOUSTIC PROPERTIES OF NEUTRON STARS.

ASTROPHYS. J., V.333, P.880-94. 1988

40-30

EPSTEIN, RICHARD I. (ESS-9); COLGATE, STIRLING A. (T-6); HAXTON, WICK C.

NEUTRINOINDUCED R-PROCESS NUCLEOSYNTHESIS

PHYS. REV. LETT., V.61, P.2038-41. 1988. 
40-31

EPSTEIN, RICHARD I. (ESS-9); BAYM, GORDON (T-DO).

VORTEX PINNING IN NEUTRON STARS.

ASTROPHYS. J., V.328, P.680-90. 1988.

40-32

FENIMORE, EDWARD E. (ESS-9); CONNER, JERRY P. (ESS.DO): EPSTEIN, RICHARD I. (ESS-9);

KLEBESADEL, RAY W. (ESS-9); LAROS, JOHN G. (ESS-9); YOSHIDA, ATSUMASA (ESS-9); FUJII, M.;

HAYASHIDA, K.; ITOH, M.; MUPAKAMI, T.; NISHIMURA, J.: YAMAGAMI, T.; KONDO, I.;

KAWAI, NOBUYUKI (ESS-9).

INTERPRETATIONS OF MULTIPLE ABSORPTION FEATURES IN A GAMMA-RAY BURST SPECTRUM.

ASTROPHYS. J., V.335, P.L71-4. 1988.

40-33

GALVEZ, MIGUEL (ESS-8): GARY, S. PETER (ESS-8); BARNES, CHRISTOPHER (X-1): WINSKE, DAN (X-1).

COMPUTER SIMULATIONS OF PLASMA EXPANSION ACROSS A MAGNETIC FIELD.

PHYS. FLUIDS, V.31, P.1554-67. 1989.

40-34

GALVEZ, MIGUEL (ESS-8); BOROVSKY, JOSEPH E. (ESS-8).

ELECTROSTATIC TWO-STREAM INSTABILITY DRIVEN BY SLAB-SHAPED ANO CYUNDAICAL BEANS

INJECTED INTO PLASMAS.

PHYS. FLUIDS, V.31. P.857-62. 1998

40-35

GALVEZ, MIGUEL (ESS-8); BARNES, CHRISTOPHER $(x-1)$.

TWO-DIMENSIONAL ELECTROSTATIC SIMULATIONS OF PLASMA PROPAGATION PERPENDICULAR TO A

MAGNETIC FIELD.

PHYS. FLUIDS, V.31, P.863-8. 1988.

40-36

GARY, S. PETER (ESS-8); MADLAND, CHRISTIAN D. (ESS-8); OMIDI, NOSAN; WINSKE, DAN (X-1).

COMPUTER SIMULATIONS OF TWOPICKUP-ION INSTABILTIES IN A COMETARY ENVIFONMENT.

J. GEOPHYS. RES., V.93A, P.9584-96. 1988.

40-37

GAPY, S. PETER (ESS-8); MADLAND, CHRISTIAN D. (ESS-8).

ELECTROMAGNETIC ION INSTABILTIES IN A COMETARY ENVIRONMENT.

J. GEOPHYS. RES., V.93A, P.235-41. 1988.

40-38

GERSTL, S. A. W. (T-DOT); ZARDECKI, ANDREW (T-DO); WISER, H. L.

NEW UV-B HANDBOOK, VOL 1.

IN: NATO ADVANCED RESEARCH WORKSHOP ON THE IMPACT OF SOLAR VL: 'AVIOLET RADIATION UPON TERAESTRIAL ECOSYSTEMS, AGRICULTURAL CAOPS, 1983, BAD WINDSHEIM, GERMANY.

PROC., P. 63-86. SPRINGER VERLAG, 1986. (OH540 .N37X NO. 8)

40-39

GIFFORD, FRANKLIN A. (ESS-7).

SIMILARITY THEORY OF THE TROPOSPHERIC TURBULENCE ENERGY SPECTAUM.

J. ATMOS. SCI., V.48, P.1370-9. 1988.

40-40

GISLER, GALEN R. (ESS-8); WOLLMAN, ERIC R. (ESS-B).

SIMULATIONS OF ELECTRICALLY POLARIZED GAAVITATIONAL CONDENSATIONS IN A GPAIN PLASMA.

PHYS. FLUIDS, V.31, P.1101-4. 1988.

40-41

GOSUNG, JOHN T. (ESS-8); BAME, SAMUEL J., JR. (ESS-8); SMITH, E. J.; BURTON, M. E.

FORWARD-REVERSE SHOCK PAIRS ASSOCIATED WITH TRANSIENT DISTURBANCES IN THE SOLAF WIND AT 1 AU.

J. GEOPHYS. RES., V.93A, P.8741-8. 1988.

40-42

GOSLING, JOHN T. (ESS-8); WINSKE, DAN (X-1); THOMSEN, MICHELLE F. (ESS-8).

NONCOPLANAR MAGNETIC FIELDS AT COLLISIONLESS SHOCKS-A TEST OF A NEW APPPOACH.

J. GEOPHYS. RES., V.93A, P.2735-40. 1988. 
40-43

GOSWAMI, J. N.; MC GUIRE, ROBERT E.; REEDY, ROBERT C. (ESS-8); LAL, DEVENDRA; JHA, R.

SOLAR FLARE PROTONS AND ALPHA PARTICLES DURING THE LAST THREE SOLAR CYCLES.

J. GEOPHYS. RES., V.93A, P.7195-205. 1988.

40.44

HILLS, JACK G. (T-6).

HYPER-VELOCITY AND TIDAL STARS FROM BINARIES DISRUPTED BY A MASSIVE GALACTIC BLACK HOLE. NATURE, V.331, P.687-9. 1988.

40.45

HJELLMING, ROBERT M.: CALOVINI, T. A.; HAN, XIAO HONG; CORDOVA, FRANCE ANNE (ESS-9).

TRANSIENT RADIO EMISSION FROM THE X-RAY NOVA ASM 2000+25.

ASTROPHYS. J., V.335, P.L75-8. 1988.

40-46

HOFFMAN, Y. (T-6); ZUREK, WOJCIECH H. (T-6)

IS THE 'GREAT ATTRACTOR' A LOOP OF COSMIC STRING?

NATURE, V.333, P.46-9. 1988.

40-47

HOFFMAN, Y. (T-6).

ON THE FORMATION AND STRUCTURE OF GALACTIC HALOS.

ASTROPHYS. J., V.328, P.489-98. 1988.

40-48

HONES, EDWARD W., JR. (ESS-8); CRAVEN, J. D.; FRANK, L. A.; PARKS, G. K.

ASSOCIATION OF PLASMA SHEET VARIATIONS WITH AURORAL CHANGES DURING SUBSTORMS.

TIC, 1988 13P. MN (LA-UR-88-2223)

40-49

HONES, EOWARD W., JP. (ESS-8); CRAVEN, J. D.; FRANK, L. A.; GALVIN, A. B.; MURPHREE, J. S.;

ELPHINSTONE, R. D.; ELPHIC, R. C.

STUDY OF A SUBSTORM ON N:AY 4. 1986.

TIC, 1988. 16P. MN (LA-UR-88-2222)

$40-50$

JACOBSON, ABRAM R. (ESS-7); CARLOS, ROBERT C. (ESS-7); BLANC, ELIZABETH.

OBSEAVATIONS OF IONOSPHERIC DISTURBANCES FOLLOWING A 5-KILOTON CHEMICAL EXPLOSION-1. PERSISTENT OSCILLATION IN THE LOWER THERMOSPHERE AFTER SHOCK PASSAGE.

RADIO SCI., V.23, P.820-30. 1988

40-51

JOHNSTONE, A.; COATES, A.; HEATH, J.; THOMSEN, MICHELLE F. (ESS-8): WILKEN, B.: JOCKERS, K.;

FORMISANO, V.: AMATA, E.: WINNINGHAM, J. DAVID; BORG, H.; BRYANT, D. A.

ALFVENIC TURBULENCE IN THE SOLAR WIND FLOW DURING THE APPROACH TO COMET PAHALLEY. IN: ESLAB SYMPOSIUM, 20TH, 1986, HEIDELBERG, GERMANY. PROC., P.25-32. SPRINGER-VERLAG. 1988. (QB723.H2 E75 1986)

40-52

JOHNSTDive, A.; GLASSMEIER, K.; ACUNA, M.; BORG, H.; BRYANT, D. A.; COATES, A.; FORMISANO, V.: HEATH, J.; MARIANI, F.; MUSMANN, G.; NEUBAUER, F.; THOMSEN, MICHELLE F. (ESS-8); WILKEN, B.; WINPINGHAM, J. DAVID.

WAVES IN THE MAGNETIC FIELD AND SOLAR WIND FLOW OUTSIDE THE BOW SHOCK AT COMET

P/HALLEY.

IN: ESLAB SYMPOSIUM, 20TH, 1986, HEIDELBERG, GERMANY. FROC., P.47-54. SPRINGER-VERLAG, 1988. (OB723.H2 E75 1986)

40-53

KADISH, ABRAHAM (ESS-7); NEBEL, R. A. (CTR-6); SHANAHAN, WILLIAM R. (X-10).

STRUCTURED PLASMOIDS FOR EXOATMOSPHERIC PROPAGATION.

LOS ALAMOS NAT!ONAL LABORATORY, 1988. 44P. (LA-11450-MS) 
40-54

KAWAI, NOBUYUKI (ESS-9); FENIMORE. EDWARD E. (ESS-9); MIDOLEDITCH, JOHN (ESS-9);

CRUDDACE, R. G.; FRITZ, G.; SNYDER, W. A.; ULMER, M. P.

X-RAY OBSERVATIONS OF THE GALACTIC CENTER BY SPARTAN 1

ASTROPHYS. J., V.330, P.130-41. 1988.

40-55

KEADY, JOHN J. (T-4): HALL, D. N.; RIDGWAY, S. T.

IRC + 10216 CIRCUMSTELLAR ENVELOPE-I. MODELS FOR THE DUST AND GAS.

ASTROPHYS. J., V.326, P.832-42. 1988.

40-56

KLEBESADEL, RAY W. (ESS-9).

HISTORY OF GAMMA-RAY BURST OBSERVATIONS.

IN: INTERNATIONAL SYMPOSIUM ON THE PHYSICS OF NEUTRON STARS AND BLACK HOLES. 1988. TOKYO, JAPAN. PROC., P.387-404. UNIVERSAL ACADEMY PRESS, 1988. (DB843.N4 158

ALSO PUBLISHED: TIC, 1988. 24P. MN (LA-UR-88-2403)

40.57

KLEIN, J.; NISHIIZUMI, KUNIHIKO; REEDY, ROBERT C. (ESS-8); ENGLERT, P.; MIDDLETON, $P$.

SIMULATION OF COSMIC-RAY PRODUCTION OF ALUMINUM-26 AND BERYLLIUM-10.

IN: LUNAR AND PLANETARY SCIENCE CONFERENCE, 19TH, 1988, HOUSTON, TEX. PAOC., P.609-12. LUNAR AND PLANETARY INSTITUTE, 1988. (OB592 .L84 1988X)

$40-53$

KOEHLER, P. E. (P-3); O'BRIEN, HAROLD A. (P-3); BOWMAN, CHATLES D. (P-3).

MEASUREMENTS OF ASTROPHYSICAL REACTION RATES FOR RADIOACTIVE SAMPLES.

IN: NUCLEAR SPECTROSCOPY OF ASTROPHYSICAL SOURCES, 1987, WASHINGTON, D.C. PROC., P.143-8. AMERICAN INSTITUTE OF PHYSICS, 1988. (OC1 .A38X NO.170)

ALSO PUBLISHED: TIC, 1987. 11P. MN (LA-UR-87-4104)

40-59

KOEHLER, P. E. (P-3); O'BRIEN, HAROLD A. (P-3).

NUCLEAR ASTROPHYSICS FROM NEUTRON CROSS-SECTION MEASUREMENTS ON RADIOACTIVE

SAMPLES.

NUCL. INSTRUM. METHODS PHYS. RES., V.B40/41, P.494-7. 1989.

ALSO PUBLISHED: TIC, 1988. 15P. MN (LA-UR-88-3177)

40-60

MC COMAS. DAVID J. (ESS-8); GOSLING, JOHN T. (ESS-8); WINTERHALTER, D.; SMITH, E. J.

INTERPLANETARY MAGNETIC FIELD ORAPING ABOUT FAST CORONAL MASS EJECTA IN THE OUTER HELIOSPHERE.

J. GEOPHYS. RES., V.93A, $.2519-26.1988$.

40-61

MUAPHY, TIMOTHY C. (ESS-7).

DEVELOPMENT AND TESTING OF KITES.

LOS ALAMOS NATIONAL LABORATORY, 1988. 44P. (LA-11329-MS)

40-62

MURPHY, TIMOTHY C. (ESS-7).

TCTAL AND DIFFERENTIAL ELECTRON COLLISION CROSS SECTIONS FOR OXYGEN AND NITROGEN.

LOS ALAMOS, 1988. 38P. (LA-11288-MS)

40-63

NAGLE, DARRAGH E. (IAP-4); BOLTON, RICHARD D. (MP-4); BURMAN, ROBERT L. (MP-4);

BUTTERFIELD, KENNETH B. (Q-2); CADY, ROBERT LLOYD (MP-4); CARLINI, ROGER D. (MP-4);

SANDBERG, V. D. (MP-4): WILLIAMS, ROBERT A. (MP-4); WILKINSON, C. A. (MP-4): CHANG, C. Y.; DINGUS, B : GOODMAN, J. A.; TALAGA, R. L.; YODH, G. B.; KRAKAUER, D. A.; GUPTA, S. K.; ELLSWORTH, R. W.;

LINSLEY, JOHN; ALLEN, R. C.

DETECTION OF 10 TO THE FOURTEENTH ELECTRON VOLTS GAMMA RAYS FROM CYGNUS X-3 DURING 1986.

IN: TEXAS SYMPOSIUM ON RELATIVISTIC ASTROPHYSICS, 13TH, 1986, CHICAGO. PROC., P.595-9.

WORLD SCIENTIFIC, 1987. (OB/460/.T49/1986X)

ALSO PUBLISHED: TELEMARK IV CONFERENCE-NEUTRINO MASSES AND NEUTRINO ASTROPHYSICS,

1987, ASHLAND, WIS. PROC., P.438-41. WORLD SCIENTIFIC, 1987. (CC793.5.N426 N49 1987X) 
40-64

NIETO, MICHAEL MARTIN (T-8); GOLDMAN, TERRENCE J. (T-5); HUGHES, RICHARD J. (T-8).

PHENOMENOLOGICAL ASPECTS OF NEW GRAVITATIONAL FORCES-IV. NEW TERRESTRIAL

EXPERIMENTS

PHYS. REV., V.38D, P.2937-43. 1988.

40-65

NISHIIIUMI, KUNIHIKO; KUBIK, PETER W.; ELMORE, DAVID; REEDY, ROBERT C. (ESS-8): ARNOLD, JAMES R. COSMOGENIC CHLORINE-36 PRODUCTION RATES IN METEORITES AND THE LUNAR SURFACE.

TIC, 1988. 30P. MN (LA-UR-88-1878)

40-6;6

OMIDI, NOJAN; AKIMOTO, KAZUHIRO (ESS-B); GURNETT, D. A.: ANDERSON, R. R.

NATURE AND THE NONLINEAR EVOLUTION OF ELECTROSTATIC WAVES ASSOCIATED WITH THE AMPTE SOLAR WIND RELEASES.

J. GEOPHYS. RES., V.93A, P.8532-44. 1988.

40-67

PHILLIFS, JOHN L. (ESS-8); LUHMANN, JANET G.; KNUDSEN, W. C.; BRACE, L. H. ASYMMETRIES IN THE LOCATION OF THE VENUS IONOPAUSE.

J. GEOPHYS. RES., V.93A, P.3927-41. 1988.

40-68

POWERS, B. J. (T-DOT); GERSTL, S. A. W. (T-DOT).

MODELING OF ATMOSPHERIC EFFECTS ON THE ANGULAR DISTRIBUTION OF A BACKSCATTERING PEAK.

IEEE TRANS. GECISCI. REMOTE SENS., V.26, P.649-59. 1988.

40-69

OUEST, KEVIN B. (X-1).

THEORY AND SIMULATION OF COLLISIONLESS PARALLEL SHOCKS.

J. GEOPHYS. RES., V.93, P.9649-80. 1988.

40-70

OUINN, P. J.; ZUREK, WOJCIECH H. (T-6).

ANGULAR MOMENTI IM DISTRIBUTION IN GALACTIC HALOS

ASTROPHYS. J., V.331, P.1-18. 1988.

40-71

RABY, STUART A. (T-8); WEST, GEOFFREY B. (T-8).

DETECTION OF GALACTIC HALO MAGNINOS VIA THEIR COHERENT INTERACTION WITH HEAVY NUCLEI. PHYS. LETT., V.200, P.547-52. 1988.

40-72

RABY, STUART A. (T-8); WEST, GEOFFREY B. (T-8).

FOURTH GENERATION NEUTRINO WITH A STANDARD HIGGS SCALAR SOLVES BOTH THE SOLAR NEUTRINO AND DARK MATTER PROBLEMS.

PHYS. LETT., V.202B, P.47-52. 1988.

40.73

RABY, STUART A. (T-8); WEST, GEOFFREY B. (T-8).

MAGNINOS-EXPERIMENTAL CONSEOUENCES AND CONSTRAINTS.

IN: TELEMARK IV CONFERENCE-NEUTRINO MASSES AND NEUTRINO ASTROPHYSICS, 1987. ASHLAND, WIS. PROC., P.182-206. WORLD SCIENTIFIC, 1987. (OC793.5.N426 N49 1987X)

ALSO PUBLISHED: TIC, 1987. 27P. MN (LA-UR-87-1255)

40-74

RABY, STUART A. (T-B).

PARTICLE PHYSICS CANDIDATES FOR THE COSMION SOLUTION TO THE SOLAR NEUTRINO PROBLEM. IN: RENCONTRE DE MORIOND, 23RD, 1988, LES ARCS, SAVOIE, FRANCE. PROC., P.467-82. EDITIONS FRONTIERES, 1988. (CC794.8.E44 E43 1988X)

ALSO PUBLISHED: TIC, 1988. 18P. MN (LA-UR-88-1741) 
$40-75$

REEDY, ROBERT C. (ESS-8).

COSMIC-RAY INTERACTIONS AND DATING OF METEORITE STRANDING SUAFACES WITH COSMOGENIC NUCLIDES.

TIC, 1988. 5P. MN (LA-UR-88-3336)

$40-76$

REEDY, ROEERT C. (ESS-8).

GAMMA-RAY AND NEUTRON SPECTROSCOPY OF PLANETARY SURFACES AND ATMOSPHERES.

IN: NUCLEAR SPECTROSCOPY OF ASTROPHYSICAL SOURCES, 1987, WASHINGTON, D.C. PROC., P.203-10. AMERICAN INSTITUTE OF PHYSICS, 1988. (OC1 .A38X NO.170)

ALSO PUBLISHED: TIC, 1987. 9P. MN (LA-UR-87-4199)

40-77

REEDY, ROBERT C. (ESS-8).

NEUTRON PRODUCTION BY COSMIC RAYS-DEPENDENCE ON PROTON ENERGY AND SPECTRA.

IN: LUNAR AND PLANETARY SCIENCE CONFERENCE, 19TH, 1988, HOUSTON, TEX. PROC., P.966-7.

LUNAR AND PLANETARY INSTITUTE, 1988. (OB592 L84 1988X)

40-78

REEDY, ROBERT C. (ESS-8).

PRODUCTS FROM COSMIC-RAY INTERACTIONS IN EXTRATERRESTRIAL MATTER-WHAT THEY TELL US ABOUT RADIATION BACKGROUNDS IN SPACE.

TIC, 1987. 7P. MN (LA-UR-88-1176)

40-79

ROSEN, SIMON PETER (T-DO): GELB, JAMES M. (T-DO).

MATTER OSCILLATIONS AND SOLAR NEUTRINOS-A REVIEW OF THE MSW EFFECT.

IN: INTERNATIONAL CONFERENCE ON HIGH ENERGY PHYSICS, 23RD, 1986, BERKELEY, CALIF. PROC., P.909-20. WORLD SCIENTIFIC, 1987. (OC793.3.H5 156 1986X)

ALSO PUBLISHED: TIC، 1986. 13P. MN (LA-UR-86-3789)

40-80

ROSEN, SIMON PETER (T-DO).

REMARKS ON THE FIRST TWO EVENTS IN THE SUPERNOVA BURST OBSEAVED BY KAMIOKANDE II.

PHYS. REV., V.37D, P.1682-4. 1988.

40-81

SANDERSON, T. R.; WENZEL, K. P.; DALY, P. W.; COWLEY, S. W. H.; HYNDS, R. J.; RICHARDSON, I. G.;

SMITH, E. J.; BAME, SAMUEL J., JR. (ESS-8): ZWICKL, RONALD D. (ESS-B).

OBSERVATIONS OF HEAVY ENERGETIC IONS FAR UPSTREAM FROM COMET PHALLEY.

IN: ESLAB SYMPOSIUM, 20TH, 1986, HEIDELBERG, GERMANY. PROC., P.125-8. SPRINGER-VERLAG, 1988. (OB723.H2 E75 1986)

ALSO PUBLSHED: TIC, 1986. 6P. MN (LA-UR-86-4331)

40-82

SMITH, BARHAM W. (ESS-5); JONES, ERIC M. (ESS-5).

MODELS FOR X-RAY EMISSION FROM TYCHO'S REMNANT.

IN: INTERNATIONAL ASTRONOMICAL UNION. COLLPQUIUM, 101ST, 1987, PENTICTON. B.C. PROC.,

P.133-6. CAMBRIDGE UNIVERSITY PRESS, 1988. (O3843.S95 157 1987X)

40-83

STANSBERRY, JOHN A. (ESS-8); GOSLING, JOHN T. (ESS-8); THOMSEN, MICHELLE F. (ESS-8);

BAME, SAMUEL J., JR. (ESS-8); SMITH. E. J.

INTERPLANETARY MAGNETIC FIELD ORIENTATIONS ASSOCIATED WITH BIDIRECTIONAL ELECTRON HEAT

FLUXES DETECTED AT ISEE 3.

J. GEOPHYS. RES., V 93A, P.1975-80. 1988.

40-84

STARRFIELD, SUMNER G. (T-6).

CLASSICAL NOVA OUTBURST.

IN: MULTIWAVELENGTH ASTROPHYSICS, F.A. CORDOVA, ED., P.159-88. CAMBRIDGE UNIVERSITY PRESS, 1988. (QBA61 MB5 1988)

ALSO PUBLISHED: TIC, 1988. 3OP. MN (LA-UR-88-1210) 
40-85

STARRFIELD, SUMNER G. (T-DO); SONNEBORN, G.; STRYKER, L. L.; SPARKS, WARREN M. (X-5);

TRURAN, JAMES W.; FERLAND, GARY; WAGNER, R. M.; GALLAGHER, J. S.; WADE, R.; WILLIAMS, ROBERT E.;

GEHRZ, ROBERT D.; NEY, E. P.; KENYON, S.; SHAVIV, GIORA; WU, C. C.

OBSERVATIONS AND SIMULATIONS OF NOVA VUL 1984 NO.2-A NOVA WITH EJECTA RICH IN OXYGEN. NEON, AND MAGNESIUM.

TIC, 1988. 15P. MN (LA-UR-88-2691)

40-86

STARRFIELD, SUMNER G. (T-DO); STRYKER, L. L.; SONNEBORN, G.; SPARKS, WARREN M. (X-5);

FERLAND, GARY; WAGNER, R. M.; WILLIAMS, ROBERT E.; GEHRZ, ROBERT D.; NEY, E. P.; KENYON, S.;

WADE, R.; TRURAN, JAMES W.; WU, C. $C$.

OBSERVATIONS OF CLASSICAL NOVAE IN OUTBURST

TIC, 1988. 15P. MN (LA-UR-88-2690)

40-87

STARRFIELD, SUMNER G. (T-DO); STRYKER, L. L.; SONNEBORN, G.; SPARKS, WARREN M. (X-5):

WAGNER, R. M. ; FERLAND, GARY; GALLAGHER, J. S.; WADE, R.; WILLIAMS, ROBERT E.; GEHRZ, ROBERT D.; NEY, E. P.; KENYON, $S$;; TRURAN, JAMES W.; WU, C. C.

OPTICAL AND ULTRAVIOLET OBSERVATIONS OF NOVA VUL 1987

TIC, 1988. 16P. MN (LA-UR-88-2692)

40-88

STARRFIELD, SUMNER G. (T-DO).

REVIEW OF THE ULTRAVIOLET STUDIES OF GALACTIC NOVAE.

TIC, 1986. 25P. MN (LA-UR-86-2642)

40-89

STARRFIELD, SUMNER G. (T-DO); STRYKER, L. L.; SONNEBORN, G; SPARKS, WARREN M. (X-5):

SION, EDWARO M.; WAGNER, R. M.; FERLAND, GARY; GALLAGHER, J. S.; WADE, R.; WILLIAMS, POBERT E.; HEATHCOTE, S.; GEHRZ, ROBERT D.; NEY, E. P.; KENYON, S.; SHAVIV, GIORA; WEHRSE, RAINER;

HAUSCHILDT, P.; TRURAN, JAMES W.; WU, C. $C$.

ULTRAVIOLET OBSERVATIONS OF LMC NOVA 1988

TIC, 1988. I1P. MN (LA-UR-88-2693)

40-90

TAKAHASHI, KAZUE (ESS-8); HONES, EDWARD W., JR. (ESS-8).

ISEE 1 AND 2 OBSERVATIONS OF ION DISTRIBUTIONS AT THE PLASMA SHEET-TAIL LOBE BOUNDARY. J. GEOPHYS. RES., V.93A, P.8558-82. 1988.

$40-91$

THOMSEN, MICHELLE F. (ESS-8); GOSLING, JOHN T. (ESS-8).

COMMENT ON "ION DISTRIBUTIONS IN THE DAYSIDE MAGNETOSHEATHS OF JUPITER AND SATURN" BY $J$.

D. RICHARDSON.

J. GEOPHYS. RES., V.93A, P.2761-2. 1988.

40-92

THOMSEN, MICHELLE F. (ESS-8); GOSLING, JOHN T. (ESS-B); BAME, SAMUEL J., JR. (ESS-8);

OUEST, KEVIN B. (X-1); RUSSELL, C. T.; FUSELIER, STEPHEN A.

ON THE ORIGIN OF HOT DIAMAGNETIC CAVITIES NEAR THE EARTH'S BOW SHOCK.

J. GEOPHYS. RES., V.93A, P.11311-25. 1988.

40-93

TSURUTANI, B. T.; BRINCA, A. L.; SMITH, E. J.; THORNE, R. M.; SCARF, F. L.; GOSLING, JOHN T. (ESS-8);

IPAVICH, FRED M.

MHD WAVES DETECTED BY ICE AT DISTANCES GREATER THAN OA EQUAL TO $28 \times 10$ TO THE 6TH POWER KM FROM COMET P/HALLEY-COMETARY OR SOLAR WIND ORIGIN?

IN: ESLAB SYMPOSIUM, 20TH, 1986, HEIDELBERG, GERMANY. PROC., P.97-102. SPRINGER-VERLAG, 1988. (OB723.H2 E75 1986)

ALSO PUBLISHED: TIC. 1986. TP. MN (LA-UR-B7-0039)

40-94

VAN RIPER, KENNETH A. (X-7).

EFFECTS OF NUCLEAR EOUATION OF STATE ON GENERAL RELATIVISTIC STELLAR CORE COLLAPSE MODELS.

ASTROPHYS. J., V.326, P.235-40. 1988 
40.95

VAN RIPEA, KENNETH A. (X-7).

MAGNETIC NEUTRON STAR ATMOSPHERES

ASTROPHYS. J., V.329, P.339-75. 1988.

40-96

VANIMAN, DAVID T. (ESS-1).

EXTRACTION OF LIGHT GASSES FROM MARS OR ITS MOONS.

TIC. 1988. 12P. MN (LA-UR-38-1282)

40-97

VANIMAN, DAVID T. (ESS-1); REEDY, ROBERT C. (ESS-8).

PLANETARY SKIN GAMES.

TIC, 1988. 6P. MN (LA-UR-88-1264)

40-98

VANIMAN, DAVID T. (ESS-1); PETTIT, DONALD R. (M-9); HEIKEN, GRANT (ESS-1).

USES OF LUNAR SULFUR.

TIC, 1988. 2OP. MN (LA-UR-88-1345)

$40-99$

WALLACE, RICHARD K. $(X-7)$

CORE HELIUM FLASH, CONVECTION, AND SURFACE ABUNDANCE ANOMALIES.

IN: SYMPOSIUM ON THE ORIGIN AND DISTAIBUT'ION OF THE ELEMENTS, 1987, NEW ORLEANS, LA.

PROC. P.377-86. (QB/450/.S97/1987) WORLD SCIE:NTIFIC. 1988.

40-100

WARK, DAVID L. (P-3); BOWLES, THOMAS J. (P-3); KNAPP, DAVID A.; MALEY, MARTIN P. (P-10);

ROBERTSON, ROBERT G. H. (P-3); WILKEASON, J. F. (P-3).

LIMIT ON ANTI-ELECTRON-NEUTRINO MASS FROM FREE MOLECULAR TRITIUM BETA DECAY.

IN: TELEMARK IV CONFERENCE-NEUTRINO MASSES AND NEUTRINO ASTROPHYSICS, 1987,

ASHLAND, WIS. PROC., P.20-7. WORLD SCIENTIFIC, 1987. (QC793.5.N426 N49 1987X)

40-101

ZINN, JOHN (ESS-7); SUTHERLAND, C. DEXTER (ESS-7); FENIMORE, EDWARD E. (ESS-9); GANGULY, S.

SOLAR FLARE OF 18 AUGUST 1979-INCOHERENT SCATTER RADAR DATA AND PHOTOCHEMICAL MODEL COMPARISONS.

LOS ALAMOS, 1988. 25P. (LA-11200-MS)

40-102

ZUREK, WOJCIECH H. (T-6).

RICHNESS DISTRIBUTION OF STRING-SEEDED ABELL CLUSTERS-ACCRETION BY A LOOP VERSUS

HIERARCHICAL CLUSTERING.

ASTROPHYS. J., V.324, P.19-34. 1988.

40.103

ZUREK, WOJCIECH H. (T-6); QUINN, P. J.; SALMON, J. K.

ROTATION OF HALOS IN OPEN AND CLOSED URIIVERSES-DIFFERENTIATED MERGING AND NATURAL SELECTION OF GALAXY TYPES.

ASTROPHYS ', V.330, P.519-34. 1988 
41-1

ALCOUFFE, RAYMOND E. (X-6); HOLM, DARRYL D. (T-7); O'ROURKE, PETEA J. (T-3).

PROCEEDINGS OF THE CONFERENCE ON NUMERICAL METHODS IN HIGH TEMPERATURE PHYSICS. LOS ALAMOS NATIONAL LABORATORY, 1988. 350P. (LA-11342-C)

41-2

ARKO, AL J. (P.10); KOELLING, D. D.; CAPASSO, C.; DEL GIUDICE, M.; OLSON, C. G.

IMAGING OF 5F DENSITIES OF STATES IN RESONANT PHOTOEMISSION MEASUREMENTS.

PHYS. REV., V.38B, P.1627-31. 1988.

41-3

ARNDT, T.; BACKE, H.; STEINHOF, A.; DYER, PEGGY (P-3); FOWLER, MALCOLM M. (INC-11);

ELLER, P. GARY (INC-4); WILHELMY, JERAY B. (INC-11).

RADIATIVE LIFETIMES OF AMERICIUM I ATOMIC LEVELS.

PHYS. REV., V.38A, P.5084-91. 1988.

41-4

AUER, LAWRENCE H. (ESS-5).

ACCELERATION OF CONVERGENCE.

IN: NUMERICAL RADIATIVE TRANSFER, W. KALKOFEN, ED., P.101-8. CAMBRIDGE UNIVERSITY PRESS, 1987. (QB/809.NB5/1987X)

41-5

BAER, H. W. (MP-4); BERGMANN, ANTON G. (MP-13); BOWMAN, J. DAVID (MP-4); IROM, FAROKH (NP-4); LEITCH, M. J. (P-2); SEFTOR, COLIN J. (MP-13); ROKNI, S. H.; ALSTER, JONAS; PIASETZKY, EU;

CLAUSEN, B. L.; LOVEMAN, ROBERT A.; PETERSON, R. J.; ULLMANN, J. L.; COMFORT, J. R.;

KNUDSON, JAMES N.; SENNHAUSER, U.

ISOBARIC-ANALOG-STATE TRANSITIONS IN PION CHARGE-EXCHANGE REACTIONS ABOVE THE

DELTA(1232) RESONANCE.

IN: PION-NUCLEUS PHYSICS-FUTURE DIRECTIONS AND NEW FACILTIES AT LAMPF, 1987, LOS

ALAMOS NATIONAL LABORATORY, PROC., P.67-78. AMERICAN INSTITUTE OF PHYSICS, 1388. (CC793.5 M42 P56 1987X)

$41-6$

BAKER, GEORGE A., JR. (T-11).

PLURALISM IN THE CRITICAL PHENOMENA OF THE ONE-DIMENSIONAL CONTINUOUS-SPIN ISING NODEL PHYS. REV. LETT., V.60, P.1844-6. 1988.

41-7

BAKER, GEORGE A., JR. (T-11); OITMAA, JAAN; VELGAKIS, M. J.

SERIES ANALYSIS OF MULTIVALUED FUNCTIONS.

PHYS. REV., V.38A, P.5316-31. 1988.

41-8

BAKER, GEORGE A., JR. (T-11).

STRING PERTURBATION THEORY.

PHYS. REV. LETT., V.61, P.1516. 1988.

41.9

BALDWIN, GEORGE C. (P-3).

SUPERLATTICE GEOMETRY FOR GAMMA-RAY LASERS.

J. QUANT. SPECTROSC. PADIAT.TRANSFER, V.40, P.685-91. 1988.

41-10

BARNES, JOHN F. (T-DO); LYON, STANFORD P. (T-1).

SESAME EQUATION OF STATE NUMBER 7530, BASALT.

LOS ALAMOS NATIONAL LABORATOAY, 1988. 1OP. (LA-11253-MS) 
41-11

BECHTEL, S. E.; FOREST, M. G.; HOLM, DARRYL D. (T-7); LIN, K. J.

1-D CLOSURE MODELS FOR SLENDER 3-D VISCOELASTIC FREE JETS-VON KARMAN FLOW GEOMETAY AND ELLIPTICAL CROSS SECTION.

TIC. 1988. 14P. MN (LA-UR-88-1742)

41-12

BEDELL, KEVIN S. (T-11); PINES, DAVID (CMS).

SPIN-FLUCTUATION-INDUCED SUPERCONDUCTIVITY IN YTTAIUM BARIUM 2 COPPER 3 OXYGEN 6.9 PHYS. REV., V.378, P.3730-3. 1988.

$41-13$

BELAK, J.; ETTERS, RICHARD D. (M-6); LE SAR, RICHARD A. (T- 1 !).

THERMODYNAMIC PROPERTIES AND EQUATION OF STATE OF DENSE FLUID NITROGEN. J. CHEM. PHYS., V.89, P.1625-33. 1988.

41-14

BENJAMIN, ROBERT F. (M-6).

EXPERIMENTAL OBSERVATIONS OF SHOCK STABILITY AND SHOCK-INDUCED TURBULENCE.

TIC, 1988. 13P. MN (LA-UR-88-4229)

41-15

BERNAPDIN, MICHAEL P. $(X-10)$.

SCALING LAWS FOR COUPLING QUANTITIES ON EMP.

IEEE TRANS. ELECTROMAGN. COMPAT., V.30, P.48-53. 1988.

$41-16$

BESNARD, DIDIER (CNLS); HARLOW, FRANCIS H. (T-3).

TURBULENCE IN MULTIPHASE FLOW. INT. J. MULTIPHASE FLOW, V.14, P.679-99. 1988.

41-17

BINDER, PHILIPPE M. (CNLS); DHUMIERES, DOMINIQUE; POU!OLL, L.

PROPERTIES OF TAGGED LATTICE FLUIDS-II. VELOCITY CORAELATION FUNCTIONS.

TIC. 1988. 7P. MN (LA-UR-88-3681)

41-18

BISHOP, ALAN R. (T-11); HOROVITZ, BARUCH; LOMDAHL, PETER S. (T-11)

CURRENT OSCILLATIONS IN NEAR-COMMENSURATE SYSTEMS.

PHYS. REV., V.388, P.4853-62. 1988.

41-19

BLAU, STEVE (T-8); VISSER, MATT (T-8); WIPF, ANDREAS (T-8).

DETERMINANTS OF CONFORMAL WAVE OPERATORS IN FOUR DIMENSIONS. PHYS. LETT., V.209E, P.209-13. 1988.

41-20

BONESS, DAVID A.; BROWN, J. MICHAEL; SHANER, J. W. (M-6).

RAREFACTION VELOCITIES IN SHOCKED LEAD. IN: SHOCK WAVES IN CONDENSED MATTER, 1987, MONTEREY. PROC., P.115-8. ELSEVIER, 1988. (CC173.4.C65 S57 1987X)

ALSO PUBLISHED: TIC, 1987. 5P. MN (LA-UR-87-2283)

41-21

BOROVSKY, JOSEPH E. (ESS-8)

INDUCED ABSORPTION OF EXTRAORDINARY (Z)-MODE) WAVES VIA ELECTRON PUMPING. PHYS. FLUIDS, V.31, P.700-2. 1988.

$41 \cdot 22$

BOWMAN, CHARLES D. (P-3).

NEW NEUTRON PHYSICS USING SPALLATION SOUACES

TIC, 1888. 28P. MN (LA-UR-88-1640) 
$41-23$

BRACKBILL, JEREMIAH U. (T-3).

RINGING INSTABILITY IN PARTICLE-IN-CELL CALCULATIONS OF LOW-SPEED FLOW. J. COMPUT. PHYS., V.75, P.469-92. 1988

$41-24$

BRACKBILL, JEREMIAH U. (X-DO); KOTHE, D. B. (X-1); RUPPEL, HANS M. (T-3).

FLIP-A LOW-DISSIPATION, PARTICLE-IN-CELL METHOD FOR FLUID FLOW.

COMPUT. PHYS. COMMUN., V.48, P. 25-38. 1988.

ALSO PUBLISHED: TIC. 1987. 24P. MN (LA-UR-87-2897)

41-25

BRAND, HELMUT R.; DORIA, MAURO M. (CNLS); PLEINER, HARALD.

GRADIENT TERMS IN THE GINZBURG-LANDAU FREE ENERGY FOR PURE AND THORIUM-DOPED

SUPERCONDUCTING UAANIUM BERYLLIUM- 13.

PHYS. REV. LETT., V.60. P.2810. 1988.

41-26

BRAND, HELMUT R.

PHASE DYNAMICS-A REVIEW AND A PERSPECTIVE

IN: INTERNATIONAL WORKSHOP ON PROPAGATION IN NONEQUILIBRIUM SYSTEMS, 1987, LES HOUCHES, FRANCE. PROC., P.206-24. SPRINGER-VERLAG, 1988. (OC150 .P76 1987X)

ALSO PUBLISHED: TIC, 1987. 33P. MN (LA-UR-87-2554)

41-27

BRAND, HELMUT R.; DORIA, MAURO M. (CNLS).

SPECIFIC HEAT AND THE NUMBER OF COMPONENTS OF THE ORDER PARAMETER IN THE 9O-K SUPERCONDUCTORS.

PHYS. REV. LETT., V.61, P.480. 1988

41-28

BRANDENBURG, RICHARD A.; CHULICK, GARY S.; MACHLEIDT, R.; PICKLESIMER, A. (P-15); THALER, R. M. ESSENTIAL MECHANISMS IN THE TRITON BINDING

PHYS. REV., V.37C, P.1245-52. 1988.

41-29

BRANDOW, BAIRD H. (T-11).

FINITE-TEMPERATURE BEHAVIOR OF THE ANDERSON LATTICE.

PHYS. REV., V.37B, P.250-60. 1988.

41-30

BRANDOW, BAIRD H. (T-11).

THEORY OF MOTT INSULATORS.

IN: NATO ADVANCED STUDY INSTITUTE ON NARROW-BAND PHENOMENA-INFLUENCE OF ELECTRONS WITH BOTH BAND AND LOCALIZED CHARACTER, 1987, NIJMEGEN, NETHERLANDS. PROC., P.97-109. PLENUM PRESS, 1988. (OC1 .N37X V.184)

ALSO PUBLISHED: TIC, 1987. 15P. MN (LA-UR-87-1755)

41-31

BRISON, J. P.; LASJAUNIAS, J. C.; RAVEX, A.; FLOUOUET, JACOUES; JACCARD, D.; FISK, ZACH.ARY (P-10);

SMITH, JAMES L. (CMS).

LOW TEMPERATURE MEASUREMENTS ON URANIUM BEAYLLIUM-13.

IN: INTERNATIONAL CONFERENCE ON HIGH TEMPERATURE SUPERCONDUCTORS AND MATERIALS AND MECHANISMS OF SUPERCONDUCTIVITY, 1988, INTERLAKEN, SWITZERLAND. PROC., P.437-8. NORTH.HOLLAND, 1988. (OC611.98.H54 157 1988X)

ALSO PUBLISHED: TIC, 1988. 3P. MN (LA-UR-88-1926)

41-32

BROWN, STUART E. (P-10); WILLIS, JEFFREYO. (P-10): ALAVI, B.; GRUNER, GEORGE.

EFFECT OF SUBSTITUTIONAL IMPURITIES ON THE LOW-TEMPERATURE SPECIFIC HEAT OF (TANTALUM

SELENIUM-4)2 IODINE.

PHYS. REV., V.37B, P.6551-4. 1988 
41-33

BUKIET, BRUCE G. (T-14); JONES, JAMES W. (T-14).

COMPETITION BETWEEN CURVATURE AND CHEMISTRY IN A SPHERICALLY EXPANDING DETONATION.

APPL. PHYS. LETT., V.52, P.1921-3. 1988.

41-34

CAMPBELL, DAVID K. (CNLS); GAMMEL, JAN T. (T-11); LOH, EUGENE Y. (T-11).

BOND-CHARGE COULOMB REPULSION IN PEIERLS.HUBBARD MODELS.

PHYS. REV., V.38B, P.12043-6. 1988.

41-35

CAMPBELL, DAVID K. (CNLS); GAMMEL, JAN T. (T-11); LOH, EUGENE Y. (T-11).

EXTENDED PEIERLS-HUBBARD MODEL-OFF-DIAGONAL TERMS

SYNTH. MET., V.27, P.A9-14. 1988.

ALSO PUBLISHED: TIC, 1988. 7P. MN (LA-UR-88-2613)

41-36

CAMPBELL, LAURENCE J. (T-11); DORIA, MAURO M. (CNLS); KOGAN, VLADIMAR G.

VORTEX LATTICE STRUCTURES IN UNIAXIAL SUPERCONDUCTORS.

PHYS. REV., V.38B, P.2439-43. 1988.

41-37

CANAVAN. GREGORY H. (P.DO); HUNTER, ROBERT O., JR.; HUNTER, A. M., II (P.16).

DEVELOPMENT AND APPLICATION OF ADVANCED HIGH ENERGY LASERS.

IN: ENERGY IN PHYSICS, WAR AND PEACE; A FESTSCHRIFT CELEBRATING EDWARD TELLER'S 8OTH BIRTHDAY, H. MARK AND L. WOOD, EDS., P.367-384. KLUWER ACADEMIC, 1988. (OC71 .E62 1988)

$4 i-38$

CHEONG, SANG-WOOK (P-10); FISK, ZACHARY (P-10); KWOK, RAYMOND S; REMEIKA, J. P.;

THOMPSON, JOE D. (P-10); GRUNER, GEORGE.

ELECTRONIC ANISOTROPY IN SINGLE.CRYSTAL LANTHANUM-2 COPPER OXYGEN-4

PHYS. REV., V.37B, P.5916-9. 1988.

41-39

CHITANVIS, SHIRISH M. (T-DOT); ZARDECKI, ANDAEW (T-DO).

EFFECT OF THERMAL BLOOMING ON PULSE PROPAGATION THROUGH VAPORIZING AEROSOLS. APPL. OPT., V.27, P.2495-501. 1988.

41-40

CHITANVIS, SHIRISH M. (T-DOT).

STIMULATED BRILLOUIN SCATTERING IN SOLID AEROSOLS.

TIC, 1988. 18P. MN (LA-UR-88-2876)

41-41

CHUNG, YUN C. (CLS-5); SHAY, T. M. (CLS-5).

FREOUENCY STABILIZATION OF A DIODE LASER TO A FABRY-PEROT INTERFERONETER. OPT. ENG., V.27, P.424-7. 1988.

41-42

CIOSLOWSKI, JERZY (T-12).

WHY DOES THE AITKEN EXTRAPOLATION OFTEN HELP TO ATTAIN CONVERGENCE IN SELF.

CONSISTENT FIELD CALCULATIONS?

J. CHEM. PHYS., V.89, P.2126-7. 1988

41-43

COGGESHALL, S. V. $(x-1)$; MEAD, W. C. $(x-1)$; JONES, ROGER D. $(x-1)$.

"FLICKER" IN SMALL SCALE LASER-PLASMA SELF-FOCUSING.

PHYS. FLUIDS, V.31, P.2750-2. 1988.

41-44

COHEN, JAMES S. (T-12).

CHARGE TRANSFER AND IONILATION IN COLLISIONS OF ALPHA-MU WITH ALL ELEMENTS.

PHYS. REV., V.37A, P.2343-8. 1988. 
41-45

COHEN, JAMES S. (T-12); STRUENSEE, MICHAEL C. (T-12).

STARK MIXING OF $2 S$ MUONIC HELIUM-II. MOLECULAR AND MULTIPLE-SCATTERING EFFECTS IN HYDROGEN.

PHYS. REV., V.38A, P.53-7. 1988.

41-46

COLTON, EUGENE P. (MP-14).

LONGITUDINAL PHASE-SPACE FORMULAS FOR STATIONARY BUCKETS

TIC. 1988. 6P. MN (LA-UH-88-1655)

$41-47$

COOKE, D. W. (MP-14); HUTSON, RICHARD L. (MP-3); KWOK, RAYMOND S.; MAEZ, M. (MP.14); PEMPP, H.; SCHILLACI, MARIO E. (MP-DO); SMITH, JAMES L. (CMS); WILLIS, JEFFREY O. (P-10); LICHTI. R. L.;

CHAN, K.-C. B.: BOEKEMA, CAROLUS; WEATHERSEY, S. P.; FLINT, J. A.; OOSTENS. J.

MUON DEPOLARIZATION AND MAGNETIC FIELD PENETRATION DEPTH IN SUPERCONDUCTING GADOUNIUM BARIUM-2 COPPER-3 OXYGEN-X.

PHYS. REV., V.37B, P.9401-4, i988.

$41-48$

COOKE, D. W. (MP. 14); HUTSON, RICHARD L. (MP-5); KWOK, RAYMOND S.; MAEZ, M. (MP.14);

REMPP, H. (MP-DO); SCHILLACI, MARIO E. (MP-DO); SMITH, JAMES L (CMS); WILLIS, JEFFREY O. (P-10);

UCHT, R. L; CHAN, K.-C. B.; BOEKEMA, CAROLUS: WEATHERSBY, S. P.: OOSTENS, J.

MUON SPIN DEPOLARIZATION IN GADOLINIUM-AND EUROPIUM BARIUM 1 COPPER 3 OXYGEN $X$.

TIC. 1988. 7P. MN (LA-UR-88-1519)

41.49

COOPER, MARTIN D. (MP-4).

MEGA EXPERIMENT [MUON DECAYS INTO AN ELECTRON AND A GAMMA RAY] EXPEAIMENT-A SEARCH FOR MU YIELDS EGAMMA.

IN: CONFERENCE ON THE INTERSECTIONS BETWEEN PARTICLE AND NUCLEAR PHYSICS, 3RD, 1988, POCKPORT, MAINE. PROC., P.845-9. AMERICAN INSTITUTE OF PHYSICS, 1988. (OC1 .A38X NO.176) ALSO PUBUSHED: TIC, 1988. 6P. MIN (LA-UR-88-1866)

41-50

COPENHAVER, CARL (ESS-8).

UNEAR THEOFY OF DRIFT-TEARING AND -INTERCHANGE MODES.

PHYS. FLUIUS, V.31, P.2937-43. 1988.

41-51

CORNELUS, WAYNE D. (AT-7).

SOURCES OF POLARIZED IONS AND ATOMS.

TIC, 1988. 22P. MN (LA-UR-88-1446)

$41-52$

CALY, BART J. (T-3).

NUMERICAL STUDY OF RAYLEIGH-TAYLOR INSTABILITY IN ALUMINUM AND STEEL PLATES. LOS ALAMOS, 1988. 31P. (LA-11192-MS)

41.53

DAVID, D. (CNLS).

HAMILTONIAN REDUCTION AND COMPLEX BEHAVIOUR IN NONLINEAR POLARIZATION DYNAMCS.

TIC, 1988. 5P. MN (LA-UA-88-2652)

41-54

DAVIS, CECIL G. (P-15).

IMPROVEMENTS TO SOIL (AN EULERIAN' HYDRODYNAMICS CODE).

LOS ALAMOS, 1988. 23P. (LA-11199-MS)

41-55

DENSKE, D.; BRAZELL, NICK; FARLEY, W. EDWIN; POGUE, E.; WARNES, R. H. (M-4)

INTENSE ELECTRON BEAM DETONATION OF INSENSITIVE ENERGETIC MATERIALS.

IN: SHOCK WAVES IN CONDENSED MATTER, 1987, MONTEREY. PROC., P.773-80. ELSEVIEA, 1988.

(OC173.4.C65 S57 1987X) 
41-56

DICK, JERRY JOEL (M-9).

STRESS-STRAIN HISTORIES IN SHOCKED POLYMETHYL METHACAYLATE.

IN: SHOCK WAVES IN CONDENSED MATTER, 1987, MONTEREY. PROC., P.301-4. ELSEVIER, 1988. (QC173.4.C65 S57 1987X)

41.57

DINGUS, RONALD S. (P-DO); GOLDMAN, SANFORD R. $(X-1)$.

PLASMA ENERGY BALANCE MODEL FOR OPTICAL-LASER-INDUCED IMPULSE IN VACUO

IN: INTERNATIONAL CONFERENCE ON LASERS, 1986, ORLANDO. PROC., P.111-22. STS PRESS,

ALSO PUBLISHED: TIC, 1986. 13P. MN (LA-UR-87-0083)

41-58

DOLL, JIMMIE D. (CLS-2); FREEMAN, DAVID L.

COMPARATIVE ANALYSIS OF STATIONARY-PHASE MONTE CARLO METHODS

J. PHYS. CHEM., V.92, P.3278-80. 1988.

41-59

DOLL, JIMMIE D. (CLS-2); FREEMAN, DAVIO L..; VOTER, ARTHUA F. (T-12).

RECENT DEVELOPMENTS IN THE THEORY OF MANY-BODY PROCESSES-IMPLICATIONS FOR THE STUDY OF SURFACE DYNAMICS.

IN: WORKSHOP ON INTERFACE PHENOMENA, 2ND, 1987, CAMPOBELLO ISLAND, CANADA. PROC., P.144-54. SPRINGER VERLAG, 1988. (OD506.A1 S68X V.12)

41-60

DOMBECK, THOMAS W. (P-3); FINCH, ANN (P-3); GPISHAM, DONALD L. (MP-7).

DETAILS OF A WATER TARGET USED IN A HIGH-CURRENT APPLICATION FOR A NEUTRINO- PHYSICS EXPERIMENT AND THE DIRECT MEASUREMENT OF THE INCIDENT PROTON BEAM BY THE INDUCED

CHANGE IN THE WATER CONDUCTIVITY.

NUCL. INSTRUM. METHODS PHYS. RES., V.A272, P.650-9. 1988

41-61

DOMBECK, THOMAS W. (P-3); FINCH, ANN (P-3); GRISHAM, DONALD L. (MP-7).

DIRECT MEASUREMENT OF A PROTON BEAM PASSING THROUGH A WATER TARGET BY THE INDUCED

CHANGE IN THE WATER CONDUCTIVITY.

IN: IEEE PARTICLE ACCELERATOA CONFERENCE, 1987, WASHINGTON, D.C. PROC., P.588-90. IEEE, 1987. (OC787.P3 137 1987)

41-62

DOMBECK, THOMAS W. (P-3); BOWLES, THOMAS J. (P-3); BROWN, ALLEN A. (P-3); BFOWN, RONALD E. (P-3): BURMAN, ROBERT L (MP-4); CLARK, DAVID A. (MP-6); CLEARWATER, SCOTT H. (AT-8);

KRUSE, HERALD W. (P-14); LEE, DAVID (MP-13); AAS, BJORNE; IGO, G.; NEWSOM, C.; BEAVIS, D.; FUNG, S. Y.; GORN, W.; POE, R. T.; VAN DALEN, G. J.; BASSALLECK, B.; DIETERLE, B.; HILL, ROBERT E. (MP-2):

KANG, J. H.; LEAVITT, C. P.; AUERBACH, L. B.; DATTA, S.; HUANG, Y.; FISK, R.; KOETKE, D.; MNWEILER, R. SEARCH FOR NEUTRINO OSCILLATIONS IN THE APPEARANCE MODE NU MU GOING TO NU E FOR NEUTRINO ENERGIES NEAR THE MUON THRESHOLD

PHYS. LETT., V.194B, P.591-6. 1987

41.53

DORIA, MAURO M. (CNLS); SATIJA, INDUGALA I. (CNLS)

QUASIPERIODICITY AND LONG-RANGE ORDER IN A MAGNETIC SYSTEM. PHYS. REV. LETT., V.60, P.444-7. 1988

41-64

DORIA, MAURO M. (CNLS); BORGES, HORTENCIO A. (P.10)

SPIN-SINGLET PAIRING MECHANISM IN MAGNON-PAIRING THEORIES PHYS. REV., V.38B, P.4999-5001. 1988.

41-65

DUBOIS, DONALD F. (T-DOT); ROSE, HARVEY A. (T-DOT); RUSSELL, DAUID A. (T-DOT)

POWER SPECTRA OF FLUCTUATIONS IN STAONG LANGMUIR TURBULENCE.

PHYS. REV. LETT., V.61, P.2209-12. 1988 
41.66

DYEA, PEGGY (P-3); SOUNDS, J. A. (P-15).

LASER SPECTROSCOPY OF THULIUM- 170.

PHYS. REV., V.38C, P.2813-7. 1988.

41-67

EARLY, JAMES WILLIANM (CLS-1),

DEFLECTION OF BARIUM ATOMS BY A STANOING-WHAVE LIGHT FIELD.

OPT. COMMUN., V.65, P.250-6. 1988.

$41-68$

ECKE, ROBERT E. (P-10); KEVREKIDIS, I. G. (CNLS).

INTERACTIONS OF RESONANCES AND GLOBAL BIFURCATIONS IN RAYLEIGH-BENARD CONVECTION. PHYS. LETT., V.131A, P.344-52. 1988.

41.69

ECKE, ROBERT E. (P-10); HAUCKE, HANS (P-10).

NOISE-INDUCED INTERMITTENCY IN THE QUASIPERIODIC REGIME OF RAYLEIGH-BENARD CONVECTION. J. STAT. PHYS., V.54, P.1153-72. 1989.

ALSO PUBLISHED: TIC, 1988. 33P. MN (LA-UR-88-3239)

$41-70$

ECKERT, JUERGEN (P-8); KUBAS, GREGORY J. (INC-4); DIANOUX, ALBEAT J.

ROTATIONAL TUNNELING OF BOUND HYDROGEN IN A TUNGSTEN COMPLEX.

J. CHEM. PHYS., V.88, P.466-8. 1988.

41-71

ENGELKE, RAY P. (M-9).

DEPENDENCE OF THE NITROGEN VIBRATIONAL POTENTIAL ON DENSITY.

J. CHEM. PHYS., V.88, P.215961. 1988.

41-72

ERPENBECK, JEROME J. (T-11).

SHEAR VISCOSITY OF THE LENNARD-JONES FLUID NEAR THE TRIPLE POINT-GREEN-KUBO RESULTS. PHYS. REV., V.3BA, P.6255-66. 1988.

41.73

FABER, VANCE (C-3); MANTEUFFEL, THOMAS A. (C-3).

LOOK AT TRANSPORT THEORY FROM THE POINT OF VIEW OF LINEAR ALGEBRA.

TIC, 1988. 26P. MN (LA-UR-88-2625)

41-74

FARMER, J. DOYNE (CNLST-7); SIDOROWICH, JOHN J. (CNLS).

CAN NEW APPROACHES TO NONLINEAR MODELING IMPROVE ECONOMIC FORECASTS?

IN: THE ECONOMY AS AN EVOLVING COMPLEX SYSTEM-PROCEEDINGS OF THE EVOLUTIONARY PATHS OF THE GLOBAL ECONOMY WORKSHOP, 1987, SANTA FE, NEW MEX. PROC., P.99-115.

ADDISON-WESLEY, 1988. (HB21 E265 1987X)

41.75

FEAREY, BRYAN L. (INC-7); PARENT, DENISE C, (CLS-2); KELLER, RICHARD A. (CLS-2);

MILLER, CHARLES M. (INC-7).

VERY HIGH RESOLUTION SATURATION SPECTROSCOPY OF LUTETIUM ISOTOPES VIA CW

SINGLE-FREOUENCY LASER RESONANCE IONIZATION MASS SPECTROMETRY.

TIC, 1987. 4P. MN (LA-UR-87-4244)

41-76

FISK, ZACHARY (CMS); HESS, D. W. (CMS); PETHICK, C. J.; PINES, DAVID (CMS); SMITH, JAMES L. (CMS);

THOMPSON, JOE D. (P-10); WILLIS, JEFFREY O. (P-10).

HEAVY ELECTRON METALS-NEW HIGHLY CORRELATED STATES OF MATTER. SCIENCE, V.239, P.33-42. 1988. 
41.77

FISK, ZACHARY (P-10); BORGES, HORTENCIO A. (P.10); MC ELFRESH, M. W. (P.10); SMITH, JAMES;

THOMPSON, JOSEPH; OTT, HANS R.; AEPPLI, G.; BUCHER, ERNST; LAMBERT, STEVEN E.:

MAPLE, MERRILL B.; BROHOLM, C.; KJEMS, J. K.

SUPERCONDUCTIVITY OF HEAVY-ELECTRON URANIUM COMPOUNDS.

TIC, 1988. 8P. MN (LA-UR-88-0788)

41-78

FOLLANSBEE, PAUL S. (MST-5).

HIGH-STRAIN-RATE DEFORMATION MECHANISMS IN COPPER AND IMPLICATIONS FOR BEHAVIOR DUAING SHOCK-WAVE DEFORMATION.

IN: SHOCK WAVES IN CONDENSED MATTER, 1987, MONTEREY. PROC., P.249-54. ELSEVIEF, 1988. (OC173.4.C65 557 1987X)

ALSO PUBLISHED: TIC, 1987, 19P. MN (LA-UR-87-2198)

41.79

FRIAR, JAMES L. (T-5).

THREE-BODY FORCES AND THE TRINUCLEONS.

IN: CEBAFISURA SUMMER WORKSHOP, 1987, NEWPORT NEWS, VA. PROC., P.210-23. CONTINUOUS ELECTRON 8EAM FACILITY, 1987. (OC/787/.P3/C4/1987X)

\section{1-80}

GAMMEL, JAN T. (T-11).

OPTICAL ABSORPTION OF THE SOLITON LATTICE IN PCLYACETYLENE REEXAMINED.

PHYS. REV., V.378, P.6517-8, 1988.

41-81

GARZON. F. H. (MEE-11); BEERY, JEROME G. (MEE-11); RAISTRICK, IAN D. (MEE-11).

AMORPHOUS-TO-CAYSTALLINE TRANSFORMATIONS IN BISMUTH-OXIDE-BASED HIGH TC

SUPERCONDUCTORS.

APPL. PHYS. LETT., V.53, P.805-7. 1988.

41-82

GITOMER, STEVEN J. $(X-1)$; BENDER, STEVEN C. (CLS-8); MC VEY, BRIAN D. $(X-1)$.

MOCE-MEDIA INTERACTIONS IN AN FEL [FREE ELECTRON LASER].

NUCL. INSTRUM. METHODS PHYS. RES., V.A272, P.610-6. 1988.

ALSO PUBLISHED: TIC, 1987, 37P. MN (LA-UR-87-3929)

$41-83$

GLIMM, JAMES; SHARP, DAVID H. (T-8).

$S$ MATRIX THEORY FOR CLASSICAL NONLINEAR PHYSICS.

FOUND. PHYS., V.16, P.125-40. 1986.

ALSO PUBLISHED: BETWEEN OUANTUM AND COSMOS, W.H. ZUREK, ED., P.42-58. PRINCETON

UNIVERSITY PRESS, 1988. (OC71.B45 1988)

41-84

GLIMM, JANES; LI, X. L.; MENIKOFF, RALPH (T-14); SHARP, DAVID H. (T-8),

STATISTICAL THEORIES OF RAYLEIGH-TAYLOR INSTABILITY FOR COMPRESSIBLE FLUIDS.

TIC, 1988. 1OP. MN (LA-UR-88-3702)

41.85

GRUNER, GEORGE

COMPETING INTERACTIONS-CHARGE DENSITY WAVES AND MMURITIES IN: CMS WORKSHOP ON COMPETING INTERACTIONS AND MICROCLUSTERS-STATICS AND DYNAMICS. i\&87. LOS ALAMOS, N.M. PROC., P.202-11. SPRINGER-VERLAG, 1988. (OC173.4.C65 C58 1987)

41-86

GUY, FPANK W. (AT-1); LAPOSTOLLE, P. M. (AT-1); WANGLER, THOMAS P. (AT-1).

INFLUENCE OF DENSITY DISTRIBUTION ON THE STABILITY OF BEAMS. IN: IEEE PARTICLE ACCELERATOR CONFERENCE, 1987, WASHINGTON. D.C. PROC., P.1149.51. IEEE, 1987. (OC787.P3 137 1987)

ALSO PUBLISHED: TIC, 1987. 4P. MN (LA-UR-87-0645) 
41-87

HAMMEL, P. CHRISTOPHER (P-10); TAKIGAWA, MASASHI (P-10); HEFFNER, ROBERT H. (P-10):

FISK, ZACHARY (P-10).

COPPER NUCLEAR QUADRUPOLE RESONANCE IN GADOLINIUM BARIUM-2 COPPER-3 OXYGEN-7-

DETERMINATION OF SITE ASSIGNMENT.

PHYS. REV., V.38B, P.2832-5. 1988.

41-88

HARLOW, FRANCIS H. (T-3).

PIC [PARTICLE-IN-CELL] AND ITS PROGENY.

COMPUT. PHYS. COMMUN., V.48, P.1-10. 1988.

ALSO PUBLISHED: TIC, 1987. 17P. MN (LA-UR-87-1862)

41.89

HODGDON. MARION L. (M-6).

APPLICATIONS OF A THEORY OF FERROMAGNETIC HYSTERESIS.

IEEE TRANS. MAGN., V.24, P.218-21. 1988.

41-90

HOLIAN, BRAD LEE (T-1).

MODELING SHOCKWAVE DEFORMATION VIA MOLECULAR DYNAMICS.

IN: SHOCK WAVES IN CONDENSED MATTER, 1987, MONTEREY. PROC., P.185-90. ELSEVIER, 1988. (CC173.4.C65 S57 1987X)

ALSO PUBLISHED: TIC, 1987. 7P. MN (LA-UR-87-2367)

41-91

HOLIAN, KATHLEEN S. (T-1)

HYDROCODE SIMULATIONS OF HYPERVELOCITY IMPACTS.

IN: SHOCK WAVES IN CONDENSED MATTER, 1987, MONTEREY. PROC., P.725-8. ELSEVIER, 1988.

(OC173.4.C65 S57 1987X)

41-92

HOLM, DARAYL D. (T-7); KUPERSHMIDT, BORIS A.

ANALOGY BETWEEN SPIN GLASSES AND YANG-MILLS FLUIDS.

J. MATH. PHYS., V.29, P.21-30, 1988.

$41-93$

HOLM, DARRYL D. (T-7).

HAMILTONIAN STRUCTURE FOR TWO-DIMENSIONAL HYDRODYNAMICS WITH NONLINEAR DISPERSION. PHYS. FLUIDS, V.31, P.2371-3. 1988.

$41-94$

HOLM, DARRYL D. (T-7).

NONLINEAR STABILITY OF IDEAL FLUID EQUILIBRIA.

TIC, 1988. 67P. MN (LA-UR-88-1743)

41-95

HOOVER, WILLIAM G.; MORAN, B.; HOLIAN, BRAD LEE (T-1); POSCH, HARALD A.; BESTIALE, S.

COMPUTER SIMULATION OF NONEQUILIBRIUM PROCESSES.

IN: SHOCK WAVES IN CONDENSED MATTER, 1987, MONTEREY. PROC., P.19i-4. ELSEVIE., 1988. (OC173.4.C65 S57 1987X)

41.96

HOROVITZ, BARUCH; BISHOP, ALAN R. (T-11); PHILLPOT, SIMON R

SEMICLASSICAL FORMALISM OF OPTICAL ABSORPTION AND BREATHERS IN POLYACETYLENE.

PHYS. REV. LETT., V.60, P.2210-3. 1988.

41-97

HOWE, STEVEN D. (ET/ETIO): HYNES, M. V. (P-15).

EXCITATION OF LAGING MEDIA USING ANTIPROTON-ANNIHILATION PRODUCTS

TIC, 1988. 11P. MN (LA-UR-88-3983)

41-90

HUGHES, RICHARD J. (T-8); NIETO, MICHAEL MARTIN (T-8); GCLDMAN, TERRENCE J. (T-5).

EOTVOS VERSUS A GALILEO EXPERIMENT-A STUDY IN TWO- VERSUS THREE-DIMENSIONAL PHYSICS.

PHYS. LETT., V. 212B, P.18-22. 1988 
$41-99$

HYMAN, JAMES M. (T-7); NOVICK-COHEN, AMY; ROSENAU, PHILIP.

MODIFIED ASYMPTOTIC APPROACH TO MODELING A DILUTE-BINARY-ALLOY SOLIDIFICATION FRONT.

PHYS. REV., V.37B, P.7603-8. 1988.

41- 100

JASON, ANDREW JOHN (AT-3); SVATON, ERNIE M. (AT-6); BLIND, BARBARA (AT-3);

HEIGHWAY, EDWARD A. (AT-6).

DESIGN OF ACHROMATIC BENDING SYSTEMS IN THE PRESENCE OF SPACE CHARGE.

IN: IEEE PARTICLE ACCELERATOR CONFERENCE, 1987, WASHINGTON, D.C. PROC., P.1767-9. IEEE, 1987. (QC787.P3 137 1987)

41-101

JOHNSON, JAMES N. (T-1); ADDESSIO, F. L. (T-3).

TENSILE PLASTICITY AND DUCTILE FRACTURE. J. APPL. PHYS., V.64, P.6699-712. 1988.

41-102

JOHNSON, MIKKEL B. (MP-DO).

INSTANTANEOUS MESON-EXCHANGE POTENTIALS ON THE LIGHT FRONT. TIC, 1988. 5P. MN (LA-UR-88-1809)

41-103

JONES, ROGER D. $(X-1)$.

KINETIC THEORY OF ELECTRON DRIFT VORTEX MODES.

PHYS. FLUIDS, V.31, P.535-7, 1988.

41-104

JONES, ROGER D. (X-1); MEAD, W. C. (X-1); COGGESHALL, S. V. $(X-1)$; ALDRICH, CHARLES H. (X-7);

NORTON, JOHN L. (X-7); POLLAK, GREGORY D. $(X-7)$; WALLACE, JON M. $(X-1)$.

SELF-FOCUSING AND FILAMENTATION OF LASER LIGHT IN HIGH $Z$ PLASMAS.

PHYS. FLUIDS, V.31, P.1249-72. 1988.

41-105

KIM, GEORGE J.

MEASUREMENT OF THE LEFT-RIGHT ASYMMETRY IN PION-PROTON RADIATIVE EXCHANGE AND

CHARGE EXCHANGE SCATTERING FROM 301 TO 625 MEVIC ON A TRANSVERSELY POLARIZED TARGET. LOS ALAMOS NATIONAL LABORATORY, 1988. 165P. (LA-11273-T)

41-106

KRAICHNAN, ROBERT H. (T-DO): CHEN, SHI-YI (CNLS).

IS THERE A STATISTICAL MECHANICS OF TURBULENCE?

TIC, 1988. 32P. MN (LA-UR-88-3703)

41-107

KUNASZ, PAUL; AUER, LAWRENCE H. (ESS-5).

SHORT CHARACTERISTIC INTEGRATION OF RADIATIVE TRANSFER PROBLEMS-FORMAL SOLUTION IN TWO-DIMENSIONAL SLABS.

J. QUANT. SPECTROSC. RADIAT. TRANSFER V.39, P.67-9. 1988.

41-108

KWAN. THOMAS J. T. (X-10); PETER, WILLIAM K. $(X-10)$.

MICROWAVE PROPAGATION THROUGH AN APERTURED COAXIAL WAVEGUIDE.

PHYS. REV., V.38A, P.4743-51. 1988.

41-109

KWAN, THOMAS J. T. (X-10); DAVIS, HAROLD A. (P-1).

NUMERICAL SIMULATIONS OF THE REDITRON.

IEEE TAANS. PLASMA SCI., V.16, P.185-91. 1988. 
$41-110$

LAPEDES, ALAN S. (T-DOT); FARBER, ROBERT M. (T-10).

SELF.OPTIMIZING, NONSYMMETRICAL NEURAL NET FOR CONTENT ADDRESSABLE MEMORY AND

PATTERN RECOGNITION.

PHYSICA, V. 22D, P. 247-259. 1986.

ALSO PUBLISHED: EVOLUTION, GAMES, AND LEARNING-MODELS FOR ADAPTATION IN MACHINES

AND NATURE. INTERNATIONAL CONFERENCE OF THE CENTER FOR NONLINEAR STUDIES, 5TH, 1985. LOS ALAMOS, N.M. PROC., P.247-259. NORTH-HOLLAND, 1986. (OP408 .C46 1985)

41-111

LAWRENCE, J. M.; CHEN, Y. Y.; THOMPSON, JOE D. (P-10).

COHERENCE IN CERIUM PALLADIUM-3.

IN: INTERNATIONAL CONFERENCE ON VALENCE FLUCTUATIONS, 5TH, 1987, 8ANGALORE, INDIA.

PROC., P.169-78. PL.ENUM, 1987. (OC/176.8/.V34/157/1987)

ALSO PUBLISHED: TIC, 1987. 11P. MN (LA-UR-87-0438)

$41-112$

LE CLAIRE, RENE J., JR. (A-4).

BEAM PROFILE EFFECTS ON NPB PERFORMANCE.

LOS ALAMOS, 1988. 15P. (LA-11228-MS)

$41-113$

LE SAR, RICHARD A. (T-11); ETTERS, RICHARD D. (M-6).

CHARACTER OF THE A.LPHA-BETA PHASE TRANSITION IN SOLID OXYGEN.

PHYS. REV., V.37B, P.5364-70. 1988.

41-114

LE SAR, RICHARD A. (T-11).

EQUATION OF STATE OF DENSE HELIUM.

PHYS. REV. LETT., V.61, P.2121-4. 1988.

41-115

LEE, PETER H. Y. (P-1): CASPERSON, DONALD E. (P-1); SCHAPPERT, GOTTFRIED T. (P-DO).

SEARCH FOR X RAYS GENERATED BY COLLISIONLESS MULTIPHOTON PROCESSES.

TIC, 1988. 15P. MN (LA-UR-88-3084)

41-116

LOH, EUGENE Y. (T-11); MARTIN, THIERRY (CNLS); PRELOVSEK, PETER (CNLS); CAMPBELL, DAVID K. (CNLS). COUPLED-SPIN-MOBILE-HOLE MODEL FOR HIGH-TC SUPERCONDUCTING OXIDES.

PHYS. REV., V.38B, P.2494-503. 1988.

41.117

LOH, EUGENE Y. (T-11); CAMPBELL, DAVID K. (CNLS).

OPTICAL ABSORPTION IN EXTENDED PEIERLS-HUBBARD MODELS.

SYNTH. MET., V.27A, P.499-508. 1988.

41-118

LOMDAHL, PETER S. (T-11); SAMUELSEN, MOGENS R.

NODULATION OF THE BREATHER FREOUENCY IN THE AC-DRIVEN SINE-GORDON SYSTEM WITH LOSS. PHYS. LETT., V.128A, P.427-32. 1988.

41-119

LUUCK, JAMES D. (T-7); BEYER, WILLIAM A. (T-7); BIEDENHARN, LAWRENCE C.: STEIN, PAUL R. (T-7).

SYMMETRIES OF SOME HYPERGEOMETRIC SERIES-IMPLICATIONS FOR 3J-AND 6J-COEFFICIENTS

IN: INTERNATIONAL COLLOOUIUM ON GROUP THEORETICAL METHOOS IN PHYSICS, 15TH, 1986, DREXEL UNIVERSITY. PROC., P.428-34. WORLD SCIENTIFIC. 1987. (OC20.7.G76 157

ALSO PUBLISHED: TIC, 1986. 8P. MN (LA-UR-86-4203)

41-120

LOWE, MARY (P-10); HAMMEL, P. CHRISTOPHER (P-10); ECKE, ROBERT E. (P-10); BEDELL, KEVIN S. (T-11);

TAKIGAWA, MASASHI (P-10).

NUCLEAR SPIN-LATTICE RELAXATION IN HELIUM 3-HELIUM 4 MIXTURES.

PHYS. REV., V.37B, P.2281-4. 1988. 
41.121

LYNCH, DIANE L. (T-12): SCHNEIDER, BARRY I. (T-12); COLLINS, LEE A. (T-11); MC KOY, V.; HUO, W. M. VIBRATIONALLY RESOLVED STUDIES OF THE 2 PI PHOTOIONIZATION OF NITRIC OXIDE.

CHEM. PHYS. LETT., V.147, P.529-34. 1988.

41-122

MAC FARLANE, ROBERT E. (T-2)

COLD MODERATOR SCATTERING KERNELS.

TIC, 1988. 25P. MN (LA-UR-88-3358)

41-123

MANUEL. A. A.; HOFFMANN, L.; SINGH, A. K; JARLBORG, T.; PETER, M.; SMITH, JAMES L. (CMS);

FISK, ZACHARY (P-10); PECORA, L. M.; EHRLICH, A. C.

POSITRON ANNIHILATION IN TITANIUM BERYLLIUN 2

TIC, 1988. 4\%. MN (LA-UR-88-3522)

41-124

MARIANER, SHLOMO (T-11); BISHOP, ALAN R. (T-11)

FRENKEL-KONTOROVA MODEL WITH NONCONVEX.INTERPARTICLE INTERACTIONS AND STRAIN

GRADIENTS

PHYS. REV., V.37B, P.9893-6. 1988

41-125

MARINUZZI, ROBERTA (MP-DO).

PROCEEDINGS OF THE TWENTY-FIRST LAMPF USERS GROUP MEETING-LOS ALAMOS NATIONAL

LABORATORY, LOS ALAMOS, NEW MEXICO NOVEMBER 9-10, 1987.

LOS ALAMOS NATIO:VAL LABORATORY, 1988. 210P. (LA-11248-C)

41-126

MARTIN, RICHARD A. (MEE-13); CLINE, MICHAEL C. (T-3); MUNTZ, E. P.; FARNHAM, T.

GAS NEUTRALIZER FLOWFIELD MODELING AND EXPERIMENTS

J. SPACECR. ROCKETS, V.25, P.286-93. 1988.

$41-127$

MARTIN, RICHARD L. (T-12); BISHOP, ALAN .1. (T-11); TESANOVIC, ZLATKO B. (T-11).

POLARIZATION-INDUCED PAIRING-AN EXCITONIC MECHANISM FOR HIGH-TEMPERATURE

SUPERCONDUCTIVITY.

IN: CHEMISTRY OF HIGH-TEMPERATURE SUPERCONDUCTORS II, 1988, LOS ANGELES. PROC., P.130-7.

AMERICAN CHEMICAL SOCIETY, 1988. (OD1 .A28X V.377)

ALSO PUBLISHED: TIC. 1988. 13P. MN (LA-UR-88-1646)

41-128

MAZOR, AVRAHAM (T-11); SROLOVITZ, DAVID J. (T-11); HAGAN, PATRICK S. (T-7); BUKIET, BRUCE G. (T-14). COLUMNAR GROWTH IN THIN FILMS.

PHYS. REV. LETT., V.60, P.424-7. 1988

41-129

MAZOR, AVRAHAM (T-11); BUKIET, BRUCE G. (T-14); SROLOVITZ, DAVID J. (T-11).

EFFECT OF VAPOR INCIDENCE ANGLE UPON THIN FILM COLUMNAR GROWTH.

TIC, 1988. 21P. MN (LA-UR-88-2451)

41-130

MAZOR, AVRAHAM (T-11); SPOLOVITZ, DAVID J. (T-11); HAGAN, PATRICK S. (T-7); BUKIET, 8RUCE G. (T-14).

THIN FILM MICROSTRUCTURES-SIMULATION AND THEORY.

IN: MODELING OF OPTICAL THIN FILMS, 1987, SAN DIEGO. PROC., P.88-94. SPIE, 1988.

ALSO PUBLISHED: TIC, 1987. 8P. MN (LA-UR-87-2679)

41-131

MC KINSTRIE, C. J. (CNLS).

BEAM-PLASMA DISPERSION RELATIONS BY LORENTZ TRANSFORMATION

PHYS. FLUIDS, V.31, P.1273-6. 1988.

41-132

MC KINSTRIE, C. J. (CNLS); DUBOIS, DONALD F. (T-DOT)

COVARIANT FORMALISM FOR WAVE PROPAGATION APPLIED TO STIMULATED RAMAN SCATTERING

PHYS. FLUIDS, V.31, P.278-87. 1988. 
41-133

MC KINSTRIE, C. J. (CNLS); RUSSELL, DAVID A. (T-DOT).

NONLINEAR FOCUSING OF COUPLED WAVES.

PHYS. REV. LETT., V.61, P.2929-32. 1988.

41-134

MC OUEEN, ROBERT G. (M-6); MARSH, STANLEY P. (M-6).

HIGH EXPLOSIVE SYSTEMS FOR EOUATION-OF-STATE STUDIES.

IN: SHOCK WAVES IN CONDENSED MATTER, 1987, MONTEREY. PROC., P.107-10. ELSEVIER, 1988. (OC173.4.C65 S57 1987X)

41-135

MEIER, M. M. (P-9); GOULDING, CHARLES A. (N-2); MORGAN, G. L. (P-15); ULLMAN, JOHN (P-3). NEUTRON YIELDS FROM STOPPING-LENGTH AND NEAR-STOPPING-LENGTH TARGETS FOF 256-MEV PROTONS.

LOS ALAMOS NATIONAL LABORATORY, 1988. 49P. (LA-11286-MS)

41-136

MERTENS, F. G.; BISHOP, ALAN R. (T-11); GOUVEA, MARIA E. (T-11); WYSIN, GARY M. (MEE-11).

DYNAMICS OF UNBOUND VORTICES IN THE 2-DIMENSIONAL XY AND ANISOTROPIC HEISENBERG MODELS.

TIC, 1988. 11P. MN (LA-UR-88-2988)

41-137

MIGLIORI, ALBERT (P-10); REAGOR, DAVID W. (P-10); PETERSON, DEAN E. (MST-5); WILLIS, JOARY (P-10);

FISK, ZACHARY (P-10); SMITH, RUSS C. (P-14).

RF AND MICROWAVE MEASUREMENTS AT LOS ALAMOS ON OXIDE SUPERCONDUCTORS.

IN: WORKSHOP ON RF-SUPERCONDUCTIVITY, 3RD, 1987, ARGONNE NATIONAL LABORATORY. PROC.. P.233-6. ARGONNE NATIONAL LABORATORY, 1988. (ANL-PHY-88-1)

41-138

MILONNI, PETER W. (T-12); ACKERHALT, JAY R. (T-12); GOGGIN, MICHAEL E. (T-12).

OUANTUM-MECHANICAL ASPECTS OF CLASSICALLY CHAOTIC DRIVEN SYSTEMS.

IN: INTERNATIONAL CONFERENCE ON MULTIPHOTON PROCESSES, 4TH, 1987, BOULDER, CO. PROC., P.245-51. CAMBRIDGE UNIVERSITY PRESS, 1988. (OC350 .C35X V.8)

ALSO PUBLISHED: TIC, 1987. BP. MN (LA-UR-87-2322)

41-139

MILONNI, PETER W. (T-12); COOK, RICHARD J.; GOGGIN, MICHAEL E. (T-12).

RADIATION PRESSURE FROM THE VACUUM-PHYSICAL INTERPRETATION OF THE CASIMIR FORCE. PHYS. REV., V.38A, P.1621-3. 1988.

41-140

MONTGOMERY, D.; PHILLIPS, LEE.

MHD TURBULENCE-RELAXATION PROCESSES AND VARIATIONAL PRINCIPLES.

TIC, 1988. 23P. MN (LA-UR-88-2600)

41-141

MOSES, ELISHA; STEINBERG, VICTOR (CNLS).

MASS TRANSPORT IN PROPAGATING PATTERNS OF CONVECTION.

TIC, 1988. 47P. MN (LA-UR-88-3680)

41-142

MOSES, RONALD W., JR. (CTR-6); HEIGHWAY, EDWARD A. (AT-6); CHRISTIAN, RICHARD S.;

DRAGT, ALEX JAMES.

SCALING LAWS FOR ABERRATIONS IN MAGNETIC QUADRUPOLE LENS SYSTEMS.

IN: IEEE PARTICLE ACCELERATOR CONFERENCE, 1987, WASHINGTON. D.C. PROC., P.1764-6. IEEE. 1987. (OC787.P3 137 1987)

ALSO PUBLISHED: TIC, 1987. 4P. MN (LA-UR-87-0297)

41-143

MUELLER, F. M. (CMS); CHEN, SHAO P. (T-11): PRUEITT, MELVIN L. (IS-2): SMITH, JAMES F. (CMS):

SMITH, JAMES L. (CMS); WOHLLEBEN, D. (CMSS).

COHERENT TWIN BOUNDARIES IN HIGH-TC SUPERCONDUCTING OXIDES

PHYS. REV., V.37B, P.5837-40. 1988. 
NEWNAM, BRIAN E. (CLS-6).

EXTENSION OF FREE-ELECTRON LASERS INTO THE EXTREME ULTRAVIOLET.

IN: FREE-ELECTRON: LASERS, 1987, LOS ANGELES. PROC., P.155-75. SPIE, 1988. (TA1677 .F73 1987X)

ALSO PUBLISHED: TIC, 1988. 22P. MN (LA-UR-89-0051)

41-145

NIETO. MICHAEL MARTIN (T-8).

PROPERTIES OF THE DKP [DUFFIN-KEMMER-PETIAU] EOUATION.

TIC, 1988. 9P. MN (LA-UR-88-2339)

41-146

NORMAN, M. R.; ALBERS, ROBERT C. (T-11); BORING, A. MICHAEL (T-11); CHRISTENSEN, N. E.

FERMI SURFACE AND EFFECTIVE MASSES FOR THE HEAVY-ELECTRON SUPERCONDUCTOR URANIUM

PLATINUM-3.

SOLID STATE COMMUN. V.68, P.245-9. 1988.

41-147

PENNEY, T.; MILLIKEN, F. P.; HOLTZBERG, FRED; FISK, ZÂCHARY (CMS).

COHERENT STATE IN THE HEAVY FERMION CERIUM COPPER-6.

IN: INTERNATIONAL CONFERENCE ON VALENCE FLUCTUATIONS, 5TH, 1987, BANGALORE, INDIA. PROC., P.77-86. PLENUM, 1987. (OC/176.8/.V34/157/1987)

41-148

PETHICK, C. J.; PINES, DAVID (CMS).

UNDERSTANDING HEAVY ELECTRON SYSTEMS.

IN: INTERNATIONAL CONFERENCE ON RECENT PROGRESS IN MANY-BODY THEORIES, 5TH, OULU, FINLAND. PROC., P.17-29. PLENUM PRESS, 1988. (OC612.S8 1496 1987)

41.149

PHIPPS, CLAUDE R., JR. (CLS-5); TURNER, THOMAS P. (CLS-6); HARRISON, RONALD F. (CLS-5);

YORK, GEORGE W. (CLS-5); OSBORNE, W. Z. (WX-11); ANDERSON, GRAYDON K. (CLS-4):

CORLSS, X. F. (CLS-5); HAYNES, L. C. (CLS-5); STEELE, H. S. (CLS-5); SPICOCHI, KENNETH C. (WX-11);

KING, T. $R$.

IMPULSE COUPLING TO TARGETS IN VACUUM BY KRYPTON FLUORIDE, HYDROGEN FLUORIDE, AND

CARBON DIOXIDE SINGLE-PULSE LASERS.

J. APPL. PHYS., V.64, P.1083-96. 1988.

41-150

PIMBLEY, GEORGE H. (T-14).

SEMIGROUP FOR LAGRANGIAN 1D ISENTROPIC FLOW.

TIC, 1988. 14P. MN (LA-UP-B8-050B)

41-151

PLOHR, BRADLEY J.; SHARP, DAVID H. (T-8).

CONSERVATIVE EULERIAN FORMULATION OF THE EQUATIONS FOR ELASTIC FLOW. ADV. APPL MATH., V.9, P.481-99. 1988.

41-152

PNEVMATIKOS, STEPHANOS.

SOUTON DYNAMICS OF HYDROGEN-BONDED NETWORKS-A POSSIBLE MECHANISM FOA PROTON CONDUCTIVITY.

PHYS. REV. LETT., V.60, P.1534-7. 1988.

41-153

POTTER, M. E.

STUDY OF NEUTRINO-ELECTRON SCATTERING AT LAMPF.

LOS ALAMOS, 1988. 181P. (LA-11182-T)

41-154

PFATT, LAWAENCE R. (CLS-2); HOFFMAN, GARY G. (CLS-2); HARRIS, ROBERT A.

STATISTICAL THEORY OF ELECTRON DENSITIES. J. CHEM. PHYS., V.88, P.1818-23. 1988. 
41-155

RINKER, GEORGE A., JR. (T-1); SOLEM, JOHNDALE C. (T-CO); BIEDENHARN, LAWRENCE C.

CALCULATION OF HARMONIC RADIATION AND NUCLEAR COUPLING ARISING FROM ATONS IN STRONG LASER FIELDS.

TIC. 1988. 9P. MN (LA-UR-B8-0269)

41.156

RINKER, GEORGE A., JR. (T-1).

SYSTEMATIC CALCULATIONS OF PLASMA TRANSPORT COEFFICIENTS FOR THE PERIODIC TABLE.

PHYS. REV., V.37A, P.1284-97, 1988.

41-157

ROBINSON, ROBERT A. (P-8).

ON THE KINEMATICS AND RESOLUTION OF SPECTROMETERS FOR NEUTRON BRILLOUIN SCATTERING.

TIC, 1988. 21P. MN (LA-UR-89-3099)

41-158

ROBISCOE, R. T. (ESS-7).

ARCING AND RF SIGNAL GENERATION DURING TARGET IRRADIATION BY A HIGH-ENERGY, PULSED

NEUTRAL PARTICLE BEAM.

LOS ALANOS, 1988. 84P. (LA-10958-MS)

41-159

ROBISCOE, R. T. (ESS-7); KADISH, ABRAHAM (ESS-7); MAIER, WILLIAM B., II (ESS-7).

LUMPED CIRCUIT MODEL FOR TRANSIENT ARC DISCHARGES.

J. APPL. PHYS., V.64, P.4355-63. 1988.

41- 160

fiose, HARVEY A. (T-DOT): DUBOIS, DONALD F. (T-DOT); RUSSELL, D. K: BEZZERIDES, BANDEL B. (X-1). EXPERIMENTAL SIGNATUPES OF LOCALIZATION IN LANGMUIR WAVE TUABULENCE.

TIC, 1988. 43P. MN (LA-UR-88-2962)

41-161

ROSEN, SIMON PETER (T-DO).

ON SEEING THE FIRST DOUBLE BETA DECAY.

COMMENTS NIJCL. PART. PHYS., V.18, P.31-54. 1988.

41-162

ROSENAU, PHILIP.

EVOLUTION AND BREAKING OF ION-ACOUSTIC WAVES

PHYS. FLUIDS, V.31, P.1317-9. 1988.

41-163

RUSSELL, DAVID A. (T-DOT): DUBOIS, DONALD F. (T-DOT); ROSE, HARVEY A. (T-DOT). NUCLEATION IN TWO-DIMENSIONAL LANGMUIR TURBULENCE.

PHYS. REV. LETT., V.60, P.581-4. 1988.

41-164

RUSSEL', GARY J. (P-9); LEGATE, G. L. (P-9); ROBINSON, HAROLD (P-9): WOODS, RICHARD (P-9). SHIELDING THE LANSCE [LOS ALAMOS NEUTRON SCATTERING CENTER] 800-MEV SPALATION NEUTRON SOURCE.

TIC, 1988. 2OP. MN (LA-UR-88-1987)

41-165

SAILOR, WILLIAM C. (P-2).

MODEL FOR ELECTRONION RECOMBINATION IN IONIZATION CHAMBERS

LOS ALAMOS NATIONAL LABORATORY. 1988. 24P. (LA-11267-MS)

41-166

SAILOR, WILLIAM C. (P-2): BYRD, R. C. (P-2); YARIV, Y.

TRACE-A MONTE CARLO CODE FOR THE EFFICIENCY AND DIFFERENTIAL EFFICIENCY OF

MULTI-ELEMENT NEUTROIN SCINTILLATOR DETECTORS.

LOS ALAMOS NATIONAL LABORATORY, 1988. 37P. (LA-11348-MS) 
41-167

SANDBERG, V. D. (MP-4).

CENTER OF BUOYANCY DEFINITION.

LOS ALAMOS NATIONAL LABORATORY, 1988. 5P. (LA-11416-MS)

41- 168

SCHMIDT, STEPHEN C. (M-6); MOORE, DAVID S. (CLS-4); SHAW, MILTON S. (T-14); JOHNSON, JAMES D. (T- 1 ).

VIBRATIONAL SPECTROSCOPY OF SHOCK-COMPRESSED FLUID NITROGEN-2 AND OXYGEN-2.

IN: SHOCK WAVES IN CONDENSED MATTER, 1987, MONTEREY. PROC., P.489-92. ELSEVIER, 1988. (OC173.4.C65 S57 1987X)

ALSO PUBLISHED: TIC, 1987. 5P. MN (LA-UR-87-2272)

41-169

SHANER, J. W. (M-6); HIXSON, ROBERT S. (M-6); WINKLER, MAX A. (M-5); BONESS, DAVID A.;

BROWN, J. MICHAEL.

BIRCH'S LAW FOR FLUID METALS.

IN: SHOCK WAVES IN CONDENSED MATTER, 1987, MONTEREY. PROC., P.135-8. ELSEVIER, 1988. (OC173.4.C65 S57 1987X)

ALSO PUBLISHED: TIC, 1987. 5P. MN (LA-UR-87-2233)

41-170

SHANER, J. W. (M-6)

GRUNEISEN GAMMA AND ACOUSTIC VELOCITY FOR SOFT SPHERE FLUIDS.

J. CHEM. PHYS., V.89, P.1616-24. 1988.

41-171

SHAW, MLTON S. (T-14).

DENSITY OF STATES TRANSFORMATION MONTE CARLO METHOD-THERMODYNAMICS OF THE LENNARD-JONES FLUID.

J. CHEM. PHYS., V.89, P.2312-23. 1988.

41-172

SHAW, MLTON S. (T-14); JOHNSON, JAMES D. (T-1).

SLOW REACTION RATE IN DETONATIONS DUE TO CARBON CLUSTERING.

IN: SHOCK WAVES IN CONDENSED MATTER, 1987, MONTEREY. PROC., P.503-6. ELSEVIER, 1988. (OC173.4C65 S57 1987X)

41-173

SHAW, MILTON S. (T-14); JOHNSON, JAMES D. (T-4).

THEORY OF DENSE MOLECILAR FLUID EQUATICNS OF STATE WITH APPLICATION TO DETONATION PRODUCTS.

IN: SYMPOSIUM (INTERNATIONAL) ON DETONATION, 8TH, 1985, ALBUQUERQUE. PREPRINTS. P.296-304. NAVAL SURFACE WEAPONS CENTER, 1986. (OD516.S924 1985XA) ALSO PUBLISHED: SYMPOSIUM (INTERNATIONAL) ON DETONATION, 8TH, 1985, ALBUQUERQUE. PROC., P.531-9. NAVAL SURFACE WEAPONS CENTER, 1986. (OD516.S924 1985X)

41-174

SHEFFIELD, STEPHEN A. (M-9).

ONSET OF SHOCK-INDUCED REACTION IN LIOUID CARBON DISULFIDE. IN: SHOCK WAVES IN CONDENSED MATTER, 1987, MONTEREY. PROC., P.463-6. ELSEVIER, 1988. (OC173.4.C65 557 1987X)

41-175

SILVER, RICHARD N. (T-11); CLARK, JOHN W. (T-11/CNLS),

FINAL STATE EFFECTS IN INCLUSIVE QUASIELASTIC ELECTRON SCATTERING FROM NUCLEI-CLUES FROM QUANTUM FLUIDS.

TIC, 1988. 1OP. MN (LA-UR-88-2507)

41.176

SILVER, RICHARD N. (T-11).

HARD CORE PERTUABATION THEORY.

TIC, 1988. 11P. MN (LA-UR-88-2896) 
41-177

SILVER, RICHARD N. (T-11).

THEORY OF DEEP INELASTIC NEUTRON SCATTERING ON QUANTUM FLUIDS.

PHYS. REV., V.37B, P.3794-7. 1988.

41-178

SILVER, RICHARD N. (T-t t).

THEORY OF DEEP INELASTIC NEUTRON SCATTERING-HARD-CORE PERTUABATION THEORY. PHYS. REV., V.38B, P.2283-96. 1988.

41-179

SILVER, RICHARD N. (T-11).

THEORY OF NEUTAON SCATTERING EXPERIMENTS ON MOMENTUM DISTRIBUTIONS IN OUANTUM

FLUIDS.

IN: INTERNATIONAL WORKSHOP ON CONDENSED MATTER PHYSICS, 11TH, 1987, OULU. PROC., P.131-42. PLENUM, 1988.

ALSO PUBLISHED: TIC, 1987. 13P. MN (LA-UR-87-2534)

41-180

SMITH, JAMES F. (CMS): WOHLLEBEN, D. (CMS).

TWIN REFINEMENT BELOW ROOM TEMPERATURE IN THE 1-2-3 HIGH TC SUPERCONDUCTORS.

Z. PHYSIK, V.728, P.323-334. 1988.

41-181

SAOLOVITZ, DAVID J. (T-11); MAZOR, AVRAHAM (T-11); BUKIET, BRUCE G. (T-14).

ANALYTICAL AND NUMERICAL MODELING OF COLUMNAR EVOLUTION IN THIN FILMS. J. VAC. SCI. TECHNOL., V.A6, P.237t-80. 1988.

41-182

SROLOVITZ, DAVID J. (T-11); GREST, G. S.; HASSOLD, G. N.; EYKHOLT, RICHARD E. (T-11).

EFFECTS OF IMPURITIES ON DOMAIN GROWTH.

IN: CMS WORKSHOP ON COMPETING INTERACTIONS AND MICROCLUSTERS-STATICS AND DYNAMICS, 1987, LOS ALAMOS, N.M. PROC.. P.254-62. SPRINGER-VERLAG, 1988. (OC173.4.C65 C58 ALSO PUBLISHED: TIC, 1987. 9P. MN (LA-UR-87-2636)

41-183

SROLOVITZ, DAVID J. (T-11); HASSOLD, G. N.; GAYDA, JOHN.

MONTE CARLO SIMULATION OF MODULATED PHASES.

IN: INTERNATIONAL SYMPOSIUM ON DYNAMICS OF ORDERING PROCESSES IN CONDENSED MATTER, 1987, KYOTO, JAPAN. PROC., P.111-120. PLENUM PRESS, 1988. (OC173.4.C65 I46 1987)

ALSO PUBLISHED: TIC, 1987. 11P. MN (LA-UR-87-2702)

41-184

SROLOVITZ, DAVID J. (T-11); MAZOR, AVRAHAM (T-11); BUKIET, BRUCE G. (T-14); HAGAN, PATRICK S. (T-7).

THEORY AND SIMULATIONS OF ZONE II MICROSTRUCTURES IN THIN FILMS.

ABSTRACT IN: J. VAC. SCI. TECHNOL., V.A6, P.1640-1. 1988.

ALSO PUBLISHED: TIC, 1987. 7P. MN (LA-UR-87-2869)

41-185

STAUDHAMMER, KARL P. (MST-6).

SUPERCONDUCTOR CONSOLIDATION.

IN: WOAKSHOP ON INDUSTRIAL APPLICATIONS OF SHOCK FROCESSING OF POWDERS, 1ST, 1988. SOCORRO, N.M. PROC. P.229-37. CENTER FOR EXPLOSIVES TECHNOLOGY RESEARCH, NEW MEXICO INSTITUTE OF MINING AND TECHNOLOGY, 1988. (TP 156.C59 W67 1988X)

41-186

STRAUB, GALEN K. (T-1); WILLS, JOHN M. (T-1); WALLACE, DUANE C. (X-4)

ELASTIC MODULI OF COPPER UNDER COMPRESSION IN: SHOCK WAVES IN CONDENSED MATTER, 1987, MONTEREY. PROC., P.207-10. ELSEVIER, 1988. (OC173.4.C65 557 1987X)

ALSO PUBLISHED: TIC, 1987. 5P. MN (LA-UR-87-2260) 
41-187

STRAUB, GALEN K. (T-1).

THERMODYNAMICS OF METALLIC SOLIDS AND FLUIDS FROM MOLECULAR DYNAMICS.

IN: ASM MATERIALS SCIENCE SEMINAR-COMPUTER SIMULATION IN MATERIALS SCIENCE, 1986, LAKE BUENA VISTA, FLA. PROC., P.79-110. ASM INTERNATIONAL, 1988. (TA/403.6/.A83/1986X)

41-188

SWIFT, GREGORY W. (P-10).

THERMOACOUSTIC ENGINES.

J. ACOUST. SOC. AM., V.84, P.1145-80. 1988.

41-189

TAN, TAI HO (M-6); OONA, HENN (M-1j).

TEMPORAL MEASUREMENTS OF SPECTRALLY RESOLVED OPTICAL EMISSION FROM CARBON DIOXIDE LASER-PRODUCED PLASMAS.

PHYS. LETT., V.130A, P.361-6. 1988

41-190

TAYLOR, F. DEAN (P-10); FARRELL, JOSEPH N. (P-10).

ISOMER SHIFT AND MAGNETIC PROPERTIES OF EUROPIUM OXYGEN UNDER PRESSURE. J. APPL. PHYS., V.63, P.4108-9. 1988.

41-191

TESANOVIC, ZLATKO B. (T-11); BISHOP, ALAN R. (T-11); MARTIN, RICHARD L. (T-12); HARRIS, C.

EXCITONIC SUPERCONDUCTIVITY IN COPPER OXIDES.

IN: ADRIATICO RESEARCH CONFERENCE AND WORKSHOP ON TOWARDS THE THEORETICAL UNDERSTANDING OF HIGH TC SUPERCONDUCTORS, 1988, TRIESTE, ITALY. WORLD SCIENTIFIC, 1988. (OC611.98.H54 P76X V.14)

ALSO PUBLISHED: TIC, 1988. 12P. MN (LA-UR-88-2549)

41-192

TESANOVIC, ZLATKO B. (T-11).

PHENOMENOLOGY OF HIGH-TC OXIDES-NEW KIND OF ANISOTROPIC SUPERCONDUCTORS. PHYS. REV., V.38B, P.2489-93. 1988.

41-193

THOMPSON, JOE D. (P-10); BORGES, HORTENCIO A. (P-10); FISK, ZACHARY (CMS); HORN, S:;

PARKS, RONALD D.; WELLS, GENE L. (CMS).

RESPONSE OF KONDO LATTICE SYSTEMS TO PRESSURE.

INTERNATIONAL CONFERENCE ON VALENCE FLUCTUATIONS, 5TH, 1987, BANGALORE, INCIA. PROC., P.151-8. PLENUM, 1987. (OC/176.8/V34/157/1987)

ALSO PUBLISHED: TIC, 1987. 9P. MN (LA-UR-87-0013)

41-194

TR!ORNE, KIP S.; ZUREK, WOJCIECH H. (T-6).

JOHN ARCHIBALD WHEELER-A FEW HIGHLISHTS OF HIS CONTRIBUTIONS TO PHYSICS.

FOUND. PHYS., V.16, P.79-88. 1986.

ALSO PUBLISHED: BETWEEN QUANTUM AND COSMOS, W.H. ZUREK, ED., P.3-13. PRINCETON

UNIVERSITY PRESS, 1988. (OC71.B45 1988)

41-195

TOKAR, ROBERT L $(X-1)$; MC VEY, BRIAN D. $(X-1)$; GOLDSTEIN, JOHN C. $(X-1)$.

SIDEBAND SUPPRESSION IN FREE-ELECTRON LASERS USING A GRATING RHOMB.

JEEE J. QUANTUM ELECTRON, V.24, P.856-63. 1988.

41-195

TONKS, DAVIS L. (T-1).

RELATION BETWEEN SHOCK STRENGTH AND STRAIN-RATE PLASTICITY AT MAXIMUM DEVIATOAIC STRESS.

IN: SHOCK WAVES IN CONDENSED MATTER, 1987, MONTEREY. PROC., P.231-4. ELSEVIER, 1988. (OC173.4.C65 S57 1987X)

ALSO P!JBLISHED: TIC, 1987. 14P. MN (LA-UR-87-2171) 
41-197

TRUGMAN, STUART A. (T-11).

INTERACTION OF HOLES IN A HUBBARD ANTIFERROMAGNET AND HIGH-TEMPERATURE

SUPERCONDUCTIVITY.

PHYS. REV., V.37B, P.1597-603. 1988.

41-198

TSIRONIS, G. P.: PNEVMATIKOS, STEPHANOS (CNLS).

PROTON CONDUCTIVITY IN QUASI-ONE-DIMENSIONAL HYDROGEN-BONDED SYSTEMS- NONLINEAR APPROACH.

PHYS. FEV., V.39B, P.7161-73. 1989.

ALSO PUBLISHED: TIC, 1988. 53P. MN (LA-UR-88-2599)

$41-199$

VISSCHER, PIETER B.; HOLIAN, BRAD LEE (T-1)

VIBRATIONAL RELAXATION RATES IN MOLECULAR FLUIDS VIA EQUILIBRIUM SIMULATION-THEAMAL SOFTENING.

IN: SHOCK WAVES IN! CONDENSED MATTER, 1987, MONTEREY. PROC., P.495-8. ELSEVIER, 1988. (OC173.4.C65 S57 1987X)

ALSO PUBLISHED: J. CHEM. PHYS., V.89, P.5128-36. 1988.

41-200

WACKERLE, JERRY D. (M-9); STACY, HOWARD L. (M-9); DALLMAN, JOHN C. (M-9).

REFRACTIVE INDEX EFFECTS FOR SHOCKED WINDOWS IN INTERFACE VELOCIMETRY.

IN: HIGH SPEED PHOTOGRAPHY, VIDEOGRAPHY, AND PHOTONICS, 5TH, 1987, SAN DIEGO. PROC., P.72-82. SPIE, 1988.

41.201

WADLINGER, E. ALAN (AT-3).

PARAMETER SCALING TO PRODUCE DIFFERENT CHARGED-PARTICLE BEÁM-TRANSFOORT SYSTEMS HAVING IDENTICAL EQUATIONS OF MOTION

LOS ALAMOS, 1988. 21P. (LA-11263-MS)

41-202

WALLACE, DUANE C. $(X-4)$.

EXTENSION OF BOLTZMANN'S THEORY TO A LIQUID.

NUCL. PHYS. B, (PROC. SUPPL.) V.5A, P.267-71. 1988.

ALSO PUBLISHED: TIC, 1988. 6P. MN (LA-UR-88-1126)

41-203

WALLACE, DUANE C. (X-4).

STATISTICAL ENTROPY AND A QUALITATIVE GAS-LIQUID PHASE DIAGRAM.

PHYS. REV., V.38A, P.469-72. 1988.

41-204

WALSH, JOHN M. (M-4).

ON THE PROBLEM OF THE OBLIOUE INTERACTION OF A DETONATION WAVE WITH AN EXPLOSIVE-METAL INTERFACE.

IN: SHOCK WAVES IN CONDENSED MATTER, 1987, MONTEREY. PROC., P.3-10. ELSEVIER, 1988.

(C)173.4.C65 S57 1987X)

41-205

WARK, J. S.; WHITLOCK, R. R.; HAUER, ALLAN (P-4); SWAIN, J. E.; SOLONE, P. J.

SHORT-PULSE X-RAY DIFFRACTION FROM LASER-SHOCKEO CRYSTALS.

IN: SHOCK WAVES IN CONDENSED MATTER, 1987, MONTEREY. PROC., P.781-6. ELSEVIER, 1988.

(OC173.4.C65 S57 1987X)

41-206

WEERTMAN, JOHANNES (MST-5); FOLLANSBEE, PAUL S. (MST-5).

DISLOCATION DYNAMICS AND PLASTIC SHOCK WAVES.

MECH. MATER., V.7, P.177-89. 1988. 
41-207

WEST, GEOFFREY B. (T-B).

GENERAL STRUCTUFE OF COMPOSITE PROPAGATORS FROM THE RENORMALIZATION GROUP AND

ANALYTICITY.

NUCL. PHYS., V.8288, P.444-60. 1987.

41-208

WILKERSON, J. F. (P-3).

LIMITS ON THE ELECTRON-ANTINEUTRINO MASS

IN: AMERICAN PHYSICAL SOCIETY. DIVISION OF PARTICLES AND FIELDS, MEETING, 3AD, 1987, SALT LAKE CITY, UTAH. PROC., P.338-47. WORLD SCIENTIFIC, 1987. (OC/793/.A47/1987X)

ALSO PUBLISHED: TIC, 1987. 11P. MN (LA-UR-87-1196)

41.209

WILKERSON, J. F. (P-3); BOWLES, THOMAS J. (P-3); KNAPP, DAVID A.; ROBERTSON, ROBERT G. H. (P-3): WARK, DAVID L. (P-3).

PROGRESS REPORT ON THE LOS ALAMMOS TRITIUM BETA DECAY EXPERIMENT.

IN: INS INTERNATIONAL SYMPOSIUM ON NEUTRINO MASS ANO RELATEO TOPICS, 16TH. 1898, UNIVERSITY OF TOKYO. PROC., P.105-14. WORLD SCIENTIFIC, 1988. (OC793.5.N426 158 ALSO PUBLISHED: TIC, 1988. 11P. MN (LA-UR-88-0830)

ALSO PUBLISHED: 5TH FORCE NEUTRINO PHYSICS-MORIOND WORKSHOP, 8TH, 1988, SAVOIE, FRANCE. PROC., P.79-88. EDITIONS FRONTIERES, 1989. (OC793 .M67 1988X)

41-210

WILLIAMS, ARTHUR (LANSCE); KWEI, GEORGE H. (LANSCE); VON DREELE, R. B. (LANSCE);

LARSON, ALLEN C. (P-8); RAISTRICK, IAN D. (MEE-11); BISH, DAVID L. (ESS-1).

JOINT $X$-RAY AND NEUTRON REFINEMENT OF THE STRUCTURE OF SUPERCONDUCTING YTTRIUM

BARIUM-2 COPPER-3 OXYGEN-7-X-THERMAL PARAMETERS, STRAIN, AND CATION DISORDER.

PHYS. REV., V.37B, P.7960-2. 1988.

41-211

WILLIS, JEFFREY O. (P-10); COOKE, D. W. (MP.14); BROWN, ROBERT D. (MP-7);

COST, JAMES RICHARD (MST-5); SMITH, JAMES F. (CMS); SMITH, JAMES L. (CMS): AIKIN, ROBERT M. (P-10); MAEZ, M. (MP-14).

PROTON RADIATION DAMAGE IN SUPERCONDUCTING EUROPIUM BARIUM-2 COPPER-3 OXYGEN $X$ AND

GADOLINIUM BARIUM-2 COPPER-3 OXYGEN $X$

APPL. PHYS. LETT., V.53, P.417-9, 1988

41-212

WILLIS, JEFFREY O. (P-10); MC ELFRESH, M. W. (P-10); THOMPSON, JOE D. (P-10); SMITH, JAMES L. (CMS); FISK, ZACHARY (P-10).

SCALING OF THE MAGNETORESISTANCE OF URANIUM BERYLLIUM-13 UNDER PRESSURE.

J. APPL. PHYS., V.64, P.5613-5. 1988.

ALSO PUBLISHED: TIC, 1988. 16P. MN (LA-UR-88-1556)

41-213

WILSON, TIMOTHY L (Q-6); NICHOLS, BILL D. (N-6); HIRT, CYRIL W.. JR; STEIN, LELAND R. (T-3).

SOLA-DM-A NUMERICAL SOLUTION ALGORITHM FOR TRANSIENT THREE-DIMENSIONAL FLOWS.

LOS ALAMOS, 1988. 91P. (LA-11161-MS)

41-214

WYSIN, GARY M. (MEE-11); SMITH, DOUGLAS L (MEE-11); REDONDO, ANTONIO (MEE-11).

PICOSECOND RESPONSE OF PHOTOEXCITEO GALLIUM ARSENIDE IN A UNIFORM ELECTRIC FIELD BY

MONTE CARLO DYNAMICS.

PHYS. REV., V.38B, P.12514-24. 1988

41.215

YAN, YITON T. (X-10); CHEN, HUDONG (CNLS)

NONLINEAR SOLUTION FOR THE OPTIMAL SHAPING OF THE DRIVING ELECTRON BEAM IN THE PLASMA WAKE-FIELD ACCELERATOR.

PHYS. REV., V.38A, P.1490-4. 1988

41.216

YANG, MING.JEN (P.DO).

MONTE CARLO CODE FOR A TIME PAOJECTION CHAMBER.

NUCL INSTAUM. METHODS PHYS. RES., V.A270, P.126-39. 1988. 
YETHIRA, M. (P-9); ROBINSON, ROBERT A. (P-8).

COMPARISON OF JERMANIUM AND COPPER ANALYZERS FOR PULSED-SOURCE CRYSTAL- ANALYZER SPECTFOMETERS.

TIC, 1988. 11P. MN (LA-UR-88-3064)

41. 218

YETHIRW, M. (P-9); ROBINSON, ROBERT A. (LANSCE).

RECENT RESULTS WITH THE LOS ALAMOS CONSTANT-O SPECTROMETER.

TiC, ise8. 11?. MN (LA-UR-88-4225)

$41-210$

YOUNG, PHILLIP G., JR. (T-2); DAVIDSON, J. WILEY (A-4); MUIR, DOUGLAS W. (T-2).

EVALUATION OF THE LITHIUM-7(N,N'T) HELIUM-4 CROSS SECTION FOR ENDF/B-VI AND APPLICATION TO UNCERTAINTY ANALYSIS.

TIC, 1988. 1OP. MN (LA-UR-88-3296)

41-220

ZARDECKI, ANDREW (T-DO); ARMSTRONG, ROBERT L.

ENEAGY BALANCE IN LASER-IRRADIATED VAPORIZING DROPIETS. APPL. OPT., V.27, P.3690-5. 1988.

41-221

ZARDECKI, ANDREW (T-DO).

GRAVITY AS A GAUGE THEORY WITH CARTAN CONNECTION. J. MATH. PHYS., V.29, P.1661-6. 1988.

41-222

ZARDECKI, ANDREW (T-DO); GERSTL, S. A. W. (T-DOT).

HIGH-ENERGY LASER-ASSISTED IMIAGING THROUGH VAPORIZING AEROSOLS IN: OPTICAL, INFRARED, AND MILLIMETER WAVE PROPAGATION ENGINEERING, 1988, ORLANDO, FLA. PROC., P.160-71. SPIE, 1988.

ALSO PUBLISHED: TIC, 1988. 13P. MN (LA-UR-88-0669)

41-223

ZARDECKI, ANDREW (T-DO); ARMSTRONG, ROBERT L.

PROPAGATION OF HIGH-ENERGY LASER BEAMS THROUGH METALLIC AEROSOLS.

TIC, 1988. 9P. MN (LA-UR-88-2875) 
42-1

ARTHUA, EDWARD D. (T-2); MADLAND, DAVID G. (T-2); GEORGE, DENISE C. (T-2)

EXTENSION OF NUCLEAR STRUCTURE DATA BASE SEARCHES FOR GAMMA-RAY LASER CANDIDATES.

TIC. 1987. 4P. MN (LA-UR-87-4214)

42-2

ARTHUR, EDWARD D. (T-DO): YOUNG, PHILLIP G., JR. (T-2); PERRY, ROBERT T.: MADLAND, DAVID G. (T-2); MAC FARLANE, ROBERT E. (T-2); LITTLE, ROBERT C. (X-6); BOZOIAN, MICHAEL (T-2);

LA BAUVE, RAPHAEL J. (T-2).

DEVELOPMENT AND BENCHMARKING OF HIGHER ENERGY NEUTRON TRANSPORT DATA LIBRARIES.

TIC. 1988. 5P. MN (LA-UR-88-1762)

42-3

ARTHUR, EDWARD D. (T-DO).

GNASH PREEOUILIBRIUM-STATISTICAL NUCLEAR MODEL CODE.

TIC, 1988. 5IP. MN (LA-UR-88-1753)

42-4

ARTHUR, EDWARD D. (T-DO); YOUNG, PHILLIP G., JR. (T-2); KALBACH-WALKER, CONSTANCE (T-2). NEW DEVELOPMENTS IN THE GNASH NUCLEAR THEORY CODE.

TIC. 1988. 5P. MN (LA-UR-88-1739)

42-5

AUERBACH, NAFTALI (MP-DO); BARTEL, JOHANN (T-9); WENES, GEERT (T-5).

CORE POLARIZATION EFFECTS IN SD-SHELL NUCLEI AND CHARGE-SYMMETRY BREAKING IN THE NUCLEAR MEAN FIELD.

PHYS. REV., V.38C, P.2921-7. 1988.

42-6

AUERBACH, NAFTALI (MP-DO); GIBES, WILLIAM R. (MP.DO); GINOCCHIO, JOSEPH N. (T.5);

KAUFMANN, WILLIAM B. (T-5).

PION-NUCLEUS DOUBLE CHARGE EXCHANGE AND THE NUCLEAR SHELL MODEL.

PHYS. REV., V.38C, P.1277-96. 1988.

42-7

AUERBACH, NAFTALI (MP-DO).

THEORY OF PION DOUBLE CHARGE EXCHANGE.

IN: PION-NUCLEUS PHYSICS-FUTURE DIRECTIONS AND NEW FACILITIES AT LAMPF, 1987, LOS ALAMOS NATIONAL LABORATORY. PROC., P.34-53. AMERICAN INSTITUTE OF PHYSICS, 1988. (OC/793.5/.M42/P56/1987X)

42-8

BAER, H. W. (MP-4).

PROBING TWONUCLEON CORRELATIONS WITH PION DOUBLE-CHARGE EXCHANGE.

IN: CONFERENCE ON THE INTERSECTIONS BETWEEN PARTICLE AND NUCLEAR PHYSICS, 3RD, 1988. ROCKPORT, MAINE. PROC., P.589-600. AMERICAN INSTITUTE OF PHYSICS, 1988. (OC1 A38X NO.176) ALSO PUBLISHED: TIC, 1988. 13P. MN (LA-UR-88-2296)

$42-9$

BLESZYNSKI, E.; BLESZYNSKI, M. (MP-4); GLAUBER, RAY J

NUCLEON-NUCLEON CORRELATIONS DETECTEU VIA PION DOUBLE-CHARGE-EXCHANGE REACTIONS.

IN: PION-NUCLEUS PHYSICS-FUTURE DIRECTIONS AND NEW FACILITIES AT LAMPF, 1897. LOS ALAMOS NATIONAL LABORATORY, PROC., P.54-66. AMERICAN INSTITUTE OF PHYSICS, 1988. (QC/7\%3.5I.M42/P56/1987X)

ALSO PUBLSHED: TIC. 1987. 14P. MN (LA.UR-87-3387) 
42-10

BOLSTERLI, MARK (T-9).

FOCK EXCHANGE IN MESON THEORIES OF NUCLEI.

IN: WINDSURFING THE FERMI SEA-INTERNATIONAL CONFERENCE AND SYMPOSIUM ON UNIFIED CONCEPTS OF MANY-DODY PROBLEMS, 1986, STATE UNIV. OF NEW YORK AT STONY BROOK. PROC., P.36-9, VOL.2. ELSEVIER, 1987.

ALSO PUBLISHED: TIC, 1986. 5P. MN (LA-UR-86-3633)

42-11

BOWMAN, CHARLES D. (P-3); BOWMAN, J. DAVID (MP-4); HERCZEG, PETER (T-5); SZYMANSKI, J.:

YUAN, VINCENT W. (AT-2); AN.9YA, JOSE MANUEL (P-3); MORTENSEN, R. (P-3); POSTMA, HANS;

DELHEIJ, PETER P. J. (P-3); BAKER, O. K. (MP.DO); GOULD, CHRIS R.; HAASE, D. G.; MITCHEL!, GARY E.:

ROBERSON, N. R.; ZHU, $X$.; MC DONALD, ARTHUR BRUCE; BENTON, DAVID; TIPPINS, BRAD; CHUPP. T.

FACIUTY FOR PARITY AND TIME REVERSAL EXPERIMENTS WITH INTENSE EPITHERMAL (EV) NEUTRON BEAMS.

TIC, 1988. 8P. MN (LA-UR-8B-3955)

42-12

BOWMAN, J. DAVID (MP-4).

TESTS OF TIME REVERSAL IN NEUTRON-NUCLEUS SCATTERING.

IN: CONFERENCE ON THE INTERSECTIONS BETWEEN PARTICLE AND NUCLEAR PHYSICS, 3RD, 1988, ROCKPORT, MAINE. PROC., P.1112-7. AMERICAN INSTITUTE OF PHYSICS, 1988. (OC1 A38X NO.176) ALSO PUBUSHED: TIC, 1988. 8P. MN (LA-UR-88-2062)

$42-13$

BOZOIAN, MICHAEL (T-2); ARTHUR, EDWARD D. (T-DO); PERRY, ROBERT T.; WILSON, WILLIAM B. (T-2); YOUNG, PHILLIP G., JR. (T-2).

CALCULATED NEUTRON-ACTIVATION CROSS SECTIONS FOR EN LESS THAN OR EOUAL TO 100 MEV FOR

A RANGE OF ACCELERATOR MATERIALS.

TIC. 1988. 6P. MN (LA-UR-88-2380)

42-14

BOZOIAN, MICHAEL (T-2); SICILIANO, EDWARD R. (T-2): SMITH, AICHARD D. (T-2).

DEVELOPMENT OF NUCLEAR MODELS FOR HIGHER ENERGY CALCULATIONS.

TIC, 1988. 9P. MN (LA-UR-88-1695)

42-15

BOZOIAN, MICHAEL (T-2); ARTHUR, EDWARD D. (T-DO); GEORGE, DENISE C. (T-2); MADLAND, DAVID G. (T-2); YOUNG, PH!LLP G., JA. (T-2)

PRELIMINARY CALCULATIONS OF MEDIUIM-ENERGY FISSION CROSS SECTIONS AND SPECTRA.

TIC, 1988. 7P. MN (LA-UR-88-3319)

42-16

BRANDENBURG, RICHARD A.; CHULICK, GARY S.; MACHLEIDT, R.; PICKLESIMER, A. (P-15); THALER, R. M. MESIC RETARDATION AND THE TRITON BINDING ENERGY.

PHYS. REV., V.38C, P.1397-402. 1988.

42-17

BRANDENBURG, RICHARD A.; CHULICK, GARY S.; KIM, YEONG E.; KLEPACKI, OAVID J.; MACHLEIDT, R.; PICKLESIMER, A. (P-15); THALER, R. M.

NUCLEAR CHARGE SYMMETRY RREAKING AND THE HYDROGEN-3 - HELIUM-3 BINDING ENERGY

DIFFERENCE.

PHYS. REV., V.37C, P.781-5. 1988

42-18

CARLSON, JOSEPH A. (T-5).

ALPHA PARTICLE STRUCTURE.

PHYS. REV., V.38C, P.1879-85. 1988.

$42-19$

CARLSON, JOSEPH A. (T-5); FRIAR, JAUES L. (T-5); PAYNE, GERALD L.

ENERG Y AND SYMMETRY OF STATES IN LIGHT NUCLEI.

PHYS. REV., V.37C, P.420-2. 1988. 
CHULICK, GARY S.; ELSTER, CHARLOTTE; MACHLEIDT, R.; PICKLESIMER, A. (P-15); THALER, R. M. NEUTRON-PROTON SCATTERING OBSERVABLES AT 325 MEV. THE EPSILON 1 PARAMETER, AND THE TENSOR FORCE.

PHYS. REV., V.37C, P.1549-53. 1988.

$$
\text { 42-21 }
$$

CLAUSEN, B. L.

PION SCATERING TO 8-MINUS STRETCHED STATES IN NICKEL-60.

LOS ALAMOS NATIONAL LABORATORY, 1988. 209P. (LA-11213-T)

42.22

COHEN, JAMES S. (T-12).

CAOSS SECTIONS FOR KINETICS OF MUONIC HELIUM CAEATED IN MUON-CATALYZED

DEUTERIUM-DEUTERIUM AND DEUTERIUM- TRITIUM FUSION.

MUON-CATAL. FUSION, V.3, P.499-510. 1988.

42-23

COHEN, JAMES S. (T-12): LEON, MELVIN (MP-DO)

NOTE ON EPITHERMAL DTMU FORMATION.

MUON-CATAL. FUSION, V.3, P.497-8. 1988.

42-24

COHEN, JAMES S. (T-12).

STRIPPING AND $X$-RAY PRODUCTION IN MUON-CATALYZED DEUTERIUM-DEUTERIUM AND

DEUTERIUMTRITIUM FUSION.

MUON-CATAL. FUSION, V.3, P.421-38. 1988.

42-25

CSERNAI, L P.; GONG, MINZHUAN; STROTTMAN, DANIEL D. (T-2).

FLUID DYNAMICAL MODEL OF ULTRARELATIVISTIC HEAVY ION COLLISIONS.

TIC, 1988. 6P. MN (LA-UR-88-0748)

$42-26$

DAVIDSON, D. R. (MP-3); REEDY, ROBERT C. (ESS-8); GREENWOOD. L. R.; SOMMER, WALTER F., JP. (NP-5); WECHSLER, M. S. (MP-3).

ADDITIONAL MEASUREMENTS OF THE RADIATION ENVIRONMENT AT THE LOS ALAMOS SPALLATION

RADIATION EFFECTS FACILITY AT LAMPF.

IN: INFLUENCE OF RADIATION ON MATERIAL PROPERTIES, 13TH, 1986, SEATTLE. PROC., P.730-40. ASTM, 1987. (TA/418.6/.154/1986XA)

42-27

DIMOPOULOS, SAVAS; RABY, STUART A. (T-8).

GEOMETRIC HIEPARCHY.

NUCL. PHYS., V.219B, P.479-512. 1983.

ALSO PUBLISHED: SUPERSYMMETRY, S. FERRARA, ED., P.824-57. ELSEVIER SCIENCE PUB., 1987. (QC174.17.S9 S972 1987)

$42-28$

DROSG, MANFRED (P-3); LISOWSKI, PAUL W. (P-3); DRAKE, DARRELL M. (ESS-8);

HARDEKOPF, ROBERT A. (AT-3); MUELLNER, S. M.

CROSS SECTIONS FOR NEUTRON-PRODUCING REACTIONS INDUCED BY 14.1 MEV NEUTRONS INCIDENT

ON UTHIUM-6, LITHIUM-7, BORON- 10, BORON-11, AND CARBON.

LOS ALAMOS NATIONAL LABORATORY, 1988. 54P. (LA-11367-MS)

42-29

DYER, PEGGY (P-3); BOUNDS, J. A. (P-15); HAIGHT, ROBEAT C. (P-15); LUK, T. S.

NUCLEAR TRANSITIONS INDUCED BY ATOMIC EXCITATIONS.

IN: SHORT AND ULTRASHORT WAVELENGTH LASERS, 1988, LOS ANGELES. PROC., P.88-91. SPIE,

42-30

ENGLAND. TALMADGE R. (T-2); ARTHUR, EDWARD D. (T-2); BRADY, MICHAELE C. (T-2);

LA BAUVE, RAPHAEL J. (T-2).

BACKGROUND RADIATION FROM FISSION PULSES.

LOS ALANHOS NATIONAL LABORATORY, 1988. 89P. (LA-11151-MS) 
42-31

ENGLAND, TALMADGE R. (T-2); BRADY, MICHAELE C. (T-2).

DELAYED NEUTRON SPECTRA BY DECAY FOR FISSIONING SYSTEMS FROM THORIUM-227 THROUGH FERMIUM-255.

TIC, 1988. 14P. MN (LA-UR-88-2140)

42-32

ENGLAND, TALMADGE R. (T-2); BRADY, MICHAELE C. (T-2).

DELAYED NEUTRON SPECTRA DECAY GROUP FOR FISSIONING SYSTEMS FROM THORIUM-227

THROUGH FERMIUM-255.

TIC. 1988. 9P. MN (LA-UR-88-0626)

$42-33$

ENGLAND, TALMADGE R. (T-2); BLACHOT, JEAN.

STATUS OF FISSION YEILD DATA.

TIC, 1988. 8P. MN (LA-UP-88-1696)

42-34

ESTES, GUY P. (X-6); SCHRANDT, ROBERT G. (X-6); KRIESE, JOHN T. (X-2).

AUTOMATED MCNP PHOTON SOURCE GENERATION FOR ARBITRARY CONFIGURATIONS OF

RADIOACTIVE MATERIALS AND FIRST. PRINCIPLES CALCULATIONS OF PHOTON DETECTOR RESPONSES.

LOS ALAMOS, 1988. 23P. (LA-11153-MS)

42-35

FAWCETT, LOUIS RAYMOND. JR. (X-DO); ROBERTS, ROGER R.; HUNTER, RAYMOND E. (X-DO).

ANALYSIS OF TRITIUM PRODUCTION IN CONCENTRIC SPHERES OF ORALLOY AND GLD IRRADIATED BY 14-MEV NEUTRONS.

LOS ALAMOS NATIONAL LABORATORY. 1988. 3OP. (LA-11110)

42-36

FAZELY. AL; ESTEP, R. J. (INC-11); GREENE, STEVEN J. (MP-10); IROM, FAROKH (MP-4);

LIU, LON CHANG (INC-11); WARREN, JOHN E. (INC-11); WATSON, DAUGLAS L. (MP-10); DHUGA, KALVIR S.;

GAANETT, R. W.; BURLEIN, M.; ZUMBRO, J. D.; ROKNI, S. H.

NONANALOG O PLUS (G.S.) GOING TO O PLUS (G.S.) TRANSITIONS IN PION DOUBLE CHARGE EXCHANGE

REACTIONS ON TELLURIUM-128 AND TELLURIUM- 130.

PHYS. LETT., V.208B, P.361-4. 1988.

42-37

FISHER, HARLO $M .(X-6)$.

XDATAP.

LOS ALAMOS, 1988. 82P. (LA-11155-MS)

42-38

FOSTER, D. GRAHAM, JR. (T-2); ARTHUR, EDWARD D. (T-2).

AVERAGE NEUTRONIC PROPERTIES OF PROMPT FISSION PRODUCTS.

LOS ALAMOS, 1982. 29P. (LA-09168-MS)

42-39

FRIAR, JANES L. (T-5); PAYNE, GERALD L.

BIPOLAR HAAMONIC REDUCTION FORMULAE.

PHYS. REV., V.38C, P.1-4, 1988.

42-40

FRIAR, JAMES L. (T-5); GIBSON, BENJAMIN F. (T-5); PAYNE, GERALD L.

HYDROGEN-3 AND HELIUM-3 SOLUTIONS FOR MOMENTUM-DEPENDENT POTENTIALS. PHYS. REV., V.37C, P.2869-72. 1988.

42-41

FRIAR, JANES L. (T-5); GIBSON, BENJAMIN F. (T-5); PAYNE, GERALD L.; COON, SIUNEY A.

SWAVE PION NUCLEON SCATTERING EFFECTS IN THE NUCLEAR THREE-BODY FORCE. FEW-BODY SYST., V.5, P.13-20. 1988. 
42-42

FRIAR, JAMES L. (T-5); GIBSON, BENJAMIN F. (T-5); LEHMAN, DONALD R.; PAYNE, GERALD L. TRINUCLEON ASYMPTOTIC NORMALIZATION CONSTANTS-A COMPAAISON OF HELIUM-3 AND HYDROGEN-3.

PHYS. REV., V.37C, P.285968. 1988.

$42-43$

FAIAR, JAMES L (T-5); GIBSON, BENJAMIN F. (T-5); PAYNE, GERALD L; TOMUSIAK, EDWARD L; KIMURA, M TRINUCLEON MAGNETIC MOMENTS-34-CHANNEL RESULTS.

PHYS. REV., V.37, P.2852-8. 1988.

42-44

GARVEY, G. T. (MP-DO).

INTERNATIONAL CONFERENCE ON SPIN OBSERVABLES OF NUCLEAR PROBES-SUMMAFY TALK

TIC, 1988. 16P. MN (LA-UR-88-2918)

42-45

GIBBS, WILLIAM R. (T-5); KAUFMANN, WILLIAM B. (T-5).

MONTE CAFLO ANALYSIS OF PION ABSORPTION.

IN: PION-NUCLEUS PHYSICS-FUTURE DIRECTIONS AND NEW FACILITIES AT LASAPF, 1837, LOS ALAMOS NATIONAL LABORATORY. PROC., P.279-87. AMERICAN INSTITUTE OF PHYSICS, 1998. (QC/793.5/.M42/P56/1987X)

ALSO PUBLISHED: TIC, 1987. 11P. MN (LA-UA-87-3206)

42-46

GIBSON, BENJAMIN F. (T-5).

DECAY OF HYPERNUCLEI.

TIC, 1988. 21P. MN (LA-UR-88-3639)

42-47

GIBSON, BENJAMIN F. (T-5); MC KELLAR, BRUCE H. J.

THREE-BODY FORCES IN THE TRINUCLEONS.

FEW-BODY SYST., V.3, P.143-70. 1988.

$42-48$

GIBSON, BENJAMIN F. (T-5); LEHMAN, DONALD R.

ZERO PLUS - ONE PLUS A=4 LAMBDA-HYPERNUCLEI BINDING ENERGY DIFFERENCES IN AN EXACT EQUATION, SEPARABLE POTENTIAL CALCULATION.

TIC, 1988. 2P. MN (LA-UR-88-0944)

$42-49$

GILMORE, JAMES S. (INC-11); RUSSELL, GARY J. (P-9); ROBINSON, HAROLD (P-9); PRAEL, RICHARD E. (X-6). FERTILE-TOFISSILE AND FISSION MEASUREMENTS FOR DEPLETED URANIUM AND THOAIUM BOMBARDED BY 800-MEV PROTONS.

NUCL. SCI. ENG., V.99, P.41-52. 1988.

$42-50$

GINOCCHIO, JOSEPH N. (T-5).

FERMION DYNAMICAL SYMMETAY AND THE NUCLEAR SHELL MODEL.

IN: INTERNATIONAL SYMPOSIUM ON PARTICLE AND NUCLEAR PHYSICS, 1985, PEKING, CHINA. PROC. P.309-21. WORLD SCIENTIFIC, 1986. (OC/793/.15614/1985)

ALSO PUBLISHED: TIC, 1985. 14P. MN (LA-UR-86-0878)

42-51

GINOCCHIO, JOSEPH N. (T-5).

PION DOUBLE CHARGE EXCHANGE AND NUCLEAR STRUCTURE.

IN: PION-NUCLEUS PHYSICS-FUTURE DIRECTIONS AND NEW FACILITES AT LAMPF, 1887, LOS

ALAMOS NATIONAL LABORATORY, PROC., P.425-37. AMERICAN INSTITUTE OF PHYSICS. 1988. (OC/793.5/.M42/P56/1987X)

ALSO PUBLISHED: TIC, 1987. 14P. MN (LA-UR-87-2909)

$42-52$

GINOCCHIO, JOSEPH N. (T-5).

PROBING NUCLEAR CORRELATIONS WITH P!ON-NUCLEUS DOUB!E CHARGE EXCHANCE.

IIC, 1988. 21P. MN (LA-UR-B8-1789) 
42-53

GOLDMAN, TERRENCE J. (T-5); MALTMAN, KIM R. (T-5); STEPHENSON, GERARD J., JR. (P-DO);

SCHMIDT, KEVIN E.

IMPORTANCE OF NUCLEON SUBSTRUCTURE IN NUCLEAR GROUND STATES. NUCL. PHYS., V.A481, P.621-67. 1988.

$42-54$

GOLDMAN, TERRENCE J. (T-5); PRESTON, DEAN L. (X-4).

PARITY VIOLATING TOTAL CROSS SECTIONS.

TIC. 1988. 17P. MN (LA-UR-88-2922)

42-55

GRAM, PETER A. M. (MP-4).

SYSTEMATICS OF INCLUSIVE DOUBLE CHARGE EXCHANGE

IN: PION-NUCLEUS PHYSICS-FUTURE DIRECTIONS AND NEW FACILITIES AT LAMPF, 1987, LOS ALAMOS NATIONAL LABORATORY. PROC., P.79-99. AMERICAN INSTITUTE OF PHYSICS. 1988. (OC/793.5/.MA2/P56/1987X)

42-56

GURSKY, JUDITH C. (P-3); BAER, H. W. (MP-4); FLICK, FRED F. (MST-6); GALLEGOS. DAN (MP-8).

PRECISION NUCLEAR TARGETS FOR DRELL-YAN CROSS SECTION MEASUREMENTS AT $800 \mathrm{GEV}$.

TIC, 1988. 20P. MN (LA-UR-88-3041)

42-57

HAIGHT, ROBERT C. (P-15); ULLMANN, J. L. (P-3); LISOWSKI, PAUL W. (P-3); WENDER, STEVE A. (P-3): NELSON, RONALD O. (P-3); SEESTROM-MORRIS, SUSAN J. (P-3); KING, NICHOLAS S. P. (P-15);

RAPAPORT, J.; ZECKER, P.; BRADY, F. P.; ROMERO, J. L.; HJORT, E. J.; SORENSON, D. S.:

DRUMMOND, J. R.; HOWELL, C. R.; TORNOW, W.

CAPABILITIES AND PLANS FOR PRE-EQUILIBRIUM NUCLEAR REACTION MEASUREMENTS AT TARGET-4 AT LAMPF.

TIC, 1988. 7P. MN (LA-UR-88-0421)

42-58

HEMPELMANN, R. (LANSCE!; RICHTER, DIETER; FAUX, O. A.; ROSS. O. K

COLLECTIVE RELAXATION, SINGLE PARTICLE MOTION AND SHORT RANGE ORDER IN ALPHA PRIME NIOBIUM DEUTERIUM X-A QUASIELASTIC NEUTRON SCATTERING STUDY.

TIC, 1988. 1OP. MN (LA-UR-88-1662)

42-59

HERCZEG, PETER (T-5).

T-VIOLATION IN NUCLEAR INTERACTIONS-AN OVERVIEW.

TIC, 1988. 19P. MN (LA-UR-88-3568)

$42 \cdot 60$

HURD, J. R.; BOSWELL, J. S.; MINEHART, R. C.; TZENG, Y.; ZIOCK, H. J.; ZIOCK, K. O. H.;

LIU, LON CHANG (INC-11): SICILIANO. EDWARD R.

REACTION (PI PLUS, PI PLUS DEUTERON) ON 6 LITHIUM AND 12 CARBON.

NUCL. PHYS., V.A475, P.743-60. 1988.

42-61

JACAK, B. V. (P-2); DOSS, K. G. R.; GUSTAFSSON, H. A.; GUTBROD, H. H.; HARRIS, J. W.; KAMPERT, K. H.; KOLB, B.; POSKANZER, A. M.; RITTER, H. G.; SCHMIDT, HANS RUDI; TEITELBAUM, L.; TINCKNELL, M.;

WEISS, S.; WIEMAN, H.

FRAGMENTATION AND FLOW IN CENTRAL CULLISIONS

TIC, 1987. 15P. MN (LA-UR-88-0153)

42-62

JACAK, B. V. (P-2).

MULTIFRAGMENTATION.

TIC. 1988. 13P. MN (LA-UR-88-2475)

$42-63$

JOHNSON, MIKKEL B. (MP-DO): WENES, GEERT (T-5).

EFFECT OF SURFACE FLUCTUATIONS ON THE NUCLEAR DENSITY.

PHYS. REV., V.38C, P.386-9. 1988. 
JOHNSON, MIKKEL B. (MP.DO).

PION SCATTERING AND NUCLEAR DYNAMICS

TIC. 1988. 28P. MN (LA-UR-88-2893)

$42-65$

JONES, K. W. (MP-10).

NUCLEAR SPIN RESPONSE STUDIES IN INELASTIC POLARIZED PROTON SCATTERING.

TIC. 1988. 15P. MN (LA-UR-88-1272)

42-66

KINNEY, E. R.

INCLUSIVE PION DOUBLE CHARGE EXCHANGE IN HELIUM-4 AT INTERMEDIATE ENERGIES

LOS ALAMOS NATIONAL LABORATORY, 1988. 347P. (LA-11417-T)

$42-67$

KOEHLER, P. E. (P-3); BOWMAN, CHARLES D. (P-DO); STEINKRUGER, FREDERICK J. (INC-11):

MOODY. DAVID C. (INC-11); HALE, GERALD M. (T-2); STARNER, JOHN W. (INC-5); WENDER, STEVE A. (P-3);

HAIGHT, ROBERT C. (P.15); LISOWSKI, PAUL W. (P-3); TALBERT, W. L., JR. (INC-11).

BERYLLIUM-7 (NEUTRON, PROTON) LITHIUM-7 CROSS SECTION FROM 25 MEV TO 13.5 KEV.

PHYS. REV., V.37C, P.917-26. 1988.

42-68

KOEHLER, P. E. (P-3); O'BRIEN, HAROLD A. (P-3).

CROSS-SECTION MEASUREMENTS FOR RADIOACTIVE SAMPLES.

TIC, 1988. 24P. MN (LA-UR-88-1526)

$42-69$

KOEHLER, P. E. (P-3); O'BRIEN, HAROLD A. (P-3).

SODIUM-22(NEUTRON, PROTON) NEON-22 AND SODIUM-22(NEUTRON, ALPHA)FLUORINE-19 CROSS

SECTIONS FAOM 25 MILLIELECTRON- VOLTS TO 35 KILOELECTRONVOLTS.

PHYS. REV., V.38C, P.2019-25. 1988.

42.70

KOZACK, R. (T-2); CLARK, B.; HAMA, S.; MISHRA, V.; KALBERMANN, G.; MERCER, R.; RAY, L.

KEMMER-DUFFIN-PETIAU FORMALISM AND INTERMEDIATE-ENERGY DEUTERON-NUCLEUS SCATTERING.

TIC, 1988. 9P. MN (LA-UR-88-2084)

$42-71$

LEE, KIEN Y. (M-1); COBURN, MICHAEL D. (M-1)

3-NITRO-1,2,4-TRIAZOL-5-ONE, A LESS SENSITIIE EXPLOSIVE.

PATENTED MAR. 29, 1998, FILED 1987. (U.S.PATENT-4,733,610)

42.72

LEHMAN, DONALD R.; MAXIMON, LEONARD C.; FRIAR, JAMES L. (T-5).

COULCMB EFFECTS IN ASYMPTOTIC NORMALIZATION CONSTANTS-A SOLUBLE MODEL.

PHYS. REV., V.37C. P.336-48. 1988

42-73

LEITCH, M. J. (P-2).

LOW-ENERGY PION DOUBLE CHARGE EXCHANGE AND NUCLEON-NUCLEON CORRELATIONS IN NUCLEI.

TIC, 1988. 24P. MN (LA-UR-88-3854)

42-74

LING, ALAN G. (MP-10).

SMALL ANGLE ELASTIC SCATTERING OF PROTONS OFF OF SPINLESS NUCLEI.

LOS ALAMOS NATIONAL LABORATORY, 1988. 247P. (LA-11330-T)

42-75

LISOWSKI, PAUL W. (P-3); ULLMANN, J. L. (P-3); BALESTRINI, SILVIO J. (P-3); CARLSON, A. D.; WASSON, O. A. NEUTRON INDUCED FICSION CROSS SECTION RATIOS FOR THORIUM-232, URANIUM-235,238.

NEPTUNIL'M-237, AND PLUTONIUM-239 FROM 1 TO 400 MEV.

TIC, 1988. 4P. MN (LA-UR-88-1813) 
42.76

MADLAND, DAVID G. (T-2).

IMPROVED CALCULATION OF THE PROMPY FISSION NEUTRON SPECTRUM FROM THE SPONTANEOUS FISSION OF CALIFORNIUM-252-PRELIMINARY IRESULTS.

TIC, 1988. 5P. MN (LA-UR-88-1925)

42-77

MADLAND, DAVID G. (T-2).

IMPROVED CALCULATION OF THE PROMPT FISSION NEUTRON SPECTRUM FROM THE SPONTANEOUS

FISSION OF 252 CALIFORNIUM.

TIC, 1988. 23P. MN (LA-UR-88-1645)

$42-78$

MADLAND, DAVID G. (T-2); NIX, JAMES R. (T-9).

NEW MODEL OF THE AVERAGE NEUTRON AND PROTON PAIRING GAPS.

NUCL. PHYS, V.A476, P.i-38. 1988.

42-79

MADLAND, DAVID G. (T-2); STROTTMAN, DANIEL D. (T-9).

NUCLEAR STRUCTURE STUDY OF THE PROPOSED GAMMA-RAY LASER CANDIDATE NUCLEUS RHENIUM-186.

TIC, 1988. 12P. MN (LA-UR-87-4297)

$42-80$

MADLAND, DAVID G. (T-2); LA BAUVE, RAPHAEL J. (T-2); NIX, JAMES R. (T-9).

RECENT IMPROVEMENTS IN THE CALCULATION OF PROMPT FISSION NEUTRON SPECTRAPRELIMINARY RESULTS.

TIC, 1988. 12P. MN (LA-UR-88-2340)

42-81

MADLAND, DAVIO G. (T-2).

RECENT RESULTS IN THE DEVELOPMENT OF A GI_OBAL MEDIUM-ENERGY NUCLEON-NUCLEUS OPTICAL-MODEL POTENTIAL.

TIC, 1988. 15P. MN (LA-UR-88-0376)

42-82

MADLAND, DAVID G. (T-2).

THEORY OF NEUTRON EMISSION IN FISSION.

TIC, 1388. 6P. MN (LA-UR-88-3342)

42-83

MC CLELLAND, JOHN B. (MP-10).

ISOVECTOR SPIN OBSERVABLES IN NUCLEAR CHARGE REACTIONS AT LAMPF. TIC, 1988. 12P. MN (LA-UR-88-1116)

42-84

MILLENER, DONALD J.

SHELL MODEL TRANSITION DENSITIES FOR ELECTRON AND PION SCATTERING.

IN: PION-NUCLEUS PHYSICS-FUTURE DIRECTIONS AND NEW FACILITIES AT LAMPF, 1997, LOS ALAMOS NATIONAL LABORATORY. PROC., P.402-24. AMERICAN INSTITUTE OF PHYSICS, 1988. (QC/793.5/.M42/P56/1987X)

42-85

MISCHKE, RICHARD E. (MP-4).

PARITY NONCONSERVATION IN PROTON SCATTERING AT HIGHER ENERGIES.

TIC, 1987. 16P. MN (LA-UR-87-2126)

ALSO PUBLISHED: CAN. J. PHYS., V.66, P.495-502. 1988.

42.86

MOLLER, PETER (T-9); NIX, JAMES R. (T-9).

NUCLEAR MASSES FROM A UNIFIED MACROSCOPIC-MICROSCOPIC MODEL.

AT. DATA NUCL. DATA TABLES, V.39, P.213-23. 1988. 
42-87

MORDECHAI, S.; AUERBACH, NAFTALI (MP-DO); BURLESON, GEORGE R.; DHUGA, KALVIR S.; DWYER, MARK: FAUCET, JOHN A.; FORTUNE, H. T.; GILMAN, R.; GREENE, STEVEN J. (MP-10); LAYMON, CHARLES; MOORE, C. FRED; MORRIS. CHRISTOPHER L. (MP-10); OAKLEY. D. S.; PLUM, MICHAEL A. (MP-10): SEESTROMMORRIS, SUSAN J. (P-3); SEIDL, PETER A.; SMITHSON, M. J.; WANG, Z. F; ZUMBRO, J. D. GIANT DIPOLE RESONANCES BUILT ON ISOBARIC ANALOG STATES IN PION DOUBLE CHARGE EXCHANGE.

PHYS. REV. LETT., V.60, P.408-11. 1988.

42-88

MORDECHAI, S.; AUERBACH, NAFTALI (MP-DO); FORTUNE, H. T.; MORAIS, CHRISTOPHER L. (MP-10); MOORE, C. FRED.

ISOSPIN SPLITTING OF THE GIANT DIPOLE BUILT ON THE ISOBARIC ANALOG STATE. PHYS. REV., V.38C, P.2709-15. 1988

$42-89$

MORDECHAI, S.; AUERBACH, NAFTALI (MP-DO); BURLEIN, M.; FORTUNE, :-1. T.; GREENE, STEVEN J. (MP-10); MOORE, C. FAED; MORAIS, CHPISTOPHER L. (MP-10); ODONNELL, J. M.; RAWOCL, M. W.; SILK, J. D.;

WATSON, D. L.; YOO, S. H.; ZUMBRO, J. D.

PION DOUBLE CHARGE EXCHANGE TO THE DOUBLE DIPOLE RESONANCE. PHYS. REV. LTRS., V.61, P.531-4. 1988.

$42-30$

MUIR, DOUGLAS W. (T-2).

NUCLEAR-DATA EVALUATION BASED ON DIRECT AND INDIRECT MEASUAEMENTS WITH GENERAL COARELATIONS.

TIC, 1988. 5P MN (LA-UR-B8-1697)

$42-91$

NIX, JAMES R. (T-9); SIERK, AANOLD J. (T-9).

MECHANISM OF DISSIPATION IN HEAVY-ION REACTIONS.

IN: ADRIATIC INTERNATIONAL CONFERENCE ON NUCLEAR PHYSICS, 6TH, 1987, DUBAOVNIK, CROATIA. PROC., P.333-40. WORLD SCIENTIFIC, 1997. (QC/794.8/,H4/A37/1987)

ALSO PUBLISHED: TIC, 1987. 9P. MN (LA-UR-87-1705)

$42-92$

OAKLEY, D. S.; SMITHSON, M. J.; MORDECHAI, S.; MOORE, C. FRED; SEIDL, PETER A.;

MORRIS, CHRISTOPHER L. (MP-10); SEESTROM-MORRIS, SUSAN J. (P-3); GILMAN, R.; ZUMBRO, J. D.; FORTUNE, H. T.; DHUGA, KALVIR S.

PION INELASTIC SCATTERING FROM TITANIUM 48,50, CHROMIUM 52, AND IRON 54,56- HIGHER-LYING

STATES.

PHYS. REV., V.37C, P.866-9. 1988.

$42-93$

OSET, EULOGIO; VICENTE-VACAS, M. J.; JOHNSON, MKKEL B. (MP-DO); STROTTMAN, DANIEL D. (T-9); FORTUNE, H. T.; GILMAN, R.

DELTA INTEPACTION CONTRIBUTION TO DOUBLE CHARGE EXCHANGE

NUCL PHYS., V.A483, P.514-38. 1988.

42-94

OSET, EULOGIO; SALCEDO, L.; STROTTMAN, DANIEL D. (T-9); VICENTE-VACAS, M. J.; GARCIA-RECIO, C.; HERNANDEZ, E.

MONTE CAALO SIMULATION OF INCLUSIVE PIONIC REACTION AROUND RESONANCE AND HIGHER

ENERGY PERSPECTIVES.

IN: PION-NUCLEUS PHYSICS-FUTUAE DIRECTIONS AND NEW FACILITIES AT LAMPF, 1997, LOS

ALAMOS NATIONAL LABORATOAY. PROC. P.100-18. AMERICAN INSTITUTE OF PHYSICS, 1988. (OC/793.5/.M42/P56/1987X)

ALSO PUBUSHED: TIC, 1987. 20P. MN (LA-UR-87-3709)

42-95

PENG, JEN CHEIH (P-2).

ASSOCIATED PRODUCTION OF HYPERNUCLEI WITH (PION, KAON) REACTION.

IN: CONFERENCE ON THE INTERSECTIONS BETWEEN PARTICLE AND NUCLEAR PHYSICS, 3AD, 1988, ROCKPORT, MAINE. PROC., P.39-53. AMERICAN INSTITUTE OF PHYSICS, 1988. (OC1 .A38X NO.176)

ALSO PUBLSHED: TIC, 1988. 16P. MN (LA-UR-88-3029) 
42-96

PENG, JEN CHEIH (P-2)

ETA-MFSON PRODUCTION EXPERIMENTS AT LAMPF.

TIC, 1986. 7P. MN (LA-UR-86-2505)

42-97

PENG, JEN CHEIH (P-2): MISHRA, CHANDRA S. (P-2); MOSS, JOEL MARSHALL (P-2):

MC GAUGHEY, PATRICK L. (P-2); KAPUSTINSKY, JON S. (P-2).

FEASIBILITY OF DETECTING B YIELDS HYDROGEN PLUS HYDROGEN MINUS IN A FIXED-TARGET

EXPERIMENT.

IN: INTERNATIONAL CONFERENCE ON MEDIUM- AND HIGH-ENERGY NUCLEAR PHYSICS, 1988, TAIPEI,

TAIWAN. PROC., P.449-59. WORLD SCIENTIFIC, 1989 (QC793.15544 1988X)

ALSO PUBLISHED: TIC, 1988. 12P. MN (LA-UR-88-3028)

42-98

PENG, JEN CHEIH (P-2); KAPUSTINSKY, JON S. (P.2); LEE, C. (T-1); LFITCH, M. J. (P. 2); LI, T. K. (N-1);

LIU, LON CHANG (INC-11); MOSS, JDEL MARSHALL (P-2); SIMMONS, JAMES E. (P-2); TANG, SHERMAN (P-2);

SMITH, C.: WHITNEY, ROY R.

OBSERVATION OF ETA MESON PRODUCTION IN THE REACTION PION MINUS AND HELIUM-3 GOES TO

ETA MESON AND TRITON.

PHYS. REV. LETT., V.58, P.2027-30. 1987.

$42-99$

PICKLESIMER, A. (P-15).

MESIC INTERACTIONS AND TRINUCLEAR PHYSICS

TIC, 1988. 16P. MN (LA-UR-88-1664)

$42-100$

PYNN, R. (LANSCE).

MATERIALS SCIENCE AT AN ADVANCED HADRON FACILITY.

IN: ADVANCED HADRON FACILITY ACCELERATOR DESIGN WORKSHOP, 1988, LOS ALAMOS NATIONAL LABORATORY. PROC., P.70-7. LOS ALAMOS NATIONAL LABORATORY, 1989. (LA-11432-C)

ALSO PUBLISHED: TIC, 1988. 9P. MN (LA-UR-88-1663)

42-101

RAMAKRISHNAN, P.; MITCHELL, GARY E.; GOULD, CHAIS R.; WENDER, STEVE A. (P-3):

AUCHAMPAUGH, GEORGE F. (P-3); LITTLE, ROBERT C. (X-6).

NEUTRON-INDUCED PHOTON PRODUCTION CROSS SECTIONS.

NUCL. SCI. ENG., V.98, P.357-64. 1988.

42-102

RAMAKRISHNAN, P.; MITCHELL, GARY E.; GOULD, CHRIS R.; WENDER, STEVE A. (P-3);

AUCHAMPAUGH, GEORGE F. (P-DO).

PHOTON PRODUCTION CROSS SECTION FOR TANTALUM 181.

NUCL. SCI. ENG., V.98, F.348-56. 1988.

42-103

RAWOOL, M. W.

MEASUREMENT OF THE SPIN CORRELATION PARAMETERS C SL AND C LL IN POLARIZED NEUTRON POLARIZED PROTON GOING TO NEUTRON PROTON SCATTERING AT ENERGIES 484, 634, AND 789 MEV. LOS ALAMOS NATIONAL LABORATORY, 1988. 178P. (LA-11387- T)

42-104

FOKNI, S. H.; BAER, H. W. (MP-4); BERGMANN, ANTON G. (MP-13); BOWMAN, J. DAVID (MP-4);

IROM, FAROKH (MP-4); LEITCH, M. J. (P-2); SEFTOR, COLIN J. (MP-13); ALSTER, JONAS; PIASETZKY, ELI;

CLAUSEN, B. L.; LOVEMAN, ROBERT A.; PETERSON, R. J.; ULLMAN, JOHN (P-3); COMFORT. J. R.;

KNUDSON, JAMES N.; SENNHAUSER, U.

ISOBARIC-ANALOG-STATE TRANSITIONS IN PION CHARGE-EXCHANGE REACTIONS ABOVE THE

DELTA(1232) RESONANCE.

PHYS. LETT., V.202B, P.35-9 1988

42-105

ROUSSIN, ROBERT W.; DUNFORD, CHARLES L.; MC KNIGHT, ROBERT; YOUNG, PHILLIP G., JR. (T-2).

STATUS OF ENDF/B-VI.

TIC. 1988. 10P. MN (LA-UR-88-3104) 
42-106

RUTHERFORD, DEBRA A.

THEORETICAL AND EXPERIMENTAL CROSS SECTIONS FOR NEUTRON REACTIONS ON ZINC-64. LOS ALAMOS, 1988. 111P. (LA-11233-T)

42-107

SEESTROM-MORRIS, SUSAN J. (P-3); DEHNKARD, DIETRICH; FRANEY, M. A.; HOLTKAMP. DAVID B. (P-3); BLILIE, C. L.; MORAIS, CHRISTOPHER L. (MP-10); ZUMBRO, J. D.; FORTUNE, H. T.

PION INELASTIC SCATTERING TO THE THREE LOWEST 2 POSITIVE STATES OF OXYGEN-18.

PHYS. REV., V.37C, P.2057-63. 1988.

42-10B

SHERA, E. BAOOKS (P-DO).

PROBING NUCLEAR CHARGE RADII AND MOMENTS WITH MUONIC ATOMS.

IN: INTERNATIONAL CONFERENCE ON NUCLEAR STRUCTURE THROUGH STATIC AND DYNAMIC MOMENTS, 1987, MELBOURNE, AUSTRALIA. PROC., P.325-8. CONFERENCE PROCEEDINGS PRESS. 1987. (CC 793.3 .SQ/15733/1987X)

42-109

SILLERUD, LAUREL O. (LS-7); VAN HULSTEYN, DAVID B. (P-14); GRIFFEY, RICHARD H.

\{CARBON-13\}-POLARIZATION TRANSFER PFOTON NMR IMAGING OF A SODIUM [CARBON-13]FORMATE PHANTOM AT 4.7 TESLA.

J. MAGN. RESON., V.76, P.380-5. 1988.

42-110

SMITH, FICHARD D. (T-2): WAMBACH, J.

DAMPING OF THE CONTINUUM PESPONSE FROM 2P-2H EXCITATIONS.

PHYS. REV., V.38C, P.100-8. 1988.

42-111

SOLEM, JOHNDALE C. (T-DO); BIEDENHARN, LAWRENCE C.

LASER COUPLING TO NUCLEI VIA COLLECTIVE ELECTRONIC OSCILLATIONS A SIMPLE HEURISTIC MODEL STUDYY.

J. QUANT. SPECTROSC. RADIAT.TRANSFER, V.40, P.707-12. 1988.

42-112

SOLEM, JOHNDALE C. (T-DO).

THEOREM RELATING SPATIAL AND TEMPORAL HARMONICS FOR NUCLEAR INTERLEVEL TRANSFER DRIVEN BY COLLECTIVE ELECTRONIC OSCILLATION.

J. QUANT. SPECTROSC. MADIAT.TRANSFER, V.dO, P.713-5. 1988.

42-113

STROTTMAN, DANIEL D. (T-9).

RELATIVISTIC HYDRODYNAMICS AND HEAVY ION REACTIONS.

TIC, 1987. 10P. MN (LA-UF-B7-3703)

42.114

TADDEUCCI, T. N. (MP-10).

EMIPIRICAL CONNECTION BETWEEN (PROTON, NEUTRON) CROSS SECTIONS AND BETA DECAY

TRANSITION STRENGTHS.

TIC, 1988. 17P. MN (LA.UR-88-2924)

42-115

TALBERT, W. L., JR. (INC-11); BUNKER, MERLE E. (INC-5).

FUTURE DIRECTIONS AND EMERGING TECHNIOUES FOR ISOL SYSTEMS.

IN: INTERNATIONAL CONFERENCE ON NUCLEAR STRUCTURE, REACTIONS, AND SYMMETRIES, 1986. DUBROVNIK. CROATIA. PROC., P.645-5i;. WORLD SCIENTIFIC, 1986. (OC/793.3/.S8/1573/1986)

ALSO PUBLISHED: TIC, 1986. 14P. MN (LA-UR-86-2759)

42-116

TALLEY, THURMAN L. (T-2); EVANS, FOSTER ( $X-7)$.

MONTE CARLO ALPHA DEPOSITION.

TIC, 1988. AP. MN (LA-UR-88-1698) 
TANAKA, NOBUYUKI (MP-10); GAZZALY, MAGDI M.; PAULETTA, G.; AAS, BJORNE; ADANS, D.; FUJISAWA, F.; GREENE, STEVEN J. (MP-10); HASAI, H.; IGO, G.; ISAGAWA, S.; ISHIMOTO, S.; IWATANI, K.;

JONES, K. W. (MP-10); LOPIANO, D.; MASAIKE, A.; NISHIYAMA, F.; OHASHI, Y.; OKIHANA, A.; OKUMI. S.;

SPERISEN, F.; WESTON, G.; WHITTEN, C. A.

SPIN DEPENDENCE OF PP INELASTICITIES AT INTERMEDIATE ENERGIES.

PHYS. REV., V.37C, P.2071-4. 1988.

42.118

THOMPSON, JOE D. (P-10); LAWSON, A. C. (MST-5); MC ELFRESH, M. W. (P-10);

SATTELBERGER, ALFRED P. (INC-4); FISK, ZACHARY (P-10).

ELASTIC NEUTRON SCATTERING IN URANIUM CARBON DEUTERIUM-11.

TIC, 1988. 9P. MN (LA-UR-88-2023)

42- 119

USSERY, LARRY E. (N-2); VIEIRA, DAVID J. (INC-11); BERLIJN, J. J. (X-2); BUTLER, GILBERT W. (INC-11); DROPESKY, BRUCE J. (INC-11); GIESLER, GREGG CARL (INC-11); IMANISHI, NOBUTSUGU;

LEITCH, M. J. (P-2): RUNDBERG, ROBERT S. (INC-11).

EXCITATION FUNCTION FOR THE PION SINGLE-CHARGE-EXCHANGE REACTION CARBON-13 (PI PLUS, PI

0) NITROGEN-13 (G.S.)

PHYS. REV., V.38C, P.2761-70. 1988.

$42-120$

WEINFELD, ZEEV; PIASETZKY, ELI; BAER, H. W. (MP-4); ZURMAN, ROBERT L. (MP-4); LEITCH, M. J. (P-2);

MORRIS, CHRISTOPHER L. (MP-10); WRIGHT, D. H.; ROKNI, S. H.; COMFORT, J. R.

LOW-ENERGY PION DOUBLE CHARGE EXCHANGE ON CALCIUM ISOTOPES.

PHYS. REV., V.37C, P.902-5. 1988.

42-121

WENES, GEERT (T-5); GINOCCHIO, JOSEPH N. (T-5).

ALGEBRAIC APPROACH TO MEDIUM ENERGY PROTON SCATTERING.

IN: INTERNATIONAL SYMPOSIUM ON CAPTURE GAMMA-RAY SPECTROSCOPY AND RELATED TOPICS, 6TH, 1987, LEUVEN, BELGIUM. PROC., P.S65-70. INSTITUTE OF PHYSICS, 1988. (OC1.157X NO.88)

42-122

WENES, GEERT (T-5); OTSUKA, TAKAHARU; GINOCCHIO, JOSEPH N. (T-5).

F-SPIN VECTOR EFFECTS IN INELASTIC ELECTRON SCATTERING FROM LOW-LYING COLLECTIVE STATES OF 154 GADOLINIUM.

PHYS. REV., V.37, P.1878-83. 1988.

42-123

WILSON, WILLIAM B. (T-2); BOZOIAN, MICHAEL (T-2); PERRY, ROBERT T.

CALCULATED ALPHA-INDUCED THICK TARGET NEUTRON YIELOS AND SPECTAA, WITH COMPARISON TO MEASURED DATA.

TIC, 1988. 6P. MN (LA-UR-88-1976)

42-124

YOUNG, PHILLIP G., JR. (T-2); ARTHUR, EDWARD D. (T-DO).

CALCULATION OF 235 URANIUM(NU,NU') CROSS SECTIONS FOR ENDF/B-VI.

TIC, 1988. 6P. MN (LA-UR-88-1740)

42-125

YOUNG, PHILLIP G., JR. (T-2).

COVARIANCE ANALYSIS OF NITROGEN PLUS LITHIUM-7 DATA FOR ENDF/B-VI.

TIC, 1988. 5P. MN (LA-UR-B8-1618) 
43-1

ALLEN, R. C.: LU, X. Q.; GOLLWITZER, K.; IGO, GEORGE J.; GULMEZ, E.; WHITTEN, C.; VAN DALEN, G. J.; LAYTER, J. G.; FUNG, S. Y: SHEN, B. C.; RISTINEN, R. A.; SMYTHE. W. R.; CARLINI, R. C.:

BLACK, JOEL KEVIN (MP-13); BURMAN, ROBERT L. (MP-4); DONAHUE, JOEY B. (MP-7): FITZGERALD, D. H. (MP-13); FOREMAN, WILL M. (MP-6); HOEHN, MARTHA V. (MP-1); KOZLOWSKI, THOMAS (MP-6); LEE, DAVID M. (MP-5); LOUIS, W. C. (MP-4); MACEK, ROBERT J. (MP-DO); MC GILL, JOHN A. (MP-13); MILNER, E. C. (MP-4); OOTHOUDT, M. A. (MP-6); SANDBERG, V. D. (MP-4); SANDERS, GARY H. (MP-4); SCHILLACI, MARIO E. (MP-DO); WERBECK, RICHARD D. (MP-7); WESTERVELT, R. (MP-4); WHITE, D. HYWEL (MP-4); DIETERLE, B.; LEAVITT, C. P.; KANG, J. H.; FPATI, W.; MANN, ALFRED K.; NEWCOMER, F. M.; VAN BERG, R.; AUERBACH, L. B.; HIGHLAND, V. L.; MC FARLANE, W. K.; SEIGEL, R. T.

PROPOSAL FOR A PRECISION TEST OF THE STANDARD MODEL BY NEUTRINO-ELECTRON SCATTERING (LARGE CERENKOV DETECTOR PROJECT).

LOS ALAMOS NATIONAL LABORATORY, 1988. 311P. (LA-11300-P)

43-2

BOLSTERLI, MARK (T-9); PARMENTOLA, JOHN A. (T-9).

NUCLEON AS A PIONIC NONTOPOLOGICAL SOLITON OR BEYOND THE CLOUDY BAG MODEL. IN: TOPICAL CONFERENCE ON NUCLEAR CHROMODYNAMICS, 1988, ARGONNE NATIONAL LABORATORY. PROC., P.63-72. WORLD SCIENTIFIC, 1988. (QC793.3.035 T66 1988)

ALSO PUBLSHED: TIC, 1988. 11P. MN (LA-UR-88-1494)

43-3

BOLSTERL, MARK (T-9): PARMENTOLA, JOHN A. (T-9).

NUCLEON AS A PIONIC SOLITON.

IN: CHIRAL SOLITONS, A REVIEW VOLUME, K. LIU, ED. P.507-36. WORLD SCIENTIFIC, 1987.

(OC/793.3/.A4/C48/1987)

\section{3-4}

BOLTON, RICHARD D. (MP-4); COOPER, MARTIN D. (MP-4); FRANK, JAMES S. (MP-4);

HALLIN, AKSEL L. (MP-4); HEUSI, P. (MP-4); HOFFMAN, CYRUS M. (MP-4); HOGAN, GARY E. (MP-4);

MARIAM, FESSHA G. (MP-4); MATIS, H. S. (MP-4); MISCHKE, RICHARD E. (MP-4); PIILONEN, LEO E. (MP-4);

SANDBERG, V. D. (MP-4); SANDERS, GARY H. (MP-4): SENNHAUSER, URS (MP-4);

WERBECK, RICHARD D. (MP-7): WILLIAMS, ROBERT A. (N-2); WILSON, STEVE L. (MP-4); HOFSTADTER, R.; HUGHES, E. BARRIE; RITTER, M.; GROSNICK, D.; WRIGHT, S. C.; HIGHLAND, V. L; MC DONOUGH, J.

SEARCH FOR RARE MUON DECAYS WITH THE CRYSTAL BOX DETECTOR.

PHYS. REV., V.38D, P.2077-101. 1988.

43-5

BONNER, B. E.; NIETO, MICHAEL MARTIN (T-B).

BASIC PHYSICS PROGRAM FOR A LOW ENERGY ANTIPROTON SOURCE IN NORTH AMERICA.

IN: RAND WORKSHOP ON ANTIPROTON SCIENCE AND TECHNOLOGY, 1987, RAND CORPORATION. PROC., P.249-82. WORLD SCIENTIFIC, 1988. (OC793.5.P72 R36 1987)

ALSO PUBLISHED: TIC, 1987. 35P. MN (LA-UR-87-3980)

43.6

BROWN, ROBERT D. (MP-7); COST, JAMES RICHARD (MST-5); KIEISNER, G. P. (MST-5); BREWER, E. G. NEUTRON IRRADIATION STUDY OF NEODYMIUM-IRON-BORON PERMANENT MAGNETS MADE FROM MELT-SPUN RIBBONS

TIC. 1988. 7P. MN (LA-UR-88-1601)

43-7

CARLINI, ROGER D. (MP-14); SIEGEL, R.

(E,P) PARITY VIOLATION PROGRAM AT CEBAF.

IN: CEBAFISURA SUMMER WORKSHOP, 1987, NEWPORT NEWS, VA. PROC., P.418-28. CONTINUOUS

ELECTRON BEAM FACILITY, 1987. (OC/787/.P3/C4/1987X)

43-8

CARLSON, JOSEPH A. (T-5); HELLER, LEON (T-5); TJON, J. A

STABILITY OF DIMESONS.

PHYS. REV., V.37D, P.744-53. 1988. 
43-9

CARRUTHERS, PETER A. (T-DO); SHIH, CHIA CHANG.

E POSITIVE - E NEGATIVE MULTIPLICITY DISTRIBUTIONS-NEGATIVE BINOMIAL OR POISSON?

TIC, 1986. 9P. MN (LA-UR-86-2706)

43-10

CARRUTHERS, PETER A. (T-DO); SHIH, CHIA CHANG.

E POSITIVE-E NEGATIVE HADHONIC MULTIPLICITY DISTRIBUTIONS.

TIC, 1986. 27P. MN (LA-UR-86-2705)

43-11

CHEONG, SANG-WOOK (P-10); THOMPSON, JOE D. (P-10); FISK, ZACHARY (P-10); GRUNER, GEORGE

MAGNETIC FIELD DEPENDENCE OF THE 3D ORDERING IN LANTHANUM-2 COPPER OXIDE-4-DELTA. SOLID STATE COMMUN., Y.66, P.1019-21, 1988.

43-12

COOPER, FREDERICK M. (T-8); GINOCCHIO, JOSEPH N. (T-5); Y.HARE, AVINASH (T-8/CNLS).

RELATIONSHIP BETWEEN SUPERSYMMETRY AND SOLVAELE POTENTIALS.

PHYS. REV., V.36D, P.2458-73. 1987.

ALSO PUBLISHED: INTERNATIONAL COLLOOUIUM ON GROUP THEORETICAL METHODS IN PHYSICS, 15TH, 1986. DREXEL UNIVERSITY. PROC., P.608-14. WORLD SCIENTIFIC, 1987. (CC20.7.G76 157

43-13

COOPER, FREDERICK M. (T-8); KHARE, AVINASH; MUSTO, RENATO; WIPF, ANDREAS (T-8).

SUPERSYMMETRY AND THE DIRAC EOUATION.

ANN. PHYS., V.187, P.1-28. 1988.

43-14

COOPER, NECIA G. (PAO); WEST, GEOFFREY B. (T-8).

PARTICLE PHYSICS A LOS ALAMOS PRIMER.

CAMBRDIGE UNIVERSITY PRESS, 1988. 199 P. (QC/793/.P358/1988)

43-15

COUSINS, R. D.; KONIGSBERG, J.; KUBIC, J.; MELESE, P.; RUBIN, P.; SLATER, W. E.; FRANK, JAMES S. (MP-4); HART, GREGORY W. (MP-4); KINNISON, W. WAYNE (P-2); LEE, DAVID M. (MP-5); MILNER, E. C. (MP-4);

SANDERS, GARY H. (MP-4); ZIOCK, HANS J. (P-3); ARISAKA, K.; KNIBBE, P.; MOLZON, W. R.; IJAHEIM, J.; WALES, W. D.; AXELROD, S.; BIERY, K. A.; BONNEAUD, G.; IRWIN, G.; LANG, K.; MARTOFF, C. J.;

OUIMETTE, D. A.; RITCHIE, J. L.; TRANG, Q. H.; WOJCICKI, S. G.; AUERBACH, L B.; BUCHHOLZ, P.; HIGHLAND, V. L.; MC FARLANE, W. K.; SIVERTZ, M. B.; CHAPMAN, MICHAEL D.; ECKHAUSE, M. (MP-4); GINKEL, JOHN F.: GUSS, PAUL P.: JOYCE, DON; KANE, JOHN R.; KENNEY, C. J.; VULCAN, WILLIAM F.; WELSH, ROBERT E.; WHYLEY, ROBERT J.; WINTER, ROLF G.

SEARCH FOR THE DECAYS K OMICRON L TO MU ETA AND K OMICRON L TO ETA ETA. PHYS. REV., V.38D, P.2914-7. 1988.

43-16

DIMOPOULOS, SAVAS; RABY, STUART A. (T-8); WILCZEK, FRANK.

PROTON DECAY IN SUPERSYMMETRIC MODELS

IN: SUPERSYMMETRY, S. FERRARA, ED., P.774-7. ELESEVIER SCIENCE PUB., 1987. (CC174.17.59 S972 $1987)$

ALSO PUBLISHED: PHYS. LETT., V.221B, P.133-6. 1982.

43-17

DOMBECK, THOMAS W. (P-3); KOETKE, D. D.; KOETKE, D. S.; FISK, R.

CW-PION FOCUSING HORN FOR LOW-ENERGY MUON NEUTRINO BEAMS.

TIC. 1988. 14P. MN (LA-UR-88-1617)

43-18

DORIA, MAURO M. (CNLS); MENIKOFF, RALPH (T-14); SHARP, DAVID H. (T-8)

MAGNETOPHONONS IN THE TWO DIMENSIONAL LIQUID STATE OF INTEAACTING CHARGED PARTICLES. PHYS. REV., V.37A., P.2605.7. 1988 
43-19

DYER, PEGGY (P-3); CAMP. J. (P-3); HOLZSCHEITER, MICHAEL H. (P-15); GRAESSLE, SCOTT.

FALLING ANTIMATTER-AN EXPERIMENT TO MEASURE THE GRAVITATIONAL ACCELERATION OF THE

ANTIPROTON.

NUCL. INSTRUM. METHODS PHYS. RES., V.B40/41, P.485-8. 1989.

ALSO PUBLISHED: TIC, 1988. 15P. MN (LA-UR-88-3193)

43-20

FRANK, JAMES S. (MP-4); HART, GREGORY W. (MP-4); KINNISON, W. WAYNE (P-2); LEE. DAVID M. (MP-5); MC KEE, ROBERT J. (P-2); MILNER, E. C. (MP-4); SANDERS, GARY H. (MP-4); ZIOCK, HANS J. (P-2); CHAPMAN, MICHAEL D.; ECKHAUSE, M. (MP-4); GINKEL, JOHN F.; GUSS, PAUL P.; JOYCE, DON; KANE, JOHN R.; KENNEY, C. J.; WELSH, ROBERT E.; WHYLEY, ROBERT J.; WINTER, ROLF G.; VULCAN, WILLIAM F.

TRACKING RANGEFINDER FOR MUONS FROM KAON DECAY.

TIC. 1988. 8P MN (LA-UR-88-3733)

43-21

FRIAR, JAMES L. (T-5).

RESULTS OF RECENT CALCULATIONS USING REALISTIC POTENTIALS

IN: EUROPEAN CONFERENCE ON FEW-BODY PHYSICS, 11TH, 1987, FONTEVRAUD. FRANCE. PROC., P.51-63. SPRINGER. VERLAG, 1987. (OC174.17.P7 F5X V.2)

ALSO PUBLISHED: TIC, 1987. 14P. MN (LA-UR-87-2797)

$49-22$

GINTCCHIO, JOSEPH N. (T-5)

SPIN QUENCHING IN THE SOB AND SPG FERMION MODELS OF COLLECTIVE MOTION.

IN: INTERNATIONAL COLLOOUIUM ON GROUP THEORETICAL METHODS IN PHYSICS, 15TH, 1986, DREXEL UNIVERSITY. PROC. P.315-8. WORLO SCIENTIFIC, 1987. (OC20.7.G76 I57 1986)

ALSO PUBLISHED: TIC, 1986. 5P. MN (LA-UR-86-4012)

43-23

GLASHAUSSER, C.; JONES, K. W. (MP-10)

POLARIZATION TRANSFER STUDIES IN INELASTIC PROTON SCATTERING AT HIGH EXCITATION ENERGIES. IN: PION-NUCLEUS PHYSICS-FUTURE DIRECTIONS AND NEW FACIUITIES AT LAMPF, 1987, LOS ALAMOS NATIONAL LABORATORY. PROC., P.373-82. AMERICAN INSTITUTE OF PHYSICS, 1988. (CC/793.5/.M42/P56/1987X)

43-24

GOGGIN, MICHAEL E. (T-12); MILONNI, PETER W. (T-12)

DRIVEN MORSE OSCILLATOR-CLASSICAL CHAOS AND OUANTUM THEORY FOR TWOFREQUENCY EXCITATION

PHYS. REV., V.38A, P.5174-81. 1988

43-25

GOLDMAN, TERRENCE J. (T-5).

ADVANCED HADRON FACILITY-PROSPECTS AND APPLICABILITY TO ANTIPROTON PRODUCTION. IN: RAND WORKSHOP ON ANTIPROTON SCIENCE AND TECHNOLOGY, 1987, RAND COAPORATION. PROC., P.123-54. WORLD SCIENTIFIC, !988. (OC793.5.P72 R36 1987)

ALSO PUBLISHED: TIC, 1987. 33P. MN (LA-UR-87-3313)

43-26

GOLDMAN, TERRENCE J. (T-5); HUGHES, RICHARD J. (T-8); NIETO, MICHAEL MARTIN (T-8)

EXCLUSION OF AN ISOSPIN FIFTH FORCE FROM KAON DECAY.

MOD. PHYS. LETT., V.3A, P.1243-9. ${ }^{4} 988$.

$43-27$

GOLDMAN, TERRENCE J. (T-5); NIETO, MICHAEL MARTIN (T-8).

FAMILY PROBLEM.

IN: PARTICLE PHYSICS-A LOS ALAMOS PRIMER (UPDATED VERSION OF LOS ALAMOS SCIENCE, NO.

11. SUMMER-FALL. 1984) N.G. COOPER AND G.B. WEST, EDS., P.115-23. CAMBRIDGE UNIVERSIT PRESS, 1988. (OC/793/P358/1988)

ALSO PUBLISHED: LOS ALAMOS SCl. NO.11. SUMMER-FALL, P.115-23. 1984 
43-28

GOLDMAN, TERRENCE J. (T-5); MALTMAN, KIM A. (T-5); SCHMIDT, KEVIN E.;

STEPHENSON, GERARD J., JR. (P-DO).

MULTIOUARK INTERACTIONS.

IN: TOPICAL CONFERENCE ON NUCLEAR CHROMODYNAMICS, 1988, ARGONNE NATIONAL LABORATORY. PROC., P.1-15. WORLD SCIENTIFIC, 1988. (QC793.3.035 T66 1989)

ALSO PUBLISHED: TIC. 1988. 16P. MN (LA-UR-88-1940)

43-29

GOLDMAN, TEARENCE J. (T-S).

STANDARD MODEL AND ITS PROBLEMS-THE PHYSICS BACKGROUND FOR AN ADVANCED HADRON FACIUTY.

PAND WORKSHOP ON ANTIPROTON SCIENCE AND TECHNOLOGY, 1987, PAND CORPORATION. PROC., P.446-74. WORLD SCIENTIFIC. 1988. (OC733.5.P72 R36 1987)

43-30

GUPTA, RANAN (T-DO).

HYERID MONTE CARLO ALGORITHM AND THE CHIRAL TAANSITION.

TIC, 1987. 10P. MN (LA-UR-87-4146)

43-31

GUPTA, RAJAN (T-DO).

INTRODUCTION TO LATTICE GAUGE THEORY.

TIC. 1987. 4TP. MN (LA-UR-88-0629)

43-32

GUPTA, RAJAN (T-DO); GURALNIK, GERALD D. (T-DO); PATEL, APOORVA; SHARPE, STEPHEN R.

ON THE FINITE-TEMPERATURE TAANSITION OF OCD

PHYS. LETT., V.201B, P.503-9. 1988.

43-33

GURALNIK, GERALD D. (T-DO): WARNOCK, TONY T.; ZEMACH, CHARLES (T-COT).

OCD ON A CPAY-THE MASSES OF ELEMENTARY PARTICLES.

IN: PARTICLE PHYSICS-A LOS ALAMOS PRIMER (UPDATED VERSION OF LOS ALAMOS SCIENCE, NO.

11, SUMMER-FALL, 1984), N.G. COOPER AND G.B. WEST, EDS., P.41-8. CAMBRIDGE UNIVERSITY

PRESS, 1968. (OC/793/.P358/1988)

ALSO PUBLISHED: LOS ALAMOS SCI., NO.11, SUMMER-FALL, P.41. 1984.

43-34

HELLER, LEON (T-5).

HEAVY MULTIQUARK STATES.

IN: ELEMENTARY STRUCTURE OF MATTEA, 1987, LES HOUCHES, FRANCE. PROC., P.35-41.

SPRINGER-VERLAG, 1988. (OC/793.3/.S8/E44/1987X)

ALSO PUBLSHED: TIC, 1987. 14P. MN (LA-UR-87-2096)

43-35

HERCZEG, PETER (T-5).

EXOTIC DECAYS OF LIGHT MESONS.

IN: WOAKSHOP ON PRODUCTION AND DECAY OF LIGHT MESONS, 1988, ECOLE POLYTECHNIQUE, PARIS, FRANCE. PROC., P.16-46. WORLD SCIENTIFIC, 1988. (OC793.5.M42 P76 1988)

ALSO PUBLISHED: TIC, 1988. 32P. MN (LA-UR-88-1857)

43-36

HOFFMAN. CYRUS M. (MP-4).

NEW LIMIT FOR THE BRANCHING RATIO FOR THE DECAY PI ZERO TO NU NU.PRIME FPOM BEAALDUMP EXPERIMENTS

PHYS. LETT., V.208B, P.149-51. 1988.

43-37

HOFFMAN, CYRUS M. (MP-4).

SEARCH FOR LEPTON-FAMILY-NUMBER NONCONSERVATION.

IN: WORKSHOP ON GRAND UNIFICATION, 7TH, 1986, TOYAMA UNIVERSITY, JAPAN. PROC., P.315-37. VHORLD SCIENTIFIC, 1987. (OC/794.6/.G7NW67/1986)

ALSO PUBLISHED: TIC, 1986. 23P. MN (LA-UR-86-1728) 
HUGHES, RICHARD J. (T-8); GOLDMAN, TERRENCE J. (T-5); NIETO, MICHAEL MARTIN (T-8).

GRAVITY IN GREENLAND.

TIC. 1988. 5P. MN (LA-UR-88-3402)

43-39

HUGHES, RICHARD J. (T-8); COLDMAN, TERRENCE J. (T-5); NIETO, MICHAEL MARTIN (T-8).

QUANTUM GRAVITY AND NEW FORCES.

IN: 5TH FORCE NEUTRINO PHYSICS-MORIOND WORKSHOP, 8TH, 1988, SAVOIE, FRANCE. PROC., P.603-7. EDITIONS FRONTIERES, 1988. (OC793 M67 1988X)

ALSO PUBLISHED: TIC, 1988. 6P. MN (LA.UR-88-0926)

43-40

HWANG, W-Y P. (P-2); MOSS, JOEL MARSHALL (P-2); PENG, JEN CHEIH (P-2).

A DEPENDENCE OF QUARKONIUM PRODUCTION IN PROTON-NUCLEUS COLLISIONS.

PHYS. REV., V.38D, P.2785-96. 1988.

43-41

HYER, DIANNE K. (P-DO); LYNN, J. E. (P-3).

NEUTRON RESONANCE RADIOGRAPHY-REPORT OF A WORKSHOP HELD AT THE LOS ALAMOS

NATIONAL LABCRATORY JUI.Y 27-29, 1987

LOS ALAMOS NATIONAL LABORATORY, 1988. 175P. (LA-11393-C)

43-42

HYNES, M. V. (P-15); CAMPBELL, LAURENCE J. (T-11).

PHYSICS WITH THERMAL ANTIPROTONS.

IN: CONFERENCE ON THE INTERSECTIONS BETWEEN PARTICLE AND NUCLEAR PHYSICS, 3RD, :368, ROCKPORT, MAINE. PROC., P.440-6. AMERICAN INSTITUTE OF PHYSICS, 1988. (OC1 .A39X NO.176) ALSO PUBLISHED: TIC, 1988. 8P. MN (LA-UR-86-2658)

43-43

JOHNSON, MIKKEL B. (MP-DO).

LIGHT-FRONT POTENTIALS FROM MESON FIELD THEORY

TIC, 1988. 5P. MN (LA-UR-88-3698)

43-44

JOHNSON, MIKKEL B. (MP-DO).

PIONS AND THE NUULEAR SPIN-ISOSPIN RESPONSE.

IN: PION-NUCLEUS PHYSICS-FUTURE DIRECTIONS AND NEW FACILITIES AT LAMPF, 1987, LOS ALAMOS NATIONAL LABORATORY. PROC., P.352-72. AMERICAN INSTITUTE OF PHYSICS, 1989. (OC/793.5/.M42/P56/1987X)

43-45

KWAN, THOMAS J. T. $(X-10)$; DAVIS, HAROLD A. (P-1); FULTON, ROBERT D. (P-1):

SHERWOOD, EUGENE G. (P-1).

EFFECTS OF MODULATED ELECTRON BEAMS AND CAVITIES ON REDITRONS

TIC, 1988. 8P. MN (LA-UR-88-2189)

43-46

LYSENKO, W..-IER P. (AT-6); OVERLEY, MARK S. (AT-3).

MOMENT INVARIANTS FOR PARTICLE BEAMS.

IN: WOAKSHOP ON LINEAF ACCELERATOF, AND BEAM OPTICS CODES, 1988, SAN DIEGO. CALIF. PROC., P.323-35. AMERICAN INSTITUTE OF PHYSICS, 1988. (OC1 .A38X NO.177)

ALSO PUBLISHED: TIC, 1988. 14P. MN (LA-UR-88-1545)

43-47

MARIANER, SHLOMO (T-11); BISHOP, ALAN R. (T-i1); POUGET, J.

FFENKEL-KONTOROVA MODEL WITH NONCONVEX INTERPARTICLE INTERACTIONS.

IN: CMS WORKSHOP ON COMPETING INTERACTIONS AND MICROCLUSTERS-STATICS AND DYNAMICS, 1987, LOS ALAMOS, N.M. PROC., P.104-9. SPFINGER-VERLAG, 1988. (OC173.4.C55 C58 ALSO PUBLISHED: TIC, 1987. 6P. MN (LA-UR-87-3333)

43-48

MC DOWELL, H. KEITH (CLS-2).

QUANTUM LANGEVIN THEORY AND THE MORI RANDOM FORCE. NUCL PHYS. B, (PROC. SUPPL.) V.5A, P.247-9. 1988 
43-49

NIETO. MICHAEL MARTIN (T-8); HUGHES, RICHARD J. (T-8).

ANTIMATTER-ITS HISTORY AND ITS PROPERTIES

IN: RAND WORKSHOP ON ANTIPROTON SCIENCE AND TECHNOLOGY, 1987, RAND CORPORATION. PROC. P.228-48. WORLD SCIENTIFIC, 1988. (QC793.5.P72 R36 1987)

ALSO PUBLISHED: TIC, 1987. 22P. MN (LA-UR-87-4119)

43-50

NIETO, MICHAEL MARTIN (T-8); GOLDMAN, TERRENCE J. (T-5); HUGHES, RICHARD J. (T-8).

INVERSE-SOUARE LAW AND QUANTUM GRAVITY.

TIC, 1988. 4P. MN (LA-UR-88-2351)

43-51

NIETO, MICHAEL MARTIN (T-8); BONNER, B. E.

LOOKING FOR NEW GRAVITATIONAL FORCES WITH ANTIPROTONS

IN: RAND WOAKSHOP ON ANTIPROTON SCIENCE AND TECHNOLOGY, 1987. RAND CORPORATION.

PROC., P.328-41. WORLD SCIENTIFIC, 1988. (OC793.5.P72 R36 1987)

ALSO PUBLISHED: TIC, 1987. 15P. MN (LA-UR-87-3941)

43-52

NIETO, MICHAEL MARTIN (T-8); GOLDMAN, TERRENCE J. (T-5); HUGHES, RICHARD J. (T-8).

NEW GRAVITATIONAL FORCES FROM QUANTUM THEORY.

TIC, 1988. 7P. MN (LA-UR-88-2563)

43-53

PENG, JEN CHEIH (P-2).

(PI, NU) AND (PI PLUS, K PLUS) REACTIONS IN NUCLEI.

IN: PION-NUCLEUS PHYSICS-FUTURE DIRECTIONS AND NEW FACILITIES AT LAMPF. 1997, LOS ALAMOS NATIONAL LABORATORY, PROC., P.160-78. AMERICAN INSTITUTE OF PHYSICS, 1988. (CC793.5.M42 P56 1987X)

43-54

PENG, JEN CHEIH (P-2).

ETA MESON PRODUCTION AT LAMPF.

IN: WORKSHOP ON PRODUCTION AND DECAY OF LIGHT MESONS, 1988, ECOLE POLYTECHNIOUE, PARIS, FRANCE. PROC., P.102-13. WORLD SCIENTIFIC, 1988 (OC793.5.M42 P76 1988)

ALSO PUBLISHED: TIC, 1988. 13P. MN (LA-UR-88-1729)

43-55

PETERSON, R. J.; STROTTMAN, DANIEL D. (T-2)

PION-NUCLEUS PHYSICS-FUTURE DIRECTIONS AND NEW FACILITIES AT LAMPF.

AMERICAN INSTITUTE OF PHYSICS, 1988. 575 P. (OC/793.5/.M42/P56/1967X)

43-56

POELAKKER, KAREN (MP-4); HUTSON, RICHARD L. (MP-5); SCHILLACI, MARIO E. (MP-DO);

VAN DYCK, OLIN B. (MP-13); WHITE, D. HYWEL (MP-4)

PROGRESS AT LANPF JANUARY-DECEMBER 1987.

LOS ALAMOS NATIONAL LABORATORY, 1988. 336P. (LA-11339-PR)

43-57

PYNN, R. (LANSCE); BOUCHER, JEAN P: REGNAULT, LOUIS P.

INELASTIC NEUTRON SCATTERING WITH POLARIZATION ANALYSIS

TIC, 1988. 21P. MN (LA-UR-88-1746)

43-58

RABY, STUART A. (T-8); WEST, GEOFFREY B. (T-8); HOFFMAN, CYRUS M. (MP-4).

ARE THERE REALLY ANY EXPERIMENTAL LIMITS ON A LIGHT HIGGS BOSON?

PHYS. REV., V.39D, P.828-33. 1989.

ALSO PUB!JSHED: TIC, 1988. 13P. MN (LA-UA-88-2667)

43-59

RABY, STUART A. (T-8); WEST, GEOFFREY B. (T-8).

BRANCHING RATIO FOR A LIGHT HIGGS BOSON TO DECAY INTO MI ' $:$ PAIRS

PHYS. REV., V.380, P.3488-501. 1988 
43-60

RABY, STUART A. (T-8); SLANSKY, RICHARD C. (T-8); WEST, GEOFFREY B. (T-8)

PARTICLE PHYSICS AND THE STANDARD MNDEL.

IN: PARTICLE PHYSICS A LOS ALAMOS PRIMER (UPDATED VERSION OF LOS ALAMOS SCIENCE. NO. 11. SUMMER-FALL, 1984), N.G. COOPER AND G.B. WEST, EDS., P.23-40. CAMBRIDGE UNIVERSITY PRESS, 1988. (OC/793/.P358/1988)

ALSO PUBLISHED: LOS ALAMOS SCI., NO.11, P.23-53. 1984.

43-61

RABY, STUART A. (T-8).

SUPEASYMMETRY AT $100 \mathrm{GEV}$.

IN: PARTICLE PHYSICS-A LOS ALAMOS PRIMEA (UPDATED VERSION OF LOS ALAMOS SCIENCE, NO. 11, SUMMER-FALL, 1984), N.G. COOPER AND G.B. WEST, EDS., P.98-113. CAMBRIDGE UNIVERSITY PRESS, 1988. (OC/793/.P358/1988)

ALSO PUBLISHED: LOS ALAMOS SCI., NO.11, SUMMER-FALL, P.98-112. 1985.

43-62

ROBERTSON, ROBERT G. H. (P-3).

PROPERTIES OF NEUTRINOS RECENT RESULTS.

IN: LAKE LOUISE WINTER INSTITUTE ON NEW FRONTIERS IN PARTICLE PHYSICS, 2ND, 1987, CHATEAU LAKE LOUISE, CANADA. PROC., P.171-206. WORLD SCIENTIFIC, 1987. (QC794.8.E44 L34 1987X)

ALSO PUBLISHED: TIC, 1987. 37P. MN (LA-UR-87-1377)

43-63

ROBERTSON, ROBERT G. H. (P-3).

REVIEW OF NEUTRINO NIASS MEASUREMENTS

TIC, 1988. 19P. MN (LA,-UR-88-2609)

43-64

ROBERTSON, ROBERT G. H. (P-3).

TRITIUM NEUTRINO MASS EXPERIMENTS.

TIC, 1988. 14P. MN (LA-UR-88-2608)

43-65

ROSEN, SIMON PETER (T-DO).

DOUBLE BETA DECAY-A THEOAETICAL OVERVIEW

TIC, 1988. 23P. MN (LA-UR-88-3620)

43-66

ROSEN, SIMON PETER (T-DO).

INTRODUCTORY REMARKS ON DOUBLE BETA DECAY AND NUCLEAA PHYSICS.

IN: INTERNATIONAL SYMPOSIUM ON NUCLEAR BETA DECAYS AND NEUTRINO, 1986, OSAKA. PROC. P.55-70. WORLD SCIENTIFIC, 1986. (QC/793.5/.N422/1587/1986)

ALSO PUBLISHED: TIC, 1986. 18P. MN (LA-UR-86-2744)

43-67

ROSEN, SIMON PETER (T-DO)

MARCH TOWARD HIGHER ENERGIES.

IN: PARTICLE PHYSICS-A LOS ALAMOS PRIMEF (UPDATED VERSION OF LOS ALAMOS SCIENCE, NO. 11, SUMMER-FALL, 1984) N.G. COOPER AND G.B. WEST, EDS., P.150-5. CAMABRIDGE UNIVERSITY PRESS, 1988. (QC/793.P358/1988)

ALSO PUBLISHED: LOS ALAMOS SCI., NO.11, SUMMER-FALL, P.146-57, 1984.

43-68

SANDERS, GARY H. (MP.13).

EXPERIMENTS TO TEST UNIFICATION SCHEMES.

IN: PARTICLE PHYSICS-A LOS ALAMOS PRIMER (UPDATED VER ION OF LOS ALANOS SCIENCE, NO. 11. SUMMER-FALL, 1984) N.G. COOPER A ND G.8. WEST, EDS., P.128-48. CAMBRIDGE UNIYERSITY PRESS, 1988. (QC/793/.P358/1988)

ALSO PUBLISHED: LOS ALAMOS SCI., NO.11. SLMMER-FALL, P.124-44. 1984.

43-69

SANDERS, GARY H. (MAP-4).

PRESENT AND FUTURE NEUTRINO PHYSICS RESEARCH AT THE LOS ALAMOS MESON PHYSICS FACILITY. TIC, 1988. 19P. MN (LA-UR-88-0892) 
43-70

SEFTOR, COLIN J. (MP-13).

RECOIL PPOTON POLARIZATION IN PI-P ELASTIC SCATTERING.

LOS ALAMOS NATIONAL LABORATORY, 1988. 194P. (LA-11389-T)

43-71

SILBAR, RICHARD R. (T-5).

INTERMEDIATE-ENERGY HADRON INTERACTIONS, II.

TIC, 1988. 10\%'. MN (LA-UR-88-3320)

43-72

SILBAR, RICHARD R. (T-5); KLOET, WILLEM M.; KISSLINGER, LEONARD S.: DUBACH, JOHN FRASER (T-5).

PION-EXCHANGE CONTRIBUTION TO THE PARITY-VIOLATING ASYMMETRY IN PROTON-PROTON

SCATIEAING.

TIC, 1988. 9P. MN (LA-UR-88-3030)

43-73

SIMMONS, LEONARD M., JR. (T-DO).

SCIENCE UNDERGROUND-THE SEARCH FOR RARE EVENTS.

IN: PARTICLE PHYSICS-A LOS ALAMOS PRIMEA (UPDATED VERSION OF LOS ALAMOS SCIENCE, NO. 11, SUMMER-FALL, 1984). N.G. COOPER AND G.B. WEST, EDS., P.166-79. CAMBRIDGE UNIVERSITY PRESS, 1988. (OC/793/.P358/1988)

ALSO PUBLISHED: LOS ALAMOS SCI., NO.11, SUMMER-FALL, P.160-?0. 1984.

43.74

SINGHAM, SHERMILA BAITO (LS-2).

COUPLED DIPOLES IN LIGHT SCATTERING BY RANDOMLY ORIENTED CHIRAL PARTICLES.

J. CHEM. PHYS., V.88, P.1522-7. 1988.

43-75

SINGHAM, SHERMILA BAITO (LS-2); BOHAEN, CRAIG F. (LS-2).

LIGHT SCATTERING BY AN ARBITRARY PARTICLE-THE SCATTERING-ORDER FORMULATION OF THE COUPLED-DIPOLE METHOD.

J. OPT. SOC. AM., V.5A, P.1867-72. 1988.

43-76

SIVIA, DEVINDER S. (T-11); SILVER, RICHARD N. (T-11),

DETERMINATION OF MOMENTUM DISTRIBUTIONS FROM DEEP INELASTIC NEUTRON SCATTEAING

EXPERIMENTS-A BAYESIAN STUDY.

TIC, 1988. 5P. MN (LA-UR-88-3851)

43-77

SLANSKY, RICHARD C. (T-8).

LECTURE NOTES FROM SIMPLE FIELD THEORIES TO THE STANDARD MODEL

IN: PARTIC - E PHYSICS-A LOS ALAMOS PRIMER (UPDATED VERSION OF LOS ALAMOS SCIENCE, NO. 11. SUMMER-FALL, 1984), N.G. COOPER AND G.B. WEST, EDS., P.54-71. CAMBRIDGE UNIVERSITY PRESS, 1988. (OC/7931.P358/1988)

ALSO PUBLISHED: LOS ALAMOS SCI., NO.11, SUMMER-FALI., P.54-71. 1984.

43-78

SLANSKY, RICHARD C. (T-B).

TOWARD A UNIFIED THEORY.

IN: PARTICLE PHYSICS-A LOS ALAMOS PRIMER (UPDATED VERSION! OF LUS ALAMOS SCIENCE, NO. 11, SUMMER-FALL, 1984) N.G. COOPER AND G.B. WEST, EDS., P.72-97. CAMBFIDGE UNIVERSITY PRESS, 1988. (OC/793.P358/1988)

ALSO PUBLISHED: LOS ALAMOS SCI., NO.11, SUMMER-FALL, P.74-95. 1984.

43-79

STEPHENSON, GERARD J., JR. (P.DO); GOLDMAN, TERRENCE J. (T-5); MALTMAN, KIM R. (T-5);

SCHMIDT, KEVINE.

EXCHANGE EFFECTS IN DOUBLE BETA DECAY.

IN: CONFERENCE ON THE INTERSECTIONS BETWEEN PARTICLE AND NUCLEAR PHYSICS, 3RD, 1008. ROCKPORT, MAINE. PROC., P.930-5. AMERICAN INSTITUTE OF PHYSICS, 1988. (CC1 .A38X NO.376)

ALSO PUBLISHED: TIC, 1988. 7P. MN (LA-UR-88-2022) 
43-80

THIESSEN, HENRY A. (MP-DO).

LAMPF II AND THE HIGH-INTENSITY FRONTIER.

IN: PARTICLE PHYSICS - A LOS ALAMOS PRIMER (UPDATED VERSION OF LOS ALAMOS SCIENCE, NO. 11. SUMMER-FALL, 1984), N.G. COOPER AND G.B. WEST, EDS., P.158-63. CAMBRIDGE UNIVERSITY PRESS, 1988. (QC/793/.P358/1988)

ALSO PUBLISHED: LOS ALAMOS SCI., NO.11, SUMMER-FALL, P.152-7. 1984.

43-81

USSERY, LARRY E. (N-2); HOLLAS, C. L. (N-2); BUTTERFIELD, KENNETH B. (N-2);

MORGADO, RICHARD E. (N-2).

THREE-DIMENSIONAL IMAGING USING TAGGED 14.7-MEV NEUTRONS.

LOS ALAMOS NATIONAL LABORATORY, 1988. 44P. (LA-11423-MS)

43.82

WEST, GEOFFREY B. (T-L, .

SCALE AND DIMENSION-FROM ANIMALS TO QUARKS.

IN: PARTICLE PHYSICS-A LOS ALAMOS PRIMER (UPDATED VERSION OF LOS ALAMOS SCIENCE. NO. 11. SUMMER-FALL, 1984) N.G. COOPER AND G.B. WEST, EDS., P.3-21. CAMBRIDGE UNIVERSITY PRESS, 1988. (OC/793/.P358/1988)

ALSO PUBLISHED: LOS ALAMOS SCI., NO.11, SUMMER-FALL, P.4-19. 1994.

43-83

WHITE, D. HYWEL (MP-4).

INTERMEDIATE ENERGY NEUTRINO PHYSICS.

TIC. 1987. 31P. MN (LA-UR-88-0097)

43-84

WHITE, D. HYWEL (MP-4).

NEUTRINO-ELECTRON SCATTERING AT LAMPF-LARGE CHERENKOV DETECTOR EXPERIMENT.

TIC, 1988. ITP. MN (LA-UR-88-3886)

43-85

WHITE, D. HYWEL (MP-4).

SUMMARY OF NEUTRINO PRESENTATIONS

IN: CONFERENCE ON THE INTERSECTIONS BETWEEN PARTICLE AND NUCLEAR PHYSICS, 3RD. 1989. ROCKPORT, MAINE. PROC., P.230-40. AMERICAN INSTITUTE OF PHYSICS, 1988. (OC1 A38X NO.176) ALSO PUBLSHED: TIC, 1988. 12P. MN (LA-UR-88-1957)

43-66

WILSON, MAHLON T. (AT-DO).

SSC-AN ENGINEERING CHALLENGE.

IN: PARTICLE PHYSICS-A LOS ALAMOS PRIMER (UPDATED VERSION OF LOS ALAMOS SCIENCE. NO.

11. SUMMER-FALL, 1984) N.G. COOPER AND G.B WEST. EDS . P. 164-5 CAMBRIDGE UNIVERSITY

PRESS, 1988. (OC/793/.P358/1988)

ALSO PUBLSHED: LOS ALAMOS SCI., NO.11, SUMMER-FALL. P.158-9. 1984.

43-87

ZAKRZEMSKI, WOICIECH J. (T- 11 ).

LOW DIMENSIONAL SIGMA MODELS.

TIC, 1988. 16P. MN (LA-UR-88-3139) 
44-1

BENAGE, JOHN F., JA. (P-1); JONES, LARRY A. (P-1); KANIA, DON R. (P-1); BEGAY, FRED (P-4).

HEATING OF A DENSE PLASMA USING A RELATIVISTIC ELECTRON BEAM. J. APPL. PHYS., V.64, P.1748-52. 1988.

44.2

BOENIG, HEINPICH J. (CTR-9); GRIBBLE, ROBERT F. (CTR-4); HAMMER, CHARLES F. (CTR-4); HUDDLESTON, S. (CTR-4); KEWISH, RALPH W., JR. (CTR-6); KONKEL, H. E. (CTR-4):

MELTON, JIMMY G. (CTR-4); REASS, WILLIAM A. (CTR-4); ROSEV, B. J. (CTR-4); THULLEN, PHILIP (ERAMFE) PRELIMINARY DESIGN OF THE ENERGY SYSTEM FOR THE ZTH EXPERIMENT.

IN: SYMPOSIUM ON FUSION ENGINEERING, 12TH, 1987, MONTEREY, CALIF. PROC., P.812-15. IEEE. 1987. (TK9204.S93 1987X)

ALSO PUBLISHED: TIC, 1987. 5P. MN (LA-UR-87-3477)

44-3

FORMIAN, PETER A. (CTR-8); JAHODA, FRANZ C. (CTR-8); MILLER, GUTHAIE (CTA-2).

DETECTION OF PLASMA EQUILIBAIUM SHIFTS WITH FIBER-OPTIC SENSING OF IMAGE CURAENTS. REV. SCI. INSTRUM., V.59, P.1500-2. 1988

44-4

HAGENSON, F: HAMMEL, JAY E. (CTR-DO): KRAKOWSKI, ROBERT A. (CTR-12): MILLER, RONALD L (CTR-12): NEBEL, R. A. (CTR-6); SCUDDER, DAVID W. (CTR-DOT); WERLEY, KENNETH A. (CTR-12).

HIGH-DENSITY Z-PINCH (HDZP) AS A FUSION NEUTRON SOURCE

IN: SYMPCSIUM ON FUSION ENGINEERING, 12TH, 1987, MONTEREY, CALIF. PROC.. P.835-9. IEEE.

1987. (TK9204.S93 1987X)

44-5

JACOBSEN. F. M.; ANDERSEN, L. H.; DEUTCH, B. I , HVELPLUND, P : KNUDSEN, H.; CHARLTON. M.:

LAPIOCCHIA, G.; HOLZSCHEITER, MICHAEL H. (P.15).

ON ANTIHYDRCGEN PRODUCTION

IN: NATO ADVANCED WOAKSHOP ON ATOMIC PHYSICS WITH POSITAONS, 1987 LONDON. ENGLAND

PROC., F.333-4i. PLENUM PRESS, 1987. (OC/793 5/.P628/N375/1987)

44-6

JARBOE. THOMAS R. (CTR-5); BARNES, CHRISTOPHEA W (CTR-5). FERNANDEZ. JUAN C. (CTR-5):

HENINS. IVARS (CTR-5); HOIDA, HIROSHI W. (CTR-5), KNOX, STEPHEN O. (CTR-5):

MARKLIN, GEORGE J. (CTR-5): WRIGHT, BRADFORD L. (CTR-3)

INTRODUCTION TO THE SPHEROMAK AND THE CTX EXPERIMENT

IN: COURSE ANC \%RRKSHOP ON PHYSIC O O IMIRRORS, REVERSED FIELD PINCHES AND COMPACT TORI, 1987, VARENHA, ITALY PROC. P.59-?3 SOUIETA ITALIANA DI FISICA, 1988 (OC718.5.P45 P48 $1987 X$ )

44-?

MILLER, GUTHRIE (CTR-2); INGRAHAM, JOHN C. (CTR-2), SCHRANK, LOUIS S. (CTR-4). IMPROVED BOLCMETRY SYSTEM FOR REVERSED FIELD PINCH RESEARCH. REV. SCI. INSTRUN., V.59, 700-8. 1988

44-8

MILLFR, GUTHRIE (CTR-2).

MODELING OF REVEASED-FIELD-PINCH MAGNETIC PROBE MEASUREMENTS

PHYS. FLUIDS. V.31, P.3330-7. 1988

44.9

NOGAR, NICHOLAS S. (CLS-2); KEATON, GREGORY L. (CLS-2); ANDERSON, JOHNNY E. (CLS-2);

TAKULA, MITCHELL (MST-3).

OPTICAL DIAGNOSTICS FOR A HIGH POWER, RF-INDUCTIVELY COUPLED PLASMA

IN: SYMPOSILIM ON 'PROCESS DIAGNOSTICS' HELD AT THE SPRING MEETING OF THE MATERIALS RESEARCH SOCIETY, 1988, RENO, NEY. PROC., P.125-30. MATERIALS RESEARCH SOCIETY, 1988. (TA401 .M37X Y.117)

ALSO PUBLUSHED: TIC, 1988. 7P. MN (LA-UR-88-1025) 
PICKRELL, M. M. (CTR-2); REASS, WILLIAM A. (CTR-4).

PASSIVE EQUILIBRIUM STUDIES ON ZT-P. LOS ALAMOS, 1987. 16P. (LA-11175-MS)

44.11

REJ, DONALD J. (CTR-3).

DYNAMIC PROCESSES IN FIELD-REVERSED-CONFIGURATION COMPACT TOROIDS.

IN: COUASE AND WORKSHOP ON PHYSICS OF MIRRORS, REVERSED FIELD PINCHES AND COMMACT TORI, 1987, VARENNA, ITALY. PROC., P.499-518. SOCIETA ITALIANA DI FISICA, 1988. (OC718.5.P45 P48 $1987 X)$

ALSO PUBLISHED: TIC, 1987. 21P. MN (LA-UR-87-2854)

44-12

SCHOENBERG, KURT F. (CTR-2); INGRAHAM, JOHN C. (CTR-2); MUNSON, CARTER P. (CTR-2);

WEBER, PAUL G. (CTR-2); BAKER, DON A. (CTR-DO); GRIBBLE, ROBERT F. (CTR-4);

HOWELL, ROBERT B. (CTR-2); MILLER, GUTHAIE (CTR-2); REASS, WILLIAM A. (CTR-4);

SCHOFIELD, ALDRED E. (CTR-2); SHINOHARA, S.; WURDEN, GLEN A. (CTR-2).

OSCILLATING FIELD CURRENT DRIVE EXPERIMENTS IN A REVERSED FIELD PINCH.

PHYS. FLUIDS, V.31, P.2285-91. 1988.

44-13

TUSZEWSKI, MICHEL G. (CTR-3); BARNES, GARRET A. (CTR-3); CHRIEN, ROBEAT E. (CTR-3);

HUGRASS, WAHEED N.; REJ, DONALD J. (CTR-3); SIEMON, RICHARD E. (CTR-3):

WRIGHT, BRADFORD L. (CTR-3).

ORIGIN OF THE ROTATION IN FIELD-REVERSED CONFIGURATIONS.

PHYS. FLUIDS, V.31, P.946-8. 1988.

44-14

WURDEN, GLEN A. (CTR-2); WEBER, PAIL G. (CTR-8); WATT, ROBERT G. (P-4); MUNSON. CARTER P. (CTR-2');

CAYTON, THOMAS E. (CTR-6); BUCHL, K.

REFUELING AND DENSITY CONTROL IN THE ZT-4OMI REVERSED FIELO PINCH

IN: COURSE AND WORKSHOP ON PHYSICS OF MRRORS, REVERSED FIELD PINCHES AND COMPACT TORI, 1987, VARENNA. ITALY. PROC., P.411-30. SOCIETA ITALIANA DI FISICA, 1988. (OC718.5.P45 P48 1987X)

ALSO PUBLISHED: TIC, 1987. 22P. MN (LA-UR-87-2985)

44-15

WURDEN, GLEN A. (CTR-2); SCHOENBERG, KURT F. (CTR-2): PICKRELL, M. M. (CTR-2);

BAKEA, DON A. (CTR-DO); BUCHENAUER, C. JERALD (CTR-2): BURKHARDT. LOUIS C. (CTR-2):

CAYTON, THOMAS E. (CTR-6); CHRIEN, ROBERT E. (CTR-3); DOWVING, JAMES N., JR. (CTR-2):

ELUS, RICHARO F:; GRIBBLE, ROBERT F. (CTR-4); HABERSTICH, ALBERT (CTR-2);

HOWELL, ROBERT B. (CTR-2): INGRAHAM, JOHN C (CTR-2): MILLER, GUTHRIE (CTR-2):

MELTON, JIMMY G. (CTR-11); MOSES, RONALD W., JR. (CTR-6); MUNSON, CARTER P. (CTR-2):

NEBEL, R. A. (CTR-6); PHILLIPS, JAMES A. (CTR-DO); REASS, WILLIAM A. (CTR-4):

SCHOFIELD, ALDAED E. (CTR-2); WEBER, PAUL G. (CTR-2); WELDON, DAVID M. (CTR-2).

RFP EXPEAIMENTS-RESULTS FROM ZT-4OM AND ZT-P.

IN: COURSE AND WOAKSHOP ON PHYSICS OF MIRAORS, REVERSED FIELD PINCHES AND COMPACT TORI, 1987, VARENNA, ITALY. PROC., P.159-82. SOCIETA ITALIANA DI FISICA, 1988. (OC718.5.P45 P48 $1987 \mathrm{X})$ 
45-1

AKIMOTO, KAZUHIRO (ESS-8); GALVEZ, MIGUEL (ESS-8); GARY, S. PETER (ESS-8);

SGRO, ANTHONY G. (CTR-6); WINSKE, DAN $(X-1)$.

PRONPT STRUCTURING OF A PLASMA EXPANDING IN AN EXTERNAL MAGNETIC FIELD.

J. GEOMAGN. GEOELECTR., V.40, P.1161-91. 1988.

45-2

LING, KUOK M. (CTR-6); MOSES, RONALD W., JA. (CTR-6).

COMPARISON OF THE APERTURE AND CHROMATIC ABERAATIONS OF MAGNETIC ROUND LENSES WITH THOSE OF OUADRUPOLE DOUBLETS.

J. APPL. PHYS., V.64, P.1640-5. 1988.

45-3

MAYO, AOBERT M.: MARKLN, GEORGE J. (CTR-6).

NUMERICAL CALCULATION OF MERCIER BETA LIMITS IN SPHEROMAKS.

PHYS. FLUIDS, V.31, P.1812-5. 1988.

45-4

MILLER, GUTHRIE (CTR-2).

RESISTIVE EVOLUTION OF PLASMAS WITH IMPERFECT MAGNETIC SURFACES.

PHYS. FLUIOS, V.31, P.1133-41. 1988.

45-5

MOSES. RONALD W., JR. (CTR-6); SCHOENBERG, KURT F. (CTR-2); BAKER, DON A. (CTR-DO).

EMPIRICAL MODELING AND THE OEPENOENCE OF REVERSED FIELD PINCH LOOP VOLTAGE ON PLASM CONDITIONS.

PHYS. FLUIDS, V.31, P.3152-5. 1988.

45-6

SCHWARZMEIER, JAMES L (CTR-6): LEWIS, H. RALPH. JR. (CTR-6).

VARTIATIONAL NATURE OF DISPERSION EQUATIONS REVISITED.

J. MATH. PHYS., V.29, P.1486-97. 1988.

45-7

SIDIKMAN, K. L.: NEBEL, R. A. (CTR-6); CALLEN, J. D.

3-D MHD SIMULATIONS OF FIELD ERRORS IN ZTH AND ZT-4O

IN: COURSE AND WORKSHOP ON PHYSICS OF MIRRORS, REVERSED FIELD PINCHES AND CCNPACT TORI, 1987. VARENNA, ITALY. PROC., P.205.20. SOCIETA ITALIANA DI FISICA, 1988. (OC718.5.P45 P40 1987X)

45-8

TUSZEWSKI, MICHEL G. (CTR-3); BARNES, GARRET A. (CTR-3): CHRIEN, ROBERT E. (CTR-3);

HUGRASS, WAHEED N.; REJ. DONALD J. (CTR-3); SIEMON, RICHARD E. (CTR-3):

WRIGHT, BAADFCAD L. (CTR-3).

ORIGIN OF THE ROTATION IN FIELD-REVERSED CONFIGUAATIONS

PHYS. FLUIDS, V.31, P.946-8. 1988. 
46-1

O'BRIEN, HAROLD A. (P-3).

UTILIZATION OF AN INTENSE BEAM OF 800 MEV PROTONS TO PREPARE RADIONUCLDES.

TIC, 1988. 2OP. MN (LA-UR-88-3294)

46-2

RINEHART, GARY H. (MST-11).

LICHT WEIGHT RADIOISOTOPIC HEATER UNIT (LWRHU) PROINUCTION FOR THE GALILEO MISSION. LOS ALAMOS, 1988. 46P. (1A-11166-MS)

46-3

RINEHAPT, GAPY H. (MST-i 1): LATIMER, TRENT W. (MST-11).

MILIWATT GENERATOR PROJECT, APRIL 1983 - MARCH 1984.

LOS ALAMOS NATIONAL LABORATORY, 1988. 31P. (LA-11220-PR)

46-4

RINEHART, GARY H. (MST-11); LATIMER, TRENT W. (MST-11)

MULWATT GENERATOR PROJECT, APRIL 1984-MARCH 1986

LOS ALAMOS NATIONAL LABOAATORY, 1988. 27P. (LA-1 1346-PR)

46-5

RINEHART, GARY H. (MST-11); LATIMER, TRENT W. (MST-11)

MLIMATT GENERATOA PROJECT, APRIL 1982 - MARCH 1983.

LOS ALAMOS NATIONAL LABC ZATORY, 1988. 37P. (LA.11217.PR) 
47.1

BARLICH, GAIL (N-4).

RECEIPTS ASSAY FACILITY INSTRUMENT CONTROL FUNCTION USERS MANUAL.

LOS ALAMOS NATIONAL LABORATORY, 1988. 58P. (LA-11383-M)

47-2

BARLICH, GAIL (N-4).

TECHNIQUES FOR DEVELOPING RELIABLE AND FUNCTIONAL MATERIALS CONTROL AND ACCOUNTING SOFTWARE.

TIC, 1988. 4P. MN (LA-UR-88-1736)

47-3

BARNES, JAMES W. (N-4); YARBRO, STEPHEN L. (MST-12).

USE OF SAFEGUARDS DATA FOR PROCESS MONITORING IN THE ADVANCED TEST LINE FOA ACTINIDE SEPARATIONS.

TIC, 1987. 8P. MN (LA-UR-88-0102)

47-4

BEEDGEN, RAINER (N-4).

STATISTICAL NEAR-REAL-TIME ACCOUNTANCY PROCEDURES APPLIED TO AGNS MINIRUN DATA USING PROSA.

LOS ALAMOS, 1988. 40P. (LA-11196-MS)

47-5

BIERI, JOHN M. (N-2); CALDWELL, JOHN T. (N-2).

MOBILE NONDESTRUCTIVE EXAMINATION AND ASSAY INSTRUMENTS.

TIC, 1988. 7P. MN (LA-UR-88-3454)

47-6

BOSLER, Geralo E. (N-1); RINARD, PHILliP M. (N-1); KLOSTERBUER, SHIRLEY F. (N-1);

PAINTER, JO ANN (N-1).

AUTOMATED METHODS FOR REAL-TIME ANALYSIS OF SPENT-FUEL MEASUREMENT DATA.

TIC, 1988. 8P. MN (LA-UR-88-2044)

47-7

BRUNSON, G. S., JR. (N-2); COOP, K. L. (N-2).

NEUTAON MUULIPLICITY MEASUREMENTS DURING LINAC INTERAOGATION OF SAFEGUARDS ACCOUNTABLE MATERIAL.

LOS ALAMOS NATIONAL LABORATORY, 1988. 21P. (LA-11340-MS)

$47-8$

DOWDY, EDWARD J. (N-2).

USDOE MOBI: $:$ NON-DESTRUCTIVE ASSAY AND EXAMINATION SYSTEM.

TIC, 1988. 13P. MN (LA-UR-88-1465)

47-9

ERKKILA, BRUCE H. (OS-2).

SECURITY FEATURES OF A NUCLEAR MATERIAL ACCOUNTING SYSTEM.

TIC, 1988. 6P. MN (LA-UR-88-1892)

47-10

FEHLAU, PAUL E. (N-2).

RUGGED, LIGHTWEIGHT, AND LONG-OPERATING HAND-HELD INSTRUMENTS FOR NEUTRON AND GAMAMA-RAY VERIFICATION MEASUREMENTS.

TIC, 1988. 1OP. MN (LA-UR-B8-2780)

47-11

FEHLAU, PAUL E. ( $(\mathbf{\%}-2)$; WIIG, GARY.

STABILIZED, HAND-HELD, GAMMA-RAY VERIFICATION INSTRUMENT FOR SPECIAL NUCLEAR MATERIALS. TIC, 1988. 7P. MN (LA-UR-88-3673) 
47-12

GUTMACHER, R. G. (N-4); CREMERS, DAVID A. (CLS-4); WACHTER, JOSEPH R. (N-1).

CONTINUOUS MEASUREMENT OF URANIUM CONCENTRATIONS WITH THE LASER SPAAK.

TIC, 1987. 4P. MN (LA-UR-B8-0099)

47-13

HAKKILA, E. ARNOLD (N-4); GUTMACHER, R. G. (N-4); WEH, R.

SECOND US FRG WORKSHOP ON NEAR-REAL-TIN:E ACCOUNTING FOR REPROCESSING PLANTS. TIC. 1988. 5P. MN (LA-UR-88-1930)

47-14

HAKKILA, E. ARNOLD (N-4); BARNES, JAMES W. (N-4); HAFER, JOHN $r$. (N-4). USE OF PROCESS INFORMATION FOR VERIFICATION OF INVENTORY IN SOLVENT EXTRACTION CONTACTORS IN NEAR-REAL-TIME ACCOUNTING FOR REPROCESSING PLANTS.

TIC, 1988. 6P. MN (LA.UR-88-1991)

47-15

JARAMILLO, GENARA R. (N-DO).

BIBLIOGRAPHY FOR SAFEGUARDS, 1980-1984.

LOS ALAMOS NATIONAL LABORATORY, 1988. 37P. (LA-11275-MS)

47-16

KEEPIN, GEORGE ROBERT, JR. (N-DO/SG).

LOS ALAMOS SAFEGUARDS PROGRAM OVERVIEW AND NDA IN SAFEGUARDS.

TIC, 1988. 32P. MN (LA-UR-88-3190)

47-17

KOEHLER, P. E. (P-3); WENDER, STEVE A. (P-3); MOSS, CALVIN E. (N-2).

EFFICIENCY OF BARIUM FLUORIDE SCINTILLATORS FOR MONOENERGETIC GAMMA RAYS. NUCL. INSTRUM. METHODS PHYS. RES., V.A265, P.596-7. 1988.

47-18

KUNO, Y.; SHIGEOKA, K.; NISHIDA, K; IKEDA, H.; HAYASHI, N.; WACHI, I; HSUE, SIN-TAO (N-Y):

SPRINKLE, JAMES K., JR. (N-1); GUNNINK, R.; RUHTER, W. D.

FIELD TEST OF NEW TASTEX SYSTEM FOR PLUTONIUM PRODUCT VERIFICATION AT THE TOKAI REPROCESSING PLANT.

TIC. 1988. 10P. MN (LA-UR-88-2627)

47-19

LI, T. K. (N-9); SIMMONDS, STANLEY M. (N-1); ENSSLIN, NORBERT (N-1); COWDER, LEO ROSS (N-1); SPRINKLE, JAMES K., JR. (N-1); PARKER, JACK L. (N-1); HYKEL, MATTHEW N. (N-1);

BOURRET, STEVEN C. (N-1); GARCIA, DAVID L. (N-1); KRESGE, J. (N-1).

HIGH-PERFORMANCE, LOW-DENSITY WASTE ASSAY SYSTEM.

TIC, 1988. 7P. MN (LA-UR-88-2043)

47-20

LIKES, R. N.; PARKER, JACK L. (N-1); GOLDMAN, AARON (N-1).

PLUTONIUM-241 HALF-LIFE DETERMINATION BY HIGH-RESOLUTION GAMMA-RAY SPECTROMIETRIC MEASUREMENT OF PLUTONIUM-241/PLUTONIUM-239 RATIOS.

LOS ALAMOS, 1988. 66P. (LA-10979-MS)

47-21

MAC ARTHUR, DUNCAN W. (N-2); ADRIAN, STEVEN; MATTHEWS, $S$. WIRE CHAMBER ASSEMBLY TECHNIQUES.

LOS ALAMOS NATIONAL LABORATORY, 1988. 15P. (LA-11237-MS)

47-22

MARKIN, JACK T. (N-4).

ALLOCATION OF INSPECTION RESOURCES FOR INTERNATIONAL SAFEGUARDS.

TIC, 1987. 7P. MN (LA-UP-88-0078)

$47-23$

MENLOVE, HOWARD O. (N-1), SAPIR, JOSEPH L (N-1)

FEASIBILITY STUDY ON VERIFICATION METHODS FOR BULK QUANTITIES OF LOW-ENAICHMENT

URANIUM TRIOXIDE POWDER.

LOS ALAMOS NATIONAL LABORATORY, 1988. 33P. (LA-11360-MS) 
MENLOVE, HOWARD O. (N-1): STEWART, JAMES E. (N-1).

NEW METHOD OF CALIBRATION AND NORMALIZATION FOR NEUTRON DETECTOR FAMILIES.

LOS ALAMOS, 1988. 55P. (LA-11229-MS)

47-25

PARKER, JACK L. (N-1); BROOKS, M. L.

ACCURATE, WIDE-RANGE URANIUM ENAICHMENT MEASUREMENTS BY GAMMA-RAY SPECTROSCOPY-I. CHARACTERIZATION OF ENRICHMENT STANDARDS II. DEMONSTRATION OF ENRICHMENT MEASUAEMENT CAPABILITY.

LOS ALAMOS NATIONAL LABORATORY, 1988. 61P. (LA.11277-MS)

47-26

PICARD, RICHARD R. (A-1).

NOTES ON THE MUF-D STATISTIC

TIC, 1987. 11P. MN (LA-UR-88-0055)

47-27

PILLAY, K. K. S. (N-4).

HOLDUP-RELATED ISSUES IN SAFEGUARDING OF NUCLEAR MATERIALS.

TIC, 1988. 11P. MN (LA-UR-88-0424)

47-28

PILLAY, K. K. S. (N-4).

INTERNATIONAL SAFEGUARDS CONCERNS OF SPENT FLEL DISPOSAL PROGRAM.

TIC, 1988. SP. MN (LA-UR-88-1570)

$47-29$

PILLAY, K. K. S. (N-4); STIRPE, DANTE (N-4); PICARD, RICH,ARD R. (́A-1).

INTERNATIONAL SAFEGUARDS FOR AN AUTOMATED MKX FUEL FABRICATION FACILITY.

LOS ALAMOS NATIONAL LABORATORY, 1988. 81P. (LA-11219)

$47-30$

PILLAY, K. K. S. (N-4).

TRACER TECHNIOUES IN ESTIMATING NUCLEAR MATERIALS HOLDUP

J. RADIOANAL. NUCL. CHEM., V.124, P.313-29. 1988.

ALSO PUBLISHED: TIC, 1987. 20P. MN (LA-UR-87-0159)

47-31

PLASSMANN, EUGENE A. (N-2); SPRIGGS, GREGORY D. (N-2).

CRITICALITY ALARM SYSTEM VERIFICATION AT THE LOS ALAMOS CRITICAL EXPERIMENTS

FACILITY-PAST EXPERIENCE AND PRESENT CÁPABILITIES

TIC, 1988. 9P. MN (LA-UR-88-3730)

47-32

REILLY, T. DOUGLAS (N-1).

METHODS AND TECHNIOUES OF NDA (NONDESTRUCTIVE ASSAY)

TIC. 1988. 41P. MN (LA-UR-88-1077)

47-33

RINARD, PHILLIP M. (N-1); BOSLER, GERALD E. (N-1).

SAFEGUARDING LWR SPENT FUEL WITH THE FORK DETECTOR.

LCS ALAMOS, 1988. 88P. (LA-1 1096-MS)

47-34

RUSSO, P. A. (N-1); MENLOVE, HOWARO O. (N-1); FIFE. KEITH W. (MST-13); WEST, MIKE H. (MST-13);

MILLER, BARBARA L. (MST-13)

EVALUATION OF THE NEUTRON SELF-INTERROGATION APPROACH FOR ASSAY OF PLUTONIUM IN HIGH-ALPHA,N MATERIALS.

TIC, 1987. 13P. MN (LA-UR-87-4275)

47-35

SAMPSON, THOMAS E. (N-1); GOLDMAN, AARON (N-1).

ANALYSIS OF NDA INSTRUMENT CALIBRATION DATA.

LOS ALAMOS NATIONAL LABORATORY, 1988. 37P. (LA-11316-MS) 
SMITH, DARAYL B. (N-DOSG); JARAMILLO, GENARA R. (N-DO).

SAFEGUARDS AND SECURITY PROGRESS REPORT JANUARY-DECEMBER 1987.

LOS ALAMOS NATIONAL. LABORATORY, 1988. 128P. (LA-11356-PR)

47-37

SMITH, DARRYL B. (N-DOSG).

SAFEGUARDS AND SECURITY PROGRESS REPORT JANUARY-DECEMBER 1996

LOS ALAMOS, 1988. 130P. (LA-11120-PR)

47-38

SMITH, SUZANNE T. (N-4).

LAVA-AN EXPERT SYSTEM FRAMEWORK FOR RISK ANALYSIS.

TIC. 1988. 28P. MN (LA-UR-88-1385)

47-39

SMITH, SUZANNE T. (N-4): TISINGER, RICHARD M., JR. (N-4). MODELING RISK ASSESSMENT FOR NUCLEAR PROCESSING PLANTS WITH LAVA [LOS ALAMOS VULNERABILITY AND RISK ASSESSMENT].

TIC. 1988. 5P. MN (LA-UR-88-1929)

47-40

SPRINKLE, JAMES K., JR. (N-1); GOLDMAN, AARON S. (N-4); RUSSO, P. A. (N-1); STOVALL, LEONARD A. (Q-1); BRUMFIELD, T. L; GUNN, C.; WATSON, D.; BEEDGEN, RAINER (N-4).

CONFIRMATOPY MEASUREMENT TECHNIQUE FOR HEU [HIGHLY ENRICHED URANIUM].

TIC. 1987. 8P. MN (LA-UR-B7-3839)

$47-41$

TAPE. JAMES W. (N-4); COULTER, C. ALTON (N-4); MARKIN, JACK T. (N-4); THOMAS, K. E. (N-4).

INTEGRATED SAFEGUARDS AND FACILITY DESIGN AND OPERATIONS.

TIC, 1987. BP. MN (LA-UR-88-0079)

47-42

TISINGER, RICHARD M., JR. (N-4); BRUMFIELD, T. L.

SECURE TRANSMISSION OF SHIPPERIRECEIVER CONFIRMATORY MEASUREMENTS.

TIC, 1988. 4P. MN (LA-UR-BB-1994)

$47-43$

TISINGER, RICHARD M., JR. (N-4).

TESTBED FOR ADVANCED MATERIALS CONTROL AND ACCOUNTING CONCEPTS

TIC, 1988. 5P. MN (LA-UR-88-1993)

47-44

VACCARO, H. S. (N-4); GOLDMAN, AARON S. (N-4).

SPECIAL NUCLEAR MATERIAL INVENTORY SAMPLING PLANS.

TIC, 1987. 7P. MN (LA-UR-88-0094)

47-45

WHITTY, WILLIAM J. (N-4); MARKIN, JACK T. (N-4); STRITTMATTER, R. B. (N-4).

INFORMATION MANAGEMENT IN THE DESIGN OF MATERIALS CONTROL AND ACCOUNTABILITY SYSTENS.

TIC. 1988. 8P. MN (LA-UR-88-1992) 
49-1

MOORE, STANLEY $W .(Q-13)$

EXPOSURE TESTING OF SOLAR ABSORBER SURFACES.

IN: MATERIALS ANC OFTICS FOR SOLAR ENERGY CONVERSION AND ADVANCED LIGHTING TECHNOLOGY HELD AT THE, INTERNATIONAL SOCIETY OF PHOTOOPTICAL ENGINEERS SYMPOSIUM, 3RD, 1986, SAN DIEGO, CALIF. PROC.. P.126-35. SPIE, 1987.

ALSO PUBLISHED: TIC, 1986. 11P. MN (LA-UR-86-2845)

49-2

WRAY, WILLIAM O. (MEE-13); PECK, CLAUDIA (A-1)

PASSIVE SOIAR DESIGN PROCEDURES FCA NAVAL INSTALLATIONS

LOS ALAMOS NATIONAL LABORATORY, 1988. 195P. (LA.11250-M) 


\section{AUTHOR INDEX}

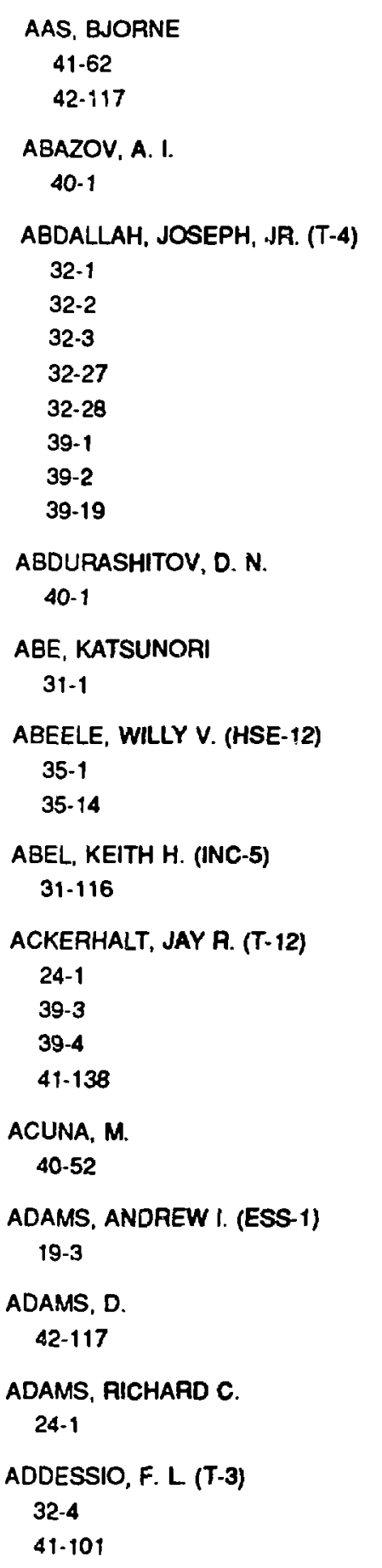

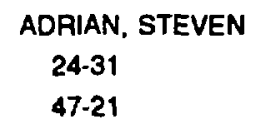

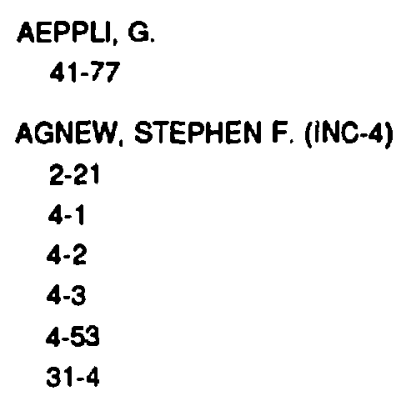

AIKIN, ROBERT M. (P-10) 41-211

AKIMOTO, KAZUHIRO (ESS-8) 40-2

40-3

40-66

45-1

AKST, IRVING B. (M-3)

$$
\text { 2-1 }
$$

ALAVI, B.

41-32

ALBERS, ROBEAT C. (T-11)

4-4

4-15

$31-10$

$39-80$

41-146

ALBRIGHT, JAMES N. (ESS-4)

20-1

38-1

38-2

$38-4$

38-5

38-6

ALCOUFFE, RAYMOND E. (X-6) 32-51

41-1
ALDISSI, MAHMOUD (MST-7)

4-3

4-53

4-62

31-4

ALDRICH, CHARLES $H$. 32-75

ALDAICH, CHARLES $H .(X-7)$ 41-104

ALESSI, VICTOR E.

17-1

ALEXANDER, $c$. 40-4

ALEXANDREAS, D. E. 40-26

ALI, M. HAGE 31-60

ALKAN, M. HAYAT

1-25

ALLEN, JANES $W$.

$$
\text { 4-54 }
$$

$39-53$

ALLEN, R. C.

40-26

40-63

43-1

ALLISON, PAUL W. (AT-2) $37-85$

37-88

ALLISON, S. W.

13-1

ALMAGUEA, JUAN ANTONIO 29-1

ALSTER, JONAS

41.5

42-104

ALTSEIMER ${ }_{2}$ JOHN H. (A-4)

$13-2$

21-1 
ALTSEIMER, JOHN H. (S-4)

10-1

39-3

AMANN, JAMES F. (MP-10) 39-64

AMATA, E.

40-16

40-51

AMATO, A.

31-78

AN, ZHI-GANG (CNLS)

32.5

ANAYA, JOSE MANUEL (P-3) 42-11

ANDER, MARK E. (ESS-3)

14-1

ANDERSEN, L $H$. 44-5

ANDERSON, ALAN N. (MP-7) $39-60$

ANDERSON, CHARLES A. (MEE-13) 16-7

ANDERSON, CHARLES A. (Q-13) 16-1

ANDERSON, GRAYDON K. (CLS-4) 41.149

ANDERSON, JAMES L. (MST-3)

29-2

29-3

29-4

$29-5$

30-1

$30-21$

ANDERSON, JOHNNYE. (CLS-2) 4.37

4-38

44-9

ANDERSON, JOSEPH M. 38-3

ANDERSON, M. P. 31-2

ANDERSON, R. R. 40-66

ANDERSON, $W$. J. 32-6
ANDERSON, WALLACE E. (MST-13) 4-70

ANDRE, S. V. 18-34

ANDREWS, ANDREW E. (A-6) 17-27

ANDREWS, GEORGE H., JR. (M-1) 2.25

ANKENY, LEE A. (C-3) 32-26

ANLAGE, S. M. 31-3

ANOSOV, O. P. 40-1

ANTONIO, ERNEST J. (HSE-12) 35-12

APEL, CHARLES T. (CHM-1) 4-5

APEL, CHARLES T. (CLS-1) 24-2

APEL, E. C. (CLS-5) 13-3

APGAR, STEWART A., III (AT-7) 37.63

APPERT, QUENTIN D. (CLS-8) 13-73

37-21

APT, KENNETH E. (CLS-DO) 17-2

ARAKAWA, E. T. 31.20

ARENDT, PAUL N. (MST-7) 31-92

ARGO, PAUL E. (ESS-7) 40-5

ARISAKA, $K$. 43-15

ARKO, AL J. (P-10)

4-6

31-57

$39-5$

$41-2$

ARMBRUSTMACHER, THEODORE J. 14-2
ARMELAGOS, GEORGE J 14f

ARMES, S. P. (MST-7) $31-4$

ARMSTRONG, ROBERT L 41-220

41-223

ARNDT, T.

4-7

41-3

ARNOLD, JAMES R.

40-65

ARONSON, MEIGAN C. (P-10)

4-14

39-6

39-7

ARTHUR, EDWARD D. (T-2)

42-1

42-30

42-38

ARTHIUR, EDWARD D. (T-DO)

18-37

42-2

42-3

$42-4$

42-13

42-15

42-124

ARTHURS, EUGENE

4-47

ASEM, ELIKPUIM $K$.

$$
\text { 1-37 }
$$

ASHERY, D.

39.8

ASPREY, LARNED B. (INC-4) 3-1

ASSMUS, $W$.

4-95

ATENCIO, L. G. (MP-10) 24-35

ATTREP, MOSES, JR. (INC-11) 3-2

14-35

$14-36$

AUCHAMFAUGH, GEORGE F (P-3) 42-101 


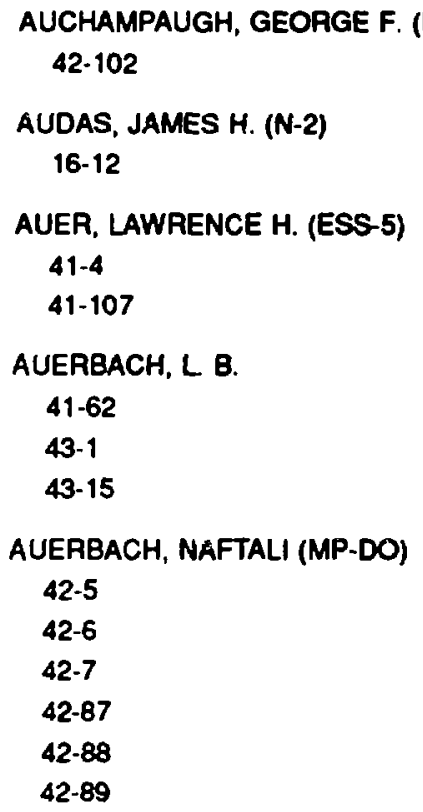

AVDEYEV, A. V. 40-1

AVEREACK, R. S. 31-73

AXELAOD, $\mathbf{S}$. 43-15

AXLER, KEITH M. (MST-5) 31-5

BAARS, RALPH E. (N-12) 27-6

BABCOCK, C. D., JA. (O-13) 16-5

BACKE. $H$.

4-7

41-3

BADALAMENTE-ALEXANDER, TIA M. 19-5

BAER, DONALD R. 31-6

BAER, H. W. (MP-4)

41-5

42-8

$42-56$

42-104

42-120

BAERISWYL, DIONYS

4-20

$39-9$

39-13
BAILEY, DAVID J. (OS-DO) 32-7

BAKER, C. 30-12

BAKER, DON A. (CTA-DO)

44-12

44-15

45-5

BAKER, GEORGE A., JR. (T-11)

$41-6$

41-7

41-8

BAKER, O. K. (MP-DO)

$39-60$

42-11

BALDIS, HECTER A. 23-6

BALDRIDGE. W. S. (ESS-1) 14-37

BALOWIN, GEORGE C. (P-3) 41-9

BALESTRINI, SILVIO J. (P-3) 42-75

BAME, SAMUEL J., JR. (ESS-8) 40-4

40-6

40-13

$40-41$

40-81

$40-83$

$40-92$

BANAR, JOSEPH C. (INC-7) 24-3

BARBOUR, J. C 31-73

BAREFIELD, J. E., II (CLS-4) 4-22

BARLETT, M. L. 39-64

BARLICH, GAIL (N-4)

47.1

47-2

BARNES. CHAISTOPHER $(x-1)$ 40-33

40-35
BAPNES, CHAISTOPHER W. (CTR-5) 29-23

$30-10$

$30-20$

32-75

446

BARNES, DANIEL C.

$29-27$

29-41

BAPNES, FAIPLEY J. (HSE-12) 35.12

BARNES, GARRET A. (CTR-3) 44-13

45-8

BARNES, JAMES $W$. (N-4)

47-3

47-14

BARNES, JOHN F. (T-DO) 41-10

BAPA, ALICE R. (HSE-8) 14-3

BAPA, SUMNER (ESS-7) 14-34

BARA, $W$. 30-12

BARAALOUGH, BAUCE L. (ESS-8) $13-6$

BARRON, MICHAEL H. (MEE-5) 24-4

BARTEL, JOHANN (T-9) 42-5

BARTH, $\mathbf{S}$ 39-10

BARTHOLDI, CATHERINE S. (CLS-9) 4-78 479

BARTHOLDI, MAPTY FRANK (LS-4) 1-7

BARTLET,, ROGER J. (P-14) $31-57$ 
BARTLIT, JOHN R. (MST-3)

29-2

29-3

29-5

29-9

29-35

30-1

30-2

30-15

30-21

BARTS, THERESE C. (AT-6)

37-11

37-12

BASS, WILLIAM M.

1.45

BASSALLEĆn, $B$.

41-62

BASTASZ, ROBERT J.

$29-44$

BASTIAN, NEIL $R$.

4-8

BATESON, M. CATHERINE 14-6

BATHKE, CHARLES G. (CTR-12) 29-6

$29-7$

29-42

29-43

$30-3$

$30-4$

$30-5$

30-19

BAUGCHUM, STEVEN L. 4-108

BAUMUNG, $K$.

27-1

BAYM, GORDON (T-DO)

40-31

BDZIL, JOHN B. (M-9)

$$
\text { 2-19 }
$$

BEATTY, $R$.

16-13

16-14

BEAVIS, $D$.

41-62

BECHTEL, S. E.

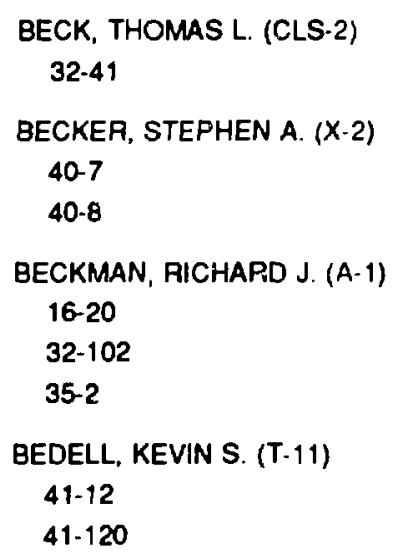

BENNETT, GLORIA A. (MEE-11)

20-2

24-6

24-7

BENNETT, JOEL G. (MEE-13)

$16-2$

16-3

16-7

29-19

32-58

BENNETT, JOEL G. (Q-13) 16-5

BENSON, MAURICE $W$. 32-8

$32-9$

BENTLEY, HAROLD $W$. 35-13

BENTON, DAVID

42-11

BERARDEZ, LEWIS

4-42

BERGANZA, JUAN

$14-9$

BERGER, MICHAEL E. (MEE-DO) 19-1

BERGMANN, ANTON G. (MP-13)

41-5

42-104

BERKBIGLEA, KATHAYN P. (C-10) 32-10

BERLIJN, J. J. (X-2)

42-119

BERNARDIN, MICHAEL P. (X-10) 41-15

BERNDT, M. E. 13-63

BERTHIER, JEAN 27-2

BERTIN, A.

32-60

BESNARD, DIDIER (CNLS) 41-16

BEST, ELAINE D. (MEE-13) 32-137

BESTIALE, $S$. 
$\underset{4-5}{\text { BEUGELSDIJK, TONY J. (MST-9) }}$

BEYER, WILLIAM A. (T-7)

41-119

BEZZERIDES, BANDEL B. $(X-1)$

41-180

BHANDARI, $P$.

16-13

16-14

BHATIA, TAROLCHAN S.

37-1

BIDDLE, RODNEY S. (AT-2)

37-118

BIEDENHARN, LAWRENCE C.

32-11

32-79

41.119

$41-155$

42-111

BIENIEWSKI, THOMAS M. (CHM-1) 13-30

BIERI, JOHN M. (N-2)

47.5

BIERY, K. A.

43-15

BIGIO, IRVING J. (CLS-5)

4-9

4-80

4-99

13-4

24.54

37-2

BILDSTEN, M. LARS

40.9

BILLEN, JAMES H. (AT-1)

13-61

$37-3$

$37-4$

37-5

37-82

BINDER, PHILIPPE M. (CNLS)

13-5

41-17

BINGAMAN, JEFF

17-1
BINNING. KIRK E. (MST-3)

30-6

$30-7$

BIRDSELL, STEVE (ESS-4)

21.5

BISH, DAVID L. (ESS-1)

4-10

14-44

14-47

31- 109

35-3

35-7

35-18

41-210

BISHOP, ALAN A. (T-11)

4-20

13-68

39-9

39-66

41-18

41-96

41-124

41-127

41-136

41-191

43-47

BISHOP, R. C.

29-27

29-41

BISOGNANO, J.

39-24

BITENSKY, MARK W. (LS-DO) $1-66$

BJORKLUND, ERIC A. (MP-1)

32-113

32-119

BLACHOT, JEAN

42-33

BLACIC, JAMES D. (ESS-3)

31-60

BLACK, J.P.

24-61

BLACK, JOEL KEVIN (MP-13)

43-1

BLAIR, LARAY S. (CLS-DO)

23-10

23-13
BLAIS, NORMAND C. (CHM-2) 2-2

BLAIS, NORIAND C. (CLS-2) 4-11

4-12

4-26

BLAISDELL, G. L. 14-4

BLAISE, JEAN

4-13

BLAKE, RICHARD L. (P-4) 24-23

BLAKE, RODGER D. (MST-4) 4-73

BLAKE, RODGER D. (MST-6) 13-22

31-51

BLANC, ELIZABETH 40-50

BLAU, P. 32-68

BLAU. STEVE (T-8) 41-19

BLESZYNSKI, $E$. 42-9

BLESZYNSKI, M. (MP-4) 42-9

BLILIE, C. L. 42-107

BLIND, BARBARA (AT-2)

37.24

37.54

41-100

BLONDIAUX, G. 31-60

BLOOMFIELD, $\mathrm{H}$.

16-13

16-14

BLUM, K. $39-27$

BLUMENAODER, $S$.

39-12

39-37

BOEKEMA, CAROLUS

41-47

$41-48$ 


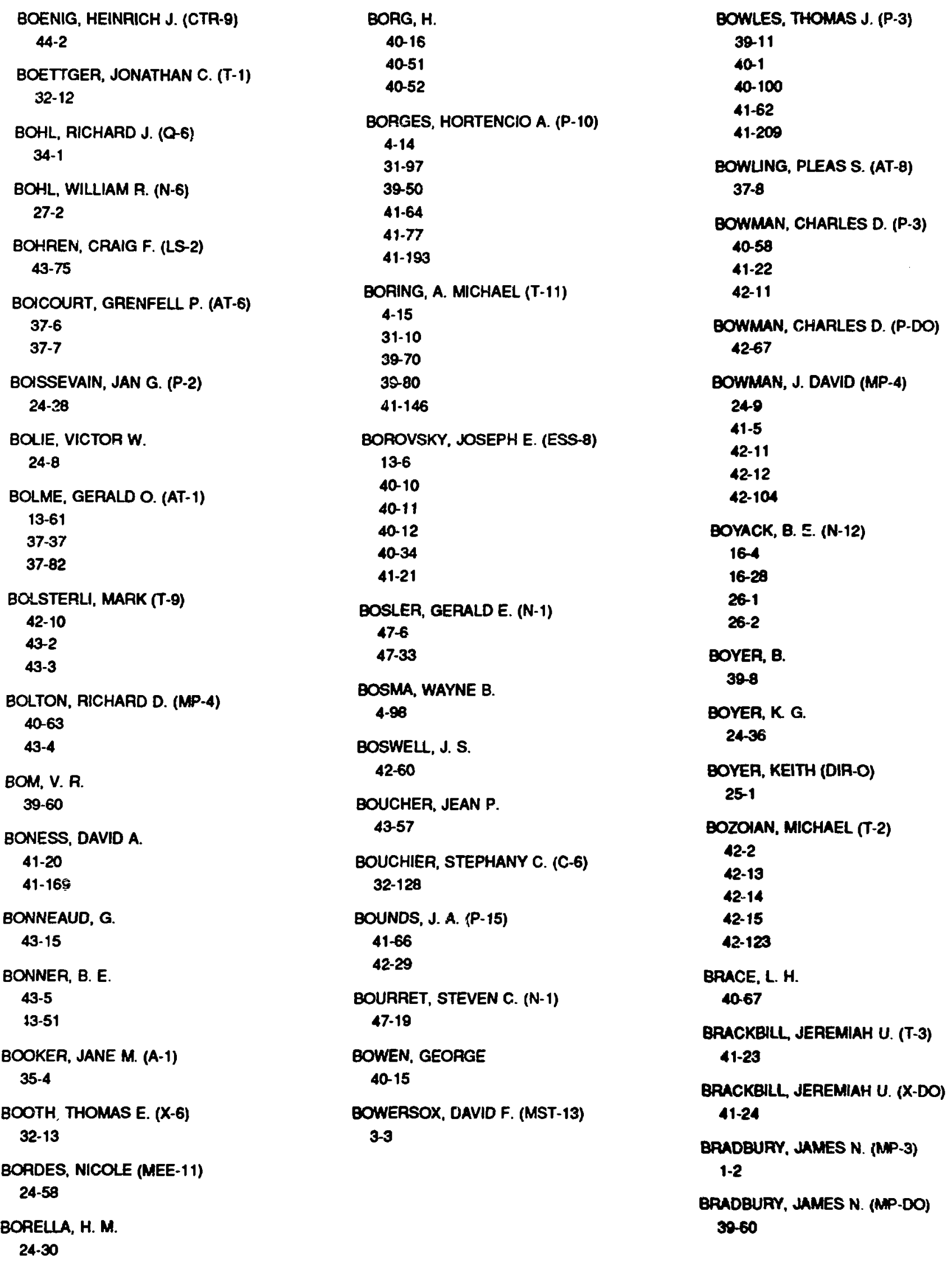




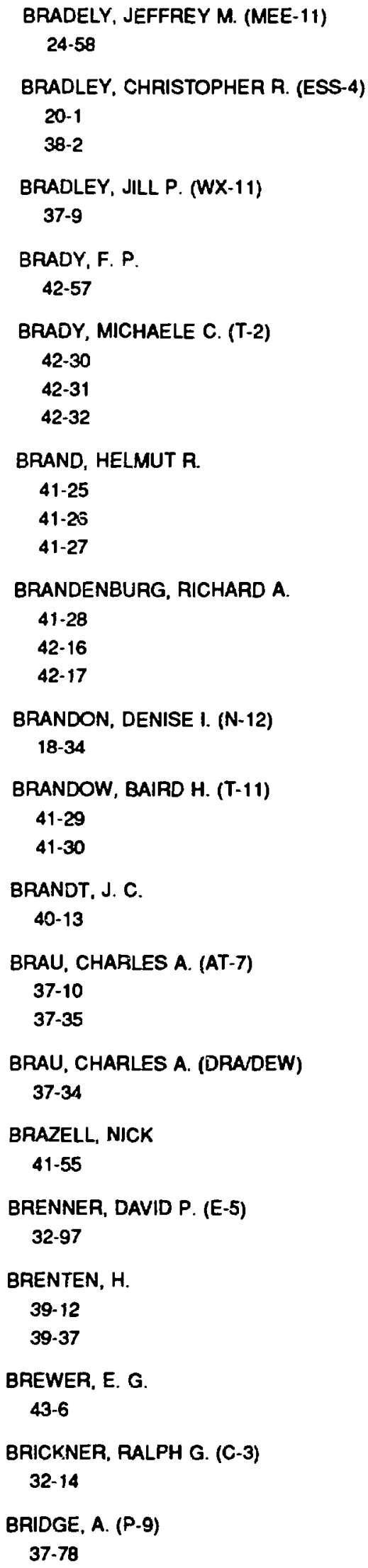

BRIESMEISTER, RICHARD A. (MST-11) 4-55

BRINCA, A. L. 40-93

BRISON, J. P. 41.31

BRIXNER, BERLYN (M-DO)

$24 \cdot 10$

24-11

BROHOLM, C. 41-77

BROOKS, F. D. 3960

BROOKS, J. 30-12

BROOKS, M. L. 47-25

BROSIUS, J. W. 40-13

BROWMAN, M. JEAN (AT-6) 32-35

$37-11$

$37-12$

$37-13$

$37-27$

BROWN, ALLEN R. (P-3) 41-62

BROWN, FRED (HSE-8) 14-3

BROWN, J. MICHAEL

$$
\text { 41-20 }
$$$$
\text { 41-169 }
$$

BROWN, JOSEPH A. (ESS-3) 14-4

BROWN, POBERT D. (MP-7)

31-12

31-113

37-60

41-211

43-6

BROWN, RONALD E. (P.3)

18-30

$41-62$

BROWN, STANLEY K. (AT-B) 37-8
BROWH, STUART E. (P-10) 3968

41-32

BROWN, WILBUR K. (P.15) 14.5

BROWNE, JOHN C. (ADDRA) 18-5

18-10

18-14

BROWNE, JOHN C. (ADR) 17-4

39-11

BRUCKNER, LAWRENCE A. (S-1) 32-15

BRUMFIELD. T. L

47-40

47.42

BRUNISH. WENDEE M. (ESS-5) 40-14 40-15

BRUNSON, G. S., JA. (N-2) 47-7

BRUSCHI, $M$. 39-60

BAYANT. D. A.

40-16

$40-51$

40-52

BRYANT, HOWARD C. 39-25

BUCHANAN, BRUCE G. 32-16

BUCHENAUER, C, JERALD (CTR-2) 44-15

BUCHER, ERNST 41-77

BUCHER, INGRID Y. (C-3) 32-17

BUCHHOL7, P. 43-15

BUCHL, K. 44-14

BUILTA, LEE A. (M-4)

24-47

$37-47$

37.56 


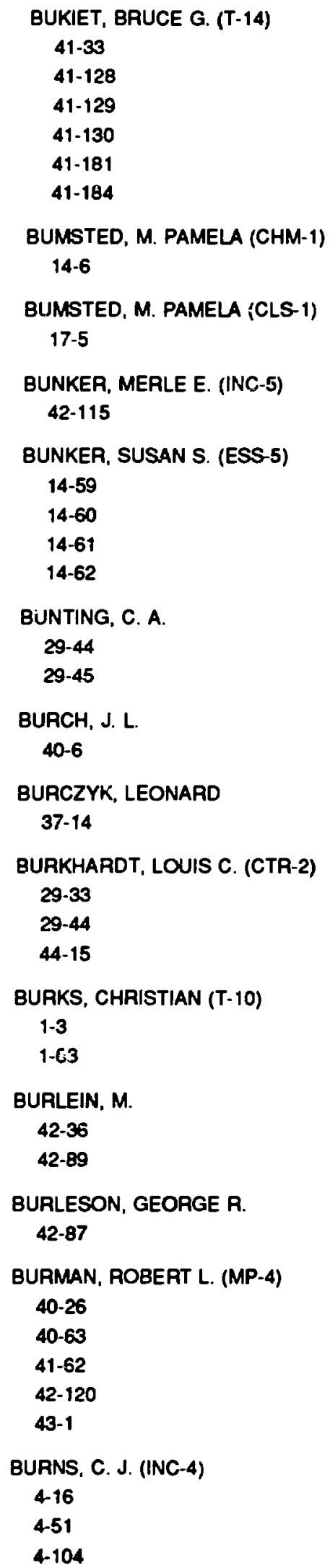

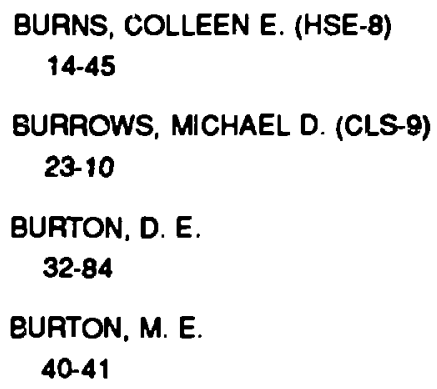

BYRD, R. C. (P-2) 41-166

BYRNES, LORIE L (MP-6) 32-109

CADWALADER, LEE C. 298

CADY, ROBERT (MP.14) 40-26

CADY, ROBERT LLOYO (MP-4) 40-63

CAFFREY, A. J. 39-60

CAGLIOSTRO, DOMINIC J. (M-4) 2-3

CAHN, JULIUS H. 40-21 40-22

CAIRD, ROBERT S., JR. (M-6) 13-23 $13-24$

CALDWELL, JOHN T. (N-2) 47-5

CALKINS, NOEL C. (WX-4) 13-64

CALLEN, J. D. 45-7

CALOVINI, T. A. 40-45

CAMERON, BEHNARO J. (MST-7) 31-82

CAMERON, R. F. 27-6

CAMP. J. (P-3)

4319

CAMPBEL, ARTHUR 24-58

CAMPBELL, ARTHUR $W$. (M-3) 2-4

CAMPBELL, BILLY M. (AT-4) 13-61 37-82 


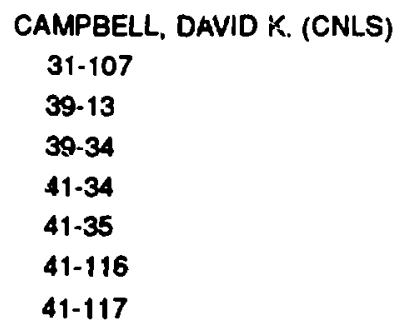

CAMPBELL, JOHN R. (5-6/C-3) 32-20

CAMPBELL, KATHERINE (A-1) 35-5

CAMPBELL, LAURENCE J. (T-11)
$32-21$
$39-14$
$41-36$
$43-42$

CAMPUZANO, J. C.

31-57

CANAVAN, GREGORY H. (P-DO) 18-1

18-2

18-3

18-4

18-5

$18-6$

18-7

18-8

18-9

18-10

18-11

18-12

18-13

18-14

41-37

CANDELARIA, LINDA M. (HSE-9) 14-49

CAPASSO, C.

$4-6$

41-2

CAPPIELLO, MICHAEL $W$. (N-6) 16-29

CAPPIELLO, MICHAEL W. (N-9) 16-17

CAPPON, JAMES $P$. 1-54

CARAMANA, EDWARD J. (CTR-6) 29.8
CARLINI, A. C. 43-1

CARLINI, ROGER D. (MP-14) 37-39

43-7

CARLINI, ROGER D. (MP-4) 40.63

CARLOS, ROBERT C. (ESS-7) 40-50

CARLSON, A. D. 42-75

CARLSON, DONALD E. (N-12) 16-13

16-14

CARLSON, JOSEPH A. (T-5)

32-22

42-18

42-19

43-8

CARLSON, RANDOLPH L. (M-4) 39-15

CARLSON, RICHARD V. (MST-3)

29-2

29-21

30-7

CARLSON, VANICE A. (LS-7) 1.62

CARLSTEN, BRUCE E. (AT-7)

37-15

37-16

37. 17

37-18

37-34

37-35

$37-43$

37.44

37-55

37-63

CAROLAR, P. G

29-44

29-45

CARPENTER, JOSEPH A., JR. 31.16

CARA, MICHAEL D.

14.7

CARRUTHERS, PETER A. (T.DO)

43-9

43-10
CARTER, DAVID H. (MST-6)

31-7

31-8

CARTER, KEITH D. (HSE-5) 22-8

CARTWRIGHT, DAVID C. (DRA/FLT) 23-3

23-10

39-16

39-48

CARTWRIGHT, DAVID C (DRP/FRA) $39-26$

$39-27$

$39-28$

CASEY, MARJORIE A. (MEE-9) 299

CASH, D. L. (MST-7)

31-115

CASH, DANIEL J. (ESS-3)

14-24

CASPERSON, DONALD E. (P-1)

13-31

41-115

CASTRO, RICHARD G. (MST-6) 31-9

CATES, MICHAEL REID

13-1

24-30

CAUSA, M. T. 39-17

CAYTON, THCMAS E. (CTR-6) 29-33

29.44

$29-45$

44-14

44-15

CEDERBERG, GAIL A. (ESS-5) 35-6

CHAMBERLAIN, R. M 32-23

CHAN, K. C. (AT-6)

13-B

$32-24$

37. 18

37-19

37-20

37.76 


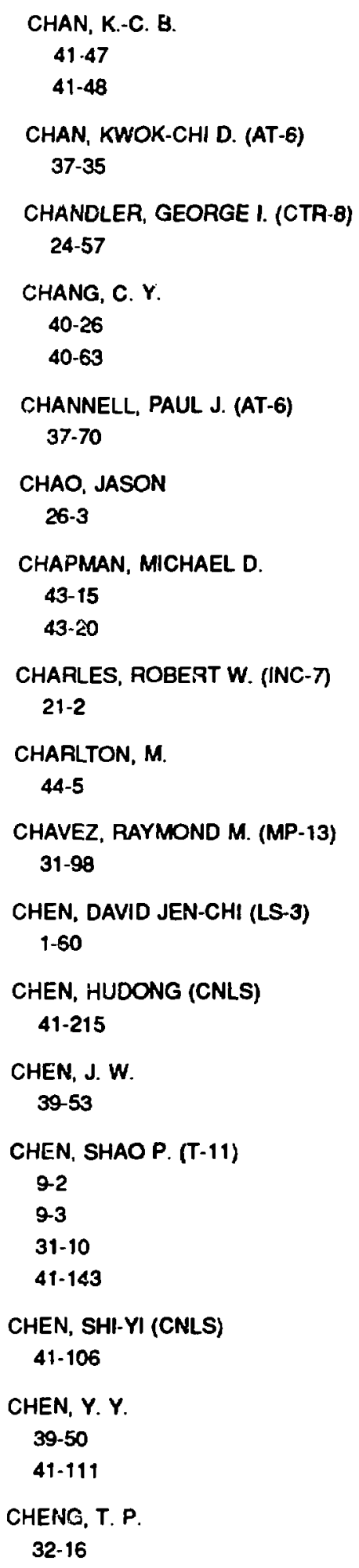

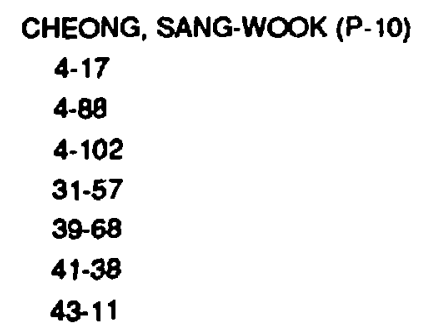

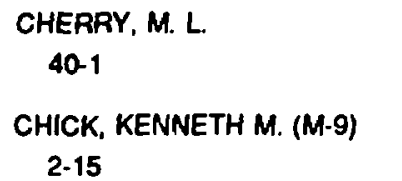

CHRISTENSEN, DANA CARL (MST-13) 3-3

CHRISTENSEN, KIRK ELDON (WX-4) 13-64

CHRISTENSEN N. E. 41-146

CHRISTENSON, K. K. 39-6

CHRISTIAN, JOHN M. (M-4) 2-16
CHRISTIAN, RICIIARD S. 41-142

CHRISTIANSEN, DAVID E. (MST-7) 6-1

CHRISTOPH, GAPY G. (C-B) 4-18

CHRISTOPH, GARY G. (P-8) 4-76

CHULICK, GARY S.

41-28

42-16

42-17

42-20

CHUNG, YUN C. (CLS-5)

$$
\text { 4-19 }
$$

24-12

24-49

41-41

CHUPP. T. 42-11

CILLIERS, W. A. 39-60

CIOSLOWSKI, JERZY (T-12) 32-25

39-18

41-42

CLARK, 8. 42-70

CLARK, DAYID A. (MP-5) 37-24

CLARK, DAVID A. (MP-6) 41-62

CLARK, OAYID A. (P.9) 39-25

CLARK, GARY L. (C-3) 32-26

CLARK, JOHN W. (T-11/CNLS) 1-5 41-175

CLARK, ROBERT EDWARD HOLMES $(X-$ 32.1 32-2 32-3 $32-27$ $32-28$ 


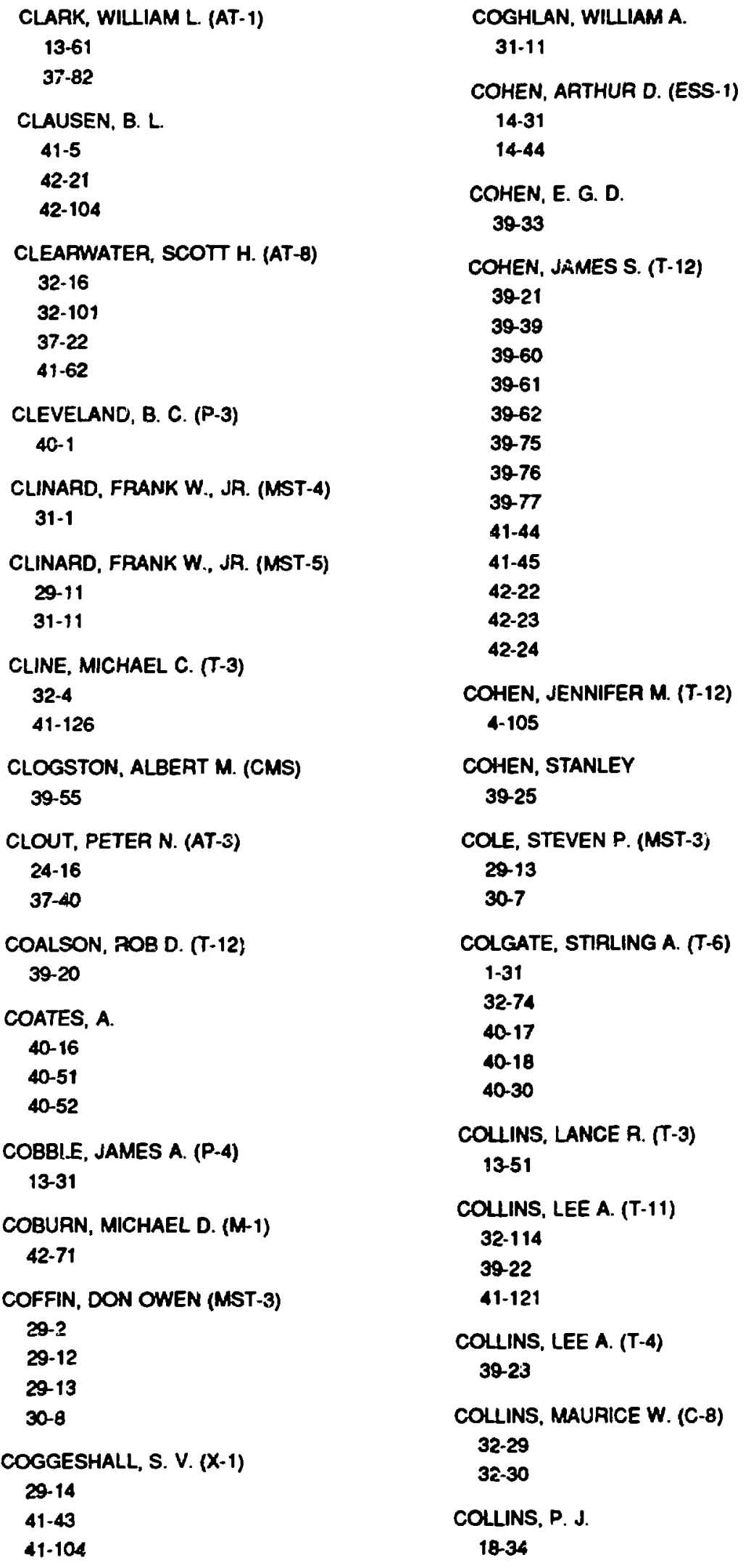

COLTON, EUGENE P. (MP-14)

37-23

$27-24$

$37-25$

39-24

41-46

COMFORT, J. R.

41-5

42-104

42-120

COMTET, $\mathrm{G}$ 39-25

CONAWAY, JOHN G. (ESS-3)

$14-8$

20-3

CONLEY, ANDREW P. (AT-3)

24-16

37-40

CONNER, JERAY P. (ESS-DO) 40-32

CONRADSON, STEVEN D. (INC-4)

4-20

4-21

$31-57$

COOK, RICHARD J. 41-130

COOK, TAVONI K. $14-58$

COOK, WLLIAM A. (WX-4) 13-13

32-31

$32-32$

32-33

COOKE, D. W. (NP-14)

24-13

41-47

$41-48$

41-211

COON, SIDNEY A.

42-41

COOP, K. L. (N-2)

$47-7$

COOPER, FREDERICK A. (T-B) $32-34$

42.12

43-13

COOPER, MANET L. (LS-A)

$1-6$ 


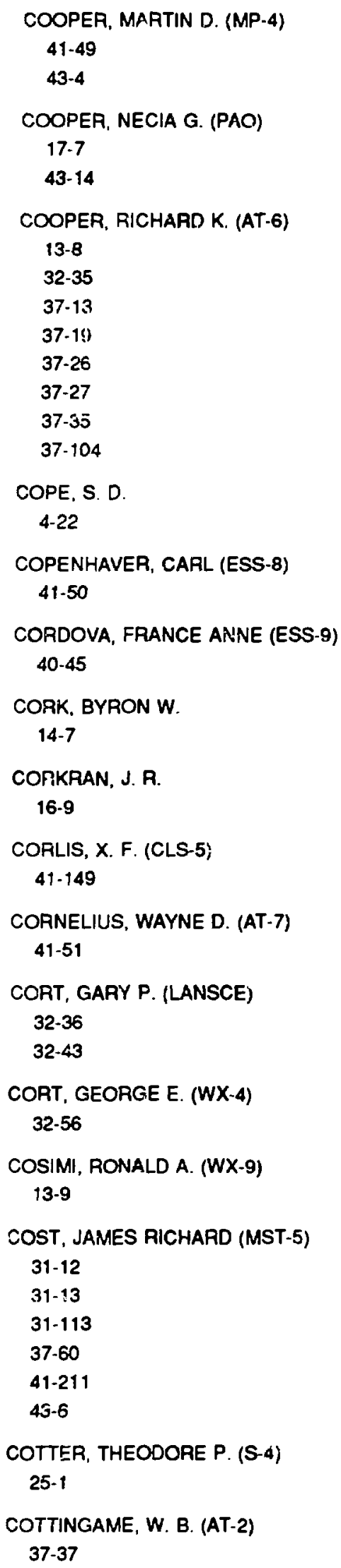

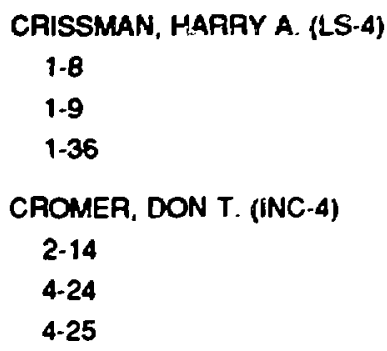

CROWE, BRIJCE M. (INC-?) 35-2

CROWLEY, W. P. 32-84

CRUDDACE, R. G. 40-54

CRUTCHER, J. K. (M-4) 37-56

CAUZ, JAMES M. (IS-12) $17-9$

CSANAK, G. Y. (T-4)

$32-28$

$39-16$

39.19

$39-26$

$39-27$

CSANAK, GEORGE (T-4) 39-28

CSERNAI, L. P. 42-25

CUL:INGFORD, HATICE S. 13-12

CUMMINGS, CHARLES E. (WX-4) 13-19 13-64

CUNDY, TERAANCE W $14-48$

CUNNINGHAM, PAUL T. (CLS-1) 4-78 4. 79

CURTIS, ELIZABETH H. (LS.7) $\uparrow .62$ 
CUSTER, DAN!EL M. (MEC-7)
30-22

CVERNA, F. (P-15)

24-37

CYRUS, TAIT

32-108

D'ANNA, JOSEPH A. (LS-3)

1-10

D'HUMIERES, DOMINIQUE

41-17

DAHLBY, JOEL W. (CLS-1)

4-28

DALESIO, LEO R. (AT-8)

37-14

DALICHAOUCH, Y.

39-53

DALLINGER, RICHARD F. 4.76

DALLMAN, JOHN C. (M-9) 2-5

41-200

DALY, BART $\$$. (T-3)

41-52

DALY, P. W.

40-81

DAMIANO, FORTUNATO A. (MST-3) 29-2

DANSHIN, S. N.

40-1

DASH, ZORA V. (ESS-4)

21-6

35-20

DATTA, $S$.

41-62

DAUELSBERG, LAWRENCE B. (AT-4) 37-59

37-61

DAVID, D. (CNLS)

41.53

DAVID, SHELLE J. (MST-11)

4-29

4.55

DAVIDSON, D. R. (MP-3)

42-25

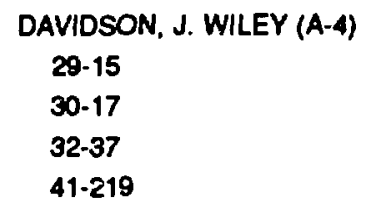

DAVIDSON, RICH FRANK (WX-4)

13-13

13-19

DAVIERWALLA, D. M.

30-17

DAVIES, G. D.

39.60

DAVIS, ALVIN H. (MST-9)

17-6

19-15

DAVIS, CECIL G. (P-15)

32-38

41-54

DAVIS, HAROLD A. (P-1)

41-109

43-45

DAVIS, K. J.

37-65

DAVIS, WILLIAM C. (M-3)

2-6

2-7

DAVIS, WILLIAM C. (M-9) 24-60

DAY, D. E.

13-22

DAY, GORDON W.

24-57

DE BRUIN, DERIK

1-60

DE HAAN, $H$.

39-60

DE KINDER, R. E., JR.

37-41

DE LONG, LANCE E.

31-20

DE PAOLO, D. J.

14-37

DEAN, SUMNER H. $(X-7)$

14.58

DEAVEN, LAAPY L. (LS-4)
DEBAUN, J. L

$31-60$

DEETER, MCHEL

13-20

DEHNHARD, DIETRICH

42-107

DEININGER, $w$.

16-13

16-14

DEL GIUDICE, $M$.

4-6

$39-5$

41-2

DELENE: JERAY G.

30-13

$30-14$

DELHAIZE, EMMANUEL (LS-3) $1-26$

DELHEIJ, PETER P. J. (P-3)

42-11

DELVIN, $W . L$

32-15

DEMSKE, D.

\$1.55

DENNIS, BERT R. (ESS-11)

14.9

DEROUIN, CHARLES R. (MEE-11)

9-8

12-3

$12-4$

DETHIER, DAVID P. (ESS-1)

14-20

DETHLOFF, L. A. (LS-1)

1-21

$1-22$

DEUPREE, ROBERT G. (ESS-5)

$14-10$

$19-2$

40-24

DEUTCH, B. I.

445

DEVANEY, MAPJORIE A. (C-8) 32-30 


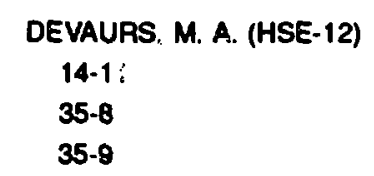

DEVENPORT, JAMES C. (BPO) 13-61

DEY, TOM N. (ESS-5) 14-57

DHUGA, KALVIR S.
$42-36$
$42-87$
$42-92$

DI BONA, P. J. 2-16

DI MARCO. JOSEPH N. (CTR-DO) 29-16

29-33

DIANOUX, ALBERT J. 4-30 41-70

DICK, CHI-M (CMS) 4-102

DICK, JERRY JOEL (M-9)

2-8 41-56

DIEKERT, GABAIELE $4-8$
DIETERLE, $B$. 41-62
43-1

DIMOPOULOS, SAYAS 42-27

43-16

DINEGAR, ROBERT H. (M-T) 13-32

DINER, RICHARD

14-35

14-36

DINGLER, ROBERT D. (AT-8) 37.14

DINGUS, $B$. 40-63

DINGUS, BRENDA L.

40-25

40-26
DINGUS, RONALD S. (P-DO) 41-57

DIRKS, RCBERT 14-6

DIXON, RAY D. (MST-6)

$13-14$

$13-49$

31-56

32-95

DOERING, CHARLES R. (CNLS) 32-39

DOERING, CHARLES R. (T-7) 32-40

DOHERTY, ROGER D.

31.80

31.81

DOLL, JIMMIE D. (CLS-2)

$32-41$

$32-42$

41-58

41-59

DOMBECK, THOMAS W. (P-3)

37-29

41-60

41-61

41-62

43-17

DONAHUE, DOUGLAS J.

40-28

DONAHUE, JOEY B. (MP-7)

$39-25$

43-1

DONAHUE, STEVE (LANSCE) $32-43$

DONALDSON, $\mathbf{W} . \mathbf{R}$. 13-4

DOOLEN, GARY D. (ADDR) 32-5

DORIA, MAURO M. (CNLS)

41.25

41-27

41-36

41-63

41-64

43-18

DOSS, JAMES D. (MP-3)

24-13
DOSS. K. G. R. 42-61

DOTSON, PAUL J. (0-9) 16-17

DOVE, RICHARD C (MEE-13)

16-2

16-3

16-7

24-14

DOWDY. EDWARD J. (N-2) 47-8

DOWELL, ILONNIE (T-1)

$39-29$

$39-30$

$39-31$

3932

DOWNEY, STEPHEN $W$. 24-47

DOWNING, JAMES N., JR. (CTR-2) 29-33

29-44

44-15

DRAGT, ALEX JAMES 41-142

DRAKE, DARRELL M. (ESS-8) 18-30

40-27

42-28

DRANSFIELD, G. D. (MEE-12) 30-22

DREICER, HARRY (CTR-DO) $29-17$ $30-9$

DREICER, JARED $S$. (A-5) 18-16 $32-44$ $32-94$

DRENNON. BARRY J. (HSE-12) 35-1

DROPESKY, BRUCE J. (INC- 1 1) 42-119

DROSG, MANFAED (P-3) 42-28

DAUMMOND, J. R. 42.57

DUBACH, JOHN FRASER (T-5) 43-72 


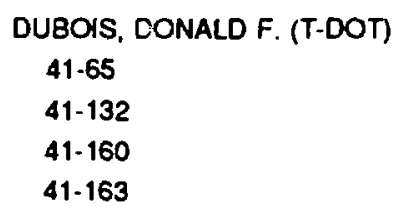

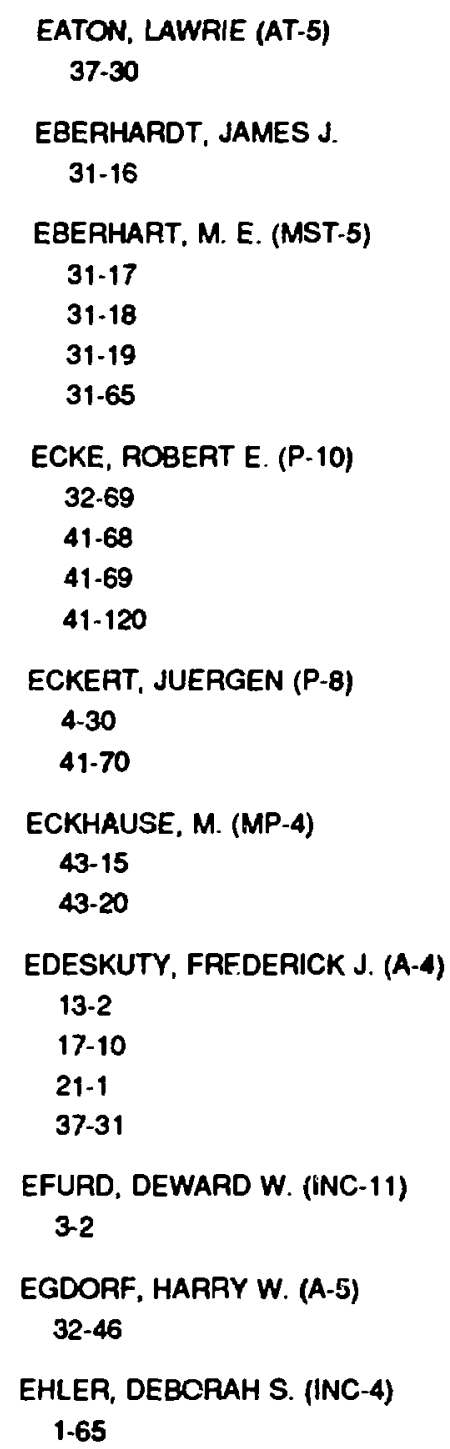

26-3

EICHELBERGEA, $\mathrm{J}$. 14-22
ELER, P. GARY (INC-4)

3-1

4.7

4-18

4-31

$4-41$

460

494

41-3

ELLOTT. C. JAMES $(X-1)$

13-75

37-42

37-44

ELЮTT, JIMMY C. (M-4) 37-56

ELLNTT, NORMAN E. (MST-7) 31-48

31-49

ELIOTT, STEVEN R. (P-3) 40-1

ELIS, AICHARD F. 29-33

4415

ELLIS, WALTON P. (CLS-2) 4-54

ELSWORTH, R. W. 40-26 40-63

ELMORE, DAVID

35-13

40-65

ELPHIC, R. C. 40-40

ELPHINSTONE, R. D. $40-49$

ELSTER, CHARLOTTE 42-20

ELSTON, WOLFGANG E. 14-12

EMBUFY, J. D. (MST-DO) $31-80$ 31-81

ENGE, H. A. 24-36

ENGELKE, RAY P. (M-9) 4-32 41.71 
ENGLAND, J. B. A. 39-60
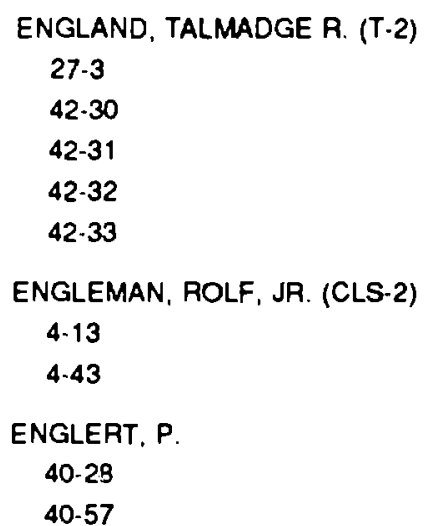

ENSSLIN, NORBERT (N-1) 47-19

ENVIRONMENTAL SURVEILLANCE GROUP (HSE-8)

22-1

EPSTEIN, RICHARD I. (ESS-9)
$\begin{aligned} & 40-29 \\ & 40-30 \\ & 40-31 \\ & 40-32\end{aligned}$

ERICKSON, GEORGE F. (CLS-6) 4-33

31-21

ERKKILA, BRUCE H. (OS-2) 47.9

EROSHKINA, L. A. 40-1

ERPENBECK. JEROME J. (T-11) 39-33

41.72

ESSINGTON, EDWARD H. (HSE- 12) 35-11

ESTEP, R. J. (INC-11)

42-36

ESTES, GUY P. $(X-6)$

4234

ESTLER, R. C.

4-34

4-84

4-85

4-93
ETTERS, RICHARD D. (M-6)

41-13

41.113

EVANS, CHRIS

13-15

EVANS, FOSTER (X-7) 42-116

EWELL, R. 16-13

$16-14$

EYKHOLT, RICHARD E. (T-11) 41-182

FABER, VANCE (C-3)

29-30

$32-47$

32.48

41-73

FAINSTEIN, $C$. 39-17

FAIRBANK, WILLIAM M., JR. 4-35

FAIRBANKS, TOM 14-13

FAIRES, LYNDA M. (CHM-1) 4-5

FAIZOV, E. L. 40-1

FARBER, ROBERT $M .(T-10)$ $1-30$

$32-72$

32-73

41-110

FARLEY, W. EDWIN 41.55

FARMER, J. DOYNE (CNLS)

$1-13$

1.14

FARMER, J. DOYNE (CNLST-7) 32-49

41.74

FARNHAM, T. 41-126

FARRAR, CHARLES R. (MEE-13) $16 \cdot 2$

16-3

16-7

24-14
FAFRELL, JOSEPH N. (P.10) 41-100

FAUCET, JOHN A. 42-87

FAUX, D. A. $42-58$

FAWCETT. LOUIS RAYMOND. JR. (X $42-35$

FAZELY, ALS

42-35

FAZ1O, MICHAEL V. (AT-5)

37-32

37-33

FEAREY, BRYAN L. (INC-7)

4-35

$4-36$

$4-37$

$4-38$

$4-39$

$4-66$

4-74

$4-85$

41-75

FEBER, ROY C., JA. (MST-3) 13-16

FEE, JAMES A. (INC-4)

1-15

$1-43$

4-112

fEHLAU, PAUL E. (N-2)

47.10

47-11

FEHLER, MICHAEL C. (ESS-4) 14-13

21-5

FEIN. RICHARD $\mathbf{S}$. 32.6

FELOMAN, DONALD W. (AT-7)

13-74

$37-34$

37.35

$37-63$

37.66

FELDMAN, R. B. (AT-6)

$37-35$

$37-63$

37-66 


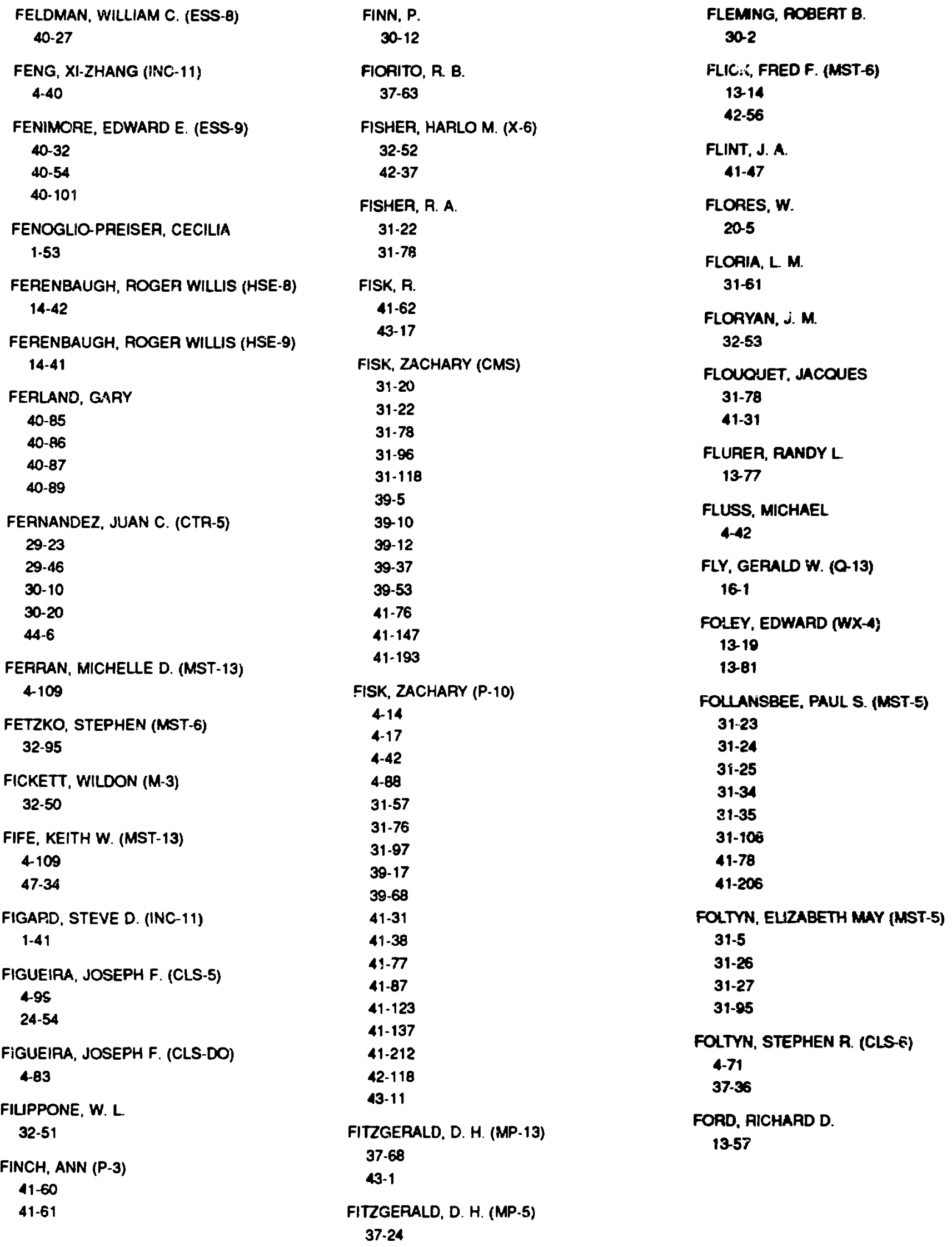


FOREMAN, LARRY R. (MST-7)

23-2

23-7

31.38

31-110

FOREMAN, WILL M. (MP-6)

43-1

FOREST, CHARLES A. (M-1)

2-23

FOREST, CHARLES A. (M!9) 2-8

FOREST, M. G.

41-11

FORMAN, PETER R. (CTR-8)

13-17

29-18

30-11

44-3

FORMISANO, $V$.

40-16

40-51

40-52

FORNWALL, MARK

32-105

FORSLUND, DAVIO W. (X-DO)

23-5

FORTGANG, CLIFFORD M. (AT-2) 37-37

FORTUNE, H. T.

42-87

42-88

42-89

42-92

42-93

42-107

FOSTER, D. GRAHAM, JF. (T-2) 42-38

FOWLER, CLARENCE M. (M-6)

13-18

13-19

13-24

13-8!

FOWLER, ERIC B. (LS-6)

35-10

FOWLER, JOHN D. (X-7) 32-54
FOWLER, MALCOLM M. (INC-11)

$$
\text { 4-7 }
$$

41.3

FRANEY, M. A.

42-107

FRANK, JAMES S. (MP-4)

43-4

43-15

43-20

FRANK, JOSEPH A. (ADIII)

20-5

FRANK, JOSEPH A.

17-11

FRANK, L. A.

40-48

40-49

FRANKE, PAUL R., JR. (ESS-DOT)

21-3

FRANTZ, CHARLES E. (MST-5)

31-122

FRASER, JOHN S. (AT-6)

37-45

FRASER, JOHN S. (AT-7)

37.20

37-34

ง7-35

37-38

FRATI, $W$.

43-1

FREDERICKSON, PAUL O. (C-3)

32-8

$32-9$

32-23

32-55

FREEMAN, DAVIO L

32-41

$32-42$

41-58

41-59

FREUND, $D$.

27-6

FREYER, JAMES P. (LS-1)

1-16

FREYER, JAMES P. (LS-4)

1.17
FRIAR, JAMES L. (T-5)

41-79

42-19

42-39

42-40

42-41

42-42

42-43

42-72

43-21

FPIEDRICHS, CARL C (AT-5) 37-13

FRIEDRICHS, CARL C. (AT-6) 37-39

FRIEDRICHS, P. B. (AT-1) 37-11

FRIES, J.

32-68

FAITZ, G.

40-54

FRITZ, JOSEPH N. (M-6) 24-15

FRY, HERBERT A. (CLS-3) 4-22

FRYDRYCH, DANIEL J. $31-6$

FUENTES, A. 39-8

FUENTES, H. R. (HSE-12) 35-11

FUGELSO, LEIF ERIK (MEE-13) 13-20

FUJII, $M$.

40-32

FUNISAWA, $F$. 42.117

FUJITA, R. K. (WX-4)

2-3

32-56

FUJITA, T

16-13

16-14

FUKA, MARY A. (E-8) 24-16

$37-40$ 


\section{FUKUSHIMA, EIICHI (INC-4) 13-21 \\ FULTON, ROBERT D. (P-1)
$43-45$}

FUNG, S. Y.

$41-62$

43-1

FUSELIER, STEPHEN A.

40-92

GAC, FRANK D. (MST-4)

13-22

GAFFNEY, E. S.

14-4

GAFFNEY, JEFFREY S. (INC- 7 )

4-65

$4-66$

$9-1$

14-14

GALLAGHER, J. $S$.

40-85

40-87

40-69

GALLACHER, T.

24-4 GALLEGOS, DAN (MP.B)
$42-56$

GALLIMORE, DAVID L. (CHM-1) 4-5

GALLIMORE, DAVID L. (CLS-1) 24-2

GALVEZ, MIGUEL (ESS-8) 40-33

40-34

40-35

45-1

GALVIN, A. B.

40-49

GAMMEL, JAN T. (T-11)

39-34

$41-34$

41-35

$41-60$

GANGULY, S.

40-101

GAFICIA, ANGEL E. (T-10)

1-18

$1-63$

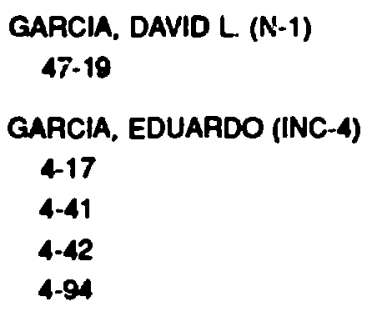

GARCIA, SAMMY R. (INC-5) 14-50

GARCIA-RECIO, C. 42-94

GARDNER, JAMIE N. (ESS-1)

14-18

14.55

21-4

33-2

GARNETT, R. $W$. 42-36

GARRITY, PATRICK J. (CNSS)

17-12

18-17

18-18

$18-19$

18-20

GARVEY, G. T. (MP-DO)
$42-44$

GARY, S. PETER (ESS-8)

$40-33$

$40-36$

40-37

45-1

GARZON, F. H. (MEE-11) 41-81

GAUTIER, MARGARET A. (HSE-9) 14-15

22-2

GAVRIN, Y. N. 40-1

GAVRON, AVIGDOR I. (P.2) 24-17

GAYDA, JOHN

41-183

GAYEVSKY, V. I.

40-1

GAZZALY, MAGDI M. 42-117

\author{
GEBHARDT, FRED \\ 37-41 \\ GEHRZ, ROBERT D. \\ $40-85$ \\ $40-86$ \\ 40-87 \\ $40-80$ \\ GEISIK, CARL (A;-2) \\ 24-18 \\ GEISSLER, $K$. \\ 13-4 \\ GEISSMAN, JOHN W. \\ 14-16 \\ GEIST, KARLHEINZ (CNLS) \\ 1.67 \\ GELB, JAMES M. (T-DO) \\ $40-70$ \\ GENIN, JCSEPH \\ 32-31 \\ 32-33 \\ GENIN, KENT E. (T-3) \\ 17-25
}

GEOFFRION, R. RONALD (MST-9) $17-6$

GEORGE, DENISE C. (T-2)

16-8

27-3

32-57

$42-1$

42-15

GEOREE, M. J. (M-4)

13-70

24-10

GEORCE, $\mathbf{S}$.

$$
\text { 4-43 }
$$

GEOAGE. TIMOTHY G. (MST-5)

31-28

31-29

34-2

343

GEOAGE, MLLAM E. (CLS-1)

4-20

GERE, SCOT B. (ACTO)

17-13 


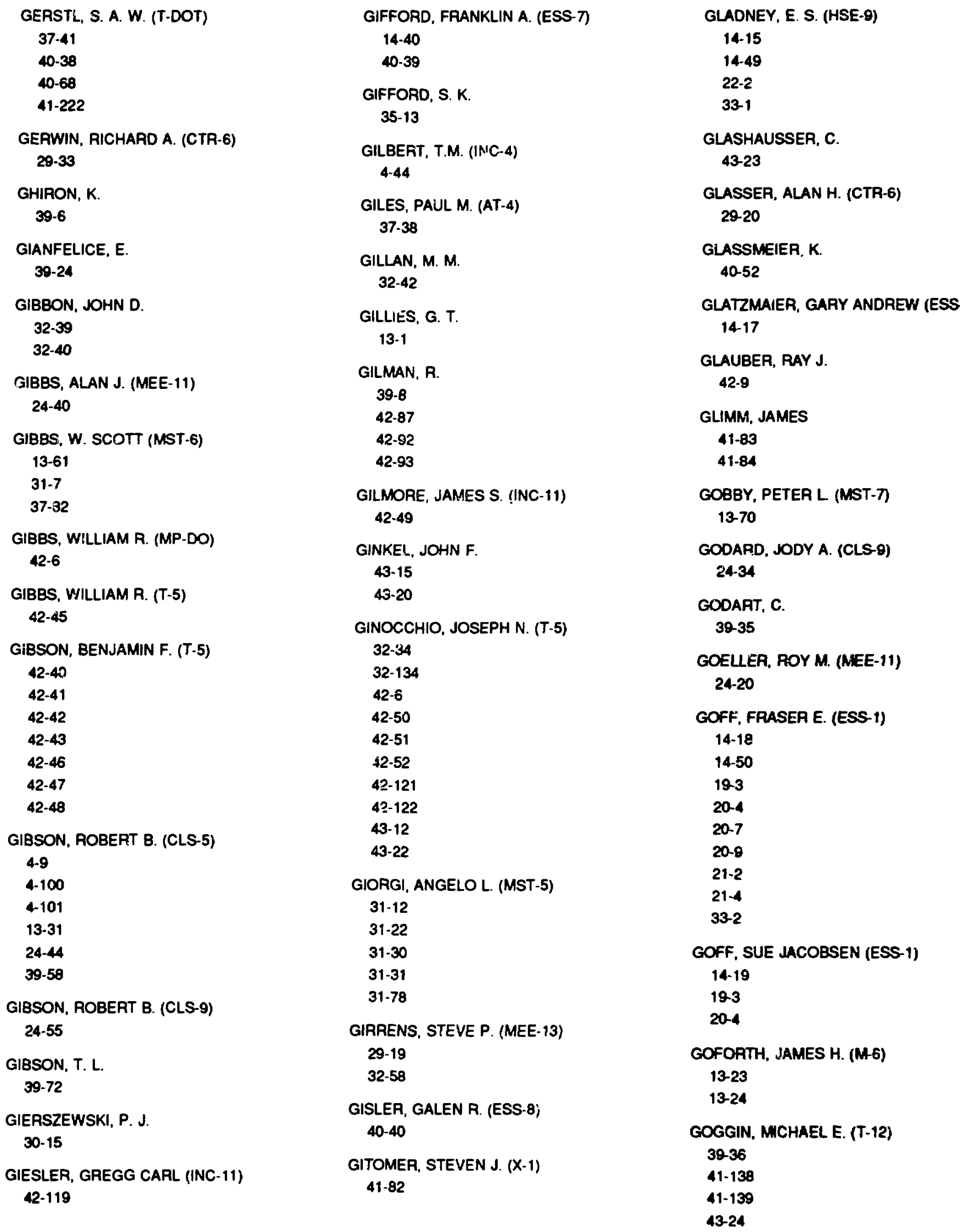

GLADNEY, E. S. (HSE-9)

14-15

14-49

22-2

33-1

GLASHAUSSER, C. 43-23

GLASSER, ALAN H. (CTR-6) 29-20

GLASSMEIER, $K$. 40-52

GLATZMAIER, GARY ANDREW (ESS 14-17

GLAUBER, RAY J. 42-9

GLIMM, JAMES

41-83

41-84

GOBBY, PETEA L (MST- 7 ) $13-70$

GODARD, JODY A. (CLS-9) 24-34

GODART, $C$. 39-35

GOELLEA, ROY M. (NEE-11) 24-20

GOFF, FRASER E. (ESS-1)

14-18

14-50

193

$20-4$

20-7

20-8

21-2

$21-4$

$33-2$

GOFF, SUE JACOBSEN (ESS-1) 14-19

193

$20-4$

GOFOATH, JAMES H. (N-6)

13-23

13-24

GOGGIN, MCHAEL E. (T-12)

39-36

41-138

41-139

43-24 
GOHAR, Y.

30-12

GOLDMAN, AARON (N-1)

$$
\text { 47.20 }
$$

47-35

GOLDMAN, AARON S. (N-4)
47-40
$47-44$

GOLDMAN, PEGGY (MEE-13)

16-2

16-3

GOLDMAN, SANFORD R. $(X-1)$ 41-57

GOLDMAN, TERRENCE J. (T-5)

14-1

40-64

41.98

42-53

42-54

$43-25$

43-26

43-27

43.28

43-29

43-38

43-39

43-50

43-52

43-79

GOLDSTEIN, BYRON (T-10)

1-19

$1-20$

GOLDSTEIN, JGHN C. $(X-1)$

13.74

37-35

37-42

37-43

37-44

37-105

37-107

41-195

GOLDSTEIN, SELMA (M-3)

2-9

GOLDSTONE. JOYCE A. (P-8) $31-30$

GOLDSTONE, PHILIP D. (P.4) 23-10

GOLLWITZER, $K$.

43-1
GONG, MINZHUAN

42-25

GONIS, A.

39-70

GONZALES, MANUEL (HSE-10) 4-28

GCNZALEZ, E. R. 12-5

GOODMAN, J. A.

40-26

$40-63$

GORE, RAYMOND A. (E-DO)

18-19

GOFN, $W$.

41-62

GORTON, MICHAEL P.

14-50

GOSLING, JOHN T. (ESS-8)

40-41

40-42

40-60

40-83

40.91

40-92

40-93

GOSNELL, TIMOTHY R. (CLS-5)

4-80

4-100

13-3

GOSWAMI, J. N.

40-43

GOTTESFELD, SHIMSHON (MEE-11)

12-1

12-2

GOULD, CHRIS R.

42-11

42-101

42-102

GOULDING, CHARLES A. (N-2)

24-4

41-135

GOUTAGNY, LUC

27-2

GOUVEA, MARIA E. (T-11)

41-136

GRADY, DEBORAH L. (LS-1)

1.10
GRADY, DENNIS E.

39-43

GRAESSLE, SCOTT

43-19

GRAF, KARL

31-98

GRAM, PETER A. M. (MP-4)

39-25

42-55

GRATTIDGE. WALT

32-68

32-105

GRAY, ALTON JAMES (MST-7) 31-116

GRAY, E. R. (AT-1)

37-4.5

37-46

GRAY, E. R. (AT-7)

37-38

GRAY, GEORGE $Y$., III (MST-5)

31-32

31-33

31-34

31-35

31-39

31-40

GRAY, NORMAN T. (P.15) 24-23

GRAYS, A.

4-43

GREEGOF, R. B

4-60

GREENE, ARTHUR E. (X-10)

13-23

13-24

GREENE, DENNIS P. (CLS-9)

4-99

23-10

24-54

GPEENE, STEVEN J. (MP-10)

42-36

42.87

42.89

42-117

GREENWADE, 1. ERIC (ESS-5) 35-6 


\section{GREENWOOD, L. R. \\ 42-26}

GREGORY, WILLIAM S. (N-6)

13-44

16-9

GREINER, NORMAN R. (CHM-1) 2-21

GREINER, NORMAN R. (CLS-1) 2-10

25-1

GREINER, SONYA E.

16-17

GREST, G. 5 .

$31-2$

41-182

GRIBBLE, ROBERT F. (CTR-4)

44-2

44.12

44-15

GRIFFEY, RICHARD H.

1-53

1-55

42.109

GRIFFITH, P.

26-1

GRIGORJEV, A. M.

40-1

GRIGSBY, CHARLES O. (ESS-1)

21-4

GRISHAM, DONALD L (MP-7)

41-60

41-61

GROSNICK, D.

43-4

GROSSMAN, $M$.

16-13

16-14

GRUETZMACHER, KATHLEEN M. (MST-3)

29-9

29-21

30-2

GRUNER, GEORGE

41-32

41-38

41-85

43-11

\section{GUFFEE, LAURA A.}

16-17

GULMEZ, E.

43-1

GUNN, C.

47-40

GLINNERSON, F. S.

$$
26-6
$$

26-7

GUNNINK, $R$.

47-18

GUNTHERODT, GUNAT
$39-12$
$39-37$

GUPTA, LAXMI C.

39-35

GUPTA, RAJAN (T-DO)

32-59

43-30

43-31

43-32

GUPTA, S. K.

40-26

40-63

GURALNIK, GERALD D. (T-DO)

43-32

43-33

GURLEY, LAWAENCE R. (LS-1)

1-21

GURLEY, LAWRENCE R. (LS-2)

1-22

GURNETT, D. A.

40-66

GURSKY, JUDITH C. (P-3)

42-56

GUSS, PAUL P.

43-15

43-20

GUSTAFSSON, H. A.

42-61

GUTBFOD, H. H.

$42-61$

GUTMACHER, R. G. (N-4)

47-12

47-13
GUY, FRANK W. (AT-1)

23-14

37-1

41-86

GUZIK, JOYCE A.

40-15

40-20

GYGAX, F. N.

39-10

HAASE, D. G.

42-11

HABBERSETT, RC (LS-4)

1-59

HABERSTICH, ALBEFTT (CTR-2) 44-15

HAFER, JOHN F. (N-4)

47-14

HAGAN, PATAICK S. (T-7)

16-10

16-11

41-128

41-130

41-184

HAGENSON, $R$.

44-4

HAGGERTY, JOHN S.

13-22

HAIDER, QUAMRUL 4-45

HAIGHT, ROBERT C. (P. 15)

42-29

42-57

42-67

HAINES, J.

30-12

HAINES, T. J.

40-26

HAKKILA. E. ARNOLO (N-4)

47-13

47.14

HALOING. JESPER (T-11) 39-38

HALE, GERALD $M$. (T-2) 29-37

39-39

39-75

42.67 
HALL. D. N. 40-55

HALL, JANE H. (P-DO) 4.24 32-60

HALLIDAY, KAPIII R.

1.23

1.53 HALLIN. AKSEL L (MP-4)
43-4

HAMA, $S$. 42-70

HAMEIRI, ELIEZER

29-1

HAMIL, ROY A.

23-5

HAMILTON, DOUGLAS J. 13.78

HA' $: M E L$, JAY E. (CTR-8) 29-20

HAMMEL, JAY E. (CTR-DO) 44-4

HAMMEL, P. CHAISTOPHEA (P-10) 41-87

41-120

HAMMER, CHARLES F. (CTR-4) 44-2

HAMMOND, JOHN H. 18-3

HAMMOND, ROBERT B. (MEE-11) 24-20

HAN, XIAO HONG

40-45

HANCOCK, A. D. 24-36

HANLON, JOHN ARTHUR (CLS-8) 23-10

HANNAH, PHIL

13-15

HANNIGAN, JAMES W. (CMS)

13-25

31-85

HANOLD, ROBERT J. (ESS-DOT) 19.6

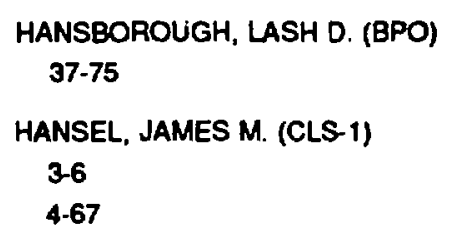

HANSEN, GORDON E. (N-2) 16-12

HANSON, DAVID E. (T-12) 24-1

HANSON, FLOYD 32-61

HANSON, KENNETH M. (M-4) 32-62

$32-63$

HARDEK, THOMAS W. (AT-3) 37-24

HAADEKOPF, ROBERT A. (AT-3) 37-24

42-28

HARGIS, PHILIP J., JR. 23-5

HARLOW, FRANCIS H. (T-3)

17-25

41. 16

41-88

HARMONY, S. C. (N-S) 26-2
HARPER, S. E. (CLS-5) 4-100

HARRADINE, DAVID M. (CLS-4) 4-46

HARAINGTON, CHARLES D. (ESS-1) 14-20 14-55

HARRINGTON, MARGYE P. (MP-6) 32-109

HARRIS, ANITA G. 14-7

HARRIS, C. 41.191

HARAIS, DAVID B. (A-4)

23-1

23-3

23-4

HARRIS. J. 0. 24-33

\author{
HARFiS, J. W. \\ 42-61 \\ HARAIS, ROBERT A. \\ $39-42$ \\ 41-154
}

HARRISON, RALPH J.

9-2

9-3

HARRISON, RONALD F. (CLS-5) 41-149

HART, GREC OAY W. (MP-4)

43-15

43-20

HARTER, WILLAM G. (T-12) 39.79

HARVEY, CAPOL $J$. 39-25

HASAI, H.

42-117

HASSANEIN, A.

30-12

HASSOLO, G. N.

41.182

$41-183$

HAUCKE. HANS (P-10) 41-69

HAUER, ALLAN (P-4)

23-5

23-6

23-10

41-205

HAUSCHILDT, P. 40-89

HAWKE, R. S.

13-19

HAWKINS, WARD L (ESS-1) 14-21

HAXTON, WICK C 40-30

HAY, P. JEFFREY (T-12) 4-57

31-10

$31-16$

39-48

HAYASHI, N.

47.18 


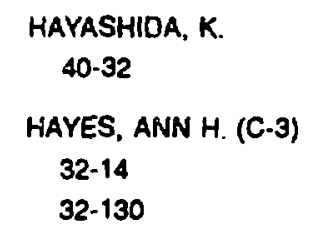

HAYES, EDWARD F.

39-40

$39-41$

HAYNES, L. C. (CLS-5)

41.149

HAYNIE, NOHN S. (HSE-1)

24-38

HEATH, J.

40.51

40-52

HEATHCOTE, $S$.

40-89

HECKER, SIEGFRIED S. (DIR)

17-1

17-14

18-21

18-22

HEDGES, ROBERT ORAIN (MEE-5) 24.4

HEDSTROM, JAMES C. (MEE-13)

24-6

24-14

HEFFNER, ROBERT H. (P-10)

41-87

HEIDENAEICH, GARY

13-36

HEIDORN, DOUGLAS B. (LS-2) 1-61

HEIDORN, DOUGLAS B. (LS-7)

$1-24$

$1-62$

HEIGHWAY, EDWARD A. (AT-6)

$$
\text { 41-100 }
$$

41-142

HEIKEN, GRANT (ESS-1)

$14-22$
$14-50$
$19-3$
$20-4$
$40-98$

HELFFRICH, J. A.

39-11
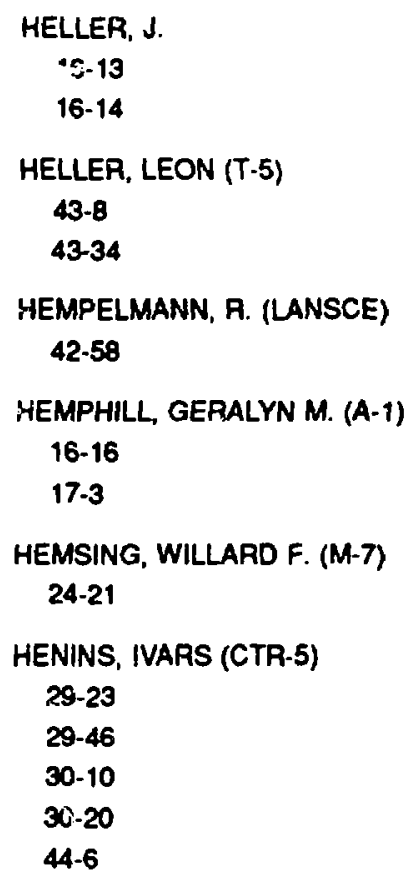

HENNEKE, K. M. (MEE-5) 24-4

HENSEL, $S$.

1-15

HERAS, HECTOR E.

$1-20$

HERCZEG, PETER (T-5)

42-11

$42-59$

43.35

HEPMES, RCBERT E.

31-115

HERNANDEZ, E.

42.94

HERRERA, JULIO

29-1

HERSKOWITZ, $M$.

16-10

16-11

HESS, D. W. (CMS)

41-76

HESS, DENNIS W.

4.70

HEUSI, P. (MP-4)

43-4

HEYSFR, JAMES W. (LS-3)

$1-46$
HIGGS, COLIN E.
$30-17$
$32-37$

HIGHLAND, V. L

43-1

43-4

43-15

HILKO, R. A.

24-36

HILL, J. O. (AT-3)

24-16

37.40

HILL, J. O. (AT-8)

37.14

HILL, ROBERT E. (MP-2) 41-62

HILLS, JACK G. (T-6)

40-19

40-44

HIRATA, SHINGO

29-2

29-35

30-21

HIROMOTO, ROBERT E. (C-3)

32-60

32-66

HIRT, CYRIL W., JR.

32-136

41-213

HIRVONEN, J. P. (MST-7) 31.47

HIRVONEN, J. P.

31-36

31-44

31.74

HITTI, 8.

$39-10$

HIXSON, ROBERT S. (M-6)

$$
\text { 41-169 }
$$

HJELLMING, ROBERT $M$. 40-45

HJELM, REX P. (P.LANSCE)

1.25

24-22

HJOAT, E. J.

42-57 
HOARD, DONALD E. (ESS-?)

14-40

14-51

HOCKADAY, MARY P. (P. 14)

24-23

HOCKETT, JOHN E., JR. (WX-5)

31-37

HODGDON, MARION L. (M-6)

32-64

41-89

HOEBERLING, ROBERT FREDERICK (AT-5)

32-115

37-32

37-33

HOEHN, MARTHA V. (MP-1)
43-1
HOFFER, JAMES K. (P-10)
$23-2$
$23-7$
$31-38$

HOFFMAN, CYAUS M. (MP-4)

43-4

43-36

43-37

43-58

HOFFMAN, D. M.

31-20

HOFFMAN, GARY G. (CLS-2)

39-42

41-154

HOFFMAN, Y. (T-6)

40-46

$40-47$

HOFFMANN, G. W. (T-10)

39-64

HOFFMANN, L.

41-123

HOFLAND, MARIANNE H.

$1-8$

HOFLER, THOMAS J. (P-10)

13-26

24-24

HOFSTADTER, $\boldsymbol{A}$.

43-4

HOGAN, GARY E. (MP-4)

43-4
HOGSET, VICTOR H. (IT-3)

18-26

HOIDA, HIROSHI W. (CTR-5)

30-10

$30-20$

44-6

HOLDEN, DANIEL N. (ESS-7) 14-23

HOLIAN, BRAD LEE (T-1)

39-43

39-44

41-90

41-95

41-199

HOLIAN, KATHLEEN S. (T-1) 41-91

HOLLAND, REDUS F. (CLS-4) 4-64

HOLLAND-MORITZ, E.

4-95

HOLLANDER, M. G. (MEE-11)

4-B1

$31 \cdot 72$

HOLLAS, C. L. (N-2)

43-81

HOLM, DARAYL D. (T-7)

29-1

32-39

$32-40$

41-1

41-11

$41-92$

41-93

41.94

HOLMAN, G. D.

40.13

HOLMES, VANNER H., JR. 24-61

HOLSINGER, RONALD F

24-36

HOLT, DAVID M.

24-25

HOLTKAMP. DAVID 8. (P-3) 42-107

HOLTZBERG, FRED

41-147
HOLZSCHEITER, MICHAEL H. (P-15) 43-19

44-5

HOMMEAU, FRANOIS

31-10.5

HONES, EDWARD W., JR. (ESS-8)

40.48

40.49

$40-90$

HONG, JUNG PYO (E-10) 32-65

HONG, JUNG PYO (MEE-10) 32-100

HONIG, EMANUEL M. (CLS-7) 13-27

13-28

HOOTEN, DAVID T. (MEC-6)

13-29

$24-46$

HOOVER, WILLIAM G.

41-85

HOAN, $S$.

41-193

HOROVITZ, BARUCH

$39-66$

41-18

41.96

HOSHINO, TSUTOMU 32-66

HOUSE, LEIGH S. (ESS-3)

14-24

HOWARD, SCOTT A.

4-10

HOWE, STEVEN D. (ERAET) 39.45

HOWE, STEVEN D. (ET/ETIO) 41-97

HOWELL, C. R.

42-57

HOWELL, JO ANN (AT-B)

3:- -14

HOWELL, ROBERT B. (CTR-2)

29-44

44-12

44-15 


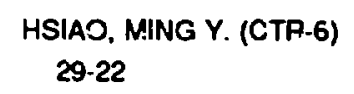

HSUE, SIN-TAO (N-1) 47-18

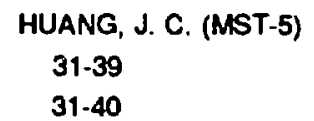

\section{HUANG, Y. \\ 41-62 \\ HUDDLESTON, S. (CTR-4) 44-2}

HUFF, JAMES R. (MEE-11:

94

96

97

HUFFMAN, J. C. 4-104

HUGHES, E. BARRIE 43-4

HUGHES, K. J. (MP-5) 37-78

HUGHES, RICHARO J. (T-8)

14-1

40-64

$41-98$

43-26

43-38

43-39

43-49

43-50

43-52

HUGHES, THCMAS P. 37-?

HUGRASS, WAHEED N.

29-10

29-36

44-:3

45-8

HULL, DONALD E. (MST-9) 13-30

HUME, M. W. 32-15

HUNG, L. S.

31-73

HUNSUCKER, R. D. 40-5
HUNTER, A. M.. $\|(P-16)$ 41-37

HUNTER, NOAMAN F. (WX-11) 13-52

HUNTER, RAYMOND E. (X-DO) 42-35

HUNTER, ROEERT O., JR. 41-37

HUO, W. M.

41-121

HURD, J. R.

42-60

HURD, JAMES W. (MP-5) 37-24

37-48

HUPLEY, GEORGE F. (MST-4) 31-94

HUTCHINSON, WILUAM B. (CLS-i) 31-5

HUTSON, RICHARD L (MP-3) 41-47

HUTSON, PICHARD L (MP.5)

37-24

37-68

41-48

43-56

HVELPLUND, $P$.

44-5

HWANG, W-Y P. (P-2)

43-40

HYATT, EDWIN C. (HSE-5) 22-8

HYER, DIANNE K. (P-DO) 24-26

43-41

HYKEL, MATTHEW N. (N-1) 47-19

HYMAN, JAMES M. (T-7)

1-31

32-74

41-99

HYNDS, R. J.

40-81
HYNES, M. V. (P.15)

39-45

$39-46$

41-97

43-42

ICHIKAWA, SCHINIC तII

27-2

IDZOREK, GEORGE C. (P-14) 24-35

ICO, G.

41-52

42-117

IGO, GEORGE J.

43-1

IKEDA, $\mathrm{H}$.

47-18

IMANISHI, NOBUTSLGU 42-119

INGRAHAM, JOHN C. (CTR-2) $29-29$

$29-33$

29-44

44-7

44-12

44-15

IPAVICH, FRED $M$. 40-93

IFOM, FAROKH (MP-4)

41-5

42-36

42-104

IRWIN, G.

43-15

ISAGAWA, S.

42- 117

ISHIMOTO, $\mathbf{s}$.

42-117

ITOH, $M$.

40-32

IVERSON, ARTHUR E. (MEE-11) 24-27

IVERSON, C. E. 24-37

IWATANI, $K$ 42-117 
JACAK, B. V. (P-2)

39-47

42-61

42-62

JACCARD, D.

31-78

41-31

JACHIM, STEPHEN (AT-5)

37-30

JACKSON, JOHN P.

4-47

JACKSON, PAUL J. (LS-3)

1-26

JACOBSEN, F. M.

44-5

JACOBSON, ABRAM R. (ESS-7) 40-50

JACOBSON, LOREN A. (MST-6)

4-48

31.41

31-42

JACOBSON, VICTORIA

32-101

JAEGER, DWIGHT L. (WX-11) 32-67

JAFFE, L.

16-13

16-14

JAHANMIR, SAID

32-105

JAHODA, FRANZ C. (CTR-8)

13-17

29-18

30-11

44-3

JAKOSKY, B. M.

40-27

JALBERT, ROLAND A. (HSE-1)

14-25

14-26

29-24

JAMESON, ROBEAT A. (AT-DO)

37-49

37-50

37-51

37-52

37-53

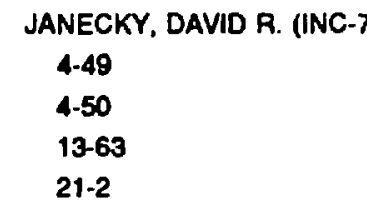

JARAMILLO, GENARA R. (N-DO)

47.15

47-36

JAPAMILLO, STEVEN A. (P.15)

24-61

JARBOE, THOMAS R. (CTR-5)

29-23

29.46

30-10

$30-20$

44-6

JARLBORG, T.

41.123

JARMER, J. J. (MP-7)

39-64

JARVINEN, GORDON D. (INC-4)

4.51

4-82

4.97

JASON, ANDREW JOHN (AT-3)
$37-24$
$37-54$
$41-100$

JENKINS, EVEAETT M. (MST-3)

30-6

$30-7$

JENKS, R. (N-6)

16-29

JENKS, RICHARD P. (N-9)

16-15

16-21

JENSEN, REED J. (ADR)

13-31

23-8

25-1

JENSEN, REED J. (P.DO)

23-9

JERMANCE, RAYMOND (ESS-11)

14-9

JEAVIS, THOMAS R. (MST-3)

31.43
JERVIS, THOMAS R. (MST-7) 31-6

$31-36$

$31-44$

$31-45$

$31-46$

31-47

31-50

$31-74$

JHA, R.

40-43

JOCKERS, $K$.

40.16

$40-51$

JOHNSON, J. 8.

144

JOHNSON, JAMES E. (T-1)

2-10

2-18

41-168

41-172

JOHNSON, JAMES D. (T-4) 41-173

JOHNSON, JAMES N. (T-1)

31-122

11-101

JOHNSON, JAMES N. (T-14)

2-23

JOHNSON, JAMES O. (ESS-6) 13-32

JOHNSON, $K$.

328

JOHNSON, K. F. (MP-10)

37-37

JOHNSON, KAYE ALLAN (MST-13)

$$
\text { 31-48 }
$$

$31-40$

JOHNSON, KAYE ALLAN (MST-4) 31-90

JOHNSON, MKKEL B. (MP-DO)

41-102

$42-63$

12-64

42-03

43-43

43-44

JOHNSON, N. F. [LS-1)

1-52 


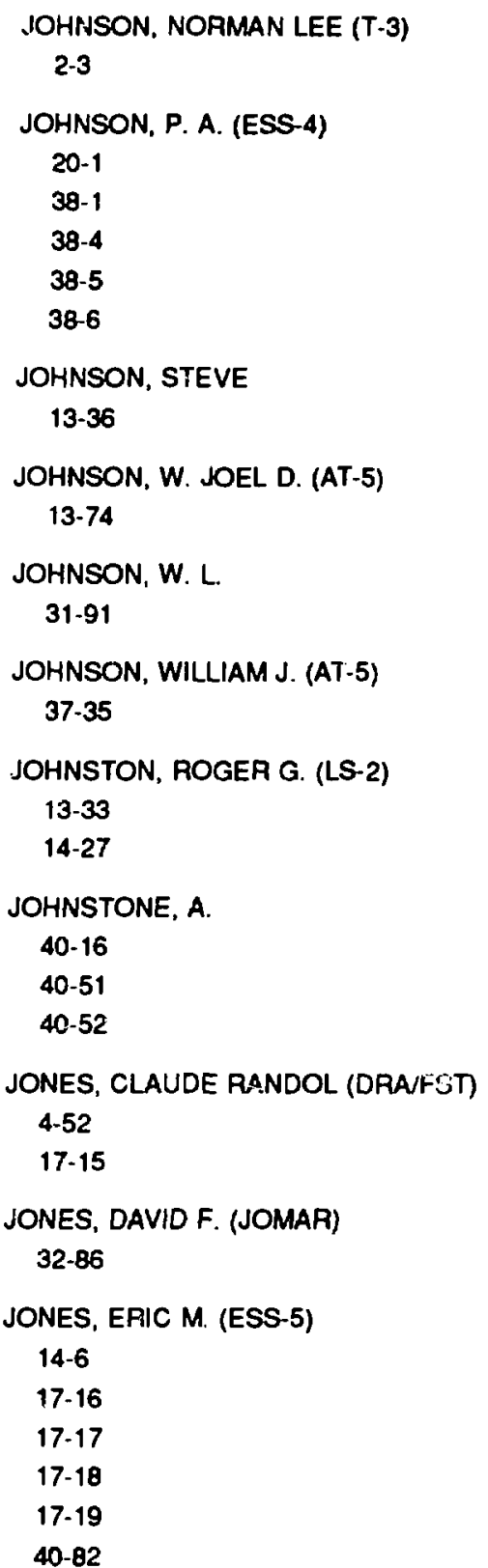

JONES, JAMES $W .(T-14)$

41-33

JONES, K. W. (MP-10)

39-64

42-65

42. 117

43-23

JONES, LARRY A. (P-1)

13-31

44- 1

JONES, LLEWELLYN H. (INOC-4)
JONES. MAPIANNE M. (CLS- 1 )
4-82

JONES, MICHAEL E. (AT-6)

37-55

JONES, MICHAEL E. (X-1)

23-11

37-57

JONES, ROGER D. $(x-1)$

29-14

$41-43$

41-103

41-104

JONES, S. E.

39-60

JORGENSEN, BETTY S. (MST-6)

4-53

JORGENSEN, J. D.

$31-30$

JOYCE, DON

43-15

43.20

JOYCE, EDWARD L., JA. (MST-7)

31-50

JULL, A. J.

40.28

JUMPEA, E. J.

$13-48$

KADIET: ABRAHAM (ESS-7)

40-53

41-159

KALASH, K. E. (WX-4)

37-87

KALBACH-WALKER, CONSTANCE (T-2) 42-4

KALBERMANN, G. 42-;0

KALIKHOV, A. V. 40-1

KALYANAM, K. M.

30.12.

KAMPERT. K. H.

42-61

KANE, JOHN R.

43-15

43-20
KANEKO, K. (CNLS) 13-34

KANG, J. H.

41-62

43-1

KANG, J. S.

4-54

KANG, MICHAEL (CLS-7) 23-10

KANIA, DON R. (P-1)

13-35

44-1

KAO, CHIH-YUE JIM (ESS-5)

14-28

$14-29$

14-39

KAPPLER, J. P. $39-35$

KAPUSTINSKY, JON S. (P-2)

24-28

42-87

$42-98$

KARIMABADI, $H$.

40-3

KARL, AOBERT RAYMOND, JR (ESS 14-30

KATSMA, K. R

26-1

KATZ, J. D. (NST-4)

6-1

31-51

$31-84$

KAUFFMAN, ERLE G. 14-35

KAUFFMAN, R. L. 23-12

KAUFFMAN, STUART A.

1-13

KAUFMANN, WILLAM B. (T-5)

42-6

42-45

KAUPPILA, T. J. (M-4)

24-47

37-56 


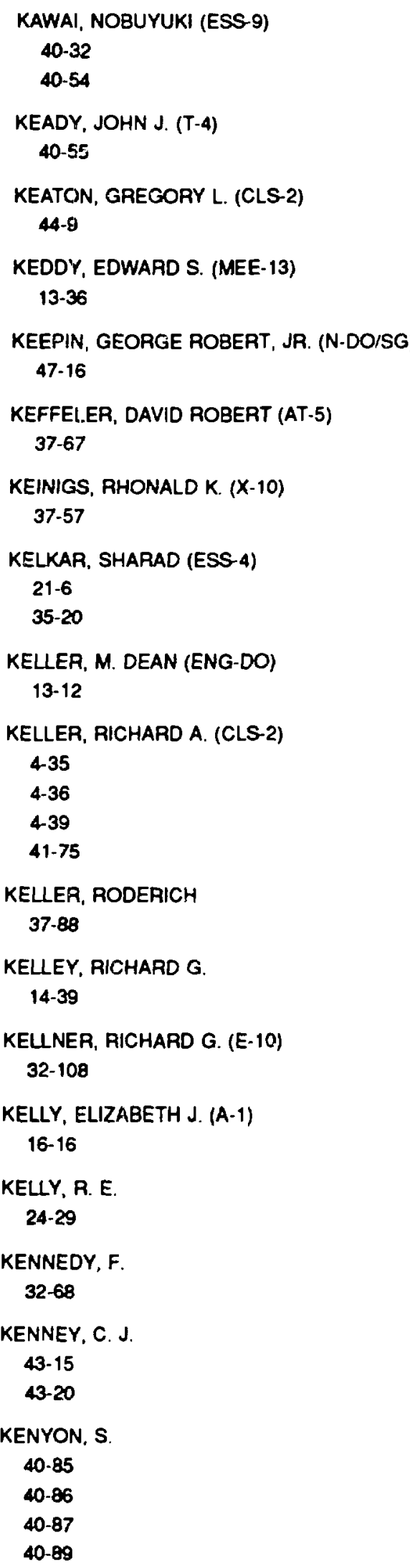

\section{KERRISK, JERRY $F$. (WX-4) \\ 13.19 \\ 13-37}

KERSHNER, JAMES D. (T-14)

2-11

2-12

KERSTIENS, DEBORA M. (AT-8) 37-14

KEVREKIDIS, I. G. (CNLS) 32.69

41.68

KEWISH, RALPH W., JR. (CTR-6) 44.2

KHARE, AVINASH

43-13

KHARE, AVINASH (T-8/CNLS) 43.12

KHERANI, NAZIR P. 29-24

KIA, T.

16-13

16-14

KIDMAN, RUSSELL B. (A-4) 40-20

KIELHOAN, W. F. 39-64

KIM, GEORGE J. 41-105

KIM, KYU C. (MST-11) 4-29

4-55

4-59

KIM, YEONG E. 42-17

KIMURA, $M$. 42.43

KINDEL, JOSEPH M. $(x-1)$

23.5

32.75

KING, CLARK

14.38

24-43

KING, JOE DAVID (P-3) 37.82
KING. NICHOLAS S. P. (P.15)
$24-36$
$24-59$
$24-62$
$37-65$
$42-57$

KING, AEBECCA L (AT-8) 37.14

KING, T. $R$.

41.149

KINNEY, E. R. 42-66

KINNISON, W. WAYNE (P-2) 43-15 43-20

KINROSS-WRIGHT, JOHN (AT-5) 37.33

KIREYEV, S. M.

40-1

KIRSCH, CLAUDIA F. (LS-7)
1.54

KIRSCH, WOLFF

1-54

KISSLINGER, LEONARD $S$.

43-72

KITTS, DAVID (C-DO) 32-30

KJEMS. J. $\mathrm{K}$.

41-77

KLEBESADEL, RAY W. (ESS-9) $40-32$

$40-56$

KLEIN. J. 40.57

KLEIN, MOAPIS M. (X-5) 24-10

24-11

KIEINSCHMNT, PHILLIP D. (MST-5) 4-56

KLEPACKI, DAVID J.

42-17

KLINCLER, OUENTIN G 24-36

KLINGNER, PHILLIP L. (CTR-5) 30.10 

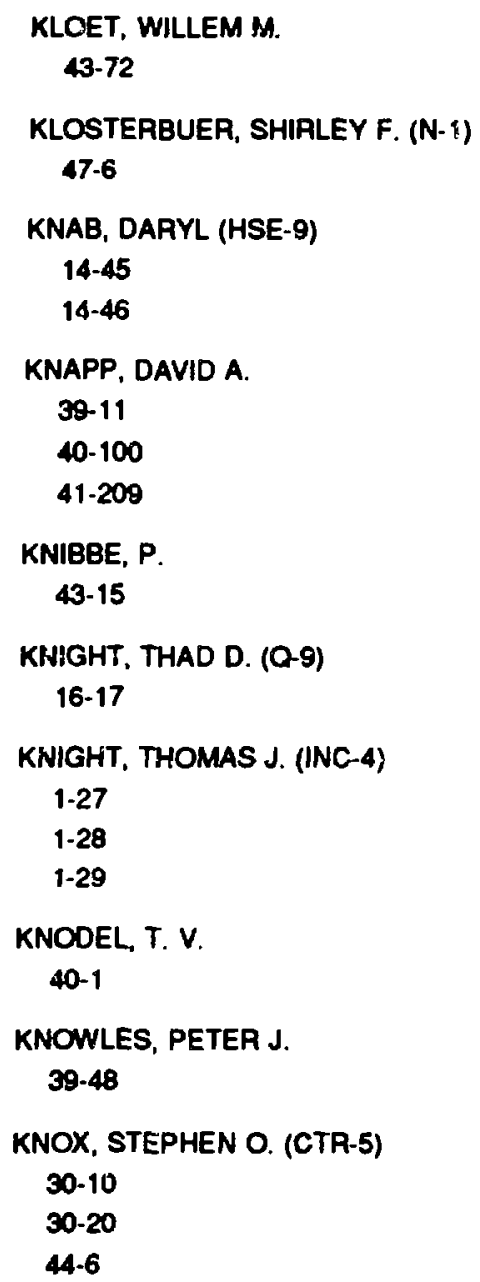

KNUDSEN, $H$.

44-5

KNUDSEN, PAUL D. (A-5) 17-24

KNUDSEN, $W . C$. 40-67

KNUDSON, ALVIN 24-58

KNUDSON, JAMES N.

$$
\text { 41-5 }
$$$$
\text { 42-104 }
$$

KNYSHENKO, I. I.

40-1

KOBER, EDWARD M. (INC-4) $4-57$

KOBER, EDWARD M. (T-14) $4-58$

\author{
KOCKS, U. FRED (CMS) \\ 31-23 \\ 31-52 \\ 31-53 \\ $31-80$ \\ 31.81 \\ 31-101 \\ KOEHLER, P. E. (P-3) \\ 40-58 \\ 40-59 \\ 42-67 \\ $42-68$ \\ 42-69 \\ 47-17
}
KOELLING, D. D.
$4-6$
$39-5$
41-2

KOETKE, D.

$$
\text { 41-62 }
$$

KOETKE, D. D.

43-17

KOETKE, D. S.

43-17

KOGAN, VLADIMAA G.

41-36

KOHARA, $T$.

39-53

KOLB, B.

42.61

KONDO, I.

40-32

KONIGSBERG, $\mathrm{J}$.

43-15

KONKEL, H. E. (CTR-4)

29-25

44-2

KOANBLUM, H. N.

23-12

KOANFELD, MARIO

1-54

KOANOUKHOV, V. N.

40-1

KOATEGAARD, B. L (CLS-8)

23-10

KOTHE, D. B. $(x-1)$

41-24
KOUZES. R. T.

40-1

KOWALSKJ, S.

24-36

KOZACK, R. (T-2)

42-70

KOZLOWSK, THOMAS (MP-6)

43-1

KOZUBAL, ANDAEW J. (AT-8)

37.14

KRAEMER, PAUL M. (LS-4)

$1-7$

$1-50$

KPAICHNAN, ROBERT H. (T-DO) 41-106

KRAKAUER, D. A.

40-26

10-63

KRAKOWSKI, ROBERT A. (CTF-12) 296

297

29-26

$29-42$

$30-3$

30-13

30-14

30-19

44-4

KPALL, NICHOLAS A.

$29-27$

KFALL, PANDY B. (LS-2)

1-47

$32-111$

KAAMEA, S. P. $(X-6)$

32-27

KRANZ, ROAERT (ESS-3)

14-57

KRAUS, ROBERT H., JR. $37-60$

KRAUSSE, GEORGE J.. III (MP-4) $13-38$

KRESGE, J. (N-1)

47-10

KRESS, NOEL D. (T-12)

32.70 
KAIESE, JOHN T. (X-2)
42-34

KRILL, $G$. 39-35

KRISTAL, RICHARD (P-4) 23-10

KROHN, BURTON J. (T-12)

$$
\text { 4-55 }
$$

4.59

KRUMHANSL, JAMES ARTHUR 1. 18

KRUPKA, MILTON C. (S-4) 38-3

K.RUSE, HERALD W. (P-14)

13-24

41-62

KUBAS, GREGORY J. (INC-4) 4-30

41-70

KUBAT-MARTIN, KIMBERLY A. (INC-4) 4-17

KURIC, J.

43-15

KUBIK, PETER $W$.

40-65

KUNASZ, PAUL

41-107

KUNO, Y. 47-18

KUPERSHMIDT, BORIS A. 41-92

KUPPERMAN, R. $H$. 18-23 18-24

KURNIT, NORMAN A. (CLS-6) 13-4

KWAK, D. 32.76

KWAN, THOMAS J. T. (X-10) 13-39

41-108

41.109

43.45

KWEI, GEORGE H. (LANSCE) 31. 109

41-210

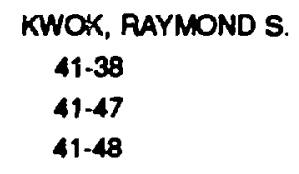

KYLE. THOMAS GAIL (IT-1) 14-39

KYRALA, GEORGE A. (P-1) 13-31

LA BAUVE, RAPHAEL J. (T-2) 16-8

18-37

27.3

32-57

42-2

42-30

42-80

\section{LA BELLE, ROBERT D. 4-35 \\ LA GATTUTA, K. J. (X-7) 39-49 \\ LACY, S. E. 31-22 \\ 31. 78} LAL, DEVENDRA
$\mathbf{4 0 - 4 3}$

LAM, D. J. 39-5

LAMBERT, STEVEN E. 39-53

$41-77$

LAN, E. H.

4-27

31-15

LANDER, G. H. 4-61

LANE, LEONARD J. 14-11

LANG, $K$. 43-15

LANGHORST, GAPY J. (HSE-12) 14-11

LANGSTON-UNKEFER, PAT J. (INC-4) $1-4$

1.12

$1-27$

1.28

1.29
LANGTON, CHRIS (CNLS) $32-71$

LAPEDES, ALAN S. (T-DOT)

$1-30$

$32-49$

32-72

32-73

41-110

LAPOSTOLE, P. M. (AT-1) 41-86

LARIOCCHIA. G. 44.5

LAROS, JOHN G. (ESS-9) 40-32

LARSON, ALLEN C. (P-8)

$$
\text { 4-18 }
$$

31-109

41-210

LARSON, ELZABETH M. (INC-4) $4-51$

$4-60$

4-81

LASINSKI, B. F.

23-12

LASJAUNIAS. J. C.

41-31

LATIMEF, TRENT W. (MST-11)

46-3

46-4

46-5

LAUGHLIN, A. WILLIAN (ESS-1)

$19-3$

$19-4$

$20-4$

$20-5$

LAVALLEE, DAVID $K$. $1-41$

LAVELLE, MORGAN J. $14-21$

LAWRENCE, GEOAGE P. (AT-3) 37-24

LAWRENCE, GEORGE P. (AT-DO) 37-58 37- 70

LAWRENCE, J. M. 39-50 41-111 


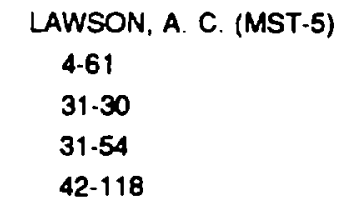

LAWTON, ROBERT $G$.

14-9

LAYMAN, LARAY R. (CLS-1)

24-2

LAYMON, CHARLES

42.87

LAYNE, SCOTT P. (CNLS)

$1-31$

$1-39$

32-74

LAYTER, J. G.

43-1

LE CLAIRE, RENE J., JR. (A-4) 18-25

41- 112

LE SAR, RICHARD A. (T-11)

31-55

39-71

41.13

41-113

41-114

LEAVITT, C. P.

41-62

43-1

LEE, B. $\boldsymbol{W}$.

$$
\text { 4-54 }
$$

$39-53$

LEE, C. (T-1)

42. 98

LEE, DAVID (MT-13)

41-62

LEE, DAVIO M. (MP-5)

43-1

43-15

43-20

LEE, KENNETH $(X-1)$

32-75

LEE, KIEN Y. (M-1)

$$
\begin{aligned}
& \text { 4-24 } \\
& 4-25 \\
& 42-71
\end{aligned}
$$

\section{LEE, MARTIN J. \\ 32-101 \\ 37-22}

LEE, PETER H. Y. (P-1)

13-31

24-44

41-115

LEE, WEN HO (X-7)

$32-76$

32-77

LEE, Y. C.

32.5

32.75

LEGATE, G. L. (P-9)

37-78

41-164

LEHMAN, DONALD R.

42-42

$42-48$

$42-72$

LEHNERT, BAUCE E. (LS-1)

1-21

$1-22$

$1-32$

$1-58$

LEISING, $G$.

31-106

LEITCH, M. J. (P.2)

41.5

$42-73$

42.98

42.104

42-119

42-120

LELLOUCHE, $\mathbf{G}$. $\mathbf{S}$. 26-1

LEMONS, ROSS A. (MEE-11) 24-20

LEON, MELVIN (MP-3)

39-61

LEON, MELVIN (MP-DO)

39-21

39-51

39-60

42-23

LESLIE, IAN H.

16-9
IESTER, C. S. (CLS-5)

4.100

LEVY, $\mathrm{S}$.

26-1

LEWIS, GARY K. (NST-6)

13-14

$31-56$

LEWIS, H. RALPH, JR. (CTR-6)

$20-20$

20-27

29-41

45-6

LEWIS, PAUL S. (MEE-3) 32-78

L., P. $39-60$

U. T. K. (N-1) $42-98$

U. T. K. (N-9)

47-19

L. X. L.

41-84

UCHTENSTADT, $\mathrm{J}$.

39-8

UCHTI, R. L.

41-47

41-48

UEPINS, RAIMOND (MST-7)

4-53

4-62

LKES, R. N.

47-20

LLES, DENNIS R. (N-9) 16-17

ULLBERG, JOHN W. (P-2) 24-28

UN, K. J.

41-11

UNDAU. I.

4.54

UNOEMUTH, I. R. (X-I) $29-20$

LNDEMUTH, 1. R. $(X-10)$

$13-23$

$13-24$ 
LINDHEIM, J.

32.23

LINC. ALAN G. (MP-10)

42.74

LING, KUOK M. (CTR-6)

29-22

45-2

LINSLEY, JOHN

40-63

LIPPELT, E.

39-10

LISKA, DONALD J. (AT-4)
37-59
$37-50$
$37-61$

LISOWSKI, PAUL W. (P-3)

42-28

42.57

42-67

42-75

LIST, A. S. (P-10)

31-57

LITTLE, CYNTHIA K. (AT-8) 37-14

LITTLE, ROBERT C. (X-6) 26-3

42-2

42-101

LITTLETON, P. E. (WX-11) 13-44

LIU, JOANNE

19-5

LIU, LON CHANG (INC-11)

4.45

42-36

42-60

42-98

LLOYD-EVANS, JEREMY (MP-4) 40.26

LOCKWOOD, FRAN 32-105

LOEBS, V. A. (MST)

37-38

LOGAN, CUNTON M. 31-1
LOGE, GARY WAYNE (CLS-4)

4-63

4-103

LOH, EUGENE Y. (T-11)

41-34

41-35

41-116

41-117

LOHE, MAX A.

32-79

LOHMANN, W. (MP-13)

31.98

LOHSEN, RICHARD A. (AT-5)

37-35

LOBL, JOHN (HSE-5)
22-8

LOMBARDI, A. (MP-14)

37-24

LOMDAHL, PETER S. (T-11)

39-38

41-18

41-118

LONDON, JERAY E. (LS-1)

1.21

$1-22$

$1-33$

$1-34$

22-3

22-4

LONG, WILLIAM H.

4-47

LOONEY, LARRY D. (P-14)

$13-40$

24-29

24-37

37-62

LOPEZ, EDWARD A. (HSE-12)

35-12

LOPIANO, D.

42-117

LOS ALAMOS NATIONAL LABORATOPY 17-20

LOUCK, JAMES D. (T-7)

32-11

32-79

41-119

LOUIE, DAVID L. Y, (MST-11)

27-4
LOUIS, W. C. (MP.S:

43-1

LOVBEFG, RALPH H.

29-20

LOVEMAN, ROBERT A.

41-5

42-104

LOWE, MARY (P-10)

39-55

41-120

LOWN, R. R.

24-36

LU. $X .0$.

43-1

LUBECK, OLAF M. (C-3)
$32-80$
$32-81$
$32-116$

LUCHT, POY A. (M-8)

13-41

LUHMANN, JANET G.

40-67

LUK, T. S.

42-29

LUKE, J. A. (LS-3)

1-60

LUMPKIN, ALEX H. (P-15)

13-74

24-62

27-1

37-35

37-63

37-64

$37-65$

LUMPKIN, ALEX H. (P-DO)

27-5

37-34

$37-66$

LUNDBERG. LYNN B. (MEE-13)

13-42

31-58

LUNOBERG. LYNN B. (a-13) 16-1

LUNDY, ARVID S. (IT-3) 18-26

LUTHER, GREG G. (CNLS) 32.02 


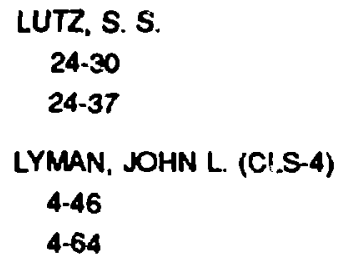

LYNCH, DIANE L. (T-12) 39-72 41-121

LYNCH, MICHAEL T. (AT-5) $37-67$

LYNCH, MICHAEL T. (BPO) 13-61

$37-75$

LYNN, DAVID K. (MEE-11) 13-78

LYNN, J. E. (P-3) 43-41

LYON, STANFORD P. (T-1) 41-10

LYONS, PETER B. (AOORA) 13-40 24-29

LYONS, PETER B. (DRANDR) $31-59$ 37-62

LYSENKO, WALTER P. (AT-6) $37-69$ 43-46

LYTLE, F. W. 4-60

MAASSEN, LARRY $W$. (ESS-1) 14-55

MAC APTHUR, DUNCAN W. (MP-4) 39-25

MAC ARTHUR, DUNCAN W. (N-2)

24-31

47-21

MAC FARLANE, ROBEAT E. (T-2) 18-27

$27-3$

41-122

42-2

MAC INNES, MARK A. (LS-3) $1-60$

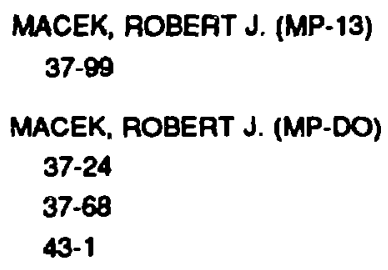

MAGGIOAE, CARL J. (CMS) 4-68

4-81

494

31-60

31-62

31-72

31-103

MAGGIORE, CARL A. (MEE-11) 4-102

MAGGIOAE, CARL J. 2458

MAHAFFY, JOHN H. (Q-9) 16-17

MAIER, WILLIAM B., "I (ESS-7) 41-159

MAILHIOT, CHAISTIAN 24-50

$39-52$

3973

MAJIMA, SUMMKO 32-66

MALENFANT, RICHARD E. (DIR-EA 7-1

MALEY, MARTIN P. (P-10) 39-11 $40-100$

MALM, JOHN G. 4-31

MALTMAN, KIM R. (T-5) 42-53 43-28 43-79

MALTRUD, HERMAN R. (WX-5) 3960

MANDELL, DAVID A. (X-7) 32-82

MANN, ALFRED $K$ 43-1

MANN, DAVD A. (ESS-1) 14-31

MANN, JOSEPH B., JR. (T- -4$)$ 32-28 39-19

MANTEUFFEL, THOMAS A. (C-3) $32-83$ $41-73$ 
MANUEL, A. A.
41-123

MANWEILER, $R$.

41-62

MANZANARES, ADBERT G. (CTR-A) 39-6

MMO, XUEYING (INC-11)

14-35

MAPLE, MERFILL $B$.

454

39.53

41.77

MARCENAT, $C$.

31-22

31-78

MARGOLIN, LEONARD G. 32-84

MARIAM, FESSHA G. (MP-4) 43-4

MARIAM, FRANK (MP-13) 37-24

MARIANER, SHLOMO (T-11) 31-61

41-124

43.47

MARIANI, $F$. 40.52

MARINUZZI, JOHN G. (DRNAMO) $1-47$

32-85

32-111

MARINUZZI, ROBERTA (MP-DO) 41-125

MARK, J. CARSON (DIRNCN) 18-28

MARKIN, JACK T. (N-4)

47-22

$47-41$

$47-45$

MARKLIN, GEOAGE J. (CTR-5) 29-23

30-10

44-6

MARKLIN, GEORGE J. (CTR-6)

29-46

30-20

45-3

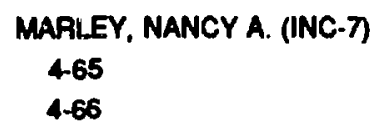

MAFOUARDT, J. H. (AT-2) 37-37

MARR, T. G. $(T-10)$
$1-31$
$1-63$
$32-74$

MARAONE, BABETTA L (LS-4) 1.35

MARRONE, BABETTA L (LS-7) 1.36

1-37

MARSH, SINCLAIR F. (MST-12) 3-4

3.5

MARSH, STANLEY P. (M-6)

13-23

13-43

41-134

MARSHALL, J. A. 40-6

MARTELL, CALVIN J. (CLS-1) 3-6

$4-67$

MAFTIN, ALBERT D. (M-8) 24.32

MAPTIN, E. RAY (AT-1)

7-3

MARTIN, E. RAY (N-2)

16-12

32-86

MARTIN, JOE A. (CLS-2)

468

4-69

4-91

31-46

31-47

31-62

MARTIN, PATAICK L (MST-5)

448

$31-42$

MARTIN, $R$.

30-15
MARTIN, RICHARD A. (MEE-13) 13-44

11-126

MARTIN, FICHAHD L. (T-12) 13-68

$32-54$

41-127

11-191

MARTIN, AONALD H. (ESE-8) $40-6$

MARTIN. THIERRY (CNLS)

41-116

MARTINEZ, FEUX A. (AT-1) $13-61$

37-82

MARTINEZ, RONALO (A-5) 32-87

MARTOFF, C. J. 43-15

MART, HARRY F., JR. (A-1) 17-21

MART, JOSEPH C. (MST-13) 4-70

MART, VIRGINIA E. (AT-8) 37-14

MASAIKE, $A$. 12-117

MASON, R. E. (MST-11) 16-18

MASON, PODNEY J. (X-9) 23-11

MATALON, $M$. 32-88

MATHER, MCHAEL W. (INC-A) 1-15 $1-38$

MATHESON, P. L. 20-11

MATHEZ, E. 31.60

MATTS. H. S. (MP-4) 43-4

MATTHEWS, ROBERT BAUCE (MST-31) 16-18 
MATTHEWS, $S$.

24-31

47.21

MAUDLIN, PAUL J. (N-6)

27-2

MAULDIN, G. $H$.

24-33

MAURO, BILLIE R. (CLS-6)

4-71

MAX, PATRICIA A. (C-10)

32-10

MAXIMON, LEONARD C. 42-72

MAXTON, PATRICK M. (P-10) 39.55

MAYER, ANTON (MST-7) 31-63

MAYER, J. W. 31-73

MAYER-KRESS, GOTTFRIED (CNLS) $1-39$

1-67

MAYO, ROBERT M. 30.20

45-3

MAZOR, AVAAHAM (T-81)
$41-128$
$41-129$
$41-130$
$41-181$
$41-184$

MAZUMDAR, SUMIT (CNLS) 39-13

MC BRYAN, OLIVER A. (C-3) 32-55 32-89

MC CABE, CHARLES W. (MP-3) 24-13

MC CALL, GENE H. (X-DO)

29-20

$29-28$

MC CLELLAND, JOHN B. (MP-10) 42-83
MC COMAS, DAVID J. (ESS-8)

13-6

40.6

$40-60$

MC COWN, ANDRES W. (CLS-9) 24-34

MC COWN, ANDREW W. (CLS-5)

4-99

24-54

MC COY, LINDA S. (LS-3)

$1-60$

MC DANIEL, PATRICK J.

34-4

MC DONALD, ARTHUR BRUCE 42-11

MC DONALD, J. W. 39-8

MC DONOUGH, J. 43-4

MC DOWELL, H. KEITH (CLS-2) 43-48

MC DOWELL, ROBIN S. (CLS-4)

4.59

4.72

MC ELFRESH, M. W. (P-10)

31-64

$39-53$

41.77

41-212

42-118

MC ELROY, JOHN F. (LS-7)

$$
1-40
$$

MC FARLANE, $W . K$.

43-1

43-15

MC GAUGHEY, PATRICK L. (P-2) 42-97

MC GILL, JOHN A. (MP-13)

37-24

37-48

43-1

MC GOWEN, MICHAEL (C-5) 32-54

MC GUIRE, RCBERT E.

40-43
MC GURN, JOHN S. (P-4)

13-70

MC HENRY, M. E. 31-65

MC INTEER, BERTHUS B. (INC-4) 4-75

MC KAY, MICHAEL D. (S-1) 32-90

MC KEE, ROBERT J. (P-2) 43-20

MC KELLAR, BRUCE H. J. 42-47

NC KENNA, KENNETH F. (BPO) 37.75

MC KERLEY, BILYY J. (MST-12) 3-3

MC KIBBEN, M. A.

$21-2$

MC KINSTRIE, C. J. (CNLS)

23-5

32-91

32.92

41-131

41-132

41-133

MC KNIGHT, ROBERT 42-105

MC KOY, V. «1-121

NC LARTY, TYCE T. (C-B) 32-29

MC LEOD. JOHN (CLS-8) 23-10

MC QUEEN, ROBERT G. (M-6) $4 \backslash-134$

MC VEY, BAIAN D. $(x-1)$

37-21

37.35

$37-42$

$37-43$

$37-44$

37-105

37-107

41-82

$41-195$

MEAD, C. ALOEN

4-11 


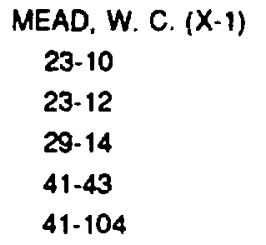

MEDINA, WALTER J. (MST-13) $31-48$

$31-49$

MEDINA, WALTER J. (MST-4) 31.99

MEEK, THOMAS T. (MST-6) 4-73

MEIER, M. M. (P.9) 41-135

MEISNER, G. P. (MST-5) 43-6

MELESE, $P$. 43-15

MELTON, JIMMY G. (CTR-11) 44-15

MELTON, JIMMY G. (CTR-4) 44-2

MENDEZ, RAUL $32 \cdot 80$

MENIKOFF, RALPH (T-14)

13-45

13-70

24-19

41-84

43-18

MENLOVE, HOWARD O. (N-1)

47-23

47-24

47-34

MENZEL, MARY TSINGOU (AT-6) 37-26

MERCER, $R$ 42-70

MERCER-SMITH, JANET A. (INC-11) 1.41

MERRIGAN, MICHAEL Á. (MEE-13;

13-16

13-36

13-46

16-19

$32-137$

\author{
MERSON, JEAN L. (AT-E) \\ 37-6 \\ MERTENS, F. G. \\ 41.136 \\ MERTS, ATHEL L. (T- 4$)$
39-23 \\ METROPOLIS, NICHOLAS (T-7) \\ 32.93 \\ METZGER, JOHN D. (A-4) \\ 16-13 \\ 16-14
}

MEYNE. JULIANNE (LS-3)

$1-7$

$1-49$

MEZENTSEVAH, S. A.

40-1

MICHELSEN, CHRISTIE D. (A-5)

18-16

32-6

$32-94$

MICHIE, ROBERT $B$.

24-1

$\mathrm{MICICH}, \mathbf{R}$.

13-61

37.82

MIDDLEDITCH, JOHN (ESS-9)

40-54

MIDDLETON, $R$.

40-57

MIGLIOAI, ALBERT (P-10)

10-3

13-26

13-47

39.55

$39-68$

41-137

MIKATARIAN, R. R. 13-48

MIKIC, 2 . 29-27

MILES, D. J. (ESS-1)

$20-7$

MILEWSKI, JOHN O. (MST-6)

13-49

$32-95$
MILLENE:R, DONALD J. 42-84

MILLEA, BARBARA L. (MST-13) 47-34

MILER, CHARLES M. (INC- 7 ) 4-35

4-36

4-37

$4-38$

$4-39$

474

4-85

$41-75$

MILER, GARY

1-54

MLLER, GEOFFREY G. (INC-11) 4-97

MILLER, GUTHRIE (CTR-2) 29-18

29-28:

29-30

29-3:1

29-44

44-3

44-7

44-8

44-12?

44-15

45-4

MILLER, RONALD L. (CTR-12)

296

$30-3$

30-16

44-4

MILER, WARREN F., JR. (DDERT) 32-96

MLLUKEN, F. P.

41-147

MILS, RENE SCOTT (AT-1) 37.110

MLLS, THOMAS R. (INC-4) 4-75

MILNER, E. C. (MP-A)

43-1

43-15

43-20 


MILONNI, PETER W. (T-12)
$39-3$
$39-4$
$39-36$
$39-56$
$39-57$
$39-58$
$41-138$
$41-139$
$43-24$

MILPOY, RICHARD D.

$29-27$

29-41

MINAI, $Y$.

4-65

MINEHAFT, R. C.

42-60

MIRIN, ART A.

29-27

MIRNOV, I. N.

40-1

MISCHKE, RACHEL (INC-4) 4-2

MISCHKE, RICHARD E. (MP-A) 37.99

$39-59$

42-85

43-4

MISDAO, M. A.

$31-60$

MISHRA, CHANDRA S. (P-2) 42-97

MISHRA. SANTOSH K. (T-10) 1-63

MISHRA, $v$. 42-70

MISKOWSKI, VINCENT M. 4-76

MITCHELL, A. W. 39-5

MITCHELL, ALAN J. (INC-11) 4-90

MITCHELL, ARTHUR C. (M6) 31.70
MITCHELL, GARY E.

42-11

42.101

42-102

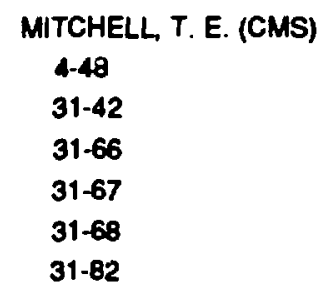

MTCHELL, T.E. 31-69

MNISZEWSKI, SUSAN MARIE (C-10) $32-5$

32-97

NOCK, $\mathrm{R}$.

32-37

MOHER, THOMAS 32-61

MOIR, DAVID C. $(M-4)$

$$
\text { 24-47 }
$$

$37-47$

37-56

MOLLEA, PETER (T-9)

42-86

MOLZON, W. $R$. 43-15

MONDT, JOHANNES P. (CTR-6) 14-32

MONEYHUN, DORA 32-105

MONSEN, SUSAN A. $13-7$

MONTGOMEAY, D.

$$
\text { 41-140 }
$$

MONTOYA, JOE G. (INC-4)

$$
\text { 4-75 }
$$

MOCDY, DAVID C. (INC-11) 42-67

MOOAE, C. FAED

$39-8$

42-87

$42-88$

$42-80$

42-92
MOOAE, DAVID S. (CLS-4)

$4-77$

$30-71$

41-168

MOORE, JAMES W. (C-3)

$32-14$

$32-80$

MOORE, LESLE M. (A-1)

$16-20$

$17-3$

MOOAE, STANLEY W. (Q-13) 49-1

MOORE, TAOY K (ESS-4) $20-6$

247

MORALES, RAUL (CLS-1)

4-78

$4-79$

MORALES, AEYNALDO (WPC-1) $18-20$

MORAN, $B$.

41-95

MORDECHA, $\mathbf{s}$.

$30-8$

42-87

42-88

$42-89$

$42-02$

MORGADO, RICHAPD E. (N-2) 43-81

MORGAN, G. L. (P-15)

41-135

MORGESON, J. DARRELL (A-5)

18-16

$32-94$

MOARIS, CHARLES E. (M-6)

31-33

34-70

MOPRIS, CHAISTOPHER L (MP-10)

$24-35$

$30-8$

$42-87$

$42-88$

42-80

42-92

42-107

42-120 


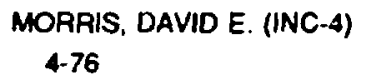

26-3

WOTLEY, FRANK E. (N-9)

16-21

MOTTERSHEAD, C. T. (AT-6)

$$
\text { 37-69 }
$$

$37-70$

MOYZIS, ROBERT K. (LS-3)

$$
1.7
$$$$
1.49
$$

MUDGETT, J. S. (LS-3) 1.60

MUELLEA, F. M. (CMS) 41-143

MUELLER, KAFL H., JR. (M-4) 2-16

MUELLER, M. H. 4-61

$$
\begin{aligned}
& \text { MUELLNER, S. M. } \\
& \text { 42-28 }
\end{aligned}
$$

MUIR, DOUGLAS W. (T-2)

30-17

41-219

$42-90$

MUKHERJEE, PRITISH (CLS-5)
$4-80$

MULFORD, ROBERT N. R. (MST-5)

$$
37
$$

$$
\begin{aligned}
& \text { MUNCK, ECKARD } \\
& 4-112
\end{aligned}
$$

MUNROE, JAMES L. (IT-3) 18-26

MUNSON, CARTER P. (CTR-2) 29-33

$29-49$

29-45

44. 12

44-14

44-15
MUNTZ, E.P. 41-126
MURAKaM!, T. 40-32
MURPHAEE, J. S. 40-49
MURPHY, CHARLES E. 14-25 $14-26$
MURPHY, DENN!S M. 29-19
MUPPHY, HUGH D. (ESS-DOT) 14-57
$19-1$
$19-6$
21-5
MURPHY, JACK 32-135
MURPHY, TIMOTHY C. (ESS-7)
14-33
32-99
40-61
40-6?

MURPAY, HUGH S. (E-11) 9-5

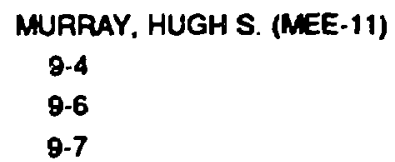

MUSGRAVE, JOHN (ESS-1) 19.3

MUSMANN, $G$.
$40-52$

MUSTO, RENATO

43-13

MYERS, C. WESLEY (ESS-1) $19-6$

MVEAS, GEAALD $(T-10)$ $1-42$

MYEPS, W H. (CHM-1) 4-5

NADER, LAURA $14-6$

NAGLE, DARRAGH E. (MP-4) 40-26

$40-63$
NAGY, ANN $(X-6)$ 16-23
NAGY, M. ANN (X-6) 16-25 $16-25$

NAITO, TAISEI $29-2$

$29-35$

$30-21$

NANCE, ROBEAT L (MST-12) 3-3

NARANJ, CLEO M. (LS-3) $1-43$

NARUSE, $Y$. 29-2 2935 30.21

NASH, $P$. 31-3 31.71

NASISE, JOSEPH E. (MST-3) 29-31 

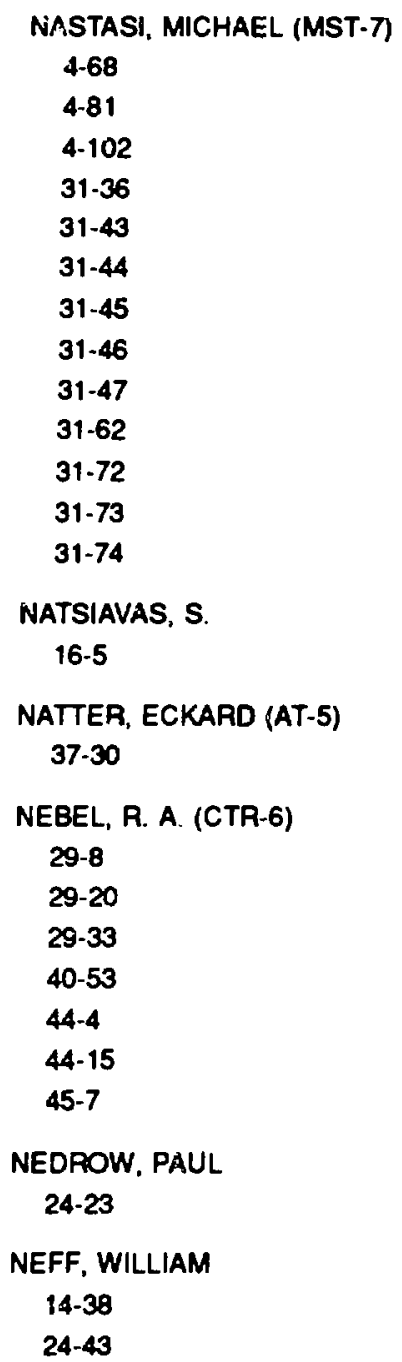

NEKIMKEN, HOWARD L. (CLS-1)

4-79

4-82

NELSON, M. A.

24-37

NELSON, R. A. (N-9)

16-17

26-4

26-5

26-6

26-7

NELSON, RALPH A. (N-9) 16-29

NELSON, RONALD O. (P-3) 13-76

42-57

NERESON, NORRIS G. (AP-2)

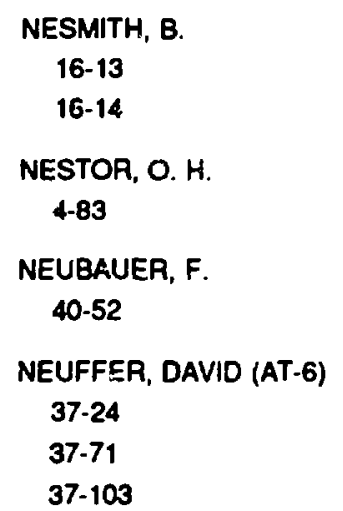

\section{NEUGEBR?.UER, $M$. 40-4}

NEUSCHAEFER, G. H. (AT-1) 37.1

NEWCOMER, F. M. 43-1

NEWELL, T.

16-13

16-14

NEWNAM, BRIAN E. (CLS-5)

31-92

37-107

NEWNAM, BRIAN E. (CLS-6)

37-35

37.72

$37-73$

37-74

37-105

41.144

NEWSOM, C.

41-62

NEY, E. P.

40-85

$40-86$

40-87

40-89

NGUYEN, DINH C. (CLS-6) 13-50

NICCUM, E. (ESS-5)

14-60

14-61

14-62

NICHOLS. BILI D. (N-6)

16-9

32-136

$41-213$
NICKOLS, JOYCE W. (LS-3) $1-60$

NICOLAENKO, BASIL (T-7) 32-40

NICOLAENKO, BASIL (T-7/CNLS) 32-39

NIEDERHOFFER, ERIC C. (INC-4) 1-43

4-8

NIEDNER, $M$. B. 40-13

NIETO. MICHAEL MARTIN (T-8)

14-1

40-64

41-98

41-145

43-5

43-26

43-27

43-38

43-39

43-49

43-50

43-51

43-52

NIEVA, G.

39-17

NINOKATA, H. (N-6)

27-2

NISHHDA, K.

47.18

NISHIIUUM, KUNIHIKO

40-57

$40-65$

NISHIMURA, J.

40-32

NISHIYAMA, F.

42.117

NITSCHE, CARMEN I. (INC-4)

4-112

NIX, JAMES R. (T-9)

42.78

$42-80$

42-86

42.91

NOEL, BRUCE W. (MEE-5)

13-1

24-30 


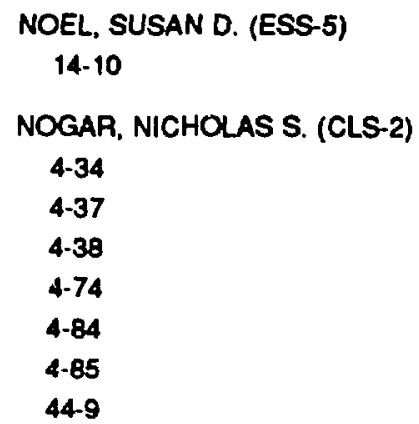

NORMAN, M. R.

41-146

NORRIS, ANDREW E. (INC-7) 35-13

NORTON, JOHN L. (X-7) 41-104

NOVICK-COHEN, AMY 41-99

NUNNALLY, WILLIAM C. (E-11) 13-27

$13-28$

NUNNALLY, WILLIAM C. 24-20

NUTTER, MURLIN J. (P-8) 24-46

NYHAN, JOHN W. (HSE-12)

$35-1$

35-14

NYITRAY, A. M. (MST-7)

34-75

O'BAIEN, HAROLD A. (P-3)

40-1

$40-58$

40.59

$42-68$

$42-69$

46-1

O'DELL, R. DOUGLAS (X-6)

16-6

16-22

16-23

16-24

16-25

16-26

O'DONNELL, J. M.

42-89

O'DONNELL, JAMES M. (LS-1) 1-44

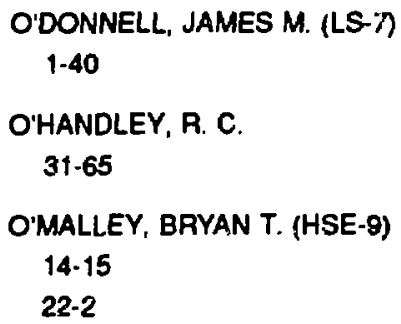

OLINGER, BARTON W. (M-6) 24-15

OLIVER, RONALD D. (ESS-3) 14-21

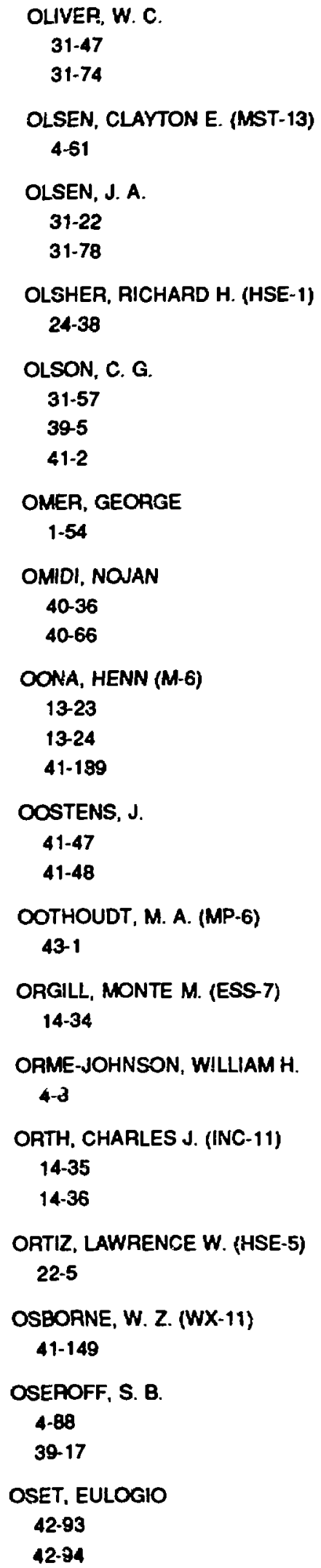




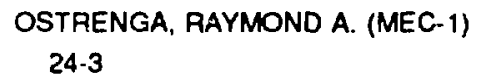
32.77

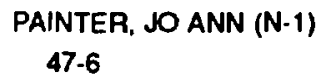

PALMER, BYRON A. (CLS-4)

$$
\text { 4-13 }
$$

4-74

PALMER, MICHAEL J. (MST-12) 3-8

PALMER, RONALD G. (N-12) 16-27

\section{PANKRATZ, MICHAEL C. (IT-3)} 18-26

PAPATHEOFANIS, B. (P-3) 13-70

PAPCUN, GEORGE JACK VICTOR (C-10) 32-5

PARENT, DENISE C. (CLS-2)

4-36

4-39

41-75

PARKER, F. (N-6)

27-2

PARKER, GREGORY A. 32-70

PARKER, JACK L. (N-1)

$47-19$

$47-20$

47.25

PARKER, JERALD V. (P-1) $13-53$

PARKER, JERALD V. (P-7)

13-19

13-54

13-55

13-56

13-81

PARKIN, DON M. (CMS)

$$
\text { 31-103 }
$$

PARKINSON, WILLIAM J. (WX-4)

6-1

38-7

PARKS, G. K.

40.48

PARKS, RONALD D. 41.193
PARMENTOLA. JOHN A. (T-9) 43-2

43-3

PARSONS, W. M. (CTR-9)

13-19

$13-54$

13-56

$13-57$

29-32

PARTER, SEYMOUR V. 32-100

PASAMEHMETOGLU, KEMAL O. (N-9) 16-17

26-4

26-5

26-6

26-7

PASTERNAK, MOSHE 4-87 39-63

PATEL, APOOAYA 43-32

PATEL, NISHEETH R. 32-65

PATTERSON, CHAIS W. (T-12) 4-59

PAULETTA, G. $39-64$

42-117

PAULIKAS, A. P. 31-57

PAULTER, NICHOLAS G. (MEE-11) 24-40 24-41

PAXSON, VERN 32-101

PAYNE, G. F. 4-91

PAYNE, GERALD L.

42-19

$42-39$

42-40

$42-41$

42-42

42-43

PECK, CLAUDIA (A-1) 17-3 492 


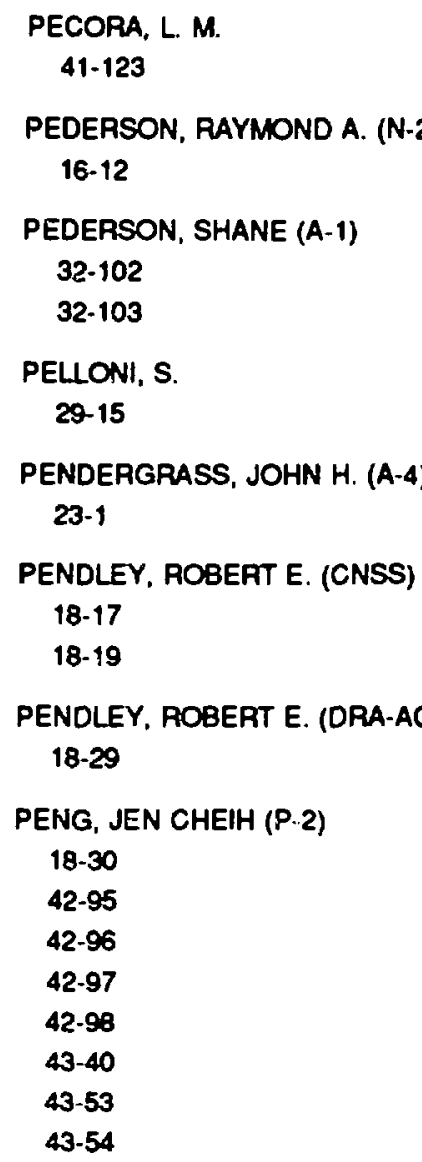

PEAFY, WILLIAM L. 1.45

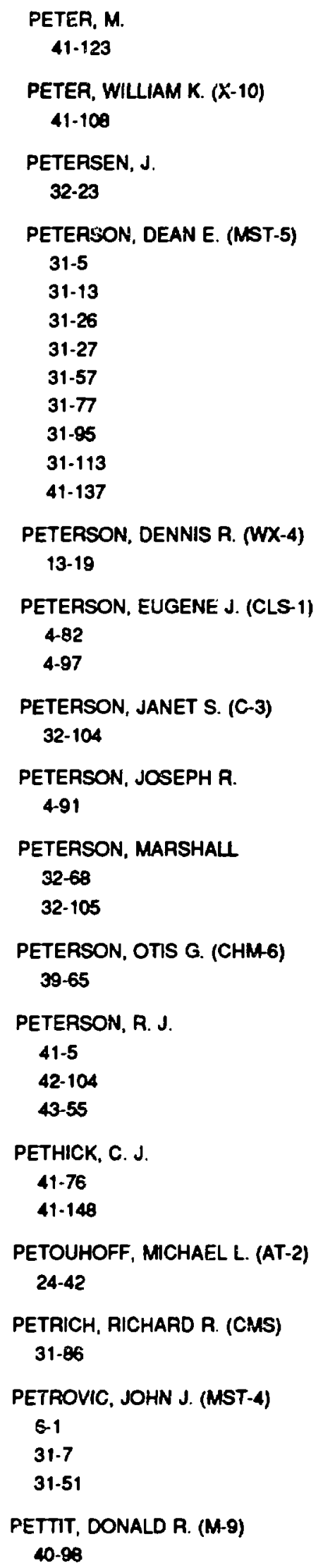

PETER, $M$.

41.123

PETER, WILLIAM K. ( $X-10)$ 41-100

PETERSEN, J. 32-23

PETERSON, DEAN E. (NST-5) 31-5

$31-13$

31-26

31-27

$31-57$

$31-77$

31-95

31.113

41-137

PETERSON, DENNIS R. $(W X-4)$ 13-19

PETERSON, EUGENE J. (CLS-1) 4-82

4-97

PETERSON, JANET S. (C-3) 32-104

PETERSON, JOSEPH $R$. 4-91

PETERSON, MARSHALL 32-68 32-105

PETERSON, OTIS G. (CHM-6) 39-65

PETERSON, R. J.

41-5

42-104

43-55

PETHICK, C. J.

41-76

$41 \cdot 148$

PETOUHOFF, MICHAEL L. (AT-2) 24-42

PETRICH, RICHARD R. (CMS) 31-86

PETROVIC, JOHN J. (MST-4)

6-1

31.7

31.51

PETTIT, DONALD R. (M-9) 40-98

PETUKHOV, V. $V$.

40-1

PFRANG, $W$.

27-6

PHARR, GEORGE M. 31-47

31-74

PHILLIPS. DAVD S. (MST-A) 6-1

PHILIPS, DAVD S. (MST-6) 2-10 31-102

PHILLIPS. MAMES A. (CTA-DO) 20-44

44-15

PHILLIPS, NOHN L (ESS-8) 40-67

PHILLIPS, LEE 41-140

PHILLIPS, MARIYN VIRCINL (CLS-1) 4-107

PHILIPS, MARYETH (HSE-O) 1415

22-2

PHILLIPS, N. E. 31-22 31-78

PHILLIPS, PICHARO LANG (C-6! 32-106

PHILIPS. WILUAM S. (ESS-4) 20-1 38-5

PHILLPOT, SIMON R. 30-68 41.96

PhIPPS, Claude A., AR. (CLS-5) 41-149

PI, T-W. 31-57

PUSETZKY, EL $30-8$ 41-5 42-104 42.120 


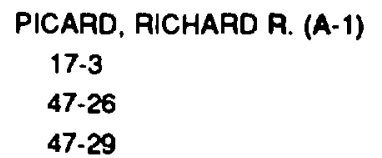

PICCININI, M. 39-60

PICKLESIMER, A. (P-15)

$39-45$

41-28

$42 \cdot 16$

$42 \cdot 17$

42-20

42.99

PICKRELL, M. M. (CTR-2)

44-10

44-15

PIERCE, C. BALLARD (P-10)

4-42

31-48

31.57

PIILONEN, LEO E. (MP-4) 43-4

PIKHULYA., O. E. 40-!

PILAT, JOSEPH F. (CNSS)

18-19

18-31

PILLAY, K. K. S. (N-4)

47-27

47-28

47-29

47-30

PIMBLEY, GEORGE H. (T-14)

32-107

41-150

PINES, DAVID (CMS)

41-12

41-76

41-148

PIRKLE, C.

16-10

16-11

PISTORESI, DENIS J.

13-73

PITCHER, ERIC J.

37-85

PLANNER, H. N. (ESS-1)

19.3

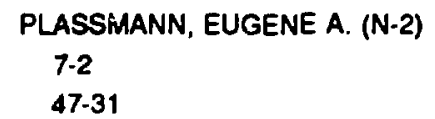

PLATTS, D. A. (CTR-5)
$30-20$

PLEINER, HARALD

41-25

PLOHR, BRADLEY $\mathrm{J}$

41-151

PLUM, MICHAEL A. (MP-10)

37-68

42-87

PLUM, MICHAEL A. (MP-5)

37-24

PNEVMATIKOS, STEPHANOS (CNLS)

41-198

PNEVMATIKOS, STEPHANOS

41-152

POE, R. T.

41-62

POELAKKER, KAREN (MP-4)

43-56

POFAHL, G.

39-12

39-37

POGUE, E.

41-55

POHORILLE, A.

4-110

POLLAK, GREGORY D. (X-7)

41-104

POLLARD, L. HOWARD

32-65

32-108

POLZER, WILFRED L. (HSE-12)

35-10

35-11

POORE, RAY V. (P-9)

32-109

POPE, LARRY E.

4-69

PORCH, WILLIAM M. (ESS-7)

14-38

14.39

$14-40$

24-43
PORTER, C. A.

18-34

POSCH, HAFALD A.

41-95

POSKANZER, A. M.

42-61

POSTMA, HANS

42-11

POTHS, JANE (INC-T)

4-35

POTTER, M. E.

40-26

41-153

POTTER, ROBERT M. (ESS-4)

1457

21-5

POTTER, WILLUAM C.

18-32

POUGET. J.

43-47

POUNOL, L

41-17

POWELL, DENNIS R. (A-5)

17-23

17.24

POWERS, B. J. (T-DOT)

4ü-68

PRAEL, RICHARD E. (X-6)

42-49

PRATT, LAWRENCE R. (CLS-2)

4-110

39-42

41-154

PAECECHTEL, D.

37-115

PREISER, CECIUIA F.

1.23

PAELOVSEK, PETER (CNLS)

41-116

PRESTON, DEAN L. (X-4) 42-54

PRESZLER, ALAN M. $29-44$

PAOBERT, M. $\mathbf{R}$.

24-30 


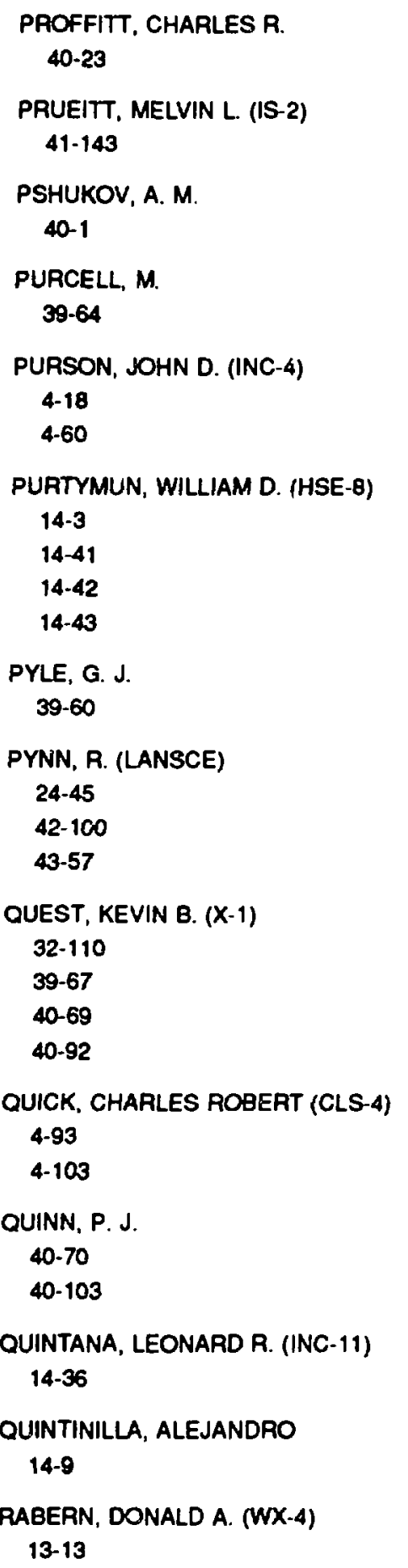

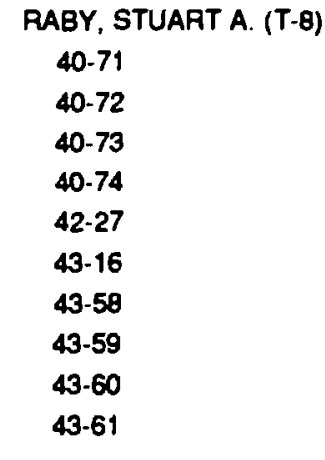

RAISTRICK, IAN D. (MEE-11)

31-109

4)-81

41-210

RAMACHANDRAN, RAM 31.3

RAMAKRISHNAN, $P$. 42-101

42-102

RAMBERG, BENNETT 18-33

RAMOS, $N$.

19-3

20-4

RAMSAY, JOHN B. (M-3) 2-13

RAMSAY, JOHN B. (M-8) 2-8

RANGARAJAN, G. 37-76

RANGEL, MANUAL (AT-5) 32-115

RANKEN, WILLIAM A. (MEE-13) 13-58

RAO, D. 4-88

RAPAPORT, J.

42.57

FATH, ALAN $R$.

13-21

RATHKE, J

13-61

37-82

RAUENZAHN, RICK (T-3) 14-57

\author{
RAVEX, A. \\ 41-31
}

RAWOOL, M. $W$.

$39-8$

42-80

42-103

RAY, FRANK A. (LS-4)
1-7
RAY, L.
42-70

RAYMONO, ROBERT, JR. (ESS-1) 14-31

$14-44$

FAYMOND, T. D.

241

REAGOR, DAVID W. (P-10)

39-68

41-137

REASS, WILUAM A (CTR-4)

44-2

44-10

44-12

44-15

REDONDO. ANTONIO (MEE-11)

9-8

$12-4$

24-27

41-214

REEDY, ROBERT C. (ESS-8) 40-28

$40-43$

40-57

40-65

40-75

40.76

40-77

40-78

40-97

42-26

AEES, L. M. $39-60$

REGNAULT, LOUIS P. 43-57

PEICHARDT, $W$.

4.95

REID, DONALD W. (AT-5)

13-50

$37-53$ 
REID, DONALD W. (AT-DO) 37- 77

REID, ROBERT S. (MEE-13)

13-46

16-19

REILLY, T. DOUGLAS (N-1) 47-32

REINOVSKY, R. E. (M-6)

13-23

13-24

REISER, CHIISTOPHER

24.1

REISFELD, MARTIN J. (LS-1)

459 REISZ, GERALD E. (ADP-2)
$32-68$

REJ, DONALD J. (CTR-3)

29.10

29-36

44-11

44-13

45-8

RENEIKA, J. P.

11-38

REMPP, H. (MP-DO)

41-40

REMPP. $H$.

41-47

REVZIN, N. YE.

40-1

RHEE, $G$.

26-2

RHOADES, JOHN (IS-8)

17-9

PHORER, AICHARD L. (MEC-6) 13-15

RICE, JAMES $K$.

24-1

RICHARDSON, I. G.

10-81

RICHARDSON, J. W., JR.

461

RICHARDSON, SHERRI A. (MST-6)

31-41
RICHTEPI, DIETER
42.58

RICHTER, $R$.

13-61

37-82

RICKETTS, C. I.

13-44

RICKETTS, RALPH L. (WX-4) 24-46

RIDGWAY, S. T.

40-55

RIGGSBY, W. STUART

1-45

RINARD, PHILLIP M. (N-1)

47-6

47-33

RINEHART, GARY H. (MST-11)

4.18

46-2

46-3

46-4

46-5

RINGLER, THOMAS J.

24-47

RINKER, GEORGE A., JR. (T-1)

41-155

41-156

RISTINEN, R. A.

43-1

RITCHIE, J. L

43-15

PITCHIE, JAMES P. (T-14)

2.14

4-58

$39-68$

RITTER, H. G.

42-61

RITTER, M.

43. 4

POACH, FRED (S-2)

38-3

ROACH, JAMES F. (S-2)

94

9-6

10-1
ROBERSON, N. R.

42-11

ROBERTS. DOUGLAS J. (A-S) 32-46

ROBERTS, JEANETTE C. (INC-11) $1-41$

ROBERTS, JEFFREY P. (CLS-5)

4-100

4.101

13-31

24-44

ROBERTS, JEFFREY P. (CLS-9) 2455

ROBERTS, PETER

14-13

ROBERTS, ROGER A.

42-35

ROBERTSON, AOBERT G. H. (P-3)

39-11

$40-100$

41-209

$43-62$

43-63

43-64

ROBINSON, BRUCE A. (ESS-4) 21-5

ROBINSON. C. PAUL (NSP) 25-1

ROBINSON, HAPOLO (P-O)

37-78

41-164

42-40

ROBINSON, N. J. (LS-3)

1-26

ROBINSON, RICHARD D. (HSE-9)

14-45

1446

ROBINSON, POBERT A. (LANSCE)

$24-45$

41-218

ROBINSON, ROBERT A. (P-B)

24-46

41-157

41-217

ROBISCOE, R. T. (ESS-7)

41-159

$41-150$ 
ROCHE, L 27-6

RODRIGUEZ, MANUEL M. (LS-3) 1.46

ROEDER, S. B. $\mathbf{w}$. 13-21

ROENSCH, F. R. (INC-11) 3-2

ROFER, C. K. (ESS-1) 35-15

ROGERS, PAMELA S. Z. (INC-7) 4.66

ROHATGI, U.S.

26-1

ROHA, DANA L (MST-5)

31-119

ROKNI, S. H.

41.5

42-36

42-104

42-120

ROKOP, DONALE J. (INC-7)

4-89

ROLLETT, ANTHONY UAVID (MST-5) 31.52

ROLLETT, ANTHONY DAVID (MST-6) 31-79

31.80

31-81

ROMERO, J. L 42-57

ROMERO, JOE A. (Q13)

16-1

ROMERO, ROBERT J. (CLS-4)

4-93

ROSATO, G.

13.61

37-82

ROSE, EVAN A. (CLS-7) 23-10

PROSE, HARVEY A. (T-DOT)

41-65

41-160

41-163
ROSE, J.

13-61

37-82

ROSEN, SIMON PETER (T-DO)

37-99

40.79

40-80

$41-161$

43-65

43-66

43-67

ROSENAU, PHILIP

29.20

$32-88$

41-99

41-162

ROSENTHAL, MARY A. (HSE-DO) 22-6

ROSEV, B. J. (CTR-4)

44-2

ROSOCHA, LOUIS A. (CLS-7)

23-10

23-13

ROSS, D. K.

42-58

ROSSEL, C.

39-53

ROTA, GIAN-CARLO (DIR OFF)

32-93

ROTHROCK, RICHARD (AT-3)

24-16

37-40

ROTHROCK, RICHARD B. (AT-8) 37-14

ROUSSIN, ROBERT W. 42-105

ROWE, M. W.

4-37

4-38

4-85

ROY. TAPPAN (CMS)

31-67

$31-69$

31-82

RUBIN, $P$.

43-15
RUFENACHT, HEINZ D.

19-3

20.4

RUHTER, W. D.

47-18

RULE, D. W. 37-63

RUMINER, MARY K. (IS-11) 17-22

RUNDBERG, ROBERT S. (INC-11) 4-90 42-119

RUPPEL, HANS M. (T-3) 41-24

RUSNAK, FAANK M. 4-112

RUSSELL, C. T. 40-92

RUSSELL, D. K. 4-22 $41-160$

RUSSELL, DAVID A. (T-DOT) 41-65

41-133

41-163

RUSSELL, GARY J. (P-9) 37-78

41-164

42-49

Russo, P. A. (N-1)

47-34

47-40

RUSTHOI, DANIEL P. (AT-2) 37-37

RUTHERFORD, DEBRA A. 42-106

RUTLEDGE, JAMES T. (ESS-4) 20-1 
RYAN, ROBERT R. (INC-4)

2-14

4-24

4-25

4-41

4-42

4-44

4-51

4-91

4-94

4-97

RYDER, R. (MP-5)

37-24

SABELLI, NORA

32-61

SAILOR, WILLIAM C. (P-2)

41-165

41.166

SALAMON, M. B.

39-6

$39-7$

SALCEDO, L.

42-94

SALMON, J. K.

40-103

SALZMAN, GARY C. (LS-2)

$1-47$

14-27

32-111

SAMADANI, R. (CLS-5)

13-3

SAMPSON, THOMAS E. (N-1) 47-35

SAMUELSEN, MOGENS $R$ 41-118

SANCHEZ, $R$.

39-17

SANDBERG, V. D. (MP-4)

40.26

40-63

41-167

43.1

43.4

SANDENAW, THOMAS A. (MST-13)

4-92

31-83

SANDER, OSCAR R. (AT-2)

37.37

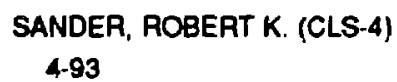

SANDERS, CLAUDIA (C-2)

32-112

32.128

SANDERS, GARY H. (MP-13)

37.99

43.68

SANDERS, GARY H. (MP-4)

43-1

43-4

43-15

43-20

43-69

SANDERS, VIRGIL E. (CLS-6) 4-71

SANDERSON, T. R. 40-81

SANDOVAL, DONALD L. (T-3) 17-25

SANEYOSHI, KEIJI 31-1

SANKEY, O. F. 31-107

SAPIR, JOSEPH L. (N-1) 47-23

SAPIR, JOSEPH L. (N-12) 18-34

SAPONAFA, ARTHUR G. (LS-2) 1-17

SATIJA, INDUBALA I. (CNLS) 41-63

SATO, I. 27-6

SATTELBERGER, ALFRED P. (INC-4)

4-16

4-21

4-44

4-104

42-118

SAUER, NANCY N. (INC-4)

4-41

4-42

4-94

SAUNDERS, JIMMY D. 24-47 saw, C. $K$. 31-84

SAXE, PAUL W. (T-12) 39-54

SAXMAN, A. C. (CLS-B) 37-21

SCARF, F. L. 40-93

SCHADE, $M$. 32.68

SCHADLER, GERHARD H. (T-11) 4-15 39-70

SCHALLER, STUART C. (MP-1) 32-113 37-79

SCHALM, ROBERT W. (OS-8) 24-48

SCHAFPERT, GOTTFRIEO T. (P-1) 13-31

SCHAPPERT, GOTTFAIED T. (P-DO) 41-115

SCHENCK, $A$. $39-10$

SCHERMER, ROBEAT I. (AT-3) 37-80

SCHIFERL, DAVID (H6) 4-32 $39-71$

SCHILLACI, MARIO E. (MP-DO) 41-47

41-48

43-1

43-56

SCHIRBER, J. E. 31-57

SCHMIDT, HANS RUDI 42.61

SCHMIDT, KEVIN E.

42.53

43-28

43-79

SCHMIDT, STEPHEN C. (M-6)

4-77

41.168 
SCHMITT, M. J.

37-44

SCHMITZ, $F$.

27-6

SCHNEIDER, BARRY I. (T-12)

32-114

39-22

39-72

41-121

SCHNEIDER, E. J. (MP-14)

37-81

SCHNURR, NORMAN M. (WX-4)

13-13

13-19

$13-60$

$37-87$

SCHOENBERG, KURT F. (CTR-2)

29-7

$29-33$

29-34

29-44

44-12

44-15

45-5

SCHOFIELD, ALDRED E. (CTR-2) 29-33

29-44

44-12

44-15

SCHOONOVER, JON R. (INC-4) 4-111

SCHOR, P. L. (LS-4)

1-17

SCHOTT, GARFY L (M-1)

2-15

4-46

SCHRAGE, DALE L. (AT-1)

13-61

37.75

37-82

SCHRANDT, ROBERT $G$. $(X-6)$

$1-48$

42.34

SCHRANK, LOUIS S. (CTR-4)

44-7

SCHAEINER, E. T.

24-33
SCHRIBER, S. O. (AT-DO)

37-1

$37-49$

37-83

SCHROEDER, N. C.

$4-89$

SCHULTZ, DAVID E. (MF-1)

$37-79$

$37-84$

37-86

SCHULTZ, S.

4-89

2917

SCHWALBE, LARAY A. (X-4)

2-16

4-47

SCHWARZ, AICARDO B. (CMS)

13.25

31-3

31-68

31-69

31-71

31-84

31-85

31-86

31-87

31-88

$31-89$

$31-90$

31-91

31-104

SCHWARZACHER-ROBINSON, TRUDE (LS-4)

$1-7$

$1-49$

$1-50$

SCHWARZMEIER, JAMES L. (CTR-6) 45-6

SCHWEITZER, L. B.

37-70

SCOGGINS, WAYNE A. (HSE-8) 22.7

SCOTT, ALWYN C. (CNLS) $1-51$

SCOTT, MARION L. (MST-7)

13.62

$31-92$

31-93

SCUDDER, DAVID W. (CTA-8)

29-20
SCUDDER, DAVID W. (CTR-DOT) $44-4$

SEBAING, ROBERT J. (LS-1)

1-32

$1-52$

SEEGER, PHIUP A. (LANSCE) 1-61

SEESTROM MORAIS, SUSAN J. (P-3) 13-76

$42-57$

42-87

42-92

42-107

SEFTOR, COUN J. (MP-13)

41-5

42-104

43-70

SEGA, RONALD $M$.

$4-47$

SEIDL, PETER A.

42-87

$42-92$

SEIGEL, R. T.

43-1

SEITZ, WENDEL LEE (M-1) 2-17

SEITZ, WENDELL LEE (M$2-8$

SEKIGUCHI, SATOSHI 32-66

SELDEN, ROBERT W. (CNSS) 18-17

$18-23$

SELDEN, ROBERT $W$. (X-DO) 18-24

SENA, J. T. (MEE-13)

13-36

SENNHAUSEA, $U$.

41-5

42-104

SENNHAUSER, URS (MP-4)

43-4

SETH, DANIEL L. (C-3)

32-48

SEVERING, ANDREA (NST-5)

4-95 


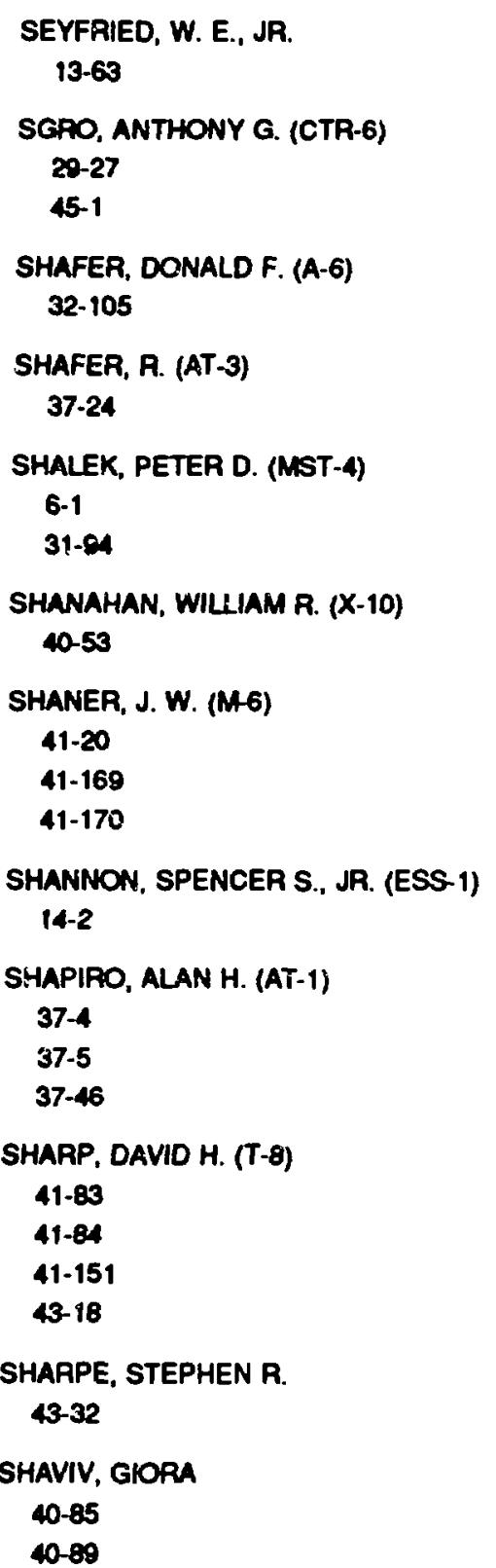

SHAW, MILTON S. (T-14)

2-18

41-168

41-171

41-172

41-173

SHAY, T. M. (CLS-5)

419

499

13-3

24-12

24-49

24-54

41.41
SHEEHEY, $P$.

29-20

SHEELY, E. V.

39.60

SHEFFIELD, RICHARD L. (AT-7)

37-17

37-38

SHEFFIELD, STEPHEN A. (M-9) 41-174

SHEINBERG, HASKELL (MST-6)

473

31-85

SHELDON, R. I. (MST-5)

3.7

31-95

SHEN, B. C.

43-1

SHEN, $2 . X$.

4-54

SHEPPARD, SCOTT

1.53

SHERA, E. BROOKS (P-DO)

4-96

42-108

SHERMAN, J. D.

37-85

SHERMAN, ROBERT H. (MST-3)

29-2

$29-35$

30-18

30-21

SHERWOOD, EUGENE G. (P-1) 43-45

SHERWOOD, KENNETH B. 16-17

SHEVENELL, LISA (ESS-1)

20-7

21-4

SHIGEOKA, K.

47-18

SHIH, C. F.

16-13

16-14

SHIH, CHIA CHANG

43-9

43-10
SHIKHIN, A. A.

40.1

SHILO, YU. I.

40.1

SHINN, N. D.

31-57

SHINOHARA, $\mathbf{S}$

44-12

SHIRE, PHILLIP R. (N-9)

16-17

26-2

SHLACHTER, JACK S. (CTR-8) 20-20

SHMAYDA, WALTER T. 22-24

SHPOUF, K. C. (MEE-5) 24-4

SHULTZ, J.

16-13

16-14

SHUMAKER, DANAE. 20-27

SHURTLEFF. J. K. 3960

SIBLEY, LEWIS $B$. 32-6

SICILIANO. EDWARD R. 42-60

SICILIANO, EDWARD R. (T-2) 42-14

SIDIKMAN, K. L

45-7

SIDOROWICH, JOHN J. (CNLS) 41-74

SIEBE. D. A. (N-12)

16-28

16-30

SIEGEL, $R$.

43-7

SIEMON, RICHARD E. (CTR-3) 22-10

29-36

44-13

45-8 


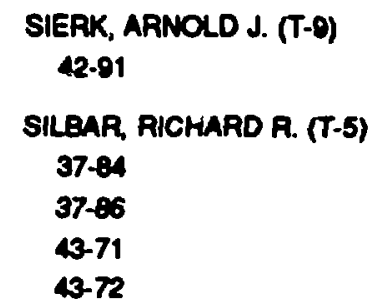

SILK, J. D.

42-80

SILLEUD, LAUREL O. (LS-2) $1-53$

SiLLERUD, LAUREL O. (LS-3) 1-23

SILIERUD, LAUREL O. (LS-7)

1.54

1-55

42-100

SILVA, ALEX A. (AT-2)

32.115

SILVER, RICHARO N. (T-11)

24-46

41-175

41-176

41.177

41-178

$41-179$

43-76

SIMMONDS, STANLEY M. (N-1) 47-19

SIMMONS, JAMES E. (P-2) 42-98

SIMMONS, LEONARD M., JR. (T-DO) 43-73

SIMMONS, MARGARET L (C-3)

32-116

32-117

$32-130$

SINPSON, M. L. 13-1

SINS, JAMES R. (WX-4)

$13-56$

13-64

SNCH, A. K. 41-123

SINGHAM, SHERMLA BAITO (LS-2) 43-74

43-75
SINHA, DIPEN N. (MEE-11) 24-40

SINORADZKI, HELEN M. (IS-11) 17-22

SION, EDWARD M. 40-80

SIPOTKIN, KAPL (T-10)

$1-45$

$1-56$

32-118

SISSINGH, R. A.

20-21

SIVERTZ, M. B.

43-15

SIVIA, DEVINDER S. (T-11)

43-76

SKAGGS, BARBARA JAN (HSE-5) 22-8

SLANSKY, RICHARD C. (T-B)

43-60

43-77

43-78

SLATER, $W$. $E$. 43-15

SLAVIN, J. A.

40-13

SLENTZ, G. E. (MEE-5)

17.24

24-4

SLYUSAREVA, YE. D.

40.1

SMITH, BARBARA F. (CLS-1)

4-82

$4-97$

SMITH, BARHAM W. (ESS-5) 40-82

SMITH, C. 42-88

SMITH, C. A.

$4-27$

31-15

SMITH, DARRY B. (N-DOSG)

47-36

47-37
SMTH, DOUGUAS L (E-11)

3052

3273

SMITH, DOUGLAS L (MEE-11)

2427

2450

41-214

SMITH, E. J.

40-4

40-13

$40-41$

$40-60$

40.81

$40-83$

$40-93$

SMITH, H. A.

18-23

18-24

SMTH, HORACE V., JR. (AT-Z) 37.87

$37-88$

SAITH, JAMES

41-77

SAMTH, JAMES F. (CMS)

$4-81$

$31-60$

31-72

41-143

41-160

41-211

SMITH, JAMES L (CMS)

4-14

$4-86$

31-20

$31-22$

31.78

31.96

31-97

31-118

39-5

39-17

39.53

41.31

41-47

$41-48$

$41-76$

41-123

41-143

41-211

41-212 


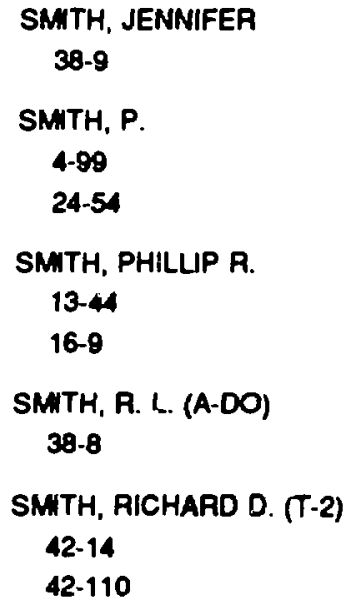

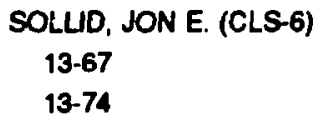

37-39

37-46

37-103

SPALEK, GEORGE (AT-3) 37-34

SPALL, $W$. DALE (LS-1)

1-21

$1-52$

SPANGLER, LEE H. (CLS-2) 498

SPANOS, J.

16-13

16-14
SPARKS, WARREN M. (X-5) 40-85 40-86 40-87 40-89

SPENCER, ROSS L (CTR-6) 20-41

SPERISEN, $F$. 42-117

SPICER, W. E. 4-54

SPICOCHI, KENNETH C. (WX-11) 41-149

SPIES, GEORGE H. (INC-4) 4-76

SPORE, JAY W. (N-9)

16-17

16-29

SPRIGGS, GREGORY D. (N-2)

7-3

16-12

47.31

SPRINGER, EDWARD A. (E-8) 32-97

SPRINGER, EVERETT P. (HSE-12) 14-11

14-48

35-8

SPRINGER, PENELOPE (INC-4) 1.15

SPRINGER, ROBERT W. (MST-H) 37-38

SPRINKLE, JAMES K., JR. (N-1) 47-18

47.19

47-40

SQUIER, G. T. A. 32.60

SRINIVASAN, S. (MEE-11)

9-8

10.2

$12-3$

$12-4$

12.5

SAINIVASAN-RAO, T. 13-4 


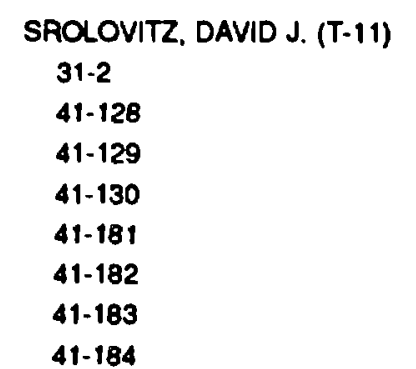

STACY, HOWARD L (M-1) $2 \cdot 17$

STACY, HOWARD L. (M-9) 2-26

$4 \uparrow-200$

STAFFORD, RONALD G. (HSE-10) 4-28

STALLINAS, E. A. (HSE-9) 14-49

STALLKAMP, $J$.

16-13

16-14

STANEK, PAUL W. (MST-6) 31.9

STANLEY, E. ANN (T-7)

$1-31$

32-74

STANLEY, JAMES T. (MST-5) 31-12

STANLEY, PATRICK M. (WX-4)

13-19

$13-29$

STANSBERRY, JOHN A. (ESS-8) 40-83

STAPLES, JOHN 37-111

STAPPAERTS, EDDY A. 4-47

STARNER, JOHN W. (INC-5) 42-67

STAROUIST, VIRGINIA L 20-8

STARAFIELD, SUMNER G. (T-6) 40-84

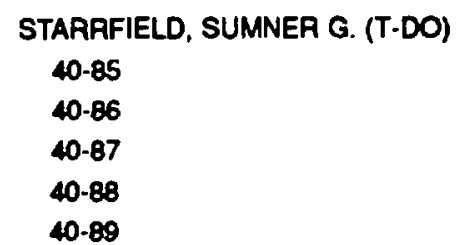

STAUDENMEIER, J. L. (CTA-6)

20-27

29-41

STAUDHAMMER, KARL P. (MST-6) 41-185

STAUDHAMMER, KARL P. (MST-7)

31-48

31-49

$31-99$

31.100

STAVERT, DOUGLAS M. (LS-1)

$1-22$

$1-58$

STEELE, H. S. (CLS-5)

4†-149

STEELE, RAYMOND DOUGLAS $(M-1)$ 13-65

STEIN, LELAND R. (T-3)

32-136

$\$ 1-213$

STEIN, PAUL R. (T-7)

41-119

STEIN, WILLIAM E. (AT-7)

13-74

37-34

37-35

37-66

STEINBERG, VICTOR (CNLS)

41-141

STEINER, J. L. (N-12)

16-28

$16-30$

STEINHOF. A.

4-7

41-3

STEINKE, ROBERT G. (N-6)

16-20

STEINKE, ROBERT G. (N-9) 16-17

STEINKRUGER, FREDERICK J. (INC-11) 42-67
STENCEL, J. R.

22-21

STEPANEK, JIRI

29-15

30-17

32-37

STEPANYUK, M. V. 40-1

STEPHENSON, GEPARD J., JP. (P-DO) 42-53

$43-28$

43.79

STEREN, L. B. 39-17

STEUERWALT, MICHAEL (X-7) 32-100

STEVENS, $J$.

16-13

16-14

STEVENS, MICHAEL F. (MST-5) 31-20

STEVENSON, ANITA P. (LS-A)

$1-9$

1.59

$1-64$

STEWART, C. C. (LS-A)

1-59

$1-64$

STEWART, DONALD S.

2-19

STEWAPT, GREGORY R. (MST-5)

31-22

31-78

STEWART, JAMES E. (N-1)

39-25

47-24

STEWART, ROBERT F.

2-14

STEWART, WALTER F. (A-4)

17.10

37-31

STINECIPHER, MARY M. (M-1)

2-20

STIRPE, DANTE (N-4)

47-20 


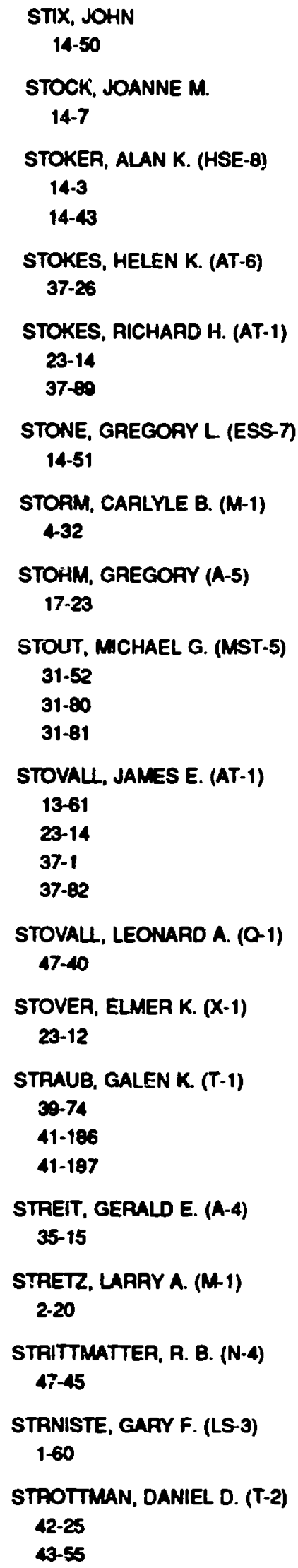

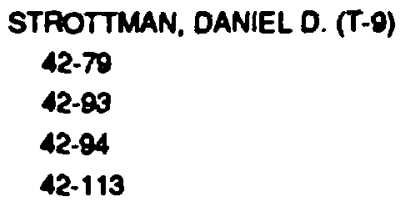

STUEWE, ROBERT B. (MP-6) 32-109

STUMPF, HENRY (N-9) 16-17

STUPIN, DAVID M. (MST-7) 24-52

STURGESS, L L (WX-5) 39-60

STUTZ, ROGER ALAN (ENG-4) $17-26$

32-121

SULLUVAN, JOHN (CLS-1) 32-16

SULLIVAN, L. HAROLD (N-6) 16-31

SUN, PENG-NIAN (INC-11) $4-40$

SUNIER, JULES $W$. (P-2) 18-30

SUTHERLAND, C. DEXTER (ESST) 40-101

SUTTON, JOHN G. (AT-8) 37-14

SVATON, ERNIE M. (AT-6) 37-54 41-100

SWAIN, GEORGE R. (MP. 14) 37-24 $37-90$
SWAN, J. E. 41-206

SWANBERG, CHANDLER 20-7

SWANSON, BASH I. (INC-4) 2-21

$4-1$

$4-2$

420

SWAATZ, BLANA K. (T-7) 32-122

SWIFT, GRECOAN W. (P-10)

8-1

$10-3$

13-26

13-47

$13-86$

41-188

SYDCPIAK, STEPHEN J. (P-A) 32-128

SZE, D. K. 30-15

SZE, ROBERT C. (CHAS5) 24.53

SZE. ROBERT C. (CLS-5)

$$
\begin{aligned}
& 4-9 \\
& 4-\infty \\
& 24-54 \\
& 31-105
\end{aligned}
$$

szrmanska. J. 42-11

TABOR, JAMES E. (C-10) 32.123

TADOEUCCI, T. N. (MPP-10) 42.114

TAFOYA, MDSE I. (Q-11) 13-7

TAGGART, DANIEL P. (CTR-3) 20-10 $22-38$

TAKAHASHI, KAZUE (ESS-O) $40-50$ 


TAKEDA, HARUNORI (AT-6)
$13-67$
$37-34$
$37-35$
$37-91$
$37-106$

TAKESHITA, TORU (CMS) 31.101

TAKIGAWA, MASASHI (P-10) 41.87

$41-120$

TALAGA, R. L.

40-26

40-63

TALBERT, W. L., JR. (INC-11) 42-67

42-115

TALBOTT, D. V. (Q-6) 13-44

TALLERICO, PAUL J. (AT-5) 37-92 37-93

TALLEY, THURMAN L (T-2) 29-37 $42-116$

TALLMAN, CHARLES A. (CLS-5) 4-100

TAN, TAI HO (M-6) 13-65 41-189

TANAKA, NOBUYUKI (MP-10) 39-64

42-117

TANG, P. K. (X-4) 2-22

TANG, P. K. $(X-7)$

2-23

2-24

TANG, SHERMAN (P-2) 42-98

TANNER, ROGER L. $9-1$ 14-14

TAPE, JAMES W. (N-4) 47-41

TATSUMI, MASAHIRO (LS-7) 1.66
TAYLOR, ANTOINETTE J. (CLS-5)

4-9

4-100

4-101

13-31

24-44

39-58

TAYLOR, ANTOINETTE J. (CLS-9) 24-55

TAYLOR, IVAN K. (MP-13) 31-98

TAYLOR, JOHN W. (ADDRA) 31-120

TAYLOR, R. DEAN (P-10) 39-63

41-190

TAYLOR, R. DEAN (WX-12) 4-87

TAYLOR, S. 39-60

TAYLOR, THOMAS N. (CLS-2) 31-102

TEITELBAUM, L 42-61

TEMMERMAN, W. M. 39-80

TEO, BOON-KENG $4-8$

TEPEAMEISTER, IGOR 38-9

TERRY, DARAELL A. (ESS-4) 38-2

TESANOVIC, ZLATKO B. (T-11) 13-68

41-127

41-191

41-192

TESMER, JOSEPH R. (P-10)

4-68

4-81

4-94

4-102

31-36

31.62

31-72

31-103
TESMER, JUDITH G. (LS-3) $1-60$

TESTEA, JEFFERSON $w$.

14-57

21-5

THALER, R. $M$.

41-28

42-16

42-17

42-20

THAYEA, D. R. (P-14)

24-37

24-56

THAYER, GAPY R. (S-4)

9-6

THEIL, ED

32.101

THERMAN, RUSSEL 32-108

THIESSEN, HENPY A. (MP-10) 37-23

THIESSEN, HENFY A. (MP-14) $37-24$

$37-68$

$37-24$

37-95

$37-96$

37.97

$37-98$

37-103

THIESSEN, HENRY A. (MP-DO) 37-90

$43-80$

THIYAGARANAN, PAPPANNAN $1-25$

THODE, LESTER E. (ADDPA)

$13-8$

37-43

$37-44$

37-104

THOMAS, K. E. (N-A) 47-41

THOMAS, KIMBEALY W. (INC-11) 35-2 35-16 
THOMPSON, JOE D. (P-10)
4-14
4-17
4-88
$31-13$
31-57
31-97
31-113
31-118
39-35
39.50
$39-53$
$39-68$
41-38
41-76
41-111
41-193
41-212
42-118
43-11

THOMPSON, JOE L. (INC-11) 35-17

THOMPSON, JOSEPH

41-77.

THOMPSON, M. CLARK (MEE-11)

24-2C

24-33

THOMSEN, MICHELLE F. (ESS-8)

40-16

40-42

40-51

40-52

$40-83$

40-91

40-92

THORN, CHARLES T. (A-6) 32-124

THORNE, KIP S.

41-194

THORNE, R. M.

40-93

THULLEN, PHILIP (ERAMFE) 44-2

TIAINEN, TUOMO J. (CMS)

31-85

31-104
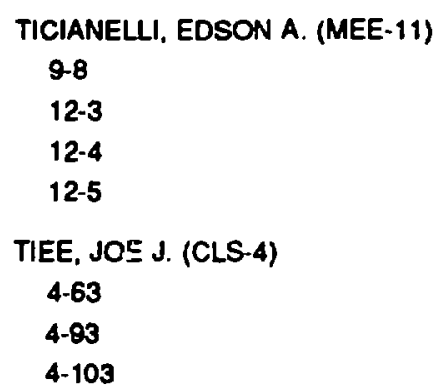

TIKHONOV, A. A. 40-1

TIMOFEYEV, P. V. 40-1

TINCKNELL, M. 42-61

TIPPINS, BRAD

42-11

TISINGER, RICHATD M., JP. (N-4)

47-39

47-42

47-43

TJON, J. A.

43-8

TOBEY, ROBERT A. (LS-1)

1.8

1.9

TOBEY, ROBERT A. (LS-3)

$1-10$

TOEVS, K. E. (LS-1)

1-32

TOKAA, ROBERT L. $(X-1)$

37-44

41-195

TOLMIE, DONALD E. (C-5)

32-125

TOMLINSON, ROBERT D. (E-10) 32-65

$32-108$

TOMS, K. W.

13-77

TOMUSIAK, EDW.ARD L 42-43

TONG, KWON-ON

13.73

TONKS, DAVIS L. :T-1\}

41-196
TOAIKACHVIL, MILTON S.

39-53

TORNEY, DAVID C. (T-10) 32-126

TORNOW, $w$. 42-57

TORSTENFELT. N. BORJE 4-90

TOVAR, $M$.

4-88

$39-17$

TRAINOR, MARY TTOODARD (A-6) 17-27

32-127

TRANG, Q. H.

43-15

TRAVIS, JOHN R. (T-3)

$2-4$

TREASE, HAROLD E. (X-7) 32-82

TREASE, L L L (AT-3)

24-16

$37-40$

TRENT, BRUCE C. (ESS-5)

$14-52$

14-55

32-84

TREWHELLA. J. (LS-2)

1-61

TREWHELLA, J. (LS-7)

1.24

$1-62$

TRIAY, INES R. (INC-1!) $4-90$

TRKULA, MITCHELL (MST-3) 44-9

TRUGMAN, STUART A. (T-19) 41-197

TRUHLAR, DONALD G.

4-11

4-12

TRURAN, JAMES $W$.

40-85

40-86

40-8?

40-89 
TSIRONIS, G. P.

41-198

TSUAUTANI, B. T.

40-93

TUCKER, ROBERT C.

32-6

TUCKER, ROBEAT W. (CNSS) 18-35

TUNG, CHANG-SHUNG (T-10)

1-57

$1-63$

TURLEY, $D$.

13-1

TURLEY, $W$. D.

24-30

TURNER, THOMAS P. (CLS-6) 41.148

TUSZEWSKI, MICHEL G. (CTR-3) 29-10

29-36

$29-38$

29-39

44-13

45-8

TWINING, BRUCE G.

$17-1$

TZENG, $Y$.

42-60

ULAM, STANISLAW M. (T-DO)

$1-48$

ULBRICH, CARLTON

14-23

ULLMAN, LOHN (P-3)

41-135

42-104

ULLMANN, J. L.

41-5

ULLMANN, J. L. (P-3)

42-57

42.75

ULMER, M. $P$.

40-54

UNKEFER, CLIFFORD J. (INC-4)

$1-65$

4-106
URHEIM, J.

43-15

USSERY, LARRY E. (N-2)
42-119
43-81

VACCARO, H. S. (N.4)

47-44

VALDEZ, FRANK O. (HSE-5) 22-5

VALDEZ, JOSEPH G. (LS-2) 1-21

VALDEZ, Y. E. (LS-1)

1-32

VALENTINE, GREG A. (ESS-5)

14-54

VALENTINI, JAMES $J$, (CHM-4) 2-2

VALLADON, $M$. $31-60$

VAN BERG, $R$. 43-1

VAN DALEN, G. J. 41.62

43-1

VAN DER SLUYS, WILLIAM G. (INC-4) 4-104

VAN DILLA, M. A.

$1-11$

VAN DYCK, OLIN B. (MP-13) 43-56

VAN EIJK, C. W. E. 39-60

VAN ETTEN, DON M. (HSE-8) 22-7

VAN GULICK, LEONARD A. (WX-11) 13-69

VAN HAAFTEN, F. W. (AT-5) 37-33

VAN HULSTEYN, DAVID B. (P-14) $1-55$

42-109

VAN RIPER, KENNETH A. $(X-\bar{\lambda})$

40-94

40-95
VAN ZEE, ROGEA D.

4.98

VANDERBORGH, NICHOLAS E. (MEE-11 9-4

VANIMAN, DAVID T. (ESS-1) 35-18

40-86

40-87

$40-88$

VANINETTI, JOE (P-14)

24-23

VANNINI, MATTEO

4-99

24.54

$31-105$

VEAL, B. W.

31-57

VEERASINGAM, $R$ 29-33

VEESER, LYNN R. (P-14)

13-70

24-19

24-57

VELGAKS, M. J.

41-7

VERETENKIN, E.P. 40-1

VEAMUL, V.M. 40.1

VICENTE-VACAS, M. J.

42-93

$42-94$

VICK, GAEG S

147

VIEIRA, DAVID J. (INC-11) 42-119

VIER, D. C 4-88

39-17

VIGIL, BENNY MANUEL (C-6) 32-129

VISSCHER. PIETER B. 41-190

VISSER, MATT (T-B)

41-19 


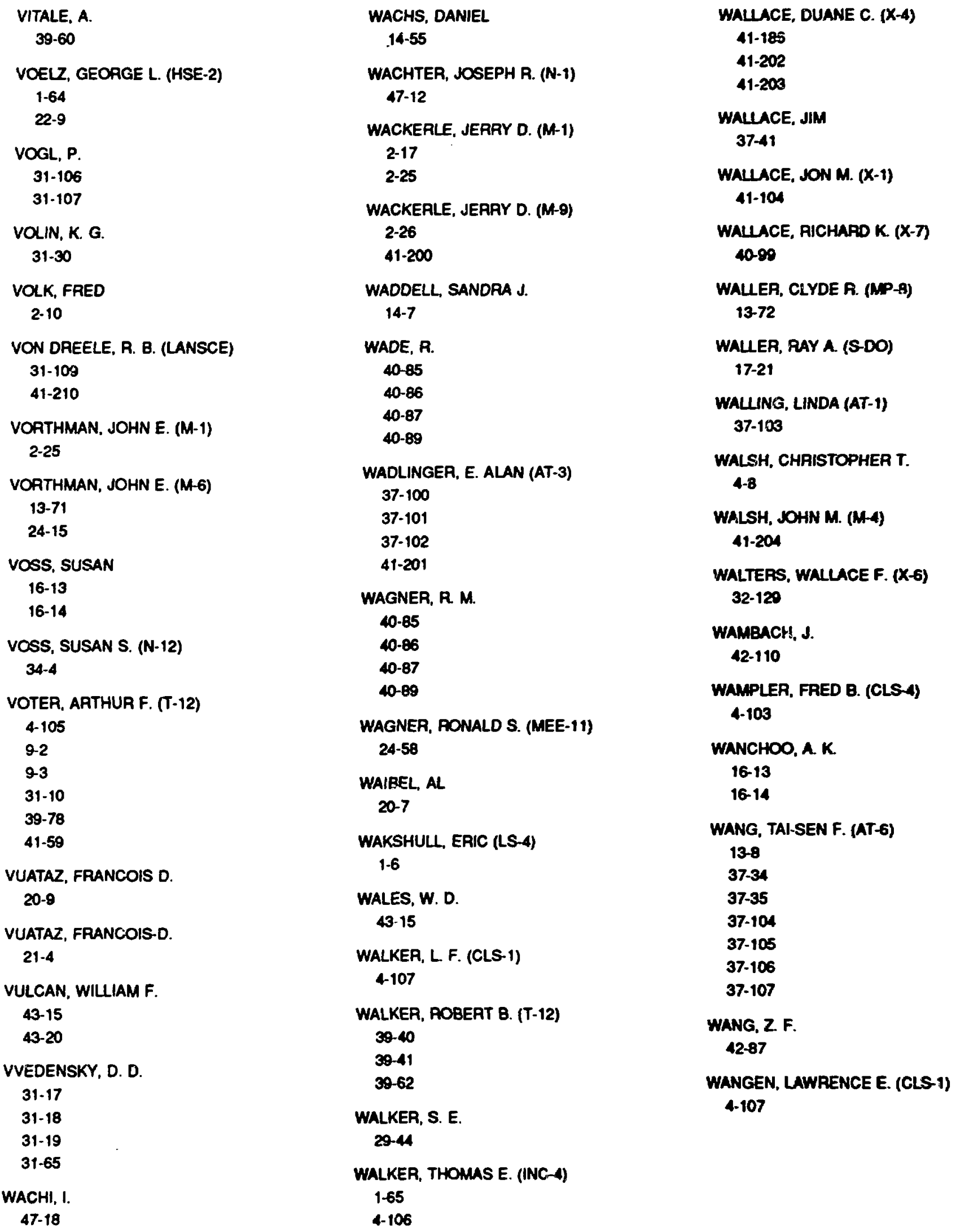




WANGLER. THOMAS P. (AT-1)
$13-61$
$23-14$
$37-1$
$37-28$
$37-80$
$37-108$
$37-100$
$37-110$
$37-111$
$41-86$

WANTUCK, PAUL JOSEPH (CLS-8) 4108 $13-73$

WARK, DAVID L (P-3)

40-1

$40-100$

$41-200$

WARK, J. S.

23-5

41-205

WARNES, R. H. (M-4)

2-3

41-55

WARNOCK, TONY T.

43-30

WARREN, JOHN E. (INC-11) 42-36

WARREN, JOHN L (AT-6) 37-26

WARREN, JOHN L. (HSE-7) 37-112

WARREN, ROGER W. (AT-7)

13-74

13-75

$37-34$

37-35

37.113

$37-114$

WASSERMAN, HARVEY J. (C-3) 451

$32-14$

32-117

$32-130$

$32-131$

WASSERMAN, HARNEY J. (INC-A) 2-14

WASSON, O. A. 42-75

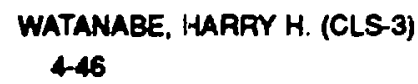

WATKINS, DAVID E. (CLS-6) $13-50$

WATSON, $D$. 47-40

WATSON, D. L 42-80

WATSON, DAUGLAS L (MP-10) 42-36

WATSON, J. MM. (AT-7) 37-34 37.35

WATT, ROBERT G. (P-4) 44-14

WEATHERSEIY, S. P.

$$
\text { 41-47 }
$$

41-48

WEBER, MICHAEL E. (CLS-6) 13.50

WEBER, PAUL G. (CTA-2)

20-33

$20-40$

$20-4$

20-45

44-12

44-15

WEBER, PAUL G. (CTR-8) 44-14

WEBSTER, R. B. (CTR-3) 20-27

WEBSTER, R. B. (CTR-6) 20-10

$20-36$

$20-41$

WECHSLER, M. S. (MP-3) 42.26

WEEKS, DAVID E. (T-12) 30-70

WEERTMAN, JOHANNES (MST-5) 31.108 $41-206$

WEH, $R$. 47.13

WEHRSE, RAINER $40-80$
WEI. S. P. 37-65

WEILAND, T. (AT-6) 37-27

WEIIAND, T. 32-35

$37-13$

WEIMACHT, DANIEL J. (MEE-13) 13.7

WEINBERGER, $P$.

4-15

39-70

$39-80$

WEINFELD, ZEEV 42-120

WEIR, DIANE R. (ADP-2) 32-132

WEISS, $S$. 42-61

WELDON, DAVID M. (CTR-2) 44-15

WELLS, GENE L (CMS) 41-193

WELSH, ROBERT E.

43-15

43-20

WELTMAN, JOHN J. (CNSS) 17-28

WENDER, STEVE A. (P-3) $13-76$

42-57

$42-67$

42.101

42-102

47-17

WENDPOFF, BURTCN (T-7)

16-32

$32-40$

32-133

WENES, GEEFT (T.5)

32-134

42-5

$42-63$

42-121

42-122

WENK, H. R. 31-101 


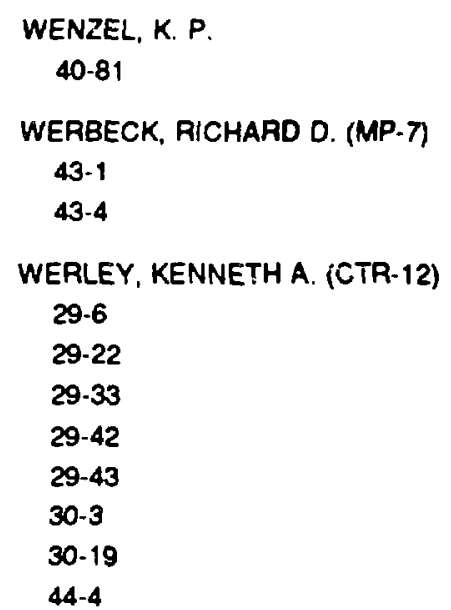

WERNER, HANS-JOACHIM 39-48

WEST, GEOFFREY B. (T-8)

40-71

40-72

$40-73$

41-207

43-14

43-58

43-59

43-60

43-82

WEST. JAMES T. (X-6) 32.135

WEST, KAREN A. (WX-4) 35-19

WEST, MIKE H. (MST-13)

$$
\text { 4.109 }
$$

47-34

WESTERVELT, DONALD R. (CNSS) $18-36$

WESTERVELT, R. (MP-4)

43-1

WESTERVELT, R. T. (E-11) 32. 109

WESTON, $\mathbf{G}$. $42 \cdot 117$

WHARTON, W. R. (LS-4)

$1-6$

WHEATLEY, JOHN C. (CMSIP-10)

10-3

13-26
WHEELER, KYLE T. (C-2)
$17-29$
$32-128$

WHETTEN, JOHN T. (ADERA)

17-1

18-6

WHITAKER, DEAN H. (AT-2) 37-87

WHITAKER, ELLIS RAY (P-9) 37-78

WHITCOMB, BRUCE $M$.

13-77

24-37

WHITE, ANDREW B., JR. (C-3) 29-30

32-133

WHITE, ANDREW B., JR. (C-DO) 32-83

WHITE, BRUCE $W$. (N-6) 16-9

WHITE, O. HYWEL (MP-4)

43-1

43-56

43-83

43-84

43-85

WHITE, L.

16-13

16-14

WHITE, POGER H. 16-12

WHITLOCK, R. R. 41-205

WHITNEY, J. W.

14-20

WHITNEY, ROY R. 42-98

WHITTEN, $C$. 43-1

WHITTEN, C. A. 42.117

WHITTY, WILLIAM J. (N-4) 47-45

WHYLEY, ROBERT $J$.

43. 15

43-20
WIEGEL, FREDEAIK W. 1.19

WIEMAN, $H$. 42-61

WIG, GARY 47-11

WILCZEK, FRANK 43-16

WILDE, DALE K (MEE-11) 13-78

WILDER, L. (M-6) 24-15

WILDER, MARK E. (LS-4) $1-8$

1.9

WILDI, PAUL 13-57

WILHELM, DIRK (N-8) 27-2

WILHELM, RICHAPD C. (MST-3) 30-7

WILHELM, RICHAPD C. (MST-9) 20-13

WLHELMY, JERPY B. (INC-11) 4-7 41-3

WILKE, MARK D. (P-15) 24-23

24-59

24-62

$37-65$

WILKEN, 8.

40-16

40-51

40-52

WILKEASON, J. F. (P-3) 39-11

40-1

40-100

41-208

41-200

WILKINSON, C. A. (NP-4) $40-26$ $10-63$

WILKINSON, GREGG S. (HSE-14) 22.10 
WILLIAMS, A. L

30-8

WILIAMS, ARTHUR (LANSCE)

$4-41$

31.109

$41-210$

WILIAMS, JOEL M. (MST-T)

17-30

31-75

31-110

$31-111$

31.112

31-116

WILIAMS, MAAY C. (HSE-9)

14-42

WILLIAMS, MICHAEL D. (A-4)

14-56

32-138

WILLIAMS, RAY E. (ESS-4)

14-57

WILLIAMS, ROBERT A. (MP-4) 40-63

WILIAMS, ROBERT A. (N-2) 43-4

WILIANS, ROBERT E.

40-85

40-86

40-87

40-89

WILLIS, JEFFREY O. (P-10)

$31-13$

31-97

$31-113$

39-53

41-32

$41-47$

$41-48$

41.76

$41-211$

$41-212$

WILIS, JOARY (P-10) 41-137

WILLMS, RICHARD S. (MST-3) 20-2

$30-12$

WILS. JOHN M. (T-1) 41.186
WILLSON, LA.

40-14

40-15

WILSON, G. E.

26-1

WILSON, MAHLON T. (AT-DO)

37-1

43-86

WILSON, MICHAEL A.

4-110

WILSON, NORMAN G. (AT-2)

37.115

WILSON, ROBERT $D$.

14-58

WILSON, STEVE L. (MP-4)

43-4

WILSON, TIMOTHY L (0-6)

32-136

41-213

WILSON, WILLIAM B. (T-2)

18-37

$27 \cdot 3$

42-13

$42 \cdot 123$

WINCHENBACH, G. L.

24-25

WINDISH, DAVID

4-47

WING, G. MLTON (C-3)

32-47

$32-48$

WINJE, R.A. (MP-14)

37.116

WINKLER, MAX A. (M-S)

31-70

41-160

WINN, KENNETH R. (CLS-4)

4-108

WINNINGHAM, J. DAVID

40-16

40-51

40.52

WINSKE, DAN $(X-1)$

40-33

40-36

40-42

$45-1$
WINSLOW, ORVILLE G. (P.9) 2460

WINTER, ROLF $G$.

43-15

43-20

WINTERHALTER, D. 4060

WIPF, ANDREAS (T-8)

32-34

41-19

43-13

WIRE, MMKE S. (MST-5) 39-12

WISER, H. L. 40-38

WOFSY, CARLA

1-20

WOHLETZ, KENNETH H. (ESS-1)

$14-12$

14-22

WOHLLEBEN, D. (CMS)

31-69

31.114

41-143

41-180

WPHLLEBEN, $D$. 4.05

WaJCICKI, S. G.

43-15

WOLFSBEAG, KURT (INC-7)

$$
\text { 4-80 }
$$

35-13

WOLLMAN, ERIC R. (ESS-8)

$40-40$

WOLOSHUN, KEITH A. (MEE-13)

13-70

32-137

WOO, L

13-77

WOODALL, DAVID M.

34-4

WOCDAOW, LISA M. (HSE-5) 22.11

WOODRUFF. SUSAN B. (X-7) 32-70 
WOODRUFF, WILLIAM H. (INC-4)

4-21

4-76

4-111

WOODS, RICHARD (P-9)

37-78

41.164

WOOLHISER, C.

13.48

WORTH, G. T. (AT-2)

37-1

37-37

WRAY, WILLIAM O. (MEE-13)

49-2

WAIGHT, BRADFORD L (CTR-3)

29-10

29-36

30-10

44-6

44-13

45-8

WRIGHT, BRADFORD L (CTR-5) 30-20

WRIGHT, D. H.

42-120

WRIGHT, JAMES C. (A-5)

17-24

WRIGHT, S. C.

43-4

WROBLESKI, DEBRA A. (MST-7)

31-111

31-115

31-116

WU, C. C.

40-85

40-86

40.87

40-89

WULFF, W.

26-1

WURDEN, GLEN A. (CTR-12) 29-33

WURDEN, GLEN A. (CTR-2)

$29-44$

29-45

44-12

44-14

44-15
WYART, J. F.

413

WYSIN, GARY M. (MEE-11)

24-27

41-136

41-214

WrSOCKI, F. J. (CTR-5)

20-23

20-46

XU, YING-TING (INC-11)

$4-40$

$20-41$

YAMADA, TETSUUI (ESS-5)

14-28

14-29

14-59

$14-60$

14-61

14-62

$32-138$

YAMAGAM, $T$.

40-32

YAMANISHI, TOSHIHIKO

20-2

29-35

30-21

YAMMZAKI, AKIO (LS-DO) $1-66$

YAN, YITON T. (X-10)

13-80

37.117

41.215

YANG, A-B.

31-57

YANG, MING-JEN (P.DO)

32-130

$41-216$

YANTS, V. E.

40.1

YARBPO, STEPHEN L (MST-12)

3-4

3-5

47.3

YARIV, $Y$. 41-166

YATES, B. $W$. 30.5
YATES, GEORGE J. (P-15)

2437

2461

$24-62$

YAVORNIK, EDMARD J. (MEE-12) $30-22$

YEARMOOO, DEIDAA D. (MST-12) 17-31

YEH, J. J.

4-54

YEN, S. P. 24-33

VETHIRN, M (P-D)

41-217

$41-218$

YODK, G. B.

40-26

$40-63$

YOO, S. H.

328

$42-60$

YORK, GEOAGE W. (CLS-5)

41.140

YOSHIDA, ATSUMASA (ESS-9)

$40-32$

YOSHIDA, $H$.

202

$20-36$

30-21

YOUNG, ANSLIE T., JA. (MST-7) 23-15

YOUNG, DAVID T. (ESS-O)

40.6

YOUNG, KATHLEEN M. (MST-13) 31-117

YOUNG, LLOVD M. (AT-1)

$13-61$

$37-35$

37.75

$37-82$

37-118 


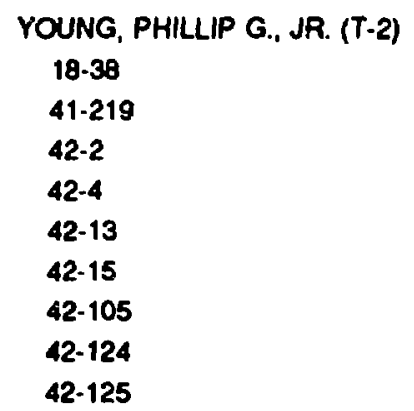

YUAN, VINCENT W. (AT-2) 39-25

42-11

ZABRISKIE, JOHN 39-60

ZAHAT, JOHN D. (X-5)

4-18

ZAKHOROV, YU. I.

40.1

ZAKAZEWSKI, WOJCIECH J. (T-11) 43-87

ZANDER, M. (AT-3)

24-16

$37-40$

ZARDECKI, ANDREW (T-DO)

37-41

40-38

41-39

41-220

41-221

41-222

41-223

ZATSEPIN, G. T.

40-1

ZAUGG, THOMAS J. (DRA-BPO) 37.75

ZBILUT, JOHN P.

$1-67$

ZECKER, $P$. 42.57

ZEMACH, CHARLES (T-DO) 32-53

ZEMACH, CHARLES (T-DOT) 43-33

ZHU, $X$. 42.11
ZIETLOW, MIRIAM H.

4-20

ZIMMERMANN, BARBARA $H$. (INC-4) 4112

ZIMMERMANN, EUGENE L. (P-1) 13-81

ZIMMERMANN, EUGENE L. (WX-4) 13-19

ZINN, JOHN (ESS-7) 40-101

ZIOCK, H. J. $42-60$

ZIOCK, HANS J. (P-2)

43.20

ZIOCK, HANS J. (P-3)

49-15

ZIOCK, K. O. H.

$42-60$

ZIRNGIEBL, ERBERHARD (MST-5)

31-118

30-12

30-37

ZIRNGIEBL, ERBERHARD (P-10) 30-55

ZOCCO, THOMAS G. (MST-5)

31-43

31- 45

$31-46$

$31-47$

31-119

200COU, A.

30-60

ZOCHER, MARC (ENG-8)

17-28

$32-121$

ZOCKER, ROY W. (MST-5)

34-5

ZUBER, N.

26-1

ZUKAS, EUGENE G. (MST-5)

31-37

31.120
ZUMBAO. J. D.

328

42-36

42-87

42.89

42-92

42-107

ZUREK, ANNA K. (MST-5)

31-121

31-122

ZUAEK, WOJCIECH H. (T-6)

40-9

$40-46$

$40-70$

40-102

40-103

41-194

ZWMCKL, RONALD D. (ESS-8) 40-13

40-81

ZWIER, TIMOTHY S.

4-oe

ZYSLER, $A$.

3017

ZVOLOSK, DEOAGE A. (T-3)

21.6

35-20 
NUMERIC INDEX

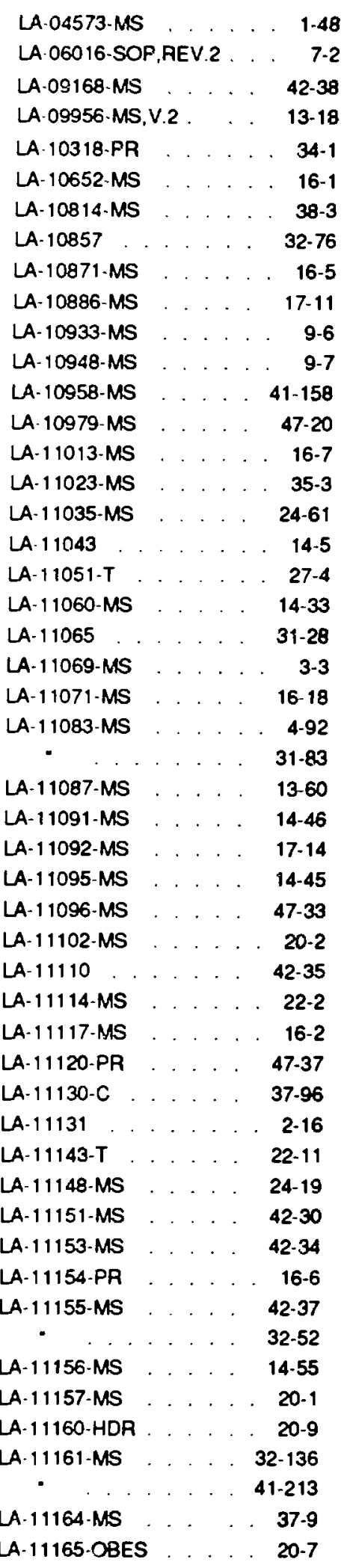

\begin{tabular}{|c|c|c|}
\hline & . & \\
\hline LA-11168-MS & . & 1.33 \\
\hline LA.11169T & & $14-52$ \\
\hline LA-11170-MS & & $31-117$ \\
\hline LA-11171-OBES & . . & 14-31 \\
\hline LA-11172-MS & . & $13-14$ \\
\hline LA-11175-MS & 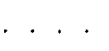 & $44-10$ \\
\hline LA-11176-OBES & & $20-8$ \\
\hline LA-11178-MS & & $23-6$ \\
\hline LA-11179-MS & . & $16-16$ \\
\hline LA-11180-P!? & . & $16-25$ \\
\hline LA.11181 & . . & $17-12$ \\
\hline LA-11182-T & . . & $41-153$ \\
\hline LA-11188-PA & . . & $16-24$ \\
\hline LA-11189-MS & $\ldots$ & 37.93 \\
\hline LA-11190-MS & $\cdots$ & $32-15$ \\
\hline LA-11191 & . . & $3-4$ \\
\hline LA-11192-MS & . . & $41-52$ \\
\hline LA-11196-MS & . & 47.4 \\
\hline LA-11198-MS & $\cdots$ & $32-17$ \\
\hline LA-11199-MS & $\ldots$ & $32-38$ \\
\hline a & . & 41.54 \\
\hline LA-11200-MS & . & $40-101$ \\
\hline LA-11202-T & . & $31-79$ \\
\hline LA-11203-MS & $\cdots$ & $29-19$ \\
\hline LA-11204-MS & $\cdots$ & $32-81$ \\
\hline LA-11206-MS & 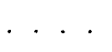 & $16-8$ \\
\hline & . & $32-57$ \\
\hline LA-11207-MS & . & $13-s$ \\
\hline LA-11208-MS & . & $16-17$ \\
\hline LA-11209-MS & & . 14-3 \\
\hline LA-11210-PR & & $14-42$ \\
\hline LA-11212-MS . & $\cdot$ & $29-22$ \\
\hline LA-11213-T & . & $42-21$ \\
\hline LA-11216-MS & . & $32-98$ \\
\hline LA-11217-PR & & $46-5$ \\
\hline LA-11218-MS & $\cdot$ & $18-31$ \\
\hline LA-11219 & 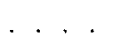 & $47-29$ \\
\hline LA-11220-PR & 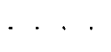 & $46-3$ \\
\hline LA-1 1223-PR & & $35-17$ \\
\hline LA-11224-MS & . & $35-20$ \\
\hline - . . & . & $21-6$ \\
\hline LA-11225-MS & . & $18-5$ \\
\hline LA-11226-MS & . & $18-10$ \\
\hline LA-11228-MS & & . 41.112 \\
\hline • & & $18-25$ \\
\hline LA-11229-MS & & 47.24 \\
\hline LA-11232-MS & & $39-74$ \\
\hline LA-11233-T & & $42-106$ \\
\hline LA-11234-MS & & $18-18$ \\
\hline LA-11235-PR & & 1.35 \\
\hline
\end{tabular}

\begin{tabular}{|c|c|c|c|c|}
\hline LA-11236 & $\cdot \cdot$ & . & $\cdot$ & $22-8$ \\
\hline LA-11237-MS & . & . . & & 47.21 \\
\hline . & . . & . & - & $24-31$ \\
\hline LA-11238-ASS & . & . & 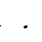 & $18-3$ \\
\hline LA-1 1239-MS & . & . & ${ }^{\circ}$ & $2-11$ \\
\hline LA-1 1243-MS & . . & . . & $e^{\circ}$ & $24-6$ \\
\hline LA-1 1246-MS & . & . . & & $35-2$ \\
\hline LA-11247-MS & . . & . . & & $17-25$ \\
\hline LA-11248-C & . . & . . & . & 41-125 \\
\hline LA-11249-PR & .. & . & . & $16-26$ \\
\hline LA-11250-M & . . & . & · . & . $\quad 49-2$ \\
\hline LA-11251-MS & . . & - & . & $19-2$ \\
\hline LA-11252-MS & - . & . & . & $13-44$ \\
\hline LA-11253-MS & . . & . & . . & $41-10$ \\
\hline LA-1 1254-MS & . - & . & . . & $16-19$ \\
\hline LA-11256 & . & . & . . & 4-109 \\
\hline LA-1 1257-PR & . . & . & • & $22-6$ \\
\hline LA-11261-MS & - & . & . & $1-34$ \\
\hline LA-11262-MS & . & ${ }^{\circ}$ & 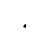 & $18-29$ \\
\hline LA-11263-MS & . . & . & . & $37-102$ \\
\hline - & . . & . & . & $41-201$ \\
\hline LA-1 1264-MS & . . & . & 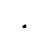 & $18-7$ \\
\hline LA-1 1265-MS & . . & . & . & $37-59$ \\
\hline LA-11267-MS & . . & . & - . & 41-165 \\
\hline LA-11271-MS & . . & . & . & $3 t-116$ \\
\hline LA-11272-MS & . . & . . & - . & $32-115$ \\
\hline LA-11273-T & - & . & $\cdot$ & 41-105 \\
\hline LA-1 1274-PR & - & . & . & $34-3$ \\
\hline LA-11275-MS & $\cdot \cdot$ & . . & . & $47-15$ \\
\hline LA-11276-MS & . . & . . & - & $14-32$ \\
\hline LA-1 1277-MS & . . & . . & . & $47-25$ \\
\hline LA-11278 & . . & . - & . & $18-19$ \\
\hline LA-11279-MS & $\cdot$ & . . & - & $18 \cdot 35$ \\
\hline LA.11280-MS & . . & . . & . & $18-20$ \\
\hline LA-1 1284-MS & .. & . . & - & $13-78$ \\
\hline LA-11285-MS & . & $\therefore$ & . & $18-4$ \\
\hline LA-1 1286-MS & $\cdot$ & . . & . & $41-135$ \\
\hline LA-11288-MS & $\cdot \cdot$ & . & . & $40-62$ \\
\hline LA-11289-MS & . . & . . & . & $35-18$ \\
\hline LA-1 1292-MS & . & . . & . & $35-7$ \\
\hline LA.11293-MS & . & . . & . & $35-5$ \\
\hline LA-11295-SR & . . & .. & . & $37-49$ \\
\hline LA-1 1296-MS & $\cdot$ & . . & . & $23-5$ \\
\hline LA-1 12S7-MS & . & . . & . & $4-107$ \\
\hline LA-11298 & - . & . . & - & $3-6$ \\
\hline LA-11300-P & $\cdot$ & . . & . & . 45-1 \\
\hline LA-11301-C & $\cdot \cdot$ & . . & . & $24-26$ \\
\hline LA-11306-ENV & • & . . & . & . $22-1$ \\
\hline LA-11309-PR & - & . . & . & . $34-2$ \\
\hline LA-11309-MS & . . & & · & . 1.21 \\
\hline LA-11312-MS & . & & . & $24-42$ \\
\hline
\end{tabular}




\begin{tabular}{|c|c|c|}
\hline US & - & 38 - \\
\hline LA-i1314-MS & $\ldots$ & $18-3$ \\
\hline LA-11315-MS & 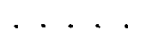 & $18-3$ \\
\hline LA-11316-MS & $\cdots$ & $47-35$ \\
\hline LA-11318-MS & ... & $18-22$ \\
\hline LA-1 1319-MS & $\cdots$ & $18-21$ \\
\hline LA-11320-MS & $\ldots$ & $13-7$ \\
\hline LA-11321-MS & . . . & $24-18$ \\
\hline LA-11322-MS & $\cdots$ & $18-11$ \\
\hline LA-11323-MS & . . . & $14-24$ \\
\hline LA-11324-M & . . . & $32-137$ \\
\hline LA-11326-MS & $\ldots$ & $13-2$ \\
\hline LA-1 1327-MS & . . & $20-6$ \\
\hline LA-11329-MS & $\ldots$. & $32-99$ \\
\hline & $\cdots$ & $40-61$ \\
\hline LA.11330-T & & $42-74$ \\
\hline LA-11332-MS & $\ldots$ & $14-43$ \\
\hline LA-11333-MS & $\ldots$ & $14-41$ \\
\hline LA-11334-MS & $\ldots$ & 32.67 \\
\hline LA-11338-MS & 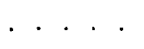 & $32-12$ \\
\hline LA-11339-PR & • & 43-56 \\
\hline LA.11340-ME & $\cdots$ & $47-7$ \\
\hline LA-11342-C. & $\ldots$ & $41-1$ \\
\hline LA-11344-MS & $\cdots$ & $18-8$ \\
\hline LA-11346-PA & $\ldots$ & $46-4$ \\
\hline LA-11348-MS & 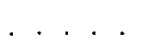 & $41-166$ \\
\hline LA-11349-MS & & $19-3$ \\
\hline LA-11352-MS & $\ldots$ & $2-22$ \\
\hline LA-1 1353-SOP & . . & $13-72$ \\
\hline LA-11354-MS & $\cdots$ & $35-10$ \\
\hline LA-11356-PR & $\cdots$ & $47-36$ \\
\hline LA-11358-PR & $\ldots$ & $16-22$ \\
\hline LA-11360-MS & . . . & $47-23$ \\
\hline LA-11361-MS & $\ldots$ & $18-2$ \\
\hline LA.1 1363-MS & $\cdots$ & 32.58 \\
\hline LA-11366-T & $\ldots$ & $31 \div 64$ \\
\hline LA-11367-MS & $\ldots$ & $42-28$ \\
\hline LA-11368-MS & $\ldots$ & $14-25$ \\
\hline LA-11371-MS & $\ldots$ & $32-31$ \\
\hline LA-1 1372-MS & ... & $32-32$ \\
\hline LA-1 1373-IIS & $\ldots$ & $24-14$ \\
\hline LA-11374-MS & $\cdots$ & $18-9$ \\
\hline LA-11375-MS & $\cdots$ & $18-13$ \\
\hline LA-11376-MS & . . & $18-1$ \\
\hline LA.11377.MS & $\cdots$ & $16-3$ \\
\hline LA-11381-MS & $\ldots$ & $4-28$ \\
\hline LA-11383-M & & $47-1$ \\
\hline LA-11384-MS & 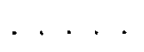 & 24-15 \\
\hline LA-11387.T & & $42-103$ \\
\hline LA. I1389.T & & $43-70$ \\
\hline LA-11390-PR & & $16-23$ \\
\hline LA-11391-MS & $\cdots$ & $14-57$ \\
\hline LA-11392-MS & $\cdots$ & $32-96$ \\
\hline LA-11393-C & & $43-41$ \\
\hline LA-11396-MS & & $38-9$ \\
\hline
\end{tabular}

\begin{tabular}{|c|c|c|}
\hline -11398-MS & . . & 35-1 \\
\hline A-11399-MS & & $18-17$ \\
\hline LA.11405 & 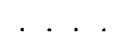 & $18-23$ \\
\hline LA-11406-MS & 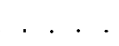 & 18.6 \\
\hline LA-11407-MS & 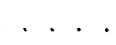 & $24-28$ \\
\hline LA-11409T & & $32-78$ \\
\hline LA-11410-MS & $\cdots$ & $19-4$ \\
\hline LA-11411-T & . . & 31.8 \\
\hline LA-11413-MS & $\ldots$ & $32-44$ \\
\hline LA-11414-MS & . . & 2.5 \\
\hline LA-11416-MS & & $41-167$ \\
\hline LA-11417-T & . & $42-66$ \\
\hline LA-11423-MS & . . & $43-81$ \\
\hline LA.11424-MS & $\ldots$ & $32-86$ \\
\hline LA-11425-T & & $32-120$ \\
\hline LA-11428-MS & & $i-32$ \\
\hline LA-11431-T & . & $40-25$ \\
\hline LA-11434-MS & . & $29-29$ \\
\hline LA-11435 & & 467 \\
\hline LA-11436-M,V.1 & $\ldots$ & $32-2$ \\
\hline - & . . & $39-2$ \\
\hline LA-11436-M,V.2 & . & $39-19$ \\
\hline - & & $32-28$ \\
\hline LA-11436-M,V.3 & 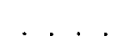 & $32-27$ \\
\hline LA-11436-M,V.4 & . & . 32.3 \\
\hline LA-11437-MS & . . & 22.3 \\
\hline LA-1 1439-MS & . & $35-15$ \\
\hline LA-11441-T & 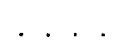 & $14-54$ \\
\hline LA-11443-PR & & $35-16$ \\
\hline LA-11450-MS & & 40.53 \\
\hline LA-11451 & $\cdot$ & $17-2$ \\
\hline LA-11453-OBES & 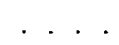 & $14-19$ \\
\hline LA-11454-MS & . . & $14-15$ \\
\hline LA-11455-MS & . & $32-56$ \\
\hline LA-1 1461-MS & & $22-4$ \\
\hline LA-11465-MS & . . & $32-131$ \\
\hline LA-11470-MS & . & $19-5$ \\
\hline LA-11475-MS & 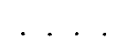 & $17-13$ \\
\hline LA-UR-82-1282 & 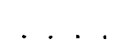 & $42-27$ \\
\hline LA-UR-83-0202 & . . & $41-37$ \\
\hline LA-UR-83-3186 & . . & $40-38$ \\
\hline LA-UR-84-0793 & . . & $1-51$ \\
\hline LA-UR-84-0987 & . & $40-55$ \\
\hline LA-UR-84-1007 & $\ldots$ & $39-69$ \\
\hline LA-UR-84-2637 & $\ldots$ & $42-60$ \\
\hline LA-UR-B4-2715 & $\ldots$ & $2-1$ \\
\hline LA-UR-84-3020 & 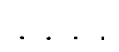 & $32-47$ \\
\hline LA-UR-84-3041 & . . & 32.49 \\
\hline LA-UR-84-3622 & . . & $32-79$ \\
\hline UA-UR-84-3982 & . & $37-99$ \\
\hline LA-UR-85-0049 & & $32-122$ \\
\hline LA-UR-85-0742 & & $2-12$ \\
\hline LA-UR-85-0769 & & 2.23 \\
\hline LA-UR-B5-0860 & & $2-2$ \\
\hline LA-UR-85-1088 & & 2.14 \\
\hline
\end{tabular}

LA-UR-85-1089

LA-UR-85-1114

LA-UR-85-1115

LA-UR-85-1122

LA-UR-85-1129

LA-UR-85-1138

LA-UR-85-1139

LA-UR-85-1140

LA-UR-85-1176

LA-UR-85-1201

LA-UR-85-1383

LA-UR-85-1390

LA-UR-85-1758

LA.UR-85-2180

LA-UR-65-2459

LA-UR-85-2460

LA-UR-85-2463

LA-UR-65-2510

LA-UR-85-2946

LA-UR-85-3071

LA-UR-85-31 10

LA-UR-85-3151

LA-UR-85-3182

LA-UA-85-3366

LA-UA-85-3550

LA.UR-85-3574

LA.UR-85-3877

LA-UP-85-3942

LA-UR-85-4037

LA-UR-85-4215

LA.UF-85-4022

LA.UR-85-4324

LA.UF-85-5000

LA.UR-85-5001

LA.UR-85-5002

LA.UR-85-5009

LA.UR-85-5004

LA-UR-85.5006

LA.UR-85-5008

LA-UR-85-5009

LA.UR-85-5011

LA-UR-85-5012

LA.UR-85-5013

LA-UR-85-5014

LA-UR-86-0036

LA-UR-86-0147

LA-UR-86-0223

LA-UR-86-0275

LA.UR-86-0355

LA-UR-86-0370

LA-UR-86-0418

LA-UR-86-0136

LA-UR-86-0471

LA.UR-86-0501

LA-UR-86-0627
41-17

2

2.

2

2.

$38-$

$38-$

38

$2-2$

2-1

47

$2-2$

33

$41-8$

$2-2$

2-1

$41-11$

24-2

$31-2$

14-1:

32-9

$1-1$.

$1-1$

$1-$

$22-4$

40:-

$45-1$

41-19

41-11c

$32-5 x$

31-16

39- 75

43-8:

$43-60$

43-35

437

43-78

43-61

43-27

$43-68$

43-67

$43-80$

43-86

43-73

39-52

$32-93$

$42-47$

$42-67$

$42-78$

$14-6$

$1-66$

$31-95$

27-5

$4-76$

31.90 
LA.UR.86-0694

LA. UR-86-0815

LA.UR-86-0878

LA.UR-86-0889

LA-UR-86-0916

LA.UR-86-0922

LA.UR-86-0925

LA-UR-86-0926

LA-UR-86-0941

LA-UR-86-0942

LA.UR-86-0943

LA-UR-86-0979

LA. UR-86-1008

LA-UR-86-1086

LA-UR-86-1151

LA.UR-B6-1160

LA-UR-86-1212

LA-UR-86-1250

LA-UR-86-1323

LA-UR-86-1405

LA.UR-86-1444

LA-UR-86-1476

LA.UR-86-1548

LA.UR-86-1606

LA-UR-86-1635

LA-UR-B6-1675

LA.UR-86-1728

LA-UR-86-1815

LA-UR-86-2044

LA.UR-86-2203

LA-UR-86-2243

LA.UR-86-2307

LA.UR-86-2319

LA.UR-86-2349

LA.UR-86-2353

LA.UR-86-2374

LA-UR-86-2475

LA.UR-86-2505

LA.UR-86-2515

LA-UR-86-2565

LA-UR.86-2638

LA.UR. 86-2642

LA-UR-8E-2705

LA.UR-86-2706

LA.UR-8E-2719

LA-UR-86-27:4

LA-UR-86-2759

LA.UR-86-2766

LA.UR-86-2779

LA-UR-86-2791

LA-UR-86-2793

LA-UR-86-2804

LA-UR-86-2845

LA-UR-86-2861

LA.UR-86-2890
39-20

10-1

24-53

37-51

4-91

37-53

32-91

37-52

24-24

1.27

42-96

37- 20

39-25

$1-58$

40-88

43-10

43-9

41-199

43-66

42-115

39-11

41-100

4-50

23-15

39-13

49.1

37.67

32-116
LA-UR-86-2910

LA-UR-86-2914

LA-UR-86-2963

24-37

LA-UR-86-3038

LA-UR-86-3049

LA-UR-86-3067

LA.UR-86-3115

LA.UR-86-3123

LA-UR-86-3124

LA-UR-86-3130

LA-UR-86-3177

LA-UR-86-3243

LA.UR-86-3244

LA-UR-86-3272

LA-UR-86-3275

LA-UR-86-3300

LA.UR-86-3319

LA-UR-86-3320

LA.UR-86-3329

LA.UR-86-3350

LA-UR-86-3382

LA-UR-86-3384

LA-UR-86-3386

LA-UR-86-3387

LA-UR-85-3407

LA-UR 86-3420

LA-UA-86-3459

ᄂ . UA-86-3480

LA-UR-86-3491

LA-UR-86-3517

LA-UR-86-3519

LA-UR-86-3520

LA-UR-86-3633

LA-UR-86-3682

LA-UR-86-3731

LA.UR-86-3762

LA-UR-86-3767

iA.UR-86-3789

LA-UR-86-3826

LA-UA-86-3862

LA-UF-86-3863

LA-UR-86-3865

LA-UR-86-3887

LA-UR-86-3889

LA-UR-86-3944

LA-UR-86-3978

LA-UR-86-3983

LA.UR-86-3993

LA-UR-86-4012

LA-UR-86-4045

LA-UR-86-4046

LA-UR-86-4088

LA-UR-66-4126

LA-UR-86-4127

LA-UR-86-4128
$40-90$

32-13

32-119

37-89

$39-65$

29-11

31-31

41-132

4-111

35-1

35-14

13-77

41.61

32.14

23-9

37-112

41-187

39-35

$31-23$

30-20

$40-24$

40-8

40-14

35-11

8.1

29-1

37-108

41-207

43-12

42-112

42-10

37.114

32-19

24-34

$17-4$

40-79

42-111

37-109

37-88

40-68

32-8

24-55

40-27

31.92

42-86

$39-29$

43-22

16-11

16-10

1.23

45-3

44-7

$29-30$
LA-UR-86-4146

LA-UR-86-4203

LA-UR-86-4218

LA-UR-86-4221

LA-UR-86-4245

LA-UR-86-4269

LA.UR-86-4306

LA-UR-86-4307

LA-UR-66-4331

LA-UR-86-4340

LA-UR-86-4341

LA-UR-86-4351

LA.UR-87-0006

LA-UR-87-0011

LA-UR-87-0012

LA-UR-87-0013

LA-UR-37-0015

LA-UR-87-0032

LA-UR-87-0039

LA-UR-87-0068

LA-UR-87-0083

LA-UR-87-0087

LA-UR-87-0157

LA-UR-87-0159

LA-UR-87-0166

LA-UR-87-0174

LA-UR-87-0184

L.UR-87-0123

LA-UR-87-0227

LA-UR-87-0231

LA-UR-87-0237

UA-UR-87-0297

LA.UR-87.0300

LA.UR-87-0303

LA-UA-87-0309

LA-UR-87-0310

LA.UR-87-0319

LA-UR-87-0342

LA-UR-87-0347

LA-UR-87-0415

LA-UR-87-0418

LA-UR-87-0432

LA-UR-87-0438

LA-UR-87-0446

LA-UR-87-0455

LA-UR-87-0501

$$
\text { - }
$$

LA-UR-87-0502

LA.UR-87-0512

LA-UR-87-0517

LA-UR-87-0554

LA-UR-87-0567

LA.UR-87-0605

LA-UR-87-0600

LA.UR-87-0633

$14-29$

41-118

$32-89$

$14-59$

$31-12$

2-15

13-14

$41-92$

40-81

$24-50$

$41-15$

$17-6$ 


\begin{tabular}{|c|c|c|}
\hline LA.UR-87-0644 & & $4-5$ \\
\hline LA.UR-87-0645 & . & \\
\hline LA.UA-87-0646 & . & $37-$ \\
\hline LA.UR-87.0647 & . & $37-11$ \\
\hline LA-UR-87.0659 & 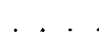 & $32-130$ \\
\hline LA.UR-87-0660 & $\ldots$ & 37.48 \\
\hline LA-UR-87-0666 & $\cdots$ & $37-5$ \\
\hline LA.UR-87-0667 & 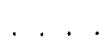 & 31 \\
\hline LA-UR-87-0679 & 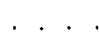 & $40-39$ \\
\hline LA-UR-87-0680 & & $41-29$ \\
\hline LA-UR-87-0688 & 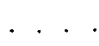 & $4-78$ \\
\hline LA-UR-87-0706 & $\ldots$ & $37-87$ \\
\hline LA-UR-87-0724 & . . & $37-6$ \\
\hline LA-UR-87-0728 & . . & $41-107$ \\
\hline LA-UR-87-0750 & . . & $24-47$ \\
\hline LA-UR-87-0763 & . . & $37-13$ \\
\hline LA-UR-87-0764 & . . . & $37-115$ \\
\hline LA-UR-87-0776 & $\cdots$ & 44-8 \\
\hline LA-UR-87-0779 & . . & $37-91$ \\
\hline LA-UR-87-0790 & $\cdots$ & $41-33$ \\
\hline LA.UR-87-0798 & . . & $39-15$ \\
\hline LA-UR-87-0809 & . & $37-47$ \\
\hline LA-UR-87-0810 & $\cdots$ & $37-56$ \\
\hline LA-UR-87-0811 & $\ldots$ & $32-113$ \\
\hline LA-UR-87-0814 & . . & 1.54 \\
\hline LA-UR-87-0815 &.. & $31-32$ \\
\hline LA-UR-87-0820 & $\ldots$ & $9-4$ \\
\hline LA-UR-87-0829 & $\cdots$ & $24-60$ \\
\hline LA-UR-87-0850 & . . & $31-87$ \\
\hline LA-UR-87-0861 & $\ldots$ & $37-110$ \\
\hline LA-UR-87-0862 & . . & $37-15$ \\
\hline LA-UR-87-0863 & ... & $37-38$ \\
\hline LA-UR-87-0864 & ... & $37-106$ \\
\hline LA-UR-87-0870 & . & $37-117$ \\
\hline LA-UR-87-0871 & . . . & $37-70$ \\
\hline LA-UR-87-0872 & . . . & $37-32$ \\
\hline LA-UR-87-0873 & . . . & 37.58 \\
\hline LA-UF-87 0877 & . & $38-4$ \\
\hline LA-UR-87-0882 & $\cdots$ & $40-23$ \\
\hline LA-UR-87-0888 & . . & $37-103$ \\
\hline LA-UR-87-0896 & $\ldots$ & $37-26$ \\
\hline LA-UR-87-0909 & . . & $37-39$ \\
\hline LA-UR-87-0911 & . . & $13-16$ \\
\hline LA.UR-87-0915 & . . & $37-94$ \\
\hline LA-UR-87-0925 & . & $37-66$ \\
\hline LA-UR-87-0936 & $\cdots$ & $41-62$ \\
\hline LA-UR-87-0963 & 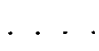 & $4-40$ \\
\hline LA.UR-87-0968 & $\cdot$ & $13-42$ \\
\hline LA-UR-87-0969 & & $13-79$ \\
\hline LA-UR-87-0986 & & $41-89$ \\
\hline LA-UR-87-1003 & 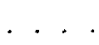 & $39-55$ \\
\hline LA-UR-87-1006 & . & 42.72 \\
\hline LA-UR-87.1022 & & $32-103$ \\
\hline LA-UR-87-1024 & & $4-72$ \\
\hline LA-UR-87-1025 & $\cdot$ & 4-10 \\
\hline
\end{tabular}

\begin{tabular}{|c|c|c|}
\hline LA-UR-87-1033 & & $12-2$ \\
\hline LA-UR-87-1041 & & \\
\hline LA-UR-87-1068 & 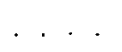 & $37-8$ \\
\hline LA-UR-87-1070 & 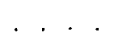 & $39-9$ \\
\hline LA-UR-87-1071 &. & $1-19$ \\
\hline LA-UR-87-1073 & . & $42-120$ \\
\hline LA-UR-87-1101 & . & 4-77 \\
\hline LA-UR-87-1111 & . & $4-3$ \\
\hline LA-UR-87-1117 & 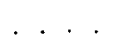 & $2-24$ \\
\hline LA-UR-87-1139 & & $42-24$ \\
\hline LA-UR-87-1155 & . & $24-56$ \\
\hline LA-UR-87-1156 & . & $37-78$ \\
\hline LA-UR-87-1163 & . & $41-80$ \\
\hline LA-UR-87-1175 & . . & $41-50$ \\
\hline LA-UR-87-1176 & $\ldots$ & $40-43$ \\
\hline LA-UR-87-1196 & $\ldots$ & $41-208$ \\
\hline LA-UR-87-1203 & . . & $40-69$ \\
\hline LA-UR-87-1238 & 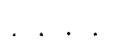 & $1-65$ \\
\hline LA-UR-87-1255 & & $40-73$ \\
\hline LA-UR-87-1259 & . & $40-47$ \\
\hline LA-UR-87-1270 & . . & $32-123$ \\
\hline LA-UR-87-1276 & $\ldots$ & $22-10$ \\
\hline LA-UR-87-1296 & $\ldots$ & $40-80$ \\
\hline LA-UR-87-1304 & $\ldots$ & $31-54$ \\
\hline LA-UR-87-1374 & . & $40-13$ \\
\hline LA-UR-87-1377 & $\cdot$ & $43-62$ \\
\hline LA-UR-87-1381 & $\ldots$ & $32-9$ \\
\hline LA-UR-87-1391 & $\ldots$ & $40-82$ \\
\hline LA-UR-87-1402 & . . & $31-121$ \\
\hline LA-UR-87-1410 & . . & $40-102$ \\
\hline LA-UR-87-1411 & . . & $40-9$ \\
\hline LA-UR-87-1429 & $\ldots$ & $32-33$ \\
\hline LA-UR-87-1437 & . & $32-107$ \\
\hline LA-UR-87-1446 & . . & 21.4 \\
\hline LA-UR-87-1450 & $\ldots$ & $1-9$ \\
\hline LA-UR-87-1488 & . . & $41-23$ \\
\hline LA-UR-87-1489 & . . & $18-24$ \\
\hline LA-UR-87-1492 & $\ldots$ & $1-40$ \\
\hline LA-UR-87-1494 & . . & $4-1$ \\
\hline LA-UR-87-1503 & $\ldots$ & $43-18$ \\
\hline LA-UR-87-1521 & $\ldots$ & $32-104$ \\
\hline LA-UR-87-1529 & . & $41-67$ \\
\hline LA-UR-87-1539 & $\ldots$ & $4-2$ \\
\hline LA-UR-87-1546 & $\ldots$ & $32-39$ \\
\hline LA-UR-87-1566 & . & $40-35$ \\
\hline LA-UR-87-1570 & $\ldots$ & $4-97$ \\
\hline LA-UR-87-1581 & & $40-11$ \\
\hline LA-UR-87-1582 & & $40-12$ \\
\hline LA-UR-87-1592 & & $41-104$ \\
\hline LA-UR-87-1594 & . . & 41.99 \\
\hline LA-UR-87-1596 & & $3-7$ \\
\hline LA-UR-87-1598 & & 40.51 \\
\hline LA-UR-87-1599 & 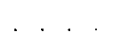 & $40-16$ \\
\hline LA-UR-87-1601 & & $40-52$ \\
\hline LA-UR-87-1631 & $\ldots$ & $32 \cdot 101$ \\
\hline
\end{tabular}

LA-UR-87-1637

LA.UR-87-1639

LA-UR-87-1640

LA-UR-87-1551

LA-UR-87-1657

LA-UR-87-1661

LA.UR-87-1662

LA.UR-87-1663

LA.UP-87-1674

LA-UR-87-1677

LA-UR-87-1681

LA-UR-87-1682

LA-UR-87-1696

LA-UR-87-1697

LA-UR-87-1702

LA-UR-87-1704

LA-UR-87-1705

LA-UR-87-1710

LA-UR-87-1715

LA-UR-87-1735

LA-UR-87-1745

LA-UR-87-1755

LA-UR-87-1763

LA-UR-87-1765

LA-UR-87-1770

LA-UR-87-1783

LA-UR-87-1803

LA-UA-87-1808

LA-UR-87-1811

LA-UR-87-1823

LA-UR-87-1843

LA-UR-87-1862

LA-UR-87-1870

LA-UR-87-1881

LA-UR-87-1889

LA-UP-87-1894

LA-UR-87-190

LA-UR-87-1909

LA-UR-87-1969

LA-UR-87-1985

LA.UR-87-1999

LA-UR-87-2096

LA-UR-87-2103

LA-UR-87-2109

LA-UR-87-2111

LA-UR-87-2124

LA-UR-87-2125

LA-UR-87-2126

LA-UR-87-2133

LA.UR-87-2134

LA.UR-87.2138

LA-UR-87-2162

LA.UR-87-2171
41-195

$43-74$

$4-93$

$1-37$

$14-62$

14-13

$14-22$

$31-43$

$24-36$

$39-16$

$40-2$

40-34

4-106

31-14

40-31

14-34

42-91

23-1

31-114

$40-21$

14-51

41-30

39-75

$31-73$

1-5

$31-30$

$30-15$

41-156

1.24

41-126

$1-52$

41-88

29-24

41-21

$1-62$

42-121

32-134

$1-49$

39-28

41-70

4-30

37.107

41-146

43-34

14-1

40-37

41-4

13-24

42-36

42-85

1-18

31-34

13-71

41-56

41.196 
LA UR $87-2180$

LA UR. 87.2192

LA UR 87.2198

LA UR.87.2199

LA. UR -87.2201

LA. UR. 87.2202

LA.UR-87.2206

LA. UR. 87.2212

LA.UR. 87.2213

LA.UP. $87-2216$

LA. UR. 87.2217

LA.UR-87-2218

LA.UR-87.2221

LA.UR-87.2225

LA UR -87-2225

LA-UR-87-2233

LA.UR-87-2249

LA-UR-87.2252

LA.UR-87-225B

LA.UR-87-2260

LA-UR-87-2268

LA-UR-87-2272

LA-UR-87-2276

LA.UR-87.2283

LA UR.87.2284

LA.UR. 87.2288

LA. UR. 87.2292

LA. UR. 87.2311

LA. UR-87.2315

LA. UR-87.2318

LA.UR- 87.2322

LA. UR. 87.2328

LA. UR. 87.2329

LA. UR. 87.2336

LA.UA. 87.2355

LA. UF. 87.2367

LA UR. 87.2378

LA. UR. 87.2383

LA. UR. 87.2386

LA.UR. $87-2393$

LA. UR. 87.2397

LA. UR-87.2414

LA.UR.87.2416

LA.UR. 87.2429

LA-UR-87.2431

LA.UR-87.2446

LA. UR. 87.2448

LA. UR. 87.2465

LA. UR. 87.2466

LA. UR-87-2481

LA. UR-87-2483

LA. UR 87.2487

LA. UR. 87.2488
41.174

24-33

14.4

42.22

41-163

41.169

$39-22$

35- 13

41-118

41-186

14-37

41-158

$31-112$

41.20

31.70

13.76

2.3

39.4

39-56

41. 154

41.138

32.23

32.55

41.204

12.4

41.90

39-36

31-97

39.46

10.2

24-10

41.43

41.113

41.189

31. 71

13.70

4.95

37-62

2. 18

41.172

42-19

31.49

$39-41$

42-122
LA. UR. 87.2494

LA. UR- $87-2500$

LA-UR-87.2506

4-110

39-62

41.41

24-12

LA-UR-87-2508

41-200

LA-UR-87-2527

45-2

LA-UR-87.2534

41. 179

LA.UR-87.2548

LA.UR.87.2549

LA.UR.87.2554

LA.UR-87.2588

LA.UR-87.2606

LA. UR. 87.2610

LA.UR-87-2616

LA.UR. 87.2618

LA.UR-87-2620

LA-UR-87-2634

LA-UR-87-2636

LA.UR-87-2637

LA.UR-87-2647

LA.UR-87-2651

LA-UR-87-2655

LA.UR-87.2656

LA-UR-87.2662

LA.UR. 87-2663

LA.UR. 87.2675

LA.UR. 87.2679

LA.UR-87-2691

LA-UR-87-2702

LA.UR. 87.2706

LA.UR-87.2709

LA.UR- 87.2719

LA-UR-8?-2729

LA.UR-87-2734

LA-UR-87-2735

LA-UR-87-2747

LA-UR.87.2754

LA. UA- $87-2768$

LA. UR-87-2772

LA.UR.87-2773

LA.UR.87.2787

LA.UR-87-2788

LA-UR-87-2797

IA.UR-87.2807

LA.UR-87.2809

LA-UR-87-2810

LA.UR-87.2814

LA.UR-87.2815

LA. UR-87.2818

LA. UR-87.2828
LA.UR-87-2829

LA.UR-87.2832

LA-UR-87-2833

LA.UR-87-2854

LA-UR-87-2863

LA-UA-87-2869

LA-UR-87-2873

LA.UR-87-2876

LA.UR-87-2878

LA.UR-87.2879

LA.UR-87.2886

LA-UR-87-2887

LA.UR-87-2889

LA.UR-87-2897

LA-UR-87-2909

LA-UA-87-2920

LA.UA.87.2928

LA-UR-87-2932.

LA-UR-87-2946

LA.UR-87-2949

LA-UR-87.2971

LA-UR-87-2981

LA-UR-87-2982

LA-UR-87-2985

LA.UR-87.2993

LA. UR-87-2994

LA-UR-87-3010

LA-UR-87.3012

LA.UR-87.3013

LA-UR-87-3021

LA-UR-87.3022

LA.UR-87.3026

LA-UR-87-3051

LA-UR-87-3052

LA-UR-B7-3059

LA-UR-87-3067

LA-UR-87-3085

LA-UR-87-3089

LA-UR-87-3090

LA-UR-87-3092

LA.UR-87-3095

LA-UR-87-3113

LA-UR-87-3121

LA.UR-87-3125

LA.UR-87-3129

LA.UR-87-3130

LA.UR-87-3131

LA.UR-87.3132

LA.UR-87.3142

LA.UR-87-3144

LA.UR-87.3966

LA-UR-87-3168

LA-UR-87-3974

U.UR.87.3176
$45-7$

31-65

31.19

44-11

40-66

41-184

31-11

13-49

41.58

$32-66$

44-6

41.28

24-41

41-24

42-51

482

41-177

4-98

14-49

1.16

41-109

13-75

42.93

44-14

40-70

40.103

40-3

2-10

$13-80$

41-128

2-9

24-52

14-23

$14-60$

40-60

45-5

21-3

24-50

32-94

37. 18

4. 13

45-8

31- 102

41. 162

43-29

32-112

$17-29$

18-16

4-24

4).190

41.12

4). 137

4-21

$1-63$

21.2 


\begin{tabular}{|c|c|c|c|c|c|c|}
\hline LA-UR-87-3177 & 37.79 & LA-UR-87-3575 & $\ldots$ & LA.UR-87-3924 & $\ldots$ & $32-111$ \\
\hline LA.UR-87-3187 & $4-37$ & LA-UR-87-3582 & $13-52$ & " & . & $1-47$ \\
\hline LA-UA-87-3205 & . . 41-120 & LA-UR-87-3590 & $\ldots$ & LA-UAF-87-3929 & . & 41-82 \\
\hline LA-UA-87-3206 & . . $42-45$ & LA-UR-87-3591 & $\ldots$ & LA-UR-87-3937 & . & $4-53$ \\
\hline LA-UR-87-3207 & . . . . 39-3 & LA-UR-87-3596 & $\ldots$ & LA-UR-87-3941 & . & 43-51 \\
\hline LA-UR-87-3209 & $\ldots$ & LA-UR-87-3597 & $\ldots$ & LA-UR-87-3950 & . . & $13-36$ \\
\hline LA-UA-87-3210 & $29-17$ & LA-UR-87-3603 & . . . & LA-UR-87-3951 & . & $4-90$ \\
\hline LA-UR-87-3217 & ... & LA-UR-87-3609 & $\cdots$ & LA-UR-87-3964 & . & $37-42$ \\
\hline LA-UR-87-3219 & . . 41-192 & LA-UR-87-3617 & $\ldots \quad 31-84$ & LA-UR-87-3974 & . & $4-46$ \\
\hline LA-UR-87-3222 & $\ldots . \quad 17-22$ & LA-UR-87-3621 & . . . . 32-129 & LA-UR-87-3979 & . . & $31-40$ \\
\hline LA-UR-E7-3230 & $\ldots \ldots 17-9$ & LA-UR-87-3623 & $\ldots \quad 40-17$ & LA-UR-87-3980 & $\ldots$ & $43-5$ \\
\hline LA-UR-87-3235 & $\ldots 40-83$ & LA-UR-87-3630 & . . $40-50$ & LA-UR-87-4002 & $\ldots$ & . $4-84$ \\
\hline LA-UR-87-3253 & $\ldots$ & LA-UR-87-3631 & . . . $39-79$ & LA-UR-87-4003 & $\ldots$ & $4-43$ \\
\hline LA-UA-87-3258 & $\ldots .37-30$ & LA-UR-87-3649 & $\ldots \quad 32-11$ & LA-UR-87-4010 & $\ldots$ & $17-30$ \\
\hline LA-UR-87-3279 & $\ldots \quad 24-49$ & LA-UR-87-3652 & $\ldots .41-131$ & LA-UR-87-4015 & $\cdots$ & $13-31$ \\
\hline LA-UR-87-3296 & $\ldots \quad 40-95$ & LA-UR-87-3654 & $\ldots 1-4$ & LA-UR-87-4016 & . . & $26-4$ \\
\hline LA-UR-87-3301 & $\ldots .32-95$ & LA-UR-87-3657 & ... 24.40 & LA-UR-87-4018 & $\ldots$ & $30-12$ \\
\hline LA-เ'9-87-3312 & $\ldots 40-71$ & LA-UR-87-3660 & $\ldots .441-149$ & LA-UR-87-4022 & $\ldots$ & $1-3$ \\
\hline LA-UR-87-3313 & $\ldots \quad 43-25$ & LA-UR-87-3664 & $\ldots 40-72$ & LA-UA-87-4033 & $\ldots$ & $4-23$ \\
\hline LA-UR-87-3325 & $\ldots \quad 44-15$ & LA-UR-87-3665 & $\ldots 40-46$ & LA-UR-87-4047 & $\cdots$ & $40-33$ \\
\hline LA-UR-87-3332 & $\ldots .39-80$ & LA-UR-87-3680 & $\ldots \quad 42-117$ & LA-UR-87-4049 & $\cdots$ & $39-76$ \\
\hline LA-UR-87-3333 & $\ldots, \quad 43-47$ & LA-UR-87-3684 & ... 41-96 & LA-UR-87-4050 & $\ldots$ & 41-45 \\
\hline LA-UR-87-3334 & $\ldots \quad 41-44$ & LA-UR-87-3686 & $\ldots$ 41-181 & LA-UR-87-4064 & $\ldots$ & $41-2$ \\
\hline LA-UR-87-3346 & $\ldots .30-2$ & LA-UR-87-3697 & . . . . 39-23 & LA-UR-87-4076 & $\ldots$ & $4-45$ \\
\hline LA-UR-87-3352 & $\ldots \ldots$ 4-15 & LA-UR-87-3700 & ... 31-66 & LA.UR-87-4079 & $\ldots$ & $23-7$ \\
\hline LA-UA-87-3354 & . . . 14-21 & LA-UR-87-3701 & ... 31-104 & $=\quad$. & $\ldots$ & . $31-38$ \\
\hline LA-UR-87-3355 & $\ldots \quad 20-3$ & LA-UR-87-3703 & $\ldots 42-113$ & LA-UR-87-4100 & $\ldots$ & $41-220$ \\
\hline$\cdot \quad \ldots$ & .... 14-8 & LA-UR-87-3709 & $\ldots \quad 42-94$ & LA-UR-87-4102 & $\ldots$ & $42-29$ \\
\hline LA-UR-87-3357 & $\ldots 40-15$ & LA-UR-87-3717 & . . 31-119 & LA-UR-87-4103 & $\ldots$ & $32-7$ \\
\hline LA-UR-87-3387 & . . . . $42-9$ & LA-UR-87-3723 & ... $41-38$ & LA-UR-87-4104 & . . & $40-58$ \\
\hline LA-UR-87.3399 & ... $29-25$ & LA-UR-87-3729 & $\ldots \quad 42-16$ & LA-UR-87-4119 & . & $43-49$ \\
\hline LA-UR-87-3407 & . . $39-38$ & LA-UR-87-3770 & $\ldots \ldots 1-28$ & LA-UR-87-4125 & . . & $19-6$ \\
\hline LA-UR-87-3418 & $\ldots \quad 42-17$ & LA-UR-87-3779 & $\ldots . .4-8$ & LA-UR-87-4129 & $\cdots$ & $14-58$ \\
\hline LA-UR-87-3423 & $\ldots .41-161$ & LA-UR-87-3780 & $\ldots$ & LA-UR-87-4138 & $\ldots$ & $41-115$ \\
\hline LA-UR-87-3426 & $\ldots \quad 30-22$ & LA-UR-87-3787 & $\ldots$ & LA-UR-87-4143 & $\ldots$ & $42-43$ \\
\hline LA-UR-87-3440 & . . . . 40-99 & LA-UR-87-3788 & ... $24-35$ & LA-UR-87-4146 & $\ldots$ & $43-30$ \\
\hline LA-UR-87-3448 & $\ldots$ & LA-UR-87-3803 & . . 31-113 & - . . & $\ldots$ & $32-59$ \\
\hline LA-UR-87-3462 & $\ldots \ldots$ & LA-UR-87-3807 & $\ldots . .4-12$ & LA-UR-87-4147 & $\cdots$ & $32-4$ \\
\hline LA-UR-87-3463 & $\ldots$ & LA-UR-87-3823 & $\ldots \quad 39-14$ & LA-UR-87-4154 & $\ldots$ & $41-59$ \\
\hline LA-UR-87-3464 & ... $30-19$ & LA-UR-87-3831 & $\ldots .41-134$ & LA-UF-87-4156 & $\cdots$ & $39-43$ \\
\hline LA-UR-87-3465 & $\ldots 30-16$ & LA-UR-87-3838 & $\ldots \quad 40-64$ & LA-UR-87-4159 & $\ldots$ & $40-4$ \\
\hline LA-UA-87-3466 & $\ldots$ & LA-UR-87-3839 & $\ldots \quad 47-40$ & LA-UR-87-4161 & $\ldots$ & 26-5 \\
\hline LA-UR-87-3467 & $\ldots 30-14$ & LA-UR-87-3844 & $31-45$ & LA-UR-87-4162 & $\ldots$ & $26-7$ \\
\hline LA-UR-87-3468 & $\ldots$ & LA-UR-87-3846 & ... & LA-UR-87-4167 & $\ldots$ & $39-70$ \\
\hline LA-UR-87-3477 & $\ldots$ & LA-UR-87-3848 & . . . $39-72$ & LA-UR-87-4170 & $\ldots$ & $41-13$ \\
\hline LA-UR-87-3494 & ... 31-69 & LA-UR-87-3854 & $\ldots \ldots 3^{35-6}$ & LA-UA-87-4171 & $\ldots$ & $26-6$ \\
\hline LA-UR-87-3498 & . . . 32-34 & LA-UR-87-3856 & $\ldots 42-110$ & LA-UR-87-4181 & $\ldots$ & $32-139$ \\
\hline LA-UR-87-3506 & ... 41-197 & LA-UR-87-3859 & $\ldots .41 .143$ & $\cdot \ldots$ & . . & $41-216$ \\
\hline LA-UR-87-3512 & . . . $39-63$ & LA.UR-87-3880 & $\ldots .41-203$ & LA-UR-87-4182 & . . & $41-32$ \\
\hline LA-UR-87-3531 & . . 31-109 & LA-UR-87-3888 & $\ldots .31-93$ & LA-UR-87-4197 & $\ldots$ & $40-18$ \\
\hline " $\quad \ldots$ & . . . 41-210 & LA.UR-87-3909 & $17-8$ & LA-UR-87-4199 & $\ldots$ & $40-76$ \\
\hline LA-UR-87-3537 & $\ldots \quad 41-71$ & LA-UR-87-3917 & $\ldots \quad 31-81$ & LA-UR-87-4209 & . . & 1.25 \\
\hline LA-UR-87-3540 & . . . 24-9 & LA-UR-87-3918 & $4-27$ & LA-UR-87-4212 & $\cdots$ & . 1.46 \\
\hline LA-UR-87-3558 & ... 24-13 & $\cdot$ & $\ldots \quad 31-15$ & LA-UR-87-4214 & & . $42-1$ \\
\hline LA-UR-87-3574 & $\ldots 31.50$ & LA-UR-87-39:22 & $\ldots .42-53$ & LA-UR-87-4218 & . & $14-53$ \\
\hline
\end{tabular}




\begin{tabular}{|c|c|c|}
\hline A-UA-87-4221 & 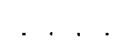 & 39-33 \\
\hline LA.UR-87-4225 & . & $44-1$ \\
\hline LA-UR-87-4231 & . . & $27-2$ \\
\hline LA-UR-87.4232 & 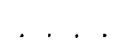 & 44-3 \\
\hline LA-UR-87-4240 & & 4.7 \\
\hline - & & $41-3$ \\
\hline LA-UR-87-4244 & $\ldots$ & $41-75$ \\
\hline LA.UR-87-4245 & 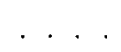 & $37-73$ \\
\hline LA-UR-87-4254 & . . & $31-39$ \\
\hline LA-UR-87-4255 & . & $32-87$ \\
\hline LA.UR-87-4256 & . & $32-46$ \\
\hline LA.UP.87-4266 & . . & $14-35$ \\
\hline LA-UR-87-4270 & 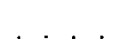 & $41-178$ \\
\hline LA-UR-87-4275 & . . & 47.34 \\
\hline LA.UR-87-4277 & $\cdot$ & $30-10$ \\
\hline LA-IJA-87-4288 & & $17-5$ \\
\hline LA-UR-87-4297 & 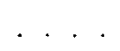 & $42-79$ \\
\hline LA-UR-87-9008 & & $33-2$ \\
\hline LA.UR-87-9057 & $\cdot$ & $14-7$ \\
\hline LA-UR-88-0003 & $\cdots$ & 4-112 \\
\hline LA-UR-88-0004 & $\ldots$ & $13-25$ \\
\hline LA-UR-88-0005 & $\ldots$ & $31-53$ \\
\hline LA-UR-88-0007 & . . & $31-101$ \\
\hline LA-UR-88-0011 & . & $41-152$ \\
\hline LA.UR-88-002? & . . & $7-3$ \\
\hline LA-UR-88-0044 & $\ldots$ & $42-42$ \\
\hline LA.UR-88-0045 & . & $31-18$ \\
\hline LA.UR-88-0048 & $\cdots$ & 3958 \\
\hline LA.UR-88-0051 & . & $41-144$ \\
\hline LA-UR-B8-0055 & . & $47-26$ \\
\hline LA-UR-88-0059 & $\ldots$ & $43-40$ \\
\hline LA-UR-88-0066 & . . & $41-139$ \\
\hline LA.UR-88-0078 & $\ldots$ & $47-22$ \\
\hline LA.UR-88-0079 & $\ldots$ & $47-41$ \\
\hline LA-UR-88-0094 & $\ldots$ & $47-44$ \\
\hline LA.UR-88-0096 & . . . & $42-40$ \\
\hline LA-UR-88-0097 & . & $43-83$ \\
\hline LA-UR-88-0099 & 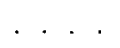 & $47 \cdot 12$ \\
\hline LA-UR-88-0100 & . & $12-5$ \\
\hline LA-UR-88-0102 & 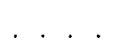 & 47.3 \\
\hline LA-UR-88-0109 & $\cdot$ & $14-36$ \\
\hline LA.UR-88-0111 & . & $32-62$ \\
\hline LA-UR-88-0113 & & $14-61$ \\
\hline LA-UR-88-0118 & 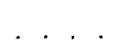 & $39-54$ \\
\hline LA-UR-88-0121 & & $12-3$ \\
\hline LA-UR-88-0122 & & 44-12 \\
\hline LA-UR-88-0125 & & 1.31 \\
\hline - & & $32-74$ \\
\hline LA-UR-88-0126 & . & $41-124$ \\
\hline LA-UR-88-0132 & . & $40-67$ \\
\hline LA.UR-88-0133 & . & $41-215$ \\
\hline LA.UR-88-0152 & & $39-26$ \\
\hline LA.UR-88-0153 & & $42-61$ \\
\hline LA-UR-88-0154 & & $23-13$ \\
\hline LA-UR-8E-0164 & & $41-108$ \\
\hline
\end{tabular}

LA-UR-88-0167

LA.UR-88-0169

LA-UR-88-0172

LA-UR-88-0177

LA-UR-88-0186

LA-UR-8B-0192

LA-UR-88-0194

41-170

43-13

... 4434

... 17-31

4-108

41-151

17-26

32-121

LA-UR-88-0196

$40-77$

LA-UR-88-0197

LA-UR-88-0205

LA-UR-88-0213

LA-UR-88-0214

LA-UR-88-0218

LA-UR-88-0224

LA-UR-8B-0250

LA-UR-88-0252

LA-UR-89-0266

LA-UR-88-0267

LA-UR-88-0269

LA-UR-88-0313

LA-UR-88-0317

LA-UR-88-0336

LA.UR-88-0344

LA-UR-88-0347

LA-UR-88-0354

LA-UR-8B-0355

LA-UR-88-0358

LA-UR-88-0361

LA-UR-88-0362

LA.UR-88-0366

LA-UR-88-0376

LA-UR-88-0383

LA-UR-88-0384

LA-UR-88-0388

LA-UR-88-0391

LA-UR-88-0392

LA-UR-88-0395

LA-UR-88-0400

LA-UR-82-0409

LA-UR-88-0413

LA.UR-88-0414

LA-UR-88-0415

LA-UR-88-0418

- ...

LA.UA-88-0421

LA-UA-88-0424

LA-UR-88-0426

LA-UR-88-0427

LA-UR-B8-0428

LA-UR-88-10443

LA-UR-88-0444

LA-UR-88-0446

LA-UR-88-0450

LA.UR-88-0460
$40-57$

$42-152$

4-41

4-94

42-101

1-8

45-1

35-8

$1-10$

1-60

41-155

13-69

A-55

26-3

1-29

41-47

31-17

37-21

$32-40$

1-44

1-38

$39-17$

42-81

39-6

39-7

$39-42$

35-12

43-4

14-17

3961

23-10

1-61

31-21

4-33

1-30

32-72

42-57

47-27

43-36

31-86

41-214

29-13

29-12

14-18

1.1

43-75
LA-UR-88-0466

LA-UR-88-0474

LA-UR-88-0477

LA-UR-88-0484

LA-UR-88-0486

LA-UR-88-0488

LA.UR-88-0508

LA-UR-88-0535

LA.UR-88-0540

LA-UR-88-0547

LA-UR-88-0555

LA-UR-88-0568

LA-UR-88-0573

LA-UR-88-0584

LA-UR-88-0588

LA-UR-88-0589

LA-UR-88-0592

LA-UR-88-0596

LA-UR-88-0597

LA-UR-88-0598

LA-UR-B8-0599

LA-UR-88-0604

LA-UR-88-0623

LA-UR-88-0625

LA-UR-8B-0626

LA-UR-8B-0629

LA-UR-8B-0633

LA-UR-8B-0635

LA-UR-83-0636

LA-UR-88-0640

- . . .

LA-UR-88-0644

LA-UR-88-0649

LA-UR-8B-0656

- . .

LA-UR-88-0669

LA-UR-88-0672

LA-UR-88-0674

LA-UR-88-0689

LA-UR-88-0693

LA-UR-88-0696

LA-UR-88-07CO

LA-UR-88-0712

UA-UR-88-0719

UA-UR-88-0720

LA-UR-89-0737

LA-UR-88-0748

LA-UR-88-0753

LA-UA-88-0758

LA-UR-88-0768

LA-UR-88-0771
$4-29$

24-45

464

40-92

$14-50$

40-28

41-150

i4-44

31-56

$17-21$

298

23-3

13-3

$1-53$

31-91

$31-9$

$3-2$

41-206

31-108

31-51

$40-29$

24-30

31-35

43-1i

$31-33$

$42-32$

43-31

13-5i

31-82

41-121

17-15

$4-52$

$31-67$

$4-25$

41-03

24-54

499

$31-105$

41-222

$30-17$

$31-60$

41-19

1-17

$13-50$

13-55

$14-16$

413

43-40

39-45

42-25

$4-81$

31-72

418

4)-171

$30-1$ 


\begin{tabular}{|c|c|c|}
\hline LA-UR-88-0781 &. & 42 \\
\hline LA.UR-88-0782 & $\cdots$ & 13 \\
\hline LA-UA-88-0788 & & $41-77$ \\
\hline LA.UR-88-0789 & 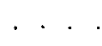 & 13.53 \\
\hline LA-UR-88-0797 & $\ldots$ & $? 4-23$ \\
\hline LA-UR-88-0798 & $\ldots$ & $24-4$ \\
\hline LA-UR-88-0801 & $\ldots$ & $41-60$ \\
\hline LA-UR-88-0809 & . . . & $31-88$ \\
\hline LA-UR-88-0811 & $\cdots$ & $29-18$ \\
\hline LA.UR-88-0814 & . . & $29-6$ \\
\hline LA-UA-88-0820 & $\ldots$ & $14-26$ \\
\hline LA-UR-88-0821 & $\ldots$ & 4-104 \\
\hline LA-UR-88-0822 & $\cdots$ & 24.44 \\
\hline LA-UR-88-0826 & . . & 40.41 \\
\hline LA-UR-88-0830 & $\cdots$ & $41-209$ \\
\hline LA-UR-88-0837 & . . & $37-72$ \\
\hline LA-UR-88-0843 & $\cdots$ & $31-29$ \\
\hline LA-UR-88-0845 & $\ldots$ & $30-6$ \\
\hline LA-UR-88-0847 & $\cdots$ & $13-46$ \\
\hline LA-UR-88-0848 & $\cdots$ & $41-188$ \\
\hline LA-UR-88-0849 & $\ldots$ & $31-110$ \\
\hline LA-UR-88-0854 & $\ldots$ & $31-68$ \\
\hline LA-UR-88-0860 & . & $42-63$ \\
\hline LA-UR-88-0863 & $\therefore$ & $42-119$ \\
\hline LA-UR-88-0865 & . . & $29-32$ \\
\hline LA-UR-88-0870 & $\cdots$ & $29-21$ \\
\hline LA-UR-88-0871 & ... & 29-31 \\
\hline LA-UR-88-0872 & 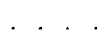 & $30-21$ \\
\hline LA-UR-88-0873 & . & $29-2$ \\
\hline LA-UR-88-0874 & . & $41-159$ \\
\hline LA-UR-88-0876 & . & $30-1$ \\
\hline LA-UR-88-0877 & . & $30-7$ \\
\hline LA-UR-88-0890 & . & $32-54$ \\
\hline LA-UR-88-0892 & $\cdots$ & $43-69$ \\
\hline LA-UR-88-0895 & . & $29-44$ \\
\hline LA.UR-88-0897 & 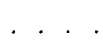 & $29-9$ \\
\hline LA-UR-88-0900 & . & $41-42$ \\
\hline LA-UR-88-0915 & . & $32-126$ \\
\hline LA-UR-88-0926 & $\ldots$ & 43-39 \\
\hline LA-UR-88-0938 & $\ldots$ & $1-50$ \\
\hline LA.UR-88-0944 & . & $42-48$ \\
\hline LA-UR-88-0947 & & $41-74$ \\
\hline LA-UR-88-0954 & . & $41-66$ \\
\hline LA.UR-88-0976 & 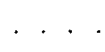 & $4-68$ \\
\hline$\cdot \quad \cdot$ & . & 31.62 \\
\hline LA-UR-88-0977 & . & $39-8$ \\
\hline LA-UR-88-0978 & . & $32-35$ \\
\hline LA-UR-88-0982 & $\cdot$ & $13-47$ \\
\hline LA-UR-88-0991 & . & $4-48$ \\
\hline$\cdot \quad .$. & $\cdots$ & $31-42$ \\
\hline LA-U:-88-0998 & . . & 43-32 \\
\hline LA-UR-88-1000 & . & $17-7$ \\
\hline LA-UR-88-1001 & $\therefore$ & $31-122$ \\
\hline LA-UR-88-1002 & & $31-46$ \\
\hline
\end{tabular}

\begin{tabular}{|c|c|c|c|c|c|}
\hline LA-UR-88-1015 & . & $31-55$ & LA-UR-88-1282 & . & 40.96 \\
\hline LA-UR-88-1023 & 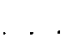 & $42-89$ & LA-UR-88-1298 & . . & $32-41$ \\
\hline LA-UR-88-1025 & & $44-9$ & LA-UR-88-1302 & 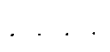 & $4-42$ \\
\hline LA-UR-88-1029 & . & $39-57$ & LA-UR-88-1303 & . & $24-25$ \\
\hline LA-UR-88-1048 & . . & 41.87 & LA-UR-88-1318 & . . & $31-48$ \\
\hline LA-UR-88-1049 & 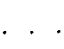 & $24-17$ & LA-UR-88-1326 & . . & $18-12$ \\
\hline LA-UR-88-1056 & & $39-48$ & LA-UR-88-1328 & $\ldots$ & 14.23 \\
\hline LA-UR-88-1058 & . & $37-86$ & LA-UR-88-1344 & $\ldots$ & $41-36$ \\
\hline LA-UR-88-1062 & . . & $39-34$ & LA-UR-88-1345 & . . & $40-98$ \\
\hline LA-UR-88-1070 & $\ldots$ & 37.77 & LA-UR-8a-1348 & . & $37-97$ \\
\hline LA-UR-88-1077 & $\ldots$ & $47-32$ & LA-UR-88-1349 & & $37-98$ \\
\hline LA-UR-88-1078 & . . & $4-44$ & LA-UR-88-1358 & $\ldots$ & $21-5$ \\
\hline LA-UR-88-1094 & . & $4-71$ & LA-UR-88-1364 & $\ldots$ & $16-15$ \\
\hline LA-UR-88-1103 & . & $29-34$ & LA-UR-88-1366 & $\ldots$ & $3-5$ \\
\hline LA-UR-88-1104 & $\ldots$ & $29-23$ & LA-UR-88-1384 & $\ldots$ & $32-133$ \\
\hline LA-UA-88-1106 & $\ldots$ & $41-64$ & LA-UR-88-1385 & . . & $47-38$ \\
\hline LA-UR-88-1107 & 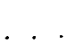 & $41-25$ & LA-LA-88-1393 & $\ldots$ & $41-185$ \\
\hline LA-UR-88-11C8 & . & $31-5$ & LA-UR-88-1394 & $\ldots$ & $32-138$ \\
\hline LA-UR-88-1110 & 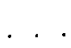 & $17-17$ & LA-UR-88-1407 & $\ldots$ & $4-35$ \\
\hline LA-UR-BB-1110-RE & & $17-16$ & LA-UR-88-1412 & . . & $4-63$ \\
\hline LA-UR-88-1115 & $\ldots$ & $41-7$ & LA-UR-88-1423 & $\ldots$ & $32-60$ \\
\hline LA-UR-88-1116 & $\ldots$ & $42-83$ & LA-UR-88-1446 & ... & $41-59$ \\
\hline LA-UR-88-1121 & $\ldots$ & $24-62$ & LA-UR-88-1450 & $\ldots$ & 24-11 \\
\hline LA-UR-88-1124 & . . & $41-18$ & LA-UR-88-1452 & $\cdots$ & $18-15$ \\
\hline LA-UR-88-1126 & $\ldots$ & $41-202$ & LA-UA-88-1458 & . . & $24-29$ \\
\hline LA-UR-88-1139 & $\ldots$ & $41-18 C$ & LA-UR-88-1461 & $\ldots$ & $40-1$ \\
\hline LA-UR-88-1146 & . & $42-41$ & LA-UR-88-1465 & $\ldots$ & $47-8$ \\
\hline LA-UR-88-1166 & . . & $32-85$ & LA.UR-88-1467 & $\ldots$ & $31-111$ \\
\hline LA-UR-88-1167 & $\ldots$ & 31.89 & LA-UR-88-1470 & . . & $32-64$ \\
\hline LA-UR-88-1168 & $\ldots$ & $31-52$ & LA-UR-88-1474 & $\ldots$ & $41-34$ \\
\hline LA-UR-88-1172 & . . & $39-77$ & LA-UR-88-1476 & $\ldots$ & $37-29$ \\
\hline LA-UR-88-1176 & $\ldots$ & $40-78$ & LA-UR-88-1482 & $\ldots$ & 43-24 \\
\hline LA.UR-88-1185 & $\ldots$ & $4-47$ & LA-UR-88-1485 & . . & 2938 \\
\hline LA-UR-88-1192 & . . & $17-19$ & LA-UR-88-1491 & . & $32-124$ \\
\hline LA-UR-88-1200 & . . & 37.84 & LA-UR-88-1494 & & $43-2$ \\
\hline LA-UR-88-1203 & $\ldots$ & 32.75 & LA-UR-88-1519 & $\ldots$ & $41-48$ \\
\hline LA-UR-88-1210 & . & $40-84$ & LA-UA-88-1525 & . . & $42-69$ \\
\hline LA-UR-88-1212 & $\ldots$ & $32-25$ & LA-UR-88-1526 & $\ldots$ & $42-68$ \\
\hline LA-UR-88-1215 & $\ldots$ & 37-105 & LA-UR-88-1550 & $\ldots$ & 4-16 \\
\hline LA-UR-88-1226 & $\ldots$ & $31-77$ & LA-UR-88-1533 & . & $9-1$ \\
\hline LA-UR-88-1229 & $\ldots$ & $13-10$ & $\cdot$ & . & $14-14$ \\
\hline LA-UR-88-1230 & . . & $29-35$ & LA-UR-88-1537 & . . & $37 \cdot 14$ \\
\hline LA-UR-88-1238 & $\ldots$ & $14-56$ & LA-UR-8B-1543 & . & $37-19$ \\
\hline LA-UR-88-1244 & . & $41-27$ & LA-UR-88-1544 & & $37-7$ \\
\hline LA-UR-88-1248 & . & $4-39$ & LA-UR-88-1545 & . . & $43-46$ \\
\hline LA-UR-88-1249 & . & $4-38$ & LA-UR-88-1546 & $\ldots$ & $37-28$ \\
\hline LA-UA-88-1250 & . & $4-36$ & LA-UR-88-1556 & $\cdots$ & $41-212$ \\
\hline LA.UR-88-1251 & $\ldots$ & 4.74 & LA-UR-BB-1560 & . & . $35-9$ \\
\hline LA-UA-88-1259 & $\ldots$ & $32-20$ & LA-UR-88-1562 & $\cdots$ & 37.111 \\
\hline LA-UR-88-1261 & $\ldots$ & $19-1$ & LA-UR-88-1569 & $\ldots$ & 4-101 \\
\hline LA-UR-88-1264 & . . & 40.97 & LA-UR-88-9570 & & $47-28$ \\
\hline LA.UR-88-1272 & & 42.65 & LA-UR-8B-158A & $\ldots$ & 1.26 \\
\hline LA-UR-83-1276 & & $\quad 39.67$ & LA-UR-88-1586 & . & $31-41$ \\
\hline$\cdot \quad$. & & $32-110$ & LA-UR-88-158: & . . & 31.100 \\
\hline LA-UR-88-1281 & & . 4.85 & LA-UA-8B-1590 & & $32-102$ \\
\hline
\end{tabular}




\begin{tabular}{|c|c|c|}
\hline LA.UR-88-1591 & & \\
\hline LA.UR-88-1598 & 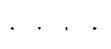 & \\
\hline LA.UR-88-1601 & & \\
\hline LA.UR. $88 \cdot 1603$ & 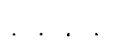 & $43-2$ \\
\hline LA-UR-88-1609 & $\cdots$ & $31-4$ \\
\hline LA-UR-88-1617 & $\cdots$ & 43 \\
\hline LA-UR-88-1618 & 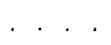 & $42-12$ \\
\hline LA.UR-88-1640 & 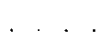 & $41-22$ \\
\hline LA.UR-88-1643 & $\cdots$ & $29-3$ \\
\hline LA-UR-88-1645 & $\cdots$ & $42-7$ \\
\hline LA.UR-88-1646 & $\cdots$ & 41-127 \\
\hline LA-UR-88-1647 & $\cdots$ & $41-211$ \\
\hline LA.UR-88- 1649 & & $41-65$ \\
\hline LA-UR-88-1650 & $\cdots$ & $42-5$ \\
\hline LA.UR. $88 \cdot 1655$ & & $41-46$ \\
\hline LA.UR-88-1662 & $\ldots$ & $42-58$ \\
\hline LA.UR-88-1663 & . . & $42-100$ \\
\hline LA-UR-88-1664 & $\cdots$ & $42-99$ \\
\hline LA-UR-B8-1671 & $\cdots$ & $39-71$ \\
\hline LA-UR-88-1672 & $\ldots$ & $39-40$ \\
\hline LA-UR-88-1675 & 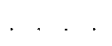 & $35-4$ \\
\hline LA.UA-88-1676 & . & $41-81$ \\
\hline LA-UR-88-1678 & $\cdots$ & 4-11 \\
\hline LA.UA-88-1682 & . . . & $37-68$ \\
\hline LA.UR-88-1687 & . & 37.83 \\
\hline LA.UR-88-1690 & . & 37.71 \\
\hline LA.UR-88-1691 & $\ldots$ & 32.77 \\
\hline LA.UR-88.1694 & & $\$ 1.114$ \\
\hline LA.UR-88-1695 & 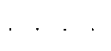 & $42-14$ \\
\hline LA.UA-88-1696 & & $42-33$ \\
\hline LA-UR-88-1697 & . & $42-90$ \\
\hline LA-UA-88-1698 & & 42.116 \\
\hline LA-UR-88-1715 & & $1-42$ \\
\hline LA-UR-88-1729 & $\cdot$ & 43-54 \\
\hline LA-UA-88-1734 &. & $39-24$ \\
\hline LA-UR-88-1736 & & $47-2$ \\
\hline LA-UA-88-1738 & & 37.41 \\
\hline LA-UR-88-1739 & . . & $42-4$ \\
\hline LA.UR-88-1740 & & $42-124$ \\
\hline LA.UR-88-1741 & & $40-74$ \\
\hline LA.UA-88-1742 & . & $41-11$ \\
\hline LA. UR-88-1743 & 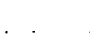 & $41-94$ \\
\hline LA-UA-88-1745 & & $42-18$ \\
\hline LA-UR-88-1746 & . & $43-57$ \\
\hline LA-UA-88-1748 & . & $39-21$ \\
\hline LA.UA-8B-1753 & & $42-3$ \\
\hline LA-UR-88-1762 & . & 42.2 \\
\hline LA-UR-88-1769 & . & 40.30 \\
\hline LA-UR-88-1788 &. & $39-39$ \\
\hline LA.UR-8B-1789 & . & $42-52$ \\
\hline LA.UR-88-1791 & . & $4-57$ \\
\hline LA-UR-88-1792 & & $32-128$ \\
\hline LA-UR-88-1797 & . & $37 \cdot 25$ \\
\hline LA-UR-8B-1800 & . & $37-24$ \\
\hline LA.UR-88-1802 & $\cdots$ & $39-51$ \\
\hline
\end{tabular}

\begin{tabular}{|c|c|c|}
\hline LA-UR & & 41-102 \\
\hline LA-UR-88-1812 & & $1-43$ \\
\hline LA-UR-88-1813 & 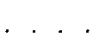 & $42-75$ \\
\hline LA-UR-88-1816 & . & $1-57$ \\
\hline LA-UR-88-1820 & . & $31-85$ \\
\hline LA-UR-88-1826 & & $37-8$ \\
\hline LA-UR-88-1827 & . & $37-69$ \\
\hline LA-UR-BB- 1829 & 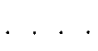 & $4-80$ \\
\hline LA-UR-88-1833 & & $42-88$ \\
\hline LA-UR-88- $\$ 836$ & & $32-82$ \\
\hline LA-UR-88-1843 & & $41-8$ \\
\hline LA.UR-88-1847 & . & $4-66$ \\
\hline LA-UR-88-1849 & . & 32.5 \\
\hline LA-UR-88-1857 & . & 43-35 \\
\hline LA-UR-88-1863 & . & $39-18$ \\
\hline LA-UA-88-1866 & . . & $41-49$ \\
\hline LA-UR-88-1878 & . . & $40-65$ \\
\hline LA-UR-88-1885 & . . & $12-1$ \\
\hline LA.UR-88-1892 & . . & $47-9$ \\
\hline LA-UR-88-1914 & . & 4-14 \\
\hline LA-UR-88-1919 & . . & $16-32$ \\
\hline LA-UR-88-1925 & $\cdots$ & $42-76$ \\
\hline LA-UR-88-1926 & . & $41-31$ \\
\hline LA-UR-88-1929 & . . & $47-39$ \\
\hline LA-UA-88-1930 & $\cdots$ & $47-13$ \\
\hline LA-UR-88-1932 & . . . & $41-98$ \\
\hline LA-UR-88-1940 & .. & $43-28$ \\
\hline LA.UR-88-1955; & . . & 41-101 \\
\hline LA-UR-88-195: & . & 43-85 \\
\hline LA-UR-88-1960) & $\ldots$ & $31-6$ \\
\hline LA-UR-88-1976 & . . & $42-123$ \\
\hline LA-UR-88-1987 & $\cdots$ & $41-164$ \\
\hline LA-UR-88-1998 & $\ldots$ & $32-43$ \\
\hline LA-UR-88-1991 & $\ldots$ & $47 \cdot 14$ \\
\hline LA-UR-88-1992 & $\cdots$ & $47-45$ \\
\hline LA-UR-88-1993 & $\cdots$ & $47-43$ \\
\hline LA-UR-88-1994 & . . & 47.42 \\
\hline LA-UR-88-1996 & $\ldots$ & $18-26$ \\
\hline LA-UR-88-2006 & $\cdots$ & $4-83$ \\
\hline LA-UR-88-2008 & $\cdots$ & $18-34$ \\
\hline LA-UR-88-2019 & $\ldots$ & $13-48$ \\
\hline LA-UR-88-2022 & . & $43-79$ \\
\hline LA-UR-88-2023 & 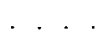 & $42-118$ \\
\hline LA.UA.88-2029 & . . & 23-11 \\
\hline LA-UFI-88-2043 & . . & 47.19 \\
\hline LA-UR-B8-2044 & . & $47-6$ \\
\hline LA-UJR-88-2045 & . & $17-10$ \\
\hline LA.UR-8B-2057 & . & $14-20$ \\
\hline LA-UR-8B-2062 & $\cdots$ & $42-12$ \\
\hline LA-UR-88-2071 & . . & $14-11$ \\
\hline LA-UR-88-2073 & & $24-58$ \\
\hline LA-UR-88-2076 & & $37-90$ \\
\hline LA-UR-88-2079 & $\cdots$ & $32-88$ \\
\hline LA.UR-88-2083 & . & 32.61 \\
\hline LA-UR-88-2084 & & $42-70$ \\
\hline
\end{tabular}

LA-UR-88-2109

LA.UR-88-2112

LA-UR-88-2128

LA.UR-88-2129

LA-UR-88-2130

LA-UP-88-2136

LA-UR-88-2140

LA-UR-88-2144

LA.UR-88-2180

I.A.UR-88-2182

LA.UR-88-2188

LA.UP-88-2189

LA-UR-88-2222

LA-UR-88-2223

LA.UR-88-2258

LA-UA-88-2260

LA-UR-88-2264

LA-UA-88-2265

LA.UR-88-2272

LA-UR-88-2277

LA-UR-88-2296

LA-UA-88-2309

LA.UR-88-2339

LA-UR-88-2340

LA-UA-88-2351

LA-UA-88-2370

LA-UR-88-2374

LA-UA-88-2376

LA-UR-88-2378

LA-UA-88-2380

LA-UR-88-2388

LA.UR-88-2391

LA.UR-88-2403

LA-UR-B8-2405

LA-UR-88-2421

LA-UR-88-2429

LA.UR-88-2439

LA-UR-88-2440

LA.UR-88-2451

LA.UR-88-2454

LA-UR-88-2458

LA-UR-88-2462

LA-UA-88-2465

LA-UR-88-2475

LA-UR-88-2482

LA-UR-88-2491

LA-UR-89-2498

4. UR-88-2507

LA.UR-88-2508

LA-UA-88-2512

LA-UR-88-2521

LA-UR-88-2531

LA-UR-88.2535

LA.UR-88-2536

LA.UR-88-2549
37-57

4-54

0.3

18-27

18-38

37-35

42-31

4-17

41-72

43-15

4-26

43-45

40-49

$40-48$

16-27

31-10

31-107

31-106

16-12

3968

42-8

31-61

41-145

42-80

43-50

18-37

37-2

14-48

4-56

42-13

29-4

32-117

40-56

13-12

475

32-21

13-20

$4-6$

4!-129

32-105

41-133

32-16

37.46

42-62

32-70

43-50

37-31

41.175

4-4

32-48

31-25

$29-10$

13-68

6-1

41. 191 


\begin{tabular}{|c|c|c|}
\hline & & \\
\hline & & \\
\hline & & \\
\hline & & \\
\hline UR.88-2572 & & \\
\hline -UR-88-2599 & & \\
\hline-2600 & & \\
\hline 608 & & \\
\hline & . . & \\
\hline-2613 & & \\
\hline & . . & \\
\hline 27 & & \\
\hline 31 & & $=$ \\
\hline 50 & & \\
\hline 2652 & & \\
\hline & & \\
\hline & & $43-4$ \\
\hline 60 & & 31.5 \\
\hline 3 & & 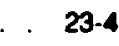 \\
\hline & & \\
\hline & & \\
\hline & & \\
\hline & & \\
\hline & & \\
\hline & & \\
\hline & & \\
\hline & . & 0 \\
\hline & & 0.8 \\
\hline & & 0.8 \\
\hline & & \\
\hline & & \\
\hline & & \\
\hline & . & $2-2$ \\
\hline & & $4-$ \\
\hline & & $4-7$ \\
\hline & & \\
\hline & & \\
\hline & & 政 \\
\hline & & \\
\hline & $\cdots$ & 3-4i \\
\hline & & 105 \\
\hline & & 26 \\
\hline & & 1.26 \\
\hline & & 29 \\
\hline & & 17.18 \\
\hline & & $4-88$ \\
\hline 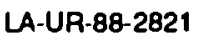 & & $9-38$ \\
\hline & & 32.11 \\
\hline & & \\
\hline & & $29-20$ \\
\hline & & $41-225$ \\
\hline -UF & & $41-4$ \\
\hline & & \\
\hline & & \\
\hline & & 29 \\
\hline
\end{tabular}

\begin{tabular}{|c|c|c|}
\hline LA-UR-88-2890 & & $29-42$ \\
\hline LA-UR-88-2893 & & $42-64$ \\
\hline LA-UR-88-2896 & & 41.176 \\
\hline LA-UR-88-2912 & & $29-28$ \\
\hline LA-UR-B8-2915 & - & 29-3 \\
\hline LA-UR-B8-2918 & & $42-44$ \\
\hline LA-UR-88-2920 & 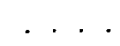 & $32-22$ \\
\hline LA-UR-88-2921 & $\ldots$ & $29-46$ \\
\hline LA-UR-88-2922 & $\ldots$ & $42-54$ \\
\hline LA-UR-88-2923 & $\cdots$ & $29-26$ \\
\hline LA-UR-88-2924 & $\ldots$ & 42.114 \\
\hline LA.UR-88-2954 & $\ldots$ & $1-15$ \\
\hline LA.UR-88-2962 & . . . & 41.160 \\
\hline LA-UR-88-2988 & . . & $41-136$ \\
\hline LA-UR-88-3006 & $\ldots$ & $37-76$ \\
\hline LA-UR-88-3008 & . & $13-61$ \\
\hline LA-UR-8B-3019 & . . & $31-75$ \\
\hline LA-UR-88-3020 & & $37-101$ \\
\hline LA-UR-88-3028 & . . & $42-97$ \\
\hline LA-UR-88-3029 & $\ldots$ & $42-95$ \\
\hline LA-UR-88-3030 & $\ldots$ & $43-72$ \\
\hline LA-UR-88-3031 & $\ldots$ & $39-59$ \\
\hline LA-UR-88-3036 & $\ldots$ & $31-47$ \\
\hline LA-UA-88-3041 & . & 42.56 \\
\hline LA-UR-88-3042 & $\ldots$ & $37-82$ \\
\hline LA-UR-88-3043 & . . . & $4-3$ \\
\hline LA-UR-88-3062 & $\ldots$ & $22-7$ \\
\hline LA-UR-88-3064 & $\ldots$ & $41-217$ \\
\hline LA-UR-88-3084 & $\ldots$ & $41-115$ \\
\hline LA-UR-88-3086 & . . . & $41-117$ \\
\hline LA-UR-88-3099 & $\ldots$ & $41-157$ \\
\hline LA-UR-89-3104 & $\ldots$ & 42-105 \\
\hline LA-UR-88-3124 & $\ldots$ & . $7-1$ \\
\hline LA-UR-88-3126 & $\ldots$ & $32-63$ \\
\hline LA-UR-88-3139 & $\ldots$ & 43-87 \\
\hline LA-UR-88-3145 &.. & $40-6$ \\
\hline LA-UR-88-3146 & . & 37.100 \\
\hline LA-UR-83-3148 & $\ldots$ & 13.73 \\
\hline LA-UR-88-3149 & . & $37-6$ \\
\hline LA-UR-88-3158 & & $26-2$ \\
\hline LA-UR-88-3176 & . . & $32-36$ \\
\hline LA-UR-88-3177 & $\ldots$ & $40-59$ \\
\hline LA-UR-88-3190 & . & $47-16$ \\
\hline LA-UR-88-3193 & $\ldots$ & 43-19 \\
\hline LA-UR-88-3205 & $\ldots$ & $37-27$ \\
\hline LA-UR-88-3206 & $\ldots$ & $32-24$ \\
\hline LA-UR-88-3219 & . & $40-22$ \\
\hline LA-UR-88-3236 & $\ldots$ & 37.75 \\
\hline LA-UR-88-3239 & $\ldots$ & $41-69$ \\
\hline LA-UR-88-3243 & & 4.49 \\
\hline LA-UR-88-3247 & $\cdots$ & $37-37$ \\
\hline LA-UA-88-3248 & 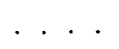 & $37-3$ \\
\hline LA-UR-88-3250 & & $3-8$ \\
\hline LA-UR-88-3251 & & 13.8 \\
\hline LA.UR-88-3252 & . . & $16-14$ \\
\hline
\end{tabular}

\begin{tabular}{|c|c|c|}
\hline A.UF & $\cdots$ & $16-13$ \\
\hline LA-UA-88-3258 & . & $37-45$ \\
\hline LA-UA-88-3259 & & $37-85$ \\
\hline LA-UR-88-3260 & 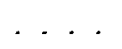 & $37-60$ \\
\hline LA-UR-88-3261 & $\ldots$ & $37-104$ \\
\hline LA-UR-88-3262 & $\ldots$ & $37-4$ \\
\hline LA-UR-B8-3263 & . . & $37-11$ \\
\hline LA-IJR-88-3264 & 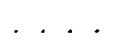 & 37.5 \\
\hline LA-UR-88-3271 & $\ldots$ & $40-20$ \\
\hline LA-UR-88-3272 & & $20-15$ \\
\hline LA-UR-88-3273 & $\ldots$ & $23-2$ \\
\hline LA-UR-88-3276 & $\ldots$ & $37-12$ \\
\hline LA-UR-88-3277 & $\cdots$ & 23-14 \\
\hline LA-UR-88-3278 & 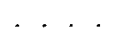 & $37-33$ \\
\hline LA-UR-88-3279 & . . & 488 \\
\hline LA-UR-88-3285 & . . & $13-34$ \\
\hline LA-UA-88-3294 & $\ldots$ & $46-1$ \\
\hline LA-UR-88-3296 & $\ldots$ & $41-219$ \\
\hline LA-UA-B8-3316 & $\ldots$ & $14-30$ \\
\hline LA-UR-88-3317 & $\ldots$ & 1400 \\
\hline LA-UR-88-3318 & 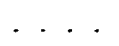 & $29-14$ \\
\hline LA-UR-88-3319 & $\cdots$ & $42-15$ \\
\hline LA-UR-89-3320 & $\cdots$ & $43-71$ \\
\hline LA-UR-88-3323 & . & $29-33$ \\
\hline LA-UR-88-3326 & $\cdots$ & $37-54$ \\
\hline LA-UR-88-3327 & $\cdots$ & $37-17$ \\
\hline LA-UR-88-3328 & - & $37-16$ \\
\hline LA-UR-88-3331 & $\cdots$ & $40-45$ \\
\hline LA-UR-88-3356 & . & 40.75 \\
\hline LA-UR-88-3341 & . . & 1430 \\
\hline LA-UR-88-3342 & . & $42-82$ \\
\hline LA-UR-88-3347 & 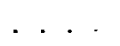 & 23-8 \\
\hline LA-UR-88-3348 & 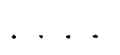 & $16-21$ \\
\hline LA-UR-88-3358 & & $41-122$ \\
\hline LA-UR-88-3363 & & $37-116$ \\
\hline LA-UR-88-3371 & & $32-37$ \\
\hline LA-UA-88-3972 & $\cdots$ & $32-18$ \\
\hline LA-UR-88-3375 & $\ldots$ & $29-16$ \\
\hline LA-UR-88-3397 & $\ldots$ & $32-71$ \\
\hline LA-UR-B8-3402 & ... & 43-38 \\
\hline LA-UR-88-3405 & 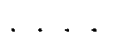 & $16-4$ \\
\hline LA-UR-88-3413 & & $31-57$ \\
\hline LA-UR-88-3414 & . & $32-100$ \\
\hline LA-UR-88-3418 & $\cdots$ & $14-27$ \\
\hline LA-UR-88-3436 & $\cdots$ & $13-62$ \\
\hline IA-UR-88-3454 & 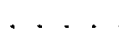 & $47-5$ \\
\hline LA-UR-88-3471 & $\cdots$ & . $17-3$ \\
\hline LA-UR-B8-3481 & $\cdots$ & $31-103$ \\
\hline LA-UR-88-3492 & & $31-60$ \\
\hline LA-UR-88-3522 & & $41-123$ \\
\hline LA-UR-88-3544 & & 1.11 \\
\hline LA-UR-89-3546 & $\cdots$ & 37.43 \\
\hline LA-UR-88-3550 & $\cdots$ & 469 \\
\hline LA-UA-88-3557 & & $16-28$ \\
\hline LA-UR-BB-3568 & & $42-50$ \\
\hline
\end{tabular}




\begin{tabular}{|c|c|c|c|}
\hline & & & \\
\hline & & & \\
\hline A. UR-88-3604 & & & \\
\hline $8-3612$ & & & \\
\hline 3 & & & \\
\hline 20 & & & \\
\hline 37 & & & \\
\hline A.UP & & & \\
\hline A.UF & & & - \\
\hline A.UP & & & \\
\hline A.UF & & & \\
\hline A.L & & & 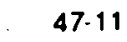 \\
\hline A.UF & & & $41-14$ \\
\hline A.UR. & & & $41-1$ \\
\hline A. UR & 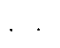 & & $4-6$ \\
\hline A.L & . & & $43-4$ \\
\hline A.UI & . & & $41-8$ \\
\hline$A-U$ & . & & $41-10$ \\
\hline A.UF & & & 47.3 \\
\hline A. $L$ & . & & 24.5 \\
\hline A.- & & & \\
\hline$A-U$ & & & \\
\hline A.U & & . & 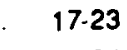 \\
\hline A.U & 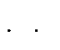 & & 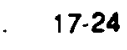 \\
\hline LA.U & 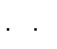 & & $\infty$ \\
\hline$L A-U$ & & & 31. \\
\hline A.U & & & $24-$ \\
\hline A.U & & 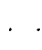 & $\cdot$ \\
\hline A.U & & & . \\
\hline A. 1 & & & . \\
\hline$A-U$ & & & 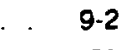 \\
\hline$A \cdot U$ & & & \\
\hline A.UR & 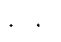 & & \\
\hline A.UR & & & \\
\hline A. UR-B & & & \\
\hline A.UF & & & \\
\hline -U & & . . & \\
\hline A.UR. & & & \\
\hline Q-UR & . & & 31.99 \\
\hline -UF & . & & ו \\
\hline 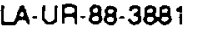 & & & \\
\hline & . & & \\
\hline & . & & \\
\hline & & . & \\
\hline -UF & & & \\
\hline Iu & & & 27 \\
\hline UA & & & 10 \\
\hline & & & \\
\hline & & & \\
\hline & & & \\
\hline 30 & & & \\
\hline & & & $41-97$ \\
\hline & & & 24-39 \\
\hline & & & 37.74 \\
\hline & & & 41.218 \\
\hline
\end{tabular}

\begin{tabular}{|c|c|c|c|}
\hline LA-UA-88-4229 & . & & $41-14$ \\
\hline LA.UR-88-4237 & . & & $13-4$ \\
\hline LA-UR-88-4257 & & & $14-9$ \\
\hline LA-UR-88-9086 & 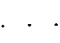 & & $40-63$ \\
\hline LA-UR-88-9096 & 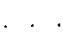 & & $22-9$ \\
\hline LA-UR-88-9098 & . & & $14-2$ \\
\hline LA-UR-88-9099 & . & & $32-108$ \\
\hline LA.UA-88-9100 & . & & $39-5$ \\
\hline LA-UA-88-9101 & . & . & $41-147$ \\
\hline LA-UR-B8-9102 & 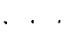 & & $39-37$ \\
\hline LA-UR-88-9103 & . & 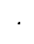 & 3912 \\
\hline LA-UR-88-9104 & . & 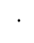 & 3910 \\
\hline LA-UR-88-9105 & . & & 31.20 \\
\hline LA-UR-88-9106 & $\cdot$ & & 41.79 \\
\hline LA-UR-88-9107 & . . & & $43-7$ \\
\hline LA-UR-88-9108 & $\cdots$ & & $18-28$ \\
\hline LA-UR-88-9110 & . . & & $18-36$ \\
\hline LA-UA-88-9111 & . . & & $31-1$ \\
\hline LA-UR-88-9112 & . . & & 42.26 \\
\hline LA.UR-88.9113 & . . & & $13-35$ \\
\hline LA-UR-88-9114 & . . . & & $43-14$ \\
\hline LA-UR-8B-9115 & . . . & & 42.55 \\
\hline LA-UR-88-9116 & . . . & . & $13-63$ \\
\hline LA.UR-88-9117 & . . . & 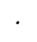 & $32-84$ \\
\hline LA-UR.88-9119 & . . & & 37.95 \\
\hline LA-UR-88-9120 & . . & & $42-84$ \\
\hline LA-UR-88-9121 & . . . & & $43-23$ \\
\hline LA-UR-88-9122 & . . & & 43-44 \\
\hline LA-UP-88-9123 & $\cdot \cdot$ & & $42-7$ \\
\hline LA.UA-88-9124 & · & & $17-28$ \\
\hline LA-UR-88-9125 & . & & $44-5$ \\
\hline LA-UR-88-9126 & $\therefore$ & . & $32-100$ \\
\hline LA-UR-88-9127 & . . . & '. & $40-100$ \\
\hline LA-UR-88-9128 & . . & . & . $34-5$ \\
\hline LA-UR-88-9129 & . & . & $34-4$ \\
\hline LA-UR-88-9130 & 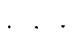 & . & $31-120$ \\
\hline LA-UR-88-9131 & . . . & . & 41.95 \\
\hline LA-UR-88-9132 & 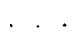 & $\cdot$ & $41-55$ \\
\hline LA-UR-88-9133 & . & . & $41-205$ \\
\hline LA.UR-88-9134 & . . & . & $32-68$ \\
\hline LA-UR-88-9135 & . . . & . & $32-6$ \\
\hline LA-UA-88.9136 & . . & . & $32-105$ \\
\hline LA-UA-88-9137 &.. & . & $41-5$ \\
\hline LA.UA-88-9138 & . . . & . & $43-53$ \\
\hline LA-UR-88-9139 & . & & $31-37$ \\
\hline LA-UR-88-9140 & $\cdot$ & . & $37-10$ \\
\hline LA-UR-88-9141 & $\cdot$ & 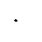 & 41.85 \\
\hline LA-UR-8B-9142 & 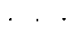 & . & 41.148 \\
\hline LA-UR-88-9143 & . & . & $31-76$ \\
\hline LA-UR-88-9144 & . & $\cdot$ & $14-47$ \\
\hline LA-UA.88-9145 & . . & 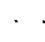 & . $27-6$ \\
\hline LA.UR.88-9146 & & & $2 T-1$ \\
\hline LA.UR.88-9147 & 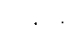 & & $19-30$ \\
\hline LALP.88-016 & & & $17-20$ \\
\hline LALP.B8.019 & & & 17.1 \\
\hline
\end{tabular}

U.S.PATENT-4.706.581 U.S.PATENT-4,718,321 U.S.PATENT-4,718,322 U.S.PATENT-4.719.375 U.S.PATENT-4,721,891 U.S.PATENT-4.721,914 U.S.PATENT-4.722,026 U.S.PATENT-4,722,201 U.S.PATENT-4,724,127 U.S.PATENT-4.726,829 U.S.PATENT-4.727,236 U.S.PATENT-4.729,318 U.S.PATENT-4.730.170 U S.PATENT-4,731,572 U.S.PATENT-4.731.840 U.S.PATENT-4,733,610 U.S.PATENT-4,734.177 U.S.PATENT $-4,735,145$ U.S.PATENT-4,743,407 U.S.PATENT $-4,745,277$ U.S.PATENT-4.755,326 U.S.PATENT-4,764,013 U.S.PATENT-4,778,263 U.S.PATENT-4.778.575 U.S.PATENT-4,780,608 USPATENT $4.783,322$ U.S.PATENT-4.784.686 . . 4.73 U.S.PATENT-4.789.537 , 31-94 U.S.PATENT-4.793.705 . . 4-96

31

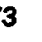

63 4-58 $29-45$ 6-30 16-29 43-76 2.73 37.65 31.99 13-1 $37-44$ $39-32$ 3931 3-B4 - 125 $37 \cdot 1$ 1.90 $39-47$ 37-23 42-11 41-97 24-39 1.218 
A. JOURNAL ARTICLES - 544 IN 152 JOURNALS

ACTA CRYSTALLOGRAPHICA - 5

ACTA METALLURGICA - 3

ADVANCES IN APPLIED MATHEMATICS - 2

AMERICAN INDUSTRIAL HYGIENE ASSOCIATION JOURNAL - 1 ANALYTICAL CHEMISTRY - 3

ANNALS OF PHYSICS - 1

ANNALS OF THE NEW YORK ACADEMY OF SCIENCES - 2

ANNUAL REVIEW OF PHYTOPATHOLOGY - 1

APPLIED OPTICS - 5

APPLIED PHYSICS LETTERS - 10

ASTROPHYSICAL JOURNAL - 15

ATOMIC DATA AND NUCLEAR DATA TABLES - 1

BIOCHEMICAL AND BIOPHYSICAL RESEARCH

COMMUNICATIONS - 1

BIOCHEMISTRY - 2

BIOPHYSICAL JOURNAL - 2

BIOTECHNIOUES - 1

BRAIN RESEARCH BULLETIN - 1

BULLETIN OF ALLOY PHASE DIAGRAMS - 3

BULLETIN OF THE AMERICAN ASTRONOMICAL SOCIETY - 1

CABIOS - 1

CANADIAN JOURNAL OF CHEMISTRY - 1

CANADIAN JOURNAL OF PHYSICS - 1

CANCER GENETICS AND CYTOGENETICS - 1

CANCER RESEARCH - 2

CARBOHYDRATE RESEARCH - 1

CHEMICAL PHYSICS LETTERS - 5

COMBUSTION AND FLAME - 1

COMMENTS ON NUCLEAR AND PARTICLE PHYSICS - 1

COMPUTER PHYSICS COMMUNICATIONS - 3

CYTOGENICS AND CELL GENETICS - 1

CYTOMETAY - $i$

CYTOMETRY SUPPLEMENT - 1

ENDOCPINOLOGY - 1

EXPERIMENTAL CELL RESEARCH - 1

FEW-BODY SYSTEMS - 2

FOUNDATIONS OF PHYSICS - 2

GEOLOGY - 1

GEOPHYSICAL AND ASTROPHYSICAL FLUID DYNAMICS - 1

GEOPHYSICAL RESEARCH LETTERS - 1

GEOSTANDAROS NEWSLETTER - 1

HAZARDOUS WASTE \& HAZARDOUS MATERIALS - 1

IEEE JOURNAL OF QUANTUM ELECTRONICS - 2

IEEE TRANSACTIONS ON ELECTROMAGNETIC COMPATIBILITY - 1

IEEE TRANSACTIONS NN F; ECTRON DEVICES - 1

IEEE TRANSACTIONS ON GEOSCIENCE AND REMOTE SENSING - 1

IEEE TRANSACTIONS ON INSTRUMENTATION AND

MEASUREMENT - 1

IEEE TRANSACTIONS ON MAGNETICS - 2

IEEE TRANSACTIONS ON PLASMA SCIENCE - 1

INORGANIC CHEMISTRY - 2

INORGANICA CHIMICA ACTA - 1

INTERNATIONAL JOURNAL OF MULTIPHASE FLOW - 1

INTERNATIONAL JOURNAL OF OUANTUM CHEMISTRY

SYMPOSIUM - 1

INTEQNATIONAL JOURNAL OF RADIATION BIOLOGY - 1
JOURNAL OF AEROSOL MEDICINE - 1

JOURNAL OF APPLIED CRYSTALLOGRAPHY - 3

JOURNAL OF APPLIED METEOROLOGY - 1

JOURNAL OF APPLIED PHYSICS - 13

JOURNAL OF ATMOSPHERIC SCIENCES - 1

JOURNAL OF BIOLOGICAL CHEMISTRY - 1

JOURNAL OF BIOMOLECULAR STRUCTURE \& DYNAMICS - 1

JOURNAL OF CARBOHYDRATE CHEMISTRY - 1

JOURNAL OF CELLULAR PHYSIOLOGY - 1

JOURNAL OF CELLULAR PLASTICS - 1

JOURNAL OF CHEMICAL PHYSICS - 23

JOURNAL OF COMPUTATIONAL PHYSICS - 4

JOURNAL OF ELECTROANALYTICAL CHEMISTRY - 1

JOURNAL OF ELECTRON MICROSCOPY TECHNIOUE - 2

JOURNAL OF FORENSIC SCIENCE - 1

JOURNAL OF FUSION ENERGY - 1

JOURNAL OF GEOMAGNETISM AND GEOELECTRICITY - 1

JOURNAL OF GEOPHYSICAL RESEARCH - 23

JOURNAL OF INTEGRAL EOUATIONS AND APPLICATIONS - 1

JOURNAL OF LESS-COMMON METALS - 8

JOURNAL OF MAGNETIC RESONANCE - 1

JOURNAL OF MATERIALS RESEARCH - 4

JOURNAL OF MATHEMATICAL PHYSICS - 4

JOURNAL OF METALS - 1

JOURNAL OF MOLECULAR SPECTROSCOPY - 3

JOURNAL OF NUCLEAR MATERIALS - 2

JOURNAL OF PHARMACOLOGY AND EXPERIMENTAL

THERAPEUTICS - 1

JOURNAL OF PHYSICAL CHEMISTRY - 4

JOURNAL OF PHYSICS B - 3

JOURNAL OF PHYSICS C - 2

JOURNAL OF POWER SOURCES - 1

JOURNAL OF QUANTITATIVE SPECTROSCOPY AND RADIATIVE

TRANSFER - 4

JOURNAL OF RADIOANALYTICAL AND NUCLEAR

CHEMISTRY - 1

JOURNAL OF REPRODUCTION AND FERTILITY - 1

JOURNAL OF SOLID STATE CHEMISTRY - 1

JOURNAL OF SPACECRAFT AND ROCKETS - 1

JOURNAL OF STATISTICAL PHYSICS - 1

JOURNAL OF THE ACOUSTICAL SOCIETY OF AMERICA - 3

JOURNAL OF THE AMERICAN CHEMICAL SOCIETY - 4

JOURNAL OF THE AMERICAN STATISTICAL ASSOCIATION - 1

JOURNAL OF THE ATMOSPHERIC SCIENCES - 1

JOURNAL OF THE ELECTROCHEMICAL SOCIETY - 3

JOURNAL OF THE OPTICAL SOCIETY OF AMERICA - 4

JOURNAL OF VACUUM SCIENCE AND TECHNOLOGY - 2

LANGMUIR - 2

LOG ANALYSIS - 1

LOS ALAMOS SCIENCE - 12

MAGNETIC RESONANCE IN MEDICINE - 2

MATERIALS SCIENCE AND ENGINEERING - 2

MATHEMATICAL BIOSCIENCES - 1

MECHANICS OF MATERIALS - 1

MODERN PHYSICS LETTERS - 1

MONTHLY WEATHER REVIEW - 1

MUON-CATALYZED FUSION - 3

NATURE - 4 
NON-LINEAR ANALYSIS; THEORY, METHODS *

APPLICATIONS - 1

NONLINEARITY - 1

NUCLEAR FUSION - $?$

NUCLEAR INSTRUMENTS AND METHODS IN PHYSICS

RESEARCH - 15

NUCLEAR PHYSICS - 8

NUCLEAR SCIENCE AND ENGINEERING - 6

NUMERICAL METHOOS FOR PARTIAL DIFFERENTIAL

EQUATIONS - 1

OPTICAL COMMUNICATIONS - 1

OPTICAL ENGINEERING - 3

OPTICS LETTERS - 3

ORGANOMETALLICS - 1

PARALLEL COMPUTING - 1

PHYSICA - 5

PHYSICA SCRIPTA - 2

PHYSICAL REVIEW - 107

PHYSICAL REVIEW : IETTERS - 23

PHYSICS OF FLUIDS - 26

PHYSICS LETTERS - 18

PHYSICS REPORTS - 1

PHYSICS TODAY - 1

PIAANT CELL REPORTS - 1

PLANT PHYSIOLOGY - 2

PROCEEDINGS OF THE AMERICAN MATHEMATICAL SOCIETY - 1

PAOCEEDINGS OF THE NATIONAL ACADEMY OF SCIENCES - 1 QUALITY PROGRESS - 1

QUARTERLY OF APPLIED MATHEMATICS - 1

RADIO SCIENCE - 2

RADIOACTIVE WASTE MANAGEMENT AND THE NUCLEAR

FUEL CYCLE - 1

RELIABILITY ENGINEERING AND SYSTEM SAFETY - 1
REVIEW OF SCIENTIFIC INSTRUMENTS - 7

SCIENCE - 2

SCRIPTA METALLUR,GICA - 3

SIAM JOURNAL ON APPLIED MATHEMATICS - 2

SIAM JOURNAL ON NUMERICAL ANALYSIS - 1

SOLAR PHYSICS - 1

SOLID STATE COMMUNICATIONS - 3

SOLVENT EXTRACTION AND ION EXCHANGE - 1

SPECTROCHIMICA ACTA - 1

SURGICAL FORUM - 1

SYNTHETIC METALS - 4

TALANTA - 1

TECHNOMETFICS - 1

WELDING JOURNAL - 2

ZEITSCHRIFT FUEA PHYSIK - 1

B. BOOKS - 4

C. CHAPTERS OF BOOKS AND ENCYCLOPEDIA ARTICLES - 29

D. PAPERS PUBLISHED IN CONFERENCE PROCEEDINGS - 423

E. ABSTRACTS OF PAPERS - 5

F. PUBLISHED STATEMENTS AT CONGAESSIONAL HEARINGS - 1

G. PATENTS - 29

H. LOS ALAMOS NATIONAL LABORATORY REPOATS - 238

1. PAPERS ISSUED AS LOS ALAMOS REPOATS BY TIC AND NTIS - 769

J. OTHER UNCLASSIFIED REPORTS - 2 